University of New Hampshire

University of New Hampshire Scholars' Repository

\title{
Surficial Geology of the Continental Shelf off New Hampshire: Morphologic Features and Surficial Sediment
}

\author{
Larry G. Ward \\ University of New Hampshire CCOM/JHC, Durham, Igward@ad.unh.edu \\ Zachary S. McAvoy \\ University of New Hampshire CCOM, Durham \\ Maxlimer Coromoto Vallee-Anziani \\ University of New Hampshire CCOM, Durham \\ Rachel C. Morrison \\ University of New Hampshire CCOM, Durham
}

Follow this and additional works at: https://scholars.unh.edu/ccom

Part of the Geology Commons, Geomorphology Commons, Oceanography Commons, and the Sedimentology Commons

\section{Recommended Citation}

Ward, L.G., McAvoy, Z.S., Vallee-Anziani, M., and Morrison, R.C., 2021, Surficial Geology of the Continental Shelf off New Hampshire: Morphologic Features and Surficial Sediment: BOEM/New Hampshire Cooperative Agreement (Contract M14AC00010) Technical Report, Department of Interior, Bureau of Ocean Energy Management, Marine Minerals Division, 45600 Woodland Road, Sterling, VA, 20166, 184pp. https://dx.doi.org/10.34051/p/2021.31

This Report is brought to you for free and open access by the Center for Coastal and Ocean Mapping at University of New Hampshire Scholars' Repository. It has been accepted for inclusion in Center for Coastal and Ocean Mapping by an authorized administrator of University of New Hampshire Scholars' Repository. For more information, please contact Scholarly.Communication@unh.edu. 

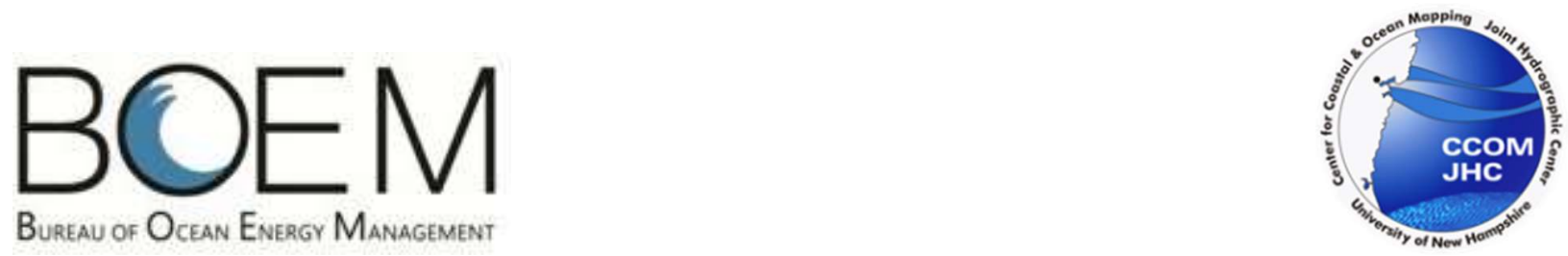

BOEM/New Hampshire Cooperative Agreement (Contract M14ACO0010) Technical Report

\section{Surficial Geology of the Continental Shelf Off New Hampshire: Morphologic Features and Surficial Sediment}

By Ward, L.G., McAvoy, Z.S., Vallee-Anziani, M., and Morrison, R.C.

University of New Hampshire Center for Coastal and Ocean Mapping/Joint Hydrographic Center 24 Colovos Road, Durham, NH 03824
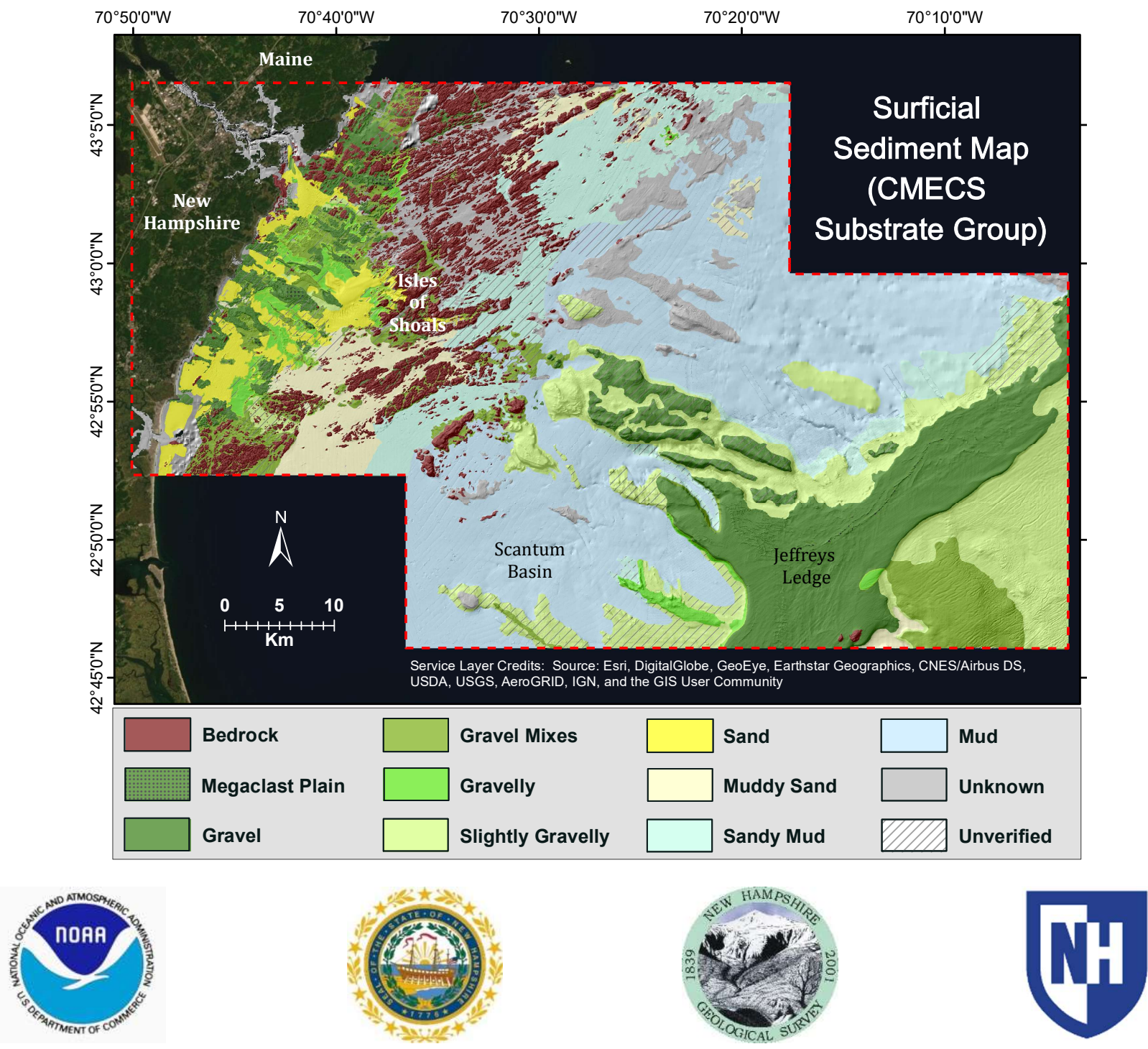


\section{Acknowledgements}

The development of the "Surficial Geology of the Continental Shelf off New Hampshire: Morphologic Features and Surficial Sediment" report and maps was supported by the Bureau of Ocean Energy Management Award Number M14AC00010 and University of New Hampshire/National Oceanic and Atmospheric Administration Joint Hydrographic Center Award Number NA15NOS4000200.

We gratefully acknowledge NOAA National Ocean Service in providing hydrographic and backscatter survey data. A number of colleagues at the University of New Hampshire Center for Coastal and Ocean Mapping/Joint Hydrographic Center provided scientific and technical support, advice, and insight including Paul Johnson (Data Management), Colleen Mitchell (Graphics Design and Editing), Will Fessenden (IT Group), and Larry Mayer, Giuseppe Masetti, Michael Bogonko, and Erin Nagel (Faculty and Research Scientists).

\section{In Memoriam}

Maxlimer Coromoto Vallee-Anziani was a valued colleague and a major contributor to the original report and maps describing the surficial geology of the continental shelf off New Hampshire that were published in 2016. This earlier work laid the foundation for the expanded report and maps presented here. Sadly, Maxlimer passed away on January 24, 2017. Her original contributions are very important to the work presented here.

\section{Map Coordinate System, Projection and Datum}

Coordinate System: WGS 1984 UTM Zone 19N

Projection: Transverse Mercator

Horizontal Datum: WGS 1984

Vertical Datum: MLLW

\section{Recommended Citation}

Ward, L.G., McAvoy, Z.S., Vallee-Anziani, M., and Morrison, R.C., 2021, Surficial Geology of the Continental Shelf off New Hampshire: Morphologic Features and Surficial Sediment: BOEM/New Hampshire Cooperative Agreement (Contract M14ACO0010) Technical Report, Department of Interior, Bureau of Ocean Energy Management, Marine Minerals Division, 45600 Woodland Road, Sterling, VA, 20166, 184pp. https://dx.doi.org/10.34051/p/2021.31

June 1, 2021 


\section{Table of Contents}

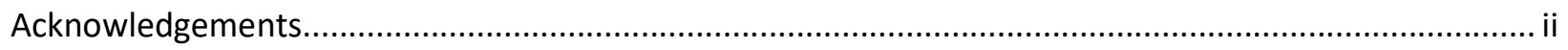

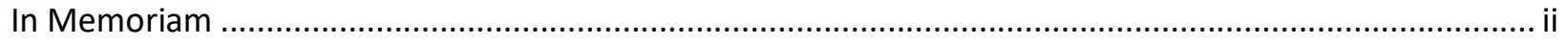

Map Coordinate System, Projection and Datum .................................................................................

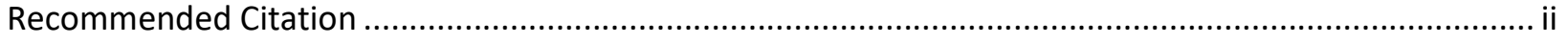

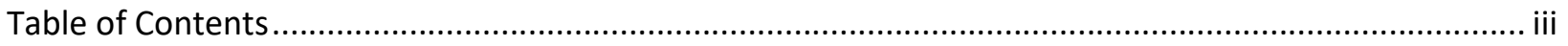

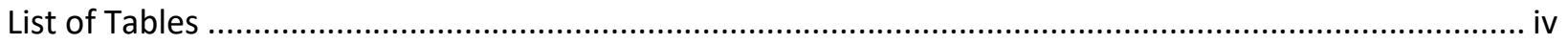

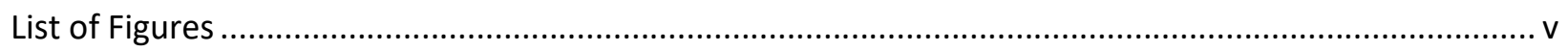

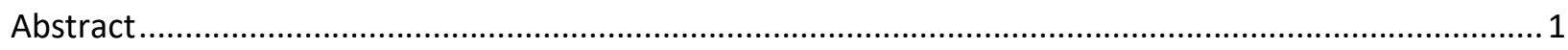

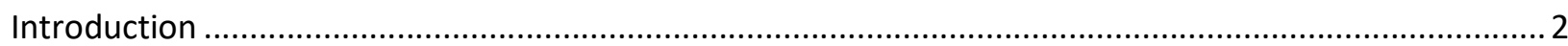

Development of the Surficial Geology Maps of the Continental Shelf off New Hampshire........................ 4

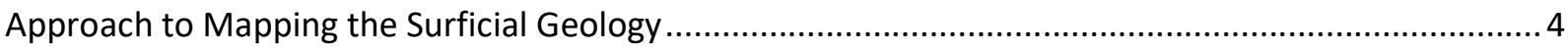

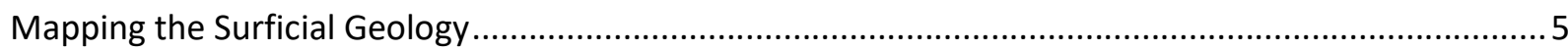

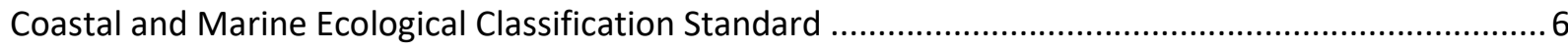

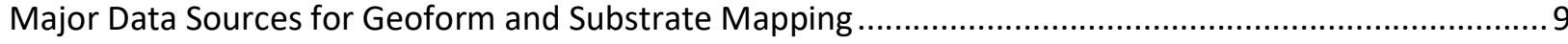

New Hampshire Continental Shelf Geophysical Database: 1971-2015 ............................................. 9

New Hampshire Continental Shelf 2016-2017 Field Campaign.......................................................... 10

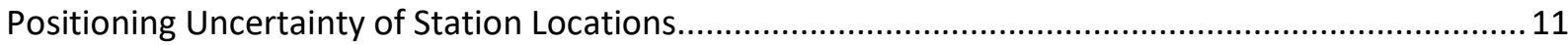

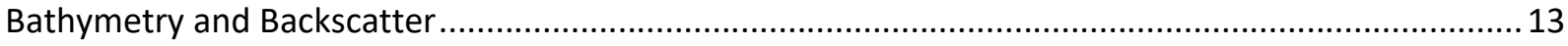

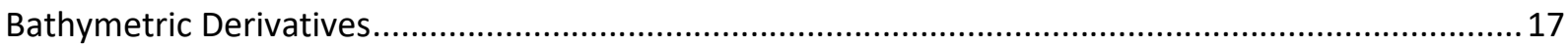

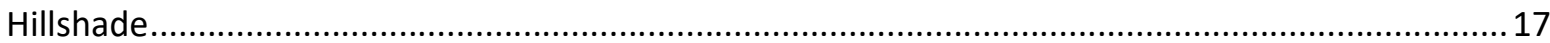

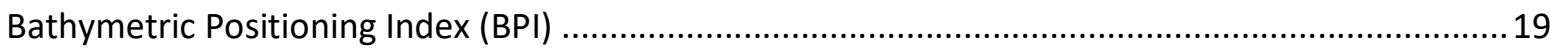

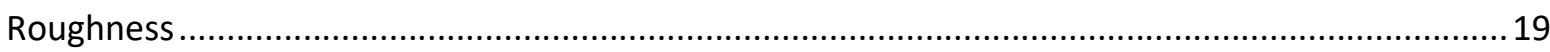

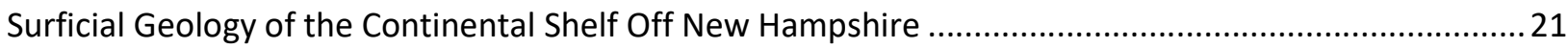

Glaciation, Sea-Level and Upland Surficial Geology in the WGOM .................................................. 21

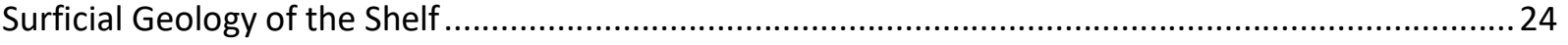

Geoforms (Physiographic Features): Origin and Characteristics ................................................24

Seafloor Sediments Based on CMECS Substrate Subclass Map.....................................................25

Seafloor Sediments Based on CMECS Substrate Group Map ......................................................25

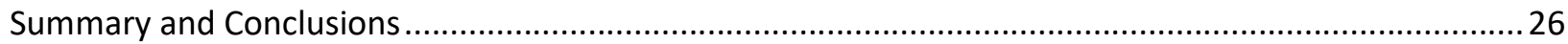

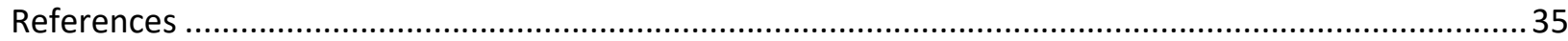

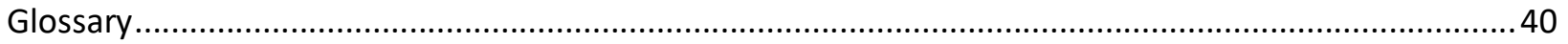


Appendix A: New Hampshire Continental Shelf Historical Geophysical Database: 1971-2015 Sediment Data

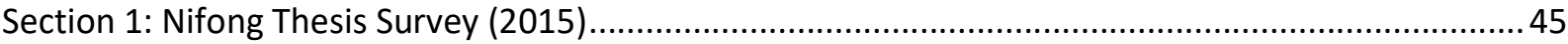

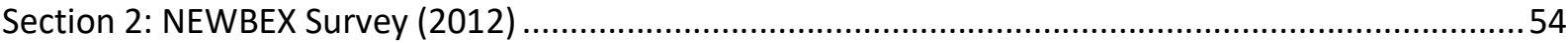

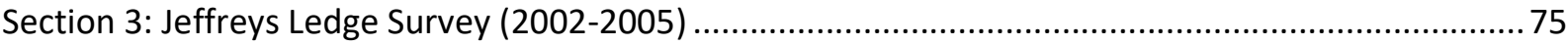

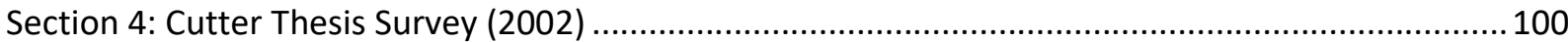

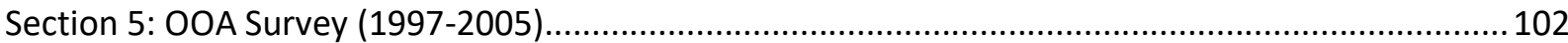

Section 6: MMS Year 2 Survey (1985), Year 4 Survey (1988), Year 8 Survey (1992), and Year 10 Survey

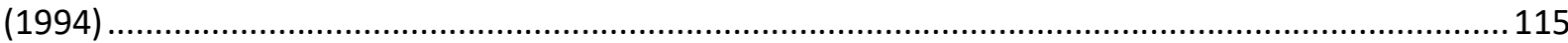

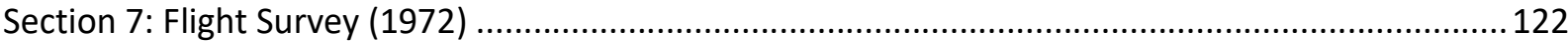

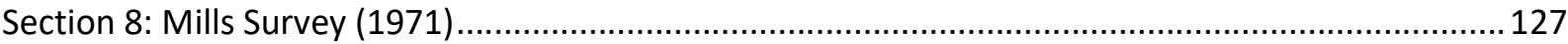

Appendix B: New Hampshire Continental Shelf 2016-2017 Field Campaign Station Locations ............... 134

Appendix C: New Hampshire Continental Shelf 2016-2017 Field Campaign: Grain Size Data ................. 141

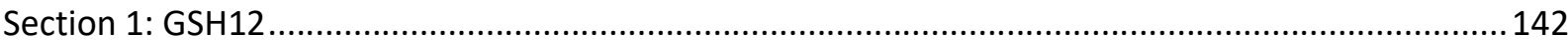

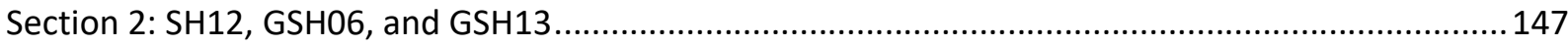

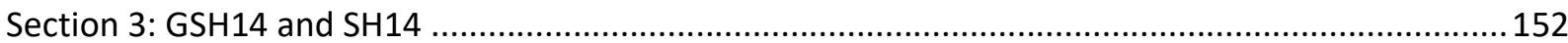

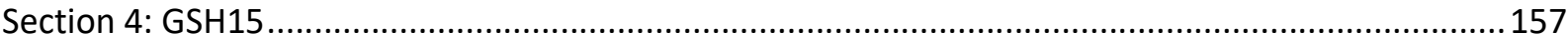

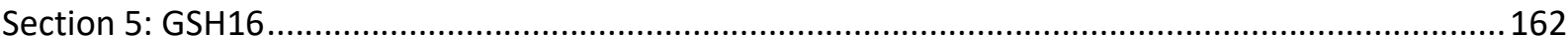

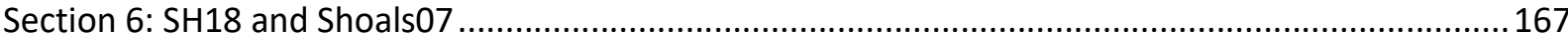

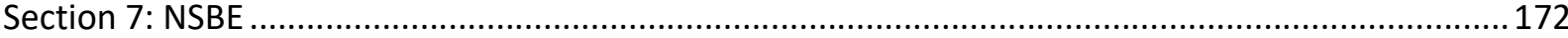

\section{List of Tables}

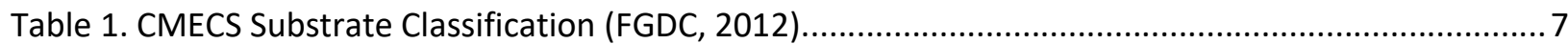

Table 2(a). Geoforms classification modified after FGDC (2012). ....................................................... 8

Table 2(b). Geoform Classification modified after Barnhardt et al. (2007) ........................................... 8

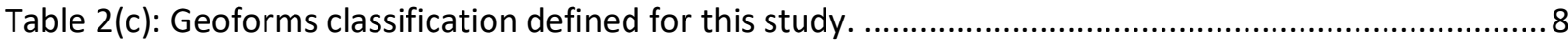

Table 3. Estimated station location uncertainty based on a review of the sampling platform's navigation system, associated position determination uncertainties, antenna location relative to equipment deployment location (offsets), and field experience 


\section{List of Figures}

Figure 1. Location map of the study area ......

Figure 2. Location map for the UNH CCOM/JHC New Hampshire shelf geophysical database consisting of sediment grain size data and classifications, vibracore logs, seismic-reflection profiles (SBP), and seafloor photographs. 10

Figure 3. Location map of the NH Shelf 2016-2017 Field Campaign bottom sediment sampling and videography stations. Station locations and sample types are shown in Appendix B......

Figure 4. High-resolution bathymetry synthesis for the Western Gulf of Maine gridded at $4 \mathrm{~m}$ from the Northeast Bathymetry and Backscatter Compilation....

Figure 5. Regional bathymetry synthesis for the Western Gulf of Maine gridded at $16 \mathrm{~m}$ from the Northeast Bathymetry and Backscatter Compilation.

Figure 6. Backscatter synthesis shown in the Western Gulf of Maine Bathymetry and Backscatter Synthesis

Figure 7. Location map of the UNH CCOM/JHC (Ocean Engineering 972) Hydrographic Field Course MBES surveys

Figure 8. Hillshade for the continental shelf off New Hampshire. The vertical exaggeration is 10x.........19

Figure 9. Bathymetry Position Index (BPI) for the continental shelf off New Hampshire.......................20

Figure 10. Seafloor roughness for the continental shelf off New Hampshire......................................20

Figure 11. Location of the $\mathrm{NH}$ shoreline (seaward edge of brown area) during the last sea-level lowstand at $-60 \mathrm{~m}, \sim 12,500$ yrs B.P.

Figure 12. Surficial geology map of the NH coastal upland

Figure 13. Major physiographic features (geoforms) map for the continental shelf off New Hampshire. The geoform terminology is based on a modification of CMECS (FGDC, 2012). See Table 2 for definitions of geoforms.

Figure 14. Geoform map for the inner continental shelf off New Hampshire ......................................29

Figure 15. Geoform map for the offshore continental shelf off New Hampshire 30

Figure 16. Surficial sediment map of the continental shelf off New Hampshire based on the CMECS classification for Geologic Substrate Subclasses. 
Figure 17. Surficial sediment map of the continental shelf off New Hampshire based on the CMECS classification for Geologic Substrate Groups.....

Figure 18. Map of Geologic Substrate Groups for the nearshore region of the continental shelf off New

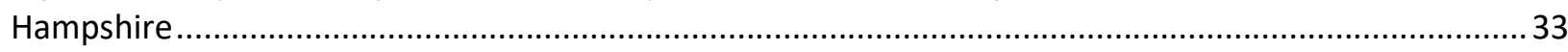

Figure 19. Map of Geologic Substrate Groups for the outer continental shelf off New Hampshire..........34 


\title{
Surficial Geology Maps of the Continental Shelf Off New Hampshire: Morphologic Features and Surficial Sediments
}

\author{
By Ward, L.G., McAvoy, Z.S., Vallee-Anziani, M., and Morrison, R.C. \\ University of New Hampshire Center for Coastal and Ocean Mapping/Joint Hydrographic Center \\ 24 Colovos Road, Durham, NH 03824
}

\section{Abstract}

The continental shelf off New Hampshire (NH) in the Western Gulf of Maine (WGOM) is extremely complex and includes extensive bedrock outcrops, marine-modified glacial deposits, marine-formed shoals, seafloor plains, and associated features that are composed of a range of sediment types from mud to gravel. Furthermore, the physiography and composition of the seafloor frequently changes dramatically over relatively short distances (tens of meters). The complexity of the WGOM seafloor results from the interplay of glaciations, sea-level fluctuations, and marine processes (waves and currents). Highresolution multibeam echosounder (MBES) bathymetry and backscatter surveys, along with ground truth consisting of archived seismic reflection profiles, bottom sediment grain size data, vibracores, and video were used to develop surficial geology maps based on the Coastal and Marine Ecological Classification Standard (CMECS). The surficial geology maps cover $\sim 3,250 \mathrm{~km}^{2}$ and extend from the coast of $\mathrm{NH}$ seaward $\sim 50 \mathrm{~km}$ to Jeffreys Ledge and depict major geoforms (physiographic features) and seafloor substrate (sediment size) classifications. CMECS provides a sound basis for classifying the texture of the seafloor; however, the geoform classifications need to be broadened for paraglacial environments in future studies.

The surficial geology maps presented here are a major refinement of the original maps produced in 2016 (see Ward et al., 2016a). The new maps reflect the results of a major field campaign conducted in 20162017 to obtain accurately located sediment samples and seafloor images to complement the original bottom sediment database. The new sites specifically targeted areas where high-resolution MBES bathymetry existed or where surficial features warranted further ground truth for evaluations. This work was designed to enhance the surficial geology mapping efforts and contribute to the development of new approaches for utilizing acoustics to remotely classify seafloor sediments and morphologic features (also supported by the University of New Hampshire Joint Hydrographic Center). The new surficial geology maps presented here depict the exposed bedrock, morphologic features, and sediment distribution on the continental shelf off $\mathrm{NH}$, revealing features of the seafloor in exceptional detail that have not been previously described.

An important finding of this study was the extent and importance of marine-modified glacial features on the WGOM continental shelf. Extensive glacial deposits including drumlins, eskers, outwash, and moraines have been eroded and modified by wave and tidal currents as sea level fluctuated over the last 12,000 years. These features are potential sources of sand and gravel for future beach nourishment projects; however, more detailed subbottom seismic surveys and vibracores are needed for verification. Also, these potential resource areas are presently too far from shore and in too great a depth of water to be easily utilized. As the demand for sand and gravel becomes more acute and technologies advance, mineral resources farther offshore and in deeper water will likely become viable. 


\section{Introduction}

A Cooperative Agreement was established in 2014 between the Bureau of Ocean Energy Management (BOEM) Marine Minerals Program (MMP), the University of New Hampshire (UNH) Center for Coastal and Ocean Mapping/Joint Hydrographic Center (CCOM/JHC), and the New Hampshire Geological Survey (NHGS) to identify potential marine mineral resources (sand and gravel) on the continental shelf off New Hampshire (NH). Part of this effort included the development of high-resolution surficial geology maps of the seafloor to help characterize bottom sediments and to provide a base for habitat or environmental assessments in the event mineral resources were found and were going to be utilized. The first set of maps was completed in 2016 (Ward et al., 2016a) and depicted exposed bedrock, major morphologic features, and the distribution of surficial sediment.

Presented here is a major update and refinement of the surficial geology maps. The maps cover an area of $\sim 3,250 \mathrm{~km}^{2}$ extending from the $\mathrm{NH}$ coast $\sim 50 \mathrm{~km}$ seaward to Jeffreys Ledge (Figure 1 ). The goal of this work was to refine the surficial geology maps by increasing the detail where possible and by applying more rigorously a standardized, widely used approach to classify the seafloor (i.e. Coastal and Marine Ecological Classification Standard or CMECS; FGDC, 2012). To achieve this goal, the surficial geology maps were updated and CMECS applied utilizing a significantly expanded database including the results of a major field campaign and additional high-resolution bathymetry and backscatter.

Included within this report are the following.

1. High-resolution surficial geology maps depicting seafloor geoforms and substrate sediment classifications of the study area (Figure 1). These maps were developed from the original archived geophysical database, the results of a new and extensive field campaign conducted in 2016-2017, and an expansion of the high-resolution bathymetry in the Western Gulf of Maine (WGOM) (Ward et al., 2021h).

2. A detailed description of the methods and protocols used to develop the surficial geology maps. See Development of the Surficial Geology Maps of the Continental Shelf off New Hampshire.

3. A Glossary of commonly used terms.

4. An extensive historical sediment grain size database built from all available studies from the continental shelf off NH carried out by investigators from UNH between 1971 and 2015. See New Hampshire Continental Shelf Historical Geophysical Database and Appendix A.

5. The geophysical database from a field campaign conducted in 2016-2017 which provided ground truth for the surficial geology mapping effort (see New Hampshire Continental Shelf 2016-2017 Field Campaign). Included here are stations and sampling history (Appendix B) and bottom sediment grain size classifications and statistics (Appendix C).

The surficial geology maps, grain size data, and seafloor photographs can be viewed at: (https://maps.ccom.unh.edu/portal/apps/webappviewer/index.html?id=28df035fe82c423cb3517295d9 bbc24c) (UNH CCOM/JHC web page "New Hampshire Shelf Surficial Geology").

This report and its associated databases, as well as additional sediment and seafloor photographs from the New Hampshire Continental Shelf 2016-2017 Field Campaign, are available digitally from the University of New Hampshire Scholars Repository (https://scholars.unh.edu/). Included are the following databases.

1. The New Hampshire Continental Shelf Geophysical Database: 2016-2017 Field Campaign Seafloor and Sample Photographs and Sediment Data which includes photographs of the 
sediment samples, seafloor photographs of locations where samples were collected, and selected grain size statistics (Ward et al., 2021b). (https://dx.doi.org/10.34051/d/2021.1)

2. The New Hampshire Continental Shelf Geophysical Database: 2016-2017 Field Campaign Stations and Sediment Data which includes the station sampling history and sediment grain size data (Ward et al., 2021c). (https://dx.doi.org/10.34051/d/2021.2)

3. The New Hampshire Continental Shelf Geophysical Database: 2016-2017 Field Campaign Seafloor Photographs which includes 855 seafloor photographs from 155 stations on the inner continental shelf off NH (Ward et al., 2021e). (https://dx.doi.org/10.34051/d/2021.5)

4. The New Hampshire Continental Shelf Historical Geophysical Database: 1971 to 2015 - Sediment Data which includes sediment grain size data from historical studies (Ward et al., 2021d). (https://dx.doi.org/10.34051/d/2021.3)

The report and databases presented here are part of a comprehensive series of reports on the bathymetry and surficial geology of the continental shelf off New Hampshire developed and supported by BOEM and UNH CCOM/JHC. Other associated major reports include the following.

1. Ward, L.G., Vallee-Anziani, M., and McAvoy, Z.S., 2016a, New Hampshire and Vicinity Continental Shelf: Morphologic Features and Surficial Sediments: BOEM/New Hampshire Cooperative Agreement (Contract M14ACO0010) Technical Report, BOEM Marine Minerals Branch, 381 Elden Street, Herndon, VA, 20170, 24 pp. (http://ccom.unh.edu/publications/new-hampshire-andvicinity-continental-shelf-morphologic-features-and-surficial)

2. Ward, L.G., Johnson, P., Nagel, E., McAvoy, Z.S., and Vallee-Anziani, M., 2016b, Western Gulf of Maine Bathymetry and Backscatter Synthesis: BOEM/New Hampshire Cooperative Agreement (Contract M14ACOO010) Technical Report, BOEM Marine Minerals Branch, 381 Elden Street, Herndon, VA, 20170, 18 pp.

Available at the UNH Scholars Repository: https://dx.doi.org/10.34051/p/2021.27

3. Ward, L.G., McAvoy, Z.S., and Vallee-Anziani, M., 2021a, New Hampshire and Vicinity Continental Shelf: Sand and Gravel Resources: BOEM/New Hampshire Cooperative Agreement (Contract M14AC00010) Technical Report, BOEM Marine Minerals Branch, 381 Elden Street, Herndon, VA, 20170, $97 \mathrm{pp}$.

Available at the UNH Scholars Repository: https://dx.doi.org/10.34051/p/2021.30

4. Ward, L.G., Morrison, R.C., McAvoy, Z.S., and Vallee-Anziani, M., 2021g, Analysis of Vibracores from the New Hampshire Continental Shelf from 1984 and 1988: BOEM/New Hampshire Cooperative Agreement (Contract M14AC00010) Technical Report, Department of Interior, Bureau of Ocean Energy Management, Marine Minerals Division, 45600 Woodland Road, Sterling, VA, 20166, $173 \mathrm{pp}$.

Available at the UNH Scholars Repository: https://dx.doi.org/10.34051/p/2021.26

5. Ward, L.G., Johnson, P., Bogonko, M., McAvoy, Z.S., and Morrison, R.C., 2021h, Northeast Bathymetry and Backscatter Compilation: Western Gulf of Maine, Southern New England, and Long Island Sound: BOEM/New Hampshire Cooperative Agreement (Contract M14ACO0010) Technical Report, Department of Interior, Bureau of Ocean Energy Management, Marine Minerals Division, 45600 Woodland Road, Sterling, VA, 20166., 23 pp.

Available at the UNH Scholars Repository: https://dx.doi.org/10.34051/p/2021.28 


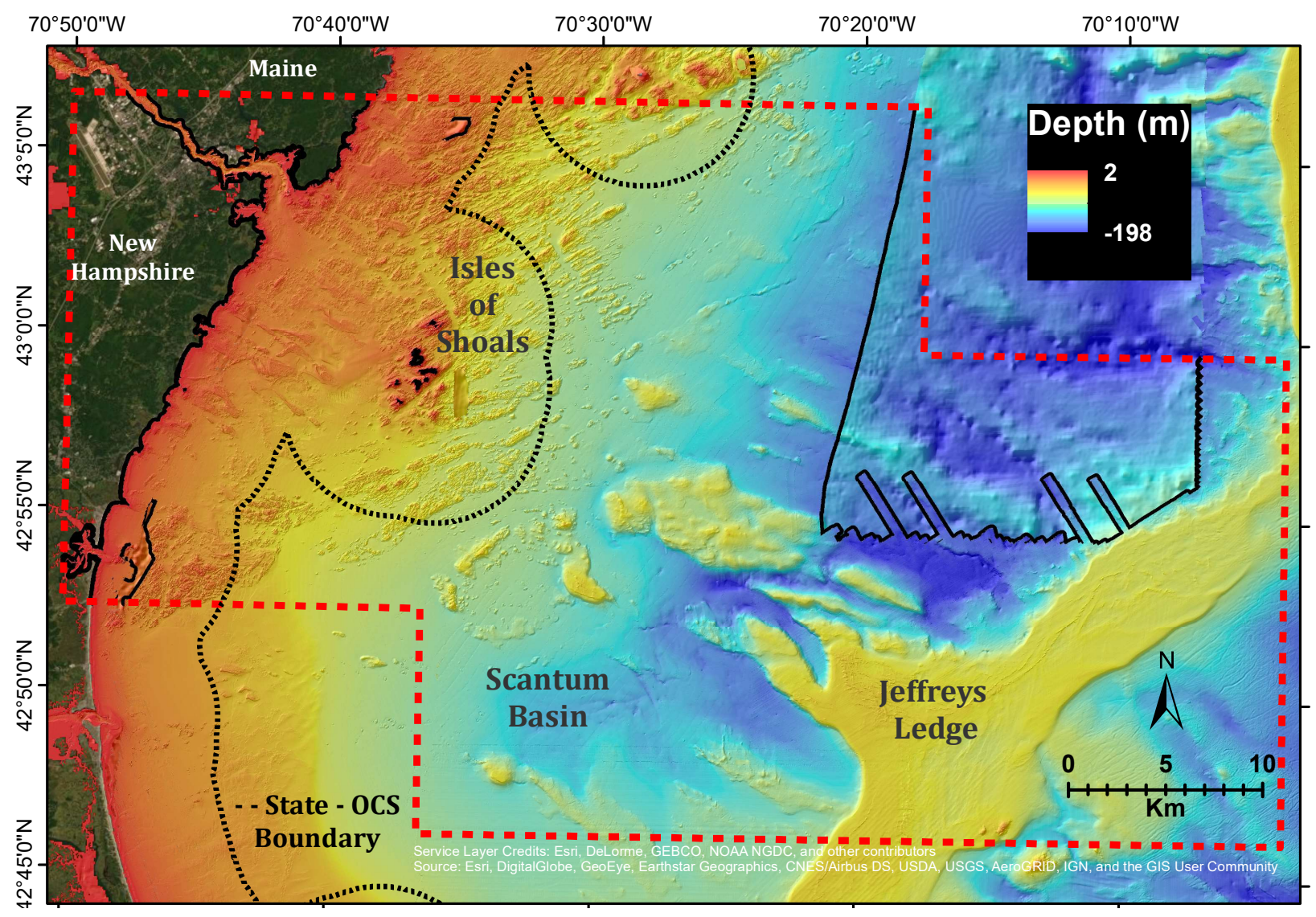

Figure 1. Location map of the study area. Surficial geology maps depicting geoforms and surficial sediments were developed for the area outlined by the red dashed line. The black dashed line is the boundary between state and federal jurisdiction. The bathymetry map was primarily developed from high-resolution multibeam surveys and lidar and is gridded at $4 \mathrm{~m}$. The exception is the area outlined in black in the upper right that is based on a compilation of lower-resolution single beam surveys and regional coverages. This area is gridded at $16 \mathrm{~m}$.

\section{Development of the Surficial Geology Maps of the Continental Shelf off New Hampshire}

The surficial geology of the continental shelf off NH is depicted in three types of maps that utilize CMECS (FGDC, 2012) including: Geoforms (major morphologic or physiographic features); Geologic Substrate Subclass; and Substrate Group. The CMECS classification and the methods used to develop the surficial geology maps are described below.

\section{Approach to Mapping the Surficial Geology}

Mapping the surficial geology of the continental shelf off $\mathrm{NH}$ used the following protocol. Geoforms and geologic substrate type (sediment) were identified and mapped based on bathymetry, bathymetric derivatives, backscatter where available, and ground truth consisting of grain size data and photographs. All of these databases were not available for the entire study area. Consequently, the best information available was used.

The seafloor was mapped at three levels depending on the availability and the quality of data: 
1. Seafloor regions where high-quality bathymetry and backscatter were available and where reliable ground truth stations with either grain size data or video existed were classified and mapped using the CMECS hierarchy. This includes geoforms, substrate subclass, and substrate group. The mapped substrates in these regions were well vetted and verified.

2. Seafloor regions where high-quality bathymetry and acoustic backscatter were available, but without reliable ground truth stations, were classified and the geoforms and substrate sediment subclass mapped with reasonable confidence. However, mapping the more detailed substrate groups was based on proximity and comparisons to other mapped regions with similar morphology and acoustic characteristics. In these regions, the substrate group was inferred, mapped, and identified with a diagonal line pattern overlay. Ultimately these areas will need ground truth for verification.

3. Seafloor regions that lack high-resolution bathymetry, backscatter, and ground truth were not mapped at this time. These regions were left transparent and the underlying hillshade displayed to show the bottom topography.

\section{Mapping the Surficial Geology}

Interpretation and mapping of the seafloor geology was done by skilled analysts with backgrounds in earth sciences and seafloor characterization. The maps were based on the following:

1. High-resolution bathymetry for almost the entire study area (Ward et al., 2021h)

2. Acoustic backscatter synthesis for $\sim 50 \%$ of the study area (Ward et al., $2021 \mathrm{~h}$ )

3. Bathymetry derivatives including hillshade, roughness, and bathymetric position index

4. An extensive geophysical database consisting of archived surficial sediment grain size data from studies conducted between 1971 and 2015 along with analog subbottom seismic profiles from the 1980 s

5. An intensive field campaign in 2016 and 2017 on the $\mathrm{NH}$ continental shelf targeting areas where additional ground truth was needed including sediment samples and seafloor photographs

The mapping procedure relied on the expertise of experienced analysts with geophysical and seafloor characterization backgrounds for several reasons related to the study area and the nature of the database. First, the study area is large $\left(\sim 3,250 \mathrm{~km}^{2}\right)$ and is a paraglacial environment. The seafloor is extremely variable with a wide range of seafloor types that frequently change over short distances. Second, the geophysical database developed for this study for ground truth is extensive, but inconsistent in spatial coverage, leaving large areas with incomplete or sparse information. Furthermore, some of the sediment grain size data are from surveys completed as early as the 1970s and the positioning has large uncertainty (discussed below). Third, the high-resolution MBES bathymetry for most of the study area is excellent. However, high quality, co-registered acoustic backscatter, which is critical to automated seafloor classification algorithms, is very limited in coverage. Therefore, after an initial assessment was done using more automated approaches to mapping the study area, such as remote or supervised classifications schemes (e.g., Erdey-Heydorn, 2008; Dorschel et al., 2014) and MBES backscatter Angular Range Analysis (Fonseca and Mayer, 2007), the use of skilled analysts was determined to be the best approach for this study.

Since the mapping effort described here was completed, an automated landform analysis algorithm (Bathymetry- and Reflectivity-Based Approach for Seafloor Segmentation: BRESS) was developed at UNH CCOM/JHC which utilizes high-resolution bathymetry to map landforms (e.g., flat, slope, ridge, valley, shoulder, footslope) (Masetti et al., 2018). BRESS has been tested on small areas of the NH continental 
shelf with good success (Ward et al., 2019) and on the U.S. Atlantic continental margin (Sowers, 2020; Sowers et al., 2020). Future work will also consider the use of machine learning approaches (e.g., Pendleton et al., 2019).

The surficial geology maps for the NH continental shelf were originally produced in ArcGIS 10 (Ward et al., 2016a). Differences in the observed bathymetric derivatives such as hillshade, roughness, BPI, and backscatter served as traceable outlines of transitions between different morphologic or sedimentologic characteristics. Polygons were manually drawn using the Create Polygon Feature tool within an editing session, resulting in $\sim 4,900$ individual polygon features. For each feature drawn, a designation of morphologic (geoform) and sediment type was entered, along with comments stating the justification for the geologic interpretation. The surficial geology map layers were composed of separate polygon feature shapefiles consisting of a geoform layer, substrate subclass layer, and substrate group layer. The map polygons were then checked again by an analyst to confirm that interpretations of map areas matched the designations within the map layers attribute table. Metadata was constructed in an Excel spreadsheet, transposed to the feature layer descriptions using ArcCatalog, and converted to XML using USGS Metadata Wizard, 2015 for ArcGIS 10.0.

A major field campaign in 2016-2017 (described below) was motivated by recognition that the original maps had significant data gaps in several important locations where higher resolution was desired. In order to incorporate the new information derived from the 2016-2017 field campaign, improve cartographic representation in the surficial geology, and re-organize the original map databases to more closely match FGDC (2012) standards for CMECS, a number of modifications were made to the original map products. The $\sim 4,900$ individual polygon features were combined using the Spatial Join Tool (Analysis Toolbox) and stored as an ESRI FGDBR. For quality assurance, a map topology was created and unsuccessful joins or previous errors such as dangles, overlapping, incompleteness, or gaps between polygons were corrected with Create Topology Tool (Data Management Toolbox). Attribute tables for the surficial map polygons were then enhanced with attribute hierarchy as defined by the CMECS proposed data structure and Crosswalk Tools version 1.0 (Office of Coastal Management, 2015).

Once in the intended format, updated WGOM bathymetry data, 2016-2017 ground truth sediment analysis results, and seafloor photos were used to verify, re-classify, or re-segment the surficial map polygons. Expanded bathymetry coverage and ground truth data also enabled expansion of the surficial geology maps to include areas closer to shore and into the nearshore of southern Maine.

Keeping in compliance with the FGDC and BOEM MMIS spatial data standards, metadata was constructed for each of the updated map and ground truth vector layers. The USGS Metadata Tools extension (USGS Metadata Wizard) for ArcGIS 10.7 was used to set field attribute definitions and domains while ArcCatalog was employed to format descriptions, contact information, and to summarize processing steps.

\section{Coastal and Marine Ecological Classification Standard}

The CMECS geologic substrate classifications are built on a simplification of the Wentworth (1922) and Folk $(1954 ; 1980)$ classification schemes (Table 1). Essentially, the size classes are grouped into a hierarchy of broader categories including Substrate Class, Substrate Group, and Substrate Subgroup. Each level provides more detailed sediment classifications. The advantage of this approach is that it allows the seafloor sediment to be mapped at different levels of detail that reflect the database. Here, the seafloor sediment is mapped at the Substrate Subclass and Substrate Group levels.

Based on an adaption of CMECS (FGDC, 2012), for this study geoforms are defined as morphologic features that are formed by geologic processes. For example, relatively large features with positive relief such as exposed bedrock, drumlins, moraines, or sandy shoals would be considered geoforms. Low-lying features 
and depressions are also included as geoforms; for example, flat or low-slope seafloor plains, sand ramps, basins, or small channels on the seafloor. The geoforms were identified by bathymetry, bathymetric derivatives, backscatter intensity, and sediment textural characteristics. The primary bathymetric derivatives used here were hillshade, $\mathrm{BPI}$, and roughness. Aspect and slope were also computed and tested, but did not provide additional information and were not used in the classifications. The definitions of the geoforms used in this study are defined in Table 2. Most of the geoform classifications used here come directly from or are slightly modified from CMECS. However, a number of classifications were not present in the CMECS and therefore were defined here.

Table 1. CMECS Substrate Classification (FGDC, 2012).

\begin{tabular}{|c|c|c|c|c|}
\hline $\begin{array}{c}\text { Substrate } \\
\text { Origin }\end{array}$ & $\begin{array}{c}\text { Substrate } \\
\text { Class }\end{array}$ & $\begin{array}{l}\text { Substrate } \\
\text { Subclass }\end{array}$ & $\begin{array}{c}\text { Substrate } \\
\text { Group }\end{array}$ & $\begin{array}{l}\text { Substrate } \\
\text { Subgroup }\end{array}$ \\
\hline \multirow{29}{*}{$\begin{array}{l}\text { Geologic } \\
\text { Substrate }\end{array}$} & Rock Substrate & Bedrock & & \\
\hline & \multirow{28}{*}{$\begin{array}{c}\text { Unconsolidated } \\
\text { Mineral } \\
\text { Substrate }\end{array}$} & \multirow{10}{*}{$\begin{array}{c}\text { Coarse } \\
\text { Unconsolidated } \\
\text { Substrate }\end{array}$} & \multirow{4}{*}{ Gravel } & Boulder \\
\hline & & & & Cobble \\
\hline & & & & Pebble \\
\hline & & & & Granule \\
\hline & & & \multirow{3}{*}{ Gravel Mixes } & Sandy Gravel \\
\hline & & & & Muddy Sandy Gravel \\
\hline & & & & Muddy Gravel \\
\hline & & & \multirow{3}{*}{ Gravelly } & Gravelly Sand \\
\hline & & & & Gravelly Muddy Sand \\
\hline & & & & Gravelly Mud \\
\hline & & \multirow{18}{*}{$\begin{array}{c}\text { Fine } \\
\text { Unconsolidated } \\
\text { Substrate }\end{array}$} & \multirow{4}{*}{ Slightly Gravelly } & Slightly Gravelly Sand \\
\hline & & & & Slightly Gravelly Muddy Sand \\
\hline & & & & Slightly Gravelly Sandy Mud \\
\hline & & & & Slightly Gravelly Mud \\
\hline & & & \multirow{5}{*}{ Sand } & Very Coarse Sand \\
\hline & & & & Coarse Sand \\
\hline & & & & Medium Sand \\
\hline & & & & Fine Sand \\
\hline & & & & Very Fine Sand \\
\hline & & & \multirow{3}{*}{ Muddy Sand } & Silty Sand \\
\hline & & & & Silty-Clayey Sand \\
\hline & & & & Clayey Sand \\
\hline & & & \multirow{3}{*}{ Sandy Mud } & Sandy Silt \\
\hline & & & & Sandy Silt-Clay \\
\hline & & & & Sandy Clay \\
\hline & & & \multirow{3}{*}{ Mud } & Silt \\
\hline & & & & Silt-Clay \\
\hline & & & & Clay \\
\hline
\end{tabular}


Table 2(a). Geoforms classification modified after FGDC (2012).

Bedforms or Bedform Field: Areas of larger bedforms or subaqueous dunes that are identifiable with bathymetry, hillshade, and backscatter. Thus, they must have wavelengths that exceed the bathymetry grid size. They are assumed to be largely composed of sand or gravelly sand and are formed by waves and currents.

Bedrock Border: Deposits of unconsolidated sediment surrounding a bedrock outcrop. They are identified by bathymetry, hillshade, roughness, and acoustic backscatter.

Channel: Linear or sinuous depressions on an otherwise flat seafloor.

Depression: Small, shallow basins or low areas of the seafloor with no natural outlet. Depressions are identified by bathymetry, BPI, and hillshade.

Ebb Tidal Delta: Subaqueous, typically fan-shaped delta composed of mostly sand and fine gravel located on the seaward side of a tidal inlet. The morphology of these features is controlled by the flood and ebb tidal currents through the adjacent tidal inlet.

Iceberg Scour Scar: Gouges or deformations of the bottom formed by movements of an iceberg dragging across the substrate. These are formed in glaciated areas and can extend for long distances.

Inlet: Narrow opening in the coast that facilitates water exchange between the ocean and adjacent enclosed bays, lagoons or marshes.

Moraine: Elongated mound or ridge-like feature often occurring as clusters on sloping seafloor or imposed on bathymetric features with positive relief. These distinct subglacial deposits are composed of unsorted, unstratified gravel mixes to megaclasts.

Ridge: Elongated, narrow, steep-sided, crested features. Identifiable in bathymetry and shaded relief.

Shoal: Relatively shallow area in a body of water, rising above adjacent seafloor. Shoals are morphologically diverse and are largely influenced by tidal or river currents. Shoals are normally composed of sand but can also be gravel mixes.

Slope: An inclined area of seafloor with a gentle change in depth over its upper and lower limits. Slopes occur at all scales and can be composed of various types of material. Primarily identified by bathymetry.

Tombolo: Sand or gravel bar or barrier that connects an island with the mainland.

Table 2(b). Geoform Classification modified after Barnhardt et al. (2007).

Nearshore Ramp: Gently sloping sandy or gravelly seafloor located offshore of a beach. The nearshore ramp generally exhibits shore-parallel bathymetric contours and is primarily covered with sand-rich sediment, although locally small exposures of ledge, cobbles, and boulders may be present.

Table 2(c): Geoforms classification defined for this study.

Bedrock Outcrop: Exposed bedrock with little sediment coverage. It is often heavily vegetated. Bedrock outcrops are identified by bathymetry, hillshade, roughness, and acoustic backscatter. The bedrock often has ridges separated by bathymetric lows or swales with sediment.

Marine-Formed Feature or Shoals: Wave- or current-formed features such as sand bars or mounds, shoals formed at lower sea levels but now submerged, and sand or gravel ridges. The marine-formed features tend 
to have relief but are generally smooth in appearance. Although defined here, the features are similar in some cases to moraine shoals or other drowned, elongated offshore ridges.

Marine-Modified Glacial Feature: Glacially formed features now submerged and altered via erosion in the marine environment. Examples include eroded drumlins, eskers, outwash, or moraines (see Glossary). In the study area, the drumlins tend to be $0.5-4 \mathrm{~km}$ in length (long axis) and are oriented northwest-southeast. Some of the larger features (up to $10 \mathrm{~km}$ ) appear to be groups of drumlins. Small glacial moraines also occur that are less than a few hundred meters in length, linear to arcuate in shape with low relief, and tend to be oriented in a northeast-southwest direction (likely De Geer moraines). Marine-modified glacial features tend to have high backscatter and roughness. The surface can appear eroded and rough, presumably due to the presence of cobble to boulder gravels.

Seafloor Plain: Large areas of the seafloor that are relatively flat or have a low slope. They can have local areas that are undulating or slightly rougher than adjacent areas. Seafloor plains tend to be composed of finer-grained sediments.

Sediment-Draped Bedrock: Bedrock covered by a thin layer of sediment. It appears as distinctive bedrock, but the texture is more subdued.

Undefined: Apparent geoforms or areas of the seafloor which cannot be identified with the available database.

\section{Major Data Sources for Geoform and Substrate Mapping}

\section{New Hampshire Continental Shelf Geophysical Database: 1971-2015}

The UNH CCOM/JHC “New Hampshire Continental Shelf Geophysical Database: 1971-2015” includes grain size data and statistics from multiple studies carried out between 1971 and 2015 . The database contains 725 samples, 24 vibracore logs (described in Ward et al., 2021g), and $1,280 \mathrm{~km}$ of analog seismicreflection profiles (Figure 2). The surveys were conducted on the $\mathrm{NH}$ continental shelf from the early $1970 \mathrm{~s}$ until 2015 by a number of investigators from UNH. Although the archived database is relatively large, the coverage is somewhat inconsistent in quality and distribution. In addition, the positioning uncertainty of the station locations varies over an order of magnitude (discussed below). The 1971-2015 sediment grain size data and classifications are given in Appendix A. A full description of the vibracores including locations, analysis methods, core logs, and sediment data can be found in Ward et al. (2021g). More information about the subbottom seismics can be found in Ward et al. (2021a). Much of the UNH database was incorporated into previous sediment syntheses by the United States Geological Survey (USGS) (Poppe et al., 2003; Reid et al., 2005). In addition, parts of the database were incorporated into the Bureau of Ocean Energy Management (BOEM) Marine Minerals Program (MMP) data portal (Marine Minerals Information System or MMIS). Several other sediment databases were utilized for the mapping effort. 


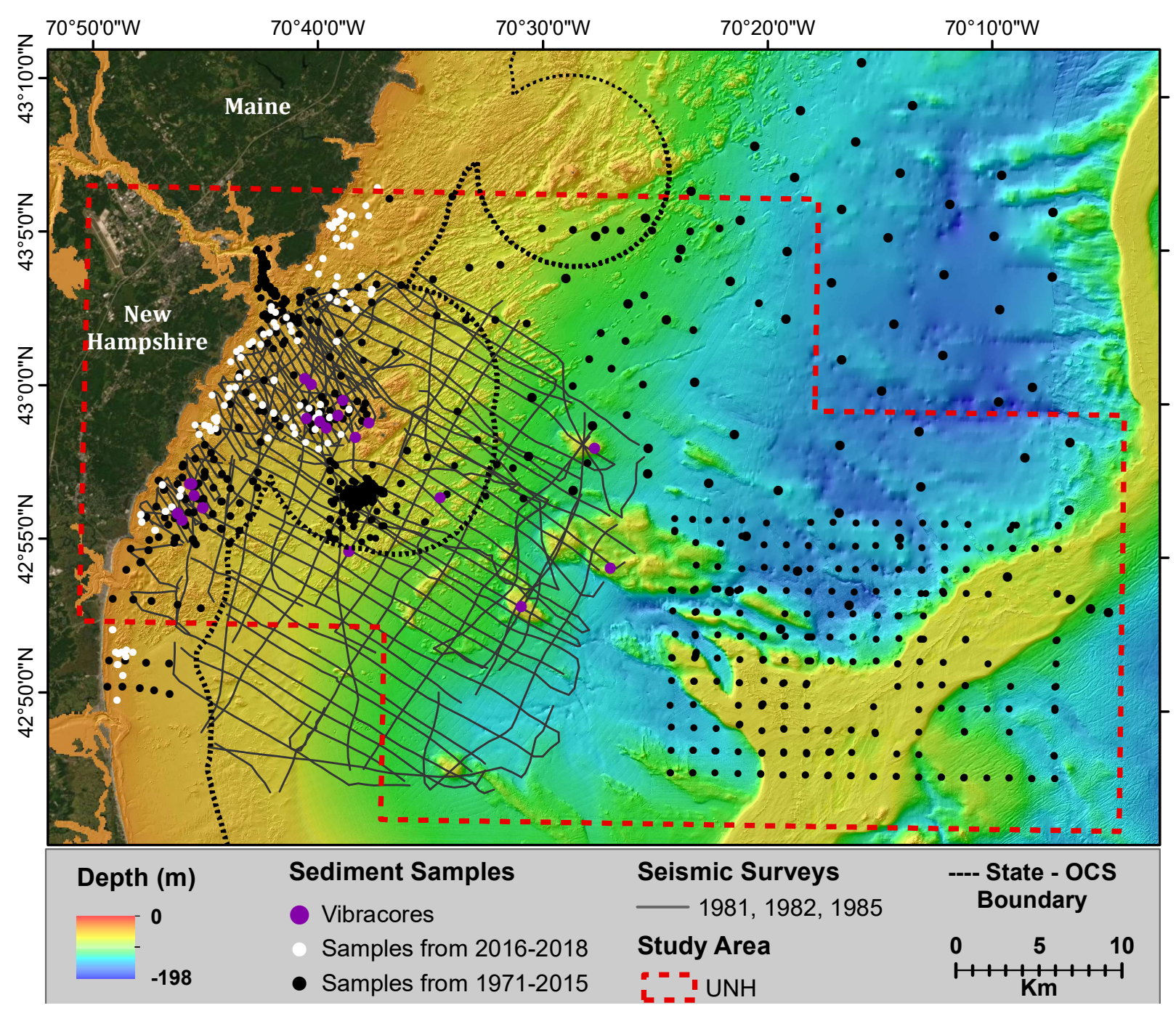

Figure 2. Location map for the UNH CCOM/JHC New Hampshire shelf geophysical database consisting of sediment grain size data and classifications, vibracore logs, seismic-reflection profiles (SBP), and seafloor photographs. Except for the SBP, the database can be viewed at:

(https://maps.ccom.unh.edu/portal/apps/webappviewer/index.htm/?id=28df035fe82c423cb3517295d9bbc24c). Most of the database is also available and can be downloaded (see the Introduction for locations).

\section{New Hampshire Continental Shelf 2016-2017 Field Campaign}

To address a gap in ground truth coverage of the seafloor, a major field campaign was conducted in 20162017 on the NH continental shelf to obtain accurately-located sediment samples and seafloor images. In total, 151 stations were occupied over thirteen one-day cruises. Seafloor video was obtained at all of the stations, while sediment samples were collected for grain size analysis at eighty-five stations (Figure 3; Appendices B and C). Not all stations occupied were sampled for sediments due to the coarseness of the substrate (e.g., bedrock or pebble-cobble bottoms). The new stations specifically targeted areas where high-resolution MBES bathymetry and backscatter existed or surficial features warranted further ground truth for evaluations. This work was designed to enhance the surficial geology mapping efforts and to 
provide needed data for an associated study being conducted in conjunction with UNH CCOM/JHC with the purpose of assessing new methods to utilize acoustics to aid in seafloor mapping (Ward et al., 2019).

The cruises were conducted aboard the UNH Research Vessel Gulf Surveyor, a $19 \mathrm{~m}$ twin screw aluminum catamaran. Bottom sediment samples were collected with a Shipek grab sampler. Seafloor video was obtained with a fabricated drop camera consisting of a Delta Vision camera frame mounted $0.4 \mathrm{~m}$ from the seafloor when in its vertical position. The base of the frame was $0.3 \mathrm{~m}$ by $0.3 \mathrm{~m}$ and was divided into $2 \mathrm{~cm}$ increments that provided a scale on all sides. The camera had a $1.2 \mathrm{~mm}$ pixel resolution (Pe'eri et al., 2013). The ship's Global Navigation Satellite System (GNSS) was recorded during sampling. The estimated accuracy of the sample positions was $\sim 30 \mathrm{~m}$ for the Shipek grab samples and $\sim 50 \mathrm{~m}$ for the video locations (discussed below). However, it is likely the positioning was more accurate than these estimates. The bottom sediments were analyzed with standard sieve and pipette analyses after Folk (1980). Statistics and initial sediment classifications were determined in Gradistat (Blott and Pye, 2001). Subsequently, the sediment samples were classified with CMECS (FGDC, 2012). Additional sediment samples previously collected by the UNH Ocean Engineering 972 Hydrographic Field Course classes in 2012, 2014, and 2018 were analyzed and pooled with the 2016-2017 Field Campaign database. The sediment grain size data is presented in Appendix C.

\section{Positioning Uncertainty of Station Locations}

Determination of the station locations where bottom sediment samples were collected was based on the position of the research vessel. This makes the reasonable assumption that the sediment sampler hung directly below the deployment location on the sampling platform (boat). However, determination of the accuracy of the position of the research vessel has changed significantly over time from the earlier studies in the database from the 1970s which used radar and dead reckoning to the highly accurate RTK GNSS used today. The uncertainty in position of the earliest studies included in the Historical Geophysical Database is not clear, but the error was estimated by the ship's crew to range from 250 to $1,000 \mathrm{~m}$ depending on distance to known points (targets). Samples using radar for positioning were included in the database, but were not used for verification or ground truth in the surficial geology maps presented in this report. Loran C was used for the sediment samples collected from the 1980 s to the early 1990 s. The absolute positioning error for Loran $\mathrm{C}$ was between 185 and $463 \mathrm{~m}$, depending on distance from shore and land obstructions (USCG Loran-C Users Handbook, accessed March 15, 2021), although the precision of the positions were significantly better. The data included in the sediment database presented here had no obstructions and were collected during good weather and sea conditions. Therefore, it is likely the positioning error was closer to the lower estimate for the accuracy of Loran $\mathrm{C}$. The most accurate positions came from the RTK or post-processed GNSS used since the early 2000s, with position uncertainty on the order of meters for the research vessel. However, for bottom samples the GNSS antenna offset has to be considered, as well as orientation of the boat. Additionally, the drift of the boat and the drag on the camera must be considered for seafloor videography and photographs. The total estimated error for RTK GNSS are on the order of $30 \mathrm{~m}$ for sediment samples and $50 \mathrm{~m}$ for videography and photographs. The uncertainty of each sample location was evaluated and a numbering system for location uncertainties assigned (described in Table 3). Uncertainty information is included in the database in Appendices $A$ and $\mathrm{C}$ and was considered in the mapping procedures (e.g., location and reliability of analyses). 


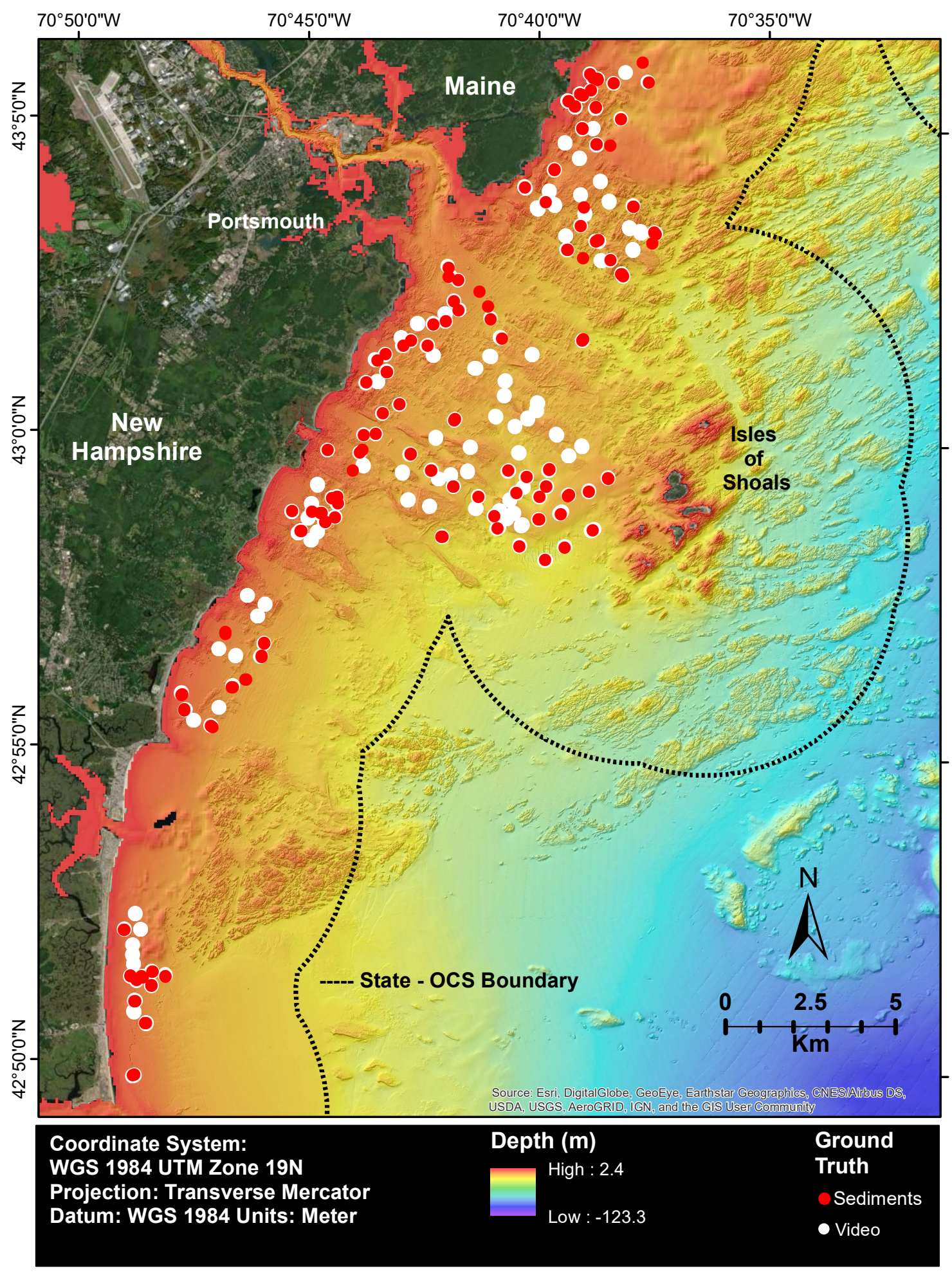

Figure 3. Location map of the NH Shelf 2016-2017 Field Campaign bottom sediment sampling and videography stations. Station locations and sample types are shown in Appendix B. The sediment data and seafloor photographs are downloadable at UNH Scholars Repository at https://dx.doi.org/10.34051/d/2021.1, https://dx.doi.org/10.34051/d/2021.2, and https://dx.doi.org/10.34051/d/2021.5, and viewable at: (https://maps.ccom.unh.edu/portal/apps/webappviewer/index.htm/?id=28df035fe82c423cb3517295d9bbc24c). 
Table 3. Estimated station location uncertainty based on a review of the sampling platform's navigation system, associated position determination uncertainties, antenna location relative to equipment deployment location (offsets), and field experience. These values are best estimates with recognition that the values are guidelines and not exact. They apply only to the databases presented in this report and are not meant to be applied to other databases. The categories were used to describe each sediment grain size sample in the Historical Geophysical Database (Appendix A) and the 2016-2017 Field Campaign (Appendix C).

\begin{tabular}{|l|l|l|}
\hline Category & System Used for Ship Positioning & Estimated Sample Location Uncertainty \\
\hline 1 & GNSS: Corrected with RTK or Postprocessing & Less than $30 \mathrm{~m}$ \\
\hline 2 & GNSS: Without Corrections & $30 \mathrm{~m}$ to $50 \mathrm{~m}$ \\
\hline 3 & Loran C & $185 \mathrm{~m}$ to $463 \mathrm{~m}$ \\
\hline 4 & Radar, Charts, Dead Reckoning & $250 \mathrm{~m}$ to $1000 \mathrm{~m}$ \\
\hline
\end{tabular}

\section{Bathymetry and Backscatter}

The primary bathymetry and backscatter databases used for seafloor mapping of the continental shelf off $\mathrm{NH}$ included the Northeast Bathymetry and Backscatter Compilation: Western Gulf of Maine, Southern New England, and Long Island (NE Compilation) (Ward et al., 2021h), and the UNH Ocean Engineering 972 Hydrographic Field Course MBES surveys from 2003 to 2018 (excluding the survey from 2011 which was located outside of the study area). The NE Compilation is an update and expansion of the WGOM Bathymetry and Backscatter Synthesis (WGOM Synthesis) (Ward et al., 2016b). The NE Compilation bathymetry synthesis incorporates all known (to the investigators) high-resolution multibeam echosounder (MBES) surveys in the WGOM including the CCOM/JHC surveys and combines them into a single map product (Figure 4). Although the extent of the high-resolution multibeam bathymetry is large, there are still major gaps in the coverage of the seafloor. In these areas, the lower resolution regional coverages that are largely based on single beam acoustic surveys fill the gaps, but the quality is far lower. While the high-resolution MBES bathymetry synthesis is gridded at $4 \mathrm{~m}$, the regional bathymetry is gridded at $16 \mathrm{~m}$ (Figure 5). However, the original bathymetry for the regional coverage was gridded at a much higher resolution (up to $90 \mathrm{~m}$ ) and was simply re-gridded in the NE Compilation. 


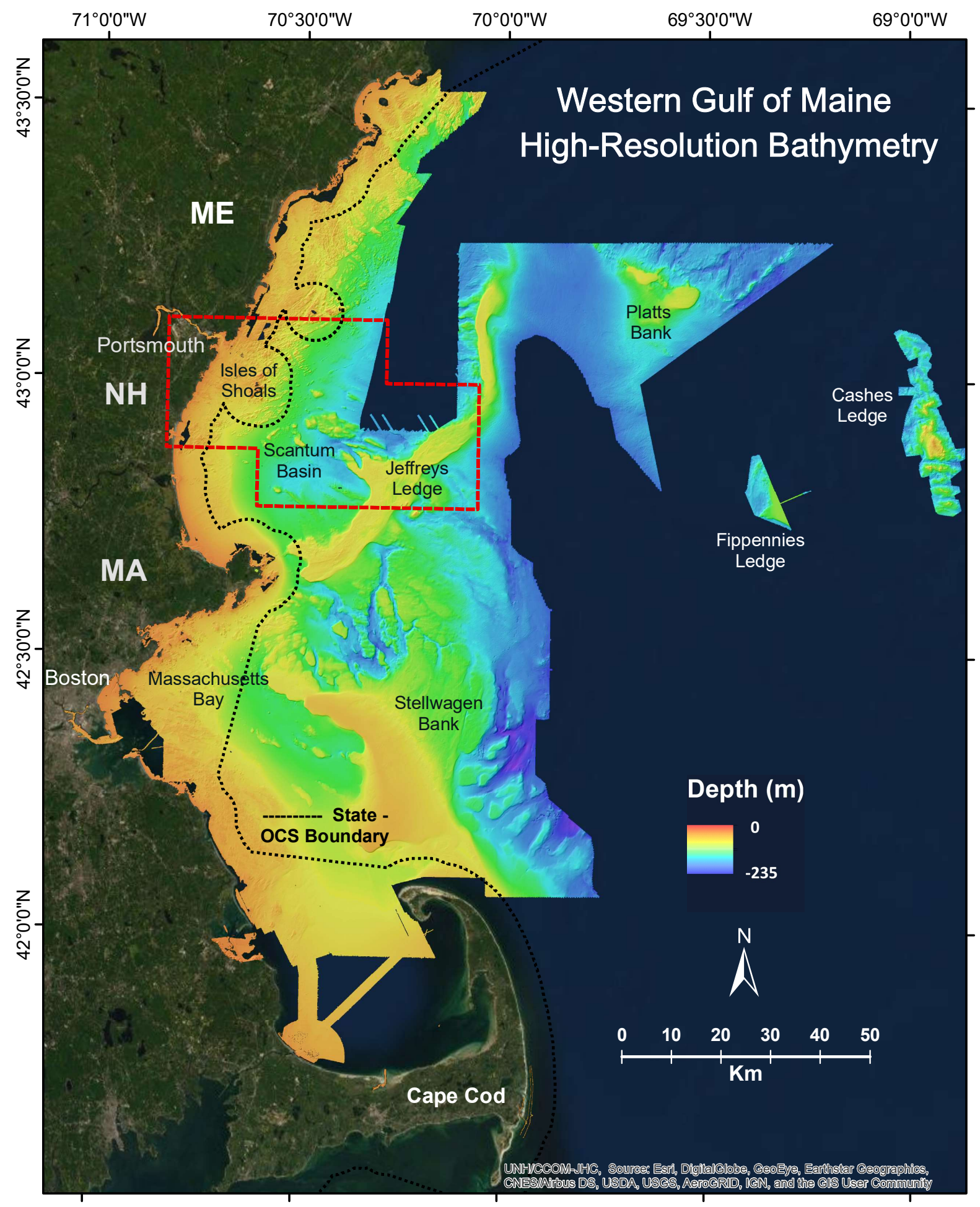

Figure 4. High-resolution bathymetry synthesis for the Western Gulf of Maine gridded at $4 \mathrm{~m}$ from the Northeast Bathymetry and Backscatter Compilation. See http://ccom.unh.edu/project/NE-bathymetry-and-backscattercompilation, assessed March 15, 2021. Surficial geology maps were developed for the area outlined with a dashed red line. 


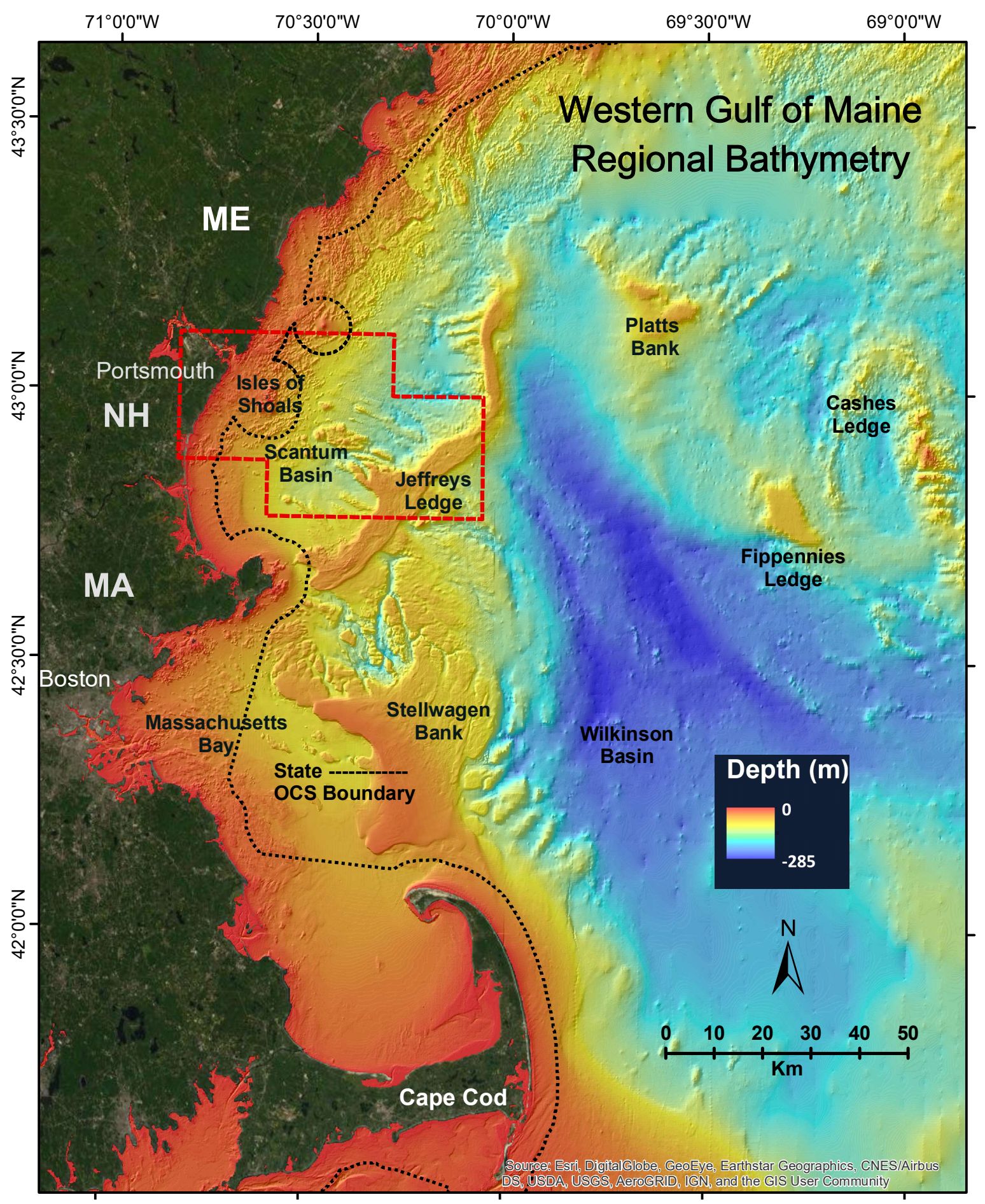

Figure 5. Regional bathymetry synthesis for the Western Gulf of Maine gridded at $16 \mathrm{~m}$ from the Northeast Bathymetry and Backscatter Compilation. See http://ccom.unh.edu/project/NE-bathymetry-and-backscattercompilation, assessed March 15, 2021. Surficial geology maps were developed for the area outlined in with a dashed red line. 
The backscatter synthesis was prepared from MBES mosaics available from National Centers for Environmental Information (NCEI), NOAA National Ocean Service (NOS), and UNH JHC/CCOM as part of the WGOM Bathymetry and Backscatter Synthesis (Figure 6; Ward et al., 2016b). The goal was to develop a single surface with backscatter surveys to help map the seafloor and predict sediment grain size. However, a review of the MBES backscatter mosaics obtained for the synthesis revealed the surveys were collected with different acoustic systems that each used different algorithms to process the backscatter. Also, the MBES was run over a wide range of frequencies. Recent studies have also shown that the algorithms used by different vendors can yield different results, and the lack of a consistent calibration method is problematic for combining or synthesizing MBES backscatter (Weber et al., 2018; Smith et al., 2020). Finally, with the exception of the UNH JHC surveys, many of the backscatter mosaics were of poor quality and needed to be reprocessed. Therefore, all backscatter surveys were converted to grayscale and the range of pixel intensity values were normalized and standardized across all surveys to create a seamless mosaic image gridded at $1.5 \mathrm{~m}$. The backscatter mosaic was very useful for identifying textural boundaries and geomorphologically significant seafloor features. However, it was not useful for predictive models.

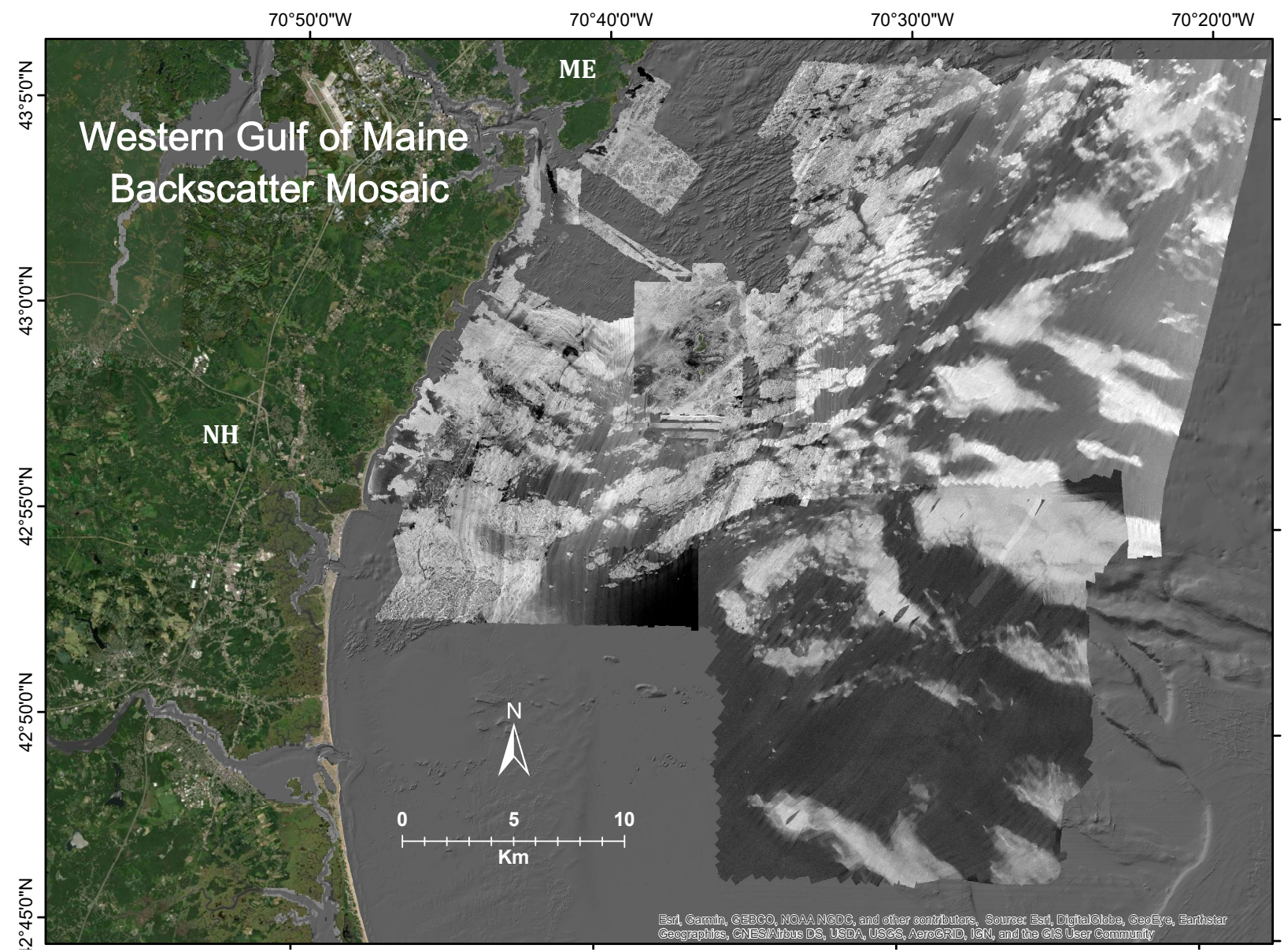

Figure 6. Backscatter synthesis shown in the Western Gulf of Maine Bathymetry and Backscatter Synthesis. Intensity is indicated by grayscale with the lighter shades indicating stronger backscatter reflection. 
Based on the results of this effort it was decided that in going forward, acoustic backscatter surveys would not be synthesized, but rather kept as separate mosaics with intensity presented in decibels if possible. The MBES surveys carried out each summer as part of the UNH CCOM/JHC (Ocean Engineering 972) Hydrographic Field Course are consistently of high quality (referred to as the CCOM/JHC Summer Hydro or $\mathrm{SH}$ in the databases). Therefore, the CCOM/JHC Summer Hydro high-resolution bathymetry was merged into the NE Compilation bathymetry synthesis described above, but was also kept as separate surveys along with the co-registered backscatter. The backscatter from the CCOM/JHC Summer Hydro surveys run prior to 2016 were included in the WGOM Synthesis described above, but also maintained as separate surveys with intensity values in decibels, except for SH2017 and SH2018 which were archived as grayscale mosaics (Figure 7). The CCOM/JHC Summer Hydro surveys proved very useful for mapping purposes, but were too limited in scope to use remote sensing applications for developing the surficial geology maps. More details of the surveys and metadata can be are found in Ward et al. (2016b) and Ward et al. (2021h). The NE Compilation is available on the UNH CCOM/JHC web site at http://ccom.unh.edu/project/NE-bathymetry-and-backscatter-compilation, accessed March 15, 2021.

\section{Bathymetric Derivatives}

Previous work has shown that several bathymetric derivatives are helpful for seafloor characterization and substrate mapping including slope, bottom roughness or rugosity, ruggedness, and bathymetric position index (BPI) (Erdey-Heydorn, 2008; Dorschel et al., 2014; Oliveira et al., 2020). Many of these derivatives have been used in supervised or unsupervised remote classifications or machine learning in the past (Diesing et al., 2014). During this study the seafloor classification and mapping was completed manually by an expert analyst. However, several bathymetric derivatives proved helpful in determining textural boundaries (e.g., roughness or rugosity) and physiographic features (e.g., hillshade and BPI). Therefore, the derivatives for the continental shelf off $\mathrm{NH}$ were developed at the highest resolution possible (and practical). The multibeam surveys for the study area were re-gridded with regard to their location and data source (original resolution). The bathymetry was divided into a nearshore and offshore region and gridded at $2 \mathrm{~m}$ and $4 \mathrm{~m}$, respectively. In the areas where only low-resolution bathymetry was available, the grid size was $8 \mathrm{~m}$.

The bathymetry was gridded using the "Mosaic to New Raster" tool from the Data Management Toolbox in ERSI ArcGIS 10.3. The new bathymetry datasets were stored in geodatabases in FGDBR (File Geodatabase Raster) format. The pixel type and depth were set to 32-bit supporting decimals (32_BIT_FLOAT), and the number of bands was set to one. The Mosaic Operator for the overlapping areas was configured to be the average value of the overlapping cells. The bathymetry was then used to create the derivatives including hillshade, BPI, and roughness.

\section{Hillshade}

The hillshade was developed by generating an imaginary light source and casting shades and shadows to create the shaded relief effect (Figure 8). A default azimuth of $315^{\circ}$ and altitude of $45^{\circ}$ were used for the illumination. The output values range from 0 to 255, with 0 representing the shadowed areas and 255 representing the brightest. The vertical exaggeration was 10x. The hillshade was very helpful in defining bathymetric features as it highlights bathymetric changes. Also, the vertical exaggerations can be altered to enhance features where needed. 


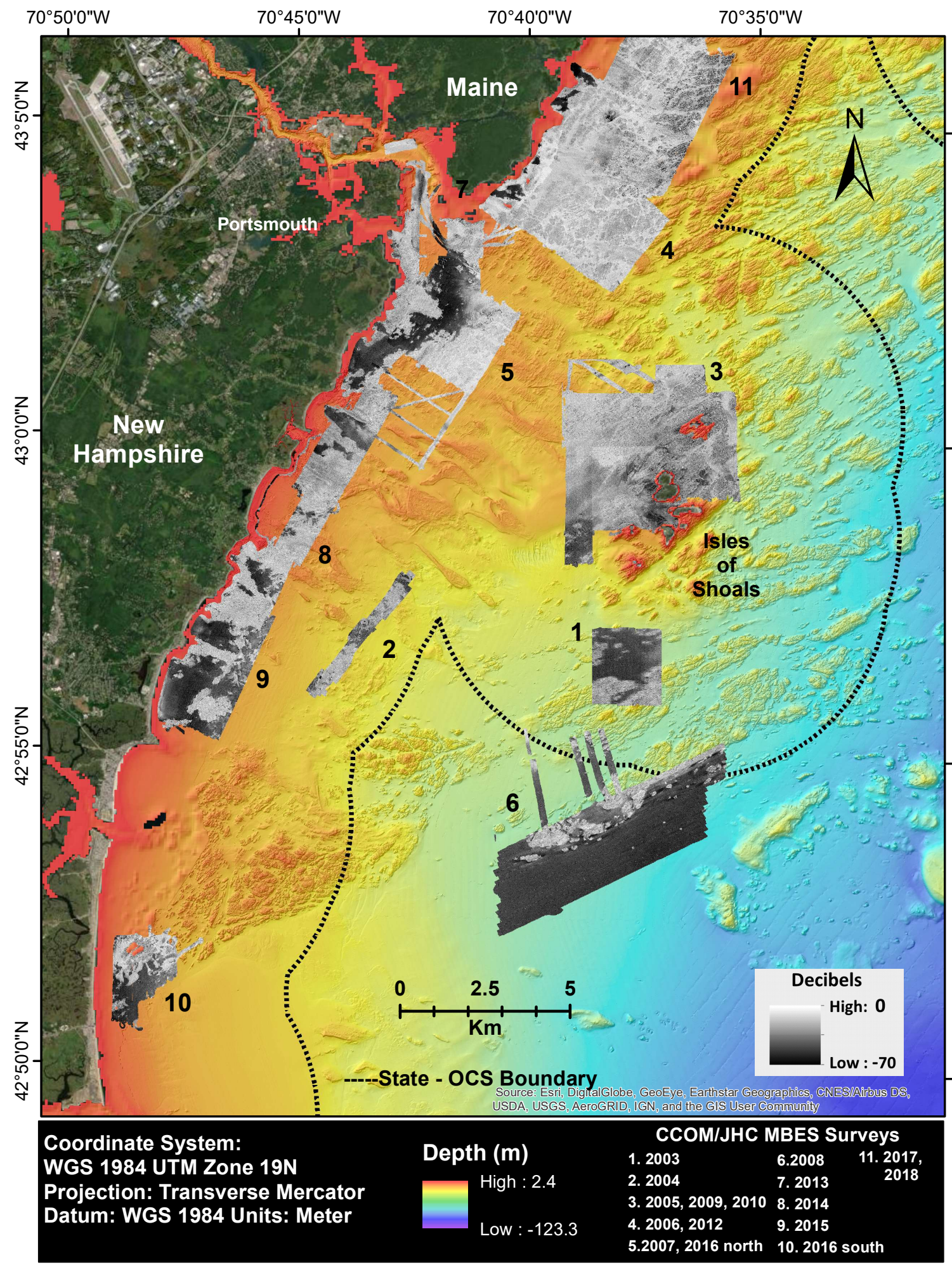

Figure 7. Location map of the UNH CCOM/JHC (Ocean Engineering 972) Hydrographic Field Course MBES surveys. The range of backscatter intensity in decibels shown in the legend is approximate due to the different MBES systems and variable frequencies used in the surveys. Also, the 2017 and 2018 backscatter surveys are in grayscale and not decibel values. For these surveys the lighter shades indicate stronger backscatter reflection or intensity. Station locations and sample types are given in Appendix B. 


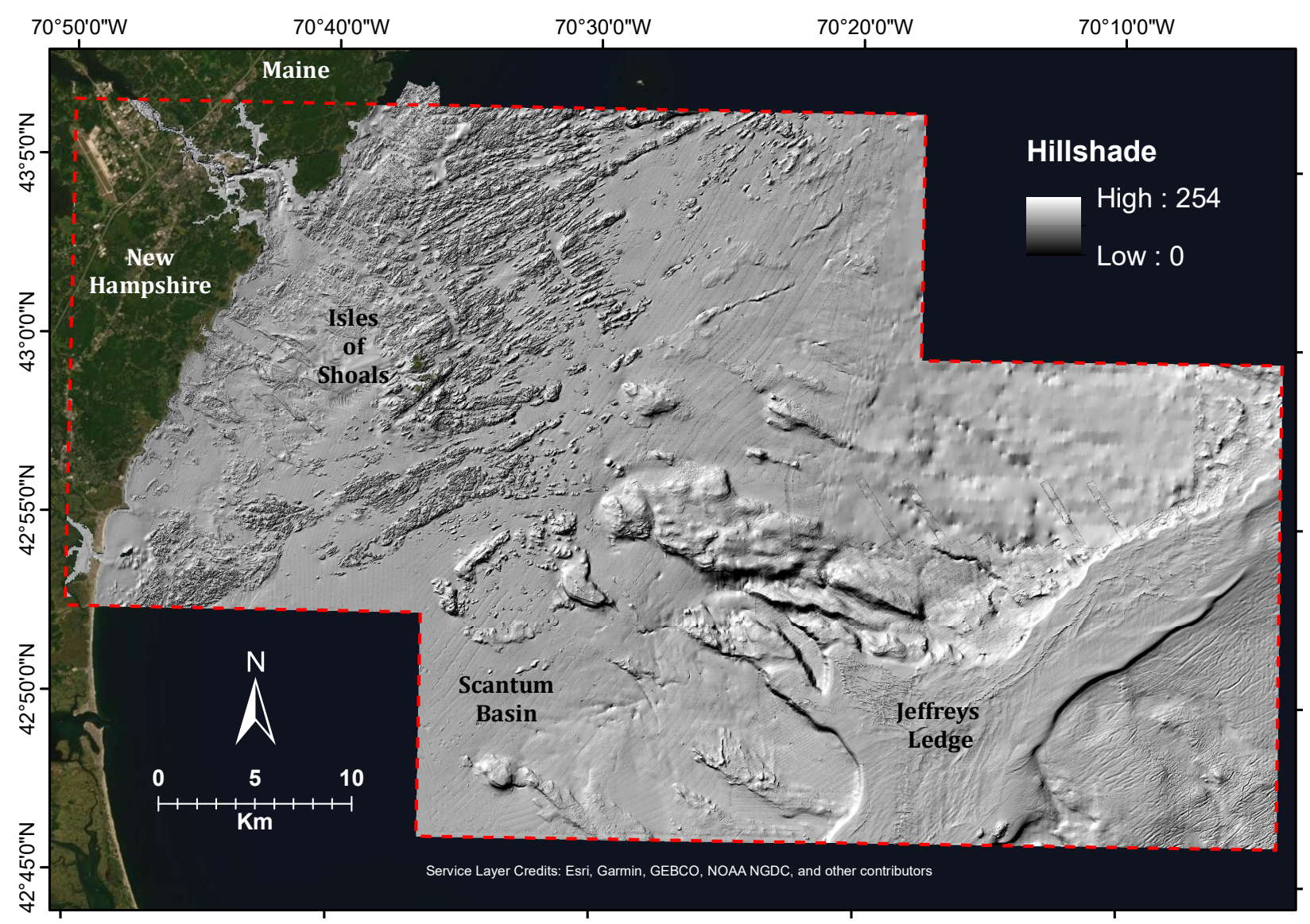

Figure 8. Hillshade for the continental shelf off New Hampshire. The vertical exaggeration is 10x.

\section{Bathymetric Positioning Index (BPI)}

BPI compares the elevation of each gridded cell in the bathymetry within the inner radius of an annulus to the mean elevation of the surrounding area defined by the outer radius of the annulus (Verfaillie et al., 2007). A negative BPI value is assigned for depressions and a positive BPI value to elevated features. ErdeyHeydorn et al. (2008) used BPI to identify seafloor features. In this study, the index was derived from bathymetric raster surfaces using the "Benthic Terrain Modeler toolbox" and was calculated for a broad scale (B-BPI). The B-BPI was applied with different inner and outer radii depending upon the resolution of the grid in order to maintain the same scale factor for both nearshore and offshore areas. The $2 \mathrm{~m}$ nearshore grid used a 20 -cell inner radius and an 800 -cell outer radius. The $4 \mathrm{~m}$ offshore grid used a $10-$ cell inner radius and a 400-cell outer radius. Therefore, both the nearshore and offshore B-BPI derivatives have a scale factor of 1,600 (Figure 9). BPI was very useful for identifying bathymetric features, especially when combined with hillshade.

\section{Roughness}

The roughness value equals the sum of the change in elevation between a central grid cell and its eight surrounding grid cells. The roughness surfaces were generated using the Surface Texture toolset from the Geomorphometry and Gradient Metric Toolbox. A circular analysis window was used with a radius of 3 cells for the Neighborhood Settings (Figure 10). The roughness proved useful for identifying changes in bottom texture and for defining boundaries. 


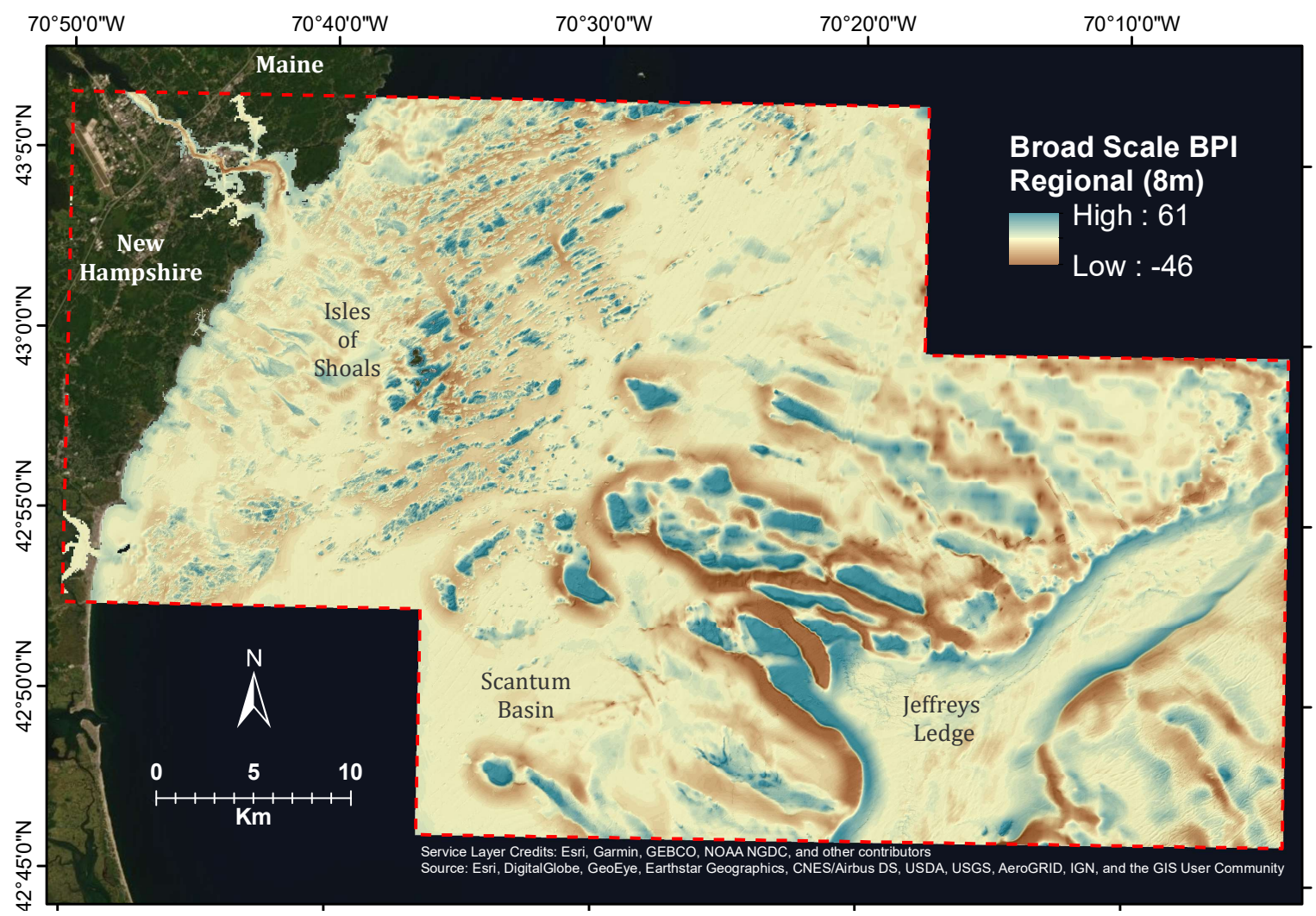

Figure 9. Bathymetry Position Index (BPI) for the continental shelf off New Hampshire.

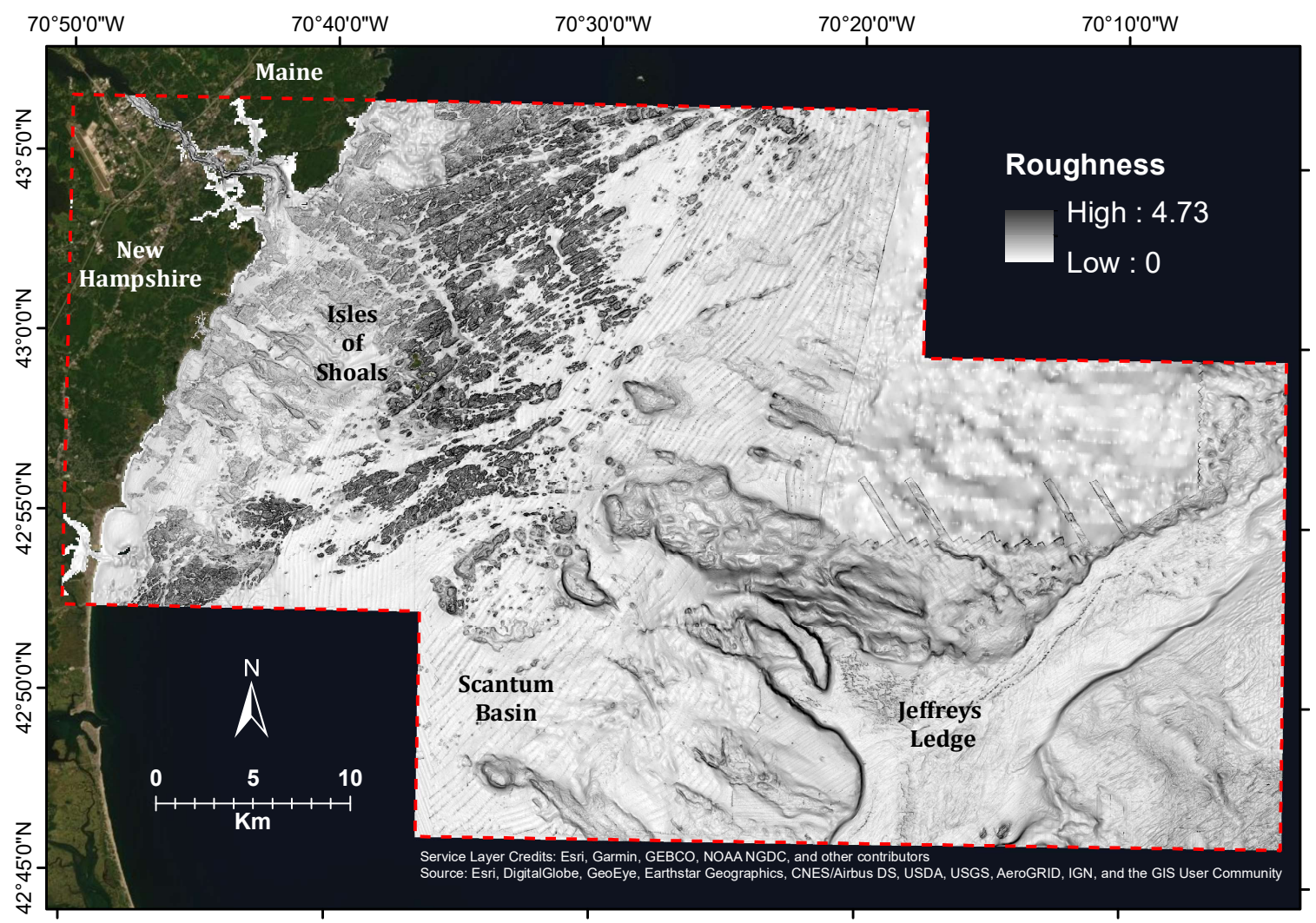

Figure 10. Seafloor roughness for the continental shelf off New Hampshire. 


\section{Surficial Geology of the Continental Shelf Off New Hampshire}

The surficial geology of the WGOM continental shelf is highly complex due to the impact of glaciations, sea-level fluctuations, marine processes, and the antecedent topography (Uchupi, 1966; Oldale and Uchupi, 1970; Oldale et al., 1973; Ballard and Uchupi, 1974; Schnitker et al., 2001; Uchupi, 2004; Barnhardt et al., 2007; and Uchupi and Bolmer, 2008). In order to describe the surficial geology of the continental shelf off $\mathrm{NH}$, help assess potential areas where sand and gravel resources may be located, provide a base for habitat and future environmental studies, and advance the understanding of the processes that shaped the seafloor, high-resolution surficial geology maps were developed that depict the geoforms and surficial sediment distribution in detail. Here, we present the surficial geology maps and make an initial assessment of the origin of many of the physiographic features based on CMECS. This includes taking into consideration the glacial and sea-level history that modified the antecedent topography and lead to many of the depositional features observed today.

\section{Glaciation, Sea-Level and Upland Surficial Geology in the WGOM}

During the last glacial period (Wisconsin), the Laurentide Ice Sheet (LIS) covered New England and the Gulf of Maine, extending to Long Island and Georges Bank. The LIS reached its maximum extent approximately 24,000 yrs B.P. (Balco and Schaefer, 2006). The upland and nearshore shelf was heavily eroded by the advancing ice sheet, exposing bedrock in many areas and leaving extensive sediment deposits including moraines, drumlins, eskers, glaciomarine deltas, submarine grounding lines, fans, kettles, tills, and stratified drift (Birch, 1984; Sinclair et al., 2018). Subsequently, the glacial deposits on what is now the inner continental shelf off $\mathrm{NH}$ were eroded by intertidal and shallow-water wave and tidal processes as the coast first underwent a transgression (marine incursion), then a regression (lowering of sea level), and a final transgression. The cause of the shift in the shoreline was driven by glacially-forced eustatic sea-level changes and isostatic adjustments of the earth's crust.

The first (late Pleistocene - early Holocene) marine incursion occurred during deglaciation when the glacier's terminus was receding and the ice front was in contact with the ocean, resulting in a submergence of the upland. The magnitude of the highstand (most landward inundation of the ocean) varied along the coast from Massachusetts to Maine due to the different ice thicknesses and levels of isostatic depression. Around 14,000 yrs B.P. the highstand extended to approximately $30 \mathrm{~m}$ above present in northern Massachusetts (Barnhardt et al., 2007), $45 \mathrm{~m}$ in NH (Birch, 1988), and $75 \mathrm{~m}$ in southern Maine (Kelley et al., 2010). Following the highstand, isostatic uplift of the crust due to the removal of the weight of the ice as it melted led to a major lowering of relative sea level (marine regression) as the rate of rebound exceeded eustatic sea-level rise. At approximately 12,500 yrs B.P. the relative or local sea level lowered to $\sim 55-60 \mathrm{~m}$ below current sea level in southern Maine (Kelley et al., 2010), and $\sim 45 \mathrm{~m}$ below current sea level in Massachusetts (Oldale et al., 1993; Barnhardt et al., 2007). As isostatic uplift began to slow, eustatic sea-level rise overtook crustal rebound leading to a relative sea-level rise and a transgression that is still occurring today. Due to the magnitude of the changes in relative sea level in the WGOM, the position of the NH shoreline migrated $\sim 40 \mathrm{~km}$ (Ward and Adams, 2001). During the maximum transgression with ice retreat, the ocean flooded inland $\sim 25 \mathrm{~km}$, and during the maximum regression, the coastline was $\sim 15 \mathrm{~km}$ seaward of its present position (Figure 11).

As a result of the glaciation and relative sea-level fluctuations, the coastal upland in the WGOM is dominated by glacial and glaciomarine deposits. This is clearly shown in surficial geology maps of the NH seacoast published by the NH Geological Survey (Bennet et al., 2004). The major features and deposits within approximately ten kilometers of the shoreline include bedrock outcrops, glacial tills, glaciomarine sediment, and Holocene (recent) deposits (Figure 12). The till deposits, which are extensive, include drumlins, eskers, and moraines. The glaciomarine deposits include wave-modified marine deltas, wave- 
formed features composed of sand and gravel, and the three facies of the Presumpscot Formation including the proximal (sandy), distal (silt and clay), and undifferentiated mixtures (sand, silt, and clay). It is reasonable to assume that these same types of deposits occur on the adjacent continental shelf.

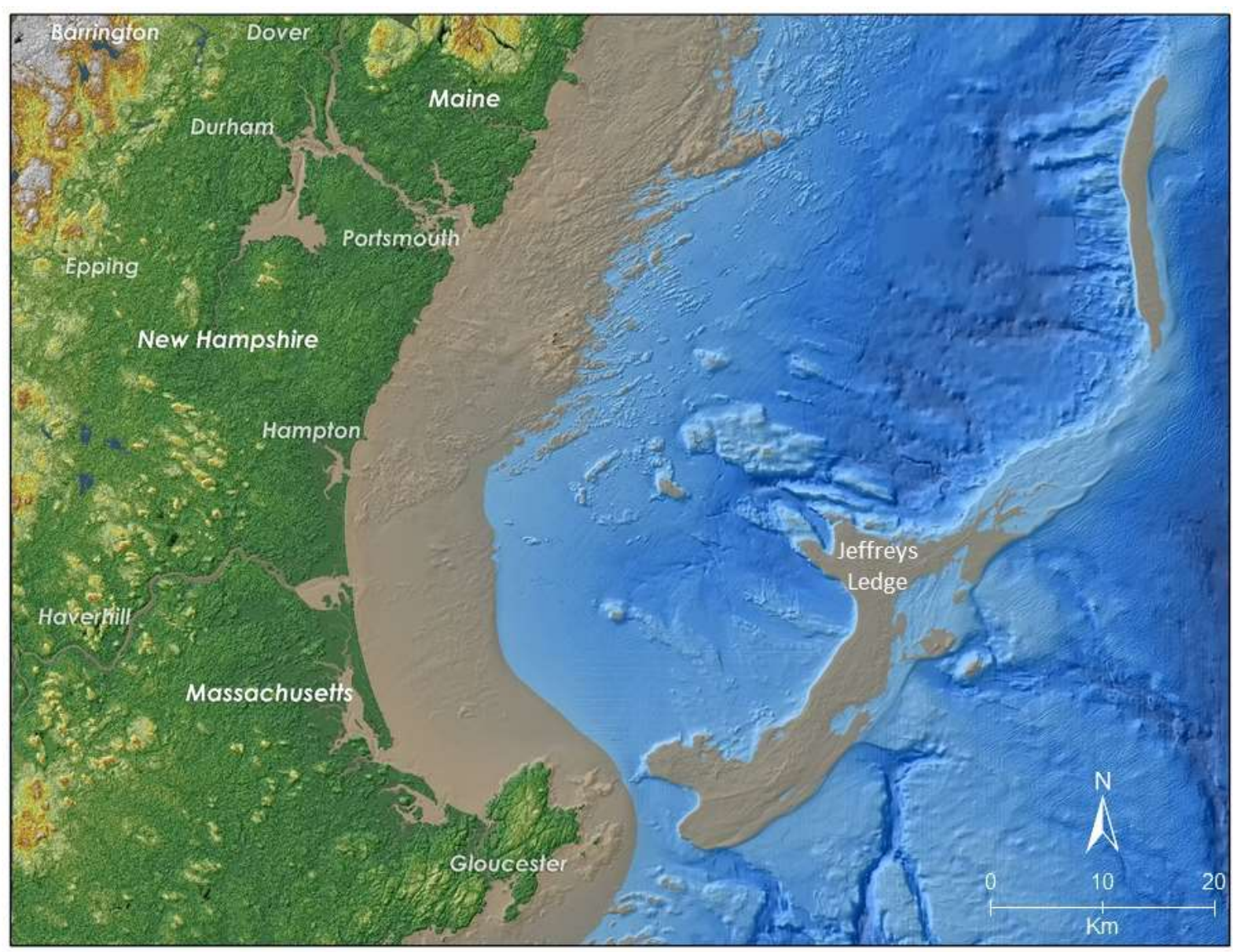

Figure 11. Location of the NH shoreline (seaward edge of brown area) during the last sea-level lowstand at -60 $m$, $\sim 12,500$ yrs B.P. (based on sea level curve from Kelley et al., 2010). During the Holocene transgression, the shoreline migrated landward reaching its present position about 4,000 B.P. (Ward and Adams, 2001). Sub-aerial exposure of the New Hampshire continental shelf and the subsequent transgression lead to extensive erosion and modification of the glacial deposits. Note the surface of Jeffreys Ledge and some of the drumlins (mounds to the west of Jeffreys Ledge) are above or near sea level during the lowstand. 


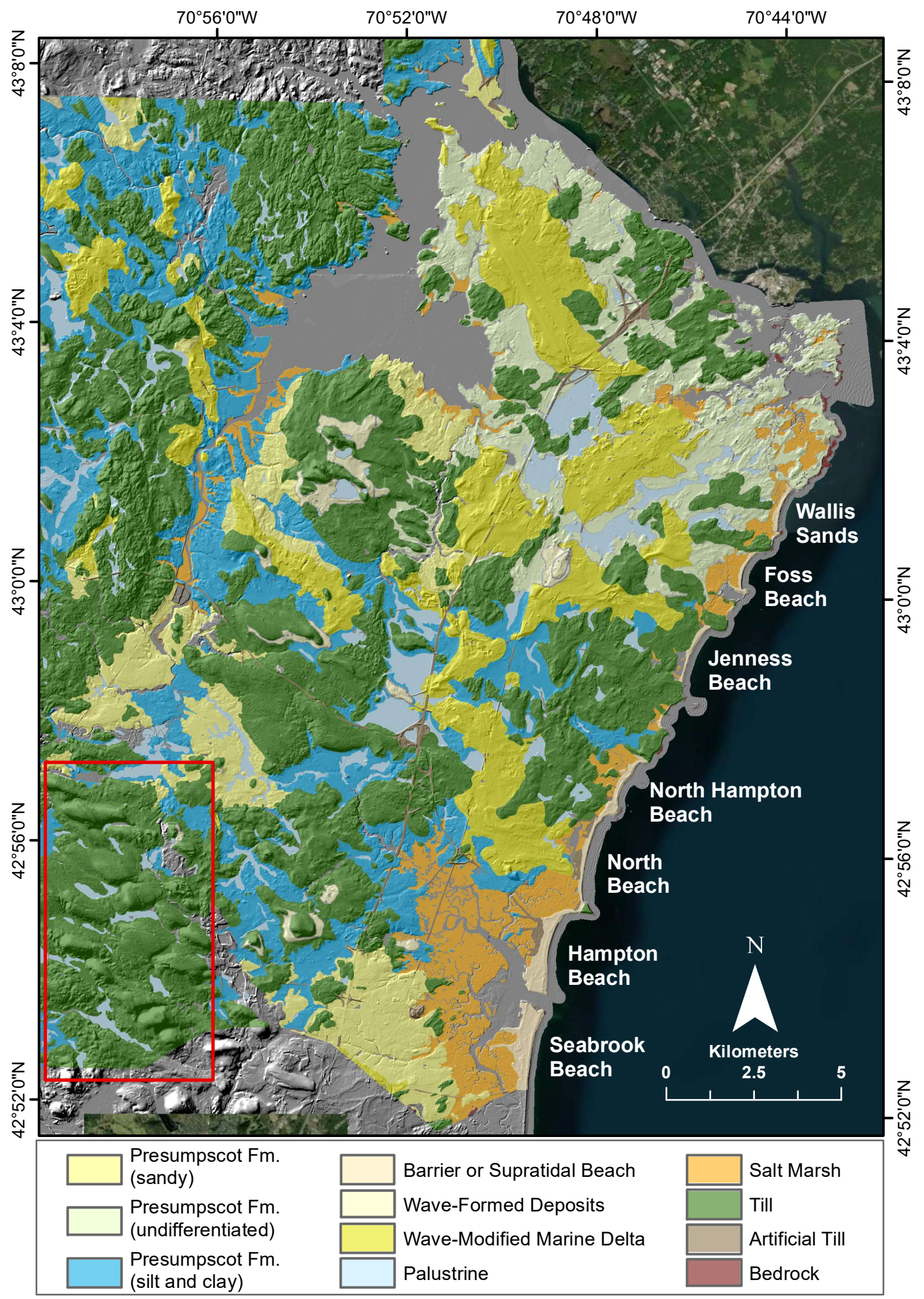

Figure 12. Surficial geology map of the NH coastal upland (modified from the NH Geological Survey digital map series; Bennett et al., 2004 http://granit.unh.edu, accessed January 2021) from Ward et al. (2021f). Note the extensive glacial deposits including tills (e.g., drumlins), Presumpscot Formation (glaciomarine), and wave-modified marine deltas. The red box in the lower left highlights an area where drumlins are also present. Definitions for terms can be found in the Glossary. 


\section{Surficial Geology of the Shelf}

High-resolution mapping of the surficial geology of the continental shelf off $\mathrm{NH}$, using the CMECS geoform and geologic substrate classifications, reveals the seafloor is primarily composed of the following: extensive megaclast platforms (cobble and boulder), many of which extend offshore from the headlands along the coast; sandy nearshore ramps which extend from the beaches; bedrock outcrops; marine shoals; large elongated mounds with twenty meters or more of relief that appear to be marine-modified drumlins; smaller linear ridges composed of sand and gravel that appear to be eroded eskers; and seafloor plains. The seafloor plains are composed of sand, gravel mixes, and gravel in shallower depths (and were exposed sub-aerially during the sea-level lowstand), while in deeper water they are composed of mud. There are also a number of large ridges or bathymetric highs in the WGOM including Jeffreys Ledge, Cashes Ledge, Fippennies Ledge, and Platts Bank. Jeffreys Ledge, which forms the seaward extent of the study area, rises up $\sim 150 \mathrm{~m}$ from the adjacent basins (i.e., Scantum Basin or Wilkinson Basin) to depths less than $50 \mathrm{~m}$ on the ridge surface (Figure 5 ).

The surficial geology maps provide a detailed view of the major physiographic features or geoforms and the general composition of the substrate. The substrate is broken into three classes including exposed bedrock, coarse and fine unconsolidated substrates, and a much more detailed description of the seafloor sediments mapped as substrate groups. These maps serve several purposes. First, the geoform maps depict the major seafloor features and also provide insights into the origin and controls of physiographic features and potential sediment types and sources (Figures 13, 14, and 15). Second, the map showing the CMECS subclass provides a very broad overview of the bedrock and the general nature of the surficial sediments from coarse-grained to fine-grained unconsolidated mineral material (Figure 16). Finally, the maps depicting the sediment groups provide the most detail of the seafloor surficial sediments (Figures 17,18 , and 19). Collectively, these maps significantly advance our understanding of the continental shelf off $\mathrm{NH}$. Each of the map types are discussed below in more detail.

It should be noted again that the size of the study area is large and, despite having an extensive database including high-resolution bathymetry, acoustic backscatter, and surficial sediment grain size data, many areas have sparse or incomplete coverages. In addition, the quality of the data varies depending on age and location. As a result, the mapping of the seafloor includes locations where the evidence is strong, locations where less information is available (shown by diagonal lines on the maps), and locations where the database is too weak at this time to allow interpretation of the seafloor (simply shown as grayscale hillshade).

\section{Geoforms (Physiographic Features): Origin and Characteristics}

The geoforms identified and classified on the continental shelf off NH show very clear trends (Figure 13). The inner NH continental shelf within $15 \mathrm{~km}$ of the coast north of Portsmouth Harbor is dominated by extensive bedrock outcrops, often separated by troughs or swales with sediment, and surrounded by bedrock borders composed of coarse sediment. The general trend of the outcropping bedrock is in a northeast-southwest direction. Landward of the Isles of Shoals and south of Portsmouth Harbor, the bedrock that had dominated the seafloor to the north transitions into sediment-draped bedrock and large sediment deposits (Figure 14). In this area, extensive marine-modified (eroded) glacial features are found. Geoforms that appear to be the roots of eroded drumlins or eskers are common, which have left megaclast deposits.

Morphologic evidence suggests that some of the marine-formed shoals developed from the erosion of drumlins or other glacial features as was observed in other paraglacial environments (Carter and Orford, 1988). The largest sandy shoal in the study area is located just landward of the Isles of Shoals (named here as the Northern Sand Body) and appears to lie between two eroded drumlins (Figure 14). It is likely the 
Northern Sand Body was formed from a wave-modified delta or marine shoal as seen on the adjacent upland. However, this is speculative and remains to be tested. Also present on the NH shelf are numerous De Geer moraines which formed by the receding ice front (Sinclair et al., 2018). The bedrock becomes more subdued south of the study area in northern Massachusetts and is ultimately buried by sandy sediment, likely from the Merrimack River.

A number of the marine-modified glacial features found close to shore also extend onshore. Particularly noticeable are marine-modified glacial deposits with megaclasts found directly off the numerous headlands along the coast. These deposits formed as the till-dominated headlands eroded during the present sea-level transgression leaving behind gravel and megaclast deposits. Also, a narrow ridge (likely an esker) originates on the inner shelf and continues onshore. The marine-formed features, as well as some of the offshore eroded drumlins, are possible targets for marine mineral resources and will be examined in greater detail in future studies.

From several kilometers seaward of the Isles of Shoals to Jeffreys Ledge, the geoforms are dominated by seafloor plains and very large marine-modified glacial features including drumlins (Figure 15). During the sea-level lowstand, the surface of Jeffreys Ledge and the nearby drumlin-like features were likely eroded by wave activity. In addition, the eroded material, which would have included sand and fine gravel, was deposited as aprons around the glacial features. A large fan-shaped structure composed of gravels to gravel mixes located on the seaward side of Jeffreys Ledge is interpreted as a subaqueous fan. The subaqueous fan resulted from subglacial discharge from beneath the glacier during the period when the ice front was close to or grounded on Jeffreys Ledge.

\section{Seafloor Sediments Based on CMECS Substrate Subclass Map}

The geologic substrate maps depict the surficial sediment composing the seafloor. The substrate subclass simply classifies the seafloor into bedrock and coarse or fine unconsolidated mineral substrate (Figure 16). The coarse unconsolidated mineral substrate includes gravel, gravel mixes, and gravelly sediment which encompass the Wentworth size classes from gravelly sand or gravelly mud to boulder gravels. The fine unconsolidated mineral substrate is composed of sediment ranging from mud to slightly gravelly sand (Table 1).

As would be expected due to the glacial history, the seafloor landward of the Isles of Shoals has extensive bedrock outcrops that tend to have coarse unconsolidated mineral substrate between the ridges. The marine-modified glacial deposits fall into this category and tend to be gravelly, although they may contain appreciable quantities of sand. The fine unconsolidated mineral substrate is found closer to shore extending off beaches and in between coarse unconsolidated mineral substrate extending off headlands.

Seaward of the Isles of Shoals, the seafloor tends to be composed of fine unconsolidated mineral substrate with the exception of the marine-modified glacial deposits (Figure 16). The glacial deposits tend to include coarse unconsolidated mineral substrate with gravel, gravel mixes, and gravelly sediment. The fine unconsolidated sediment is typically muddy to sandy mud but can be coarser depending on proximity to glacial features or bedrock.

\section{Seafloor Sediments Based on CMECS Substrate Group Map}

The geologic substrate group describes the composition of the unconsolidated mineral sediments of the seafloor in much greater detail than the substrate subclass (Figure 17). The substrate groups are closely aligned to the Wentworth scale and have the advantage of simplifying the Wentworth size classes by combining ranges.

The seafloor of the inner continental shelf landward of the Isles of Shoals is extremely heterogeneous due to the mixture of bedrock, marine-modified glacial features, and marine-formed features or shoals (Figure 
18). Here the seafloor is frequently composed of gravel mixes interspersed with gravel and associated with eroded glacial features such as drumlins or eskers. Close by are gravelly mixes to gravelly sediments, which are frequently gravelly sands. The exposed bedrock has gravel mixes to gravelly sediments in the troughs between the bedrock outcrops as well. The nearshore regions have relatively large areas of sand which are found close to shore on nearshore ramps. Sandy areas are also found farther offshore associated with eroded glacial features or marine-formed shoals. One large marine-formed feature, the Northern Sand Body (Figure 18), is a potential source of sand for beach nourishment and was described in Ward et al. (2021a).

The outer continental shelf seaward of the Isles of Shoals is somewhat complex as a result of marinemodified glacial features providing coarse sediments intermixed with muds (Figure 19). The surface of Jeffreys Ledge is composed of gravel and gravel mixes (e.g., sandy gravel), while the adjacent seafloor is mostly gravelly (gravelly sands, gravelly muddy sand, or gravelly mud). Jeffreys Ledge was likely a grounding line as the ice front retreated in the Gulf of Maine, which left extensive glacial deposits. During the last sea-level lowstand, the surface of Jeffreys Ledge was exposed to shallow water processes (Figures 5 and 11), eroding the glacial deposits and forming a slightly gravelly apron around much of Jeffreys Ledge.

At least one large sand deposit occurs in the offshore area on top of an eroded drumlin (Figure 19). This could be a potential marine mineral resource in the future when sand and gravel mining become economically feasible for deposits farther from shore in deeper water (Ward et al., 2021a). Based on the number of large marine-modified glacial features, there are likely several more viable sand and gravel deposits offshore. Note that several of the marine-modified glacial features are not mapped (shown as gray hillshade) because their composition is presently unknown. Away from the glacial features, the seafloor is mostly muddy, transitioning into a sandy mud closer to land.

\section{Summary and Conclusions}

The purpose of the work presented here was three-fold. First, to develop high-resolution surficial geology maps of the complex paraglacial continental shelf off $\mathrm{NH}$ in the Western Gulf of Maine (WGOM) in order to aid in marine mineral resource (sand and gravel) identification, evaluation, and required environmental studies for any sites that may be proposed for sand and gravel extraction. Secondly, to apply a standardized, widely used approach to classify the seafloor (i.e. the Coastal and Marine Ecological Classification Standard or CMECS; FGDC, 2012). The challenge here was adapting the CMECS classification to a paraglacial environment. Third, to advance our ability to utilize re-purposed, archived geophysical data by combining the older data with new, high-resolution acoustic surveys and applying modern technologies such as GIS platforms, data processing programs, and new algorithms. Future work will also evaluate the use of machine learning (Pendleton et al., 2019).

High-resolution mapping of the surficial geology of the continental shelf off $\mathrm{NH}$ and extending to Jeffreys Ledge (an area of $\sim 3,250 \mathrm{~km}^{2}$ ), using the CMECS geoform and geologic substrate classifications, reveals the seafloor is primarily composed of extensive bedrock outcrops, large megaclast platforms (cobble and boulder), marine-modified drumlins and eskers, sandy nearshore ramps, marine shoals, and seafloor plains. These features are composed of sand, gravel mixes, and gravel in shallower depths transitioning to mud in deeper water. The largest feature in the study area is Jeffreys Ledge, which has up to 150 $\mathrm{m}$ of relief over the nearby basins and depths less than $50 \mathrm{~m}$ on the ridge surface.

Understanding the glacial history of the region, sea-level changes in the WGOM, and the surficial geology of the adjacent upland provides insights on how the geoforms and substrate on the continental shelf off $\mathrm{NH}$ were likely formed and modified over time. The sandy seafloor plain sediment found in the nearshore shelf (within approximately 15 kilometers of the coast) is likely sourced from reworked marine-modified 
glaciomarine delta or proximal sandy glaciomarine deposits. Also, very common on the inner shelf are gravel mixes to gravels that are likely the result of erosion of eskers or till moraines. Large elongated megaclast deposits composed of cobbles and boulders are interpreted as the remnants of drumlins that were eroded by marine processes during the Late Pleistocene sea-level transgression, the early Holocene regression, and the Holocene transgression which is continuing today. Finally, De Geer moraines are common on the inner shelf. Farther offshore below the relative sea-level lowstand that occurred $\sim 12,000$ yrs B.P., glacial features such as drumlins have not been altered by marine processes as extensively as those on the inner shelf. The exceptions are features that have high relief such as large drumlins that were winnowed by wave action during the last sea-level lowstand. The surface of Jeffreys Ledge was also modified by shallow water processes during the sea-level lowstand, producing wave-formed features that can be seen in the high-resolution bathymetry.

Evaluation of potential marine mineral resources on the $\mathrm{NH}$ shelf identified several sites where sand and gravel resources may be suitable for beach nourishment projects to enhance coastal resiliency. These deposits are discussed in detail in Ward et al. (2021a) and include a large marine shoal (see Figures 14 and 18) and the eroded surface of a drumlin (see Figure 19). These potential resource areas are presently too far from shore and in too great a depth of water to be easily utilized. However, as the demand for sand and gravel becomes more acute and technologies advance, mineral resources farther offshore and in deeper water likely will become viable. 


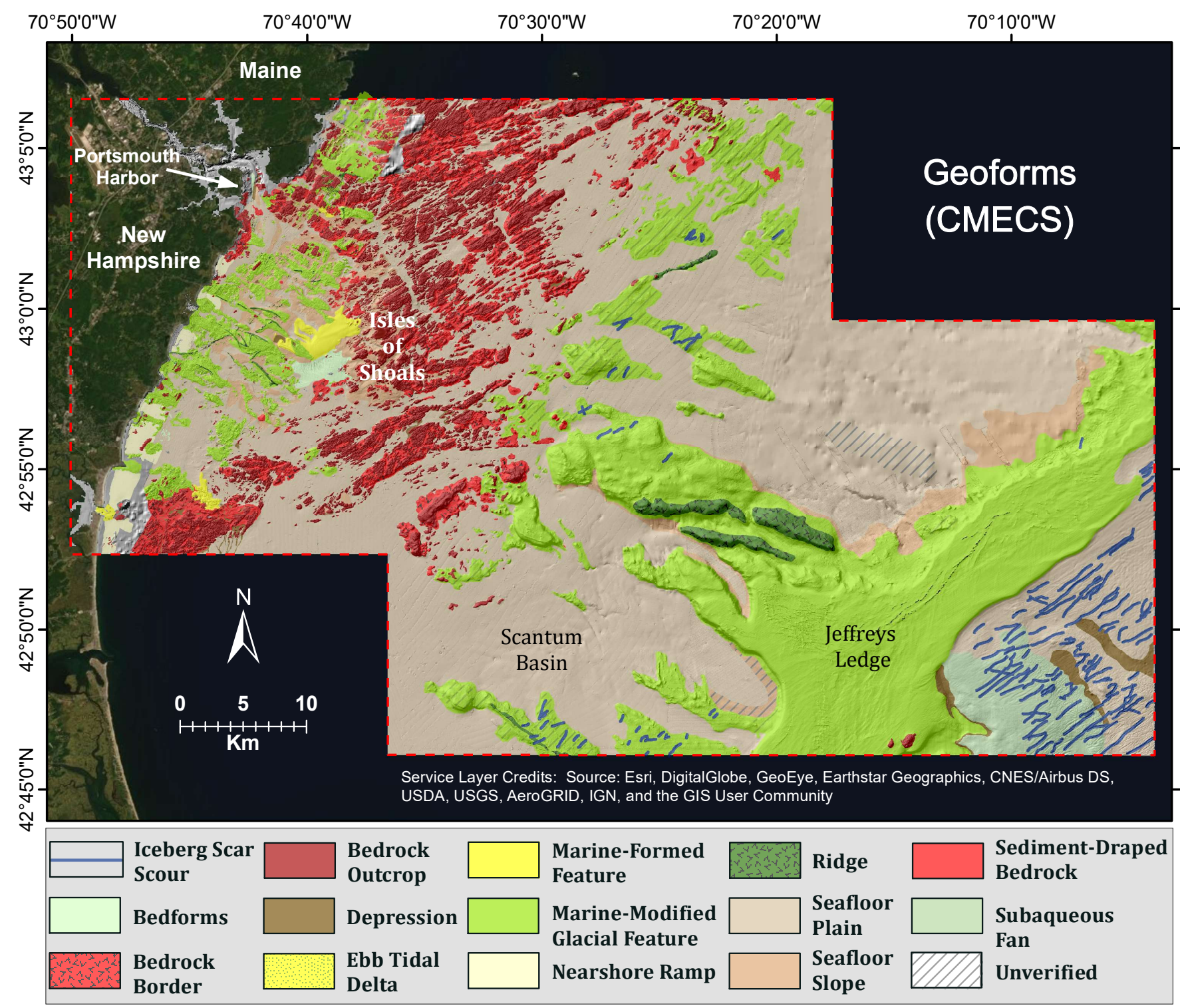

Figure 13. Major physiographic features (geoforms) map for the continental shelf off New Hampshire. The geoform terminology is based on a modification of CMECS (FGDC, 2012). See Table 2 for definitions of geoforms. 


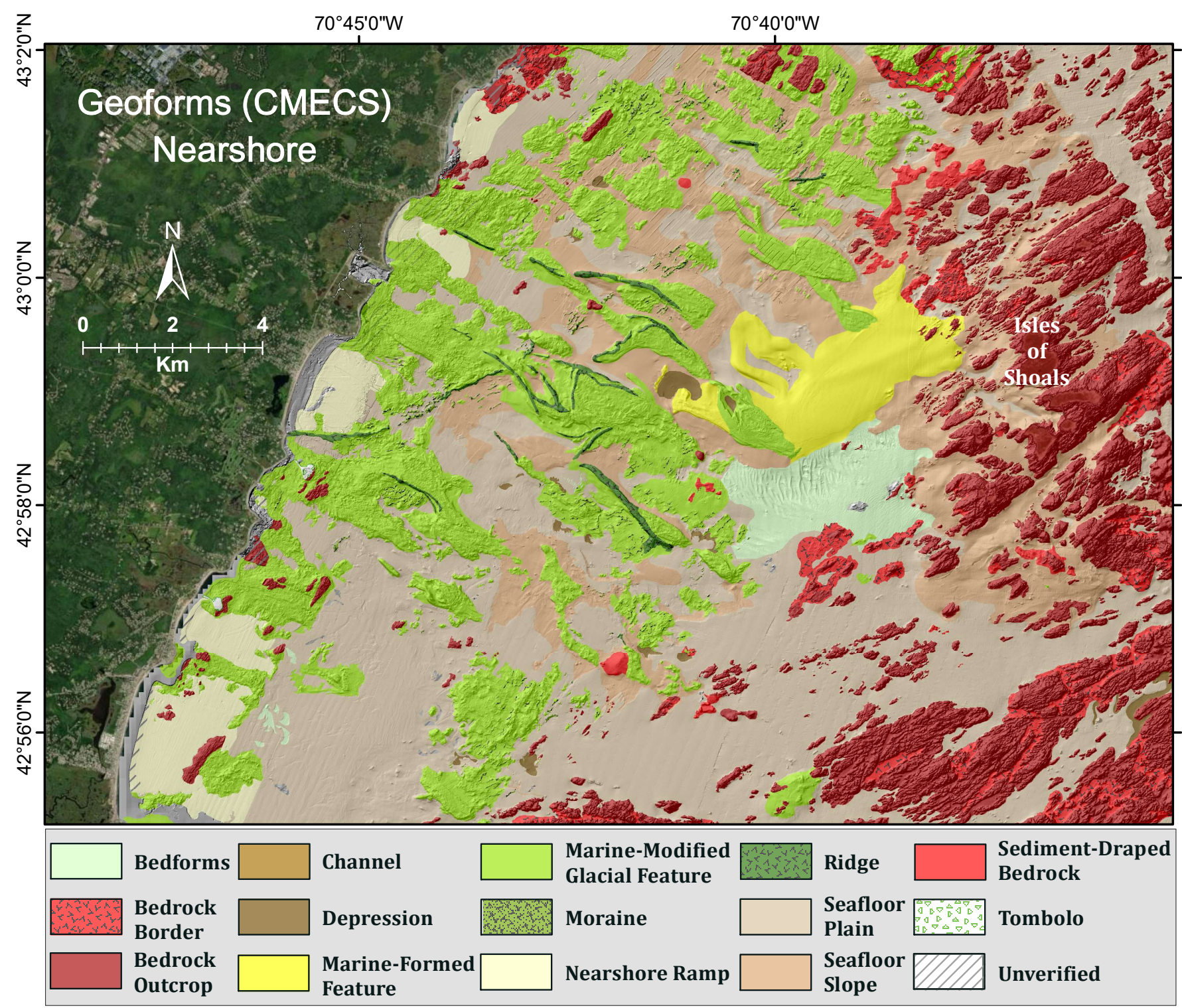

Figure 14. Geoform map for the inner continental shelf off New Hampshire. The large yellow feature (Marine-Formed Feature) is potentially a sand and fine gravel resource (see Ward et al., 2021a). The ridges are likely eroded eskers. 


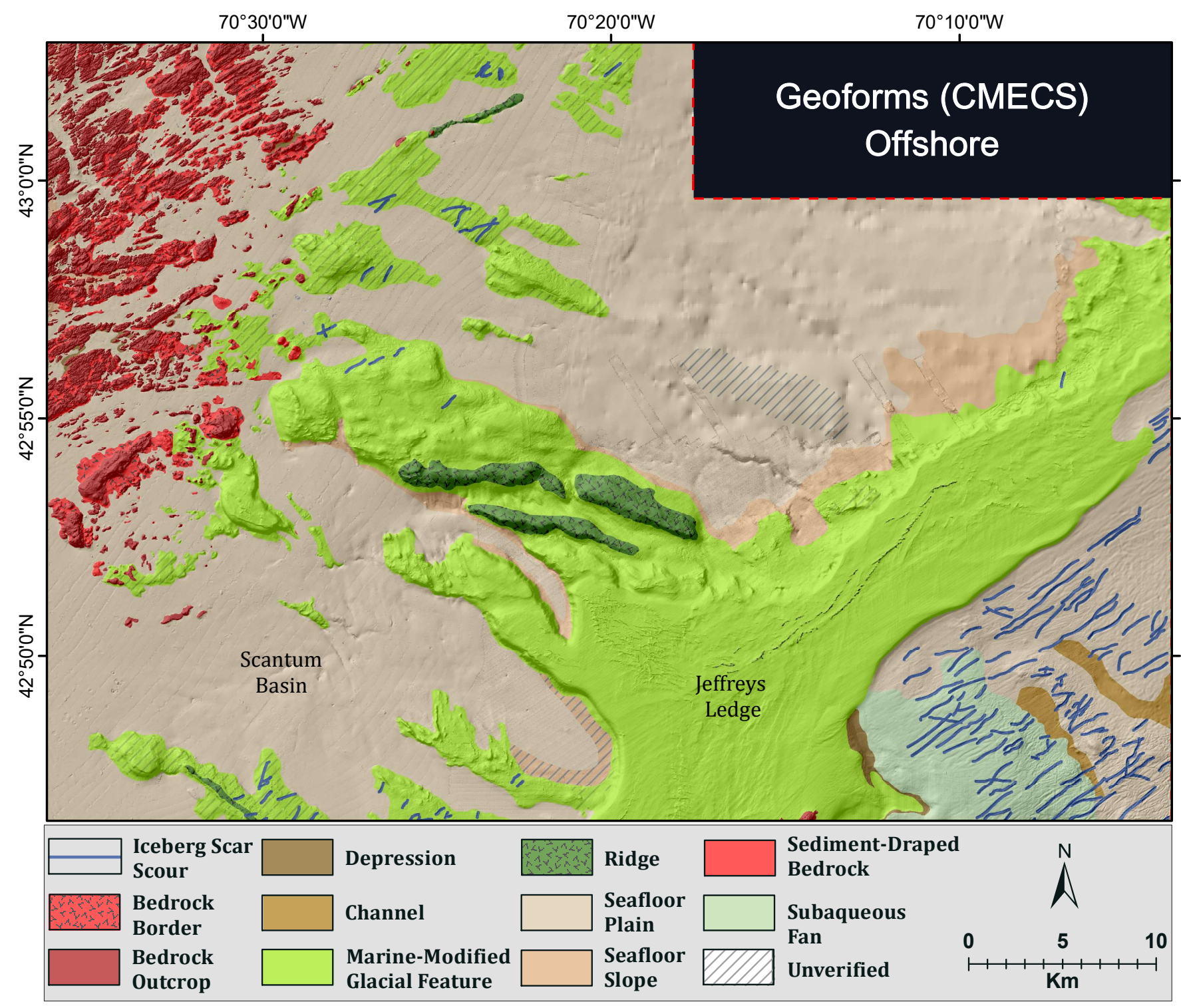

Figure 15. Geoform map for the offshore continental shelf off New Hampshire. The mounds trending northeast-southwest have the same orientation as drumlins on the NH seacoast (see Figure 12). 


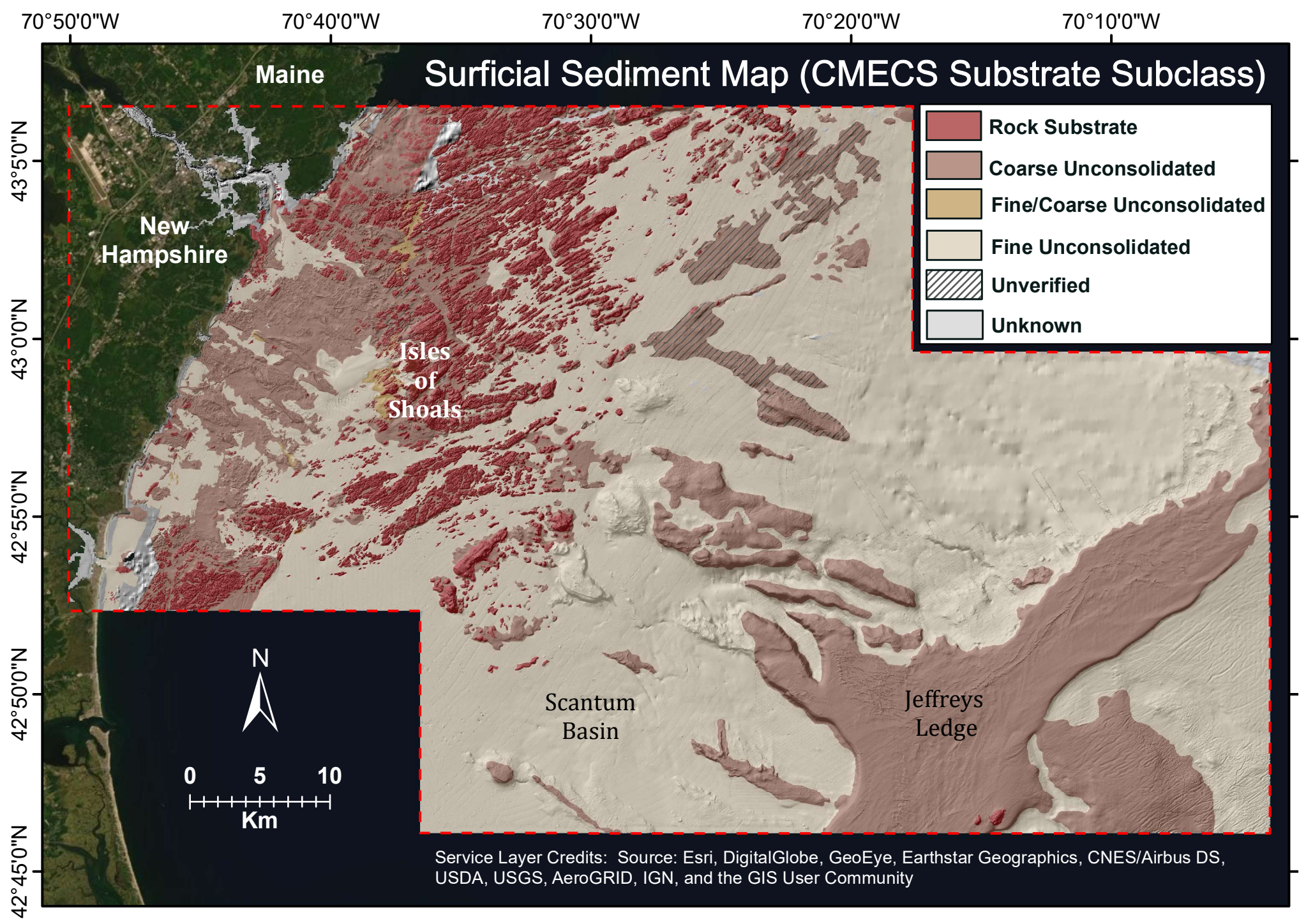

Figure 16. Surficial sediment map of the continental shelf off New Hampshire based on the CMECS classification for Geologic Substrate Subclasses (FGDC, 2012). 


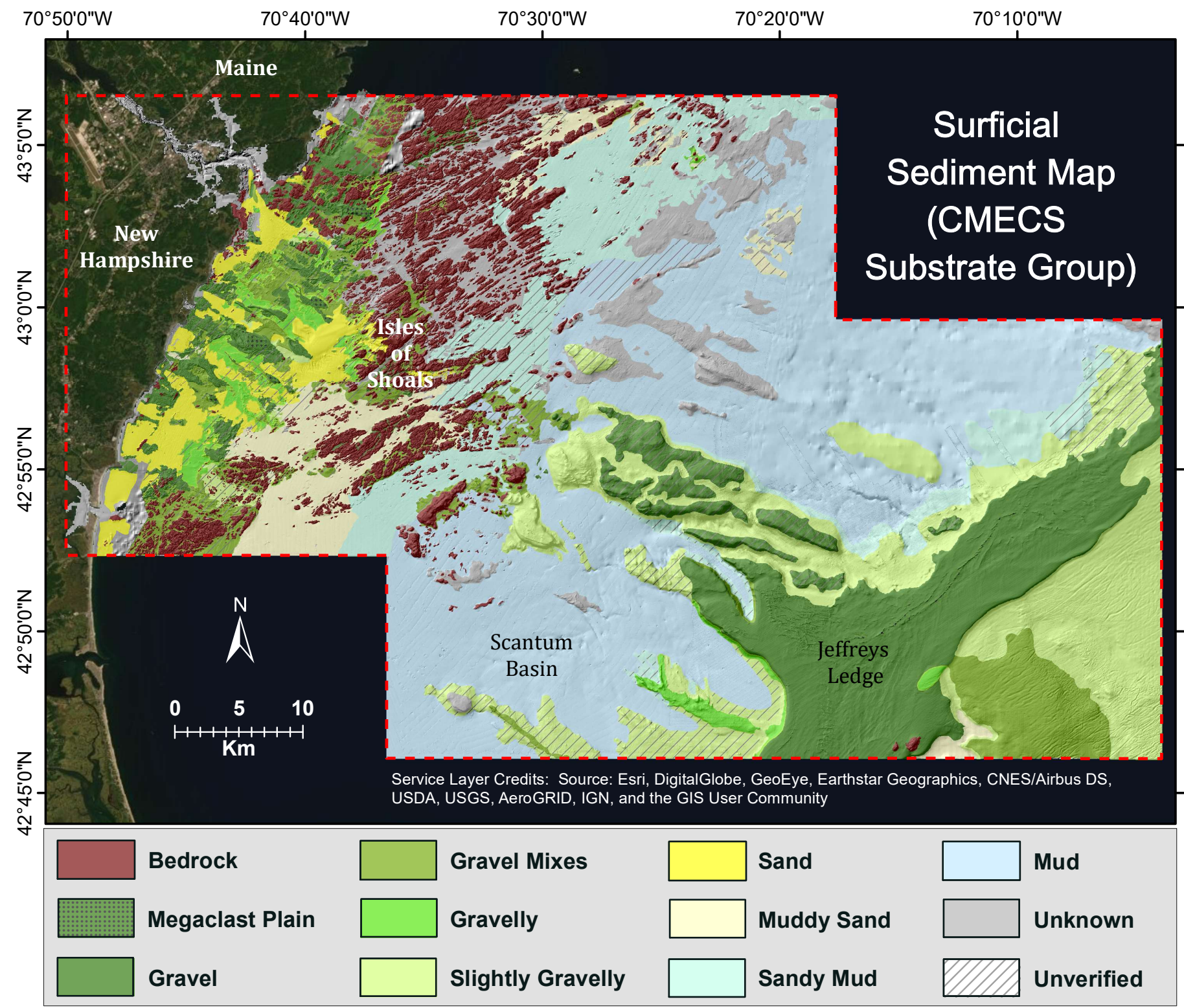

Figure 17. Surficial sediment map of the continental shelf off New Hampshire based on the CMECS classification for Geologic Substrate Groups (FGDC, 2012). 


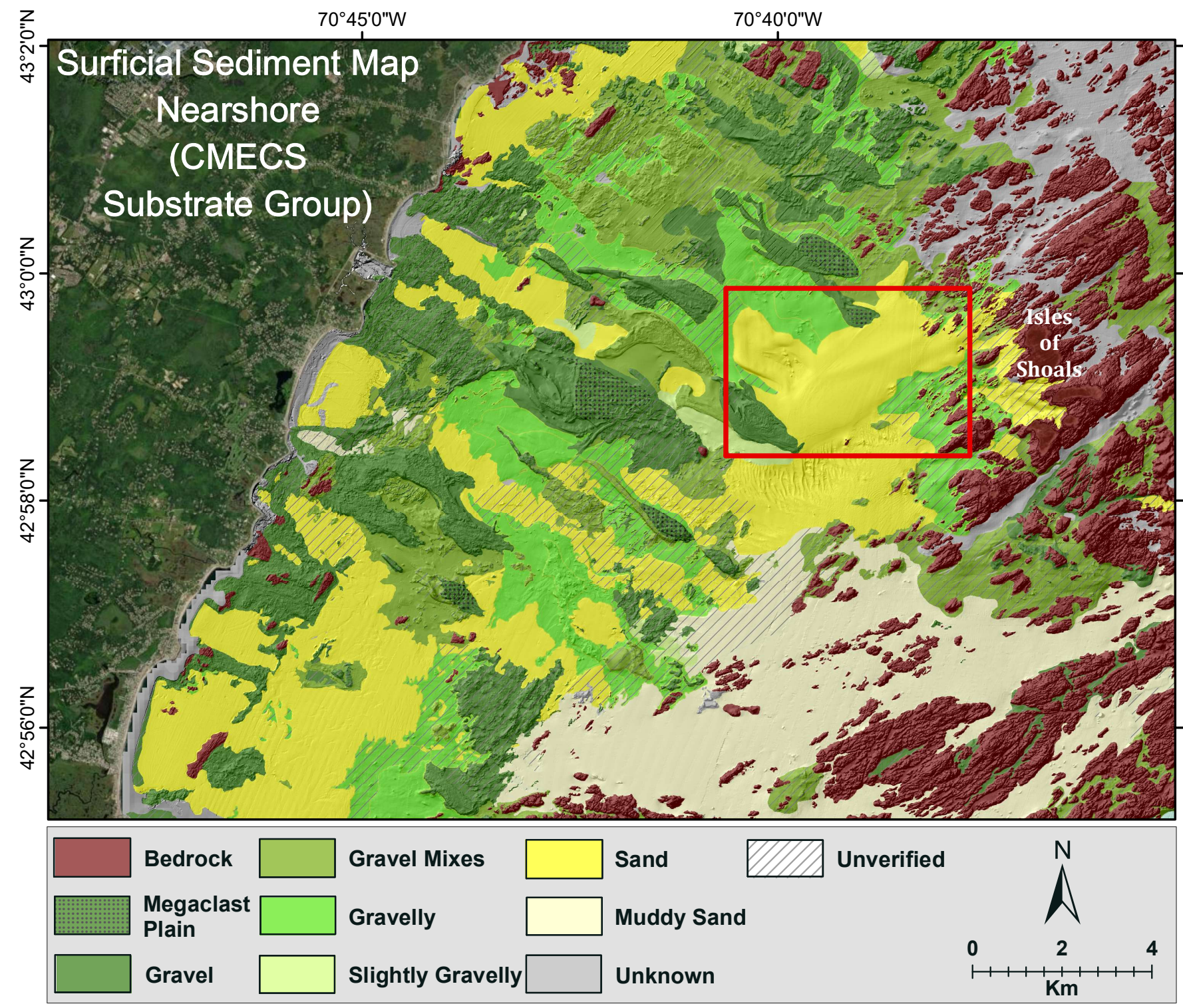

Figure 18. Map of Geologic Substrate Groups for the nearshore region of the continental shelf off New Hampshire. Note the large sand body (Northern Sand Body) immediately to the east of the Isles of Shoals (red box) which is a potential sand source for beach nourishment (Ward et al., 2021a). 


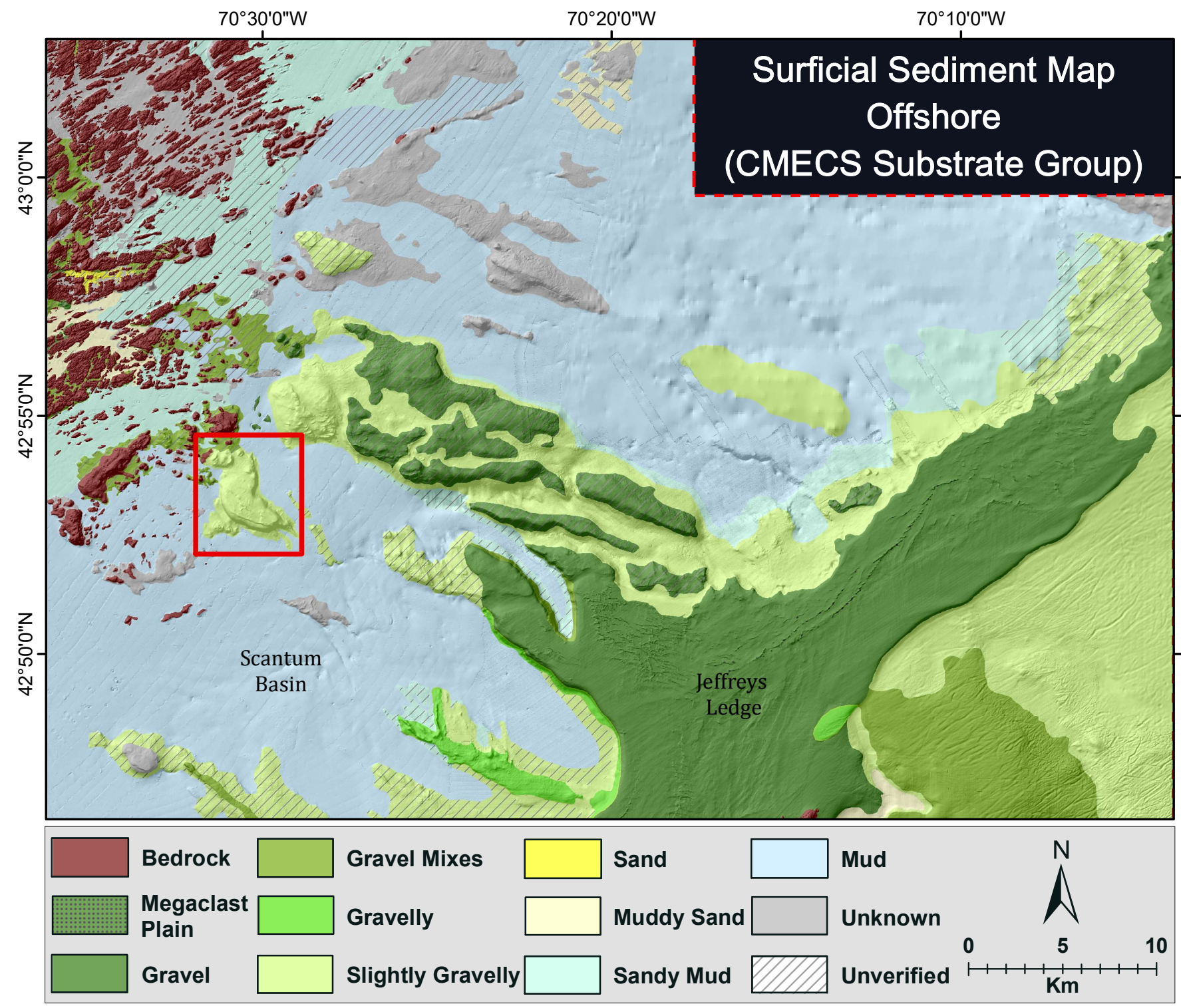

Figure 19. Map of Geologic Substrate Groups for the outer continental shelf off New Hampshire. The red box outlines a marine-modified drumlin that is a potential sand and gravel resource (see Ward et al., 2016a; 2021a) 


\section{References}

Balco, G., and Schaefer, J.M., 2006, Cosmogenic-nuclide and varve chronologies for the deglaciation of southern New England: Quaternary Geology volume 1, pp. 15-28.

Ballard, R.D. and E. Uchupi, 1974, Geology of Gulf of Maine: American Association of Petroleum Geologists Bulletin 58: 1156-1158 (No. 6, Part II of II).

Barnhardt, W.A., Andrews, B.D., Ackerman, S.D., Baldwin, W.E., and Hein, C.J., 2007, High-resolution geologic mapping of the inner continental shelf: Cape Ann to Salisbury Beach, Massachusetts: U.S. Geological Survey Open-file Report 2007-1373, variously paged. Accessed May 2019, available online at http://pubs.usgs.gov/of/2007/1373/

Bennett, D.S., Chormann, F.H., Jr., Koteff, C., and Wunsch, D.R., 2004, Conversion of surficial geologic maps to digital format in the Seacoast Region of New Hampshire: Digital Mapping Techniques '04-Workshop Proceedings, Portland, Oregon, May 16-19, 2004: U.S. Geological Survey OpenFile Report 2004-1451, 220 pp. (NHGS surficial geology digital map series; https://www.granit.unh.edu/, accessed January 2021)

Birch, F.S., 1984, A geophysical study of sedimentary deposits on the inner continental shelf of New Hampshire: Northeastern Geology, volume 6, number 4, pp. 207-221.

Birch, F.S., 1988, Sediments of the inner continental shelf: first- and second-year projects in New Hampshire: M.C. Hunt, D.C. Radcliff, S. Doenges and C. Condon (eds.), Proceedings of the First Symposium on the Studies Related to Continental Margins - A Summary of Year-One and YearTwo Activities, pp. 242-251, U.S. Department of Interior, Minerals Management Service Continental Margins Program and Association of American State Geologists Continental Margins Committee.

Blott, S.J. and Pye, K., 2001, Gradistat: A grain size distribution and statistics package for the analysis of unconsolidated sediments: Earth Surface Processes and Landforms, v. 26, no. 11, pp. 1237-1248. DOI: 10.1002/esp.261, Accessed March 1, 2020: http://www.kpal.co.uk/gradistat.html

Carter, R.W.G. and Orford, J.D., 1988, Conceptual model of coarse clastic barrier formation from multiple sediment sources: The Geographical Review, vol. 78, pp.221-239. DOI: 10.2307/214179

Diesing, M., Green, S.L., Stephens, D., and Link, R.M., Stewart, H.A., and Dove, D., 2004, Mapping seabed sediment: Comparison of manual, geostatistical, object-based image analysis and machine learning approaches, Continental Shelf Research, vol. 84, pp. 107-119. http://dx.doi.org/10.1016/j.csr.2014.05.004

Dorschel, B., Gutt, J., Piepenburg, D., and Arndt, J.E., 2014, The influence of the geomorphological and sedimentological settings on the distribution of epibenthic assemblages on a flat topped hill on the over-deepened shelf of the western Weddell Sea (Southern Ocean): Biosciences 11: 37973817. doi: 10.5194/bg-11-3797-2014

Erdey-Heydorn, M.D., 2008, An ArcGIS seabed characterization toolbox developed for investigating benthic habitats: Marine Geodesy 31: 318-358. doi: 10.1080/01490410802466819

FGDC (Federal Geographic Data Committee, Marine and Coastal Spatial Data Subcommittee), 2012, Coastal and Marine Ecological Classification Standard: FGDC-STD-018-2012, Washington, DC, 343 pp. https://www.fgdc.gov/standards/projects/cmecs-folder/CMECS_Version_06-2012_FINAL.pdf 
Folk, R.L., 1954, The distinction between grain size and mineral composition in sedimentary-rock nomenclature: The Journal of Geology, vol. 62, number 4, pp. 344-359. DOI: 10.1086/626171

Folk, R.L., 1980, Petrology of sedimentary rocks: Hemphill Publishing Company, Austin, TX. 182 pp. Accessed May 2019, available online at https://repositories.lib.utexas.edu/handle/2152/22930

Fonseca, L. and Mayer, L., 2007, Remote estimation of seafloor properties through the application Angular Range Analysis to multibeam sonar data: Marine Geophysical Research 28: 119-126. DOI 10.1007/s11001-007-9019-4

Kelley, J.T., Belknap, D.F., and Claesson, S., 2010, Drowned coastal deposits with associated archaeological remains from a sea-level "slowstand": Northwestern Gulf of Maine, USA: Geology 38 (8): 695698. DOI: $10.1130 / G 31002.1$

Masetti, G., Mayer, L.A., and Ward, L.G., 2018, A bathymetry- and reflectivity-based approach for seafloor segmentation: Geosciences 8(1),14. https://doi:10.3390/geosciences8010014

NOAA OCS Strategic Plan 2020-2024; https://nauticalcharts.noaa.gov/about/docs/about/ocs-strategicplan-fy20-24.pdf, accessed March 15, 2021.

Office of Coastal Management, 2015, Coastal and Marine Ecological Classification Standard Crosswalk Tools version 1.0. https://coast.noaa.gov/digitalcoast/tools/cmecs-crosswalk.html

Oldale, R.N. and Uchupi, E., 1970, The glaciated shelf off Northeastern United States: United States Geological Survey Professional Paper 700B, pp B167-B173.

Oldale, R.N., Uchupi, E., and Prada, L.E., 1973, Sedimentary Framework of the Western Gulf of Maine and Southeastern Massachusetts Offshore Area: U.S. Geological Survey Professional Paper 757, 10 pp.

Oldale, R.N, Colman, S.M., and Jones, J., 1993, Radiocarbon ages from two submerged strandline features in the Western Gulf of Maine and a sea-level curve for the northeastern Massachusetts coastal region: Quaternary Research, volume 40, pp. 38-45. https://doi.org/10.1006/qres.1993.1054

Oliveira, N. de, Bastos, A.C., Quaresma, V. da S., Vieira, F.V., 2020, The use of Benthic Terrain Modeler (BTM) in the characterization of continental shelf habitats, Geo-Marine Letters, vol. 40, pp. 10871097, https://doi.org/10.1007/s00367-020-00642-y

Pe'eri, S., McLeod, A., Lavoie, P., Ackerman, S., Gardner, J., and Parrish, C., 2013, Field calibration and validation of remote-sensing surveys: International Journal of Remote Sensing 34(18): 64236436. DOI: 10.1080/01431161.2013.800655

Pendleton, E.A., Sweeny, E.M., and Brothers, L.L., 2019, Optimizing an inner-continental shelf geologic framework investigation through data repurposing and machine learning: Geosciences, 9(9), 231. doi: 10.3390/geosciences9050231

Poppe, L.J., Paskevich, V.F., Williams, S.J., Hastings, M.E., Kelley, J.T., Belknap, D.F., Ward, L.G., FitzGerald, D.M., and Larsen, P.F., 2003, update 2004, Surficial Sediment Data from the Gulf of Maine, Georges Bank, and Vicinity: A GIS Compilation: U.S. Geological Survey Open-File Report 03-001. Accessed May 2019, available online at https://pubs.usgs.gov/of/2003/of03-001/index.htm

Reid, J.M., Reid, J.A., Jenkins, C.J., Hastings, M.E., Williams, S.J., and Poppe, L.J, 2005, usSEABED: Atlantic coast offshore surficial sediment data release: U.S. Geological Survey Data Series 118, version 1.0. Online at https://pubs.usgs.gov/ds/2005/118/ (doi.org/10.3133/ds118) 
Schnitker, D., Belknap, D.F., Bacchus, T., Friez, J.K., Lusardi, B.A., and Popek, D.M., 2001, Deglaciation of the Gulf of Maine. In: Weddle, T.K. and Retelle, M.J. (eds.), Deglacial History and Relative SeaLevel Changes, Northern New England and Adjacent Canada: Geological Society of America Special Paper 351, pp. 9-34.

Sinclair, S.N., Licciardi, J.M., Campbell, S.W., and Madore, B.M., 2018, Character and origin of De Geer moraines in the Seacoast region of New Hampshire, USA: Journal of Quaternary Science, volume 33, number 2, pp. 225-237, ISSN 0267-8179, DOI:10.1002/jqs.3017.

Smith, M., Masetti, G., Mayer, L.A., Malik, M.A., Augustin, J.-M., Poncelet, C., and Parnum, I., 2020, Open Backscatter Toolchain (OpenBST) - A community-vetted workflow for backscatter processing, Canadian Hydrographic Conference. Quebec City, Quebec, Canada, 2020. http://ccom.unh.edu/sites/default/files/publications/chc2020_openbst_abstract.pdf

Sowers, D., 2020, Utilizing Extended Continental Shelf (ECS) and Ocean Exploration Mapping Data for Standardized Marine Ecological Classification of the U.S. Atlantic Margin, PhD Thesis, University of New Hampshire, Durham, 150 pp.

Sowers, D.C., Masetti, G., Mayer, L.A., Johnson, P., Gardner J.V. and Armstrong, A.A., 2020, Standardized geomorphic classification of seafloor within the United States Atlantic canyons and continental margin: Frontiers in Marine Science 7: 9. doi:10.3389/fmars.2020.00009

Uchupi, E., 1966, Structural framework of the Gulf of Maine: Journal of Geophysical Research 71: 30133028.

Uchupi, E., 2004, The Stellwagen Bank region off eastern Massachusetts: A Wisconsin glaciated Cenozoic sand bank/delta?: Marine Geology 204: 325-347.

Uchupi, E. and Bolmer, S.T., 2008, Geologic evolution of the Gulf of Maine: Earth-Science Reviews 91: 2276. doi:10.1016/j.earscirev.2008.09.002

USCG (United States Coast Guard), Loran-C User's Handbook, accessed March 15, 2021, https://www.navcen.uscg.gov/?pageName=loranHandBook

USGS Metadata Wizard, accessed May 1, 2019. https://www.sciencebase.gov/catalog/item/50ed7aa4e4b0438b00db080a

Verfaillie, E., Doornenbal, P., Mitchell, A.J., White, J., and Van Lancker, V., 2007, The bathymetric position index (BPI) as a support tool for habitat mapping: MESH Mapping European Seabed Habitats, p. 14. Accessed May 2019, available online at http://www.emodnetseabedhabitats.eu/pdf/gmhm4_bathymetric_position_index_(bpi).pdf

Ward, L.G. and Adams, J.R., 2001, A Preliminary Assessment of Tidal Flooding along the New Hampshire Coast: Past, Present and Future: New Hampshire Office of Emergency Management and the Office of State Planning Coastal Program, $2 \frac{1}{2}$ Beacon Street, Concord, NH. 56 pp., (UNH CMB/JEL Report Series Number 54). https://scholars.unh.edu/faculty pubs/649/

Ward, L.G., Vallee-Anziani, M., and McAvoy, Z.S., 2016a, New Hampshire and Vicinity Continental Shelf: Morphologic Features and Surficial Sediments: BOEM/New Hampshire Cooperative Agreement (Contract M14ACOO010) Technical Report, BOEM Marine Minerals Branch, 381 Elden Street, Herndon, VA, 20170, 24 pp. http://ccom.unh.edu/publications/new-hampshire-and-vicinitycontinental-shelf-morphologic-features-and-surficial 
Ward, L.G., Johnson, P., Nagel, E., McAvoy, Z.S., and Vallee-Anziani, M., 2016b, Western Gulf of Maine Bathymetry and Backscatter synthesis: BOEM/New Hampshire Cooperative Agreement (Contract M14ACO0010) Technical Report, BOEM Marine Minerals Branch, 381 Elden Street, Herndon, VA, 20170, 18 pp. https://dx.doi.org/10.34051/p/2021.27

Ward, L., McAvoy, Z., Masetti, G., and Morrison, R., 2019, High resolution mapping of morphologic features and seafloor sediments of the New Hampshire and Vicinity Continental Shelf, Western Gulf of Maine, Abstracts, Geological Society of America (GSA) Annual Meeting, Northeastern Section, Portland, ME, March 17-19. https://gsa.confex.com/gsa/2019NE/meetingapp.cgi/Paper/328549

Ward, L.G., McAvoy, Z.S., and Vallee-Anziani, M., 2021a, New Hampshire and Vicinity continental Shelf: Sand and Gravel Resources: BOEM/New Hampshire Cooperative Agreement (Contract M14ACO0010) Technical Report, BOEM Marine Minerals Branch, 381 Elden Street, Herndon, VA, 20170, 97 pp. https://dx.doi.org/10.34051/p/2021.30

Ward, L.G., Morrison, R.C., and McAvoy, Z.S., 2021b, New Hampshire Continental Shelf Geophysical Database: 2016-2017 Field Campaign - Seafloor and Sample Photographs and Sediment Data. University of New Hampshire Center for Coastal and Ocean Mapping/Joint Hydrographic Center (CCOM/JHC), 24 Colovos Road, Durham 03824. UNH Scholars Repository (https://scholars.unh.edu). https://dx.doi.org/10.34051/d/2021.1

Ward, L.G., Morrison, R.C., and McAvoy, Z.S., 2021c, New Hampshire Continental Shelf Geophysical Database: 2016-2017 Field Campaign - Stations and Sediment Data. University of New Hampshire Center for Coastal and Ocean Mapping/Joint Hydrographic Center (CCOM/JHC), 24 Colovos Road, Durham, NH 03824. UNH Scholars Repository (https://scholars.unh.edu). https://dx.doi.org/10.34051/d/2021.2

Ward, L.G., Morrison, R.C., and McAvoy, Z.S., 2021d, New Hampshire Continental Shelf Historical Geophysical Database: 1971 to 2015 - Sediment Data. University of New Hampshire Center for Coastal and Ocean Mapping/Joint Hydrographic Center (CCOM/JHC), 24 Colovos Road, Durham, 03824. UNH Scholars Repository (https://scholars.unh.edu).

https://dx.doi.org/10.34051/d/2021.3

Ward, L.G., Morrison, R.C., and McAvoy, Z.S., 2021e, New Hampshire Continental Shelf Geophysical Database: 2016-2017 Field Campaign - Seafloor Photographs. University of New Hampshire Center for Coastal and Ocean Mapping/Joint Hydrographic Center (CCOM/JHC), 24 Colovos Road, Durham, NH 03824. UNH Scholars Repository (https://scholars.unh.edu). https://dx.doi.org/10.34051/d/2021.5

Ward, L.G., Corcoran, N.W., McAvoy, Z.S., and Morrison, R.C., 2021f, Seasonal Changes in Sediment Grain Size of New Hampshire Beaches: BOEM/New Hampshire Cooperative Agreement (Contract M14AC00010) Technical Report, Department of Interior, Bureau of Ocean Energy Management, Marine Minerals Division, 45600 Woodland Road, Sterling, VA, 20166, 200 pp.

Ward, L.G., Morrison, R.C., McAvoy, Z.S., and Vallee-Anziani, M., 2021g, Analysis of Vibracores from the New Hampshire Continental Shelf from 1984 and 1988: BOEM/New Hampshire Cooperative Agreement (Contract M14AC00010) Technical Report, Department of Interior, Bureau of Ocean Energy Management, Marine Minerals Division, 45600 Woodland Road, Sterling, VA, 20166, 173 pp. https://dx.doi.org/10.34051/p/2021.26 
Ward, L.G., Johnson, P., Bogonko, M., McAvoy, Z.S., and Morrison, R.C., 2021h, Northeast Bathymetry and Backscatter Compilation: Western Gulf of Maine, Southern New England, and Long Island Sound: BOEM/ New Hampshire Cooperative Agreement (Contract M14ACO0010) Technical Report, Department of Interior, Bureau of Ocean Energy Management, Marine Minerals Division, 45600 Woodland Road, Sterling, VA, 20166., 23 pp. https://dx.doi.org/10.34051/p/2021.28

Weber, T.C., Rice, G., and Smith, M., 2018, Toward a standard line for use in multibeam echo sounder calibration. Marine Geophysical Research, vol. 39, pp. 75-87. https://doi.org/10.1007/s11001$\underline{017-9334-3}$

Wentworth, C., 1922, A scale of grade and class terms for clastic sediments: The Journal of Geology, v. 30, no. 5, pp. 377-392. Accessed May 2019, https://www.jstor.org/stable/30063207 


\section{Glossary}

Backscatter: The measure of a signal/sound reflected back in its originating direction based on the amount of sound reflected by the seafloor and received by a sonar. Provides information on hardness and roughness of bottom; typically a stronger signal indicates a harder seafloor, whereas a weaker signal indicates a softer seafloor.

Barrier Beach: Narrow and elongated beach built by waves, currents, and winds that rises above the hightide level and extends generally parallel to the upland. Separated from the upland by a lagoon or marsh.

Bedrock: General term for the rock that is part of the crust. It is not unattached (like boulders are). Can be buried by sediment or exposed.

Datum: Reference system or an approximation of the Earth's surface against which positional measurements can be made (e.g., latitude, longitude, and elevation). See geodetic datum.

Dead Reckoning: Determination of the position of a ship based on known initial position, velocity and distance covered.

Delta: Landform that develops by the deposition of sediment flowing water (e.g., river, glacial stream) enters a larger water body (e.g. ocean, lake, estuary).

Drumlin: A low, smoothly rounded, elongated and oval hill, mound, or ridge of compact glacial till, built under the glacial ice and shaped by its flow. The longer axis is parallel to the direction of movement of the ice. Composed of a large range of sediment from boulders to mud.

Ellipsoid: A flattened sphere used to represent the geometric model of the Earth (e.g. the Earth is not completely round and is slightly flattened at the poles); a mathematical model of the Earth to represent horizontal positions on maps and charts (versus the topographic or actual visible surface of the Earth).

Erode (for beaches): To remove sediment by the action of current, waves, or wind.

Esker: A long, low, narrow, sinuous, steep-sided ridge or mound composed of irregularly stratified sand and gravel that was deposited by a subglacial stream or in an ice tunnel. Eskers, unlike drumlins, are stratified accumulations of sand and gravel.

Eustatic Sea Level, Also Global: Change in the level of the ocean irrespective of the land.

Fan: Triangle-shaped accumulation of sediments, called alluvium. Formed as flowing water interacts with sloped topography.

Geodetic Datum: Provides a reference surface (such as sea level) from which all locations on Earth can be defined with coordinates; system developed to assist surveyors, navigators, and to create maps by translating Earth's three-dimensional surface to two-dimensional coordinates.

Geoid: The true zero surface of the Earth for measuring elevations defined by Earth's gravity. The geoid surface must be modeled. Mean sea level is a close approximation. 
Geometric Datum: Coordinate system for collection of positions relative to an ellipsoid model of the Earth.

GNSS (Global Navigation Satellite System): A general term describing any satellite constellation that provides positioning, navigation, and timing on a global basis. GPS is the most prevalent GNSS.

Ground Truth: Validation of data by direct observation or sampling in the field.

Grounding Line: The zone where a glacier transitions from lying above land to floating on an open water body (e.g. ice shelf).

Hillshade: A technique for visualizing terrain through a 3D grayscale representation by creating an illumination effect of a surface based on elevation variations.

Horizontal Datum: Coordinate system for positions on Earth (e.g., latitude and longitude).

IGS08: Geodetic datum used by surveyors, engineers, and mapping professionals to measure locations (latitude and longitude) and elevations to the Earth's surface throughout the world. Referenced to an ellipsoid.

Intertidal: Area of a beach between high water and low water. Also called foreshore.

Isostatic: Change in land level relative to the sea, causing localized changes.

Kettle: Depression in a glacial outwash drift formed when a glacier retreats and melts.

Lag Deposits: Coarse-grained material that is left after currents, waves or wind have winnowed or eroded the finer material.

MLLW (Mean Lower Low Water): Average height of all the lower low water levels recorded at a given location over a 19-year period (epoch) or a computed equivalent period.

MSL (Mean Sea Level): Average water levels for all stages of the tide over a 19-year period (epoch) at a given location.

Megaclasts: Larger clasts (fragments of rock). Usually refers to cobbles and boulders.

Megaclast Platform: Flat or gently sloping surface composed of megaclasts.

Morphology: The external structure and form of landforms.

Multibeam Echosounder (MBES): Sonar system that emits sound waves from directly beneath a ship's hull, and produces acoustic waves in a narrow fan shape. Used to obtain bathymetric coverage of the seafloor. 
NAD83 (North American Datum of 1983): Geodetic datum used by surveyors, engineers, and mapping professionals to measure locations (latitude and longitude) and elevations of the Earth's surface in the United States. Referenced to an ellipsoid.

NAVD88 (North American Vertical Datum of 1988): Vertical datum used by surveyors, engineers, and mapping professionals to measure and relate elevations to the Earth's surface.

Outwash: Deposit of sand and gravel (glacial sediments) at the terminus of a glacier carried by glacial streams.

Palustrine: Relating to any non-tidal and non-saline inland wetland (marsh, swamp, bog).

Paraglacial: Regions effected by previous glaciations.

Presumpscot Formation: Glacial marine sediments of late Pleistocene age. It is widespread along the Maine and New Hampshire coastlines and inland along major river valleys.

Projection: System of mathematics and geometry to transfer locations on the Earth onto a flat piece of paper (a map).

Regression (coast): A seaward migration of the coast as sea level falls.

Regression (sea level): Referred to in this report as a lowering of sea level.

Sediment: Fragmental material that originates from breaking down rocks.

Shoaling (Sediment): Buildup of sediment due to deposition.

Shoaling (Waves): Alteration of a wave as it proceeds from deep water into shallow water. There is an initial decrease in height of the incoming wave, followed by an increase in height until it breaks.

Stratified Drift: Well-sorted and stratified layers of sand and gravel deposited by glacial meltwater.

Subbottom Seismics: Sonar system that operates by emitting a low-frequency energy signal that penetrates through the seafloor (unlike a multibeam echosounder which measures the surface of the seafloor) to characterize different layers of sediment or rock under the seafloor. The time it takes for the sound to return to the vessel is used to determine the thickness and position of these layers.

Subsidence: Sinking of the Earth's crust relative to the surrounding area.

Tidal Datum: Standard elevation framework used to track local water levels as measured by a tidal gauging station.

Tidal Inlet: An inlet through a barrier beach which water flows alternately landward with the rising tide and seaward with the falling tide. 
Till or Glacial Till: Unsorted and unstratified sediment deposited by a glacier. Generally unconsolidated, deposited directly by and underneath a glacier without subsequent reworking by water from the glacier, and consisting of a heterogeneous mixture of clay, sand, gravel, and boulders varying widely in size and shape.

Transgression (coast): Causing a landward migration of the coast as sea level rises.

Transgression (sea level): In this report refers to a rise in sea level.

Uplift: Rising of the Earth's crust relative to the surrounding area.

Vertical Datum: Measures elevation above a reference surface.

Vibracore: Method for retrieving continuous, undisturbed sediment core samples by driving a tube into the subsurface with a vibrating device. Can be limited by the composition and hardness of the seafloor.

WGS84 (World Geodetic System 1984): Geodetic datum used by surveyors, engineers, and mapping professionals to measure locations (latitude and longitude) and elevations to the Earth's surface throughout the world. Referenced to an ellipsoid. 


\section{Appendix A: New Hampshire Continental Shelf Historical Geophysical Database: 1971-2015 Sediment Data}

Sediment grain size data from the UNH CCOM/JHC Historical Geophysical Database (surveys conducted on the NH continental shelf between 1971 and 2015) are presented here. In total, grain size data was available for 725 bottom sediment samples. Included for each sample when available are sample identification information, station and sample characteristics, sediment classifications, grain size statistics, and grain size distribution. Not all of these parameters were avaialble for every study.

The surveys are ordered from the most recent surveys and ending with the surveys from the 1970 s. The sediment grain size data is grouped by the principal investigator or associated publication (e.g., Wilson, Nifong, etc.) or research program name (e.g., NEWBEX, OOA, etc.). The cruises are divided into separate sections [e.g., Nifong Thesis (1), Nifong Thesis (2), etc.]. Data for each cruise is spread across four pages (where complete grain size data was available) or two pages (where limited grain size data was available), with each sample identified in the first column by the Sample ID.

Samples were analyzed with standard sieve and pipette analyses after Folk (1980). The sediment grain size classifications include: CMECS (Coastal and Marine Ecological Classification Standard; FGDC, 2012); Gradistat (Blott and Pye, 2001); and Wentworth (Wentworth, 1922; described in Folk, 1954, 1980). Statistics are based on the phi scale and include the graphic mean, sorting, skewness, and kurtosis (Folk, 1980). Each sample has a "Reliability Ranking for Positioning" which gives an estimate of the uncertainty of the location of the sample classified from 1 to 4 . Explanation of uncertainty and the numbering system is given in "Positioning Uncertainty of Station Locations" and in Table 3.

The historical sediment grain size data is available for viewing at the UNH CCOM/JHC web site: (https://maps.ccom.unh.edu/portal/apps/webappviewer/index.html?id=28df035fe82c423cb3517295d9 bbc24c). The New Hampshire Continental Shelf Historical Geophysical Database: 1971 to 2015 Sediment Data is also available as a Microsoft Excel file from the University of New Hampshire Scholars Repository https://dx.doi.org/10.34051/d/2021.3. 
Section 1: Nifong Thesis Survey (2015) 
Nifong Thesis (2015) (1): Identification, Location, and Description

\begin{tabular}{|c|c|c|c|c|c|c|c|c|}
\hline Sample ID & $\begin{array}{l}\text { Original } \\
\text { Sample ID }\end{array}$ & $\begin{array}{c}\text { Latitude } \\
\text { WGS84 }\end{array}$ & $\begin{array}{l}\text { Longitude } \\
\text { WGS84 }\end{array}$ & $\begin{array}{l}\text { Reliability } \\
\text { Ranking for } \\
\text { Positioning }\end{array}$ & $\begin{array}{c}\text { Water } \\
\text { Depth } \\
\text { (m) }\end{array}$ & $\begin{array}{l}\text { Sample } \\
\text { Collected }\end{array}$ & $\begin{array}{c}\text { Sampler } \\
\text { Type }\end{array}$ & $\begin{array}{c}\text { Total Wt } \\
\text { (gm) }\end{array}$ \\
\hline Sta_1_A & Sta_1_A & 43.075752 & -70.709218 & 1 & 15.2 & $5 / 27 / 2015$ & Shipex & 265.39 \\
\hline Sta_1_B & Sta_1_B & 43.075303 & -70.709677 & 1 & 15.2 & $5 / 27 / 2015$ & Shipex & 259.77 \\
\hline Sta_2_A & Sta_2_A & 43.073203 & -70.704790 & 1 & 15.5 & $5 / 27 / 2015$ & Shipex & 725.93 \\
\hline Sta_2_B & Sta_2_B & 43.073645 & -70.704790 & 1 & 15.5 & $5 / 27 / 2015$ & Shipex & 539.34 \\
\hline Sta 3 A A & Sta_3_A & 43.065938 & -70.705078 & 1 & 12.2 & $5 / 27 / 2015$ & Shipex & 158.42 \\
\hline Sta_3_B & Sta_3_B & 43.066050 & -70.705273 & 1 & 12.2 & $5 / 27 / 2015$ & Shipex & 263.77 \\
\hline Sta_4_A.1 & Sta_4_A & 43.058923 & -70.701907 & 1 & 10.7 & $5 / 27 / 2015$ & Shipex & 278.99 \\
\hline Sta_4_A.2 & Sta_4_A & 43.058923 & -70.701907 & 1 & 10.7 & $5 / 27 / 2015$ & Shipex & 504.93 \\
\hline Sta_4_B & Sta_4_B & 43.059307 & -70.702338 & 1 & 10.7 & $5 / 27 / 2015$ & Shipex & 291.65 \\
\hline Sta_5_A & Sta_5_A & 43.055795 & -70.698737 & 1 & 12.2 & $5 / 28 / 2015$ & Shipex & 154.56 \\
\hline Sta_5_B & Sta_5_B & 43.055558 & -70.698700 & 1 & 12.2 & $5 / 28 / 2015$ & Shipex & 136.01 \\
\hline Sta_6_A & Sta_6_A & 43.049352 & -70.688253 & 1 & 18.3 & $5 / 29 / 2015$ & Shipex & 273.69 \\
\hline Sta_7_A & Sta_7_A & 43.045998 & -70.694900 & 1 & 14.6 & $5 / 29 / 2015$ & Shipex & 66.75 \\
\hline Sta_7_B & Sta_7_B & 43.045973 & -70.694802 & 1 & 14.6 & $5 / 29 / 2015$ & Shipex & 197.51 \\
\hline Sta_8_A & Sta_8_A & 43.040050 & -70.682387 & 1 & 20.4 & $5 / 27 / 2015$ & Shipex & 182.42 \\
\hline Sta_8_B & Sta_8_B & 43.039337 & -70.682270 & 1 & 20.4 & $5 / 27 / 2015$ & Shipex & 273.72 \\
\hline Sta_9_A & Sta_9_A & 43.036523 & -70.677572 & 1 & 24.4 & $5 / 27 / 2015$ & Shipex & 504.25 \\
\hline Sta_9_B & Sta_9_B & 43.036458 & -70.677442 & 1 & 24.4 & $5 / 27 / 2015$ & Shipex & 539.45 \\
\hline Sta_10_A & Sta_10_A & 43.037565 & -70.688847 & 1 & 18.9 & $5 / 29 / 2015$ & Shipex & 101.14 \\
\hline Sta_10_B & Sta_10_B & 43.037552 & -70.688630 & 1 & 18.9 & $5 / 29 / 2015$ & Shipex & 196.75 \\
\hline Sta_10_C & Sta_10_C & 43.037730 & -70.688890 & 1 & 18.9 & $5 / 29 / 2015$ & Shipex & 173.26 \\
\hline
\end{tabular}


Nifong Thesis (2015) (1): Sediment Classifications

\begin{tabular}{|c|c|c|c|c|c|c|c|c|c|}
\hline Sample ID & $\begin{array}{l}\text { CMECS Substrate } \\
\text { Component } \\
\text { Group (Specific) }\end{array}$ & $\begin{array}{l}\text { CMECS Substrate } \\
\text { Component } \\
\text { Subgroup (Specific) }\end{array}$ & $\begin{array}{l}\text { Textural Group } \\
\text { from \%GSM } \\
\text { (Gradistat) }\end{array}$ & $\begin{array}{l}\text { Textural Group } \\
\text { from \%SZC } \\
\text { (Gradistat) }\end{array}$ & $\begin{array}{l}\text { Sediment Name from } \\
\text { \%GSM } \\
\text { and Mode (Gradistat) }\end{array}$ & $\begin{array}{l}\text { Sediment Name from } \\
\text { \%GSM and Mode } \\
\text { (Wentworth Scale) }\end{array}$ & $\begin{array}{l}\text { Sediment } \\
\text { Classification from } \\
\text { Mean Phi (Gradistat) }\end{array}$ & $\begin{array}{l}\text { Classification } \\
\text { from Mean Phi } \\
\text { (Wentworth) }\end{array}$ & $\begin{array}{l}\text { Sorting } \\
\text { (Gradistat) }\end{array}$ \\
\hline Sta_1_A & Pebble Mixes & Sandy Pebble Gravel & Sandy Gravel & N/A & Sandy Coarse Gravel & Sandy Pebble Gravel & Very Coarse Sand & $\begin{array}{l}\text { Very Coarse } \\
\text { Sand }\end{array}$ & $\begin{array}{l}\text { Very Poorly } \\
\text { Sorted }\end{array}$ \\
\hline Sta_1_B & Pebble Mixes & Sandy Pebble Gravel & Sandy Gravel & $\mathrm{N} / \mathrm{A}$ & Sandy Coarse Gravel & Sandy Pebble Gravel & Very Coarse Sand & $\begin{array}{l}\text { Very Coarse } \\
\text { Sand }\end{array}$ & $\begin{array}{l}\text { Very Poorly } \\
\text { Sorted }\end{array}$ \\
\hline Sta_2_A & Pebble Mixes & Sandy Pebble Gravel & Sandy Gravel & $\mathrm{N} / \mathrm{A}$ & Sandy Coarse Gravel & Sandy Pebble Gravel & Fine Gravel & Pebble Gravel & $\begin{array}{l}\text { Very Poorly } \\
\text { Sorted }\end{array}$ \\
\hline Sta_2 B & Pebble Mixes & Sandy Pebble Gravel & Sandy Gravel & N/A & Sandy Coarse Gravel & Sandy Pebble Gravel & Fine Gravel & Pebble Gravel & $\begin{array}{l}\text { Very Poorly } \\
\text { Sorted }\end{array}$ \\
\hline Sta_3_A & Slightly Granuley & $\begin{array}{l}\text { Slightly Granuley } \\
\text { Medium Sand }\end{array}$ & $\begin{array}{l}\text { Slightly Gravelly } \\
\text { Sand }\end{array}$ & $\mathrm{N} / \mathrm{A}$ & $\begin{array}{l}\text { Slightly Very Fine Gravelly } \\
\text { Medium Sand }\end{array}$ & $\begin{array}{l}\text { Slightly Granular } \\
\text { Medium Sand }\end{array}$ & Medium Sand & Medium Sand & $\begin{array}{l}\text { Moderatley } \\
\text { Sorted }\end{array}$ \\
\hline Sta_3_B & Slightly Granuley & $\begin{array}{l}\text { Slightly Granuley } \\
\text { Medium Sand }\end{array}$ & $\begin{array}{l}\text { Slightly Gravelly } \\
\text { Sand }\end{array}$ & $\mathrm{N} / \mathrm{A}$ & $\begin{array}{l}\text { Slightly Very Fine Gravelly } \\
\text { Medium Sand }\end{array}$ & $\begin{array}{l}\text { Slightly Granular } \\
\text { Medium Sand }\end{array}$ & Medium Sand & Medium Sand & $\begin{array}{l}\text { Moderately } \\
\text { Well Sorted }\end{array}$ \\
\hline Sta_4_A.1 & Pebble Mixes & Sandy Pebble Gravel & Sandy Gravel & $\mathrm{N} / \mathrm{A}$ & Sandy Medium Gravel & Sandy Pebble Gravel & Very Fine Gravel & Granule Gravel & $\begin{array}{l}\text { Very Poorly } \\
\text { Sorted }\end{array}$ \\
\hline Sta_4_A.2 & Pebble Mixes & Sandy Pebble Gravel & Sandy Gravel & $\mathrm{N} / \mathrm{A}$ & Sandy Coarse Gravel & Sandy Pebble Gravel & Very Fine Gravel & Granule Gravel & $\begin{array}{l}\text { Very Poorly } \\
\text { Sorted }\end{array}$ \\
\hline Sta 4 B B & Pebble Mixes & Sandy Pebble Gravel & Sandy Gravel & N/A & Sandy Coarse Gravel & Sandy Pebble Gravel & Fine Gravel & Pebble Gravel & $\begin{array}{l}\text { Very Poorly } \\
\text { Sorted }\end{array}$ \\
\hline Sta 5 A & Slightly Granuley & $\begin{array}{l}\text { Slightly Granuley Fine } \\
\text { Sand }\end{array}$ & $\begin{array}{l}\text { Slightly Gravelly } \\
\text { Sand }\end{array}$ & N/A & $\begin{array}{l}\text { Slightly Very Fine Gravelly } \\
\text { Fine Sand }\end{array}$ & $\begin{array}{l}\text { Slightly Granular Fine } \\
\text { Sand }\end{array}$ & Fine Sand & Fine Sand & Well Sorted \\
\hline Sta 5 B & Slightly Pebbly & $\begin{array}{l}\text { Slightly Pebbly Fine } \\
\text { Sand }\end{array}$ & $\begin{array}{l}\text { Slightly Gravelly } \\
\text { Sand }\end{array}$ & N/A & $\begin{array}{l}\text { Slightly Fine Gravelly Fine } \\
\text { Sand }\end{array}$ & $\begin{array}{l}\text { Slightly Pebbly Fine } \\
\text { Sand }\end{array}$ & Fine Sand & Fine Sand & Well Sorted \\
\hline Sta_ 6 A & Pebble Mixes & Sandy Pebble Gravel & Sandy Gravel & $\mathrm{N} / \mathrm{A}$ & Sandy Medium Gravel & Sandy Pebble Gravel & Very Fine Gravel & Granule Gravel & $\begin{array}{l}\text { Very Poorly } \\
\text { Sorted }\end{array}$ \\
\hline Sta_7_A & Slightly Granuley & $\begin{array}{l}\text { Slightly Granuley Very } \\
\text { Fine Sand }\end{array}$ & $\begin{array}{l}\text { Slightly Gravelly } \\
\text { Sand }\end{array}$ & $\mathrm{N} / \mathrm{A}$ & $\begin{array}{l}\text { Slightly Very Fine Gravelly } \\
\text { Very Fine Sand }\end{array}$ & $\begin{array}{l}\text { Slightly Granular Very } \\
\text { Fine Sand }\end{array}$ & Fine Sand & Fine Sand & Well Sorted \\
\hline Sta_7 B & Slightly Pebbly & $\begin{array}{l}\text { Slightly Pebbly Very } \\
\text { Fine Sand }\end{array}$ & $\begin{array}{l}\text { Slightly Gravelly } \\
\text { Sand }\end{array}$ & N/A & $\begin{array}{l}\text { Slightly Medium Gravelly } \\
\text { Very Fine Sand }\end{array}$ & $\begin{array}{l}\text { Slightly Pebbly Very } \\
\text { Fine Sand }\end{array}$ & Very Fine Sand & Very Fine Sand & Well Sorted \\
\hline Sta_8_A & Slightly Granuley & $\begin{array}{l}\text { Slightly Granuley Fine } \\
\text { Sand }\end{array}$ & $\begin{array}{l}\text { Slightly Gravelly } \\
\text { Sand }\end{array}$ & $\mathrm{N} / \mathrm{A}$ & $\begin{array}{l}\text { Slightly Fine Gravelly Fine } \\
\text { Sand }\end{array}$ & $\begin{array}{l}\text { Slightly Granular Fine } \\
\text { Sand }\end{array}$ & Fine Sand & Fine Sand & $\begin{array}{l}\text { Moderately } \\
\text { Sorted }\end{array}$ \\
\hline Sta 8 B & Pebbly & Pebbly Medium Sand & Gravelly Sand & $\mathrm{N} / \mathrm{A}$ & $\begin{array}{l}\text { Medium Gravelly Medium } \\
\text { Sand }\end{array}$ & Pebbly Medium Sand & Coarse Sand & Coarse Sand & Poorly Sorted \\
\hline Sta_9_A & Pebble Mixes & Sandy Pebble Gravel & Sandy Gravel & $\mathrm{N} / \mathrm{A}$ & Sandy Coarse Gravel & Sandy Pebble Gravel & Very Fine Gravel & Granule Gravel & $\begin{array}{l}\text { Very Poorly } \\
\text { Sorted }\end{array}$ \\
\hline Sta_9_B & Pebble Mixes & Sandy Pebble Gravel & Sandy Gravel & $\mathrm{N} / \mathrm{A}$ & Sandy Medium Gravel & Sandy Pebble Gravel & Very Fine Gravel & Granule Gravel & Poorly Sorted \\
\hline Sta_10_A & Pebble Mixes & Sandy Pebble Gravel & Sandy Gravel & N/A & Sandy Medium Gravel & Sandy Pebble Gravel & Very Fine Gravel & Granule Gravel & $\begin{array}{l}\text { Very Poorly } \\
\text { Sorted }\end{array}$ \\
\hline Sta_10_B & Pebble Mixes & Sandy Pebble Gravel & Sandy Gravel & $\mathrm{N} / \mathrm{A}$ & Sandy Coarse Gravel & Sandy Pebble Gravel & Very Fine Gravel & Granule Gravel & $\begin{array}{l}\text { Very Poorly } \\
\text { Sorted }\end{array}$ \\
\hline Sta_10_C & Pebbly & Pebbly Fine Sand & Gravelly Sand & N/A & Medium Gravelly Fine Sand & Pebbly Fine Sand & Fine Sand & Fine Sand & Poorly Sorted \\
\hline
\end{tabular}




\section{Nifong Thesis (2015) (1): Grain Size Statistics}

\begin{tabular}{|c|c|c|c|c|c|c|c|c|c|c|c|c|c|c|c|c|c|c|c|}
\hline Sample ID & $\begin{array}{c}\text { Gravel } \\
\%\end{array}$ & $\begin{array}{c}\text { Sand } \\
\%\end{array}$ & $\begin{array}{c}\text { Mud } \\
\%\end{array}$ & $\begin{array}{c}\text { Sand } \\
\%\end{array}$ & $\begin{array}{c}\text { Silt } \\
\%\end{array}$ & $\begin{array}{c}\text { Clay } \\
\% \\
\end{array}$ & Modes & $\begin{array}{c}\text { Mode } 1 \\
\text { (phi) }\end{array}$ & $\begin{array}{c}\text { Mode } 2 \\
\text { (phi) }\end{array}$ & $\begin{array}{l}D_{10} \\
\text { (phi) }\end{array}$ & $\begin{array}{c}D_{10} \\
(\mathrm{~mm})\end{array}$ & $\begin{array}{c}D_{50} \\
\text { (phi) }\end{array}$ & $\begin{array}{r}D_{50} \\
(\mathrm{~mm})\end{array}$ & $\begin{array}{c}\text { Mean } \\
\text { Size } \\
\text { (phi) }\end{array}$ & $\begin{array}{c}\text { Mean } \\
\text { Size } \\
(\mathrm{mm})\end{array}$ & $\begin{array}{c}\text { Sorting } \\
\text { (phi) }\end{array}$ & Skewness & Kurtosis & LOI \% \\
\hline Sta_1_A & 39.5 & 59.6 & 0.9 & & & & B & & & -4.13 & 17.51 & 0.78 & 0.58 & -0.28 & 1.21 & 2.41 & -0.55 & 0.61 & 0.89 \\
\hline Sta_1_B & 37.2 & 62.5 & 0.3 & & & & $B$ & & & -4.41 & 21.26 & 1.07 & 0.48 & -0.46 & 1.38 & 2.52 & -0.74 & 0.61 & 3.18 \\
\hline Sta_2_A & 77.9 & 21.6 & 0.5 & & & & B & & & -5.17 & 36.00 & -4.12 & 17.39 & -2.70 & 6.48 & 2.59 & 0.69 & 0.61 & $\mathrm{~N} / \mathrm{A}$ \\
\hline Sta_2_B & 69.6 & 29.7 & 0.7 & & & & $B$ & & & -5.05 & 33.13 & -3.37 & 10.34 & -2.23 & 4.68 & 2.66 & 0.53 & 0.61 & 1.89 \\
\hline Sta_3_A & 2.3 & 96.6 & 1.1 & & & & U & & & -0.20 & 1.15 & 1.30 & 0.41 & 1.13 & 0.46 & 0.87 & -0.31 & 0.61 & 1.46 \\
\hline Sta_3_B & 0.6 & 98.5 & 0.9 & & & & $U$ & & & 0.95 & 0.52 & 1.70 & 0.31 & 1.64 & 0.32 & 0.51 & -0.22 & 0.61 & 0.92 \\
\hline Sta_4_A.1 & 59.6 & 39.9 & 0.5 & & & & B & & & -4.05 & 16.56 & -2.13 & 4.38 & -1.41 & 2.65 & 2.41 & 0.37 & 0.61 & 0.79 \\
\hline Sta_4_A.2 & 53.2 & 46.1 & 0.7 & & & & $B$ & & & -4.36 & 20.53 & -1.58 & 2.99 & -1.28 & 2.43 & 2.65 & 0.17 & 0.61 & $\mathrm{~N} / \mathrm{A}$ \\
\hline Sta_4_B & 71.4 & 27.9 & 0.7 & & & & B & & & -4.86 & 28.98 & -3.59 & 12.03 & -2.33 & 5.01 & 2.65 & 0.62 & 0.61 & 1.28 \\
\hline Sta_5_A & 0.1 & 98.4 & 1.5 & & & & $U$ & & & 2.49 & 0.18 & 2.96 & 0.13 & 2.95 & 0.13 & 0.39 & -0.13 & 0.61 & 0.64 \\
\hline Sta_5_B & 0.7 & 97.8 & 1.5 & & & & U & & & 2.43 & 0.19 & 2.93 & 0.13 & 2.93 & 0.13 & 0.41 & -0.12 & 0.61 & 0.73 \\
\hline Sta_6_A & 62.0 & 37.0 & 1.0 & & & & B & & & -3.62 & 12.30 & -1.93 & 3.81 & -1.32 & 2.50 & 2.12 & 0.38 & 0.61 & 0.74 \\
\hline Sta_7_A & 0.0 & 98.7 & 1.3 & & & & U & & & 2.30 & 0.20 & 3.08 & 0.12 & 3.00 & 0.13 & 0.44 & -0.35 & 0.61 & 0.72 \\
\hline Sta_7_B & 0.3 & 98.3 & 1.4 & & & & $U$ & & & 2.36 & 0.19 & 3.09 & 0.12 & 3.01 & 0.12 & 0.45 & -0.29 & 0.61 & 0.66 \\
\hline Sta_8_A & 2.2 & 96.9 & 1.0 & & & & $U$ & & & 1.26 & 0.42 & 2.23 & 0.21 & 2.23 & 0.21 & 0.73 & -0.09 & 0.61 & 0.66 \\
\hline Sta_8_B & 16.6 & 82.7 & 0.7 & & & & $B$ & & & -3.33 & 10.06 & 1.69 & 0.31 & 0.91 & 0.53 & 1.94 & -0.63 & 0.61 & 0.58 \\
\hline Sta_9_A & 48.1 & 51.3 & 0.5 & & & & $B$ & & & -4.67 & 25.46 & -0.71 & 1.64 & -1.23 & 2.35 & 2.58 & -0.20 & 0.61 & 0.55 \\
\hline Sta_9_B & 49.1 & 50.4 & 0.5 & & & & $\mathrm{~B}$ & & & -3.62 & 12.30 & -0.93 & 1.91 & -1.07 & 2.10 & 1.99 & -0.07 & 0.66 & 0.65 \\
\hline Sta_10_A & 67.5 & 31.5 & 1.0 & & & & B & & & -4.79 & 27.67 & -3.40 & 10.56 & -1.70 & 3.25 & 3.17 & 0.65 & 0.51 & $\mathrm{~N} / \mathrm{A}$ \\
\hline Sta_10_B & 64.2 & 34.2 & 1.6 & & & & B & & & -4.65 & 25.11 & -3.43 & 10.78 & -1.61 & 3.05 & 3.13 & 0.70 & 0.52 & 1.07 \\
\hline Sta_10_C & 7.5 & 91.3 & 1.2 & & & & U & & & 3.22 & 0.11 & 3.33 & 0.10 & 2.22 & 0.21 & 1.41 & -0.38 & 1.45 & 0.57 \\
\hline
\end{tabular}




\section{Nifong Thesis (2015) (1): Grain Size Distribution}

\begin{tabular}{|c|c|c|c|c|c|c|c|c|c|c|c|c|c|c|c|c|c|c|c|c|c|}
\hline$n p$ & $\begin{array}{c}\text { Class } \\
\% \\
\text { phi } \\
-5.5\end{array}$ & $\begin{array}{c}\text { Class } \\
\% \\
\text { phi } \\
-5.0\end{array}$ & $\begin{array}{c}\text { Class } \\
\% \\
\text { phi } \\
-4.5\end{array}$ & $\begin{array}{c}\text { Class } \\
\% \\
\text { phi } \\
-4.0\end{array}$ & $\begin{array}{c}\text { Class } \\
\% \\
\text { phi } \\
-3.5\end{array}$ & $\begin{array}{c}\text { Class } \\
\% \\
\text { phi } \\
-3.0\end{array}$ & $\begin{array}{c}\text { Class } \\
\% \\
\text { phi } \\
-2.5\end{array}$ & $\begin{array}{c}\text { Class } \\
\% \\
\text { phi } \\
-2.0\end{array}$ & $\begin{array}{c}\text { Class } \\
\% \\
\text { phi } \\
-1.5\end{array}$ & $\begin{array}{c}\text { Class } \\
\% \\
\text { phi } \\
-1.0\end{array}$ & $\begin{array}{c}\text { Class } \\
\% \\
\text { phi } \\
-0.5\end{array}$ & $\begin{array}{c}\text { Class } \\
\% \\
\text { phi } \\
0.0\end{array}$ & $\begin{array}{c}\text { Class } \\
\% \\
\text { phi } \\
0.5\end{array}$ & $\begin{array}{c}\text { Class } \\
\% \\
\text { phi } \\
1.0\end{array}$ & $\begin{array}{c}\text { Class } \\
\% \\
\text { phi } \\
1.5\end{array}$ & $\begin{array}{c}\text { Class } \\
\% \\
\text { phi } \\
2.0\end{array}$ & $\begin{array}{c}\text { Class } \\
\% \\
\text { phi } \\
2.5\end{array}$ & $\begin{array}{c}\text { Class } \\
\% \\
\text { phi } \\
3.0\end{array}$ & $\begin{array}{c}\text { Class } \\
\% \\
\text { phi } \\
3.5\end{array}$ & $\begin{array}{c}\text { Class } \\
\% \\
\text { phi } \\
4.0\end{array}$ & $\begin{array}{c}\text { Class } \\
\% \\
\text { phi } \\
>4.0\end{array}$ \\
\hline Sta_1_A & 0.00 & 0.00 & 4.10 & 8.20 & 4.60 & 6.50 & 4.60 & 4.20 & 4.00 & 3.40 & 2.90 & 2.20 & 2.50 & 5.10 & 11.30 & 22.70 & 10.50 & 1.60 & 0.60 & 0.10 & 0.90 \\
\hline Sta_1_B & 0.00 & 0.00 & 7.00 & 18.00 & 6.10 & 2.30 & 2.10 & 0.70 & 0.50 & 0.50 & 0.60 & 0.80 & 1.70 & 6.80 & 21.30 & 26.50 & 4.40 & 0.30 & 0.10 & 0.00 & 0.30 \\
\hline Sta_2_A & 0.00 & 16.00 & 21.20 & 17.00 & 12.50 & 3.40 & 3.40 & 1.70 & 1.50 & 1.30 & 1.10 & 1.00 & 1.30 & 2.60 & 5.00 & 7.40 & 2.40 & 0.40 & 0.20 & 0.10 & 0.50 \\
\hline ta_2_B & 0.00 & 11.70 & 10.00 & 17.00 & 8.80 & 9.90 & 4.00 & 3.60 & 2.60 & 2.00 & 1.50 & 1.40 & 1.80 & 3.50 & 6.70 & 10.30 & 3.60 & 0.50 & 0.30 & 0.10 & 0.70 \\
\hline ta_3_A & 0.00 & 0.00 & 0.00 & 0.00 & 0.00 & 0.00 & 0.10 & 0.30 & 0.40 & 1.50 & 3.80 & 6.80 & 8.60 & 14.80 & 22.70 & 31.40 & 7.60 & 0.60 & 0.30 & 0.10 & 1.00 \\
\hline Sta_3_B & 0.00 & 0.00 & 0.00 & 0.00 & 0.00 & 0.20 & 0.00 & 0.10 & 0.10 & 0.20 & 0.60 & 1.20 & 2.20 & 6.00 & 18.50 & 52.40 & 16.40 & 0.90 & 0.20 & 0.10 & 0.90 \\
\hline Sta_4_A.1 & 0.00 & 0.00 & 0.00 & 11.10 & 15.10 & 11.50 & 7.90 & 5.90 & 4.90 & 3.20 & 2.50 & 2.10 & 2.20 & 3.40 & 7.10 & 15.00 & 3.50 & 2.50 & 1.40 & 0.20 & 0.50 \\
\hline Sta_4_A.2 & 0.00 & 0.00 & 6.60 & 13.20 & 12.70 & 5.50 & 5.30 & 4.10 & 3.30 & 2.60 & 2.10 & 1.90 & 2.10 & 3.90 & 8.00 & 16.50 & 4.60 & 3.20 & 3.30 & 0.40 & 0.70 \\
\hline Sta_4_B & 0.00 & 0.00 & 40.80 & 7.40 & 2.20 & 4.00 & 4.30 & 4.20 & 4.80 & 3.70 & 2.40 & 1.60 & 1.40 & 2.30 & 6.20 & 7.80 & 2.40 & 1.80 & 1.80 & 0.20 & 0.70 \\
\hline Sta_5_A & 0.00 & 0.00 & 0.00 & 0.00 & 0.00 & 0.00 & 0.00 & 0.00 & 0.00 & 0.00 & 0.00 & 0.10 & 0.10 & 0.20 & 0.40 & 2.30 & 5.70 & 44.70 & 43.10 & 1.80 & 1.60 \\
\hline Sta_5_B & 0.00 & 0.00 & 0.00 & 0.00 & 0.00 & 0.20 & 0.30 & 0.10 & 0.10 & 0.10 & 0.10 & 0.10 & 0.10 & 0.30 & 0.60 & 2.80 & 5.90 & 45.90 & 40.00 & 1.90 & 1.50 \\
\hline Sta_6_A & 0.00 & 0.00 & 0.00 & 0.00 & 13.50 & 15.00 & 12.70 & 7.80 & 7.70 & 5.30 & 4.90 & 4.20 & 4.50 & 4.80 & 4.90 & 7.30 & 4.20 & 0.80 & 1.00 & 0.40 & 1.00 \\
\hline Sta_7_A & 0.00 & 0.00 & 0.00 & 0.00 & 0.00 & 0.00 & 0.00 & 0.00 & 0.00 & 0.00 & 0.00 & 0.10 & 0.20 & 0.40 & 0.90 & 3.00 & 8.40 & 28.00 & 53.60 & 4.10 & 1.30 \\
\hline Sta_7_B & 0.00 & 0.00 & 0.00 & 0.00 & 0.00 & 0.20 & 0.00 & 0.00 & 0.00 & 0.00 & 0.00 & 0.10 & 0.10 & 0.30 & 0.70 & 2.70 & 7.50 & 28.40 & 53.20 & 5.40 & 1.40 \\
\hline Sta_8_A & 0.00 & 0.00 & 0.00 & 0.00 & 0.30 & 0.40 & 0.40 & 0.50 & 0.30 & 0.30 & 0.30 & 0.40 & 1.10 & 2.70 & 6.40 & 20.90 & 32.60 & 22.30 & 9.10 & 1.00 & 1.00 \\
\hline Sta_8_B & 0.00 & 0.00 & 0.00 & 2.10 & 7.10 & 2.50 & 2.00 & 1.10 & 0.80 & 0.90 & 0.90 & 1.10 & 2.50 & 6.60 & 12.60 & 25.20 & 22.80 & 8.50 & 2.30 & 0.30 & 0.70 \\
\hline Sta_9_A & 0.00 & 0.00 & 16.00 & 3.30 & 5.90 & 8.90 & 5.60 & 3.70 & 2.70 & 2.20 & 3.30 & 4.90 & 7.60 & 10.60 & 9.40 & 7.10 & 4.80 & 2.50 & 0.80 & 0.10 & 0.60 \\
\hline Sta_9_B & 0.00 & 0.00 & 0.00 & 4.50 & 7.30 & 13.20 & 7.10 & 6.60 & 5.30 & 5.00 & 6.40 & 7.40 & 9.20 & 11.00 & 8.00 & 4.70 & 2.30 & 1.00 & 0.40 & 0.10 & 0.50 \\
\hline Sta_10_A & 0.00 & 0.00 & 26.80 & 1.90 & 18.70 & 13.20 & 1.90 & 2.30 & 1.40 & 1.30 & 0.70 & 0.40 & 0.50 & 0.80 & 1.20 & 2.30 & 3.80 & 7.00 & 12.60 & 2.20 & 1.00 \\
\hline Sta_10_B & 0.00 & 0.00 & 14.80 & 20.20 & 14.00 & 8.80 & 2.90 & 1.10 & 1.30 & 1.20 & 1.00 & 0.60 & 0.60 & 1.40 & 2.20 & 3.00 & 3.40 & 6.00 & 13.20 & 2.70 & 1.60 \\
\hline Sta_10_C & 0.00 & 0.00 & 0.00 & 0.00 & 2.20 & 1.30 & 0.50 & 1.10 & 1.30 & 1.00 & 0.80 & 0.70 & 1.50 & 4.90 & 9.40 & 14.20 & 14.60 & 16.40 & 24.60 & 4.20 & 1.30 \\
\hline
\end{tabular}


Nifong Thesis (2015) (2): Identification, Location, and Description

\begin{tabular}{|c|c|c|c|c|c|c|c|c|}
\hline Sample ID & $\begin{array}{c}\text { Original } \\
\text { Sample ID }\end{array}$ & $\begin{array}{c}\text { Latitude } \\
\text { WGS84 } \\
\end{array}$ & $\begin{array}{c}\text { Longitude } \\
\text { WGS84 }\end{array}$ & $\begin{array}{l}\text { Reliability } \\
\text { Ranking for } \\
\text { Positioning } \\
\end{array}$ & $\begin{array}{c}\text { Water } \\
\text { Depth } \\
(\mathrm{m}) \\
\end{array}$ & $\begin{array}{c}\text { Sample } \\
\text { Collected }\end{array}$ & $\begin{array}{c}\text { Sampler } \\
\text { Type }\end{array}$ & $\begin{array}{c}\text { Total Wt } \\
(\mathrm{gm})\end{array}$ \\
\hline Sta_11_A & Sta_11_A & 43.026837 & -70.687220 & 1 & 24.4 & $5 / 29 / 2015$ & Shipex & 212.43 \\
\hline Sta_11_B & Sta_11_B & 43.026850 & -70.687130 & 1 & 24.4 & $5 / 29 / 2015$ & Shipex & 40.34 \\
\hline Sta_11_C & Sta_11_C & 43.026507 & -70.687193 & 1 & 24.4 & $5 / 29 / 2015$ & Shipex & 348.24 \\
\hline Sta_12_A & Sta_12_A & 43.031727 & -70.704225 & 1 & 15.2 & $5 / 28 / 2015$ & Shipex & 190.55 \\
\hline Sta_12_B & Sta_12_B & 43.031635 & -70.704800 & 1 & 15.2 & $5 / 28 / 2015$ & Shipex & 185.28 \\
\hline Sta_14_A & Sta_14_A & 43.041070 & -70.701710 & 1 & 18.3 & $5 / 28 / 2015$ & Shipex & 250.51 \\
\hline Sta_14_B & Sta_14_B & 43.041110 & -70.702105 & 1 & 18.3 & $5 / 28 / 2015$ & Shipex & 285.42 \\
\hline Sta_15_A & Sta_15_A & 43.049682 & -70.705518 & 1 & 15.2 & $5 / 29 / 2015$ & Shipex & 220.39 \\
\hline Sta_15_B & Sta_15_B & 43.049537 & -70.705527 & 1 & 15.2 & $5 / 29 / 2015$ & Shipex & 264.96 \\
\hline Sta_16_A & Sta_16_A & 43.042942 & -70.689025 & 1 & 17.7 & $5 / 29 / 2015$ & Shipex & 153.03 \\
\hline Sta_16_B & Sta_16_B & 43.042750 & -70.689062 & 1 & 17.7 & $5 / 29 / 2015$ & Shipex & 117.92 \\
\hline Sta_17_B & Sta_17_B & 43.047613 & -70.676675 & 1 & 20.4 & $5 / 27 / 2015$ & Shipex & 408.15 \\
\hline Sta_19_A & Sta_19_A & 43.022295 & -70.695493 & 1 & 21.3 & $5 / 29 / 2015$ & Shipex & 401.83 \\
\hline Sta_19_B & Sta_19_B & 43.022295 & -70.695628 & 1 & 21.3 & $5 / 29 / 2015$ & Shipex & 389.30 \\
\hline Sta_20_A & Sta_20_A & 43.031153 & -70.693498 & 1 & 17.7 & $5 / 28 / 2015$ & Shipex & 97.99 \\
\hline Sta_20_B & Sta_20_B & 43.031133 & -70.693543 & 1 & 17.7 & $5 / 28 / 2015$ & Shipex & 103.52 \\
\hline Sta_21_A & Sta_21_A & 43.040017 & -70.694092 & 1 & 15.2 & $5 / 28 / 2015$ & Shipex & 195.70 \\
\hline Sta_21_B & Sta_21_B & 43.040122 & -70.694452 & 1 & 15.2 & $5 / 28 / 2015$ & Shipex & 113.32 \\
\hline Sta_22_A & Sta_22_A & 43.037393 & -70.682190 & 1 & 20.7 & $5 / 27 / 2015$ & Shipex & 381.74 \\
\hline Sta_22_B & Sta_22_B & 43.037060 & -70.681973 & 1 & 20.7 & $5 / 27 / 2015$ & Shipex & 432.33 \\
\hline Sta_23_A & Sta_23_A & 43.055077 & -70.707808 & 1 & 15.2 & $5 / 28 / 2015$ & Shipex & 336.24 \\
\hline Sta_23_B & Sta_23_B & 43.054655 & -70.708142 & 1 & 15.2 & $5 / 28 / 2015$ & Shipex & 363.49 \\
\hline
\end{tabular}


Nifong Thesis (2015) (2): Sediment Classifications

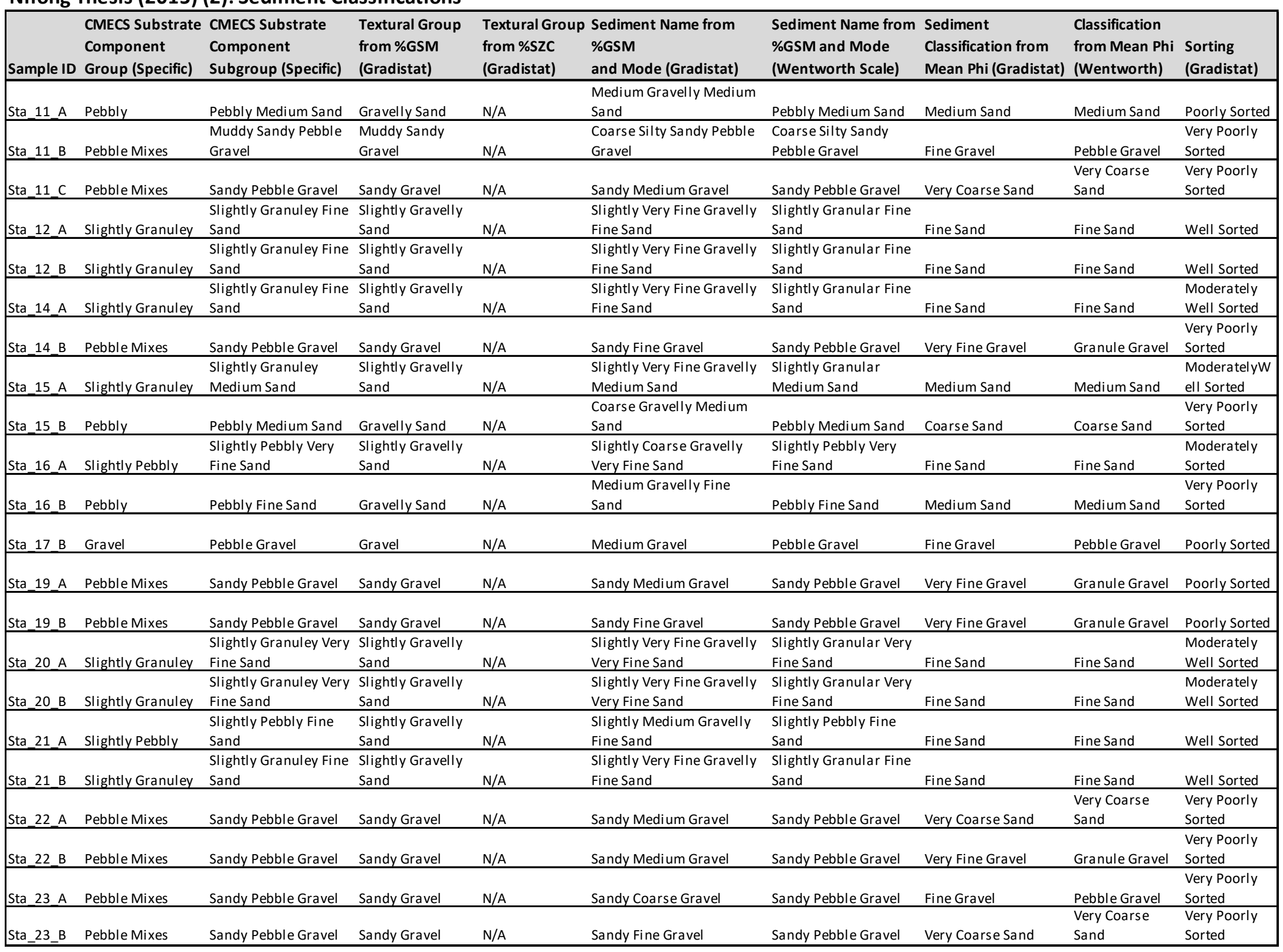




\section{Nifong Thesis (2015) (2): Grain Size Statistics}

\begin{tabular}{|c|c|c|c|c|c|c|c|c|c|c|c|c|c|c|c|c|c|c|c|}
\hline Sample ID & $\begin{array}{c}\text { Gravel } \\
\%\end{array}$ & $\begin{array}{c}\text { Sand } \\
\%\end{array}$ & $\begin{array}{c}\text { Mud } \\
\%\end{array}$ & $\begin{array}{c}\text { Sand } \\
\%\end{array}$ & $\begin{array}{c}\text { Silt } \\
\% \\
\end{array}$ & $\begin{array}{c}\text { Clay } \\
\% \\
\end{array}$ & Modes & $\begin{array}{c}\text { Mode } 1 \\
\text { (phi) }\end{array}$ & $\begin{array}{c}\begin{array}{c}\text { Mode } 2 \\
\text { (phi) }\end{array} \\
\end{array}$ & $\begin{array}{c}\mathrm{D}_{10} \\
\text { (phi) }\end{array}$ & $\begin{array}{r}D_{10} \\
(\mathrm{~mm})\end{array}$ & $\begin{array}{c}\mathrm{D}_{50} \\
\text { (phi) }\end{array}$ & $\begin{array}{r}D_{50} \\
(\mathrm{~mm}) \\
\end{array}$ & $\begin{array}{c}\text { Mean } \\
\text { Size } \\
\text { (phi) }\end{array}$ & $\begin{array}{c}\text { Mean } \\
\text { Size } \\
(\mathrm{mm}) \\
\end{array}$ & $\begin{array}{c}\text { Sorting } \\
\text { (phi) }\end{array}$ & Skewness & Kurtosis & LOI \% \\
\hline Sta_11_A & 6.1 & 93.0 & 0.9 & & & & U & & & 0.34 & 0.79 & 1.69 & 0.31 & 1.65 & 0.32 & 1.02 & -0.31 & 2.26 & 0.51 \\
\hline Sta_11_B & 78.9 & 18.5 & 2.6 & & & & $\mathrm{~B}$ & & & -4.36 & 20.53 & -3.30 & 9.85 & -2.29 & 4.90 & 2.28 & -0.64 & 1.19 & 0.80 \\
\hline Sta_11_C & 42.0 & 57.2 & 0.8 & & & & $\mathrm{~B}$ & & & -3.74 & 13.38 & 0.56 & 0.68 & -0.35 & 1.28 & 2.37 & -0.42 & 0.60 & 0.97 \\
\hline Sta_12_A & 0.4 & 97.7 & 1.9 & & & & U & & & 2.16 & 0.22 & 2.80 & 0.14 & 2.81 & 0.14 & 0.47 & -0.05 & 1.15 & 0.63 \\
\hline Sta_12_B & 0.1 & 97.8 & 2.2 & & & & $U$ & & & 2.20 & 0.22 & 2.79 & 0.14 & 2.82 & 0.14 & 0.45 & 0.04 & 1.15 & 0.61 \\
\hline Sta_14_A & 0.2 & 98.4 & 1.4 & & & & $U$ & & & 1.62 & 0.33 & 2.32 & 0.20 & 2.36 & 0.19 & 0.57 & 0.03 & 1.38 & 0.62 \\
\hline Sta_14_B & 57.9 & 40.9 & 1.2 & & & & $\mathrm{~B}$ & & & -4.54 & 23.26 & -1.65 & 3.14 & -1.14 & 2.20 & 2.69 & 0.23 & 0.78 & 0.76 \\
\hline Sta_15_A & 3.2 & 96.0 & 0.7 & & & & $u$ & & & 0.87 & 0.55 & 1.90 & 0.27 & 1.85 & 0.28 & 0.69 & -0.31 & 1.80 & 0.93 \\
\hline Sta_15_B & 19.2 & 80.2 & 0.5 & & & & $\mathrm{~B}$ & & & -4.03 & 16.34 & 1.72 & 0.30 & 0.56 & 0.68 & 2.17 & -0.77 & 1.78 & 0.69 \\
\hline Sta_16_A & 4.4 & 94.2 & 1.4 & & & & U & & & 1.52 & 0.35 & 2.88 & 0.14 & 2.72 & 0.15 & 0.96 & -0.46 & 1.66 & 0.67 \\
\hline Sta_16_B & 15.6 & 83.4 & 1.0 & & & & $\mathrm{~B}$ & & & -3.72 & 13.18 & 2.57 & 0.17 & 1.74 & 0.30 & 2.14 & -0.69 & 2.03 & 0.68 \\
\hline Sta_17_B & 85.8 & 13.7 & 0.4 & & & & $\mathrm{~B}$ & & & -3.99 & 15.89 & -3.09 & 8.51 & -2.72 & 6.61 & 1.61 & 0.47 & 1.59 & 1.35 \\
\hline Sta_19_A & 66.2 & 33.5 & 0.4 & & & & $\mathrm{~B}$ & & & -3.73 & 13.27 & -2.26 & 4.79 & -1.61 & 3.05 & 1.99 & 0.44 & 0.72 & 1.52 \\
\hline Sta_19_B & 73.5 & 26.1 & 0.4 & & & & $\mathrm{~B}$ & & & -3.35 & 10.20 & -2.12 & 4.35 & -1.68 & 3.20 & 1.63 & 0.42 & 1.06 & 0.67 \\
\hline Sta_20_A & 0.2 & 97.6 & 2.2 & & & & $U$ & & & 2.10 & 0.23 & 3.05 & 0.12 & 2.96 & 0.13 & 0.57 & -0.29 & 1.25 & 0.47 \\
\hline Sta_20_B & 0.8 & 97.2 & 2.0 & & & & U & & & 2.10 & 0.23 & 3.02 & 0.12 & 2.94 & 0.13 & 0.57 & -0.28 & 1.26 & 0.71 \\
\hline Sta_21_A & 1.5 & 96.5 & 2.0 & & & & $\mathrm{U}$ & & & 2.48 & 0.18 & 2.94 & 0.13 & 2.95 & 0.13 & 0.45 & -0.03 & 1.10 & 0.71 \\
\hline Sta_21_B & 0.0 & 97.4 & 2.5 & & & & U & & & 2.47 & 0.18 & 2.86 & 0.14 & 2.90 & 0.13 & 0.43 & 0.09 & 1.13 & 0.78 \\
\hline Sta_22_A & 42.3 & 57.3 & 0.4 & & & & B & & & -4.51 & 22.78 & 0.27 & 0.83 & -0.27 & 1.20 & 2.65 & -0.29 & 0.66 & 0.52 \\
\hline Sta_22_B & 53.1 & 45.8 & 1.1 & & & & $\mathrm{~B}$ & & & -4.20 & 18.38 & -1.60 & 3.03 & -1.16 & 2.23 & 2.60 & 0.21 & 0.65 & 0.59 \\
\hline Sta_23_A & 76.7 & 22.7 & 0.6 & & & & $\mathrm{~B}$ & & & -5.32 & 39.95 & -4.64 & 24.93 & -2.68 & 6.43 & 2.96 & 0.82 & 1.05 & 0.90 \\
\hline Sta 23 B & 49.2 & 49.9 & 0.8 & & & & B & & & -4.21 & 18.51 & 0.88 & 0.54 & -0.80 & 1.74 & 2.67 & 0.02 & 0.58 & 0.70 \\
\hline
\end{tabular}




\section{Nifong Thesis (2015) (2): Grain Size Distribution}

\begin{tabular}{|c|c|c|c|c|c|c|c|c|c|c|c|c|c|c|c|c|c|c|c|c|c|}
\hline & $\begin{array}{c}\text { Class } \\
\% \\
\text { phi }\end{array}$ & $\begin{array}{c}\text { Class } \\
\% \\
\text { phi }\end{array}$ & $\begin{array}{c}\text { Class } \\
\% \\
\text { phi }\end{array}$ & $\begin{array}{c}\text { Class } \\
\% \\
\text { phi }\end{array}$ & $\begin{array}{c}\text { Class } \\
\% \\
\text { phi }\end{array}$ & $\begin{array}{c}\text { Class } \\
\% \\
\text { phi }\end{array}$ & $\begin{array}{c}\text { Class } \\
\% \\
\text { phi }\end{array}$ & $\begin{array}{c}\text { Class } \\
\% \\
\text { phi }\end{array}$ & $\begin{array}{c}\text { Class } \\
\% \\
\text { phi }\end{array}$ & $\begin{array}{c}\text { Class } \\
\% \\
\text { phi }\end{array}$ & $\begin{array}{c}\text { Class } \\
\% \\
\text { phi }\end{array}$ & $\begin{array}{c}\text { Class } \\
\% \\
\text { phi }\end{array}$ & $\begin{array}{c}\text { Class } \\
\% \\
\text { phi }\end{array}$ & $\begin{array}{c}\text { Class } \\
\% \\
\text { phi }\end{array}$ & $\begin{array}{c}\text { Class } \\
\% \\
\text { phi }\end{array}$ & $\begin{array}{c}\text { Class } \\
\% \\
\text { phi }\end{array}$ & $\begin{array}{c}\text { Class } \\
\% \\
\text { phi }\end{array}$ & $\begin{array}{c}\text { Class } \\
\% \\
\text { phi }\end{array}$ & $\begin{array}{c}\text { Class } \\
\% \\
\text { phi }\end{array}$ & $\begin{array}{c}\text { Class } \\
\% \\
\text { phi }\end{array}$ & $\begin{array}{c}\text { Class } \\
\% \\
\text { phi }\end{array}$ \\
\hline Sample ID & -5.5 & -5.0 & -4.5 & -4.0 & -3.5 & -3.0 & -2.5 & -2.0 & -1.5 & -1.0 & -0.5 & 0.0 & 0.5 & 1.0 & 1.5 & 2.0 & 2.5 & 3.0 & 3.5 & 4.0 & $>4.0$ \\
\hline Sta_11_A & 0.00 & 0.00 & 0.00 & 0.00 & 0.50 & 2.20 & 0.70 & 1.30 & 0.70 & 0.70 & 1.20 & 1.30 & 2.10 & 5.60 & 19.00 & 37.60 & 19.00 & 5.00 & 1.80 & 0.40 & 0.90 \\
\hline Sta_11_B & 0.00 & 0.00 & 0.00 & 38.00 & 5.20 & 18.20 & 8.20 & 4.20 & 3.30 & 1.80 & 1.30 & 1.00 & 1.70 & 2.60 & 3.60 & 4.30 & 1.90 & 0.70 & 1.00 & 0.40 & 2.60 \\
\hline Sta_11_C & 0.00 & 0.00 & 0.00 & 3.10 & 13.80 & 8.00 & 8.50 & 3.60 & 2.80 & 2.20 & 1.80 & 1.80 & 3.30 & 9.10 & 14.60 & 14.00 & 6.60 & 3.60 & 2.00 & 0.40 & 0.80 \\
\hline Sta_12_A & 0.00 & 0.00 & 0.00 & 0.00 & 0.00 & 0.00 & 0.00 & 0.10 & 0.10 & 0.20 & 0.20 & 0.20 & 0.30 & 0.60 & 1.20 & 2.70 & 13.30 & 50.30 & 25.80 & 3.10 & 1.90 \\
\hline Sta_12_B & 0.00 & 0.00 & 0.00 & 0.00 & 0.00 & 0.00 & 0.00 & 0.00 & 0.00 & 0.10 & 0.10 & 0.10 & 0.20 & 0.40 & 0.90 & 2.40 & 14.20 & 52.50 & 23.60 & 3.30 & 2.20 \\
\hline Sta_14_A & 0.00 & 0.00 & 0.00 & 0.00 & 0.00 & 0.00 & 0.00 & 0.00 & 0.10 & 0.10 & 0.20 & 0.40 & 0.80 & 1.90 & 3.50 & 11.70 & 45.70 & 25.00 & 8.00 & 1.20 & 1.40 \\
\hline Sta_14_B & 0.00 & 0.00 & 11.30 & 3.40 & 5.30 & 7.20 & 7.70 & 9.70 & 8.00 & 5.40 & 4.70 & 3.90 & 3.90 & 4.50 & 3.80 & 3.80 & 6.40 & 5.30 & 3.60 & 0.90 & 1.20 \\
\hline Sta_15_A & 0.00 & 0.00 & 0.00 & 0.00 & 0.70 & 0.30 & 0.40 & 0.40 & 0.50 & 0.90 & 1.10 & 1.30 & 1.80 & 3.40 & 8.30 & 39.00 & 35.80 & 4.00 & 1.30 & 0.10 & 0.70 \\
\hline Sta_15_B & 0.00 & 0.00 & 0.00 & 10.60 & 3.00 & 0.80 & 1.10 & 1.20 & 1.20 & 1.30 & 1.60 & 1.60 & 2.00 & 3.20 & 8.10 & 31.70 & 28.40 & 2.80 & 0.80 & 0.10 & 0.50 \\
\hline Sta_16_A & 0.00 & 0.00 & 0.00 & 1.40 & 0.40 & 1.00 & 0.30 & 0.40 & 0.50 & 0.40 & 0.50 & 0.40 & 0.60 & 0.60 & 3.10 & 7.70 & 14.10 & 24.30 & 37.00 & 5.80 & 1.50 \\
\hline Sta_16_B & 0.00 & 0.00 & 0.00 & 6.10 & 7.20 & 0.20 & 0.70 & 0.20 & 0.60 & 0.60 & 0.60 & 0.40 & 0.70 & 1.30 & 4.40 & 9.60 & 13.60 & 20.90 & 27.30 & 4.50 & 1.10 \\
\hline Sta_17_B & 0.00 & 0.00 & 6.00 & 3.70 & 22.40 & 22.00 & 13.00 & 8.40 & 6.30 & 4.00 & 2.90 & 1.70 & 1.30 & 1.00 & 1.40 & 2.20 & 2.10 & 0.60 & 0.40 & 0.20 & 0.40 \\
\hline Sta_19_A & 0.00 & 0.00 & 0.00 & 3.00 & 13.20 & 20.20 & 9.50 & 8.80 & 6.30 & 5.20 & 4.60 & 3.90 & 4.20 & 5.90 & 6.20 & 4.90 & 2.10 & 0.90 & 0.60 & 0.10 & 0.40 \\
\hline Sta_19_B & 0.00 & 0.00 & 0.00 & 0.00 & 5.90 & 14.80 & 17.30 & 16.00 & 11.30 & 8.10 & 5.60 & 3.30 & 3.20 & 4.60 & 4.10 & 2.70 & 1.20 & 0.70 & 0.60 & 0.20 & 0.40 \\
\hline Sta_20_A & 0.00 & 0.00 & 0.00 & 0.00 & 0.00 & 0.00 & 0.00 & 0.00 & 0.10 & 0.20 & 0.10 & 0.20 & 0.30 & 0.80 & 1.60 & 4.70 & 9.30 & 28.10 & 44.70 & 7.70 & 2.20 \\
\hline Sta_20_B & 0.00 & 0.00 & 0.00 & 0.00 & 0.00 & 0.00 & 0.00 & 0.30 & 0.30 & 0.20 & 0.20 & 0.30 & 0.40 & 0.70 & 1.40 & 4.30 & 9.40 & 30.30 & 43.00 & 7.20 & 2.00 \\
\hline Sta_21_A & 0.00 & 0.00 & 0.00 & 0.00 & 1.10 & 0.10 & 0.00 & 0.10 & 0.10 & 0.10 & 0.10 & 0.10 & 0.10 & 0.10 & 0.30 & 2.00 & 5.50 & 45.50 & 38.00 & 4.80 & 2.00 \\
\hline Sta_21_B & 0.00 & 0.00 & 0.00 & 0.00 & 0.00 & 0.00 & 0.00 & 0.00 & 0.00 & 0.00 & 0.00 & 0.10 & 0.10 & 0.20 & 0.50 & 2.40 & 6.80 & 54.00 & 29.70 & 3.60 & 2.60 \\
\hline Sta_22_A & 0.00 & 4.50 & 5.70 & 0.00 & 4.20 & 11.70 & 7.70 & 3.50 & 2.80 & 2.20 & 2.30 & 2.90 & 4.50 & 6.70 & 7.60 & 9.80 & 10.60 & 7.90 & 4.20 & 0.80 & 0.40 \\
\hline Sta_22_B & 0.00 & 0.00 & 8.60 & 2.40 & 14.30 & 11.20 & 7.10 & 4.30 & 2.80 & 2.40 & 2.60 & 3.20 & 5.10 & 7.00 & 6.70 & 7.20 & 6.10 & 4.60 & 2.70 & 0.60 & 1.10 \\
\hline Sta_23_A & 0.00 & 30.60 & 27.90 & 2.00 & 7.60 & 2.90 & 2.70 & 1.20 & 1.00 & 0.80 & 0.60 & 0.60 & 0.70 & 1.20 & 1.70 & 4.00 & 9.10 & 3.50 & 1.10 & 0.20 & 0.60 \\
\hline Sta_23_B & 0.00 & 0.00 & 6.00 & 7.20 & 9.00 & 4.60 & 8.80 & 6.60 & 3.80 & 3.20 & 3.20 & 2.90 & 3.20 & 3.40 & 3.30 & 8.00 & 16.40 & 6.90 & 2.30 & 0.40 & 0.80 \\
\hline
\end{tabular}


Section 2: NEWBEX Survey (2012) 
NEWBEX Survey (2012) (1): Identification, Location, and Description

\begin{tabular}{|c|c|c|c|c|c|c|c|c|}
\hline Sample ID & $\begin{array}{c}\text { Original } \\
\text { Sample ID }\end{array}$ & $\begin{array}{c}\text { Latitude } \\
\text { WGS84 }\end{array}$ & $\begin{array}{c}\text { Longitude } \\
\text { WGS84 }\end{array}$ & $\begin{array}{l}\text { Reliability } \\
\text { Ranking for } \\
\text { Positioning }\end{array}$ & $\begin{array}{c}\text { Water } \\
\text { Depth } \\
\text { (m) }\end{array}$ & $\begin{array}{c}\text { Sample } \\
\text { Collected }\end{array}$ & $\begin{array}{c}\text { Sampler } \\
\text { Type }\end{array}$ & $\begin{array}{c}\text { Total Wt } \\
\text { (gm) }\end{array}$ \\
\hline NB_01 & 15_S1 & 43.070682 & -70.704077 & 1 & 15.1 & $11 / 26 / 2012$ & Shipek & 72.18 \\
\hline NB_02 & 15_S2 & 43.070710 & -70.704057 & 1 & 15.1 & $11 / 26 / 2012$ & Shipek & 106.22 \\
\hline NB_03 & 14_S1 & 43.068822 & -70.704358 & 1 & & $11 / 26 / 2012$ & Shipek & 35.04 \\
\hline NB_04 & 14_S2 & 43.068815 & -70.704428 & 1 & & $11 / 26 / 2012$ & Shipek & 60.59 \\
\hline NB_05 & 13_S1 & 43.066312 & -70.704792 & 1 & 12.9 & $11 / 26 / 2012$ & Shipek & 55.80 \\
\hline NB_06 & $13 \_\mathrm{S} 2$ & 43.066407 & -70.704713 & 1 & 12.9 & $11 / 26 / 2012$ & Shipek & 51.50 \\
\hline NB_07 & 12_S1 & 43.063998 & -70.705130 & 1 & 15.5 & $11 / 26 / 2012$ & Shipek & 52.08 \\
\hline NB_08 & 12_S2 & 43.064040 & -70.705173 & 1 & 15.5 & $11 / 26 / 2012$ & Shipek & 66.19 \\
\hline NB_09 & 11_S1 & 43.060667 & -70.705672 & 1 & 18.2 & $11 / 26 / 2012$ & Shipek & 67.01 \\
\hline NB_10 & 11_S2 & 43.060570 & -70.705683 & 1 & 18.2 & $11 / 26 / 2012$ & Shipek & 68.66 \\
\hline NB_11 & 10_S1 & 43.058133 & -70.705605 & 1 & & $11 / 26 / 2012$ & Shipek & 71.14 \\
\hline NB_12 & 10_S2 & 43.058135 & -70.705778 & 1 & & $11 / 26 / 2012$ & Shipek & 52.10 \\
\hline NB_13 & 09_S1 & 43.059418 & -70.703545 & 1 & 18.1 & $11 / 26 / 2012$ & Shipek & 638.28 \\
\hline NB_14 & 09_S2 & 43.059173 & -70.703467 & 1 & 18.1 & $11 / 26 / 2012$ & Shipek & 91.10 \\
\hline NB_15 & 09_S3 & 43.059500 & -70.703622 & 1 & 18.1 & $11 / 26 / 2012$ & Shipek & 55.60 \\
\hline NB_16 & 08_S1 & 43.057327 & -70.702315 & 1 & 14.1 & $11 / 26 / 2012$ & Shipek & 194.16 \\
\hline NB_17 & 08_S2 & 43.057295 & -70.702267 & 1 & 14.1 & $11 / 26 / 2012$ & Shipek & 206.30 \\
\hline NB_18 & 07_S1 & 43.055378 & -70.701928 & 1 & 13.6 & $11 / 26 / 2012$ & Shipek & 85.74 \\
\hline NB_19 & 07_S2 & 43.055353 & -70.701882 & 1 & 13.6 & $11 / 26 / 2012$ & Shipek & 89.20 \\
\hline NB_20 & 07_S3 & 43.055312 & -70.701822 & 1 & 13.6 & $11 / 26 / 2012$ & Shipek & 269.49 \\
\hline
\end{tabular}


NEWBEX Survey (2012) (1): Sediment Classifications

\begin{tabular}{|c|c|c|c|c|c|c|c|c|c|}
\hline Sample ID & $\begin{array}{l}\text { CMECS Substrate } \\
\text { Component } \\
\text { Group (Specific) }\end{array}$ & $\begin{array}{l}\text { CMECS Substrate } \\
\text { Component } \\
\text { Subgroup (Specific) }\end{array}$ & $\begin{array}{l}\text { Textural Group } \\
\text { from \%GSM } \\
\text { (Gradistat) }\end{array}$ & $\begin{array}{l}\text { Textural Group } \\
\text { from \%SZC } \\
\text { (Gradistat) }\end{array}$ & $\begin{array}{l}\text { Sediment Name from } \\
\text { \%GSM } \\
\text { and Mode (Gradistat) }\end{array}$ & $\begin{array}{l}\text { Sediment Name from } \\
\text { \%GSM and Mode } \\
\text { (Wentworth Scale) }\end{array}$ & $\begin{array}{l}\text { Sediment } \\
\text { Classification from } \\
\text { Mean Phi (Gradistat) }\end{array}$ & $\begin{array}{l}\text { Classification } \\
\text { from Mean Phi } \\
\text { (Wentworth) }\end{array}$ & $\begin{array}{l}\text { Sorting } \\
\text { (Gradistat) }\end{array}$ \\
\hline NB_01 & Pebble Mixes & Sandy Pebble Gravel & Sandy Gravel & $\mathrm{N} / \mathrm{A}$ & Sandy Fine Gravel & Sandy Pebble Gravel & Coarse Sand & Coarse Sand & Poorly Sorted \\
\hline NB_02 & Pebble Mixes & Sandy Pebble Gravel & Sandy Gravel & N/A & Sandy Coarse Gravel & Sandy Pebble Gravel & Very Fine Gravel & Granule Gravel & $\begin{array}{l}\text { Very Poorly } \\
\text { Sorted }\end{array}$ \\
\hline NB_03 & Slightly Granuley & $\begin{array}{l}\text { Slightly Granuley } \\
\text { Medium Sand }\end{array}$ & $\begin{array}{l}\text { Slightly Gravelly } \\
\text { Sand }\end{array}$ & $\mathrm{N} / \mathrm{A}$ & $\begin{array}{l}\text { Slightly Very Fine Gravelly } \\
\text { Medium Sand }\end{array}$ & $\begin{array}{l}\text { Slightly Granular } \\
\text { Medium Sand }\end{array}$ & Medium Sand & Medium Sand & $\begin{array}{l}\text { Moderately } \\
\text { Sorted }\end{array}$ \\
\hline NB_04 & Granuley & $\begin{array}{l}\text { Granuley Medium } \\
\text { Sand }\end{array}$ & Gravelly Sand & $\mathrm{N} / \mathrm{A}$ & $\begin{array}{l}\text { Very Fine Gravelly Medium } \\
\text { Sand }\end{array}$ & $\begin{array}{l}\text { Granular Medium } \\
\text { Sand }\end{array}$ & Medium Sand & Medium Sand & $\begin{array}{l}\text { Moderately } \\
\text { Sorted }\end{array}$ \\
\hline NB_05 & Granuley & $\begin{array}{l}\text { Granuley Medium } \\
\text { Sand }\end{array}$ & Gravelly Sand & $\mathrm{N} / \mathrm{A}$ & $\begin{array}{l}\text { Very Fine Gravelly Medium } \\
\text { Sand }\end{array}$ & $\begin{array}{l}\text { Granular Medium } \\
\text { Sand }\end{array}$ & Medium Sand & Medium Sand & Poorly Sorted \\
\hline NB_06 & Slightly Granuley & $\begin{array}{l}\text { Slightly Granuley } \\
\text { Medium Sand }\end{array}$ & $\begin{array}{l}\text { Slightly Gravelly } \\
\text { Sand }\end{array}$ & $\mathrm{N} / \mathrm{A}$ & $\begin{array}{l}\text { Slightly Very Fine Gravelly } \\
\text { Medium Sand }\end{array}$ & $\begin{array}{l}\text { Slightly Granular } \\
\text { Medium Sand } \\
\end{array}$ & Medium Sand & Medium Sand & $\begin{array}{l}\text { Moderately } \\
\text { Sorted }\end{array}$ \\
\hline NB_07 & Granuley & $\begin{array}{l}\text { Granuley Medium } \\
\text { Sand }\end{array}$ & Gravelly Sand & $\mathrm{N} / \mathrm{A}$ & $\begin{array}{l}\text { Very Fine Gravelly Medium } \\
\text { Sand }\end{array}$ & $\begin{array}{l}\text { Granular Medium } \\
\text { Sand }\end{array}$ & Medium Sand & Medium Sand & $\begin{array}{l}\text { Moderately } \\
\text { Sorted }\end{array}$ \\
\hline NB_08 & Slightly Pebbly & $\begin{array}{l}\text { Slightly Pebbly } \\
\text { Medium Sand }\end{array}$ & $\begin{array}{l}\text { Slightly Gravelly } \\
\text { Sand }\end{array}$ & $\mathrm{N} / \mathrm{A}$ & $\begin{array}{l}\text { Slightly Fine Gravelly } \\
\text { Medium Sand }\end{array}$ & $\begin{array}{l}\text { Slightly Pebbly } \\
\text { Medium Sand }\end{array}$ & Medium Sand & Medium Sand & Well Sorted \\
\hline NB_09 & Pebbly & Pebbly Medium Sand & Gravelly Sand & N/A & $\begin{array}{l}\text { Medium Gravelly Medium } \\
\text { Sand }\end{array}$ & Pebbly Medium Sand & Medium Sand & Medium Sand & Poorly Sorted \\
\hline NB_10 & Pebbly & Pebbly Medium Sand & Gravelly Sand & $\mathrm{N} / \mathrm{A}$ & $\begin{array}{l}\text { Coarse Gravelly Medium } \\
\text { Sand }\end{array}$ & Pebbly Medium Sand & Coarse Sand & Coarse Sand & $\begin{array}{l}\text { Very Poorly } \\
\text { Sorted }\end{array}$ \\
\hline NB_11 & Granuley & $\begin{array}{l}\text { Granuley Medium } \\
\text { Sand }\end{array}$ & Gravelly Sand & $\mathrm{N} / \mathrm{A}$ & $\begin{array}{l}\text { Very Fine Gravelly Medium } \\
\text { Sand }\end{array}$ & $\begin{array}{l}\text { Granular Medium } \\
\text { Sand }\end{array}$ & Medium Sand & Medium Sand & Poorly Sorted \\
\hline NB_12 & Granuley & $\begin{array}{l}\text { Granuley Medium } \\
\text { Sand }\end{array}$ & Gravelly Sand & $\mathrm{N} / \mathrm{A}$ & $\begin{array}{l}\text { Very Fine Gravelly Medium } \\
\text { Sand }\end{array}$ & $\begin{array}{l}\text { Granular Medium } \\
\text { Sand }\end{array}$ & Medium Sand & Medium Sand & $\begin{array}{l}\text { Moderately } \\
\text { Sorted }\end{array}$ \\
\hline NB_13 & Gravel & Pebble Gravel & Gravel & $\mathrm{N} / \mathrm{A}$ & Very Coarse Gravel & Pebble Gravel & Coarse Gravel & Pebble Gravel & Poorly Sorted \\
\hline NB_14 & Gravel & Pebble Gravel & Gravel & $\mathrm{N} / \mathrm{A}$ & Coarse Gravel & Pebble Gravel & Coarse Gravel & Pebble Gravel & Poorly Sorted \\
\hline NB_15 & Pebble Mixes & Sandy Pebble Gravel & Sandy Gravel & $\mathrm{N} / \mathrm{A}$ & Sandy Coarse Gravel & Sandy Pebble Gravel & Fine Gravel & Pebble Gravel & $\begin{array}{l}\text { Very Poorly } \\
\text { Sorted }\end{array}$ \\
\hline NB_16 & Pebble Mixes & Sandy Pebble Gravel & Sandy Gravel & $\mathrm{N} / \mathrm{A}$ & Sandy Coarse Gravel & Sandy Pebble Gravel & Fine Gravel & Pebble Gravel & $\begin{array}{l}\text { Very Poorly } \\
\text { Sorted }\end{array}$ \\
\hline NB_17 & Pebble Mixes & Sandy Pebble Gravel & Sandy Gravel & $\mathrm{N} / \mathrm{A}$ & Sandy Coarse Gravel & Sandy Pebble Gravel & Fine Gravel & Pebble Gravel & $\begin{array}{l}\text { Very Poorly } \\
\text { Sorted }\end{array}$ \\
\hline NB_18 & Pebble Mixes & Sandy Pebble Gravel & Sandy Gravel & N/A & Sandy Medium Gravel & Sandy Pebble Gravel & Very Fine Gravel & Granule Gravel & $\begin{array}{l}\text { Very Poorly } \\
\text { Sorted }\end{array}$ \\
\hline NB_19 & Pebbly & Pebbly Medium Sand & Gravelly Sand & N/A & $\begin{array}{l}\text { Medium Gravelly Medium } \\
\text { Sand }\end{array}$ & Pebbly Medium Sand & Medium Sand & Medium Sand & Poorly Sorted \\
\hline NB_20 & Pebble Mixes & Sandy Pebble Gravel & Sandy Gravel & $\mathrm{N} / \mathrm{A}$ & Sandy Medium Gravel & Sandy Pebble Gravel & Fine Gravel & Pebble Gravel & $\begin{array}{l}\text { Very Poorly } \\
\text { Sorted }\end{array}$ \\
\hline
\end{tabular}




\section{NEWBEX Survey (2012) (1): Grain Size Statistics}

\begin{tabular}{|c|c|c|c|c|c|c|c|c|c|c|c|c|c|c|c|c|c|c|c|}
\hline Sample ID & $\begin{array}{c}\text { Gravel } \\
\%\end{array}$ & $\begin{array}{c}\text { Sand } \\
\%\end{array}$ & $\begin{array}{c}\text { Mud } \\
\%\end{array}$ & $\begin{array}{c}\text { Sand } \\
\% \\
\end{array}$ & $\begin{array}{l}\text { Silt } \\
\%\end{array}$ & $\begin{array}{c}\text { Clay } \\
\% \\
\end{array}$ & Modes & $\begin{array}{c}\text { Mode } 1 \\
\text { (phi) }\end{array}$ & $\begin{array}{c}\text { Mode } 2 \\
\text { (phi) }\end{array}$ & $\begin{array}{l}D_{10} \\
\text { (phi) }\end{array}$ & $\begin{array}{c}D_{10} \\
(\mathrm{~mm})\end{array}$ & $\begin{array}{l}D_{50} \\
\text { (phi) }\end{array}$ & $\begin{array}{l}D_{50} \\
(\mathrm{~mm})\end{array}$ & $\begin{array}{c}\text { Mean } \\
\text { Size } \\
\text { (phi) }\end{array}$ & $\begin{array}{l}\text { Mean } \\
\text { Size } \\
(\mathrm{mm})\end{array}$ & $\begin{array}{c}\text { Sorting } \\
\text { (phi) }\end{array}$ & Skewness & Kurtosis & LOI \% \\
\hline NB_01 & 32.9 & 66.4 & 0.6 & & & & B & 1.75 & -3.74 & -2.24 & 4.73 & -2.96 & 7.77 & 0.01 & 0.99 & 1.99 & -0.50 & 0.70 & 1.32 \\
\hline NB_02 & 51.9 & 47.7 & 0.3 & & & & $\mathrm{~B}$ & -4.24 & 1.75 & -4.29 & 19.52 & -1.33 & 2.51 & -1.28 & 2.43 & 2.43 & 0.04 & 0.50 & 0.96 \\
\hline NB_03 & 4.4 & 95.2 & 0.4 & & & & U & 1.75 & & -0.15 & 1.11 & 1.38 & 0.38 & 1.24 & 0.42 & 0.82 & -0.39 & 1.44 & 0.85 \\
\hline NB_04 & 5.9 & 93.9 & 0.3 & & & & $\mathrm{U}$ & 1.75 & & -0.54 & 1.46 & 1.31 & 0.40 & 1.09 & 0.47 & 1.00 & -0.38 & 1.24 & 1.29 \\
\hline NB_05 & 10.5 & 89.0 & 0.5 & & & & $\mathrm{U}$ & 1.75 & & -1.06 & 2.08 & 1.48 & 0.36 & 1.06 & 0.48 & 1.20 & -0.56 & 1.39 & 2.02 \\
\hline NB_06 & 4.0 & 95.4 & 0.6 & & & & U & 1.75 & & 0.04 & 0.97 & 1.58 & 0.33 & 1.43 & 0.37 & 0.86 & -0.39 & 1.56 & 0.85 \\
\hline NB_07 & 9.1 & 90.2 & 0.7 & & & & U & 1.75 & & -0.11 & 1.08 & 1.84 & 0.28 & 1.80 & 0.29 & 0.99 & -0.39 & 3.18 & 0.91 \\
\hline NB_08 & 1.8 & 97.6 & 0.6 & & & & U & 1.75 & & 1.36 & 0.39 & 1.89 & 0.27 & 1.92 & 0.26 & 0.43 & -0.03 & 1.07 & 0.97 \\
\hline NB_09 & 16.5 & 82.7 & 0.8 & & & & U & 1.75 & & -2.47 & 5.56 & 1.85 & 0.28 & 1.02 & 0.49 & 1.80 & -0.71 & 3.33 & 0.88 \\
\hline NB_10 & 25.7 & 73.8 & 0.5 & & & & B & 1.75 & -4.24 & -4.03 & 16.31 & 1.74 & 0.30 & 0.30 & 0.81 & 2.39 & -0.77 & 0.83 & 0.95 \\
\hline NB_11 & 6.3 & 93.1 & 0.6 & & & & U & 1.75 & & -0.46 & 1.38 & 1.66 & 0.32 & 1.39 & 0.38 & 1.03 & -0.50 & 1.51 & 4.06 \\
\hline NB_12 & 5.0 & 94.3 & 0.7 & & & & U & 1.75 & & -0.08 & 1.06 & 1.77 & 0.29 & 1.58 & 0.33 & 0.97 & -0.41 & 1.70 & 0.95 \\
\hline NB_13 & 92.1 & 7.6 & 0.4 & & & & B & -5.24 & -3.74 & -5.37 & 41.38 & -4.79 & 27.70 & -4.43 & 21.54 & 1.61 & 0.67 & 1.90 & 0.38 \\
\hline NB_14 & 91.3 & 8.2 & 0.6 & & & & $\mathrm{U}$ & -4.24 & & -4.43 & 21.53 & -4.20 & 18.34 & -4.20 & 18.34 & 1.12 & 0.46 & 9.54 & 1.41 \\
\hline NB_15 & 76.4 & 22.8 & 0.8 & & & & U & -4.24 & & -4.37 & 20.71 & -3.69 & 12.92 & -2.11 & 4.32 & 2.58 & 0.79 & 1.00 & 1.11 \\
\hline NB_16 & 68.4 & 31.1 & 0.6 & & & & B & -4.73 & 1.75 & -4.83 & 28.48 & -4.06 & 16.71 & -2.41 & 5.30 & 2.68 & 0.78 & 0.56 & 0.42 \\
\hline NB_17 & 65.0 & 34.6 & 0.3 & & & & B & -4.73 & 1.75 & -4.85 & 28.84 & -4.05 & 16.61 & -2.37 & 5.17 & 2.74 & 0.77 & 0.51 & 0.78 \\
\hline NB_18 & 53.7 & 44.7 & 1.7 & & & & $\mathrm{~B}$ & 1.75 & -4.24 & -4.15 & 17.74 & -1.71 & 3.27 & -1.21 & 2.31 & 2.52 & 0.26 & 0.58 & 0.89 \\
\hline NB_20 & 70.9 & 28.5 & 0.6 & & & & T & -5.24 & -3.24 & -5.21 & 36.89 & -2.87 & 7.29 & -2.14 & 4.39 & 2.79 & 0.34 & 0.77 & 0.31 \\
\hline
\end{tabular}




\section{NEWBEX Survey (2012) (1): Grain Size Distribution}

\begin{tabular}{|c|c|c|c|c|c|c|c|c|c|c|c|c|c|c|c|c|c|c|c|c|c|}
\hline & $\begin{array}{c}\text { Class } \\
\% \\
\text { phi } \\
-5.5\end{array}$ & $\begin{array}{c}\text { Class } \\
\% \\
\text { phi } \\
-5.0\end{array}$ & $\begin{array}{c}\text { Class } \\
\% \\
\text { phi } \\
-4.5\end{array}$ & $\begin{array}{c}\text { Class } \\
\% \\
\text { phi } \\
-4.0\end{array}$ & $\begin{array}{c}\text { Class } \\
\% \\
\text { phi } \\
-3.5\end{array}$ & $\begin{array}{c}\text { Class } \\
\% \\
\text { phi } \\
-3.0\end{array}$ & $\begin{array}{c}\text { Class } \\
\% \\
\text { phi } \\
-2.5\end{array}$ & $\begin{array}{c}\text { Class } \\
\% \\
\text { phi } \\
-2.0\end{array}$ & $\begin{array}{c}\text { Class } \\
\% \\
\text { phi } \\
-1.5\end{array}$ & $\begin{array}{c}\text { Class } \\
\% \\
\text { phi } \\
-1.0\end{array}$ & $\begin{array}{c}\text { Class } \\
\% \\
\text { phi } \\
-0.5\end{array}$ & $\begin{array}{c}\text { Class } \\
\% \\
\text { phi } \\
0.0\end{array}$ & $\begin{array}{c}\text { Class } \\
\% \\
\text { phi } \\
0.5\end{array}$ & $\begin{array}{c}\text { Class } \\
\% \\
\text { phi } \\
1.0\end{array}$ & $\begin{array}{c}\text { Class } \\
\% \\
\text { phi } \\
1.5\end{array}$ & $\begin{array}{c}\text { Class } \\
\% \\
\text { phi } \\
2.0\end{array}$ & $\begin{array}{c}\text { Class } \\
\% \\
\text { phi } \\
2.5\end{array}$ & $\begin{array}{c}\text { Class } \\
\% \\
\text { phi } \\
3.0\end{array}$ & $\begin{array}{c}\text { Class } \\
\% \\
\text { phi } \\
3.5\end{array}$ & $\begin{array}{c}\text { Class } \\
\% \\
\text { phi } \\
4.0\end{array}$ & $\begin{array}{c}\text { Class } \\
\% \\
\text { phi } \\
>4.0\end{array}$ \\
\hline Sample ID & -5.5 & -5.0 & -4.5 & -4.0 & -3.5 & -3.0 & & -2.0 & -1.5 & -1.0 & -0.5 & 0.0 & 0.5 & 1.0 & & 2.0 & & & & & \\
\hline NB_01 & 0.00 & 0.00 & 0.00 & 0.00 & 9.41 & 0.00 & 7.20 & 7.29 & 4.52 & 4.50 & 4.18 & 3.57 & 5.32 & 8.55 & 14.42 & 23.04 & 5.91 & 0.99 & 0.29 & 0.14 & 0.64 \\
\hline NB_02 & 0.00 & 0.00 & 0.00 & 24.53 & 7.11 & 5.31 & 5.52 & 3.16 & 3.46 & 2.84 & 2.56 & 2.60 & 3.70 & 6.69 & 11.06 & 16.32 & 3.86 & 0.66 & 0.20 & 0.09 & 0.34 \\
\hline NB_03 & 0.00 & 0.00 & 0.00 & 0.00 & 0.00 & 0.00 & 1.21 & 1.00 & 0.80 & 1.41 & 2.85 & 3.89 & 5.34 & 11.22 & 28.93 & 36.00 & 6.29 & 0.51 & 0.12 & 0.06 & 0.36 \\
\hline NB_04 & 0.00 & 0.00 & 0.00 & 0.00 & 0.00 & 0.00 & 0.43 & 0.41 & 1.66 & 3.36 & 4.66 & 4.92 & 6.64 & 12.77 & 24.19 & 30.38 & 7.29 & 1.29 & 1.25 & 0.45 & 0.29 \\
\hline NB_05 & 0.00 & 0.00 & 0.00 & 0.00 & 0.00 & 0.00 & 1.83 & 2.33 & 2.17 & 4.19 & 3.92 & 3.68 & 4.72 & 8.97 & 18.87 & 34.80 & 11.78 & 1.28 & 0.33 & 0.61 & 0.53 \\
\hline NB_06 & 0.00 & 0.00 & 0.00 & 0.00 & 0.00 & 0.00 & 0.87 & 0.22 & 0.76 & 2.10 & 2.65 & 3.08 & 4.09 & 8.45 & 21.24 & 37.85 & 14.74 & 2.45 & 0.64 & 0.22 & 0.64 \\
\hline NB_07 & 0.00 & 0.00 & 0.00 & 0.00 & 0.00 & 3.39 & 0.23 & 2.06 & 2.26 & 1.15 & 0.66 & 0.32 & 0.60 & 1.40 & 7.48 & 44.90 & 29.49 & 4.48 & 0.68 & 0.21 & 0.69 \\
\hline NB_08 & 0.00 & 0.00 & 0.00 & 0.00 & 0.00 & 0.70 & 0.50 & 0.25 & 0.05 & 0.28 & 0.26 & 0.43 & 0.62 & 1.47 & 7.53 & 47.92 & 34.52 & 4.07 & 0.64 & 0.14 & 0.62 \\
\hline NB_09 & 0.00 & 0.00 & 0.00 & 0.00 & 3.26 & 4.47 & 2.21 & 2.99 & 2.21 & 1.34 & 0.81 & 0.77 & 0.87 & 1.44 & 4.80 & 34.92 & 31.34 & 6.37 & 1.15 & 0.27 & 0.81 \\
\hline NB_10 & 0.00 & 0.00 & 0.00 & 10.62 & 1.90 & 4.64 & 3.05 & 2.52 & 1.52 & 1.47 & 0.61 & 0.63 & 0.74 & 1.27 & 4.73 & 33.92 & 26.36 & 4.31 & 0.99 & 0.25 & 0.46 \\
\hline NB_11 & 0.00 & 0.00 & 0.00 & 0.00 & 0.00 & 0.00 & 0.19 & 1.17 & 1.78 & 3.20 & 3.50 & 3.51 & 4.45 & 6.98 & 12.64 & 37.65 & 21.24 & 2.61 & 0.39 & 0.10 & 0.60 \\
\hline NB_12 & 0.00 & 0.00 & 0.00 & 0.00 & 0.00 & 0.00 & 0.17 & 0.33 & 1.68 & 2.83 & 2.67 & 2.78 & 3.77 & 5.18 & 10.98 & 36.64 & 25.90 & 4.71 & 1.33 & 0.36 & 0.68 \\
\hline NB_13 & 0.00 & 42.45 & 20.06 & 2.08 & 15.14 & 7.20 & 2.23 & 1.50 & 0.80 & 0.61 & 0.49 & 0.37 & 0.32 & 0.31 & 0.52 & 2.15 & 1.60 & 1.05 & 0.56 & 0.18 & 0.37 \\
\hline NB_14 & 0.00 & 0.00 & 0.00 & 84.06 & 3.24 & 1.54 & 0.65 & 1.17 & 0.29 & 0.32 & 0.29 & 0.24 & 0.20 & 0.20 & 0.32 & 1.46 & 1.84 & 1.70 & 1.55 & 0.35 & 0.59 \\
\hline NB_15 & 0.00 & 0.00 & 0.00 & 42.93 & 11.78 & 8.21 & 4.40 & 4.18 & 2.39 & 2.49 & 1.36 & 0.93 & 0.73 & 0.89 & 1.47 & 6.56 & 5.30 & 2.96 & 2.23 & 0.43 & 0.77 \\
\hline NB_16 & 0.00 & 0.00 & 33.93 & 18.47 & 7.24 & 1.64 & 2.02 & 1.41 & 1.64 & 2.00 & 1.88 & 1.77 & 1.92 & 2.82 & 5.18 & 10.82 & 3.34 & 1.99 & 1.13 & 0.23 & 0.58 \\
\hline NB_17 & 0.00 & 0.00 & 38.70 & 12.73 & 7.88 & 0.28 & 1.00 & 1.72 & 1.88 & 0.85 & 0.95 & 1.36 & 1.89 & 3.06 & 5.89 & 12.97 & 3.89 & 2.38 & 1.81 & 0.43 & 0.34 \\
\hline NB_18 & 0.00 & 0.00 & 0.00 & 14.44 & 5.98 & 12.18 & 11.91 & 3.87 & 2.87 & 2.40 & 1.92 & 1.79 & 2.27 & 3.56 & 5.93 & 16.39 & 7.71 & 3.49 & 1.36 & 0.22 & 1.71 \\
\hline NB_19 & 0.00 & 0.00 & 0.00 & 0.00 & 5.54 & 1.58 & 0.00 & 0.66 & 0.59 & 0.87 & 3.29 & 2.95 & 5.07 & 9.38 & 14.60 & 34.08 & 13.13 & 5.08 & 1.99 & 0.34 & 0.85 \\
\hline NB_20 & 0.00 & 17.94 & 2.79 & 4.97 & 9.55 & 12.01 & 10.51 & 5.33 & 4.59 & 3.19 & 2.26 & 1.91 & 2.20 & 3.04 & 3.70 & 8.49 & 4.19 & 1.90 & 0.66 & 0.11 & 0.66 \\
\hline
\end{tabular}


NEWBEX Survey (2012) (2): Identification, Location, and Description

\begin{tabular}{|c|c|c|c|c|c|c|c|c|}
\hline Sample ID & $\begin{array}{c}\text { Original } \\
\text { Sample ID }\end{array}$ & $\begin{array}{c}\text { Latitude } \\
\text { WGS84 }\end{array}$ & $\begin{array}{c}\text { Longitude } \\
\text { WGS84 }\end{array}$ & $\begin{array}{l}\text { Reliability } \\
\text { Ranking for } \\
\text { Positioning }\end{array}$ & $\begin{array}{c}\text { Water } \\
\text { Depth } \\
\text { (m) }\end{array}$ & $\begin{array}{c}\text { Sample } \\
\text { Collected }\end{array}$ & $\begin{array}{c}\text { Sampler } \\
\text { Type }\end{array}$ & $\begin{array}{c}\text { Total Wt } \\
\text { (gm) }\end{array}$ \\
\hline NB_21 & 06_S1 & 43.055967 & -70.699415 & 1 & 12.6 & $11 / 26 / 2012$ & Shipek & 59.13 \\
\hline NB_22 & 06_S2 & 43.056038 & -70.699355 & 1 & 12.6 & $11 / 26 / 2012$ & Shipek & 74.80 \\
\hline NB_23 & 05_S1 & 43.054482 & -70.698567 & 1 & 13.2 & $11 / 26 / 2012$ & Shipek & 59.81 \\
\hline NB_24 & 05_S2 & 43.054552 & -70.698233 & 1 & 13.2 & $11 / 26 / 2012$ & Shipek & 88.40 \\
\hline NB_25 & 04_S1 & 43.052222 & -70.697197 & 1 & 13.0 & $11 / 26 / 2012$ & Shipek & 51.17 \\
\hline NB_26 & 04_S2 & 43.052003 & -70.697673 & 1 & 13.0 & $11 / 26 / 2012$ & Shipek & 80.40 \\
\hline NB_27 & 03_S1 & 43.053682 & -70.695457 & 1 & 15.0 & $11 / 26 / 2012$ & Shipek & 119.50 \\
\hline NB_28 & 03_S2 & 43.053648 & -70.695433 & 1 & 15.0 & $11 / 26 / 2012$ & Shipek & 277.59 \\
\hline NB_29 & 01_S1 & 43.050700 & -70.694400 & 1 & 14.8 & $11 / 26 / 2012$ & Shipek & 116.11 \\
\hline NB_30 & 01_S2 & 43.050600 & -70.694300 & 1 & 14.8 & $11 / 26 / 2012$ & Shipek & 97.50 \\
\hline NB_31 & A1_S1 & 43.072623 & -70.705797 & 1 & 16.8 & $7 / 3 / 2013$ & Shipek & 138.67 \\
\hline NB_32 & A1_S2 & 43.072590 & -70.705530 & 1 & 16.8 & $7 / 3 / 2013$ & Shipek & 141.29 \\
\hline NB_33 & B1_S1 & 43.068607 & -70.705162 & 1 & 15.2 & $7 / 3 / 2013$ & Shipek & 43.79 \\
\hline NB_34 & B1_S2 & 43.068588 & -70.704493 & 1 & 15.2 & $7 / 3 / 2013$ & Shipek & 46.32 \\
\hline NB_35 & C1_S1 & 43.064702 & -70.704343 & 1 & & $7 / 3 / 2013$ & Shipek & 31.74 \\
\hline NB_36 & C1_S2 & 43.064862 & -70.704485 & 1 & & $7 / 3 / 2013$ & Shipek & 32.84 \\
\hline NB_37 & D1_S1 & 43.061678 & -70.704100 & 1 & 17.7 & $7 / 3 / 2013$ & Shipek & 129.10 \\
\hline NB_38 & D1_S2 & 43.061887 & -70.704170 & 1 & 17.7 & $7 / 3 / 2013$ & Shipek & 317.99 \\
\hline NB_39 & F1_S1 & 43.057322 & -70.703735 & 1 & 16.8 & $7 / 3 / 2013$ & Shipek & 556.15 \\
\hline NB_40 & F1_S2 & 43.057767 & -70.703087 & 1 & 16.8 & $7 / 3 / 2013$ & Shipek & 448.61 \\
\hline
\end{tabular}


NEWBEX Survey (2012) (2): Sediment Classifications

\begin{tabular}{|c|c|c|c|c|c|c|c|c|c|}
\hline Sample ID & $\begin{array}{l}\text { CMECS Substrate } \\
\text { Component } \\
\text { Group (Specific) }\end{array}$ & $\begin{array}{l}\text { CMECS Substrate } \\
\text { Component } \\
\text { Subgroup (Specific) }\end{array}$ & $\begin{array}{l}\text { Textural Group } \\
\text { from \%GSM } \\
\text { (Gradistat) }\end{array}$ & $\begin{array}{l}\text { Textural Group } \\
\text { from \%SZC } \\
\text { (Gradistat) }\end{array}$ & $\begin{array}{l}\text { Sediment Name from } \\
\text { \%GSM } \\
\text { and Mode (Gradistat) }\end{array}$ & $\begin{array}{l}\text { Sediment Name from } \\
\text { \%GSM and Mode } \\
\text { (Wentworth Scale) }\end{array}$ & $\begin{array}{l}\text { Sediment } \\
\text { Classification from } \\
\text { Mean Phi (Gradistat) }\end{array}$ & $\begin{array}{l}\text { Classification } \\
\text { from Mean Phi } \\
\text { (Wentworth) }\end{array}$ & $\begin{array}{l}\text { Sorting } \\
\text { (Gradistat) }\end{array}$ \\
\hline NB_21 & Slightly Pebbly & $\begin{array}{l}\text { Slightly Pebbly Fine } \\
\text { Sand }\end{array}$ & $\begin{array}{l}\text { Slightly Gravelly } \\
\text { Sand }\end{array}$ & $\mathrm{N} / \mathrm{A}$ & $\begin{array}{l}\text { Slightly Fine Gravelly Fine } \\
\text { Sand }\end{array}$ & $\begin{array}{l}\text { Slightly Pebbly Fine } \\
\text { Sand }\end{array}$ & Fine Sand & Fine Sand & Well Sorted \\
\hline NB_22 & Slightly Pebbly & $\begin{array}{l}\text { Slightly Pebbly Fine } \\
\text { Sand }\end{array}$ & $\begin{array}{l}\text { Slightly Gravelly } \\
\text { Sand }\end{array}$ & N/A & $\begin{array}{l}\text { Slightly Fine Gravelly Fine } \\
\text { Sand }\end{array}$ & $\begin{array}{l}\text { Slightly Pebbly Fine } \\
\text { Sand }\end{array}$ & Fine Sand & Fine Sand & Well Sorted \\
\hline NB_23 & Slightly Pebbly & $\begin{array}{l}\text { Slightly Pebbly Fine } \\
\text { Sand }\end{array}$ & $\begin{array}{l}\text { Slightly Gravelly } \\
\text { Sand }\end{array}$ & $\mathrm{N} / \mathrm{A}$ & $\begin{array}{l}\text { Slightly Medium Gravelly } \\
\text { Fine Sand }\end{array}$ & $\begin{array}{l}\text { Slightly Pebbly Fine } \\
\text { Sand }\end{array}$ & Fine Sand & Fine Sand & Well Sorted \\
\hline NB_24 & Slightly Granuley & $\begin{array}{l}\text { Slightly Granuley Fine } \\
\text { Sand }\end{array}$ & $\begin{array}{l}\text { Slightly Gravelly } \\
\text { Sand }\end{array}$ & $\mathrm{N} / \mathrm{A}$ & $\begin{array}{l}\text { Slightly Very Fine Gravelly } \\
\text { Fine Sand }\end{array}$ & $\begin{array}{l}\text { Slightly Granular Fine } \\
\text { Sand }\end{array}$ & Fine Sand & Fine Sand & Well Sorted \\
\hline NB_25 & Slightly Granuley & $\begin{array}{l}\text { Slightly Granuley Fine } \\
\text { Sand }\end{array}$ & $\begin{array}{l}\text { Slightly Gravelly } \\
\text { Sand }\end{array}$ & $\mathrm{N} / \mathrm{A}$ & $\begin{array}{l}\text { Slightly Very Fine Gravelly } \\
\text { Fine Sand }\end{array}$ & $\begin{array}{l}\text { Slightly Granular Fine } \\
\text { Sand }\end{array}$ & Fine Sand & Fine Sand & Well Sorted \\
\hline NB_26 & Slightly Granuley & $\begin{array}{l}\text { Slightly Granuley Fine } \\
\text { Sand }\end{array}$ & $\begin{array}{l}\text { Slightly Gravelly } \\
\text { Sand }\end{array}$ & $\mathrm{N} / \mathrm{A}$ & $\begin{array}{l}\text { Slightly Very Fine Gravelly } \\
\text { Fine Sand }\end{array}$ & $\begin{array}{l}\text { Slightly Granular Fine } \\
\text { Sand }\end{array}$ & Very Fine Sand & Very Fine Sand & Well Sorted \\
\hline NB_27 & Pebble Mixes & Sandy Pebble Gravel & Sandy Gravel & N/A & Sandy Coarse Gravel & Sandy Pebbly Gravel & Fine Gravel & Pebble Gravel & $\begin{array}{l}\text { Very Poorly } \\
\text { Sorted }\end{array}$ \\
\hline NB_28 & Gravel & Pebble Gravel & Gravel & $\mathrm{N} / \mathrm{A}$ & Coarse Gravel & Pebbly Gravel & Medium Gravel & Pebble Gravel & Poorly Sorted \\
\hline NB_29 & Pebbly & Pebbly Fine Sand & Gravelly Sand & $\mathrm{N} / \mathrm{A}$ & $\begin{array}{l}\text { Medium Gravelly Fine } \\
\text { Sand }\end{array}$ & Pebbly Fine Sand & Coarse Sand & Coarse Sand & $\begin{array}{l}\text { Very Poorly } \\
\text { Sorted }\end{array}$ \\
\hline NB_30 & Pebbly & Pebbly Fine Sand & Gravelly Sand & N/A & $\begin{array}{l}\text { Medium Gravelly Fine } \\
\text { Sand }\end{array}$ & Pebbly Fine Sand & Coarse Sand & Coarse Sand & $\begin{array}{l}\text { Very Poorly } \\
\text { Sorted }\end{array}$ \\
\hline NB_31 & Pebble Mixes & Sandy Pebble Gravel & Sandy Gravel & N/A & Sandy Very Coarse Gravel & Sandy Pebble Gravel & Medium Gravel & Pebble Gravel & $\begin{array}{l}\text { Very Poorly } \\
\text { Sorted }\end{array}$ \\
\hline NB_32 & Pebble Mixes & Sandy Pebble Gravel & Sandy Gravel & $\mathrm{N} / \mathrm{A}$ & Sandy Coarse Gravel & Sandy Pebble Gravel & Fine Gravel & Pebble Gravel & $\begin{array}{l}\text { Very Poorly } \\
\text { Sorted }\end{array}$ \\
\hline NB_33 & Granuley & $\begin{array}{l}\text { Granuley Medium } \\
\text { Sand }\end{array}$ & Gravelly Sand & N/A & $\begin{array}{l}\text { Very Fine Gravelly Medium } \\
\text { Sand }\end{array}$ & $\begin{array}{l}\text { Granular Medium } \\
\text { Sand }\end{array}$ & Medium Sand & Medium Sand & $\begin{array}{l}\text { Moderately } \\
\text { Sorted }\end{array}$ \\
\hline NB_34 & Slightly Granuley & $\begin{array}{l}\text { Slightly Granuley } \\
\text { Medium Sand }\end{array}$ & $\begin{array}{l}\text { Slightly Gravelly } \\
\text { Sand }\end{array}$ & $\mathrm{N} / \mathrm{A}$ & $\begin{array}{l}\text { Slightly Very Fine Gravelly } \\
\text { Medium Sand }\end{array}$ & $\begin{array}{l}\text { Slightly Granular } \\
\text { Medium Sand }\end{array}$ & Medium Sand & Medium Sand & $\begin{array}{l}\text { Moderately } \\
\text { Well Sorted }\end{array}$ \\
\hline NB_35 & Granuley & $\begin{array}{l}\text { Granuley Medium } \\
\text { Sand }\end{array}$ & Gravelly Sand & $\mathrm{N} / \mathrm{A}$ & $\begin{array}{l}\text { Very Fine Gravelly Medium } \\
\text { Sand }\end{array}$ & $\begin{array}{l}\text { Granular Medium } \\
\text { Sand }\end{array}$ & Coarse Sand & Coarse Sand & Poorly Sorted \\
\hline NB_36 & Granuley & $\begin{array}{l}\text { Granuley Medium } \\
\text { Sand }\end{array}$ & Gravelly Sand & $\mathrm{N} / \mathrm{A}$ & $\begin{array}{l}\text { Very Fine Gravelly Medium } \\
\text { Sand }\end{array}$ & $\begin{array}{l}\text { Granular Medium } \\
\text { Sand }\end{array}$ & Medium Sand & Medium Sand & $\begin{array}{l}\text { Moderately } \\
\text { Sorted }\end{array}$ \\
\hline NB_37 & Pebble Mixes & Sandy Pebble Gravel & Sandy Gravel & $\mathrm{N} / \mathrm{A}$ & Sandy Medium Gravel & Sandy Pebble Gravel & Fine Gravel & Pebble Gravel & $\begin{array}{l}\text { Very Poorly } \\
\text { Sorted }\end{array}$ \\
\hline NB_38 & Pebble Mixes & Sandy Pebble Gravel & Sandy Gravel & N/A & Sandy Medium Gravel & Sandy Pebble Gravel & Fine Gravel & Pebble Gravel & $\begin{array}{l}\text { Very Poorly } \\
\text { Sorted }\end{array}$ \\
\hline NB_39 & Pebble Mixes & Sandy Pebble Gravel & Sandy Gravel & N/A & Sandy Medium Gravel & Sandy Pebble Gravel & Fine Gravel & Pebble Gravel & $\begin{array}{l}\text { Very Poorly } \\
\text { Sorted }\end{array}$ \\
\hline NB_40 & Gravel & Pebble Gravel & Gravel & N/A & Very Coarse Gravel & Pebble Gravel & Very Coarse Gravel & Pebble Gravel & $\begin{array}{l}\text { Moderately } \\
\text { Sorted }\end{array}$ \\
\hline
\end{tabular}




\section{NEWBEX Survey (2012) (2): Grain Size Statistics}

\begin{tabular}{|c|c|c|c|c|c|c|c|c|c|c|c|c|c|c|c|c|c|c|c|}
\hline Sample ID & $\begin{array}{c}\text { Gravel } \\
\% \\
\end{array}$ & $\begin{array}{c}\text { Sand } \\
\% \\
\end{array}$ & $\begin{array}{c}\text { Mud } \\
\% \\
\end{array}$ & $\begin{array}{c}\text { Sand } \\
\%\end{array}$ & $\begin{array}{c}\text { Silt } \\
\% \\
\end{array}$ & $\begin{array}{c}\text { Clay } \\
\% \\
\end{array}$ & Modes & $\begin{array}{c}\text { Mode } 1 \\
\text { (phi) }\end{array}$ & $\begin{array}{c}\text { Mode } 2 \\
\text { (phi) }\end{array}$ & $\begin{array}{l}D_{10} \\
\text { (phi) }\end{array}$ & $\begin{array}{c}\mathrm{D}_{10} \\
(\mathrm{~mm})\end{array}$ & $\begin{array}{r}D_{50} \\
\text { (phi) }\end{array}$ & $\begin{array}{c}\mathrm{D}_{50} \\
(\mathrm{~mm})\end{array}$ & $\begin{array}{c}\text { Mean } \\
\text { Size } \\
\text { (phi) }\end{array}$ & $\begin{array}{c}\text { Mean } \\
\text { Size } \\
(\mathrm{mm})\end{array}$ & $\begin{array}{c}\text { Sorting } \\
\text { (phi) }\end{array}$ & Skewness & Kurtosis & LOI \% \\
\hline NB_21 & 1.9 & 96.9 & 1.2 & & & & U & 2.74 & & 2.40 & 0.19 & 2.89 & 0.14 & 2.91 & 0.13 & 0.41 & -0.05 & 1.02 & 0.62 \\
\hline NB_22 & 0.6 & 98.7 & 0.7 & & & & U & 3.24 & & 2.50 & 0.18 & 2.97 & 0.13 & 2.96 & 0.13 & 0.38 & -0.14 & 0.90 & 0.64 \\
\hline NB_23 & 1.3 & 96.4 & 2.2 & & & & $U$ & 2.74 & & 2.15 & 0.23 & 2.88 & 0.14 & 2.90 & 0.13 & 0.48 & -0.13 & 1.19 & 0.57 \\
\hline NB_24 & 0.6 & 98.5 & 0.9 & & & & $U$ & 3.24 & & 2.11 & 0.23 & 3.03 & 0.12 & 2.96 & 0.13 & 0.49 & -0.39 & 1.15 & 0.62 \\
\hline NB_25 & 0.5 & 97.9 & 1.5 & & & & U & 3.24 & & 2.33 & 0.20 & 2.96 & 0.13 & 2.95 & 0.13 & 0.47 & -0.17 & 1.18 & 0.64 \\
\hline NB_26 & 0.5 & 98.3 & 1.3 & & & & U & 3.24 & & 2.51 & 0.18 & 3.08 & 0.12 & 3.02 & 0.12 & 0.39 & -0.31 & 0.95 & 0.62 \\
\hline NB_27 & 75.8 & 23.6 & 0.7 & & & & U & -4.24 & & -4.37 & 20.71 & -3.46 & 10.97 & -2.11 & 4.32 & 2.60 & 0.73 & 1.04 & 0.92 \\
\hline NB_28 & 90.3 & 9.4 & 0.3 & & & & U & -5.24 & & -5.28 & 38.83 & -4.16 & 17.81 & -3.92 & 15.18 & 1.76 & 0.46 & 1.59 & \\
\hline NB_29 & 29.6 & 69.5 & 0.9 & & & & B & 3.24 & 2.24 & -3.63 & 12.38 & 2.02 & 0.25 & 0.63 & 0.65 & 2.72 & -0.64 & 0.61 & 0.61 \\
\hline NB_30 & 24.6 & 74.2 & 1.2 & & & & $\mathrm{~B}$ & 3.24 & 2.24 & -3.32 & 9.97 & 2.16 & 0.22 & 0.79 & 0.58 & 2.63 & -0.65 & 0.74 & 0.65 \\
\hline NB_31 & 77.7 & 22.2 & 0.1 & & & & $\mathrm{~B}$ & -5.24 & -4.24 & -5.35 & 40.79 & -4.13 & 17.45 & -3.02 & 8.09 & 2.46 & 0.61 & 0.82 & 0.90 \\
\hline NB_32 & 75.2 & 24.5 & 0.3 & & & & B & -4.24 & 1.75 & -4.33 & 20.11 & -3.55 & 11.67 & -2.39 & 5.23 & 2.14 & 0.72 & 0.82 & \\
\hline NB_33 & 5.6 & 93.8 & 0.5 & & & & U & 1.75 & & -0.52 & 1.44 & 1.28 & 0.41 & 1.05 & 0.48 & 0.99 & -0.38 & 1.12 & 1.60 \\
\hline NB_34 & 1.8 & 97.9 & 0.3 & & & & $U$ & 1.25 & & 0.25 & 0.84 & 1.23 & 0.43 & 1.19 & 0.44 & 0.64 & -0.22 & 1.15 & 1.10 \\
\hline NB_35 & 17.5 & 81.9 & 0.6 & & & & B & 1.75 & -0.74 & -1.38 & 2.61 & 0.44 & 0.74 & 0.39 & 0.77 & 1.34 & -0.09 & 0.74 & 2.70 \\
\hline NB_36 & 5.1 & 94.3 & 0.6 & & & & $\mathrm{U}$ & 1.75 & & -0.45 & 1.37 & 1.52 & 0.35 & 1.17 & 0.45 & 0.99 & -0.54 & 1.16 & 1.50 \\
\hline NB_37 & 74.3 & 24.2 & 1.4 & & & & B & -3.74 & 1.75 & -4.33 & 20.08 & -3.71 & 13.10 & -2.03 & 4.08 & 2.63 & 0.82 & 0.80 & 2.60 \\
\hline NB_38 & 72.1 & 27.0 & 1.0 & & & & $\mathrm{~B}$ & -3.74 & 1.75 & -4.58 & 23.97 & -3.36 & 10.30 & -2.02 & 4.07 & 2.60 & 0.64 & 0.71 & \\
\hline NB_40 & 97.6 & 2.2 & 0.2 & & & & U & -5.74 & & -5.88 & 58.89 & -5.49 & 44.85 & -5.06 & 33.36 & 0.94 & 0.68 & 1.00 & \\
\hline
\end{tabular}




\section{NEWBEX Survey (2012) (2): Grain Size Distribution}

\begin{tabular}{|c|c|c|c|c|c|c|c|c|c|c|c|c|c|c|c|c|c|c|c|c|c|}
\hline & $\begin{array}{c}\text { Class } \\
\% \\
\text { phi } \\
-5.5\end{array}$ & $\begin{array}{c}\text { Class } \\
\% \\
\text { phi } \\
-5.0\end{array}$ & $\begin{array}{c}\text { Class } \\
\% \\
\text { phi } \\
-4.5\end{array}$ & $\begin{array}{c}\text { Class } \\
\% \\
\text { phi } \\
-4.0\end{array}$ & $\begin{array}{c}\text { Class } \\
\% \\
\text { phi } \\
-3.5\end{array}$ & $\begin{array}{c}\text { Class } \\
\% \\
\text { phi } \\
-3.0\end{array}$ & $\begin{array}{c}\text { Class } \\
\% \\
\text { phi } \\
-2.5\end{array}$ & $\begin{array}{c}\text { Class } \\
\% \\
\text { phi } \\
-2.0\end{array}$ & $\begin{array}{c}\text { Class } \\
\% \\
\text { phi } \\
-1.5\end{array}$ & $\begin{array}{c}\text { Class } \\
\% \\
\text { phi } \\
-1.0\end{array}$ & $\begin{array}{c}\text { Class } \\
\% \\
\text { phi } \\
-0.5\end{array}$ & $\begin{array}{c}\text { Class } \\
\% \\
\text { phi } \\
0.0\end{array}$ & $\begin{array}{c}\text { Class } \\
\% \\
\text { phi } \\
0.5\end{array}$ & $\begin{array}{c}\text { Class } \\
\% \\
\text { phi } \\
1.0\end{array}$ & $\begin{array}{c}\text { Class } \\
\% \\
\text { phi } \\
1.5\end{array}$ & $\begin{array}{c}\text { Class } \\
\% \\
\text { phi } \\
2.0\end{array}$ & $\begin{array}{c}\text { Class } \\
\% \\
\text { phi } \\
2.5\end{array}$ & $\begin{array}{c}\text { Class } \\
\% \\
\text { phi } \\
3.0\end{array}$ & $\begin{array}{c}\text { Class } \\
\% \\
\text { phi } \\
3.5\end{array}$ & $\begin{array}{c}\text { Class } \\
\% \\
\text { phi } \\
4.0\end{array}$ & $\begin{array}{c}\text { Class } \\
\% \\
\text { phi } \\
>4.0\end{array}$ \\
\hline Sample ID & -5.5 & & -4.5 & -4.0 & & -3.0 & & -2.0 & -1.5 & -1.0 & -0.5 & 0.0 & & 1.0 & 1.5 & 2.0 & 2.5 & 3.0 & & & $>4.0$ \\
\hline NB_21 & 0.00 & 0.00 & 0.00 & 0.00 & 0.00 & 0.00 & 0.00 & 1.72 & 0.12 & 0.09 & 0.03 & 0.04 & 0.06 & 0.09 & 0.29 & 2.02 & 6.64 & 49.83 & 34.67 & 3.18 & 1.23 \\
\hline NB_22 & 0.00 & 0.00 & 0.00 & 0.00 & 0.00 & 0.00 & 0.00 & 0.32 & 0.13 & 0.14 & 0.09 & 0.07 & 0.05 & 0.07 & 0.20 & 1.44 & 5.61 & 44.50 & 44.67 & 1.99 & 0.72 \\
\hline NB_23 & 0.00 & 0.00 & 0.00 & 0.00 & 0.00 & 0.68 & 0.24 & 0.06 & 0.19 & 0.15 & 0.07 & 0.15 & 0.17 & 0.40 & 0.95 & 4.60 & 7.29 & 45.61 & 34.53 & 2.64 & 2.26 \\
\hline NB_24 & 0.00 & 0.00 & 0.00 & 0.00 & 0.00 & 0.00 & 0.00 & 0.14 & 0.18 & 0.29 & 0.25 & 0.20 & 0.36 & 0.69 & 1.28 & 4.92 & 7.55 & 31.04 & 49.05 & 3.18 & 0.87 \\
\hline NB_25 & 0.00 & 0.00 & 0.00 & 0.00 & 0.00 & 0.00 & 0.00 & 0.00 & 0.31 & 0.23 & 0.17 & 0.13 & 1.25 & 0.30 & 0.72 & 2.84 & 5.81 & 41.07 & 41.19 & 4.45 & 1.52 \\
\hline NB_26 & 0.00 & 0.00 & 0.00 & 0.00 & 0.00 & 0.00 & 0.00 & 0.40 & 0.00 & 0.06 & 0.06 & 0.10 & 0.07 & 0.15 & 0.41 & 1.72 & 4.88 & 32.61 & 54.85 & 3.42 & 1.28 \\
\hline NB_27 & 0.00 & 0.00 & 0.00 & 42.98 & 6.21 & 13.18 & 3.65 & 4.98 & 2.68 & 2.11 & 1.69 & 1.58 & 1.53 & 1.81 & 1.83 & 2.88 & 1.96 & 2.43 & 6.58 & 1.27 & 0.66 \\
\hline NB_28 & 0.00 & 24.14 & 16.91 & 13.18 & 15.53 & 6.78 & 7.37 & 2.66 & 2.07 & 1.62 & 1.22 & 1.00 & 0.76 & 0.67 & 0.61 & 0.88 & 0.52 & 1.23 & 2.00 & 0.54 & 0.32 \\
\hline NB_29 & 0.00 & 0.00 & 0.00 & 4.91 & 7.08 & 8.56 & 3.15 & 2.11 & 2.24 & 1.56 & 1.03 & 0.97 & 1.42 & 2.60 & 3.54 & 10.33 & 15.30 & 15.25 & 16.15 & 2.91 & 0.90 \\
\hline NB_30 & 0.00 & 0.00 & 0.00 & 0.00 & 6.65 & 9.70 & 1.67 & 1.99 & 2.71 & 1.93 & 1.55 & 1.34 & 1.77 & 3.31 & 3.65 & 8.74 & 14.60 & 12.95 & 23.29 & 2.95 & 1.20 \\
\hline NB_31 & 0.00 & 36.16 & 0.00 & 18.66 & 4.31 & 4.88 & 5.42 & 3.96 & 2.21 & 2.08 & 2.22 & 2.22 & 2.72 & 4.03 & 5.33 & 4.59 & 0.72 & 0.20 & 0.09 & 0.05 & 0.14 \\
\hline NB_32 & 0.00 & 0.00 & 0.00 & 31.14 & 21.31 & 8.11 & 6.15 & 3.64 & 2.39 & 2.44 & 2.62 & 2.36 & 2.84 & 4.17 & 5.36 & 5.63 & 1.04 & 0.29 & 0.14 & 0.08 & 0.30 \\
\hline NB_33 & 0.00 & 0.00 & 0.00 & 0.00 & 0.00 & 0.00 & 0.80 & 0.67 & 1.01 & 3.17 & 4.70 & 5.78 & 8.35 & 12.97 & 22.49 & 30.31 & 8.21 & 0.75 & 0.18 & 0.09 & 0.53 \\
\hline NB_34 & 0.00 & 0.00 & 0.00 & 0.00 & 0.00 & 0.00 & 0.00 & 0.90 & 0.16 & 0.73 & 1.58 & 3.14 & 6.88 & 19.23 & 37.54 & 26.00 & 3.13 & 0.28 & 0.06 & 0.04 & 0.32 \\
\hline NB_35 & 0.00 & 0.00 & 0.00 & 0.00 & 0.00 & 0.94 & 1.30 & 1.61 & 4.13 & 9.55 & 12.47 & 11.53 & 9.60 & 9.47 & 11.11 & 20.28 & 5.87 & 0.93 & 0.40 & 0.20 & 0.61 \\
\hline NB_36 & 0.00 & 0.00 & 0.00 & 0.00 & 0.00 & 0.00 & 0.00 & 1.23 & 1.29 & 2.62 & 4.46 & 5.93 & 6.57 & 9.39 & 16.60 & 40.03 & 10.10 & 0.87 & 0.23 & 0.11 & 0.56 \\
\hline NB_37 & 0.00 & 0.00 & 0.00 & 30.75 & 34.29 & 4.23 & 2.04 & 1.35 & 0.68 & 1.01 & 0.53 & 0.44 & 0.49 & 0.73 & 1.91 & 7.69 & 6.07 & 3.54 & 2.17 & 0.63 & 1.46 \\
\hline NB_38 & 0.00 & 0.00 & 12.47 & 13.11 & 22.06 & 9.44 & 7.27 & 3.65 & 2.21 & 1.85 & 1.26 & 1.19 & 1.29 & 1.98 & 3.61 & 8.56 & 5.22 & 2.27 & 1.20 & 0.37 & 0.99 \\
\hline NB_39 & 0.00 & 22.85 & 0.00 & 21.62 & 22.43 & 8.58 & 2.13 & 1.07 & 0.48 & 0.29 & 0.29 & 0.38 & 0.51 & 0.80 & 1.37 & 5.46 & 5.03 & 3.76 & 2.03 & 0.46 & 0.45 \\
\hline NB_40 & 49.90 & 9.38 & 16.18 & 6.64 & 7.57 & 5.02 & 1.85 & 0.77 & 0.22 & 0.09 & 0.09 & 0.09 & 0.08 & 0.09 & 0.15 & 0.45 & 0.35 & 0.48 & 0.31 & 0.08 & 0.22 \\
\hline
\end{tabular}


NEWBEX Survey (2012) (3): Identification, Location, and Description

\begin{tabular}{|c|c|c|c|c|c|c|c|c|}
\hline Sample ID & $\begin{array}{c}\text { Original } \\
\text { Sample ID }\end{array}$ & $\begin{array}{c}\text { Latitude } \\
\text { WGS84 }\end{array}$ & $\begin{array}{c}\text { Longitude } \\
\text { WGS84 }\end{array}$ & $\begin{array}{l}\text { Reliability } \\
\text { Ranking for } \\
\text { Positioning }\end{array}$ & $\begin{array}{c}\text { Water } \\
\text { Depth } \\
\text { (m) }\end{array}$ & $\begin{array}{c}\text { Sample } \\
\text { Collected }\end{array}$ & $\begin{array}{c}\text { Sampler } \\
\text { Type }\end{array}$ & $\begin{array}{c}\text { Total Wt } \\
\text { (gm) }\end{array}$ \\
\hline NB_41 & G1_S1 & 43.055922 & -70.701318 & 1 & 13.7 & $7 / 3 / 2013$ & Shipek & 179.28 \\
\hline NB_42 & G1_S2 & 43.055930 & -70.701290 & 1 & 13.7 & $7 / 3 / 2013$ & Shipek & 240.13 \\
\hline NB_43 & H1_S1 & 43.054240 & -70.698332 & 1 & 12.8 & $7 / 3 / 2013$ & Shipek & 45.20 \\
\hline NB_44 & H1_S2 & 43.053720 & -70.698718 & 1 & 12.8 & $7 / 3 / 2013$ & Shipek & 50.20 \\
\hline NB_45 & I1_S1 & 43.052695 & -70.696708 & 1 & 12.2 & $7 / 3 / 2013$ & Shipek & 36.07 \\
\hline NB_46 & I1_S2 & 43.052448 & -70.696595 & 1 & 12.2 & $7 / 3 / 2013$ & Shipek & 41.31 \\
\hline NB_47 & J1_S1 & 43.051265 & -70.693852 & 1 & 12.8 & $7 / 3 / 2013$ & Shipek & 157.31 \\
\hline NB_48 & A1_S1 & 43.072758 & -70.705955 & 1 & 15.1 & $10 / 21 / 2013$ & Shipek & 1047.50 \\
\hline NB_49 & A1_S2 & 43.072748 & -70.705960 & 1 & 15.1 & $10 / 21 / 2013$ & Shipek & 812.85 \\
\hline NB_50 & B1_S1 & 43.069007 & -70.705178 & 1 & 13.5 & $10 / 21 / 2013$ & Shipek & 69.40 \\
\hline NB_51 & B1_S2 & 43.068913 & -70.705202 & 1 & 13.5 & $10 / 21 / 2013$ & Shipek & 76.50 \\
\hline NB_52 & C1_S1 & 43.064830 & -70.704493 & 1 & 15.6 & $10 / 21 / 2013$ & Shipek & 68.57 \\
\hline NB_53 & C1_S2 & 43.064998 & -70.704240 & 1 & 15.6 & $10 / 21 / 2013$ & Shipek & 75.30 \\
\hline NB_54 & D1_S1 & 43.061995 & -70.703853 & 1 & 20.4 & $10 / 21 / 2013$ & Shipek & 509.58 \\
\hline NB_55 & D1_S2 & 43.061993 & -70.703935 & 1 & 20.4 & $10 / 21 / 2013$ & Shipek & 429.26 \\
\hline NB_56 & E1_S1 & 43.059927 & -70.703615 & 1 & 19.7 & $10 / 21 / 2013$ & Shipek & 76.90 \\
\hline NB_57 & E1_S2 & 43.060033 & -70.703532 & 1 & 19.7 & $10 / 21 / 2013$ & Shipek & 462.60 \\
\hline NB_58 & F1_S1 & 43.057682 & -70.703418 & 1 & 16.9 & $10 / 21 / 2013$ & Shipek & 384.60 \\
\hline NB_59 & F1_S2 & 43.057737 & -70.703315 & 1 & 16.9 & $10 / 21 / 2013$ & Shipek & 727.30 \\
\hline NB_60 & G1_S1 & 43.056003 & -70.701455 & 1 & 15.1 & $10 / 21 / 2013$ & Shipek & 229.40 \\
\hline
\end{tabular}


NEWBEX Survey (2012) (3): Sediment Classifications

\begin{tabular}{|c|c|c|c|c|c|c|c|c|c|}
\hline Sample ID & $\begin{array}{l}\text { CMECS Substrate } \\
\text { Component } \\
\text { Group (Specific) } \\
\end{array}$ & $\begin{array}{l}\text { CMECS Substrate } \\
\text { Component } \\
\text { Subgroup (Specific) }\end{array}$ & $\begin{array}{l}\text { Textural Group } \\
\text { from \%GSM } \\
\text { (Gradistat) }\end{array}$ & $\begin{array}{l}\text { Textural Group } \\
\text { from \%SZC } \\
\text { (Gradistat) } \\
\end{array}$ & $\begin{array}{l}\text { Sediment Name from } \\
\% G S M \\
\text { and Mode (Gradistat) }\end{array}$ & $\begin{array}{l}\text { Sediment Name from } \\
\text { \%GSM and Mode } \\
\text { (Wentworth Scale) }\end{array}$ & $\begin{array}{l}\text { Sediment } \\
\text { Classification from } \\
\text { Mean Phi (Gradistat) }\end{array}$ & $\begin{array}{l}\text { Classification } \\
\text { from Mean Phi } \\
\text { (Wentworth) }\end{array}$ & $\begin{array}{l}\text { Sorting } \\
\text { (Gradistat) }\end{array}$ \\
\hline NB_41 & Pebble Mixes & Sandy Pebble Gravel & Sandy Gravel & $\mathrm{N} / \mathrm{A}$ & Sandy Coarse Gravel & Sandy Pebble Gravel & Fine Gravel & Pebble Gravel & $\begin{array}{l}\text { Very Poorly } \\
\text { Sorted }\end{array}$ \\
\hline NB_42 & Pebble Mixes & Sandy Pebble Gravel & Sandy Gravel & $\mathrm{N} / \mathrm{A}$ & Sandy Coarse Gravel & Sandy Pebble Gravel & Fine Gravel & Pebble Gravel & $\begin{array}{l}\text { Very Poorly } \\
\text { Sorted }\end{array}$ \\
\hline NB_43 & Slightly Pebbly & $\begin{array}{l}\text { Slightly Pebbly Fine } \\
\text { Sand }\end{array}$ & $\begin{array}{l}\text { Slightly Gravelly } \\
\text { Sand }\end{array}$ & $\mathrm{N} / \mathrm{A}$ & $\begin{array}{l}\text { Slightly Fine Gravelly Fine } \\
\text { Sand }\end{array}$ & $\begin{array}{l}\text { Slightly Pebbly Fine } \\
\text { Sand }\end{array}$ & Fine Sand & Fine Sand & $\begin{array}{l}\text { Moderately } \\
\text { Well Sorted }\end{array}$ \\
\hline NB_44 & Slightly Pebbly & $\begin{array}{l}\text { Slightly Pebbly Fine } \\
\text { Sand }\end{array}$ & $\begin{array}{l}\text { Slightly Gravelly } \\
\text { Sand }\end{array}$ & $\mathrm{N} / \mathrm{A}$ & $\begin{array}{l}\text { Slightly Medium Gravelly } \\
\text { Fine Sand }\end{array}$ & $\begin{array}{l}\text { Slightly Pebbly Fine } \\
\text { Sand }\end{array}$ & Fine Sand & Fine Sand & Well Sorted \\
\hline NB_45 & Slightly Pebbly & $\begin{array}{l}\text { Slightly Pebbly Very } \\
\text { Fine Sand }\end{array}$ & $\begin{array}{l}\text { Slightly Gravelly } \\
\text { Sand }\end{array}$ & $\mathrm{N} / \mathrm{A}$ & $\begin{array}{l}\text { Slightly Fine Gravelly Very } \\
\text { Fine Sand }\end{array}$ & $\begin{array}{l}\text { Slightly Pebbly Very } \\
\text { Fine Sand }\end{array}$ & Fine Sand & Fine Sand & $\begin{array}{l}\text { Moderately } \\
\text { Well Sorted }\end{array}$ \\
\hline NB_46 & Slightly Granuley & $\begin{array}{l}\text { Slightly Granuley Very } \\
\text { Fine Sand }\end{array}$ & $\begin{array}{l}\text { Slightly Gravelly } \\
\text { Sand }\end{array}$ & N/A & $\begin{array}{l}\text { Slightly Very Fine Gravelly } \\
\text { Very Fine Sand }\end{array}$ & $\begin{array}{l}\text { Slightly Granular Very } \\
\text { Fine Sand }\end{array}$ & Fine Sand & Fine Sand & $\begin{array}{l}\text { Moderately } \\
\text { Well Sorted }\end{array}$ \\
\hline NB_47 & Gravel & Pebble Gravel & Gravel & $\mathrm{N} / \mathrm{A}$ & Coarse Gravel & Pebble Gravel & Medium Gravel & Pebble Gravel & Poorly Sorted \\
\hline NB_48 & Pebble Mixes & Sandy Pebble Gravel & Sandy Gravel & $\mathrm{N} / \mathrm{A}$ & Sandy Coarse Gravel & Sandy Pebble Gravel & Fine Gravel & Pebble Gravel & $\begin{array}{l}\text { Very Poorly } \\
\text { Sorted }\end{array}$ \\
\hline NB_49 & Pebble Mixes & Sandy Pebble Gravel & Sandy Gravel & N/A & Sandy Coarse Gravel & Sandy Pebble Gravel & Very Fine Gravel & Granule Gravel & $\begin{array}{l}\text { Very Poorly } \\
\text { Sorted }\end{array}$ \\
\hline NB_50 & Granuley & $\begin{array}{l}\text { Granuley Medium } \\
\text { Sand }\end{array}$ & Gravelly Sand & N/A & $\begin{array}{l}\text { Very Fine Gravelly Medium } \\
\text { Sand }\end{array}$ & $\begin{array}{l}\text { Granular Medium } \\
\text { Sand }\end{array}$ & Coarse Sand & Coarse Sand & Poorly Sorted \\
\hline NB_51 & Granuley & $\begin{array}{l}\text { Granuley Medium } \\
\text { Sand }\end{array}$ & Gravelly Sand & $\mathrm{N} / \mathrm{A}$ & $\begin{array}{l}\text { Very Fine Gravelly Medium } \\
\text { Sand }\end{array}$ & $\begin{array}{l}\text { Granular Medium } \\
\text { Sand }\end{array}$ & Coarse Sand & Coarse Sand & Poorly Sorted \\
\hline NB_52 & Granuley & $\begin{array}{l}\text { Granuley Medium } \\
\text { Sand }\end{array}$ & Gravelly Sand & $\mathrm{N} / \mathrm{A}$ & $\begin{array}{l}\text { Very Fine Gravelly Medium } \\
\text { Sand }\end{array}$ & $\begin{array}{l}\text { Granular Medium } \\
\text { Sand }\end{array}$ & Coarse Sand & Coarse Sand & $\begin{array}{l}\text { Moderately } \\
\text { Sorted }\end{array}$ \\
\hline NB_53 & Granuley & $\begin{array}{l}\text { Granuley Medium } \\
\text { Sand }\end{array}$ & Gravelly Sand & $\mathrm{N} / \mathrm{A}$ & $\begin{array}{l}\text { Very Fine Gravelly Medium } \\
\text { Sand }\end{array}$ & $\begin{array}{l}\text { Granular Medium } \\
\text { Sand }\end{array}$ & Coarse Sand & Coarse Sand & Poorly Sorted \\
\hline NB_54 & Gravel & Pebble Gravel & Gravel & N/A & Very Coarse Gravel & Pebble Gravel & Coarse Gravel & Pebble Gravel & Poorly Sorted \\
\hline NB_55 & Pebble Mixes & Sandy Pebble Gravel & Sandy Gravel & $\mathrm{N} / \mathrm{A}$ & Sandy Coarse Gravel & Sandy Pebble Gravel & Fine Gravel & Pebble Gravel & $\begin{array}{l}\text { Very Poorly } \\
\text { Sorted }\end{array}$ \\
\hline NB_56 & Pebble Mixes & Sandy Pebble Gravel & Sandy Gravel & N/A & Sandy Coarse Gravel & Sandy Pebble Gravel & Fine Gravel & Pebble Gravel & $\begin{array}{l}\text { Very Poorly } \\
\text { Sorted }\end{array}$ \\
\hline NB_57 & Gravel & Pebble Gravel & Gravel & $\mathrm{N} / \mathrm{A}$ & Very Coarse Gravel & Pebble Gravel & Coarse Gravel & Pebble Gravel & $\begin{array}{l}\text { Moderately } \\
\text { Well Sorted }\end{array}$ \\
\hline NB_58 & Gravel & Pebble Gravel & Gravel & N/A & Coarse Gravel & Pebble Gravel & Medium Gravel & Pebble Gravel & Poorly Sorted \\
\hline NB_59 & Pebble Mixes & Sandy Pebble Gravel & Sandy Gravel & N/A & Sandy Medium Gravel & Sandy Pebble Gravel & Fine Gravel & Pebble Gravel & $\begin{array}{l}\text { Very Poorly } \\
\text { Sorted }\end{array}$ \\
\hline NB_60 & Pebbly & Pebbly Fine Sand & Gravelly Sand & N/A & $\begin{array}{l}\text { Medium Gravelly Fine } \\
\text { Sand }\end{array}$ & Pebbly Fine Sand & Medium Sand & Medium Sand & Poorly Sorted \\
\hline
\end{tabular}




\section{NEWBEX Survey (2012) (3): Grain Size Statistics}

\begin{tabular}{|c|c|c|c|c|c|c|c|c|c|c|c|c|c|c|c|c|c|c|c|}
\hline Sample ID & $\begin{array}{c}\text { Gravel } \\
\%\end{array}$ & $\begin{array}{c}\text { Sand } \\
\%\end{array}$ & $\begin{array}{c}\text { Mud } \\
\%\end{array}$ & $\begin{array}{c}\text { Sand } \\
\% \\
\end{array}$ & $\begin{array}{l}\text { Silt } \\
\%\end{array}$ & $\begin{array}{c}\text { Clay } \\
\% \\
\end{array}$ & Modes & $\begin{array}{c}\text { Mode } 1 \\
\text { (phi) }\end{array}$ & $\begin{array}{c}\text { Mode } 2 \\
\text { (phi) }\end{array}$ & $\begin{array}{l}D_{10} \\
\text { (phi) }\end{array}$ & $\begin{array}{c}D_{10} \\
(\mathrm{~mm})\end{array}$ & $\begin{array}{l}D_{50} \\
\text { (phi) }\end{array}$ & $\begin{array}{l}D_{50} \\
(\mathrm{~mm})\end{array}$ & $\begin{array}{c}\text { Mean } \\
\text { Size } \\
\text { (phi) }\end{array}$ & $\begin{array}{l}\text { Mean } \\
\text { Size } \\
(\mathrm{mm})\end{array}$ & $\begin{array}{c}\text { Sorting } \\
\text { (phi) }\end{array}$ & Skewness & Kurtosis & LOI \% \\
\hline NB_41 & 77.9 & 21.5 & 0.6 & & & & $\mathrm{~B}$ & -4.24 & 2.24 & -4.72 & 26.28 & -4.06 & 16.68 & -2.23 & 4.68 & 2.77 & 0.82 & 2.51 & \\
\hline NB_42 & 66.4 & 32.9 & 0.7 & & & & $\mathrm{~B}$ & -4.73 & 2.24 & -5.21 & 37.12 & -4.38 & 20.88 & -2.41 & 5.30 & 3.06 & 0.79 & 0.51 & \\
\hline NB_43 & 1.4 & 97.7 & 0.9 & & & & U & 2.74 & & 1.84 & 0.28 & 2.86 & 0.14 & 2.80 & 0.14 & 0.58 & -0.29 & 1.27 & 0.60 \\
\hline NB_44 & 1.0 & 97.8 & 1.2 & & & & U & 2.74 & & 2.11 & 0.23 & 2.89 & 0.14 & 2.89 & 0.13 & 0.48 & -0.16 & 1.16 & 0.60 \\
\hline NB_45 & 2.6 & 95.8 & 1.7 & & & & U & 3.24 & & 1.90 & 0.27 & 2.97 & 0.13 & 2.92 & 0.13 & 0.64 & -0.32 & 1.59 & 0.70 \\
\hline NB_46 & 1.6 & 97.1 & 1.3 & & & & U & 3.24 & & 2.07 & 0.24 & 2.99 & 0.13 & 2.95 & 0.13 & 0.56 & -0.26 & 1.39 & 0.60 \\
\hline NB_47 & 89.7 & 10.1 & 0.3 & & & & $\mathrm{U}$ & -4.73 & & -4.87 & 29.28 & -3.92 & 15.15 & -3.65 & 12.53 & 1.58 & 0.49 & 1.61 & \\
\hline NB_48 & 65.0 & 34.4 & 0.6 & & & & $\mathrm{~B}$ & -4.24 & 1.25 & -4.63 & 24.74 & -3.06 & 8.33 & -2.10 & 4.30 & 2.41 & 0.49 & 0.58 & 0.98 \\
\hline NB_49 & 54.3 & 45.1 & 0.6 & & & & $\mathrm{~B}$ & -4.24 & 1.75 & -4.71 & 26.19 & -1.63 & 3.09 & -1.52 & 2.87 & 2.53 & 0.04 & 0.55 & 0.75 \\
\hline NB_50 & 7.2 & 92.6 & 0.2 & & & & U & 1.25 & & -0.77 & 1.70 & 1.08 & 0.47 & 0.85 & 0.56 & 1.02 & -3.39 & 1.00 & 0.90 \\
\hline NB_51 & 7.6 & 92.0 & 0.4 & & & & U & 1.75 & & -0.80 & 1.74 & 1.17 & 0.44 & 0.90 & 0.54 & 1.09 & -0.38 & 0.95 & 1.33 \\
\hline NB_52 & 5.0 & 94.5 & 0.5 & & & & U & 1.25 & & -0.72 & 1.65 & 0.81 & 0.57 & 0.68 & 0.62 & 0.97 & -0.20 & 0.85 & 2.10 \\
\hline NB_53 & 11.3 & 88.1 & 0.6 & & & & U & 1.75 & & -1.13 & 2.19 & 1.21 & 0.43 & 0.81 & 0.57 & 1.26 & -0.45 & 0.90 & 1.29 \\
\hline NB_54 & 87.0 & 12.6 & 0.4 & & & & $\mathrm{U}$ & -5.24 & & -5.38 & 41.59 & -4.87 & 29.14 & -4.26 & 19.13 & 1.79 & 0.76 & 1.93 & 1.19 \\
\hline NB_55 & 61.0 & 38.0 & 1.0 & & & & B & -5.24 & -4.24 & -5.19 & 36.58 & -3.46 & 10.98 & -2.18 & 4.52 & 2.94 & 0.54 & 0.54 & 0.92 \\
\hline NB_56 & 72.2 & 26.2 & 1.6 & & & & B & -4.73 & -3.74 & -4.88 & 29.45 & -4.49 & 22.47 & -2.49 & 5.60 & 2.82 & 0.89 & 0.55 & \\
\hline NB_57 & 96.4 & 3.5 & 0.1 & & & & $\mathrm{U}$ & -5.24 & & -5.43 & 42.96 & -5.16 & 35.63 & -4.98 & 31.49 & 0.53 & 0.61 & 2.26 & \\
\hline NB_58 & 85.7 & 13.6 & 0.7 & & & & $\mathrm{U}$ & -4.24 & & -4.79 & 27.63 & -4.10 & 17.20 & -3.68 & 12.81 & 1.74 & 0.66 & 2.97 & \\
\hline NB_60 & 12.3 & 86.7 & 1.0 & & & & B & 2.24 & -3.24 & -2.40 & 5.27 & 1.99 & 0.25 & 1.80 & 0.29 & 1.34 & -0.52 & 3.52 & 0.66 \\
\hline
\end{tabular}




\section{NEWBEX Survey (2012) (3): Grain Size Distribution}

\begin{tabular}{|c|c|c|c|c|c|c|c|c|c|c|c|c|c|c|c|c|c|c|c|c|c|}
\hline Sample ID & $\begin{array}{c}\text { Class } \\
\% \\
\text { phi } \\
-5.5\end{array}$ & $\begin{array}{c}\text { Class } \\
\% \\
\text { phi } \\
-5.0\end{array}$ & $\begin{array}{c}\text { Class } \\
\% \\
\text { phi } \\
-4.5\end{array}$ & $\begin{array}{c}\text { Class } \\
\% \\
\text { phi } \\
-4.0\end{array}$ & $\begin{array}{c}\text { Class } \\
\% \\
\text { phi } \\
-3.5\end{array}$ & $\begin{array}{c}\text { Class } \\
\% \\
\text { phi } \\
-3.0\end{array}$ & $\begin{array}{c}\text { Class } \\
\% \\
\text { phi } \\
-2.5\end{array}$ & $\begin{array}{c}\text { Class } \\
\% \\
\text { phi } \\
-2.0\end{array}$ & $\begin{array}{c}\text { Class } \\
\% \\
\text { phi } \\
-1.5\end{array}$ & $\begin{array}{c}\text { Class } \\
\% \\
\text { phi } \\
-1.0\end{array}$ & $\begin{array}{c}\text { Class } \\
\% \\
\text { phi } \\
-0.5\end{array}$ & $\begin{array}{c}\text { Class } \\
\% \\
\text { phi } \\
0.0\end{array}$ & $\begin{array}{c}\text { Class } \\
\% \\
\text { phi } \\
0.5\end{array}$ & $\begin{array}{c}\text { Class } \\
\% \\
\text { phi } \\
1.0\end{array}$ & $\begin{array}{c}\text { Class } \\
\% \\
\text { phi } \\
1.5\end{array}$ & $\begin{array}{c}\text { Class } \\
\% \\
\text { phi } \\
2.0\end{array}$ & $\begin{array}{c}\text { Class } \\
\% \\
\text { phi } \\
2.5\end{array}$ & $\begin{array}{c}\text { Class } \\
\% \\
\text { phi } \\
3.0\end{array}$ & $\begin{array}{c}\text { Class } \\
\% \\
\text { phi } \\
3.5\end{array}$ & $\begin{array}{c}\text { Class } \\
\% \\
\text { phi } \\
4.0\end{array}$ & $\begin{array}{c}\text { Class } \\
\% \\
\text { phi } \\
>4.0\end{array}$ \\
\hline NB_41 & 0.00 & 0.00 & 18.82 & 35.60 & 17.47 & 4.73 & 0.42 & 0.26 & 0.40 & 0.17 & 0.06 & 0.14 & 0.29 & 0.50 & 0.99 & 4.76 & 8.24 & 4.65 & 1.65 & 0.23 & 0.63 \\
\hline NB_42 & 0.00 & 18.52 & 29.05 & 11.62 & 4.08 & 1.89 & 0.18 & 0.63 & 0.21 & 0.22 & 0.24 & 0.32 & 0.59 & 1.14 & 2.07 & 8.02 & 11.76 & 6.40 & 2.16 & 0.24 & 0.66 \\
\hline NB_43 & 0.00 & 0.00 & 0.00 & 0.00 & 0.00 & 0.00 & 0.82 & 0.00 & 0.43 & 0.15 & 0.24 & 0.36 & 0.51 & 0.82 & 1.89 & 6.99 & 8.05 & 40.73 & 34.88 & 3.18 & 0.95 \\
\hline NB_44 & 0.00 & 0.00 & 0.00 & 0.00 & 0.00 & 0.82 & 0.00 & 0.07 & 0.02 & 0.10 & 0.01 & 0.06 & 0.19 & 0.41 & 1.09 & 5.42 & 7.92 & 43.37 & 35.82 & 3.53 & 1.18 \\
\hline NB_45 & 0.00 & 0.00 & 0.00 & 0.00 & 0.00 & 0.00 & 1.40 & 0.55 & 0.12 & 0.48 & 0.17 & 0.45 & 0.69 & 0.92 & 1.51 & 4.61 & 6.04 & 34.87 & 40.98 & 5.52 & 1.68 \\
\hline NB_46 & 0.00 & 0.00 & 0.00 & 0.00 & 0.00 & 0.69 & 0.00 & 0.08 & 0.46 & 0.37 & 0.15 & 0.38 & 0.64 & 0.91 & 1.44 & 4.06 & 5.69 & 35.83 & 42.19 & 5.79 & 1.33 \\
\hline NB_47 & 0.00 & 0.00 & 46.69 & 0.00 & 21.63 & 9.75 & 4.02 & 3.37 & 2.48 & 1.71 & 1.38 & 1.02 & 1.21 & 1.19 & 1.41 & 1.66 & 0.81 & 0.58 & 0.62 & 0.18 & 0.29 \\
\hline NB_48 & 0.00 & 0.00 & 14.12 & 18.87 & 11.87 & 5.82 & 5.42 & 3.91 & 2.66 & 2.28 & 2.94 & 3.04 & 3.86 & 6.08 & 8.37 & 8.13 & 1.42 & 0.35 & 0.14 & 0.09 & 0.63 \\
\hline NB_49 & 0.00 & 4.89 & 9.45 & 14.15 & 4.43 & 5.35 & 4.58 & 4.29 & 3.95 & 3.18 & 3.60 & 3.66 & 4.62 & 7.51 & 10.45 & 12.37 & 2.28 & 0.43 & 0.12 & 0.07 & 0.60 \\
\hline NB_50 & 0.00 & 0.00 & 0.00 & 0.00 & 0.00 & 0.00 & 0.13 & 1.31 & 2.24 & 3.55 & 6.08 & 7.19 & 9.85 & 15.89 & 24.66 & 24.26 & 4.12 & 0.36 & 0.11 & 0.06 & 0.18 \\
\hline NB_51 & 0.00 & 0.00 & 0.00 & 0.00 & 0.00 & 0.00 & 0.21 & 1.10 & 1.94 & 4.33 & 6.14 & 7.84 & 9.34 & 12.33 & 19.38 & 28.25 & 7.57 & 0.87 & 0.21 & 0.11 & 0.39 \\
\hline NB_52 & 0.00 & 0.00 & 0.00 & 0.00 & 0.00 & 0.00 & 0.22 & 0.35 & 1.02 & 3.42 & 9.28 & 11.39 & 13.24 & 17.79 & 21.98 & 17.16 & 2.89 & 0.48 & 0.18 & 0.10 & 0.49 \\
\hline NB_53 & 0.00 & 0.00 & 0.00 & 0.00 & 0.00 & 0.00 & 1.43 & 1.97 & 2.97 & 4.93 & 7.53 & 7.29 & 7.74 & 10.06 & 14.64 & 30.84 & 8.63 & 1.00 & 0.24 & 0.11 & 0.62 \\
\hline NB_54 & 0.00 & 45.28 & 20.65 & 5.15 & 5.61 & 2.56 & 6.12 & 0.63 & 0.52 & 0.52 & 0.54 & 0.41 & 0.41 & 0.55 & 1.72 & 5.06 & 2.50 & 0.93 & 0.35 & 0.11 & 0.39 \\
\hline NB_55 & 0.00 & 17.23 & 5.68 & 14.97 & 11.84 & 4.84 & 2.24 & 1.62 & 1.45 & 1.41 & 1.49 & 1.27 & 1.35 & 1.92 & 4.30 & 13.98 & 8.70 & 3.24 & 1.30 & 0.30 & 0.88 \\
\hline NB_56 & 0.00 & 0.00 & 50.44 & 0.00 & 13.35 & 3.73 & 0.62 & 1.79 & 1.43 & 0.80 & 0.70 & 0.68 & 0.61 & 0.93 & 2.34 & 9.28 & 6.64 & 3.85 & 1.00 & 0.14 & 1.65 \\
\hline NB_57 & 0.00 & 76.47 & 5.28 & 11.52 & 2.21 & 0.24 & 0.36 & 0.11 & 0.06 & 0.14 & 0.16 & 0.15 & 0.13 & 0.12 & 0.22 & 0.91 & 0.78 & 0.65 & 0.28 & 0.09 & 0.12 \\
\hline NB_58 & 0.00 & 0.00 & 25.95 & 30.65 & 18.20 & 6.91 & 1.83 & 0.99 & 0.63 & 0.56 & 0.49 & 0.32 & 0.33 & 0.37 & 0.56 & 2.88 & 3.68 & 2.86 & 1.86 & 0.25 & 0.69 \\
\hline NB_59 & 15.84 & 0.00 & 6.73 & 5.72 & 17.98 & 17.19 & 7.11 & 2.87 & 1.41 & 1.26 & 0.96 & 0.74 & 0.74 & 0.86 & 1.52 & 4.85 & 5.56 & 4.30 & 3.18 & 0.36 & 0.82 \\
\hline NB 60 & 0.00 & 0.00 & 0.00 & 0.00 & 2.86 & 6.35 & 0.64 & 0.84 & 0.84 & 0.79 & 0.80 & 0.72 & 0.90 & 1.36 & 3.62 & 31.01 & 35.48 & 8.21 & 4.24 & 0.31 & 1.04 \\
\hline
\end{tabular}


NEWBEX Survey (2012) (4): Identification, Location, and Description

\begin{tabular}{|c|c|c|c|c|c|c|c|c|}
\hline Sample ID & $\begin{array}{c}\text { Original } \\
\text { Sample ID }\end{array}$ & $\begin{array}{c}\text { Latitude } \\
\text { WGS84 }\end{array}$ & $\begin{array}{c}\text { Longitude } \\
\text { WGS84 }\end{array}$ & $\begin{array}{l}\text { Reliability } \\
\text { Ranking for } \\
\text { Positioning }\end{array}$ & $\begin{array}{c}\text { Water } \\
\text { Depth } \\
\text { (m) }\end{array}$ & $\begin{array}{c}\text { Sample } \\
\text { Collected }\end{array}$ & $\begin{array}{c}\text { Sampler } \\
\text { Type }\end{array}$ & $\begin{array}{c}\text { Total Wt } \\
\text { (gm) }\end{array}$ \\
\hline NB_61 & G1_S2 & 43.055973 & -70.701463 & 1 & 15.1 & $10 / 21 / 2013$ & Shipek & 56.00 \\
\hline NB_62 & G1_S3 & 43.055930 & -70.701277 & 1 & 15.1 & $10 / 21 / 2013$ & Shipek & 121.10 \\
\hline NB_63 & H1_S1 & 43.053930 & -70.698463 & 1 & 14.0 & $10 / 21 / 2013$ & Shipek & 95.40 \\
\hline NB_64 & H1_S2 & 43.053878 & -70.698375 & 1 & 14.0 & $10 / 21 / 2013$ & Shipek & 52.80 \\
\hline NB_65 & I1_S1 & 43.052573 & -70.696462 & 1 & 14.2 & $10 / 21 / 2013$ & Shipek & 58.36 \\
\hline NB_66 & I1_S2 & 43.052545 & -70.696427 & 1 & 14.2 & $10 / 21 / 2013$ & Shipek & 64.80 \\
\hline NB_67 & J1_S1 & 43.050712 & -70.695082 & 1 & 14.0 & $10 / 21 / 2013$ & Shipek & 326.90 \\
\hline NB_68 & J1_S2 & 43.050690 & -70.695130 & 1 & 14.0 & $10 / 21 / 2013$ & Shipek & 223.50 \\
\hline NB_69 & O1_S1 & 43.050693 & -70.675228 & 1 & 22.0 & $10 / 21 / 2013$ & Shipek & 488.50 \\
\hline NB_70 & T1_S1 & 43.066780 & -70.704848 & 1 & 14.5 & $10 / 21 / 2013$ & Shipek & 59.79 \\
\hline NB_71 & T1_S2 & 43.066902 & -70.704893 & 1 & 14.5 & $10 / 21 / 2013$ & Shipek & 54.20 \\
\hline NB_72 & A1_S1 & 43.072490 & -70.705650 & 1 & 15.0 & $12 / 17 / 2013$ & Shipek & 255.72 \\
\hline NB_73 & A1_S2 & 43.072763 & -70.705948 & 1 & 15.0 & $12 / 17 / 2013$ & Shipek & 353.30 \\
\hline NB_74 & B1_S1 & 43.068882 & -70.704958 & 1 & 15.6 & $12 / 17 / 2013$ & Shipek & 49.90 \\
\hline NB_75 & B1_S2 & 43.068873 & -70.705045 & 1 & 15.6 & $12 / 17 / 2013$ & Shipek & 66.76 \\
\hline NB_76 & C1_S1 & 43.064935 & -70.704415 & 1 & & $12 / 17 / 2013$ & Shipek & 55.20 \\
\hline NB_77 & C1_S2 & 43.064872 & -70.704533 & 1 & & $12 / 17 / 2013$ & Shipek & 80.80 \\
\hline NB_78 & D1_S1 & 43.061908 & -70.704035 & 1 & 21.5 & $12 / 17 / 2013$ & Shipek & 515.20 \\
\hline NB_79 & D1_S2 & 43.061715 & -70.703923 & 1 & 21.5 & $12 / 17 / 2013$ & Shipek & 342.80 \\
\hline NB_80 & E1-2_S1 & 43.059975 & -70.703493 & 1 & 20.1 & $12 / 17 / 2013$ & Shipek & 206.20 \\
\hline
\end{tabular}


NEWBEX Survey (2012) (4): Sediment Classifications

\begin{tabular}{|c|c|c|c|c|c|c|c|c|c|}
\hline Sample ID & $\begin{array}{l}\text { CMECS Substrate } \\
\text { Component } \\
\text { Group (Specific) }\end{array}$ & $\begin{array}{l}\text { CMECS Substrate } \\
\text { Component } \\
\text { Subgroup (Specific) }\end{array}$ & $\begin{array}{l}\text { Textural Group } \\
\text { from \%GSM } \\
\text { (Gradistat) }\end{array}$ & $\begin{array}{l}\text { Textural Group } \\
\text { from \%SZC } \\
\text { (Gradistat) }\end{array}$ & $\begin{array}{l}\text { Sediment Name from } \\
\% G S M \\
\text { and Mode (Gradistat) }\end{array}$ & $\begin{array}{l}\text { Sediment Name from } \\
\text { \%GSM and Mode } \\
\text { (Wentworth Scale) }\end{array}$ & $\begin{array}{l}\text { Sediment } \\
\text { Classification from } \\
\text { Mean Phi (Gradistat) }\end{array}$ & $\begin{array}{l}\text { Classification } \\
\text { from Mean Phi } \\
\text { (Wentworth) }\end{array}$ & $\begin{array}{l}\text { Sorting } \\
\text { (Gradistat) }\end{array}$ \\
\hline NB_61 & Pebbly & Pebbly Fine Sand & Gravelly Sand & N/A & $\begin{array}{l}\text { Medium Gravelly Fine } \\
\text { Sand }\end{array}$ & Pebbly Fine Sand & Fine Sand & Fine Sand & Poorly Sorted \\
\hline NB_62 & Pebble Mixes & Sandy Pebble Gravel & Sandy Gravel & $\mathrm{N} / \mathrm{A}$ & Sandy Coarse Gravel & Sandy Pebble Gravel & Very Fine Gravel & Granule Gravel & $\begin{array}{l}\text { Very Poorly } \\
\text { Sorted }\end{array}$ \\
\hline NB_63 & Pebbly & Pebbly Very Fine Sand & Gravelly Sand & $\mathrm{N} / \mathrm{A}$ & $\begin{array}{l}\text { Coarse Gravelly Very Fine } \\
\text { Sand }\end{array}$ & Pebbly Very Fine Sand & Fine Sand & Fine Sand & Poorly Sorted \\
\hline NB_64 & Slightly Granuley & $\begin{array}{l}\text { Slightly Granuley Very } \\
\text { Fine Sand }\end{array}$ & $\begin{array}{l}\text { Slightly Gravelly } \\
\text { Sand }\end{array}$ & $\mathrm{N} / \mathrm{A}$ & $\begin{array}{l}\text { Slightly Very Fine Gravelly } \\
\text { Very Fine Sand }\end{array}$ & $\begin{array}{l}\text { Slightly Granular Very } \\
\text { Fine Sand }\end{array}$ & Fine Sand & Fine Sand & Well Sorted \\
\hline NB_65 & Slightly Pebbly & $\begin{array}{l}\text { Slightly Pebbly Very } \\
\text { Fine Sand }\end{array}$ & $\begin{array}{l}\text { Slightly Gravelly } \\
\text { Sand }\end{array}$ & $\mathrm{N} / \mathrm{A}$ & $\begin{array}{l}\text { Slightly Fine Gravelly Very } \\
\text { Fine Sand }\end{array}$ & $\begin{array}{l}\text { Slightly Pebbly Very } \\
\text { Fine Sand }\end{array}$ & Very Fine Sand & Very Fine Sand & $\begin{array}{l}\text { Moderately } \\
\text { Well Sorted }\end{array}$ \\
\hline NB_66 & Pebbly & Pebbly Very Fine Sand & Gravelly Sand & $\mathrm{N} / \mathrm{A}$ & $\begin{array}{l}\text { Medium Gravelly Very Fine } \\
\text { Sand }\end{array}$ & Pebbly Very Fine Sand & Fine Sand & Fine Sand & Poorly Sorted \\
\hline NB_67 & Pebble Mixes & Sandy Pebble Gravel & Sandy Gravel & $\mathrm{N} / \mathrm{A}$ & Sandy Medium Gravel & Sandy Pebble Gravel & Very Coarse Sand & $\begin{array}{l}\text { Very Coarse } \\
\text { Sand }\end{array}$ & $\begin{array}{l}\text { Very Poorly } \\
\text { Sorted }\end{array}$ \\
\hline NB_68 & Pebble Mixes & Sandy Pebble Gravel & Sandy Gravel & N/A & Sandy Medium Gravel & Sandy Pebble Gravel & Very Coarse Sand & $\begin{array}{l}\text { Very Coarse } \\
\text { Sand }\end{array}$ & $\begin{array}{l}\text { Very Poorly } \\
\text { Sorted }\end{array}$ \\
\hline NB_69 & Pebble Mixes & Sandy Pebble Gravel & Sandy Gravel & $\mathrm{N} / \mathrm{A}$ & Sandy Medium Gravel & Sandy Pebble Gravel & Very Fine Gravel & Granule Gravel & Poorly Sorted \\
\hline NB_70 & Granuley & $\begin{array}{l}\text { Granuley Medium } \\
\text { Sand }\end{array}$ & Gravelly Sand & $\mathrm{N} / \mathrm{A}$ & $\begin{array}{l}\text { Very Fine Gravelly Medium } \\
\text { Sand }\end{array}$ & $\begin{array}{l}\text { Granular Medium } \\
\text { Sand }\end{array}$ & Medium Sand & Medium Sand & $\begin{array}{l}\text { Moderately } \\
\text { Sorted }\end{array}$ \\
\hline NB_71 & Granuley & $\begin{array}{l}\text { Granuley Medium } \\
\text { Sand }\end{array}$ & Gravelly Sand & $\mathrm{N} / \mathrm{A}$ & $\begin{array}{l}\text { Very Fine Gravelly Medium } \\
\text { Sand }\end{array}$ & $\begin{array}{l}\text { Granular Medium } \\
\text { Sand }\end{array}$ & Medium Sand & Medium Sand & $\begin{array}{l}\text { Moderately } \\
\text { Sorted }\end{array}$ \\
\hline NB_72 & Pebble Mixes & Sandy Pebble Gravel & Sandy Gravel & N/A & Sandy Medium Gravel & Sandy Pebble Gravel & Fine Gravel & Pebble Gravel & $\begin{array}{l}\text { Very Poorly } \\
\text { Sorted }\end{array}$ \\
\hline NB_73 & Pebble Mixes & Sandy Pebble Gravel & Sandy Gravel & $\mathrm{N} / \mathrm{A}$ & Sandy Very Coarse Gravel & Sandy Pebble Gravel & Very Fine Gravel & Granule Gravel & $\begin{array}{l}\text { Very Poorly } \\
\text { Sorted }\end{array}$ \\
\hline NB_74 & Granuley & $\begin{array}{l}\text { Granuley Medium } \\
\text { Sand }\end{array}$ & Gravelly Sand & $\mathrm{N} / \mathrm{A}$ & $\begin{array}{l}\text { Very Fine Gravelly Medium } \\
\text { Sand }\end{array}$ & $\begin{array}{l}\text { Granular Medium } \\
\text { Sand }\end{array}$ & Medium Sand & Medium Sand & $\begin{array}{l}\text { Moderately } \\
\text { Sorted }\end{array}$ \\
\hline NB 75 & Slightly Granuley & $\begin{array}{l}\text { Slightly Granul ey } \\
\text { Medium Sand }\end{array}$ & $\begin{array}{l}\text { Slightly Gravelly } \\
\text { Sand }\end{array}$ & N/A & $\begin{array}{l}\text { Slightly Very Fine Gravelly } \\
\text { Medium Sand }\end{array}$ & $\begin{array}{l}\text { Slightly Granular } \\
\text { Medium Sand }\end{array}$ & Medium Sand & Medium Sand & $\begin{array}{l}\text { Moderately } \\
\text { Sorted }\end{array}$ \\
\hline NB_76 & Granuley & $\begin{array}{l}\text { Granuley Medium } \\
\text { Sand }\end{array}$ & Gravelly Sand & $\mathrm{N} / \mathrm{A}$ & $\begin{array}{l}\text { Very Fine Gravelly Medium } \\
\text { Sand }\end{array}$ & $\begin{array}{l}\text { Granular Medium } \\
\text { Sand }\end{array}$ & Coarse Sand & Coarse Sand & Poorly Sorted \\
\hline NB_77 & Slightly Granuley & $\begin{array}{l}\text { Slightly Granul ey } \\
\text { Medium Sand }\end{array}$ & $\begin{array}{l}\text { Slightly Gravelly } \\
\text { Sand }\end{array}$ & $\mathrm{N} / \mathrm{A}$ & $\begin{array}{l}\text { Slightly Very Fine Gravelly } \\
\text { Medium Sand }\end{array}$ & $\begin{array}{l}\text { Slightly Granular } \\
\text { Medium Sand }\end{array}$ & Medium Sand & Medium Sand & $\begin{array}{l}\text { Moderately } \\
\text { Sorted }\end{array}$ \\
\hline NB_78 & Pebble Mixes & Sandy Pebble Gravel & Sandy Gravel & $\mathrm{N} / \mathrm{A}$ & Sandy Coarse Gravel & Sandy Pebble Gravel & Fine Gravel & Pebble Gravel & $\begin{array}{l}\text { Very Poorly } \\
\text { Sorted }\end{array}$ \\
\hline NB_79 & Gravel & Pebble Gravel & Gravel & $\mathrm{N} / \mathrm{A}$ & Coarse Gravel & Pebble Gravel & Coarse Gravel & Pebble Gravel & Well Sorted \\
\hline NB_80 & Gravel & Pebble Gravel & Gravel & N/A & Coarse Gravel & Pebble Gravel & Coarse Gravel & Pebble Gravel & Poorly Sorted \\
\hline
\end{tabular}


NEWBEX Survey (2012) (4): Grain Size Statistics

\begin{tabular}{|c|c|c|c|c|c|c|c|c|c|c|c|c|c|c|c|c|c|c|c|}
\hline Sample ID & $\begin{array}{c}\text { Gravel } \\
\% \\
\end{array}$ & $\begin{array}{c}\text { Sand } \\
\% \\
\end{array}$ & $\begin{array}{c}\text { Mud } \\
\% \\
\end{array}$ & $\begin{array}{c}\text { Sand } \\
\%\end{array}$ & $\begin{array}{c}\text { Silt } \\
\% \\
\end{array}$ & $\begin{array}{c}\text { Clay } \\
\% \\
\end{array}$ & Modes & $\begin{array}{c}\text { Mode } 1 \\
\text { (phi) }\end{array}$ & $\begin{array}{c}\text { Mode } 2 \\
\text { (phi) }\end{array}$ & $\begin{array}{l}D_{10} \\
\text { (phi) }\end{array}$ & $\begin{array}{c}\mathrm{D}_{10} \\
(\mathrm{~mm})\end{array}$ & $\begin{array}{r}D_{50} \\
\text { (phi) }\end{array}$ & $\begin{array}{c}\mathrm{D}_{50} \\
(\mathrm{~mm})\end{array}$ & $\begin{array}{c}\text { Mean } \\
\text { Size } \\
\text { (phi) }\end{array}$ & $\begin{array}{c}\text { Mean } \\
\text { Size } \\
(\mathrm{mm})\end{array}$ & $\begin{array}{c}\text { Sorting } \\
\text { (phi) }\end{array}$ & Skewness & Kurtosis & LOI \% \\
\hline NB_61 & 8.2 & 90.3 & 1.5 & & & & U & 2.24 & & 0.56 & 0.68 & 2.05 & 0.24 & 2.01 & 0.25 & 1.15 & -0.41 & 3.67 & 0.77 \\
\hline NB_62 & 55.1 & 43.9 & 1.0 & & & & $\mathrm{~B}$ & -4.24 & 2.24 & -4.37 & 20.61 & -3.50 & 11.28 & -1.78 & 3.44 & 2.82 & 0.76 & 0.48 & 1.42 \\
\hline NB_63 & 7.6 & 88.0 & 4.4 & & & & $U$ & 3.24 & & 1.64 & 0.32 & 3.01 & 0.12 & 2.88 & 0.14 & 1.41 & -0.55 & 4.42 & 0.64 \\
\hline NB_64 & 0.5 & 98.1 & 1.4 & & & & $U$ & 3.24 & & 2.17 & 0.22 & 3.00 & 0.13 & 2.95 & 0.13 & 0.47 & -0.32 & 1.11 & 0.63 \\
\hline NB_65 & 1.4 & 97.1 & 1.5 & & & & U & 3.24 & & 2.30 & 0.20 & 3.11 & 0.12 & 3.02 & 0.12 & 0.51 & -0.39 & 1.41 & 0.70 \\
\hline NB_66 & 10.2 & 88.5 & 1.3 & & & & $\mathrm{~B}$ & 3.24 & -3.74 & -1.27 & 2.41 & 3.03 & 0.12 & 2.75 & 0.15 & 1.47 & -0.71 & 3.96 & 0.70 \\
\hline NB_67 & 50.8 & 48.1 & 1.0 & & & & $\mathrm{~B}$ & -3.74 & 3.24 & -3.85 & 14.39 & -1.15 & 2.21 & -0.64 & 1.55 & 2.76 & 0.23 & 0.55 & 0.59 \\
\hline NB_68 & 48.7 & 50.0 & 1.3 & & & & B & -3.74 & 3.24 & -3.84 & 14.28 & -0.77 & 1.70 & -0.47 & 1.39 & 2.80 & 0.13 & 0.53 & 0.79 \\
\hline NB_69 & 68.1 & 30.8 & 1.1 & & & & U & -1.24 & & -3.90 & 14.94 & -1.82 & 3.54 & -1.85 & 3.62 & 1.74 & 0.07 & 0.90 & 0.90 \\
\hline NB_70 & 5.5 & 93.9 & 0.6 & & & & $\mathrm{U}$ & 1.75 & & -0.08 & 1.06 & 1.62 & 0.33 & 1.45 & 0.37 & 0.90 & -0.45 & 1.75 & 1.27 \\
\hline NB_71 & 6.1 & 93.3 & 0.6 & & & & U & 1.75 & & -0.22 & 1.17 & 1.65 & 0.32 & 1.60 & 0.33 & 0.84 & -0.36 & 2.05 & 0.88 \\
\hline NB_72 & 73.7 & 26.0 & 0.3 & & & & B & -5.24 & -3.74 & -5.28 & 38.96 & -3.33 & 10.05 & -2.64 & 6.23 & 2.49 & 0.39 & 0.68 & 0.80 \\
\hline NB_73 & 54.4 & 45.2 & 0.4 & & & & $\mathrm{~B}$ & -5.24 & 1.25 & -5.20 & 36.81 & -1.78 & 3.42 & -1.83 & 3.55 & 2.68 & -0.01 & 0.54 & 0.61 \\
\hline NB_74 & 5.1 & 94.5 & 0.4 & & & & $U$ & 1.75 & & -0.15 & 1.11 & 1.58 & 0.33 & 1.39 & 0.38 & 0.89 & -0.45 & 1.66 & 1.28 \\
\hline NB_75 & 4.7 & 94.7 & 0.6 & & & & U & 1.75 & & -0.28 & 1.21 & 1.40 & 0.38 & 1.21 & 0.43 & 0.91 & -0.85 & 1.33 & 1.30 \\
\hline NB_76 & 8.5 & 91.0 & 0.6 & & & & $\mathrm{U}$ & 1.75 & & -0.88 & 1.85 & 1.07 & 0.48 & 0.84 & 0.56 & 1.15 & -0.30 & 0.93 & 1.61 \\
\hline NB_77 & 2.5 & 96.7 & 0.8 & & & & U & 1.75 & & 0.48 & 0.72 & 1.66 & 0.32 & 1.57 & 0.34 & 0.73 & -0.34 & 1.53 & 0.96 \\
\hline NB_78 & 72.1 & 27.2 & 0.7 & & & & $\mathrm{~B}$ & -4.73 & 1.75 & -4.81 & 28.11 & -4.02 & 16.18 & -2.35 & 5.08 & 2.71 & 0.77 & 0.60 & 0.71 \\
\hline NB_80 & 92.8 & 6.9 & 0.3 & & & & U & -4.73 & & -4.92 & 30.32 & -4.70 & 26.05 & -4.70 & 26.05 & 1.08 & 0.46 & 9.71 & \\
\hline
\end{tabular}




\section{NEWBEX Survey (2012) (4): Grain Size Distribution}

\begin{tabular}{|c|c|c|c|c|c|c|c|c|c|c|c|c|c|c|c|c|c|c|c|c|c|}
\hline & $\begin{array}{c}\text { Class } \\
\% \\
\text { phi } \\
-5.5\end{array}$ & $\begin{array}{c}\text { Class } \\
\% \\
\text { phi } \\
-5.0\end{array}$ & $\begin{array}{c}\text { Class } \\
\% \\
\text { phi } \\
-4.5\end{array}$ & $\begin{array}{c}\text { Class } \\
\% \\
\text { phi } \\
-4.0\end{array}$ & $\begin{array}{c}\text { Class } \\
\% \\
\text { phi } \\
-3.5\end{array}$ & $\begin{array}{c}\text { Class } \\
\% \\
\text { phi } \\
-3.0\end{array}$ & $\begin{array}{c}\text { Class } \\
\% \\
\text { phi } \\
-2.5\end{array}$ & $\begin{array}{c}\text { Class } \\
\% \\
\text { phi } \\
-2.0\end{array}$ & $\begin{array}{c}\text { Class } \\
\% \\
\text { phi } \\
-1.5\end{array}$ & $\begin{array}{c}\text { Class } \\
\% \\
\text { phi } \\
-1.0\end{array}$ & $\begin{array}{c}\text { Class } \\
\% \\
\text { phi } \\
-0.5\end{array}$ & $\begin{array}{c}\text { Class } \\
\% \\
\text { phi } \\
0.0\end{array}$ & $\begin{array}{c}\text { Class } \\
\% \\
\text { phi } \\
0.5\end{array}$ & $\begin{array}{c}\text { Class } \\
\% \\
\text { phi } \\
1.0\end{array}$ & $\begin{array}{c}\text { Class } \\
\% \\
\text { phi } \\
1.5\end{array}$ & $\begin{array}{c}\text { Class } \\
\% \\
\text { phi } \\
2.0\end{array}$ & $\begin{array}{c}\text { Class } \\
\% \\
\text { phi } \\
2.5\end{array}$ & $\begin{array}{c}\text { Class } \\
\% \\
\text { phi } \\
3.0\end{array}$ & $\begin{array}{c}\text { Class } \\
\% \\
\text { phi } \\
3.5\end{array}$ & $\begin{array}{c}\text { Class } \\
\% \\
\text { phi } \\
4.0\end{array}$ & $\begin{array}{c}\text { Class } \\
\% \\
\text { phi } \\
>4.0\end{array}$ \\
\hline Sample ID & -5.5 & -5.0 & -4.5 & -4.0 & -3.5 & -3.0 & -2.5 & -2.0 & -1.5 & -1.0 & -0.5 & 0.0 & 0.5 & 1.0 & 1.5 & 2.0 & & 3.0 & & & \\
\hline NB_61 & 0.00 & 0.00 & 0.00 & 0.00 & 3.40 & 1.57 & 2.33 & 0.46 & 0.25 & 0.22 & 0.38 & 0.61 & 0.62 & 1.12 & 3.32 & 31.07 & 40.77 & 8.44 & 3.66 & 0.25 & 1.52 \\
\hline NB_62 & 0.00 & 0.00 & 0.00 & 40.15 & 10.06 & 1.89 & 1.09 & 0.71 & 0.48 & 0.69 & 0.40 & 0.27 & 0.39 & 0.55 & 1.37 & 8.01 & 19.07 & 8.99 & 4.56 & 0.31 & 1.02 \\
\hline NB_63 & 0.00 & 0.00 & 0.00 & 3.98 & 1.43 & 1.66 & 0.16 & 0.08 & 0.07 & 0.19 & 0.15 & 0.15 & 0.18 & 0.31 & 0.70 & 3.31 & 6.45 & 30.74 & 43.97 & 2.01 & 4.48 \\
\hline NB_64 & 0.00 & 0.00 & 0.00 & 0.00 & 0.00 & 0.00 & 0.00 & 0.12 & 0.14 & 0.28 & 0.17 & 0.21 & 0.27 & 0.46 & 1.01 & 4.52 & 7.77 & 35.18 & 45.77 & 2.68 & 1.42 \\
\hline NB_65 & 0.00 & 0.00 & 0.00 & 0.00 & 0.00 & 0.00 & 0.00 & 0.87 & 0.31 & 0.18 & 0.33 & 0.40 & 0.47 & 0.54 & 0.91 & 2.75 & 5.06 & 25.08 & 56.54 & 5.01 & 1.56 \\
\hline NB_66 & 0.00 & 0.00 & 0.00 & 0.00 & 8.92 & 0.00 & 0.24 & 0.46 & 0.20 & 0.40 & 0.58 & 0.59 & 0.59 & 0.66 & 1.06 & 3.14 & 5.47 & 24.59 & 47.86 & 3.90 & 1.32 \\
\hline NB_67 & 0.00 & 0.00 & 0.00 & 4.77 & 17.63 & 11.28 & 6.26 & 4.83 & 3.33 & 2.73 & 2.77 & 2.39 & 3.47 & 5.23 & 3.72 & 4.61 & 5.94 & 6.05 & 12.43 & 1.51 & 1.05 \\
\hline NB_68 & 0.00 & 0.00 & 0.00 & 3.91 & 19.11 & 9.65 & 6.39 & 3.98 & 3.13 & 2.56 & 2.80 & 2.48 & 3.70 & 5.23 & 3.70 & 4.98 & 5.68 & 5.94 & 13.30 & 2.14 & 1.32 \\
\hline NB_69 & 0.00 & 0.00 & 0.00 & 7.79 & 11.46 & 10.82 & 7.25 & 8.98 & 10.78 & 11.04 & 10.25 & 7.23 & 4.63 & 2.74 & 2.02 & 2.12 & 1.20 & 0.28 & 0.15 & 0.13 & 1.13 \\
\hline NB_70 & 0.00 & 0.00 & 0.00 & 0.00 & 0.00 & 0.00 & 0.20 & 0.96 & 1.97 & 2.41 & 2.28 & 2.63 & 3.41 & 7.33 & 18.46 & 41.45 & 16.01 & 1.58 & 0.60 & 0.10 & 0.59 \\
\hline NB_71 & 0.00 & 0.00 & 0.00 & 0.00 & 0.00 & 0.00 & 0.40 & 1.51 & 1.84 & 2.37 & 2.42 & 2.69 & 3.10 & 1.25 & 20.98 & 42.42 & 17.68 & 2.09 & 0.57 & 0.12 & 0.56 \\
\hline NB_72 & 0.00 & 24.73 & 8.51 & 0.00 & 13.44 & 10.27 & 5.70 & 4.46 & 3.75 & 2.86 & 2.76 & 3.24 & 3.55 & 5.08 & 5.83 & 4.61 & 0.64 & 0.15 & 0.11 & 0.05 & 0.27 \\
\hline NB_73 & 0.00 & 17.78 & 8.58 & 3.74 & 6.71 & 4.61 & 4.10 & 3.20 & 2.96 & 2.70 & 3.27 & 4.40 & 5.41 & 8.98 & 11.44 & 9.64 & 1.54 & 0.29 & 0.18 & 0.07 & 0.42 \\
\hline NB_74 & 0.00 & 0.00 & 0.00 & 0.00 & 0.00 & 0.41 & 0.59 & 0.84 & 1.49 & 1.77 & 2.74 & 3.13 & 4.05 & 7.85 & 20.25 & 39.78 & 14.27 & 1.55 & 0.72 & 0.20 & 0.38 \\
\hline NB_75 & 0.00 & 0.00 & 0.00 & 0.00 & 0.00 & 0.00 & 0.17 & 0.76 & 1.45 & 2.35 & 3.37 & 4.42 & 5.76 & 11.71 & 24.73 & 33.20 & 9.85 & 0.96 & 0.46 & 0.22 & 0.61 \\
\hline NB_76 & 0.00 & 0.00 & 0.00 & 0.00 & 0.00 & 0.00 & 0.00 & 1.11 & 2.91 & 4.46 & 6.75 & 8.01 & 9.54 & 15.09 & 15.82 & 25.13 & 9.01 & 0.90 & 0.55 & 0.17 & 0.57 \\
\hline NB_77 & 0.00 & 0.00 & 0.00 & 0.00 & 0.00 & 0.00 & 0.34 & 0.29 & 0.60 & 1.25 & 1.88 & 2.43 & 3.30 & 7.35 & 18.10 & 43.20 & 17.54 & 1.97 & 0.76 & 0.18 & 0.81 \\
\hline NB_78 & 0.00 & 0.00 & 29.93 & 20.76 & 9.15 & 3.10 & 3.30 & 2.33 & 2.05 & 1.46 & 1.20 & 0.95 & 0.94 & 1.47 & 3.41 & 9.88 & 6.20 & 1.83 & 1.11 & 0.24 & 0.71 \\
\hline NB_79 & 0.00 & 0.00 & 84.41 & 5.38 & 4.74 & 0.80 & 0.36 & 0.30 & 0.28 & 0.27 & 0.20 & 0.17 & 0.12 & 0.14 & 0.32 & 1.05 & 0.68 & 0.26 & 0.23 & 0.08 & 0.21 \\
\hline NB_80 & 0.00 & 0.00 & 89.59 & 0.00 & 0.00 & 0.53 & 0.38 & 0.78 & 0.83 & 0.73 & 0.54 & 0.41 & 0.29 & 0.26 & 0.46 & 1.87 & 1.47 & 0.77 & 0.65 & 0.15 & 0.30 \\
\hline
\end{tabular}


NEWBEX Survey (2012) (5): Identification, Location, and Description

\begin{tabular}{|lcccccccc|}
\hline Sample ID & $\begin{array}{c}\text { Original } \\
\text { Sample ID }\end{array}$ & $\begin{array}{c}\text { Latitude } \\
\text { WGS84 }\end{array}$ & $\begin{array}{c}\text { Longitude } \\
\text { WGS84 }\end{array}$ & $\begin{array}{c}\text { Reliability } \\
\text { Ranking for } \\
\text { Positioning }\end{array}$ & $\begin{array}{c}\text { Water } \\
\text { Depth } \\
(\mathbf{m})\end{array}$ & $\begin{array}{c}\text { Sample } \\
\text { Collected }\end{array}$ & $\begin{array}{c}\text { Sampler } \\
\text { Type }\end{array}$ & $\begin{array}{c}\text { Total Wt } \\
\text { (gm) }\end{array}$ \\
\hline NB_81 & E1-2_S2 & 43.059800 & -70.703518 & 1 & 20.1 & $12 / 17 / 2013$ & Shipek & 156.30 \\
\hline NB_82 & F1_S1 & 43.057613 & -70.702950 & 1 & 16.2 & $12 / 17 / 2013$ & Shipek & 456.10 \\
\hline NB_83 & F1_S2 & 43.057757 & -70.703085 & 1 & 16.2 & $12 / 17 / 2013$ & Shipek & 496.10 \\
\hline NB_84 & G1_S1 & 43.055538 & -70.701807 & 1 & 14.5 & $12 / 17 / 2013$ & Shipek & 647.70 \\
\hline NB_85 & G1_S2 & 43.055523 & -70.701680 & 1 & 14.5 & $12 / 17 / 2013$ & Shipek & 132.90 \\
\hline NB_86 & H1_S1 & 43.054142 & -70.698687 & 1 & 13.9 & $12 / 17 / 2013$ & Shipek & 69.50 \\
\hline NB_87 & H1_S2 & 43.053968 & -70.698858 & 1 & 13.9 & $12 / 17 / 2013$ & Shipek & 56.00 \\
\hline NB_88 & I1_S1 & 43.052637 & -70.696713 & 1 & 13.8 & $12 / 17 / 2013$ & Shipek & 54.10 \\
\hline NB_89 & I1_S2 & 43.052622 & -70.696753 & 1 & 13.8 & $12 / 17 / 2013$ & Shipek & 61.20 \\
\hline NB_90 & J1_S1 & 43.050997 & -70.694450 & 1 & 14.4 & $12 / 17 / 2013$ & Shipek & 76.20 \\
\hline NB_91 & J1_S2 & 43.051042 & -70.694473 & 1 & 14.4 & $12 / 17 / 2013$ & Shipek & 64.10 \\
\hline NB_92 & N1_S1 & 43.050658 & -70.676677 & 1 & 18.9 & $12 / 17 / 2013$ & Shipek & 84.70 \\
\hline NB_93 & N1_S2 & 43.050590 & -70.676718 & 1 & 18.9 & $12 / 17 / 2013$ & Shipek & 43.70 \\
\hline
\end{tabular}


NEWBEX Survey (2012) (5): Sediment Classifications

\begin{tabular}{|c|c|c|c|c|c|c|c|c|c|}
\hline Sample ID & $\begin{array}{l}\text { CMECS Substrate } \\
\text { Component } \\
\text { Group (Specific) }\end{array}$ & $\begin{array}{l}\text { CMECS Substrate } \\
\text { Component } \\
\text { Subgroup (Specific) }\end{array}$ & $\begin{array}{l}\text { Textural Group } \\
\text { from \%GSM } \\
\text { (Gradistat) }\end{array}$ & $\begin{array}{l}\text { Textural Group } \\
\text { from \%SZC } \\
\text { (Gradistat) }\end{array}$ & $\begin{array}{l}\text { Sediment Name from } \\
\% G S M \\
\text { and Mode (Gradistat) }\end{array}$ & $\begin{array}{l}\text { Sediment Name from } \\
\text { \%GSM and Mode } \\
\text { (Wentworth Scale) }\end{array}$ & $\begin{array}{l}\text { Sediment } \\
\text { Classification from } \\
\text { Mean Phi (Gradistat) }\end{array}$ & $\begin{array}{l}\text { Classification } \\
\text { from Mean Phi } \\
\text { (Wentworth) }\end{array}$ & $\begin{array}{l}\text { Sorting } \\
\text { (Gradistat) }\end{array}$ \\
\hline NB_81 & Gravel & Pebble Gravel & Gravel & N/A & Coarse Gravel & Pebble Gravel & Coarse Gravel & Pebble Gravel & Poorly Sorted \\
\hline NB 82 & Gravel & Pebble Gravel & Gravel & N/A & Medium Gravel & Pebble Gravel & Fine Gravel & Pebble Gravel & $\begin{array}{l}\text { Very Poorly } \\
\text { Sorted }\end{array}$ \\
\hline NB_83 & Gravel & Pebble Gravel & Gravel & N/A & Coarse Gravel & Pebble Gravel & Fine Gravel & Pebble Gravel & $\begin{array}{l}\text { Very Poorly } \\
\text { Sorted }\end{array}$ \\
\hline NB_ 84 & Pebble Mixes & Sandy Pebble Gravel & Sandy Gravel & N/A & Sandy Coarse Gravel & Sandy Pebble Gravel & Very Coarse Sand & $\begin{array}{l}\text { Very Coarse } \\
\text { Sand }\end{array}$ & $\begin{array}{l}\text { Very Poorly } \\
\text { Sorted }\end{array}$ \\
\hline NB_85 & Pebbly & Pebbly Medium Sand & Gravelly Sand & N/A & $\begin{array}{l}\text { Coarse Gravelly Medium } \\
\text { Sand }\end{array}$ & Pebbly Medium Sand & Medium Sand & Medium Sand & Poorly Sorted \\
\hline NB_86 & Slightly Pebbly & $\begin{array}{l}\text { Slightly Pebbly Very } \\
\text { Fine Sand }\end{array}$ & $\begin{array}{l}\text { Slightly Gravelly } \\
\text { Sand }\end{array}$ & N/A & $\begin{array}{l}\text { Slightly Fine Gravelly Very } \\
\text { Fine Sand }\end{array}$ & $\begin{array}{l}\text { Slightly Pebbly Very } \\
\text { Fine Sand }\end{array}$ & Fine Sand & Fine Sand & Well Sorted \\
\hline NB_87 & Slightly Pebbly & $\begin{array}{l}\text { Slightly Pebbly Fine } \\
\text { Sand }\end{array}$ & $\begin{array}{l}\text { Slightly Gravelly } \\
\text { Sand }\end{array}$ & $\mathrm{N} / \mathrm{A}$ & $\begin{array}{l}\text { Slightly Fine Gravelly Fine } \\
\text { Sand }\end{array}$ & $\begin{array}{l}\text { Slightly Pebbly Fine } \\
\text { Sand }\end{array}$ & Fine Sand & Fine Sand & Well Sorted \\
\hline NB_88 & Pebbly & Pebbly Very Fine Sand & Gravelly Sand & $\mathrm{N} / \mathrm{A}$ & $\begin{array}{l}\text { Medium Gravelly Very Fine } \\
\text { Sand }\end{array}$ & Pebbly Very Fine Sand & Fine Sand & Fine Sand & Poorly Sorted \\
\hline NB_89 & Pebbly & Pebbly Very Fine Sand & Gravelly Sand & N/A & $\begin{array}{l}\text { Coarse Gravelly Very Fine } \\
\text { Sand }\end{array}$ & Pebbly Very Fine Sand & Fine Sand & Fine Sand & $\begin{array}{l}\text { Moderately } \\
\text { Sorted }\end{array}$ \\
\hline NB_90 & Pebble Mixes & Sandy Pebble Gravel & Sandy Gravel & N/A & Sandy Coarse Gravel & Sandy Pebble Gravel & Coarse Sand & Coarse Sand & $\begin{array}{l}\text { Very Poorly } \\
\text { Sorted }\end{array}$ \\
\hline NB_91 & Slightly Pebbly & $\begin{array}{l}\text { Slightly Pebbly Fine } \\
\text { Sand }\end{array}$ & $\begin{array}{l}\text { Slightly Gravelly } \\
\text { Sand }\end{array}$ & $\mathrm{N} / \mathrm{A}$ & $\begin{array}{l}\text { Slightly Fine Gravelly Fine } \\
\text { Sand }\end{array}$ & $\begin{array}{l}\text { Slightly Pebbly Fine } \\
\text { Sand }\end{array}$ & Fine Sand & Fine Sand & $\begin{array}{l}\text { Moderately } \\
\text { Sorted }\end{array}$ \\
\hline NB_92 & Gravel & Pebble Gravel & Gravel & N/A & Medium Gravel & Pebble Gravel & Fine Gravel & Pebble Gravel & Poorly Sorted \\
\hline NB_93 & Gravel & Pebble Gravel & Gravel & N/A & Medium Gravel & Pebble Gravel & Medium Gravel & Pebble Gravel & Poorly Sorted \\
\hline
\end{tabular}




\section{NEWBEX Survey (2012) (5): Grain Size Statistics}

\begin{tabular}{|c|c|c|c|c|c|c|c|c|c|c|c|c|c|c|c|c|c|c|c|}
\hline Sample ID & $\begin{array}{c}\text { Gravel } \\
\% \\
\end{array}$ & $\begin{array}{c}\text { Sand } \\
\%\end{array}$ & $\begin{array}{c}\text { Mud } \\
\% \\
\end{array}$ & $\begin{array}{c}\text { Sand } \\
\% \\
\end{array}$ & $\begin{array}{c}\text { Silt } \\
\% \\
\end{array}$ & $\begin{array}{c}\text { Clay } \\
\% \\
\end{array}$ & Modes & $\begin{array}{c}\text { Mode } 1 \\
\text { (phi) }\end{array}$ & $\begin{array}{c}\text { Mode } 2 \\
\text { (phi) }\end{array}$ & $\begin{array}{c}D_{10} \\
\text { (phi) }\end{array}$ & $\begin{array}{c}D_{10} \\
(\mathrm{~mm})\end{array}$ & $\begin{array}{c}\mathrm{D}_{50} \\
\text { (phi) }\end{array}$ & $\begin{array}{c}D_{50} \\
(\mathrm{~mm})\end{array}$ & $\begin{array}{c}\text { Mean } \\
\text { Size } \\
\text { (phi) }\end{array}$ & $\begin{array}{c}\text { Mean } \\
\text { Size } \\
(\mathrm{mm})\end{array}$ & $\begin{array}{c}\text { Sorting } \\
\text { (phi) }\end{array}$ & Skewness & Kurtosis & LOI \% \\
\hline NB_81 & 90.3 & 9.4 & 0.4 & & & & $\mathrm{U}$ & -4.73 & & -4.92 & 30.21 & -4.68 & 25.60 & -4.62 & 24.52 & 1.21 & 0.62 & 9.57 & \\
\hline NB_82 & 82.9 & 16.4 & 0.7 & & & & $\mathrm{~B}$ & -3.24 & -4.73 & -4.67 & 25.44 & -2.96 & 7.80 & -2.66 & 6.32 & 2.11 & 0.37 & 1.72 & \\
\hline NB_83 & 82.6 & 16.9 & 0.5 & & & & B & -4.73 & -3.74 & -4.79 & 27.68 & -3.64 & 12.46 & -2.82 & 7.07 & 2.28 & 0.61 & 1.39 & \\
\hline NB_84 & 41.1 & 58.6 & 0.3 & & & & $\mathrm{~B}$ & 1.75 & -4.24 & -4.44 & 21.71 & 0.54 & 0.69 & -0.57 & 1.49 & 2.64 & -0.50 & 0.53 & 0.50 \\
\hline NB_85 & 11.5 & 88.0 & 0.5 & & & & $\mathrm{~B}$ & 1.75 & -4.24 & -4.01 & 16.12 & 1.76 & 0.30 & 1.54 & 0.34 & 1.50 & -0.55 & 3.31 & 0.59 \\
\hline NB_86 & 0.7 & 98.5 & 0.8 & & & & $U$ & 3.24 & & 2.35 & 0.20 & 3.00 & 0.12 & 2.96 & 0.13 & 0.42 & -0.27 & 1.01 & 0.54 \\
\hline NB_87 & 0.3 & 99.1 & 0.6 & & & & $\mathrm{U}$ & 3.24 & & 2.34 & 0.20 & 2.94 & 0.13 & 2.93 & 0.13 & 0.41 & -0.16 & 0.97 & 0.55 \\
\hline NB_88 & 11.5 & 87.7 & 0.9 & & & & U & 3.24 & & -2.69 & 6.47 & 3.04 & 0.12 & 2.73 & 0.15 & 1.47 & -0.73 & 3.93 & 0.69 \\
\hline NB_89 & 5.1 & 93.9 & 1 & & & & $\mathrm{U}$ & 3.24 & & 1.73 & 0.30 & 3.06 & 0.12 & 2.94 & 0.13 & 0.95 & -0.59 & 2.89 & 0.58 \\
\hline NB_90 & 35.6 & 63.8 & 0.7 & & & & $\mathrm{~B}$ & -4.24 & 3.24 & -4.31 & 19.85 & 1.88 & 0.27 & 0.30 & 0.81 & 3.05 & -0.62 & 0.45 & 0.73 \\
\hline NB_91 & 0.8 & 98.1 & 1 & & & & $U$ & 3.24 & & 1.52 & 0.35 & 2.64 & 0.16 & 2.56 & 0.17 & 0.76 & -0.22 & 0.83 & 0.67 \\
\hline NB_92 & 84.0 & 15.6 & 0.4 & & & & $\mathrm{U}$ & -3.74 & & -3.74 & 13.32 & -2.49 & 5.60 & -2.35 & 5.11 & 1.21 & 0.21 & 0.82 & \\
\hline NB_93 & 89.7 & 9.9 & 0.4 & & & & U & -3.74 & & -4.24 & 18.94 & -3.73 & 13.28 & -3.42 & 10.71 & 1.12 & 0.64 & 4.27 & \\
\hline
\end{tabular}


NEWBEX Survey (2012) (5): Grain Size Distribution

\begin{tabular}{|c|c|c|c|c|c|c|c|c|c|c|c|c|c|c|c|c|c|c|c|c|c|}
\hline Sample ID & $\begin{array}{c}\text { Class } \\
\% \\
\text { phi } \\
-5.5 \\
\end{array}$ & $\begin{array}{c}\text { Class } \\
\% \\
\text { phi } \\
-5.0 \\
\end{array}$ & $\begin{array}{c}\text { Class } \\
\% \\
\text { phi } \\
-4.5 \\
\end{array}$ & $\begin{array}{c}\text { Class } \\
\% \\
\text { phi } \\
-4.0 \\
\end{array}$ & $\begin{array}{c}\text { Class } \\
\% \\
\text { phi } \\
-3.5 \\
\end{array}$ & $\begin{array}{c}\text { Class } \\
\% \\
\text { phi } \\
-3.0 \\
\end{array}$ & $\begin{array}{c}\text { Class } \\
\% \\
\text { phi } \\
-2.5 \\
\end{array}$ & $\begin{array}{c}\text { Class } \\
\% \\
\text { phi } \\
-2.0 \\
\end{array}$ & $\begin{array}{c}\text { Class } \\
\% \\
\text { phi } \\
-1.5 \\
\end{array}$ & $\begin{array}{c}\text { Class } \\
\% \\
\text { phi } \\
-1.0 \\
\end{array}$ & $\begin{array}{c}\text { Class } \\
\% \\
\text { phi } \\
-0.5 \\
\end{array}$ & $\begin{array}{c}\text { Class } \\
\% \\
\text { phi } \\
0.0 \\
\end{array}$ & $\begin{array}{c}\text { Class } \\
\% \\
\text { phi } \\
0.5\end{array}$ & $\begin{array}{c}\text { Class } \\
\% \\
\text { phi } \\
1.0 \\
\end{array}$ & $\begin{array}{c}\text { Class } \\
\% \\
\text { phi } \\
1.5\end{array}$ & $\begin{array}{c}\text { Class } \\
\% \\
\text { phi } \\
2.0\end{array}$ & $\begin{array}{c}\text { Class } \\
\% \\
\text { phi } \\
2.5 \\
\end{array}$ & $\begin{array}{c}\text { Class } \\
\% \\
\text { phi } \\
3.0 \\
\end{array}$ & $\begin{array}{c}\text { Class } \\
\% \\
\text { phi } \\
3.5\end{array}$ & $\begin{array}{c}\text { Class } \\
\% \\
\text { phi } \\
4.0\end{array}$ & $\begin{array}{c}\text { Class } \\
\% \\
\text { phi } \\
>4.0 \\
\end{array}$ \\
\hline NB_81 & 0.00 & 0.00 & 82.16 & 4.52 & 1.09 & 0.57 & 0.21 & 0.29 & 0.72 & 0.71 & 0.45 & 0.36 & 0.23 & 0.25 & 0.57 & 2.68 & 2.23 & 1.29 & 1.09 & 0.22 & 0.36 \\
\hline NB_82 & 0.00 & 0.00 & 15.95 & 2.87 & 14.19 & 15.90 & 14.98 & 11.26 & 5.58 & 2.20 & 1.20 & 0.72 & 0.53 & 0.66 & 1.37 & 3.76 & 3.24 & 2.64 & 2.02 & 0.29 & 0.66 \\
\hline NB_83 & 0.00 & 0.00 & 26.42 & 9.99 & 19.39 & 8.74 & 8.31 & 5.17 & 3.32 & 1.23 & 0.98 & 0.64 & 0.55 & 0.71 & 1.27 & 3.78 & 3.55 & 2.75 & 2.33 & 0.35 & 0.52 \\
\hline NB_84 & 0.00 & 0.00 & 8.63 & 14.66 & 4.56 & 5.12 & 2.37 & 1.47 & 2.04 & 2.19 & 2.76 & 2.48 & 3.22 & 5.38 & 8.71 & 21.29 & 10.04 & 2.73 & 1.78 & 0.21 & 0.34 \\
\hline NB_85 & 0.00 & 0.00 & 0.00 & 10.24 & 0.56 & 0.00 & 0.00 & 0.22 & 0.08 & 0.39 & 0.69 & 1.14 & 2.14 & 4.68 & 9.22 & 39.17 & 23.81 & 4.70 & 2.28 & 0.18 & 0.48 \\
\hline NB_86 & 0.00 & 0.00 & 0.00 & 0.00 & 0.00 & 0.00 & 0.26 & 0.17 & 0.17 & 0.12 & 0.10 & 0.12 & 0.14 & 0.25 & 0.61 & 3.10 & 6.64 & 38.00 & 47.16 & 2.37 & 0.78 \\
\hline NB_87 & 0.00 & 0.00 & 0.00 & 0.00 & 0.00 & 0.00 & 0.31 & 0.00 & 0.00 & 0.02 & 0.04 & 0.03 & 0.07 & 0.19 & 0.53 & 3.10 & 8.01 & 42.90 & 42.29 & 1.93 & 0.59 \\
\hline NB_88 & 0.00 & 0.00 & 0.00 & 0.00 & 6.69 & 3.04 & 0.46 & 0.31 & 0.45 & 0.51 & 0.47 & 0.50 & 0.55 & 0.58 & 0.93 & 2.68 & 4.77 & 23.71 & 49.22 & 4.23 & 0.90 \\
\hline NB_89 & 0.00 & 0.00 & 0.00 & 1.80 & 0.00 & 0.91 & 0.65 & 0.39 & 0.83 & 0.56 & 0.60 & 0.57 & 0.60 & 0.70 & 1.03 & 3.00 & 5.24 & 26.26 & 51.64 & 4.21 & 1.02 \\
\hline NB_90 & 0.00 & 0.00 & 0.00 & 27.81 & 7.47 & 0.00 & 0.00 & 0.00 & 0.13 & 0.17 & 0.30 & 0.28 & 0.77 & 2.31 & 4.28 & 8.60 & 9.88 & 10.85 & 23.69 & 2.78 & 0.67 \\
\hline NB_91 & 0.00 & 0.00 & 0.00 & 0.00 & 0.00 & 0.00 & 0.34 & 0.17 & 0.27 & 0.05 & 0.21 & 0.24 & 0.66 & 2.09 & 5.20 & 15.36 & 19.92 & 17.89 & 32.73 & 3.83 & 1.06 \\
\hline NB_92 & 0.00 & 0.00 & 0.00 & 0.00 & 19.40 & 13.49 & 17.14 & 12.43 & 11.24 & 10.31 & 8.25 & 3.75 & 1.41 & 0.66 & 0.43 & 0.60 & 0.38 & 0.08 & 0.04 & 0.02 & 0.37 \\
\hline NB_93 & 0.00 & 0.00 & 0.00 & 20.04 & 57.40 & 2.77 & 3.44 & 3.26 & 1.33 & 1.46 & 1.81 & 2.21 & 2.09 & 1.30 & 0.73 & 0.82 & 0.64 & 0.18 & 0.08 & 0.03 & 0.41 \\
\hline
\end{tabular}


Section 3: Jeffreys Ledge Survey (2002-2005) 
Jeffreys Ledge Survey (2002-2005) (1): Identification, Location, and Description

\begin{tabular}{|c|c|c|c|c|c|c|c|c|}
\hline Sample ID & $\begin{array}{c}\text { Original } \\
\text { Sample ID }\end{array}$ & $\begin{array}{c}\text { Latitude } \\
\text { WGS84 }\end{array}$ & $\begin{array}{c}\text { Longitude } \\
\text { WGS84 }\end{array}$ & $\begin{array}{l}\text { Reliability } \\
\text { Ranking for } \\
\text { Positioning }\end{array}$ & $\begin{array}{c}\text { Water } \\
\text { Depth } \\
\text { (m) }\end{array}$ & $\begin{array}{c}\text { Sample } \\
\text { Collected }\end{array}$ & $\begin{array}{c}\text { Sampler } \\
\text { Type }\end{array}$ & $\begin{array}{c}\text { Total Wt } \\
\text { (gm) }\end{array}$ \\
\hline JL-01 & JL00 & 42.933620 & -70.371710 & 1 & 128 & 15-Jul-02 & $\mathrm{BC}$ & 19.90 \\
\hline $\mathrm{JL}-02$ & JL01 & 42.934240 & -70.396590 & 1 & 119 & 15-Jul-02 & $\mathrm{BC}$ & 27.10 \\
\hline $\mathrm{JL}-03$ & JL02 & 42.933740 & -70.383470 & 1 & 124 & $7 / 15 / 2002$ & $\mathrm{BC}$ & 16.90 \\
\hline $\mathrm{JL}-04$ & JL03 & 42.933700 & -70.358520 & 1 & 137 & $7 / 15 / 2002$ & $\mathrm{BC}$ & 16.70 \\
\hline $\mathrm{JL}-05$ & JL04 & 42.933870 & -70.346840 & 1 & 144 & $7 / 15 / 2002$ & $B C$ & 17.60 \\
\hline $\mathrm{JL}-06$ & JL05 & 42.932320 & -70.328670 & 1 & 146 & $7 / 30 / 2002$ & $\mathrm{BC}$ & 20.90 \\
\hline JL-07 & JL07 & 42.933220 & -70.296080 & 1 & 143 & $7 / 30 / 2002$ & $B C$ & 25.90 \\
\hline $\mathrm{JL}-08$ & JL08 & 42.933230 & -70.279750 & 1 & 139 & $7 / 30 / 2002$ & $\mathrm{BC}$ & 31.00 \\
\hline JL-09 & JL09 & 42.933300 & -70.262600 & 1 & 141 & $7 / 30 / 2002$ & $\mathrm{BC}$ & 33.90 \\
\hline $\mathrm{JL}-10$ & JL10 & 42.933080 & -70.248090 & 1 & 148 & $8 / 19 / 2002$ & $\mathrm{BC}$ & 15.20 \\
\hline $\mathrm{JL}-11$ & JL11 & 42.933480 & -70.230810 & 1 & 174 & $8 / 19 / 2002$ & $\mathrm{BC}$ & 11.30 \\
\hline $\mathrm{JL}-12$ & $\mathrm{JL} 12$ & 42.933550 & -70.213270 & 1 & 157 & $8 / 19 / 2002$ & $\mathrm{BC}$ & 12.70 \\
\hline $\mathrm{JL}-13$ & JL13a & 42.933620 & -70.196750 & 1 & 130 & $8 / 19 / 2002$ & $\mathrm{BC}$ & 15.40 \\
\hline JL-14 & $\mathrm{JL} 13 \mathrm{~b}$ & 42.933250 & -70.197400 & 1 & 130 & $8 / 11 / 2005$ & $\mathrm{SH}$ & 17.50 \\
\hline $\mathrm{JL}-15$ & $\mathrm{JL} 14 \mathrm{a}$ & 42.933210 & -70.179770 & 1 & 128 & $8 / 19 / 2002$ & $\mathrm{BC}$ & 26.30 \\
\hline JL-16 & JL16 & 42.933390 & -70.144080 & 1 & 113 & $9 / 19 / 2002$ & $\mathrm{BC}$ & 38.30 \\
\hline JL-17 & JL16b & 42.930900 & -70.148530 & 1 & 115 & $8 / 11 / 2005$ & $\mathrm{SH}$ & 28.40 \\
\hline JL-18 & JL20 & 42.921250 & -70.380660 & 1 & 101 & $7 / 26 / 2002$ & $\mathrm{SH}$ & 65.90 \\
\hline JL-19 & JL21 & 42.920770 & -70.362540 & 1 & 138 & $7 / 26 / 2002$ & $\mathrm{SH}$ & 27.50 \\
\hline JL-20 & JL22 & 42.925130 & -70.346770 & 1 & 143 & $7 / 15 / 2002$ & $\mathrm{BC}$ & 14.20 \\
\hline
\end{tabular}


Jeffreys Ledge Survey (2002-2005) (1): Sediment Classifications

\begin{tabular}{|c|c|c|c|c|c|c|c|c|c|}
\hline Sample ID & $\begin{array}{l}\text { CMECS Substrate } \\
\text { Component } \\
\text { Group (Specific) }\end{array}$ & $\begin{array}{l}\text { CMECS Substrate } \\
\text { Component } \\
\text { Subgroup (Specific) }\end{array}$ & $\begin{array}{l}\text { Textural Group } \\
\text { from \%GSM } \\
\text { (Gradistat) }\end{array}$ & $\begin{array}{l}\text { Textural Group } \\
\text { from \%SZC } \\
\text { (Gradistat) }\end{array}$ & $\begin{array}{l}\text { Sediment Name from } \\
\text { \%GSM } \\
\text { and Mode (Gradistat) }\end{array}$ & $\begin{array}{l}\text { Sediment Name from } \\
\text { \%GSM and Mode } \\
\text { (Wentworth Scale) }\end{array}$ & $\begin{array}{l}\text { Sediment } \\
\text { Classification from } \\
\text { Mean Phi (Gradistat) }\end{array}$ & $\begin{array}{l}\text { Classification } \\
\text { from Mean Phi } \\
\text { (Wentworth) }\end{array}$ & $\begin{array}{l}\text { Sorting } \\
\text { (Gradistat) }\end{array}$ \\
\hline $\mathrm{JL}-01$ & Mud & Silt-Clay & Mud & Mud & Mud & Mud & Clay & Clay & $\begin{array}{l}\text { Very Poorly } \\
\text { Sorted }\end{array}$ \\
\hline $\mathrm{JL}-02$ & Mud & Silt-Clay & Mud & Mud & Mud & Mud & Very Fine Silt & Clay & $\begin{array}{l}\text { Very Poorly } \\
\text { Sorted }\end{array}$ \\
\hline $\mathrm{JL}-03$ & Mud & Silt-Clay & Mud & Mud & Mud & Mud & Very Fine Silt & Clay & $\begin{array}{l}\text { Very Poorly } \\
\text { Sorted }\end{array}$ \\
\hline $\mathrm{JL}-04$ & Mud & Silt-Clay & Mud & Mud & Mud & Mud & Clay & Clay & $\begin{array}{l}\text { Very Poorly } \\
\text { Sorted }\end{array}$ \\
\hline $\mathrm{JL}-05$ & Mud & Silt-Clay & Mud & Mud & Mud & Mud & Clay & Clay & $\begin{array}{l}\text { Very Poorly } \\
\text { Sorted }\end{array}$ \\
\hline $\mathrm{JL}-06$ & Mud & Clay & Mud & Mud & Mud & Mud & Clay & Clay & $\begin{array}{l}\text { Very Poorly } \\
\text { Sorted }\end{array}$ \\
\hline $\mathrm{JL}-07$ & Slightly Granuley & $\begin{array}{l}\text { Slightly Granuley Very } \\
\text { Fine Sandy Clay }\end{array}$ & $\begin{array}{l}\text { Slightly Gravelly } \\
\text { Sandy Mud }\end{array}$ & Sandy Mud & $\begin{array}{l}\text { Slightly Very Fine Gravelly } \\
\text { Very Fine Sandy Mud }\end{array}$ & $\begin{array}{l}\text { Slightly Granular Very } \\
\text { Fine Sandy Mud }\end{array}$ & Very Fine Silt & Clay & $\begin{array}{l}\text { Very Poorly } \\
\text { Sorted }\end{array}$ \\
\hline $\mathrm{JL}-08$ & Slightly Pebbly & $\begin{array}{l}\text { Slightly Pebbly Very } \\
\text { Fine Sandy Clay }\end{array}$ & $\begin{array}{l}\text { Slightly Gravelly } \\
\text { Sandy Mud }\end{array}$ & $\mathrm{N} / \mathrm{A}$ & $\begin{array}{l}\text { Slightly Medium Gravelly } \\
\text { Very Fine Sandy Mud }\end{array}$ & $\begin{array}{l}\text { Slightly Pebbly Very } \\
\text { Fine Sandy Mud }\end{array}$ & Fine Silt & Very Fine Silt & $\begin{array}{l}\text { Extremely } \\
\text { Poorly Sorted }\end{array}$ \\
\hline $\mathrm{JL}-09$ & Slightly Granuley & $\begin{array}{l}\text { Slightly Granuley Very } \\
\text { Fine Sandy Clay }\end{array}$ & $\begin{array}{l}\text { Slightly Gravelly } \\
\text { Sandy Mud }\end{array}$ & Sandy Mud & $\begin{array}{l}\text { Slightly Very Fine Gravelly } \\
\text { Very Fine Sandy Mud }\end{array}$ & $\begin{array}{l}\text { Slightly Granular Very } \\
\text { Fine Sandy Mud }\end{array}$ & Fine Silt & Very Fine Silt & $\begin{array}{l}\text { Extremely } \\
\text { Poorly Sorted }\end{array}$ \\
\hline $\mathrm{JL}-10$ & Pebbly & Pebbly Clay & Gravelly Mud & $\mathrm{N} / \mathrm{A}$ & Medium Gravelly Mud & Pebbly Mud & Fine Silt & Very Fine Silt & $\begin{array}{l}\text { Extremely } \\
\text { Poorly Sorted }\end{array}$ \\
\hline $\mathrm{JL}-11$ & Mud & Clay & Mud & Mud & Mud & Mud & Clay & Clay & $\begin{array}{l}\text { Very Poorly } \\
\text { Sorted }\end{array}$ \\
\hline $\mathrm{JL}-12$ & Mud & Clay & Mud & Mud & Mud & Mud & Clay & Clay & $\begin{array}{l}\text { Very Poorly } \\
\text { Sorted }\end{array}$ \\
\hline $\mathrm{JL}-13$ & Slightly Granuley & $\begin{array}{l}\text { Slightly Granuley Very } \\
\text { Fine Sandy Silt-Clay }\end{array}$ & $\begin{array}{l}\text { Slightly Gravelly } \\
\text { Sandy Mud }\end{array}$ & Sandy Mud & $\begin{array}{l}\text { Slightly Very Fine Gravelly } \\
\text { Very Fine Sandy Mud }\end{array}$ & $\begin{array}{l}\text { Slightly Granular Very } \\
\text { Fine Sandy Mud }\end{array}$ & Very Fine Silt & Clay & $\begin{array}{l}\text { Very Poorly } \\
\text { Sorted }\end{array}$ \\
\hline $\mathrm{JL}-14$ & Sandy Mud & Sandy Silt-Clay & Sandy Mud & Sandy Mud & Very Fine Sandy Mud & Very Fine Sandy Mud & Fine Silt & Very Fine Silt & $\begin{array}{l}\text { Very Poorly } \\
\text { Sorted }\end{array}$ \\
\hline $\mathrm{JL}-15$ & Slightly Granuley & $\begin{array}{l}\text { Slightly Granuley Very } \\
\text { Fine Sandy Silt-Clay }\end{array}$ & $\begin{array}{l}\text { Slightly Gravelly } \\
\text { Sandy Mud }\end{array}$ & Sandy Mud & $\begin{array}{l}\text { Slightly Very Fine Gravelly } \\
\text { Very Fine Sandy Mud }\end{array}$ & $\begin{array}{l}\text { Slightly Granular Very } \\
\text { Fine Sandy Mud }\end{array}$ & Fine Silt & Very Fine Silt & $\begin{array}{l}\text { Very Poorly } \\
\text { Sorted }\end{array}$ \\
\hline $\mathrm{JL}-16$ & Slightly Granuley & $\begin{array}{l}\text { Slightly Granul ey Silty- } \\
\text { Clayey Fine Sand }\end{array}$ & $\begin{array}{l}\text { Slightly Gravelly } \\
\text { Muddy Sand }\end{array}$ & N/A & $\begin{array}{l}\text { Slightly Very Fine Gravelly } \\
\text { Muddy Fine Sand }\end{array}$ & $\begin{array}{l}\text { Slightly Granular } \\
\text { Muddy Fine Sand }\end{array}$ & Fine Sand & Fine Sand & $\begin{array}{l}\text { Very Poorly } \\
\text { Sorted }\end{array}$ \\
\hline $\mathrm{JL}-17$ & Granuley & $\begin{array}{l}\text { Granuley Silty-Clayey } \\
\text { Very Fine Sand }\end{array}$ & $\begin{array}{l}\text { Gravelly Muddy } \\
\text { Sand }\end{array}$ & $\mathrm{N} / \mathrm{A}$ & $\begin{array}{l}\text { Very Fine Gravelly Muddy } \\
\text { Very Fine Sand }\end{array}$ & $\begin{array}{l}\text { Granular Muddy Very } \\
\text { Fine Sand }\end{array}$ & Fine Sand & Fine Sand & $\begin{array}{l}\text { Very Poorly } \\
\text { Sorted }\end{array}$ \\
\hline $\mathrm{JL}-18$ & Pebbly & $\begin{array}{l}\text { Pebbly Silty-Clayey } \\
\text { Fine Sand }\end{array}$ & $\begin{array}{l}\text { Gravelly Muddy } \\
\text { Sand }\end{array}$ & $\mathrm{N} / \mathrm{A}$ & $\begin{array}{l}\text { Medium Gravelly Muddy } \\
\text { Fine Sand }\end{array}$ & $\begin{array}{l}\text { Pebbly Muddy Fine } \\
\text { Sand }\end{array}$ & Medium Sand & Medium Sand & $\begin{array}{l}\text { Extremely } \\
\text { Poorly Sorted }\end{array}$ \\
\hline $\mathrm{JL}-19$ & Mud & Silt-Clay & Mud & Mud & Mud & Mud & Very Fine Silt & Clay & $\begin{array}{l}\text { Very Poorly } \\
\text { Sorted }\end{array}$ \\
\hline $\mathrm{JL}-20$ & Mud & Silt-Clay & Mud & Mud & Mud & Mud & Clay & Clay & $\begin{array}{l}\text { Very Poorly } \\
\text { Sorted }\end{array}$ \\
\hline
\end{tabular}




\section{Jeffreys Ledge Survey (2002-2005) (1): Grain Size Statistics}

\begin{tabular}{|c|c|c|c|c|c|c|c|c|c|c|c|c|c|c|c|c|c|c|c|}
\hline Sample ID & $\begin{array}{c}\text { Gravel } \\
\%\end{array}$ & $\begin{array}{c}\text { Sand } \\
\%\end{array}$ & $\begin{array}{c}\text { Mud } \\
\%\end{array}$ & $\begin{array}{c}\text { Sand } \\
\%\end{array}$ & $\begin{array}{c}\text { Silt } \\
\%\end{array}$ & $\begin{array}{c}\text { Clay } \\
\%\end{array}$ & Modes & $\begin{array}{c}\text { Mode } 1 \\
\text { (phi) }\end{array}$ & $\begin{array}{c}\text { Mode } 2 \\
\text { (phi) }\end{array}$ & $\begin{array}{l}D_{10} \\
\text { (phi) }\end{array}$ & $\begin{array}{c}D_{10} \\
(\mathrm{~mm})\end{array}$ & $\begin{array}{l}D_{50} \\
\text { (phi) }\end{array}$ & $\begin{array}{l}\mathrm{D}_{50} \\
(\mathrm{~mm})\end{array}$ & $\begin{array}{c}\text { Mean } \\
\text { Size } \\
\text { (phi) }\end{array}$ & $\begin{array}{l}\text { Mean } \\
\text { Size } \\
(\mathrm{mm})\end{array}$ & $\begin{array}{l}\text { Sorting } \\
\text { (phi) }\end{array}$ & Skewness & Kurtosis & LOI \% \\
\hline JL-01 & 0.0 & 0.9 & 99.1 & 0.9 & 39.7 & 59.4 & U & 7.50 & & 5.36 & 0.02 & 8.84 & 0.00 & 9.08 & 0.00 & 2.95 & 0.09 & 0.75 & 11.26 \\
\hline JL-02 & 0.0 & 1.8 & 98.2 & 1.8 & 42.9 & 55.3 & U & 7.50 & & 5.04 & 0.03 & 8.55 & 0.00 & 8.81 & 0.00 & 3.07 & 0.10 & 0.74 & 9.50 \\
\hline JL-03 & 0.0 & 1.0 & 99.0 & 1.0 & 42.4 & 56.6 & $\mathrm{U}$ & 6.50 & & 5.12 & 0.03 & 8.64 & 0.00 & 8.89 & 0.00 & 3.03 & 0.10 & 0.74 & 9.03 \\
\hline JL-04 & 0.0 & 0.5 & 99.5 & 0.5 & 36.5 & 63.0 & $\mathrm{U}$ & 7.50 & & 5.63 & 0.02 & 9.07 & 0.00 & 9.27 & 0.00 & 2.84 & 0.08 & 0.77 & 8.36 \\
\hline JL-05 & 0.0 & 0.4 & 99.6 & 0.4 & 33.6 & 66.0 & $\mathrm{U}$ & 8.48 & & 5.99 & 0.02 & 9.36 & 0.00 & 9.47 & 0.00 & 2.77 & 0.04 & 0.76 & 7.96 \\
\hline JL-06 & 0.0 & 1.1 & 98.9 & 1.1 & 29.1 & 69.8 & $\mathrm{U}$ & 6.47 & & 6.02 & 0.02 & 9.57 & 0.00 & 9.50 & 0.00 & 3.16 & 0.04 & 0.89 & 7.85 \\
\hline JL-07 & 0.6 & 19.5 & 79.9 & 19.5 & 25.4 & 54.5 & $\mathrm{~B}$ & 8.48 & 3.75 & 2.55 & 0.17 & 8.45 & 0.00 & 8.08 & 0.00 & 4.00 & -0.15 & 0.76 & 6.56 \\
\hline JL-08 & 4.5 & 18.5 & 77.0 & 18.5 & 24.2 & 52.8 & B & 8.48 & 12.52 & 1.92 & 0.26 & 8.26 & 0.00 & 7.84 & 0.00 & 4.34 & -0.19 & 0.87 & 8.30 \\
\hline JL-09 & 0.4 & 28.2 & 71.4 & 28.2 & 20.9 & 50.5 & $\mathrm{~B}$ & 3.24 & 8.48 & 2.47 & 0.18 & 8.05 & 0.00 & 7.70 & 0.00 & 4.06 & -0.10 & 0.66 & 6.97 \\
\hline JL-10 & 6.6 & 20.7 & 72.8 & 20.6 & 21.2 & 51.6 & $\mathrm{~T}$ & 8.48 & 3.24 & 0.85 & 0.56 & 8.16 & 0.00 & 7.50 & 0.01 & 4.83 & -0.27 & 0.89 & 6.49 \\
\hline JL-11 & 0.0 & 1.6 & 98.4 & 1.6 & 28.0 & 70.4 & U & 8.48 & & 6.30 & 0.01 & 9.44 & 0.00 & 9.68 & 0.00 & 2.63 & 0.07 & 0.83 & 8.84 \\
\hline JL-12 & 0.0 & 2.5 & 97.5 & 2.5 & 29.6 & 67.9 & U & 8.48 & & 5.93 & 0.02 & 9.35 & 0.00 & 9.51 & 0.00 & 2.80 & 0.02 & 0.86 & 8.54 \\
\hline JL-13 & 0.2 & 17.3 & 82.5 & 17.3 & 30.8 & 51.7 & $\mathrm{~B}$ & 3.73 & 8.48 & 3.61 & 0.08 & 8.16 & 0.00 & 8.06 & 0.00 & 3.59 & -0.01 & 0.69 & 6.76 \\
\hline JL-14 & 0.0 & 21.6 & 78.4 & 21.6 & 28.9 & 49.5 & B & 3.73 & 8.48 & 3.36 & 0.10 & 7.96 & 0.00 & 7.90 & 0.00 & 3.69 & 0.00 & 0.68 & 5.30 \\
\hline JL-15 & 0.1 & 26.0 & 73.9 & 26.0 & 30.5 & 43.4 & B & 3.73 & 8.48 & 3.32 & 0.10 & 7.23 & 0.01 & 7.54 & 0.01 & 3.60 & 0.15 & 0.65 & 5.74 \\
\hline JL-16 & 4.9 & 79.8 & 15.3 & 79.8 & 5.2 & 10.1 & U & 3.24 & & -0.38 & 1.30 & 2.12 & 0.23 & 2.07 & 0.24 & 2.70 & 0.21 & 1.99 & 1.70 \\
\hline JL-17 & 9.7 & 72.1 & 18.2 & 72.1 & 6.2 & 12.0 & $\mathrm{U}$ & 3.24 & & -0.96 & 1.94 & 2.31 & 0.20 & 2.54 & 0.17 & 3.40 & 0.26 & 1.97 & 1.70 \\
\hline JL-18 & 26.4 & 55.5 & 18.1 & 55.5 & 6.5 & 11.6 & $\mathrm{~B}$ & -3.74 & 2.24 & -3.64 & 12.45 & 1.68 & 0.31 & 1.34 & 0.39 & 4.57 & 0.08 & 1.49 & 2.46 \\
\hline JL-19 & 0.0 & 10.0 & 90.0 & 10.0 & 36.7 & 53.3 & $\mathrm{U}$ & 3.73 & & 4.01 & 0.06 & 8.36 & 0.00 & 8.49 & 0.00 & 3.34 & 0.05 & 0.74 & 10.70 \\
\hline JL-20 & 0.0 & 0.5 & 99.5 & 0.5 & 34.5 & 65.0 & U & 7.50 & & 5.83 & 0.02 & 9.35 & 0.00 & 9.44 & 0.00 & 2.80 & 0.02 & 0.77 & 8.71 \\
\hline
\end{tabular}




\section{Jeffreys Ledge Survey (2002-2005) (1): Grain Size Distribution}

\begin{tabular}{|c|c|c|c|c|c|c|c|c|c|c|c|c|c|c|c|c|c|c|c|c|c|c|c|c|c|c|c|c|c|c|c|c|}
\hline Sample ID & $\begin{array}{c}\text { Class } \\
\% \\
\text { phi } \\
-5.5\end{array}$ & $\begin{array}{c}\text { Class } \\
\% \\
\text { phi } \\
-5.0 \\
\end{array}$ & $\begin{array}{c}\text { Class } \\
\% \\
\text { phi } \\
-4.5 \\
\end{array}$ & $\begin{array}{c}\text { Class } \\
\% \\
\text { phi } \\
-4.0 \\
\end{array}$ & $\begin{array}{c}\text { Class } \\
\% \\
\text { phi } \\
-3.5 \\
\end{array}$ & $\begin{array}{c}\text { Class } \\
\% \\
\text { phi } \\
-3.0 \\
\end{array}$ & $\begin{array}{c}\text { Class } \\
\% \\
\text { phi } \\
-2.5 \\
\end{array}$ & $\begin{array}{c}\text { Class } \\
\% \\
\text { phi } \\
-2.0 \\
\end{array}$ & $\begin{array}{c}\text { Class } \\
\% \\
\text { phi } \\
-1.5 \\
\end{array}$ & $\begin{array}{c}\text { Class } \\
\% \\
\text { phi } \\
-1.0 \\
\end{array}$ & $\begin{array}{c}\text { Class } \\
\% \\
\text { phi } \\
-0.5 \\
\end{array}$ & $\begin{array}{c}\text { Class } \\
\% \\
\text { phi } \\
0.0 \\
\end{array}$ & $\begin{array}{c}\text { Class } \\
\% \\
\text { phi } \\
0.5 \\
\end{array}$ & $\begin{array}{c}\text { Class } \\
\% \\
\text { phi } \\
1.0 \\
\end{array}$ & $\begin{array}{c}\text { Class } \\
\% \\
\text { phi } \\
1.5 \\
\end{array}$ & $\begin{array}{c}\text { Class } \\
\% \\
\text { phi } \\
2.0 \\
\end{array}$ & $\begin{array}{c}\text { Class } \\
\% \\
\text { phi } \\
2.5 \\
\end{array}$ & $\begin{array}{c}\text { Class } \\
\% \\
\text { phi } \\
3.0 \\
\end{array}$ & $\begin{array}{c}\text { Class } \\
\% \\
\text { phi } \\
3.5 \\
\end{array}$ & $\begin{array}{c}\text { Class } \\
\% \\
\text { phi } \\
4.0 \\
\end{array}$ & $\begin{array}{c}\text { Class } \\
\% \\
\text { phi } \\
>4.0 \\
\end{array}$ & $\begin{array}{c}\text { Class } \\
\% \\
\text { phi } \\
5.0\end{array}$ & $\begin{array}{c}\text { Class } \\
\% \\
\text { phi } \\
6.0 \\
\end{array}$ & $\begin{array}{c}\text { Class } \\
\% \\
\text { phi } \\
7.0 \\
\end{array}$ & $\begin{array}{c}\text { Class } \\
\% \\
\text { phi } \\
8.0 \\
\end{array}$ & $\begin{array}{c}\text { Class } \\
\% \\
\text { phi } \\
9.0 \\
\end{array}$ & $\begin{array}{c}\text { Class } \\
\% \\
\text { phi } \\
10.0 \\
\end{array}$ & $\begin{array}{c}\text { Class } \\
\% \\
\text { phi } \\
>10.0 \\
\end{array}$ & $\begin{array}{c}\text { Class } \\
\% \\
\text { phi } \\
11.0 \\
\end{array}$ & $\begin{array}{c}\text { Class } \\
\% \\
\text { phi } \\
12.0 \\
\end{array}$ & $\begin{array}{c}\text { Class } \\
\% \\
\text { phi } \\
13.0 \\
\end{array}$ & $\begin{array}{c}\text { S Class } \\
\% \\
\text { phi } \\
14.0 \\
\end{array}$ \\
\hline L-01 & 0.00 & 0.00 & 0.00 & 0.00 & 0.00 & 0.00 & 0.00 & 0.00 & 0.00 & 0.00 & 0.00 & 0.00 & 0.00 & 0.00 & 0.00 & 0.00 & 0.00 & 0.00 & 0.00 & 0.80 & & 5.79 & 9.82 & 11.46 & 612.67 & 710.91 & 8.06 & & 10.12 & 10.12 & 210.12 & 210.12 \\
\hline $\mathrm{L}-02$ & 0.00 & 0.00 & 0.00 & 0.00 & 0.00 & 0.00 & 0.00 & 0.00 & 0.00 & 0.00 & 0.00 & 0.00 & 0.00 & 0.00 & 0.00 & 0.00 & 0.00 & 0.00 & 0.00 & 1.72 & & 7.96 & 11.59 & 911.32 & 212.05 & 59.40 & 8.42 & & 9.39 & 9.39 & 9.39 & $\begin{array}{l}9 \\
9\end{array}$ \\
\hline $\mathrm{LL}-03$ & 0.00 & 0.00 & 0.00 & 0.00 & 0.00 & 0.00 & 0.00 & 0.00 & 0.00 & 0.00 & 0.00 & 0.00 & 0.00 & 0.00 & 0.00 & 0.00 & 0.00 & 0.00 & 0.00 & 0.96 & & 7.81 & 10.89 & 912.46 & 611.30 & 9.88 & 8.26 & & 9.61 & 9.61 & 9.61 & $1 \quad 9.61$ \\
\hline JL-04 & 0.00 & 0.00 & 0.00 & 0.00 & 0.00 & 0.00 & 0.00 & 0.00 & 0.00 & 0.00 & 0.00 & 0.00 & 0.00 & 0.00 & 0.00 & 0.00 & 0.00 & 0.00 & 0.00 & 0.49 & & 3.94 & 8.96 & 10.63 & 312.93 & 312.10 & 9.26 & & 10.42 & 10.42 & 210.42 & 210.42 \\
\hline $\mathrm{JL}-05$ & 0.00 & 0.00 & 0.00 & 0.00 & 0.00 & 0.00 & 0.00 & 0.00 & 0.00 & 0.00 & 0.00 & 0.00 & 0.00 & 0.00 & 0.00 & 0.00 & 0.00 & 0.00 & 0.00 & 0.35 & & 2.70 & 7.07 & 11.73 & 312.10 & 11.96 & 10.62 & & 10.87 & 10.87 & 710.87 & 710.87 \\
\hline JL-06 & 0.00 & 0.00 & 0.00 & 0.00 & 0.00 & 0.00 & 0.00 & 0.00 & 0.00 & 0.00 & 0.00 & 0.00 & 0.00 & 0.00 & 0.00 & 0.00 & 0.00 & 0.00 & 0.00 & 1.02 & & 5.05 & 3.01 & 17.15 & $\begin{array}{ll}5 \quad 3.92 \\
\end{array}$ & 13.23 & 11.05 & & 11.39 & 11.39 & 911.39 & $911.3 \mathrm{~s}$ \\
\hline LL-07 & 0.00 & 0.00 & 0.00 & 0.00 & 0.00 & 0.00 & 0.00 & 0.00 & 0.22 & 0.40 & 0.22 & 0.49 & 0.95 & 1.51 & 1.79 & 2.07 & 2.09 & 2.35 & 3.24 & 4.77 & & 7.35 & 9.11 & 1.61 & 7.35 & 9.74 & 9.38 & & 8.85 & 8.85 & 58.84 & 8.84 \\
\hline $\mathrm{JL}-08$ & 0.00 & 0.00 & 0.00 & 0.00 & 0.00 & 3.56 & 0.00 & 0.41 & 0.40 & 0.17 & 0.33 & 0.37 & 0.62 & 1.00 & 1.49 & 1.97 & 2.18 & 2.70 & 3.48 & 4.34 & & 3.45 & 4.08 & 6.36 & 10.32 & 210.55 & 8.31 & & 8.48 & 8.48 & 38.48 & $\begin{array}{l}3 \quad 8.48 \\
\end{array}$ \\
\hline JL-09 & 0.00 & 0.00 & 0.00 & 0.00 & 0.00 & 0.00 & 0.00 & 0.00 & 0.00 & 0.39 & 0.35 & 0.17 & 0.72 & 1.13 & 1.63 & 2.35 & 3.27 & 6.39 & 7.27 & 4.93 & & 2.11 & 2.76 & 6.28 & 39.75 & 9.97 & 7.70 & & 8.21 & 8.21 & 8.21 & 18.21 \\
\hline IL-10 & 0.00 & 0.00 & 0.00 & 0.00 & 0.00 & 4.68 & 0.00 & 1.09 & 0.00 & 0.79 & 0.53 & 0.71 & 1.05 & 1.65 & 2.05 & 2.25 & 2.07 & 2.44 & 3.97 & 3.93 & & 2.27 & 3.55 & 5.79 & 9.60 & 9.87 & 9.18 & & 8.13 & 8.13 & 8.13 & $\begin{array}{l}3 \quad 8.13 \\
\end{array}$ \\
\hline JL-11 & 0.00 & 0.00 & 0.00 & 0.00 & 0.00 & 0.00 & 0.00 & 0.00 & 0.00 & 0.00 & 0.00 & 0.00 & 0.00 & 0.00 & 0.00 & 0.00 & 0.00 & 0.00 & 0.00 & 1.54 & & 2.21 & 3.98 & 7.70 & 14.17 & 714.92 & 11.78 & & 10.92 & 10.92 & 210.92 & 210.92 \\
\hline JL-12 & 0.00 & 0.00 & 0.00 & 0.00 & 0.00 & 0.00 & 0.00 & 0.00 & 0.00 & 0.00 & 0.00 & 0.00 & 0.00 & 0.00 & 0.00 & 0.00 & 0.00 & 0.00 & 0.00 & 2.50 & & 3.47 & 4.37 & 8.55 & 513.19 & 13.63 & 11.70 & & 10.65 & 10.65 & 510.65 & 510.65 \\
\hline $\mathrm{JL}-13$ & 0.00 & 0.00 & 0.00 & 0.00 & 0.00 & 0.00 & 0.00 & 0.00 & 0.00 & 0.16 & 0.09 & 0.10 & 0.12 & 0.09 & 0.23 & 0.44 & 0.57 & 1.18 & 4.35 & 10.08 & & 8.95 & 5.34 & 6.67 & 9.90 & 10.29 & 8.50 & & 8.24 & 8.24 & $\begin{array}{r}8.24 \\
\end{array}$ & $\begin{array}{l}4 \quad 8.24 \\
\end{array}$ \\
\hline $\mathrm{JL}-14$ & 0.00 & 0.00 & 0.00 & 0.00 & 0.00 & 0.00 & 0.00 & 0.00 & 0.00 & 0.00 & 0.04 & 0.20 & 0.29 & 0.46 & 0.70 & 1.05 & 1.08 & 2.12 & 5.31 & 10.22 & & 7.67 & 5.61 & 6.49 & 9.18 & 9.44 & 8.15 & & 8.00 & 8.00 & 8.00 & 8.00 \\
\hline JL-15 & 0.00 & 0.00 & 0.00 & 0.00 & 0.00 & 0.00 & 0.00 & 0.00 & 0.00 & 0.09 & 0.15 & 0.06 & 0.17 & 0.22 & 0.42 & 0.63 & 0.94 & 2.22 & 7.63 & 13.45 & & 11.29 & 5.12 & 5.71 & 8.43 & 8.58 & 6.39 & & 7.13 & 7.13 & 7.13 & 7.13 \\
\hline JL-16 & 0.00 & 0.00 & 0.00 & 0.00 & 0.00 & 0.00 & 0.00 & 0.34 & 2.06 & 2.51 & 4.09 & 4.62 & 6.15 & 7.89 & 9.06 & 10.68 & 10.40 & 11.94 & 10.93 & 4.05 & & 1.37 & 0.91 & 0.95 & 2.00 & 1.93 & 2.09 & & 1.51 & 1.51 & 1.51 & 1.51 \\
\hline JL-17 & 0.00 & 0.00 & 0.00 & 0.00 & 0.00 & 0.00 & 0.00 & 2.94 & 3.07 & 3.69 & 3.65 & 4.04 & 4.80 & 6.09 & 7.42 & 9.35 & 7.59 & 10.77 & 11.81 & 6.56 & & 1.67 & 1.25 & 1.06 & 2.25 & 2.06 & 2.01 & & 1.99 & 1.99 & 1.99 & $\begin{array}{ll}9 & 1.99 \\
\end{array}$ \\
\hline $\mathrm{JL}-18$ & 0.00 & 0.00 & 0.00 & 0.00 & 14.23 & 5.82 & 0.68 & 1.20 & 2.06 & 2.40 & 2.48 & 2.33 & 3.48 & 5.06 & 6.76 & 9.47 & 10.13 & 10.26 & 4.40 & 1.10 & & 1.28 & 1.28 & 1.79 & 2.15 & 2.28 & 1.56 & & 1.95 & 1.95 & 1.95 & $5 \quad 1.95$ \\
\hline $\mathrm{JL}-19$ & 0.00 & 0.00 & 0.00 & 0.00 & 0.00 & 0.00 & 0.00 & 0.00 & 0.00 & 0.00 & 0.00 & 0.00 & 0.00 & 0.00 & 0.00 & 0.00 & 0.00 & 0.00 & 0.00 & 9.88 & & 7.44 & 8.43 & 10.23 & 310.63 & 39.19 & 7.32 & & 9.22 & 9.22 & 9.22 & 9.22 \\
\hline JL-20 & 0.00 & 0.00 & 0.00 & 0.00 & 0.00 & 0.00 & 0.00 & 0.00 & 0.00 & 0.00 & 0.00 & 0.00 & 0.00 & 0.00 & 0.00 & 0.00 & 0.00 & 0.00 & 0.00 & 0.47 & & 3.55 & 7.27 & 10.99 & 912.74 & 10.85 & 11.23 & & 10.73 & 10.73 & B 10.73 & 310.73 \\
\hline
\end{tabular}


Jeffreys Ledge Survey (2002-2005) (2): Identification, Location, and Description

\begin{tabular}{|c|c|c|c|c|c|c|c|c|}
\hline Sample ID & $\begin{array}{c}\text { Original } \\
\text { Sample ID }\end{array}$ & $\begin{array}{c}\text { Latitude } \\
\text { WGS84 }\end{array}$ & $\begin{array}{l}\text { Longitude } \\
\text { WGS84 }\end{array}$ & $\begin{array}{l}\text { Reliability } \\
\text { Ranking for } \\
\text { Positioning }\end{array}$ & $\begin{array}{c}\text { Water } \\
\text { Depth } \\
\text { (m) }\end{array}$ & $\begin{array}{l}\text { Sample } \\
\text { Collected }\end{array}$ & $\begin{array}{c}\text { Sampler } \\
\text { Type }\end{array}$ & $\begin{array}{c}\text { Total Wt } \\
\text { (gm) }\end{array}$ \\
\hline $\mathrm{JL}-21$ & JL23 & 42.920510 & -70.330420 & 1 & 156 & $7 / 30 / 2002$ & $B C$ & 16.90 \\
\hline $\mathrm{JL}-22$ & $\mathrm{JL} 24$ & 42.920660 & -70.313830 & 1 & 154 & $7 / 30 / 2002$ & $\mathrm{BC}$ & 15.60 \\
\hline $\mathrm{JL}-23$ & $\mathrm{JL} 25$ & 42.920750 & -70.297350 & 1 & 168 & $7 / 30 / 2002$ & $\mathrm{BC}$ & 15.50 \\
\hline $\mathrm{JL}-24$ & JL26 & 42.920510 & -70.279930 & 1 & 152 & $7 / 30 / 2002$ & $\mathrm{BC}$ & 28.40 \\
\hline $\mathrm{JL}-25$ & $\mathrm{JL} 27$ & 42.920480 & -70.262640 & 1 & 143 & $7 / 30 / 2002$ & $\mathrm{BC}$ & 32.60 \\
\hline $\mathrm{JL}-26$ & JL28 & 42.920980 & -70.247670 & 1 & 128 & $8 / 19 / 2002$ & $\mathrm{BC}$ & 22.50 \\
\hline JL-27 & JL29 & 42.920700 & -70.229820 & 1 & 132 & $8 / 19 / 2002$ & $B C$ & 20.00 \\
\hline $\mathrm{JL}-28$ & JL30 & 42.920280 & -70.213860 & 1 & 177 & $8 / 19 / 2002$ & $B C$ & 13.10 \\
\hline JL-29 & $\mathrm{JL} 31$ & 42.920970 & -70.197230 & 1 & 139 & $8 / 19 / 2002$ & $\mathrm{BC}$ & 24.10 \\
\hline $\mathrm{JL}-30$ & JL32 & 42.920500 & -70.180290 & 1 & 124 & $8 / 19 / 2002$ & $\mathrm{BC}$ & 25.30 \\
\hline JL-31 & JL33 & 42.920790 & -70.163120 & 1 & 119 & $9 / 19 / 2002$ & $\mathrm{BC}$ & 52.00 \\
\hline $\mathrm{JL}-32$ & JL34b & 42.920650 & -70.146640 & 1 & 100 & $9 / 19 / 2002$ & $\mathrm{BC}$ & 155.90 \\
\hline JL-34 & JL39 & 42.908630 & -70.364150 & 1 & 108 & $7 / 26 / 2002$ & $\mathrm{SH}$ & 55.10 \\
\hline $\mathrm{JL}-35$ & JL40 & 42.907390 & -70.346320 & 1 & 137 & $7 / 31 / 2002$ & $\mathrm{BC}$ & 17.10 \\
\hline $\mathrm{JL}-36$ & JL41 & 42.908380 & -70.329830 & 1 & 163 & $7 / 31 / 2002$ & $\mathrm{BC}$ & 19.00 \\
\hline $\mathrm{JL}-37$ & JL42 & 42.908260 & -70.313180 & 1 & 161 & $7 / 31 / 2002$ & $\mathrm{BC}$ & 18.90 \\
\hline $\mathrm{JL}-38$ & $\mathrm{JL} 43$ & 42.907950 & -70.296030 & 1 & 161 & $7 / 31 / 2002$ & $\mathrm{BC}$ & 12.90 \\
\hline JL-39 & JL44 & 42.908290 & -70.275020 & 1 & 146 & $7 / 31 / 2002$ & $B C$ & 17.10 \\
\hline $\mathrm{JL}-40$ & JL45 & 42.908040 & -70.262160 & 1 & 150 & $7 / 31 / 2002$ & $B C$ & 13.50 \\
\hline $\mathrm{JL}-41$ & JL46 & 42.909010 & -70.248160 & 1 & 157 & $8 / 26 / 2002$ & $\mathrm{BC}$ & 18.60 \\
\hline JL-42 & JL47 & 42.908380 & -70.230680 & 1 & 153 & $8 / 26 / 2002$ & $B C$ & 10.70 \\
\hline
\end{tabular}


Jeffreys Ledge Survey (2002-2005) (2): Sediment Classifications

\begin{tabular}{|c|c|c|c|c|c|c|c|c|c|}
\hline Sample ID & $\begin{array}{l}\text { CMECS Substrate } \\
\text { Component } \\
\text { Group (Specific) }\end{array}$ & $\begin{array}{l}\text { CMECS Substrate } \\
\text { Component } \\
\text { Subgroup (Specific) }\end{array}$ & $\begin{array}{l}\text { Textural Group } \\
\text { from \%GSM } \\
\text { (Gradistat) }\end{array}$ & $\begin{array}{l}\text { Textural Group } \\
\text { from \%SZC } \\
\text { (Gradistat) }\end{array}$ & $\begin{array}{l}\text { Sediment Name from } \\
\text { \%GSM } \\
\text { and Mode (Gradistat) }\end{array}$ & $\begin{array}{l}\text { Sediment Name from } \\
\text { \%GSM and Mode } \\
\text { (Wentworth Scale) }\end{array}$ & $\begin{array}{l}\text { Sediment } \\
\text { Classification from } \\
\text { Mean Phi (Gradistat) }\end{array}$ & $\begin{array}{l}\text { Classification } \\
\text { from Mean Phi } \\
\text { (Wentworth) }\end{array}$ & $\begin{array}{l}\text { Sorting } \\
\text { (Gradistat) }\end{array}$ \\
\hline $\mathrm{JL}-21$ & Mud & Silt-Clay & Mud & Mud & Mud & Mud & Clay & Clay & $\begin{array}{l}\text { Very Poorly } \\
\text { Sorted }\end{array}$ \\
\hline $\mathrm{JL}-22$ & Mud & Clay & Mud & Mud & Mud & Mud & Clay & Clay & $\begin{array}{l}\text { Very Poorly } \\
\text { Sorted }\end{array}$ \\
\hline $\mathrm{JL}-23$ & Mud & Silt-Clay & Mud & Mud & Mud & Mud & Clay & Clay & $\begin{array}{l}\text { Very Poorly } \\
\text { Sorted }\end{array}$ \\
\hline $\mathrm{JL}-24$ & Sandy Mud & Sandy Clay & Sandy Mud & Sandy Mud & Very Fine Sandy Mud & Very Fine Sandy Mud & Very Fine Silt & Clay & $\begin{array}{l}\text { Very Poorly } \\
\text { Sorted }\end{array}$ \\
\hline $\mathrm{JL}-25$ & Slightly Granuley & $\begin{array}{l}\text { Slightly Granuley Very } \\
\text { Fine Sandy Silt-Clay }\end{array}$ & $\begin{array}{l}\text { Slightly Gravelly } \\
\text { Sandy Mud }\end{array}$ & Sandy Mud & $\begin{array}{l}\text { Slightly Very Fine Gravelly } \\
\text { Very Fine Sandy Mud }\end{array}$ & $\begin{array}{l}\text { Slightly Granular Very } \\
\text { Fine Sandy Mud }\end{array}$ & Fine Silt & Very Fine Silt & $\begin{array}{l}\text { Very Poorly } \\
\text { Sorted }\end{array}$ \\
\hline $\mathrm{JL}-26$ & Slightly Granuley & $\begin{array}{l}\text { Slightly Granuley Very } \\
\text { Fine Sandy Clay }\end{array}$ & $\begin{array}{l}\text { Slightly Gravelly } \\
\text { Sandy Mud }\end{array}$ & $\mathrm{N} / \mathrm{A}$ & $\begin{array}{l}\text { Slightly Very Fine Gravelly } \\
\text { Very Fine Sandy Mud }\end{array}$ & $\begin{array}{l}\text { Slightly Granular Very } \\
\text { Fine Sandy Mud }\end{array}$ & Medium Silt & Fine Silt & $\begin{array}{l}\text { Extremely } \\
\text { Poorly Sorted }\end{array}$ \\
\hline $\mathrm{JL}-27$ & Slightly Granuley & $\begin{array}{l}\text { Slightly Granuley Very } \\
\text { Fine Sandy Clay }\end{array}$ & $\begin{array}{l}\text { Slightly Gravelly } \\
\text { Sandy Mud }\end{array}$ & Sandy Mud & $\begin{array}{l}\text { Slightly Very Fine Gravelly } \\
\text { Very Fine Sandy Mud }\end{array}$ & $\begin{array}{l}\text { Slightly Granular Very } \\
\text { Fine Sandy Mud }\end{array}$ & Fine Silt & Very Fine Silt & $\begin{array}{l}\text { Very Poorly } \\
\text { Sorted }\end{array}$ \\
\hline $\mathrm{JL}-28$ & Mud & Clay & Mud & Mud & Mud & Mud & Clay & Clay & $\begin{array}{l}\text { Very Poorly } \\
\text { Sorted }\end{array}$ \\
\hline $\mathrm{JL}-29$ & Slightly Granuley & $\begin{array}{l}\text { Slightly Granuley Very } \\
\text { Fine Sandy Silt-Clay }\end{array}$ & $\begin{array}{l}\text { Slightly Gravelly } \\
\text { Sandy Mud }\end{array}$ & Sandy Mud & $\begin{array}{l}\text { Slightly Very Fine Gravelly } \\
\text { Very Fine Sandy Mud }\end{array}$ & $\begin{array}{l}\text { Slightly Granular Very } \\
\text { Fine Sandy Mud }\end{array}$ & Fine Silt & Very Fine Silt & $\begin{array}{l}\text { Very Poorly } \\
\text { Sorted }\end{array}$ \\
\hline $\mathrm{JL}-30$ & Sandy Mud & Sandy Silt-Clay & Sandy Mud & Sandy Mud & Very Fine Sandy Mud & Very Fine Sandy Mud & Medium Silt & Fine Silt & $\begin{array}{l}\text { Very Poorly } \\
\text { Sorted }\end{array}$ \\
\hline $\mathrm{JL}-31$ & Slightly Granuley & $\begin{array}{l}\text { Slightly Granul ey Silty- } \\
\text { Clayey Very Fine Sand }\end{array}$ & $\begin{array}{l}\text { - Slightly Gravelly } \\
\text { Muddy Sand }\end{array}$ & Muddy Sand & $\begin{array}{l}\text { Slightly Very Fine Gravelly } \\
\text { Muddy Very Fine Sand }\end{array}$ & $\begin{array}{l}\text { Slightly Granular } \\
\text { Muddy Very Fine Sand }\end{array}$ & Very Coarse Silt & Coarse Silt & $\begin{array}{l}\text { Very Poorly } \\
\text { Sorted }\end{array}$ \\
\hline $\mathrm{JL}-32$ & Pebble Mixes & $\begin{array}{l}\text { Clayey Sandy Pebble } \\
\text { Gravel }\end{array}$ & $\begin{array}{l}\text { Muddy Sandy } \\
\text { Gravel }\end{array}$ & $\mathrm{N} / \mathrm{A}$ & $\begin{array}{l}\text { Muddy Sandy Coarse } \\
\text { Gravel }\end{array}$ & $\begin{array}{l}\text { Muddy Sandy Pebble } \\
\text { Gravel }\end{array}$ & Very Coarse Sand & $\begin{array}{l}\text { Very Coarse } \\
\text { Sand }\end{array}$ & $\begin{array}{l}\text { Very Poorly } \\
\text { Sorted }\end{array}$ \\
\hline $\mathrm{JL}-34$ & Pebbly & $\begin{array}{l}\text { Pebbly Silty-Clayey } \\
\text { Very fine Sand }\end{array}$ & $\begin{array}{l}\text { Gravelly Muddy } \\
\text { Sand }\end{array}$ & $\mathrm{N} / \mathrm{A}$ & $\begin{array}{l}\text { Medium Gravelly Muddy } \\
\text { Very Fine Sand }\end{array}$ & $\begin{array}{l}\text { Pebbly Muddy Very } \\
\text { Fine Sand }\end{array}$ & Very Fine Sand & Very Fine Sand & $\begin{array}{l}\text { Extremely } \\
\text { Poorly Sorted }\end{array}$ \\
\hline $\mathrm{JL}-35$ & Sandy Mud & Sandy Clay & Sandy Mud & Sandy Mud & Very Fine Sandy Mud & Very Fine Sandy Mud & Very Fine Silt & Clay & $\begin{array}{l}\text { Very Poorly } \\
\text { Sorted }\end{array}$ \\
\hline $\mathrm{JL}-36$ & Mud & Clay & Mud & Mud & Mud & Mud & Clay & Clay & $\begin{array}{l}\text { Very Poorly } \\
\text { Sorted }\end{array}$ \\
\hline $\mathrm{JL}-37$ & Mud & Silt-Clay & Mud & Mud & Mud & Mud & Clay & Clay & $\begin{array}{l}\text { Very Poorly } \\
\text { Sorted }\end{array}$ \\
\hline $\mathrm{JL}-38$ & Mud & Clay & Mud & Mud & Mud & Mud & Clay & Clay & $\begin{array}{l}\text { Very Poorly } \\
\text { Sorted }\end{array}$ \\
\hline $\mathrm{JL}-39$ & Mud & Clay & Mud & Mud & Mud & Mud & Clay & Clay & $\begin{array}{l}\text { Very Poorly } \\
\text { Sorted }\end{array}$ \\
\hline $\mathrm{JL}-40$ & Mud & Clay & Mud & Mud & Mud & Mud & Clay & Clay & $\begin{array}{l}\text { Very Poorly } \\
\text { Sorted }\end{array}$ \\
\hline JL-41 & Mud & Silt-Clay & Mud & Mud & Mud & Mud & Clay & Clay & $\begin{array}{l}\text { Very Poorly } \\
\text { Sorted }\end{array}$ \\
\hline JL-42 & Mud & Silt-Clay & Mud & Mud & Mud & Mud & Clay & Clay & $\begin{array}{l}\text { Very Poorly } \\
\text { Sorted }\end{array}$ \\
\hline
\end{tabular}


Jeffreys Ledge Survey (2002-2005) (2): Grain Size Statistics

\begin{tabular}{|c|c|c|c|c|c|c|c|c|c|c|c|c|c|c|c|c|c|c|c|}
\hline Sample ID & $\begin{array}{c}\text { Gravel } \\
\% \\
\end{array}$ & $\begin{array}{c}\text { Sand } \\
\%\end{array}$ & $\begin{array}{c}\text { Mud } \\
\%\end{array}$ & $\begin{array}{c}\text { Sand } \\
\%\end{array}$ & $\begin{array}{c}\text { Silt } \\
\%\end{array}$ & $\begin{array}{c}\text { Clay } \\
\%\end{array}$ & Modes & $\begin{array}{c}\text { Mode } 1 \\
\text { (phi) }\end{array}$ & $\begin{array}{c}\text { Mode } 2 \\
\text { (phi) }\end{array}$ & $\begin{array}{r}D_{10} \\
\text { (phi) }\end{array}$ & $\begin{array}{c}D_{10} \\
(\mathrm{~mm})\end{array}$ & $\begin{array}{r}\mathrm{D}_{50} \\
\text { (phi) }\end{array}$ & $\begin{array}{c}D_{50} \\
(\mathrm{~mm})\end{array}$ & $\begin{array}{c}\text { Mean } \\
\text { Size } \\
\text { (phi) }\end{array}$ & $\begin{array}{c}\text { Mean } \\
\text { Size } \\
(\mathrm{mm}) \\
\end{array}$ & $\begin{array}{c}\text { Sorting } \\
\text { (phi) }\end{array}$ & Skewness & Kurtosis & LOI \% \\
\hline $\mathrm{JL}-21$ & 0.0 & 1.9 & 98.1 & 1.9 & 32.8 & 65.3 & U & 7.50 & & 5.77 & 0.02 & 9.38 & 0.00 & 9.46 & 0.00 & 2.83 & 0.01 & 0.77 & 8.51 \\
\hline JL-22 & 0.0 & 0.6 & 99.4 & 0.6 & 30.5 & 68.9 & U & 8.48 & & 6.13 & 0.01 & 9.51 & 0.00 & 9.63 & 0.00 & 2.70 & 0.03 & 0.79 & 8.55 \\
\hline $\mathrm{JL}-23$ & 0.0 & 2.6 & 97.4 & 2.6 & 31.2 & 66.2 & U & 8.48 & & 5.68 & 0.02 & 9.31 & 0.00 & 9.43 & 0.00 & 2.87 & 0.01 & 0.83 & 7.67 \\
\hline JL-24 & 0.0 & 10.9 & 89.1 & 10.9 & 28.9 & 60.2 & B & 8.48 & 3.73 & 3.92 & 0.07 & 8.80 & 0.00 & 8.78 & 0.00 & 3.32 & -0.03 & 0.86 & 11.41 \\
\hline JL-25 & 0.1 & 30.1 & 69.8 & 30.1 & 25.4 & 44.4 & B & 3.73 & 8.48 & 2.91 & 0.13 & 7.33 & 0.01 & 7.46 & 0.01 & 3.82 & 0.05 & 0.69 & 4.39 \\
\hline JL-26 & 2.8 & 34.6 & 62.6 & 34.6 & 20.0 & 42.6 & B & 3.24 & 8.48 & 1.31 & 0.40 & 7.07 & 0.01 & 6.95 & 0.01 & 4.34 & -0.05 & 0.76 & 5.35 \\
\hline $\mathrm{JL}-27$ & 0.3 & 27.8 & 71.9 & 27.8 & 22.0 & 49.9 & B & 3.24 & 8.48 & 2.56 & 0.17 & 7.99 & 0.00 & 7.71 & 0.00 & 3.98 & -0.09 & 0.68 & 5.84 \\
\hline $\mathrm{JL}-28$ & 0.0 & 0.8 & 99.2 & 0.8 & 29.6 & 69.6 & U & 8.48 & & 6.19 & 0.01 & 9.44 & 0.00 & 9.63 & 0.00 & 2.67 & 0.05 & 0.82 & 8.33 \\
\hline JL-29 & 0.1 & 24.5 & 75.5 & 24.5 & 29.7 & 45.8 & B & 3.73 & 8.48 & 3.21 & 0.11 & 7.51 & 0.01 & 7.65 & 0.00 & 3.68 & 0.08 & 0.65 & 6.20 \\
\hline $\mathrm{JL}-30$ & 0.0 & 40.1 & 59.9 & 40.1 & 30.5 & 29.4 & U & 3.73 & & 3.24 & 0.11 & 4.61 & 0.04 & 6.26 & 0.01 & 3.32 & 0.69 & 0.79 & 4.33 \\
\hline JL-31 & 0.2 & 71.3 & 28.6 & 71.3 & 10.7 & 17.9 & U & 3.24 & & 2.17 & 0.22 & 3.29 & 0.10 & 4.79 & 0.04 & 3.13 & 0.71 & 1.87 & 2.80 \\
\hline $\mathrm{JL}-32$ & 39.2 & 51.9 & 8.9 & 51.9 & 2.6 & 6.3 & B & -4.24 & 2.24 & -4.10 & 17.18 & 0.35 & 0.78 & -0.21 & 1.16 & 3.68 & 0.02 & 1.03 & 1.70 \\
\hline JL-34 & 16.1 & 55.7 & 28.2 & 55.7 & 10.2 & 18.0 & B & 3.24 & -3.74 & -3.00 & 7.99 & 3.13 & 0.11 & 3.54 & 0.09 & 4.83 & 0.14 & 1.80 & 2.88 \\
\hline $\mathrm{JL}-35$ & 0.0 & 15.3 & 84.7 & 15.3 & 25.5 & 59.2 & B & 3.73 & 8.48 & 3.66 & 0.08 & 8.79 & 0.00 & 8.43 & 0.00 & 3.63 & -0.11 & 0.66 & 6.84 \\
\hline $\mathrm{JL}-36$ & 0.0 & 0.9 & 99.1 & 0.9 & 31.8 & 67.3 & U & 8.48 & & 5.04 & 0.03 & 9.54 & 0.00 & 9.26 & 0.00 & 3.13 & -0.11 & 0.84 & 8.17 \\
\hline JL-37 & 0.0 & 0.8 & 99.2 & 0.8 & 32.4 & 66.8 & U & 7.50 & & 6.07 & 0.01 & 9.51 & 0.00 & 9.59 & 0.00 & 2.73 & 0.02 & 0.76 & 7.83 \\
\hline $\mathrm{JL}-38$ & 0.0 & 0.5 & 99.5 & 0.5 & 30.6 & 68.9 & $U$ & 8.48 & & 6.20 & 0.01 & 9.46 & 0.00 & 9.62 & 0.00 & 2.68 & 0.05 & 0.79 & 8.31 \\
\hline JL-39 & 0.0 & 0.8 & 99.2 & 0.8 & 30.1 & 69.1 & U & 8.48 & & 6.19 & 0.01 & 9.49 & 0.00 & 9.64 & 0.00 & 2.68 & 0.04 & 0.79 & 8.75 \\
\hline JL-40 & 0.0 & 1.0 & 99.0 & 1.0 & 30.2 & 68.8 & U & 7.50 & & 6.11 & 0.01 & 9.52 & 0.00 & 9.64 & 0.00 & 2.70 & 0.02 & 0.80 & 8.43 \\
\hline $\mathrm{JL}-41$ & 0.0 & 1.9 & 98.1 & 1.9 & 31.2 & 66.9 & U & 8.48 & & 5.77 & 0.02 & 9.31 & 0.00 & 9.45 & 0.00 & 2.83 & 0.02 & 0.83 & 8.48 \\
\hline $\mathrm{JL}-42$ & 0.0 & 5.9 & 94.1 & 5.9 & 30.7 & 63.4 & B & 7.50 & 3.73 & 4.56 & 0.04 & 9.27 & 0.00 & 9.23 & 0.00 & 3.11 & -0.07 & 0.86 & 7.40 \\
\hline
\end{tabular}




\section{Jeffreys Ledge Survey (2002-2005) (2): Grain Size Distribution}

\begin{tabular}{|c|c|c|c|c|c|c|c|c|c|c|c|c|c|c|c|c|c|c|c|c|c|c|c|c|c|c|c|c|c|c|c|c|}
\hline Sample ID & $\begin{array}{c}\text { Class } \\
\% \\
\text { phi } \\
-5.5\end{array}$ & $\begin{array}{c}\text { Class } \\
\% \\
\text { phi } \\
-5.0\end{array}$ & $\begin{array}{c}\text { Class } \\
\% \\
\text { phi } \\
-4.5 \\
\end{array}$ & $\begin{array}{c}\text { Class } \\
\% \\
\text { phi } \\
-4.0 \\
\end{array}$ & $\begin{array}{c}\text { Class } \\
\% \\
\text { phi } \\
-3.5\end{array}$ & $\begin{array}{c}\text { Class } \\
\% \\
\text { phi } \\
-3.0\end{array}$ & $\begin{array}{c}\text { Class } \\
\% \\
\text { phi } \\
-2.5 \\
\end{array}$ & $\begin{array}{c}\text { Class } \\
\% \\
\text { phi } \\
-2.0\end{array}$ & $\begin{array}{c}\text { Class } \\
\% \\
\text { phi } \\
-1.5\end{array}$ & $\begin{array}{c}\text { Class } \\
\% \\
\text { phi } \\
-1.0\end{array}$ & $\begin{array}{c}\text { Class } \\
\% \\
\text { phi } \\
-0.5\end{array}$ & $\begin{array}{c}\text { Class } \\
\% \\
\text { phi } \\
0.0\end{array}$ & $\begin{array}{c}\text { Class } \\
\% \\
\text { phi } \\
0.5 \\
\end{array}$ & $\begin{array}{c}\text { Class } \\
\% \\
\text { phi } \\
1.0 \\
\end{array}$ & $\begin{array}{c}\text { Class } \\
\% \\
\text { phi } \\
1.5\end{array}$ & $\begin{array}{c}\text { Class } \\
\% \\
\text { phi } \\
2.0\end{array}$ & $\begin{array}{c}\text { Class } \\
\% \\
\text { phi } \\
2.5\end{array}$ & $\begin{array}{c}\text { Class } \\
\% \\
\text { phi } \\
3.0\end{array}$ & $\begin{array}{c}\text { Class } \\
\% \\
\text { phi } \\
3.5\end{array}$ & $\begin{array}{c}\text { Class } \\
\% \\
\text { phi } \\
4.0\end{array}$ & $\begin{array}{c}\text { Class } \\
\% \\
\text { phi } \\
>4.0\end{array}$ & $\begin{array}{c}\text { Class } \\
\% \\
\text { phi } \\
5.0\end{array}$ & $\begin{array}{c}\text { Class } \\
\% \\
\text { phi } \\
6.0\end{array}$ & $\begin{array}{c}\text { Class } \\
\% \\
\text { phi } \\
7.0\end{array}$ & $\begin{array}{c}\text { Class } \\
\% \\
\text { phi } \\
8.0\end{array}$ & $\begin{array}{c}\text { Class } \\
\% \\
\text { phi } \\
9.0\end{array}$ & $\begin{array}{c}\text { Class } \\
\% \\
\text { phi } \\
10.0\end{array}$ & $\begin{array}{c}\text { Class } \\
\% \\
\text { phi } \\
>10.0\end{array}$ & $\begin{array}{c}\text { Class } \\
\% \\
\text { phi } \\
11.0\end{array}$ & $\begin{array}{c}\text { Class } \\
\% \\
\text { phi } \\
12.0\end{array}$ & $\begin{array}{c}\text { Class } \\
\% \\
\text { phi } \\
13.0\end{array}$ & $\begin{array}{c}\text { S Class } \\
\% \\
\text { phi } \\
14.0 \\
\end{array}$ \\
\hline L-21 & 0.00 & 0.00 & 0.00 & 0.00 & 0.00 & 0.00 & 0.00 & 0.00 & 0.00 & 0.00 & 0.00 & 0.00 & 0.00 & 0.00 & 0.00 & 0.00 & 0.00 & 0.00 & 0.00 & 1.88 & & 2.37 & 7.49 & 10.51 & 112.44 & 411.46 & 9.62 & & 11.06 & 611.06 & 511.06 & 611.06 \\
\hline L-22 & 0.00 & 0.00 & 0.00 & 0.00 & 0.00 & 0.00 & 0.00 & 0.00 & 0.00 & 0.00 & 0.00 & 0.00 & 0.00 & 0.00 & 0.00 & 0.00 & 0.00 & 0.00 & 0.00 & 0.63 & & 2.02 & 6.22 & 9.16 & 13.07 & 713.39 & 10.38 & & 11.28 & 811.28 & 311.28 & 811.28 \\
\hline L-23 & 0.00 & 0.00 & 0.00 & 0.00 & 0.00 & 0.00 & 0.00 & 0.00 & 0.00 & 0.00 & 0.00 & 0.00 & 0.00 & 0.00 & 0.00 & 0.00 & 0.00 & 0.00 & 0.00 & 2.59 & & 3.71 & 5.51 & 9.38 & 312.67 & 712.86 & 9.96 & & 10.83 & 310.83 & 10.83 & 310.83 \\
\hline $\mathrm{JL}-24$ & 00 & 0.00 & 0.00 & 0.00 & 0.00 & 0.00 & 0.00 & 0.00 & 0.00 & 0.00 & 0.01 & 0.01 & 0.03 & 0.09 & 0.20 & 0.32 & 0.37 & 0.71 & 3.01 & 6.06 & & 4.45 & 4.60 & 7.68 & 312.24 & +12.43 & 8.89 & & 9.73 & 9.73 & 9.73 & $\begin{array}{l}3 \quad 9.73 \\
\end{array}$ \\
\hline JL-25 & 0.00 & 0.00 & 0.00 & 0.00 & 0.00 & 0.00 & 0.00 & 0.00 & 0.00 & 0.10 & 0.20 & 0.35 & 0.48 & 0.72 & 1.12 & 1.71 & 2.24 & 3.68 & 8.72 & 10.81 & & 6.26 & 4.65 & 6.22 & 8.33 & 9.09 & 7.14 & & 7.05 & 7.05 & 7.05 & $5 \quad 7.05$ \\
\hline L-26 & 0.00 & 0.00 & 0.00 & 0.00 & 0.00 & 0.00 & 0.00 & 1.04 & 0.76 & 1.05 & 0.80 & 0.87 & 1.48 & 2.21 & 2.87 & 3.49 & 3.63 & 4.97 & 8.47 & 5.75 & & 3.17 & 3.46 & 5.46 & 7.90 & 8.16 & 7.99 & & 6.63 & 6.63 & 6.63 & $\begin{array}{l}3 \quad 6.63 \\
\end{array}$ \\
\hline L-27 & 00 & 0.00 & 0.00 & 0.00 & 0.00 & 0.00 & 0.00 & 0.00 & 0.24 & 0.09 & 0.25 & 0.30 & 0.51 & 1.03 & 1.80 & 2.44 & 2.66 & 4.03 & 7.93 & 6.78 & & 3.28 & 3.96 & 6.11 & 8.69 & 9.94 & 8.76 & & 7.80 & 7.80 & 7.80 & 7.80 \\
\hline L-28 & 00 & 0.00 & 0.00 & 0.00 & 0.00 & 0.00 & 0.00 & 0.00 & 0.00 & 0.00 & 0.00 & 0.00 & 0.00 & 0.00 & 0.00 & 0.00 & 0.00 & 0.00 & 0.00 & 0.78 & & 2.71 & 4.95 & 8.46 & 513.45 & 514.25 & 11.74 & & 10.92 & 210.92 & 10.92 & 210.92 \\
\hline $\mathrm{JL}-29$ & 0.00 & 0.00 & 0.00 & 0.00 & 0.00 & 0.00 & 0.00 & 0.00 & 0.00 & 0.05 & 0.02 & 0.01 & 0.04 & 0.06 & 0.15 & 0.54 & 1.46 & 4.20 & 7.82 & 10.03 & & 10.28 & 34.95 & 6.09 & 8.44 & 8.69 & 7.21 & & 7.49 & 7.49 & 7.49 & 7.49 \\
\hline JL-30 & 0.00 & 0.00 & 0.00 & 0.00 & 0.00 & 0.00 & 0.00 & 0.00 & 0.00 & 0.00 & 0.00 & 0.01 & 0.04 & 0.06 & 0.10 & 0.22 & 0.46 & 2.78 & 12.42 & 23.88 & & 16.55 & 4.64 & 4.32 & 5.03 & 5.41 & 4.66 & & 4.86 & 4.86 & 4.86 & $5 \quad 4.86$ \\
\hline JL-31 & 0.00 & 0.00 & 0.00 & 0.00 & 0.00 & 0.00 & 0.00 & 0.00 & 0.09 & 0.07 & 0.19 & 0.45 & 0.85 & 1.28 & 1.73 & 3.03 & 6.59 & 20.19 & 25.03 & 311.90 & & 3.44 & 1.84 & $\begin{array}{r}2.42 \\
\end{array}$ & 2.97 & 3.34 & 3.55 & & 2.76 & 2.76 & 2.76 & 2.76 \\
\hline JL-32 & 00 & 0.00 & 0.00 & 12.69 & 9.23 & 3.16 & 3.08 & 3.73 & 3.86 & 3.48 & 3.73 & 3.88 & 4.47 & 5.00 & 5.76 & 7.73 & 8.44 & 8.49 & 3.14 & 1.26 & & 0.61 & 0.42 & 0.66 & 0.93 & 1.20 & 1.36 & & 0.92 & 0.92 & 0.92 & 0.92 \\
\hline JL-34 & 0.00 & 0.00 & 0.00 & 0.00 & 10.00 & 0.00 & 2.13 & 1.94 & 1.18 & 0.83 & 0.91 & 0.94 & 1.18 & 1.96 & 2.63 & 3.73 & 4.87 & 12.46 & 19.38 & $\begin{array}{l}37.62 \\
\end{array}$ & & 2.51 & 2.33 & 2.63 & 2.79 & 3.55 & 2.98 & & 2.87 & 2.87 & 2.87 & 2.87 \\
\hline JL-35 & 0.00 & 0.00 & 0.00 & 0.00 & 0.00 & 0.00 & 0.00 & 0.00 & 0.00 & 0.00 & 0.05 & 0.02 & 0.01 & 0.02 & 0.05 & 0.11 & 0.31 & 1.45 & 5.15 & 8.02 & & 8.69 & 9.37 & 6.59 & 0.91 & 11.27 & 8.49 & & 9.88 & 9.88 & 9.88 & $\begin{array}{l}39.88 \\
\end{array}$ \\
\hline JL-36 & 0.00 & 0.00 & 0.00 & 0.00 & 0.00 & 0.00 & 0.00 & 0.00 & 0.00 & 0.00 & 0.00 & 0.00 & 0.00 & 0.00 & 0.00 & 0.00 & 0.00 & 0.00 & 0.00 & 0.79 & & 8.95 & 10.16 & $\begin{array}{ll}5 & 0.21 \\
\end{array}$ & 12.48 & 812.06 & 9.87 & & 11.37 & 711.37 & 11.37 & 711.37 \\
\hline $\mathrm{JL}-37$ & 0.00 & 0.00 & 0.00 & 0.00 & 0.00 & 0.00 & 0.00 & 0.00 & 0.00 & 0.00 & 0.00 & 0.00 & 0.00 & 0.00 & 0.00 & 0.00 & 0.00 & 0.00 & 0.00 & 0.74 & & 1.58 & 7.00 & 10.32 & 213.52 & 211.33 & 10.53 & & 11.25 & 511.25 & 11.25 & 511.25 \\
\hline IL-38 & 0.00 & 0.00 & 0.00 & 0.00 & 0.00 & 0.00 & 0.00 & 0.00 & 0.00 & 0.00 & 0.00 & 0.00 & 0.00 & 0.00 & 0.00 & 0.00 & 0.00 & 0.00 & 0.00 & 0.49 & & 2.14 & 5.37 & 10.04 & 413.12 & 213.82 & 10.39 & & 11.16 & 611.16 & 11.16 & 611.16 \\
\hline $\mathrm{JL}-39$ & 0.00 & 0.00 & 0.00 & 0.00 & 0.00 & 0.00 & 0.00 & 0.00 & 0.00 & 0.00 & 0.00 & 0.00 & 0.00 & 0.00 & 0.00 & 0.00 & 0.00 & 0.00 & 0.00 & 0.81 & & 1.81 & 5.64 & $\begin{array}{r}9.27 \\
\end{array}$ & 13.36 & 513.71 & 10.56 & & 11.21 & 111.21 & 11.21 & 111.21 \\
\hline $\mathrm{JL}-40$ & 0.00 & 0.00 & 0.00 & 0.00 & 0.00 & 0.00 & 0.00 & 0.00 & 0.00 & 0.00 & 0.00 & 0.00 & 0.00 & 0.00 & 0.00 & 0.00 & 0.00 & 0.00 & 0.00 & 1.01 & & 2.22 & 5.81 & 8.73 & 313.42 & 212.57 & 11.69 & & 11.14 & 411.14 & 11.14 & 411.14 \\
\hline JL-41 & 0.00 & 0.00 & 0.00 & 0.00 & 0.00 & 0.00 & 0.00 & 0.00 & 0.00 & 0.00 & 0.00 & 0.00 & 0.00 & 0.00 & 0.00 & 0.00 & 0.00 & 0.00 & 0.00 & 1.81 & & 4.05 & 5.45 & 9.33 & 312.42 & 213.38 & 10.73 & & 10.71 & 110.71 & 10.71 & 110.71 \\
\hline JL-42 & 0.00 & 0.00 & 0.00 & 0.00 & 0.00 & 0.00 & 0.00 & 0.00 & 0.00 & 0.00 & 0.00 & 0.00 & 0.00 & 0.00 & 0.00 & 0.00 & 0.00 & 0.00 & 0.00 & 5.82 & & 7.44 & 3.04 & 8.24 & 12.03 & $\begin{array}{ll}3 & 9.97\end{array}$ & 11.56 & & 10.48 & 810.48 & 10.48 & 810.48 \\
\hline
\end{tabular}


Jeffreys Ledge Survey (2002-2005) (3): Identification, Location, and Description

\begin{tabular}{|c|c|c|c|c|c|c|c|c|}
\hline Sample ID & $\begin{array}{c}\text { Original } \\
\text { Sample ID }\end{array}$ & $\begin{array}{c}\text { Latitude } \\
\text { WGS84 }\end{array}$ & $\begin{array}{c}\text { Longitude } \\
\text { WGS84 }\end{array}$ & $\begin{array}{l}\text { Reliability } \\
\text { Ranking for } \\
\text { Positioning }\end{array}$ & $\begin{array}{c}\text { Water } \\
\text { Depth } \\
\text { (m) }\end{array}$ & $\begin{array}{c}\text { Sample } \\
\text { Collected }\end{array}$ & $\begin{array}{c}\text { Sampler } \\
\text { Type }\end{array}$ & $\begin{array}{c}\text { Total Wt } \\
\text { (gm) }\end{array}$ \\
\hline $\mathrm{JL}-43$ & $\mathrm{JL48}$ & 42.908510 & -70.213760 & 1 & 146 & $8 / 26 / 2002$ & $\mathrm{BC}$ & 11.50 \\
\hline $\mathrm{JL}-44$ & JL48b & 42.908280 & -70.213470 & 1 & 149 & $8 / 11 / 2005$ & $\mathrm{SH}$ & 8.40 \\
\hline $\mathrm{JL}-45$ & JL49 & 42.908730 & -70.196880 & 1 & 121 & $8 / 26 / 2002$ & $\mathrm{BC}$ & 19.20 \\
\hline $\mathrm{JL}-46$ & JL49b & 42.908330 & -70.196670 & 1 & 120 & $8 / 11 / 2005$ & $\mathrm{SH}$ & 23.20 \\
\hline $\mathrm{JL}-47$ & JL50b & 42.908880 & -70.180740 & 1 & 99 & $8 / 26 / 2002$ & $\mathrm{BC}$ & 49.40 \\
\hline $\mathrm{JL}-48$ & JL60 & 42.895970 & -70.313350 & 1 & 150 & $7 / 31 / 2002$ & $\mathrm{BC}$ & 18.00 \\
\hline JL-49 & JL61 & 42.895720 & -70.297090 & 1 & 161 & $7 / 31 / 2002$ & $B C$ & 18.60 \\
\hline $\mathrm{JL}-50$ & JL62 & 42.895970 & -70.279680 & 1 & 163 & $7 / 31 / 2002$ & $\mathrm{BC}$ & 12.30 \\
\hline JL-51 & JL63 & 42.895590 & -70.263130 & 1 & 165 & $7 / 31 / 2002$ & $\mathrm{BC}$ & 15.50 \\
\hline $\mathrm{JL}-52$ & JL64 & 42.896400 & -70.247020 & 1 & 156 & $8 / 26 / 2002$ & $\mathrm{BC}$ & 13.30 \\
\hline $\mathrm{JL}-53$ & JL65 & 42.896220 & -70.230940 & 1 & 135 & $8 / 26 / 2002$ & $\mathrm{BC}$ & 11.20 \\
\hline JL-54 & JL66 & 42.895790 & -70.213960 & 1 & 123 & $8 / 26 / 2002$ & $\mathrm{BC}$ & 23.00 \\
\hline $\mathrm{JL}-55$ & JL66b & 42.895600 & -70.210330 & 1 & 124 & $8 / 11 / 2005$ & $\mathrm{SH}$ & 20.80 \\
\hline JL-56 & $\mathrm{JL74b}$ & 42.883930 & -70.379680 & 1 & 101 & $6 / 28 / 2004$ & $\mathrm{BC}$ & 37.30 \\
\hline $\mathrm{JL}-57$ & JL75a & 42.883450 & -70.364070 & 1 & 119 & $8 / 9 / 2002$ & $\mathrm{BC}$ & 35.10 \\
\hline JL-58 & JL75b & 42.883450 & -70.363800 & 1 & 117 & $6 / 28 / 2004$ & $\mathrm{BC}$ & 18.70 \\
\hline JL-59 & JL76 & 42.883380 & -70.347320 & 1 & 135 & $8 / 9 / 2002$ & $\mathrm{BC}$ & 35.90 \\
\hline $\mathrm{JL}-60$ & JL79 & 42.883540 & -70.297680 & 1 & 130 & $8 / 2 / 2002$ & $\mathrm{BC}$ & 34.10 \\
\hline JL-61 & JL80 & 42.883430 & -70.280190 & 1 & 166 & $8 / 2 / 2002$ & $\mathrm{BC}$ & 16.50 \\
\hline JL-62 & JL81 & 42.883810 & -70.264010 & 1 & 155 & $8 / 2 / 2002$ & $\mathrm{BC}$ & 26.60 \\
\hline
\end{tabular}


Jeffreys Ledge Survey (2002-2005) (3): Sediment Classifications

\begin{tabular}{|c|c|c|c|c|c|c|c|c|c|}
\hline Sample ID & $\begin{array}{l}\text { CMECS Substrate } \\
\text { Component } \\
\text { Group (Specific) }\end{array}$ & $\begin{array}{l}\text { CMECS Substrate } \\
\text { Component } \\
\text { Subgroup (Specific) }\end{array}$ & $\begin{array}{l}\text { Textural Group } \\
\text { from \%GSM } \\
\text { (Gradistat) }\end{array}$ & $\begin{array}{l}\text { Textural Group } \\
\text { from } \% S Z C \\
\text { (Gradistat) } \\
\end{array}$ & $\begin{array}{l}\text { Sediment Name from } \\
\text { \%GSM } \\
\text { and Mode (Gradistat) }\end{array}$ & $\begin{array}{l}\text { Sediment Name from } \\
\text { \%GSM and Mode } \\
\text { (Wentworth Scale) }\end{array}$ & $\begin{array}{l}\text { Sediment } \\
\text { Classification from } \\
\text { Mean Phi (Gradistat) }\end{array}$ & $\begin{array}{l}\text { Classification } \\
\text { from Mean Phi } \\
\text { (Wentworth) }\end{array}$ & $\begin{array}{l}\text { Sorting } \\
\text { (Gradistat) }\end{array}$ \\
\hline $\mathrm{JL}-43$ & Mud & Silt-Clay & Mud & Mud & Mud & Mud & Clay & Clay & $\begin{array}{l}\text { Very Poorly } \\
\text { Sorted }\end{array}$ \\
\hline $\mathrm{JL}-44$ & Mud & Silt-Clay & Mud & Mud & Mud & Mud & Clay & Clay & $\begin{array}{l}\text { Very Poorly } \\
\text { Sorted }\end{array}$ \\
\hline $\mathrm{JL}-45$ & Muddy Sand & $\begin{array}{l}\text { Silty-Clayey Very Fine } \\
\text { Sand }\end{array}$ & Muddy Sand & Muddy Sand & Muddy Very Fine Sand & Muddy Very Fine Sand & Coarse Silt & Medium Silt & $\begin{array}{l}\text { Very Poorly } \\
\text { Sorted }\end{array}$ \\
\hline $\mathrm{JL}-46$ & Slightly Granuley & $\begin{array}{l}\text { Slightly Granul ey Silty- } \\
\text { Clayey Very Fine Sand }\end{array}$ & $\begin{array}{l}\text { - Slightly Gravelly } \\
\text { Muddy Sand }\end{array}$ & Muddy Sand & $\begin{array}{l}\text { Slightly Very Fine Gravelly } \\
\text { Muddy Very Fine Sand }\end{array}$ & $\begin{array}{l}\text { Slightly Granular } \\
\text { Muddy Very Fine Sand }\end{array}$ & Coarse Silt & Medium Silt & $\begin{array}{l}\text { Very Poorly } \\
\text { Sorted }\end{array}$ \\
\hline $\mathrm{JL}-47$ & Pebbly & $\begin{array}{l}\text { Pebbly Clayey Fine } \\
\text { Sand }\end{array}$ & $\begin{array}{l}\text { Gravelly Muddy } \\
\text { Sand }\end{array}$ & $\mathrm{N} / \mathrm{A}$ & $\begin{array}{l}\text { Fine Gravelly Muddy Fine } \\
\text { Sand }\end{array}$ & $\begin{array}{l}\text { Pebbly Muddy Fine } \\
\text { Sand }\end{array}$ & Fine Sand & Fine Sand & $\begin{array}{l}\text { Very Poorly } \\
\text { Sorted }\end{array}$ \\
\hline $\mathrm{JL}-48$ & Sandy Mud & Sandy Silt-Clay & Sandy Mud & Sandy Mud & Very Fine Sandy Mud & Very Fine Sandy Mud & Fine Silt & Very Fine Silt & $\begin{array}{l}\text { Very Poorly } \\
\text { Sorted }\end{array}$ \\
\hline $\mathrm{JL}-49$ & Mud & Silt-Clay & Mud & Mud & Mud & Mud & Clay & Clay & $\begin{array}{l}\text { Very Poorly } \\
\text { Sorted }\end{array}$ \\
\hline $\mathrm{JL}-50$ & Mud & Clay & Mud & Mud & Mud & Mud & Clay & Clay & $\begin{array}{l}\text { Very Poorly } \\
\text { Sorted }\end{array}$ \\
\hline $\mathrm{JL}-51$ & Mud & Clay & Mud & Mud & Mud & Mud & Clay & Clay & $\begin{array}{l}\text { Very Poorly } \\
\text { Sorted }\end{array}$ \\
\hline $\mathrm{JL}-52$ & Sandy Mud & Sandy Silt-Clay & Sandy Mud & Sandy Mud & Very Fine Sandy Mud & Very Fine Sandy Mud & Fine Silt & Very Fine Silt & $\begin{array}{l}\text { Very Poorly } \\
\text { Sorted }\end{array}$ \\
\hline $\mathrm{JL}-53$ & Sandy Mud & Sandy Silt-Clay & Sandy Mud & Sandy Mud & Very Fine Sandy Mud & Very Fine Sandy Mud & Fine Silt & Very Fine Silt & $\begin{array}{l}\text { Very Poorly } \\
\text { Sorted }\end{array}$ \\
\hline $\mathrm{JL}-54$ & Slightly Pebbly & $\begin{array}{l}\text { Slightly Pebbly Silty- } \\
\text { Clayey Very Fine Sand }\end{array}$ & $\begin{array}{l}\text { Slightly Gravelly } \\
\text { Muddy Sand }\end{array}$ & $\mathrm{N} / \mathrm{A}$ & $\begin{array}{l}\text { Slightly Fine Gravelly } \\
\text { Muddy Very Fine Sand }\end{array}$ & $\begin{array}{l}\text { Slightly Pebbly Muddy } \\
\text { Very Fine Sand }\end{array}$ & Coarse Silt & Medium Silt & $\begin{array}{l}\text { Very Poorly } \\
\text { Sorted }\end{array}$ \\
\hline $\mathrm{JL}-55$ & Slightly Granuley & $\begin{array}{l}\text { Slightly Granul ey Silty- } \\
\text { Clayey Very Fine Sand }\end{array}$ & $\begin{array}{l}\text { - Slightly Gravelly } \\
\text { Muddy Sand }\end{array}$ & Muddy Sand & $\begin{array}{l}\text { Slightly Very Fine Gravelly } \\
\text { Muddy Very Fine Sand }\end{array}$ & $\begin{array}{l}\text { Slightly Granular } \\
\text { Muddy Very Fine Sand }\end{array}$ & Very Coarse Silt & Coarse Silt & $\begin{array}{l}\text { Very Poorly } \\
\text { Sorted }\end{array}$ \\
\hline $\mathrm{JL}-56$ & Slightly Granuley & $\begin{array}{l}\text { Slightly Granuley Silty- } \\
\text { Clayey Fine Sand }\end{array}$ & $\begin{array}{l}\text { - Slightly Gravelly } \\
\text { Muddy Sand }\end{array}$ & N/A & $\begin{array}{l}\text { Slightly Very Fine Gravelly } \\
\text { Muddy Fine Sand }\end{array}$ & $\begin{array}{l}\text { Slightly Granular } \\
\text { Muddy Fine Sand }\end{array}$ & Fine Sand & Fine Sand & $\begin{array}{l}\text { Very Poorly } \\
\text { Sorted }\end{array}$ \\
\hline $\mathrm{JL}-57$ & Slightly Granuley & $\begin{array}{l}\text { Slightly Granuley Silty- } \\
\text { Clayey Very Fine Sand }\end{array}$ & $\begin{array}{l}\text { - Slightly Gravelly } \\
\text { Muddy Sand }\end{array}$ & Muddy Sand & $\begin{array}{l}\text { Slightly Very Fine Gravelly } \\
\text { Muddy Very Fine Sand }\end{array}$ & $\begin{array}{l}\text { Slightly Granular } \\
\text { Muddy Very Fine Sand }\end{array}$ & Very Coarse Silt & Coarse Silt & $\begin{array}{l}\text { Very Poorly } \\
\text { Sorted }\end{array}$ \\
\hline $\mathrm{JL}-58$ & Muddy Sand & $\begin{array}{l}\text { Silty-Clayey Very Fine } \\
\text { Sand }\end{array}$ & Muddy Sand & Muddy Sand & Muddy Very Fine Sand & Muddy Very Fine Sand & Very Coarse Silt & Coarse Silt & $\begin{array}{l}\text { Very Poorly } \\
\text { Sorted }\end{array}$ \\
\hline $\mathrm{JL}-59$ & Muddy Sand & $\begin{array}{l}\text { Silty-Clayey Very Fine } \\
\text { Sand }\end{array}$ & Muddy Sand & Muddy Sand & Muddy Very Fine Sand & Muddy Very Fine Sand & Very Coarse Silt & Coarse Silt & $\begin{array}{l}\text { Very Poorly } \\
\text { Sorted }\end{array}$ \\
\hline $\mathrm{JL}-60$ & Slightly Granuley & $\begin{array}{l}\text { Slightly Granul ey Silty- } \\
\text { Clayey Very Fine Sand }\end{array}$ & $\begin{array}{l}\text { - Slightly Gravelly } \\
\text { Muddy Sand }\end{array}$ & Muddy Sand & $\begin{array}{l}\text { Slightly Very Fine Gravelly } \\
\text { Muddy Very Fine Sand }\end{array}$ & $\begin{array}{l}\text { Slightly Granular } \\
\text { Muddy Very Fine Sand }\end{array}$ & Coarse Silt & Medium Silt & $\begin{array}{l}\text { Very Poorly } \\
\text { Sorted }\end{array}$ \\
\hline $\mathrm{JL}-61$ & Mud & Silt-Clay & Mud & Mud & Mud & Mud & Clay & Clay & $\begin{array}{l}\text { Very Poorly } \\
\text { Sorted }\end{array}$ \\
\hline $\mathrm{JL}-62$ & Sandy Mud & Sandy Silt-Clay & Sandy Mud & Sandy Mud & Very Fine Sandy Mud & Very Fine Sandy Mud & Fine Silt & Very Fine Silt & $\begin{array}{l}\text { Very Poorly } \\
\text { Sorted }\end{array}$ \\
\hline
\end{tabular}




\section{Jeffreys Ledge Survey (2002-2005) (3): Grain Size Statistics}

\begin{tabular}{|c|c|c|c|c|c|c|c|c|c|c|c|c|c|c|c|c|c|c|c|}
\hline Sample ID & $\begin{array}{c}\text { Gravel } \\
\% \\
\end{array}$ & $\begin{array}{c}\text { Sand } \\
\% \\
\end{array}$ & $\begin{array}{c}\text { Mud } \\
\% \\
\end{array}$ & $\begin{array}{c}\text { Sand } \\
\% \\
\end{array}$ & $\begin{array}{c}\text { Silt } \\
\% \\
\end{array}$ & $\begin{array}{c}\text { Clay } \\
\% \\
\end{array}$ & Modes & $\begin{array}{c}\text { Mode } 1 \\
\text { (phi) }\end{array}$ & $\begin{array}{c}\text { Mode } 2 \\
\text { (phi) }\end{array}$ & $\begin{array}{l}D_{10} \\
\text { (phi) }\end{array}$ & $\begin{array}{c}D_{10} \\
(\mathrm{~mm}) \\
\end{array}$ & $\begin{array}{l}\mathrm{D}_{50} \\
\text { (phi) }\end{array}$ & $\begin{array}{c}D_{50} \\
(\mathrm{~mm}) \\
\end{array}$ & $\begin{array}{c}\text { Mean } \\
\text { Size } \\
\text { (phi) }\end{array}$ & $\begin{array}{c}\text { Mean } \\
\text { Size } \\
(\mathrm{mm})\end{array}$ & $\begin{array}{c}\text { Sorting } \\
\text { (phi) }\end{array}$ & Skewness & Kurtosis & LOI \% \\
\hline JL-43 & 0.0 & 4.6 & 95.4 & 4.6 & 33.0 & 62.4 & $U$ & 7.50 & & 4.84 & 0.03 & 9.09 & 0.00 & 9.10 & 0.00 & 3.11 & -0.03 & 0.85 & 8.00 \\
\hline$J L-44$ & 0.0 & 4.6 & 95.4 & 4.6 & 33.2 & 62.2 & $U$ & 8.48 & & 4.76 & 0.04 & 9.00 & 0.00 & 9.04 & 0.00 & 3.14 &.-0.15 & 0.83 & 7.10 \\
\hline JL-45 & 0.0 & 53.1 & 46.9 & 53.1 & 20.8 & 26.1 & $U$ & 3.24 & & 3.02 & 0.12 & 3.92 & 0.07 & 5.77 & 0.02 & 3.32 & 0.77 & 0.86 & 3.50 \\
\hline JL-46 & 0.1 & 50.4 & 49.6 & 50.4 & 22.2 & 27.4 & $\mathrm{~B}$ & 3.73 & 7.50 & 2.92 & 0.13 & 3.98 & 0.06 & 5.82 & 0.02 & 3.37 & 0.75 & 0.83 & 2.50 \\
\hline $\mathrm{JL}-47$ & 6.9 & 83.6 & 9.5 & 83.6 & 3.0 & 6.5 & $\mathrm{~B}$ & 2.74 & -2.24 & -0.05 & 1.03 & 2.59 & 0.17 & 2.33 & 0.20 & 2.26 & -0.09 & 4.04 & 1.40 \\
\hline JL-48 & 0.0 & 22.3 & 77.7 & 22.3 & 28.5 & 49.2 & $\mathrm{~B}$ & 3.73 & 7.50 & 3.46 & 0.09 & 7.93 & 0.00 & 7.89 & 0.00 & 3.62 & 0.03 & 0.65 & 6.68 \\
\hline JL-49 & 0.0 & 1.8 & 98.2 & 1.8 & 32.0 & 66.2 & $U$ & 6.50 & & 6.18 & 0.01 & 9.39 & 0.00 & 9.53 & 0.00 & 2.76 & 0.03 & 0.78 & 8.08 \\
\hline JL-50 & 0.0 & 0.7 & 99.3 & 0.7 & 30.7 & 68.6 & $U$ & 7.50 & & 6.16 & 0.01 & 9.48 & 0.00 & 9.62 & 0.00 & 2.68 & 0.04 & 0.80 & 8.61 \\
\hline JL-51 & 0.0 & 0.8 & 99.2 & 0.8 & 31.5 & 67.7 & $U$ & 7.50 & & 6.10 & 0.01 & 9.50 & 0.00 & 9.61 & 0.00 & 2.71 & 0.02 & 0.78 & 8.11 \\
\hline JL-52 & 0.0 & 17.3 & 82.7 & 17.3 & 37.1 & 45.6 & $B$ & 3.73 & 7.50 & 3.55 & 0.09 & 7.72 & 0.00 & 7.90 & 0.00 & 3.59 & 0.09 & 0.75 & 6.80 \\
\hline JL-53 & 0.0 & 30.4 & 69.6 & 30.4 & 26.3 & 43.3 & $B$ & 3.73 & 7.50 & 3.44 & 0.09 & 7.21 & 0.01 & 7.51 & 0.01 & 3.56 & 0.15 & 0.64 & 5.60 \\
\hline JL-54 & 3.5 & 51.4 & 45.1 & 51.4 & 18.9 & 26.2 & B & 3.24 & -2.24 & 2.73 & 0.15 & 3.86 & 0.07 & 5.69 & 0.02 & 3.41 & 0.72 & 0.88 & 4.00 \\
\hline JL-55 & 0.1 & 68.4 & 31.5 & 68.4 & 16.4 & 15.1 & $U$ & 3.24 & & 2.71 & 0.15 & 3.64 & 0.08 & 4.77 & 0.04 & 2.62 & 0.73 & 2.65 & 2.30 \\
\hline JL-56 & 2.1 & 82.0 & 15.9 & 82.0 & 5.8 & 10.1 & $U$ & 2.24 & & 0.80 & 0.57 & 2.34 & 0.20 & 2.55 & 0.17 & 2.31 & 0.40 & 3.60 & 1.60 \\
\hline JL-57 & 0.1 & 78.3 & 21.6 & 78.3 & 7.9 & 13.7 & $U$ & 3.24 & & 2.36 & 0.19 & 3.19 & 0.11 & 4.31 & 0.05 & 2.62 & 0.74 & 3.66 & 2.30 \\
\hline JL-58 & 0.0 & 70.0 & 30.0 & 70.0 & 11.0 & 19.0 & $\mathrm{U}$ & 3.24 & & 2.48 & 0.18 & 3.34 & 0.10 & 4.90 & 0.03 & 3.07 & 0.77 & 1.39 & 2.80 \\
\hline JL-59 & 0.0 & 73.8 & 26.2 & 73.8 & 10.6 & 15.6 & $U$ & 3.24 & & 2.64 & 0.16 & 3.42 & 0.09 & 4.72 & 0.04 & 2.71 & 0.78 & 3.17 & 2.71 \\
\hline JL-60 & 0.4 & 62.6 & 37.0 & 62.6 & 14.3 & 22.7 & $U$ & 3.24 & & 2.69 & 0.15 & 3.63 & 0.08 & 5.41 & 0.02 & 3.22 & 0.78 & 0.98 & 3.32 \\
\hline JL-61 & 0.0 & 2.8 & 97.2 & 2.8 & 33.0 & 64.0 & $U$ & 8.48 & & 5.33 & 0.02 & 9.13 & 0.00 & 9.26 & 0.00 & 2.97 & 0.02 & 0.83 & 8.30 \\
\hline JLL-62 & 0.0 & 22.3 & 77.7 & 22.3 & 29.9 & 47.8 & B & 3.73 & 8.48 & 3.58 & 0.08 & 7.77 & 0.00 & 7.85 & 0.00 & 3.58 & 0.07 & 0.63 & 5.97 \\
\hline
\end{tabular}




\section{Jeffreys Ledge Survey (2002-2005) (3): Grain Size Distribution}

\begin{tabular}{|c|c|c|c|c|c|c|c|c|c|c|c|c|c|c|c|c|c|c|c|c|c|c|c|c|c|c|c|c|c|c|c|c|}
\hline Sample ID & $\begin{array}{c}\text { Class } \\
\% \\
\text { phi } \\
-5.5\end{array}$ & $\begin{array}{c}\text { Class } \\
\% \\
\text { phi } \\
-5.0\end{array}$ & $\begin{array}{c}\text { Class } \\
\% \\
\text { phi } \\
-4.5\end{array}$ & $\begin{array}{c}\text { Class } \\
\% \\
\text { phi } \\
-4.0\end{array}$ & $\begin{array}{c}\text { Class } \\
\% \\
\text { phi } \\
-3.5\end{array}$ & $\begin{array}{c}\text { Class } \\
\% \\
\text { phi } \\
-3.0\end{array}$ & $\begin{array}{c}\text { Class } \\
\% \\
\text { phi } \\
-2.5\end{array}$ & $\begin{array}{c}\text { Class } \\
\% \\
\text { phi } \\
-2.0\end{array}$ & $\begin{array}{c}\text { Class } \\
\% \\
\text { phi } \\
-1.5\end{array}$ & $\begin{array}{c}\text { Class } \\
\% \\
\text { phi } \\
-1.0\end{array}$ & $\begin{array}{c}\text { Class } \\
\% \\
\text { phi } \\
-0.5\end{array}$ & $\begin{array}{c}\text { Class } \\
\% \\
\text { phi } \\
0.0\end{array}$ & $\begin{array}{c}\text { Class } \\
\% \\
\text { phi } \\
0.5\end{array}$ & $\begin{array}{c}\text { Class } \\
\% \\
\text { phi } \\
1.0\end{array}$ & $\begin{array}{c}\text { Class } \\
\% \\
\text { phi } \\
1.5\end{array}$ & $\begin{array}{c}\text { Class } \\
\% \\
\text { phi } \\
2.0\end{array}$ & $\begin{array}{c}\text { Class } \\
\% \\
\text { phi } \\
2.5\end{array}$ & $\begin{array}{c}\text { Class } \\
\% \\
\text { phi } \\
3.0\end{array}$ & $\begin{array}{c}\text { Class } \\
\% \\
\text { phi } \\
3.5\end{array}$ & $\begin{array}{c}\text { Class } \\
\% \\
\text { phi } \\
4.0\end{array}$ & $\begin{array}{c}\text { Class } \\
\% \\
\text { phi } \\
>4.0\end{array}$ & $\begin{array}{c}\text { Class } \\
\% \\
\text { phi } \\
5.0\end{array}$ & $\begin{array}{c}\text { Class } \\
\% \\
\text { phi } \\
6.0\end{array}$ & $\begin{array}{c}\text { Class } \\
\% \\
\text { phi } \\
7.0\end{array}$ & $\begin{array}{c}\text { Class } \\
\% \\
\text { phi } \\
8.0\end{array}$ & $\begin{array}{c}\text { Class } \\
\% \\
\text { phi } \\
9.0\end{array}$ & $\begin{array}{c}\text { Class } \\
\% \\
\text { phi } \\
10.0\end{array}$ & $\begin{array}{c}\text { Class } \\
\% \\
\text { phi } \\
>10.0\end{array}$ & $\begin{array}{c}\text { Class } \\
\% \\
\text { phi } \\
11.0\end{array}$ & $\begin{array}{c}\text { Class } \\
\% \\
\text { phi } \\
12.0\end{array}$ & $\begin{array}{c}\text { Class } \\
\% \\
\text { phi } \\
13.0\end{array}$ & $\begin{array}{c}\text { Class } \\
\% \\
\text { phi } \\
14.0\end{array}$ \\
\hline JL-43 & 0.00 & 0.00 & 0.00 & 0.00 & 0.00 & 0.00 & 0.00 & 0.00 & 0.00 & 0.00 & 0.00 & 0.00 & 0.00 & 0.00 & 0.00 & 0.00 & 0.00 & 0.00 & 0.00 & 4.57 & & 6.51 & 6.47 & 7.55 & 12.50 & 11.15 & 10.50 & & 10.19 & 910.19 & 910.19 & 910.19 \\
\hline L-44 & 0.00 & 0.00 & 0.00 & 0.00 & 0.00 & 0.00 & 0.00 & 0.00 & 0.00 & 0.00 & 0.00 & 0.08 & 0.02 & 0.04 & 0.07 & 0.06 & 0.06 & 0.22 & 0.71 & 3.29 & & 7.28 & 6.44 & 7.94 & 11.52 & 211.93 & 9.61 & & 10.19 & 910.19 & 910.19 & 910.19 \\
\hline L-45 & 0.00 & 0.00 & 0.00 & 0.00 & 0.00 & 0.00 & 0.00 & 0.00 & 0.00 & 0.00 & 0.00 & 0.03 & 0.10 & 0.12 & 0.25 & 0.53 & 1.22 & 6.89 & 21.96 & 21.93 & & 9.21 & 3.16 & 4.05 & 5.44 & 5.04 & 3.99 & & 4.27 & 4.27 & 4.27 & $\begin{array}{l}7 \quad 4.27 \\
\end{array}$ \\
\hline L-46 & 00 & 0.00 & 0.00 & 0.00 & 0.00 & 0.00 & 0.00 & 0.00 & 0.00 & 0.07 & 0.10 & 0.10 & 0.28 & 0.30 & 0.49 & 0.80 & 1.44 & 7.54 & 18.80 & 20.43 & & 9.85 & 3.02 & 1.08 & $\begin{array}{l}38.30 \\
\end{array}$ & 5.02 & 4.85 & & 4.39 & 4.39 & 4.39 & $\begin{array}{l}9 \\
9\end{array}$ \\
\hline JL-47 & 0.00 & 0.00 & 0.00 & 0.00 & 0.00 & 0.00 & 0.00 & 6.15 & 0.00 & 0.75 & 1.47 & 1.82 & 2.33 & 2.98 & 3.59 & 7.29 & 16.64 & 30.77 & 14.46 & 2.29 & & 0.77 & 0.54 & 0.68 & 1.03 & 1.29 & 1.46 & & 0.93 & 0.93 & $\begin{array}{l}3 \quad 0.93 \\
\end{array}$ & $\begin{array}{l}3 \quad 0.93 \\
\end{array}$ \\
\hline JL-48 & 0.00 & 0.00 & 0.00 & 0.00 & 0.00 & 0.00 & 0.00 & 0.00 & 0.00 & 0.00 & 0.04 & 0.03 & 0.03 & 0.03 & 0.04 & 0.11 & 0.27 & 1.73 & 7.95 & 12.03 & & 6.62 & 4.85 & 5.85 & 11.20 & 9.51 & 7.93 & & 7.95 & 7.95 & 7.95 & $5 \quad 7.95$ \\
\hline L-49 & 0.00 & 0.00 & 0.00 & 0.00 & 0.00 & 0.00 & 0.00 & 0.00 & 0.00 & 0.00 & 0.00 & 0.00 & 0.00 & 0.00 & 0.00 & 0.00 & 0.00 & 0.00 & 0.00 & 1.76 & & 2.90 & 3.06 & 13.20 & 012.93 & 311.83 & 10.54 & & 10.95 & 510.95 & 510.95 & 510.95 \\
\hline L-50 & 00 & 0.00 & 0.00 & 0.00 & 0.00 & 0.00 & 0.00 & 0.00 & 0.00 & 0.00 & 0.00 & 0.00 & 0.00 & 0.00 & 0.00 & 0.00 & 0.00 & 0.00 & 0.00 & 0.69 & & 2.20 & 5.61 & 9.31 & 13.58 & 312.77 & 11.67 & & 11.05 & 511.05 & 511.05 & 511.05 \\
\hline JL-51 & 0.00 & 0.00 & 0.00 & 0.00 & 0.00 & 0.00 & 0.00 & 0.00 & 0.00 & 0.00 & 0.00 & 0.00 & 0.00 & 0.00 & 0.00 & 0.00 & 0.00 & 0.00 & 0.00 & 0.82 & & 2.13 & 6.12 & 9.53 & 33.71 & 12.14 & 10.75 & & 11.20 & 11.20 & 011.20 & $\begin{array}{ll}011.20 \\
\end{array}$ \\
\hline JL-52 & 0.00 & 0.00 & 0.00 & 0.00 & 0.00 & 0.00 & 0.00 & 0.00 & 0.00 & 0.00 & 0.00 & 0.02 & 0.05 & 0.07 & 0.16 & 0.48 & 0.74 & 1.64 & 5.60 & 8.48 & & 6.56 & 4.37 & 10.94 & 415.31 & 2.07 & 11.01 & & 8.13 & 8.13 & $\begin{array}{l}38.13 \\
\end{array}$ & $\begin{array}{l}3 \quad 8.13 \\
\end{array}$ \\
\hline JL-53 & 0.00 & 0.00 & 0.00 & 0.00 & 0.00 & 0.00 & 0.00 & 0.00 & 0.00 & 0.00 & 0.00 & 0.07 & 0.10 & 0.07 & 0.15 & 0.39 & 0.67 & 1.69 & 7.44 & 19.71 & & 6.90 & 4.54 & 6.50 & 8.37 & 7.79 & 8.10 & & 6.88 & 6.88 & $\begin{array}{ll}3 & 6.88\end{array}$ & $\begin{array}{ll}3 & 6.88\end{array}$ \\
\hline JL-54 & 0.00 & 0.00 & 0.00 & 0.00 & 0.00 & 0.00 & 0.00 & 3.52 & 0.00 & 0.00 & 0.08 & 0.04 & 0.14 & 0.16 & 0.27 & 0.57 & 1.44 & 7.66 & 21.84 & +19.12 & & 7.81 & 2.37 & 4.12 & 4.62 & 4.84 & 4.65 & & 4.19 & 4.19 & 4.19 & 4.19 \\
\hline JL-55 & 0.00 & 0.00 & 0.00 & 0.00 & 0.00 & 0.00 & 0.00 & 0.00 & 0.00 & 0.14 & 0.31 & 0.23 & 0.42 & 0.58 & 0.75 & 1.09 & 1.88 & 10.10 & 25.87 & 27.09 & & 9.90 & 2.02 & 1.95 & 2.57 & 2.50 & 2.74 & & 2.47 & 2.47 & 2.47 & 2.47 \\
\hline $\mathrm{JL}-56$ & 0.00 & 0.00 & 0.00 & 0.00 & 0.00 & 0.00 & 0.95 & 0.00 & 0.67 & 0.53 & 1.40 & 1.60 & 2.61 & 3.70 & 6.69 & 15.71 & 22.43 & 20.54 & $\begin{array}{r}5.47 \\
\end{array}$ & 1.83 & & 1.69 & 1.21 & 1.19 & 7. 1.70 & 2.05 & 1.70 & & 1.59 & $\begin{array}{r}1.59 \\
\end{array}$ & $\begin{array}{l}9 \\
0\end{array}$ & $\begin{array}{l}9 \\
9\end{array}$ \\
\hline JL-57 & 0.00 & 0.00 & 0.00 & 0.00 & 0.00 & 0.00 & 0.00 & 0.00 & 0.00 & 0.05 & 0.06 & 0.18 & 0.36 & 0.50 & 0.93 & 2.27 & 7.38 & 26.10 & 29.94 & +10.63 & & 2.19 & 1.31 & 1.62 & 2.74 & 2.84 & 2.27 & & 2.16 & 2.16 & $5 \quad 2.16$ & $5 \quad 2.16$ \\
\hline JL-58 & 0.00 & 0.00 & 0.00 & 0.00 & 0.00 & 0.00 & 0.00 & 0.00 & 0.00 & 0.00 & 0.01 & 0.11 & 0.13 & 0.21 & 0.48 & 1.82 & 7.03 & 21.71 & 25.98 & 312.45 & & 3.27 & 2.17 & 2.30 & 3.59 & 3.56 & 3.48 & & 2.93 & 2.93 & 2.93 & 2.93 \\
\hline JL-59 & 0.00 & 0.00 & 0.00 & 0.00 & 0.00 & 0.00 & 0.00 & 0.00 & 0.00 & 0.00 & 0.07 & 0.21 & 0.28 & 0.43 & 0.69 & 1.23 & 2.61 & 14.22 & 34.39 & 19.68 & & 3.78 & 1.55 & 2.20 & 3.04 & 3.15 & 2.76 & & 2.43 & 2.43 & 2.43 & 2.43 \\
\hline $\mathrm{JL}-60$ & 0.00 & 0.00 & 0.00 & 0.00 & 0.00 & 0.00 & 0.00 & 0.00 & 0.23 & 0.16 & 0.13 & 0.17 & 0.34 & 0.51 & 0.84 & 1.37 & 2.24 & 9.71 & 28.81 & 18.39 & & 4.75 & 2.61 & 2.70 & 4.34 & 4.35 & 3.81 & & 3.64 & $\begin{array}{r}\quad 3.64 \\
\end{array}$ & $\begin{array}{l}+\quad 3.64 \\
\end{array}$ & $\begin{array}{l}4 \quad 3.64 \\
\end{array}$ \\
\hline $\mathrm{JL}-61$ & 0.00 & 0.00 & 0.00 & 0.00 & 0.00 & 0.00 & 0.00 & 0.00 & 0.00 & 0.00 & 0.00 & 0.00 & 0.00 & 0.00 & 0.00 & 0.00 & 0.00 & 0.00 & 0.00 & 2.74 & & 5.10 & 6.79 & 9.07 & 12.34 & +12.37 & 9.71 & & 10.47 & 710.47 & 710.47 & 710.47 \\
\hline JL-62 & 0.00 & 0.00 & 0.00 & 0.00 & 0.00 & 0.00 & 0.00 & 0.00 & 0.00 & 0.00 & 0.02 & 0.03 & 0.03 & 0.02 & 0.03 & 0.05 & 0.12 & 0.85 & 5.67 & 15.41 & & 9.83 & 4.97 & 5.99 & 9.14 & 9.68 & 6.74 & & 7.86 & 7.86 & 7.86 & 7.86 \\
\hline
\end{tabular}


Jeffreys Ledge Survey (2002-2005) (4): Identification, Location, and Description

\begin{tabular}{|c|c|c|c|c|c|c|c|c|}
\hline Sample ID & $\begin{array}{c}\text { Original } \\
\text { Sample ID }\end{array}$ & $\begin{array}{c}\text { Latitude } \\
\text { WGS84 }\end{array}$ & $\begin{array}{c}\text { Longitude } \\
\text { WGS84 }\end{array}$ & $\begin{array}{l}\text { Reliability } \\
\text { Ranking for } \\
\text { Positioning }\end{array}$ & $\begin{array}{c}\text { Water } \\
\text { Depth } \\
\text { (m) }\end{array}$ & $\begin{array}{l}\text { Sample } \\
\text { Collected }\end{array}$ & $\begin{array}{c}\text { Sampler } \\
\text { Type }\end{array}$ & $\begin{array}{c}\text { Total Wt } \\
\text { (gm) }\end{array}$ \\
\hline $\mathrm{JL}-63$ & JL82 & 42.883390 & -70.245870 & 1 & 154 & $9 / 6 / 2002$ & $B C$ & 13.00 \\
\hline JL-64 & JL83b & 42.883300 & -70.229820 & 1 & 115 & $9 / 6 / 2002$ & $\mathrm{BC}$ & 29.30 \\
\hline JL-65 & JL91 & 42.871180 & -70.394530 & 1 & & $6 / 28 / 2004$ & $\mathrm{BC}$ & 12.60 \\
\hline JL-66 & JL92a & 42.870700 & -70.379890 & 1 & 148 & $10 / 2 / 2002$ & $\mathrm{BC}$ & 25.80 \\
\hline JL-67 & JL92b & 42.871520 & -70.380270 & 1 & & $6 / 28 / 2004$ & $B C$ & 9.80 \\
\hline $\mathrm{JL}-68$ & JL93 & 42.871270 & -70.363230 & 1 & 95 & $6 / 28 / 2004$ & $\mathrm{BC}$ & 37.10 \\
\hline JL-69 & JL94b & 42.870790 & -70.346760 & 1 & 104 & $10 / 2 / 2002$ & $\mathrm{BC}$ & 121.70 \\
\hline $\mathrm{JL}-70$ & JL96 & 42.871040 & -70.313410 & 1 & 117 & $10 / 2 / 2002$ & $\mathrm{BC}$ & 62.30 \\
\hline $\mathrm{JL}-71$ & JL98 & 42.871170 & -70.280470 & 1 & 148 & $8 / 2 / 2002$ & $\mathrm{BC}$ & 30.80 \\
\hline $\mathrm{JL}-72$ & JL99 & 42.871410 & -70.263670 & 1 & 119 & $8 / 2 / 2002$ & $\mathrm{BC}$ & 37.40 \\
\hline $\mathrm{JL}-73$ & JL100 & 42.870470 & -70.246580 & 1 & 119 & $9 / 6 / 2002$ & $\mathrm{BC}$ & 23.00 \\
\hline $\mathrm{JL}-74$ & JL101 & 42.870910 & -70.229570 & 1 & 101 & $9 / 6 / 2002$ & $B C$ & 35.20 \\
\hline JL-75 & JL108c & 42.871080 & -70.113730 & 1 & 95 & $11 / 17 / 2004$ & $\mathrm{BC}$ & 23.10 \\
\hline $\mathrm{JL}-76$ & JL109a & 42.858280 & -70.396860 & 1 & 79 & $10 / 2 / 2002$ & $\mathrm{BC}$ & 131.90 \\
\hline JL-77 & JL110f & 42.861450 & -70.379150 & 1 & 68 & $6 / 28 / 2004$ & $\mathrm{SH}$ & 36.70 \\
\hline $\mathrm{JL}-78$ & JL111 & 42.858550 & -70.363420 & 1 & 124 & $6 / 28 / 2004$ & $\mathrm{BC}$ & 6.90 \\
\hline $\mathrm{JL}-79$ & JL112 & 42.858330 & -70.346680 & 1 & 57 & $6 / 28 / 2004$ & $\mathrm{SH}$ & 48.40 \\
\hline JL-80 & JL113c & 42.858620 & -70.329370 & 1 & 88 & $6 / 28 / 2004$ & $\mathrm{SH}$ & 9.40 \\
\hline $\mathrm{JL}-81$ & JL116 & 42.858170 & -70.279850 & 1 & 95 & $9 / 6 / 2002$ & $\mathrm{BC}$ & 68.10 \\
\hline $\mathrm{JL}-82$ & JL117 & 42.858030 & -70.263240 & 1 & 101 & $9 / 6 / 2002$ & $\mathrm{BC}$ & 49.10 \\
\hline JL-83 & JL118 & 42.858250 & -70.246240 & 1 & 91 & $9 / 6 / 2002$ & $B C$ & 67.00 \\
\hline
\end{tabular}


Jeffreys Ledge Survey (2002-2005) (4): Sediment Classifications

\begin{tabular}{|c|c|c|c|c|c|c|c|c|c|}
\hline Sample ID & $\begin{array}{l}\text { CMECS Substrate } \\
\text { Component } \\
\text { Group (Specific) }\end{array}$ & $\begin{array}{l}\text { CMECS Substrate } \\
\text { Component } \\
\text { Subgroup (Specific) }\end{array}$ & $\begin{array}{l}\text { Textural Group } \\
\text { from \%GSM } \\
\text { (Gradistat) }\end{array}$ & $\begin{array}{l}\text { Textural Group } \\
\text { from \%SZC } \\
\text { (Gradistat) }\end{array}$ & $\begin{array}{l}\text { Sediment Name from } \\
\text { \%GSM } \\
\text { and Mode (Gradistat) }\end{array}$ & $\begin{array}{l}\text { Sediment Name from } \\
\text { \%GSM and Mode } \\
\text { (Wentworth Scale) }\end{array}$ & $\begin{array}{l}\text { Sediment } \\
\text { Classification from } \\
\text { Mean Phi (Gradistat) }\end{array}$ & $\begin{array}{l}\text { Classification } \\
\text { from Mean Phi } \\
\text { (Wentworth) }\end{array}$ & $\begin{array}{l}\text { Sorting } \\
\text { (Gradistat) }\end{array}$ \\
\hline $\mathrm{JL}-63$ & Sandy Mud & Sandy Silt-Clay & Sandy Mud & Sandy Mud & Very Fine Sandy Mud & Very Fine Sandy Mud & Medium Silt & Fine Silt & $\begin{array}{l}\text { Very Poorly } \\
\text { Sorted }\end{array}$ \\
\hline $\mathrm{JL}-64$ & Slightly Granuley & $\begin{array}{l}\text { Slightly Granuley Silty- } \\
\text { Clayey Very Fine Sand }\end{array}$ & $\begin{array}{l}\text { - Slightly Gravelly } \\
\text { Muddy Sand }\end{array}$ & Muddy Sand & $\begin{array}{l}\text { Slightly Very Fine Gravelly } \\
\text { Muddy Very Fine Sand }\end{array}$ & $\begin{array}{l}\text { Slightly Granular } \\
\text { Muddy Very Fine Sand }\end{array}$ & Very Coarse Silt & Coarse Silt & $\begin{array}{l}\text { Very Poorly } \\
\text { Sorted }\end{array}$ \\
\hline $\mathrm{JL}-65$ & Sandy Mud & Sandy Silt-Clay & Sandy Mud & Sandy Mud & Very Fine Sandy Mud & Very Fine Sandy Mud & Fine Silt & Very Fine Silt & $\begin{array}{l}\text { Very Poorly } \\
\text { Sorted }\end{array}$ \\
\hline $\mathrm{JL}-66$ & Mud & Silt-Clay & Mud & Mud & Mud & Mud & Clay & Clay & $\begin{array}{l}\text { Very Poorly } \\
\text { Sorted }\end{array}$ \\
\hline $\mathrm{JL}-67$ & Mud & Silt-Clay & Mud & Mud & Mud & Mud & Very Coarse Silt & Clay & $\begin{array}{l}\text { Very Poorly } \\
\text { Sorted }\end{array}$ \\
\hline $\mathrm{JL}-68$ & Slightly Granuley & $\begin{array}{l}\text { Slightly Granuley } \\
\text { Clayey Fine Sand }\end{array}$ & $\begin{array}{l}\text { Slightly Gravelly } \\
\text { Muddy Sand }\end{array}$ & Muddy Sand & $\begin{array}{l}\text { Slightly Very Fine Gravelly } \\
\text { Muddy Fine Sand }\end{array}$ & $\begin{array}{l}\text { Slightly Granular } \\
\text { Muddy Fine Sand }\end{array}$ & Fine Sand & Fine Sand & $\begin{array}{l}\text { Very Poorly } \\
\text { Sorted }\end{array}$ \\
\hline $\mathrm{JL}-69$ & Slightly Granuley & $\begin{array}{l}\text { Slightly Granul ey Silty- } \\
\text { Clayey Very Fine Sand }\end{array}$ & $\begin{array}{l}\text { - Slightly Gravelly } \\
\text { Muddy Sand }\end{array}$ & Muddy Sand & $\begin{array}{l}\text { Slightly Very Fine Gravelly } \\
\text { Muddy Very Fine Sand }\end{array}$ & $\begin{array}{l}\text { Slightly Granular } \\
\text { Muddy Very Fine Sand }\end{array}$ & Very Fine Sand & Very Fine Sand & Poorly Sorted \\
\hline $\mathrm{JL}-70$ & Muddy Sand & $\begin{array}{l}\text { Silty-Clayey Very Fine } \\
\text { Sand }\end{array}$ & Muddy Sand & Muddy Sand & Muddy Very Fine Sand & Muddy Very Fine Sand & Very Coarse Silt & Coarse Silt & $\begin{array}{l}\text { Very Poorly } \\
\text { Sorted }\end{array}$ \\
\hline $\mathrm{JL}-71$ & Slightly Granuley & $\begin{array}{l}\text { Slightly Granuley Very } \\
\text { Fine Sandy Silt-Clay }\end{array}$ & $\begin{array}{l}\text { Slightly Gravelly } \\
\text { Sandy Mud }\end{array}$ & Sandy Mud & $\begin{array}{l}\text { Slightly Very Fine Gravelly } \\
\text { Very Fine Sandy Mud }\end{array}$ & $\begin{array}{l}\text { Slightly Granular Very } \\
\text { Fine Sandy Mud }\end{array}$ & Fine Silt & Very Fine Silt & $\begin{array}{l}\text { Very Poorly } \\
\text { Sorted }\end{array}$ \\
\hline $\mathrm{JL}-72$ & Pebbly & $\begin{array}{l}\text { Pebbly Silty-Clayey } \\
\text { Very Fine Sand }\end{array}$ & $\begin{array}{l}\text { Gravelly Muddy } \\
\text { Sand }\end{array}$ & N/A & $\begin{array}{l}\text { Medium Gravelly Muddy } \\
\text { Very Fine Sand }\end{array}$ & $\begin{array}{l}\text { Pebbly Muddy Very } \\
\text { Fine Sand }\end{array}$ & Very Coarse Silt & Coarse Silt & $\begin{array}{l}\text { Very Poorly } \\
\text { Sorted }\end{array}$ \\
\hline $\mathrm{JL}-73$ & Slightly Granuley & $\begin{array}{l}\text { Slightly Granuley Silty- } \\
\text { Clayey Very Fine Sand }\end{array}$ & $\begin{array}{l}\text { - Slightly Gravelly } \\
\text { Muddy Sand }\end{array}$ & Muddy Sand & $\begin{array}{l}\text { Slightly Very Fine Gravelly } \\
\text { Muddy Very Fine Sand }\end{array}$ & $\begin{array}{l}\text { Slightly Granular } \\
\text { Muddy Very Fine Sand }\end{array}$ & Very Coarse Silt & Coarse Silt & $\begin{array}{l}\text { Very Poorly } \\
\text { Sorted }\end{array}$ \\
\hline $\mathrm{JL}-74$ & Granuley & Granuley Fine Sand & Gravelly Sand & N/A & $\begin{array}{l}\text { Very Fine Gravelly Fine } \\
\text { Sand }\end{array}$ & Granular Fine Sand & Fine Sand & Fine Sand & Poorly Sorted \\
\hline $\mathrm{JL}-75$ & Granuley & $\begin{array}{l}\text { Granuley Medium } \\
\text { Sand }\end{array}$ & Gravelly Sand & $\mathrm{N} / \mathrm{A}$ & $\begin{array}{l}\text { Very Fine Gravelly Medium } \\
\text { Sand }\end{array}$ & $\begin{array}{l}\text { Granular Medium } \\
\text { Sand }\end{array}$ & Coarse Sand & Coarse Sand & Poorly Sorted \\
\hline $\mathrm{JL}-76$ & Slightly Pebbly & $\begin{array}{l}\text { Slightly Pebbly Fine } \\
\text { Sand }\end{array}$ & $\begin{array}{l}\text { Slightly Gravelly } \\
\text { Sand }\end{array}$ & $\mathrm{N} / \mathrm{A}$ & $\begin{array}{l}\text { Slightly Fine Gravelly Fine } \\
\text { Sand }\end{array}$ & $\begin{array}{l}\text { Slightly Pebbly Fine } \\
\text { Sand }\end{array}$ & Medium Sand & Medium Sand & Poorly Sorted \\
\hline $\mathrm{JL}-77$ & Gravel & Pebble Gravel & Gravel & $\mathrm{N} / \mathrm{A}$ & Coarse Gravel & Pebble Gravel & Fine Gravel & Pebble Gravel & Poorly Sorted \\
\hline $\mathrm{JL}-78$ & Mud & Silt-Clay & Mud & Mud & Mud & Mud & Clay & Clay & $\begin{array}{l}\text { Very Poorly } \\
\text { Sorted }\end{array}$ \\
\hline JL-79 & Pebble Mixes & Sandy Pebble Gravel & Sandy Gravel & $\mathrm{N} / \mathrm{A}$ & Sandy Medium Gravel & Sandy Pebble Gravel & Very Fine Gravel & Granule Gravel & $\begin{array}{l}\text { Very Poorly } \\
\text { Sorted }\end{array}$ \\
\hline $\mathrm{JL}-80$ & Sand & Very Fine Sand & Sand & Sand & Very Fine Sand & Very Fine Sand & Very Fine Sand & Very Fine Sand & Poorly Sorted \\
\hline $\mathrm{JL}-81$ & Pebbly & Pebbly Medium Sand & Gravelly Sand & $\mathrm{N} / \mathrm{A}$ & $\begin{array}{l}\text { Very Fine Gravelly Medium } \\
\text { Sand }\end{array}$ & Pebbly Medium Sand & Coarse Sand & Coarse Sand & $\begin{array}{l}\text { Very Poorly } \\
\text { Sorted }\end{array}$ \\
\hline JL-82 & Slightly Granuley & $\begin{array}{l}\text { Slightly Granuley Fine } \\
\text { Sand }\end{array}$ & $\begin{array}{l}\text { Slightly Gravelly } \\
\text { Sand }\end{array}$ & Sand & $\begin{array}{l}\text { Slightly Very Fine Gravelly } \\
\text { Fine Sand }\end{array}$ & $\begin{array}{l}\text { Slightly Granular Fine } \\
\text { Sand }\end{array}$ & Fine Sand & Fine Sand & Poorly Sorted \\
\hline JL-83 & Slightly Granuley & $\begin{array}{l}\text { Slightly Granuley Fine } \\
\text { Sand }\end{array}$ & $\begin{array}{l}\text { Slightly Gravelly } \\
\text { Sand }\end{array}$ & Sand & $\begin{array}{l}\text { Slightly Very Fine Gravelly } \\
\text { Fine Sand }\end{array}$ & $\begin{array}{l}\text { Slightly Granular Fine } \\
\text { Sand }\end{array}$ & Fine Sand & Fine Sand & $\begin{array}{l}\text { Moderately } \\
\text { Sorted }\end{array}$ \\
\hline
\end{tabular}


Jeffreys Ledge Survey (2002-2005) (4): Grain Size Statistics

\begin{tabular}{|c|c|c|c|c|c|c|c|c|c|c|c|c|c|c|c|c|c|c|c|}
\hline Sample ID & $\begin{array}{c}\text { Gravel } \\
\%\end{array}$ & $\begin{array}{c}\text { Sand } \\
\%\end{array}$ & $\begin{array}{c}\text { Mud } \\
\%\end{array}$ & $\begin{array}{c}\text { Sand } \\
\%\end{array}$ & $\begin{array}{c}\text { Silt } \\
\%\end{array}$ & $\begin{array}{c}\text { Clay } \\
\%\end{array}$ & Modes & $\begin{array}{c}\text { Mode } 1 \\
\text { (phi) }\end{array}$ & $\begin{array}{c}\text { Mode } 2 \\
\text { (phi) }\end{array}$ & $\begin{array}{l}D_{10} \\
\text { (phi) }\end{array}$ & $\begin{array}{c}D_{10} \\
(\mathrm{~mm})\end{array}$ & $\begin{array}{l}D_{50} \\
\text { (phi) }\end{array}$ & $\begin{array}{l}D_{50} \\
(\mathrm{~mm})\end{array}$ & $\begin{array}{c}\text { Mean } \\
\text { Size } \\
\text { (phi) }\end{array}$ & $\begin{array}{c}\text { Mean } \\
\text { Size } \\
(\mathrm{mm})\end{array}$ & $\begin{array}{l}\text { Sorting } \\
\text { (phi) }\end{array}$ & Skewness & Kurtosis & LOI \% \\
\hline JL-63 & 0.0 & 43.4 & 56.6 & 43.4 & 24.7 & 31.9 & u & 3.73 & & 3.20 & 0.11 & 4.58 & 0.04 & 6.25 & 0.01 & 3.36 & 0.68 & 0.74 & 3.90 \\
\hline JL-64 & 0.6 & 69.4 & 30.0 & 69.4 & 11.0 & 19.0 & U & 3.24 & & 1.76 & 0.30 & 3.24 & 0.11 & 4.76 & 0.04 & 3.37 & 0.65 & 1.53 & 3.00 \\
\hline JL-65 & 0.0 & 26.2 & 73.8 & 26.2 & 25.7 & 48.1 & B & 3.24 & 8.48 & 3.09 & 0.12 & 7.80 & 0.00 & 7.71 & 0.00 & 3.76 & 0.01 & 0.64 & 5.60 \\
\hline JL-66 & 0.0 & 5.2 & 94.8 & 5.2 & 33.4 & 61.4 & U & 8.48 & & 4.95 & 0.03 & 8.86 & 0.00 & 9.06 & 0.00 & 3.03 & 0.03 & 0.88 & 9.10 \\
\hline JL-67 & 0.0 & 7.6 & 92.4 & 7.6 & 31.4 & 61.0 & B & 9.48 & 3.73 & 4.56 & 0.04 & 8.84 & 0.00 & 8.53 & 0.00 & 2.80 & -0.13 & 1.23 & 7.10 \\
\hline JL-68 & 0.2 & 83.3 & 16.5 & 83.3 & 5.0 & 11.5 & U & 2.74 & & 1.30 & 0.41 & 2.71 & 0.15 & 2.95 & 0.13 & 2.28 & 0.44 & 3.21 & 2.30 \\
\hline JL-69 & 0.0 & 87.2 & 12.8 & 87.2 & 4.5 & 8.3 & U & 3.24 & & 2.14 & 0.23 & 3.12 & 0.12 & 3.15 & 0.11 & 1.61 & 0.33 & 4.51 & 1.50 \\
\hline JL-70 & 0.0 & 71.9 & 28.1 & 71.9 & 11.6 & 16.5 & $\mathrm{U}$ & 3.24 & & 2.82 & 0.14 & 3.50 & 0.09 & 4.90 & 0.03 & 2.74 & 0.81 & 2.82 & 2.20 \\
\hline JL-71 & 0.2 & 30.8 & 69.0 & 30.8 & 28.0 & 41.0 & B & 3.73 & 8.48 & 3.35 & 0.10 & 6.75 & 0.01 & 7.33 & 0.01 & 3.57 & 0.25 & 0.64 & 5.28 \\
\hline JL-72 & 5.4 & 64.2 & 30.4 & 64.2 & 12.6 & 17.8 & B & 3.24 & -3.24 & 1.58 & 0.33 & 3.45 & 0.09 & 4.82 & 0.04 & 3.66 & 0.47 & 2.50 & 3.06 \\
\hline JL-73 & 0.0 & 71.6 & 28.4 & 71.6 & 13.2 & 15.2 & U & 3.24 & & 2.84 & 0.14 & 3.60 & 0.08 & 4.79 & 0.04 & 2.58 & 0.77 & 2.93 & 2.40 \\
\hline JL-74 & 6.3 & 86.7 & 7.0 & 86.7 & 2.3 & 4.7 & U & 2.74 & & -0.14 & 1.10 & 2.46 & 0.18 & 2.14 & 0.23 & 1.89 & -0.15 & 3.17 & 1.60 \\
\hline JL-75 & 6.2 & 90.8 & 3.1 & 90.8 & 0.5 & 2.6 & U & 1.25 & & -0.63 & 1.55 & 0.91 & 0.53 & 0.82 & 0.57 & 1.06 & -0.15 & 1.07 & 0.70 \\
\hline JL-76 & 2.3 & 90.7 & 7.0 & 90.7 & 1.8 & 5.2 & U & 2.24 & & 0.37 & 0.77 & 1.90 & 0.27 & 1.82 & 0.28 & 1.77 & 0.20 & 2.77 & 0.90 \\
\hline JL-77 & 85.4 & 14.3 & 0.3 & 14.3 & & & U & -4.24 & & -4.35 & 20.36 & -3.53 & 11.54 & -2.98 & 7.91 & 1.67 & 0.61 & 1.07 & 0.40 \\
\hline JL-78 & 0.0 & 1.6 & 98.4 & 1.6 & 32.2 & 66.2 & U & 8.48 & & 5.58 & 0.02 & 9.32 & 0.00 & 9.39 & 0.00 & 2.88 & 0.00 & 0.81 & 8.00 \\
\hline JL-79 & 69.9 & 29.2 & 0.9 & 29.2 & & & B & -3.24 & 1.75 & -3.75 & 13.45 & -2.62 & 6.13 & -1.66 & 3.15 & 2.13 & 0.59 & 0.72 & 0.70 \\
\hline JL-80 & 0.0 & 91.4 & 8.6 & 91.4 & 3.9 & 4.7 & U & 3.24 & & 2.49 & 0.18 & 3.13 & 0.11 & 3.15 & 0.11 & 1.12 & 0.36 & 3.19 & 1.30 \\
\hline JL-81 & 14.0 & 80.3 & 5.7 & 80.3 & 1.7 & 4.0 & U & 1.25 & & -1.44 & 2.71 & 0.83 & 0.56 & 0.80 & 0.58 & 2.06 & 0.08 & 1.55 & 0.80 \\
\hline JL-82 & 0.4 & 92.1 & 7.4 & 92.1 & 3.0 & 4.4 & U & 2.74 & & 1.59 & 0.33 & 2.56 & 0.17 & 2.56 & 0.17 & 1.26 & 0.25 & 3.08 & 1.20 \\
\hline JL-83 & 0.8 & 94.6 & 4.6 & 94.6 & 1.3 & 3.3 & $\mathrm{u}$ & 2.24 & & 1.17 & 0.45 & 2.18 & 0.22 & 2.16 & 0.22 & 0.78 & -0.05 & 1.69 & 0.90 \\
\hline
\end{tabular}




\section{Jeffreys Ledge Survey (2002-2005) (4): Grain Size Distribution}

\begin{tabular}{|c|c|c|c|c|c|c|c|c|c|c|c|c|c|c|c|c|c|c|c|c|c|c|c|c|c|c|c|c|c|c|c|c|}
\hline Sample ID & $\begin{array}{c}\text { Class } \\
\% \\
\text { phi } \\
-5.5\end{array}$ & $\begin{array}{c}\text { Class } \\
\% \\
\text { phi } \\
-5.0\end{array}$ & $\begin{array}{c}\text { Class } \\
\% \\
\text { phi } \\
-4.5 \\
\end{array}$ & $\begin{array}{c}\text { Class } \\
\% \\
\text { phi } \\
-4.0 \\
\end{array}$ & $\begin{array}{c}\text { Class } \\
\% \\
\text { phi } \\
-3.5\end{array}$ & $\begin{array}{c}\text { Class } \\
\% \\
\text { phi } \\
-3.0\end{array}$ & $\begin{array}{c}\text { Class } \\
\% \\
\text { phi } \\
-2.5 \\
\end{array}$ & $\begin{array}{c}\text { Class } \\
\% \\
\text { phi } \\
-2.0\end{array}$ & $\begin{array}{c}\text { Class } \\
\% \\
\text { phi } \\
-1.5\end{array}$ & $\begin{array}{c}\text { Class } \\
\% \\
\text { phi } \\
-1.0\end{array}$ & $\begin{array}{c}\text { Class } \\
\% \\
\text { phi } \\
-0.5\end{array}$ & $\begin{array}{c}\text { Class } \\
\% \\
\text { phi } \\
0.0\end{array}$ & $\begin{array}{c}\text { Class } \\
\% \\
\text { phi } \\
0.5 \\
\end{array}$ & $\begin{array}{c}\text { Class } \\
\% \\
\text { phi } \\
1.0\end{array}$ & $\begin{array}{c}\text { Class } \\
\% \\
\text { phi } \\
1.5\end{array}$ & $\begin{array}{c}\text { Class } \\
\% \\
\text { phi } \\
2.0\end{array}$ & $\begin{array}{c}\text { Class } \\
\% \\
\text { phi } \\
2.5\end{array}$ & $\begin{array}{c}\text { Class } \\
\% \\
\text { phi } \\
3.0\end{array}$ & $\begin{array}{c}\text { Class } \\
\% \\
\text { phi } \\
\mathbf{3 . 5}\end{array}$ & $\begin{array}{c}\text { Class } \\
\% \\
\text { phi } \\
4.0\end{array}$ & $\begin{array}{c}\text { Class } \\
\% \\
\text { phi } \\
>4.0\end{array}$ & $\begin{array}{c}\text { Class } \\
\% \\
\text { phi } \\
5.0\end{array}$ & $\begin{array}{c}\text { Class } \\
\% \\
\text { phi } \\
6.0\end{array}$ & $\begin{array}{c}\text { Class } \\
\% \\
\text { phi } \\
7.0\end{array}$ & $\begin{array}{c}\text { Class } \\
\% \\
\text { phi } \\
8.0\end{array}$ & $\begin{array}{c}\text { Class } \\
\% \\
\text { phi } \\
9.0\end{array}$ & $\begin{array}{c}\text { Class } \\
\% \\
\text { phi } \\
10.0\end{array}$ & $\begin{array}{c}\text { Class } \\
\% \\
\text { phi } \\
>10.0\end{array}$ & $\begin{array}{c}\text { Class } \\
\% \\
\text { phi } \\
11.0\end{array}$ & $\begin{array}{c}\text { Class } \\
\% \\
\text { phi } \\
12.0\end{array}$ & $\begin{array}{c}\text { Class } \\
\% \\
\text { phi } \\
13.0\end{array}$ & $\begin{array}{c}\text { Class } \\
\% \\
\text { phi } \\
14.0\end{array}$ \\
\hline LL-63 & 0.00 & 0.00 & 0.00 & 0.00 & 0.00 & 0.00 & 0.00 & 0.00 & 0.00 & 0.00 & 0.00 & 0.02 & 0.02 & 0.03 & 0.03 & 0.15 & 0.34 & 2.84 & 15.51 & 24.36 & & 11.52 & 2.88 & 7.64 & 2.73 & 5.91 & 6.03 & & 4.99 & 4.99 & 4.99 & $\begin{array}{l}9 \\
9\end{array}$ \\
\hline L-64 & 0.00 & 0.00 & 0.00 & 0.00 & 0.00 & 0.00 & 0.00 & 0.00 & 0.27 & 0.36 & 0.75 & 0.82 & 1.20 & 2.00 & 2.58 & 3.86 & 7.52 & 19.25 & 22.17 & 9.20 & & 3.77 & 1.81 & 2.35 & 3.19 & 3.56 & 3.41 & & 2.98 & $\begin{array}{r}3.98 \\
\end{array}$ & 2.98 & $\begin{array}{l}3 \quad 2.98 \\
\end{array}$ \\
\hline L-65 & 0.00 & 0.00 & 0.00 & 0.00 & 0.00 & 0.00 & 0.00 & 0.00 & 0.00 & 0.00 & 0.05 & 0.02 & 0.08 & 0.09 & 0.15 & 0.27 & 0.97 & 6.43 & 10.07 & 7.97 & & 6.36 & 4.23 & 6.44 & 8.69 & 9.20 & 7.98 & & 7.76 & 7.76 & 7.76 & 7.76 \\
\hline JL-66 & 0.00 & 0.00 & 0.00 & 0.00 & 0.00 & 0.00 & 0.00 & 0.00 & 0.00 & 0.00 & 0.07 & 0.05 & 0.01 & 0.01 & 0.01 & 0.03 & 0.06 & 0.35 & 1.50 & 3.10 & & 5.14 & 5.82 & 9.75 & 12.74 & 412.82 & 10.84 & & 9.43 & 9.43 & 9.43 & $\begin{array}{l}3 \quad 9.43 \\
\end{array}$ \\
\hline JL-67 & 0.00 & 0.00 & 0.00 & 0.00 & 0.00 & 0.00 & 0.00 & 0.00 & 0.00 & 0.00 & 0.02 & 0.01 & 0.04 & 0.03 & 0.07 & 0.09 & 0.15 & 0.73 & 2.50 & 3.92 & & 4.33 & 6.37 & 9.13 & 11.57 & 712.75 & 26.05 & & 5.55 & 5.55 & 5.55 & 5.55 \\
\hline LL-68 & 0.00 & 0.00 & 0.00 & 0.00 & 0.00 & 0.00 & 0.00 & 0.00 & 0.00 & 0.22 & 0.59 & 0.71 & 1.56 & 3.19 & 6.11 & 12.01 & 14.53 & 324.74 & 15.51 & 4.29 & & 1.13 & 0.42 & 1.51 & 1.98 & 2.20 & 2.21 & & 1.77 & 1.77 & 1.77 & $\begin{array}{l}7 \quad 1.77 \\
\end{array}$ \\
\hline L-69 & 0.00 & 0.00 & 0.00 & 0.00 & 0.00 & 0.00 & 0.00 & 0.00 & 0.00 & 0.01 & 0.38 & 0.69 & 1.02 & 1.40 & 1.94 & 2.75 & 6.13 & 26.62 & 35.84 & 10.44 & & 1.46 & 0.67 & 0.89 & 1.43 & 1.73 & 1.96 & & 1.15 & 1.15 & 1.15 & $5 \quad 1.15$ \\
\hline L-70 & 00 & 0.00 & 0.00 & 0.00 & 0.00 & 0.00 & 0.00 & 0.00 & 0.00 & 0.00 & 0.05 & 0.06 & 0.08 & 0.13 & 0.27 & 0.62 & 1.60 & 10.90 & 34.94 & 23.20 & & 5.78 & 1.37 & 1.97 & 2.53 & 3.18 & 2.51 & & 2.70 & 2.70 & 2.70 & $\begin{array}{r}2.70 \\
\end{array}$ \\
\hline $\mathrm{JL}-71$ & 0.00 & 0.00 & 0.00 & 0.00 & 0.00 & 0.00 & 0.00 & 0.00 & 0.00 & 0.17 & 0.01 & 0.02 & 0.05 & 0.06 & 0.05 & 0.14 & 0.40 & 2.26 & 9.37 & 18.28 & & 10.79 & 4.01 & 5.83 & 7.56 & 7.62 & 6.06 & & 6.83 & 6.83 & 6.83 & $\begin{array}{l}3 \quad 6.83 \\
\end{array}$ \\
\hline $\mathrm{JL}-72$ & 0.00 & 0.00 & 0.00 & 0.00 & 0.00 & 4.93 & 0.00 & 0.00 & 0.21 & 0.30 & 0.34 & 0.42 & 0.69 & 1.05 & 1.62 & 2.51 & 3.81 & 11.68 & 323.91 & 18.11 & & 5.07 & 1.79 & 2.49 & 3.24 & 3.34 & 2.89 & & 2.90 & 2.90 & 2.90 & 2.90 \\
\hline JL-73 & 0.00 & 0.00 & 0.00 & 0.00 & 0.00 & 0.00 & 0.00 & 0.00 & 0.00 & 0.02 & 0.02 & 0.04 & 0.06 & 0.18 & 0.30 & 0.61 & 1.56 & 10.41 & 29.75 & 28.62 & & 6.90 & 1.61 & 2.03 & 2.66 & 2.79 & 2.85 & & 2.40 & 2.40 & 2.40 & 2.40 \\
\hline JL-74 & 0.00 & 0.00 & 0.00 & 0.00 & 0.00 & 0.00 & 0.00 & 1.54 & 2.75 & 2.01 & 2.12 & 2.19 & 2.89 & 3.42 & 4.04 & 8.17 & 21.59 & 31.67 & 8.73 & 1.87 & & 0.78 & 0.47 & 0.54 & 0.48 & 0.74 & 1.05 & & 0.74 & $\begin{array}{r}0.74 \\
\end{array}$ & 0.74 & $\begin{array}{l}4 \quad 0.74 \\
\end{array}$ \\
\hline JL-75 & 0.00 & 0.00 & 0.00 & 0.00 & 0.00 & 0.00 & 0.00 & 0.99 & 2.09 & 3.08 & 5.32 & 9.16 & 14.61 & 17.85 & 20.42 & 17.73 & 4.12 & 0.88 & 0.48 & 0.21 & & 0.02 & 0.15 & 0.11 & 0.19 & 0.45 & 0.58 & 1.55 & & & & \\
\hline $\mathrm{JL}-76$ & 0.00 & 0.00 & 0.00 & 0.00 & 0.00 & 0.00 & 1.06 & 0.12 & 0.49 & 0.67 & 1.04 & 2.04 & 6.09 & 8.24 & 12.18 & 22.67 & 21.34 & 13.84 & $\begin{array}{r}2.69 \\
\end{array}$ & 0.51 & & 0.29 & 0.38 & 0.46 & 0.71 & 0.72 & 1.02 & & 0.86 & 0.86 & 0.86 & $5 \quad 0.86$ \\
\hline JL-77 & 0.00 & 0.00 & 0.00 & 35.26 & 16.09 & 14.93 & 3.90 & 2.94 & 8.07 & 4.19 & 3.08 & 1.90 & 1.68 & 1.42 & 1.54 & 1.82 & 1.40 & 1.13 & 0.26 & 0.07 & 0.35 & & & & & & & & & & & \\
\hline JL-78 & 0.00 & 0.00 & 0.00 & 0.00 & 0.00 & 0.00 & 0.00 & 0.00 & 0.00 & 0.00 & 0.00 & 0.02 & 0.02 & 0.02 & 0.03 & 0.03 & 0.06 & 0.22 & 0.44 & 0.69 & & 4.42 & 7.09 & 9.41 & 11.36 & 512.30 & 11.29 & & 10.65 & 510.65 & 510.65 & $5 \quad 10.6$ \\
\hline JL-79 & 0.00 & 0.00 & 0.00 & 0.00 & 20.48 & 19.56 & 13.37 & 9.38 & 5.10 & 2.00 & 2.14 & 2.43 & 3.11 & 4.06 & 4.70 & 6.40 & 5.10 & 1.06 & 0.12 & 0.05 & 0.96 & & & & & & & & & & & \\
\hline $\mathrm{JL}-80$ & 0.00 & 0.00 & 0.00 & 0.00 & 0.00 & 0.00 & 0.00 & 0.00 & 0.00 & 0.00 & 0.15 & 0.24 & 0.43 & 0.49 & 0.54 & 1.12 & 5.86 & 31.50 & 36.54 & 14.52 & & 1.86 & 0.69 & 0.53 & 0.80 & 1.22 & 1.17 & & 0.58 & 0.58 & 0.58 & $\begin{array}{l}3 \quad 0.58 \\
\end{array}$ \\
\hline JL-81 & 0.00 & 0.00 & 0.00 & 0.00 & 0.00 & 3.52 & 1.38 & 2.24 & 2.42 & 4.49 & 7.34 & 9.15 & 11.29 & 12.19 & 12.27 & 11.59 & 6.87 & 5.20 & 2.92 & 1.46 & & 0.40 & 0.39 & 0.42 & 0.53 & 0.75 & 0.74 & & 0.62 & 0.62 & 0.62 & $2 \quad 0.62$ \\
\hline JL-82 & 0.00 & 0.00 & 0.00 & 0.00 & 0.00 & 0.00 & 0.00 & 0.00 & 0.23 & 0.19 & 0.28 & 0.59 & 1.32 & 2.11 & 3.42 & 9.88 & 26.53 & 34.34 & 11.09 & 2.56 & & 1.29 & 0.49 & 0.56 & 0.72 & 1.03 & 1.07 & & 0.58 & 0.58 & 0.58 & 0.58 \\
\hline JL-83 & 0.00 & 0.00 & 0.00 & 0.00 & 0.00 & 0.00 & 0.00 & 0.24 & 0.21 & 0.36 & 0.44 & 1.37 & 2.37 & 3.09 & 5.73 & 21.95 & 37.95 & 18.40 & 2.71 & 0.60 & & 0.44 & 0.21 & 0.27 & 0.37 & 0.56 & 0.76 & & 0.50 & 0.50 & 0.50 & 0.50 \\
\hline
\end{tabular}


Jeffreys Ledge Survey (2002-2005) (5): Identification, Location, and Description

\begin{tabular}{|c|c|c|c|c|c|c|c|c|}
\hline Sample ID & $\begin{array}{c}\text { Original } \\
\text { Sample ID }\end{array}$ & $\begin{array}{c}\text { Latitude } \\
\text { WGS84 }\end{array}$ & $\begin{array}{l}\text { Longitude } \\
\text { WGS84 }\end{array}$ & $\begin{array}{l}\text { Reliability } \\
\text { Ranking for } \\
\text { Positioning }\end{array}$ & $\begin{array}{c}\text { Water } \\
\text { Depth } \\
\text { (m) }\end{array}$ & $\begin{array}{l}\text { Sample } \\
\text { Collected }\end{array}$ & $\begin{array}{c}\text { Sampler } \\
\text { Type }\end{array}$ & $\begin{array}{c}\text { Total Wt } \\
\text { (gm) }\end{array}$ \\
\hline JL-84 & JL120 & 42.858150 & -70.214030 & 1 & 57 & $9 / 23 / 2004$ & SH & 177.20 \\
\hline JL-85 & JL121 & 42.858250 & -70.196920 & 1 & 58 & $9 / 23 / 2004$ & SH & 140.10 \\
\hline JL-86 & JL123 & 42.858000 & -70.163650 & 1 & 59 & $9 / 23 / 2004$ & SH & 287.90 \\
\hline JL-87 & JL126 & 42.858300 & -70.113500 & 1 & 105 & $11 / 17 / 2004$ & $\mathrm{BC}$ & 34.40 \\
\hline JL-88 & JL127 & 42.846080 & -70.396630 & 1 & 134 & $9 / 13 / 2002$ & $\mathrm{BC}$ & 7.20 \\
\hline JL-89 & JL129 & 42.845980 & -70.363550 & 1 & 62 & $6 / 28 / 2004$ & SH & 19.50 \\
\hline JL-90 & JL131 & 42.846820 & -70.328720 & 1 & 53 & $6 / 28 / 2004$ & SH & \\
\hline JL-91 & JL138 & 42.845720 & -70.213530 & 1 & 56 & $9 / 23 / 2004$ & SH & 284.70 \\
\hline JL-92 & JL139 & 42.845720 & -70.197430 & 1 & 54 & $9 / 23 / 2004$ & $\mathrm{SH}$ & 125.00 \\
\hline JL-93 & JL142b & 42.845960 & -70.146720 & 1 & 108 & $10 / 8 / 2002$ & $\mathrm{BC}$ & 73.20 \\
\hline JL-94 & JL144a & 42.845970 & -70.113370 & 1 & 115 & $9 / 30 / 2002$ & $\mathrm{BC}$ & 97.80 \\
\hline JL-95 & JL144b & 42.845450 & -70.114050 & 1 & 115 & $11 / 17 / 2004$ & $\mathrm{BC}$ & 23.00 \\
\hline JL-96 & JL145 & 42.832730 & -70.397640 & 1 & 140 & $8 / 7 / 2002$ & $\mathrm{BC}$ & 12.90 \\
\hline JL-97 & JL146 & 42.833420 & -70.380430 & 1 & 130 & $9 / 13 / 2002$ & $\mathrm{BC}$ & 10.20 \\
\hline JL-98 & JL151 & 42.833120 & -70.296620 & 1 & 54 & $9 / 21 / 2004$ & $\mathrm{SH}$ & 190.50 \\
\hline JL-99 & JL154 & 42.833520 & -70.247520 & 1 & 54 & $9 / 21 / 2004$ & $\mathrm{SH}$ & 167.30 \\
\hline JL-100 & JL156 & 42.832600 & -70.214230 & 1 & 54 & $9 / 23 / 2004$ & SH & 204.60 \\
\hline JL-101 & JL157 & 42.833120 & -70.197500 & 1 & 90 & $8 / 26 / 2005$ & SH & 24.90 \\
\hline JL-102 & JL159 & 42.833200 & -70.163730 & 1 & 108 & $11 / 17 / 2004$ & $B C$ & 51.10 \\
\hline JL-103 & JL160 & 42.833400 & -70.147530 & 1 & 123 & $11 / 17 / 2004$ & $\mathrm{BC}$ & 28.00 \\
\hline JL-104 & JL162a & 42.833580 & -70.113580 & 1 & 130 & $9 / 30 / 2002$ & $B C$ & 68.30 \\
\hline
\end{tabular}


Jeffreys Ledge Survey (2002-2005) (5): Sediment Classifications

\begin{tabular}{|c|c|c|c|c|c|c|c|c|c|}
\hline Sample ID & $\begin{array}{l}\text { CMECS Substrate } \\
\text { Component } \\
\text { Group (Specific) }\end{array}$ & $\begin{array}{l}\text { CMECS Substrate } \\
\text { Component } \\
\text { Subgroup (Specific) }\end{array}$ & $\begin{array}{l}\text { Textural Group } \\
\text { from \%GSM } \\
\text { (Gradistat) }\end{array}$ & $\begin{array}{l}\text { Textural Group } \\
\text { from \%SZC } \\
\text { (Gradistat) }\end{array}$ & $\begin{array}{l}\text { Sediment Name from } \\
\text { \%GSM } \\
\text { and Mode (Gradistat) }\end{array}$ & $\begin{array}{l}\text { Sediment Name from } \\
\text { \%GSM and Mode } \\
\text { (Wentworth Scale) }\end{array}$ & $\begin{array}{l}\text { Sediment } \\
\text { Classification from } \\
\text { Mean Phi (Gradistat) }\end{array}$ & $\begin{array}{l}\text { Classification } \\
\text { from Mean Phi } \\
\text { (Wentworth) } \\
\end{array}$ & $\begin{array}{l}\text { Sorting } \\
\text { (Gradistat) } \\
\end{array}$ \\
\hline $\mathrm{JL}-84$ & Pebble Mixes & Sandy Pebble Gravel & Sandy Gravel & N/A & Sandy Medium Gravel & Sandy Pebble Gravel & Fine Gravel & Pebble Gravel & $\begin{array}{l}\text { Very Poorly } \\
\text { Sorted }\end{array}$ \\
\hline $\mathrm{JL}-85$ & Gravel & Pebble Gravel & Gravel & $\mathrm{N} / \mathrm{A}$ & Coarse Gravel & Pebble Gravel & Medium Gravel & Pebble Gravel & Poorly Sorted \\
\hline $\mathrm{JL}-86$ & Pebble Mixes & Sandy Pebble Gravel & Sandy Gravel & $\mathrm{N} / \mathrm{A}$ & Sandy Very Coarse Gravel & Sandy Pebble Gravel & Medium Gravel & Pebble Gravel & $\begin{array}{l}\text { Very Poorly } \\
\text { Sorted }\end{array}$ \\
\hline $\mathrm{JL}-87$ & Slightly Granuley & $\begin{array}{l}\text { Slightly Granuley Fine } \\
\text { Sand }\end{array}$ & $\begin{array}{l}\text { Slightly Gravelly } \\
\text { Sand }\end{array}$ & Sand & $\begin{array}{l}\text { Slightly Very Fine Gravelly } \\
\text { Fine Sand }\end{array}$ & $\begin{array}{l}\text { Slightly Granular Fine } \\
\text { Sand }\end{array}$ & Fine Sand & Fine Sand & Poorly Sorted \\
\hline $\mathrm{JL}-88$ & Mud & Silt-Clay & Mud & Mud & Mud & Mud & Clay & Clay & $\begin{array}{l}\text { Very Poorly } \\
\text { Sorted }\end{array}$ \\
\hline $\mathrm{JL}-89$ & Granuley & $\begin{array}{l}\text { Granuley Medium } \\
\text { Sand }\end{array}$ & Gravelly Sand & N/A & $\begin{array}{l}\text { Very Fine Gravelly Medium } \\
\text { Sand }\end{array}$ & $\begin{array}{l}\text { Granular Medium } \\
\text { Sand }\end{array}$ & Medium Sand & Medium Sand & Poorly Sorted \\
\hline $\mathrm{JL}-90$ & Gravel & Pebble Gravel & Gravel & N/A & Fine Gravel & Pebble Gravel & Fine Gravel & Pebble Gravel & $\begin{array}{l}\text { Moderately } \\
\text { Sorted }\end{array}$ \\
\hline JL-91 & Pebble Mixes & Sandy Pebble Gravel & Sandy Gravel & $\mathrm{N} / \mathrm{A}$ & Sandy Very Coarse Gravel & Sandy Pebble Gravel & Medium Gravel & Pebble Gravel & $\begin{array}{l}\text { Very Poorly } \\
\text { Sorted }\end{array}$ \\
\hline $\mathrm{JL}-92$ & Gravel & Pebble Gravel & Gravel & $\mathrm{N} / \mathrm{A}$ & Coarse Gravel & Pebble Gravel & Medium Gravel & Pebble Gravel & Poorly Sorted \\
\hline $\mathrm{JL}-93$ & Slightly Pebbly & $\begin{array}{l}\text { Slightly Pebbly Fine } \\
\text { Sand }\end{array}$ & $\begin{array}{l}\text { Slightly Gravelly } \\
\text { Sand }\end{array}$ & $\mathrm{N} / \mathrm{A}$ & $\begin{array}{l}\text { Slightly Medium Gravelly } \\
\text { Fine Sand }\end{array}$ & $\begin{array}{l}\text { Slightly Pebbly Fine } \\
\text { Sand }\end{array}$ & Fine Sand & Fine Sand & Poorly Sorted \\
\hline $\mathrm{JL}-94$ & Pebbly & Pebbly Fine Sand & Gravelly Sand & $\mathrm{N} / \mathrm{A}$ & Medium Gravelly Fine Sand & Pebbly Fine Sand & Fine Sand & Fine Sand & $\begin{array}{l}\text { Very Poorly } \\
\text { Sorted }\end{array}$ \\
\hline $\mathrm{JL}-95$ & Pebbly & $\begin{array}{l}\text { Pebbly Clayey Fine } \\
\text { Sand }\end{array}$ & $\begin{array}{l}\text { Gravelly Muddy } \\
\text { Sand }\end{array}$ & N/A & $\begin{array}{l}\text { Medium Gravelly Muddy } \\
\text { Fine Sand }\end{array}$ & $\begin{array}{l}\text { Pebbly Muddy Fine } \\
\text { Sand }\end{array}$ & Very Fine Sand & Very Fine Sand & $\begin{array}{l}\text { Very Poorly } \\
\text { Sorted }\end{array}$ \\
\hline JL-96 & Mud & Silt-Clay & Mud & Mud & Mud & Mud & Clay & Clay & $\begin{array}{l}\text { Very Poorly } \\
\text { Sorted }\end{array}$ \\
\hline JL-97 & Mud & Silt-Clay & Mud & Mud & Mud & Mud & Very Fine Silt & Clay & $\begin{array}{l}\text { Very Poorly } \\
\text { Sorted }\end{array}$ \\
\hline $\mathrm{JL}-98$ & Gravel & Pebble Gravel & Gravel & $\mathrm{N} / \mathrm{A}$ & Very Coarse Gravel & Pebble Gravel & Coarse Gravel & Pebble Gravel & Poorly Sorted \\
\hline JL-99 & Pebble Mixes & $\begin{array}{l}\text { Clayey Sandy Pebble } \\
\text { Gravel }\end{array}$ & $\begin{array}{l}\text { Muddy Sandy } \\
\text { Gravel }\end{array}$ & N/A & $\begin{array}{l}\text { Muddy Sandy Coarse } \\
\text { Gravel }\end{array}$ & $\begin{array}{l}\text { Muddy Sandy Pebble } \\
\text { Gravel }\end{array}$ & Medium Gravel & Pebble Gravel & $\begin{array}{l}\text { Very Poorly } \\
\text { Sorted }\end{array}$ \\
\hline $\mathrm{JL}-100$ & Pebble Mixes & Sandy Pebble Gravel & Sandy Gravel & N/A & Sandy Medium Gravel & Sandy Pebble Gravel & Medium Gravel & Pebble Gravel & $\begin{array}{l}\text { Very Poorly } \\
\text { Sorted }\end{array}$ \\
\hline $\mathrm{JL}-101$ & Pebbly & Pebbly Medium Sand & Gravelly Sand & $\mathrm{N} / \mathrm{A}$ & Fine Gravelly Medium Sand & Pebbly Medium Sand & Coarse Sand & Coarse Sand & Poorly Sorted \\
\hline $\mathrm{JL}-102$ & Sand & Fine Sand & Sand & Sand & Fine Sand & Fine Sand & Fine Sand & Fine Sand & Poorly Sorted \\
\hline $\mathrm{JL}-103$ & Muddy Sand & $\begin{array}{l}\text { Silty-Clayey Very Fine } \\
\text { Sand }\end{array}$ & Muddy Sand & Muddy Sand & Muddy Very Fine Sand & Muddy Very Fine Sand & Very Fine Sand & Very Fine Sand & Poorly Sorted \\
\hline JL-104 & Pebbly & $\begin{array}{l}\text { Pebbly Silty-Clayey } \\
\text { Very Fine Sand }\end{array}$ & $\begin{array}{l}\text { Gravelly Muddy } \\
\text { Sand }\end{array}$ & N/A & $\begin{array}{l}\text { Coarse Gravelly Muddy } \\
\text { Very Fine Sand }\end{array}$ & $\begin{array}{l}\text { Pebbly Muddy Very } \\
\text { Fine Sand }\end{array}$ & Medium Sand & Medium Sand & $\begin{array}{l}\text { Very Poorly } \\
\text { Sorted }\end{array}$ \\
\hline
\end{tabular}


Jeffreys Ledge Survey (2002-2005) (5): Grain Size Statistics

\begin{tabular}{|c|c|c|c|c|c|c|c|c|c|c|c|c|c|c|c|c|c|c|c|}
\hline Sample ID & $\begin{array}{c}\text { Gravel } \\
\%\end{array}$ & $\begin{array}{c}\text { Sand } \\
\%\end{array}$ & $\begin{array}{c}\text { Mud } \\
\%\end{array}$ & $\begin{array}{c}\text { Sand } \\
\%\end{array}$ & $\begin{array}{c}\text { Silt } \\
\%\end{array}$ & $\begin{array}{c}\text { Clay } \\
\%\end{array}$ & Modes & $\begin{array}{c}\text { Mode } 1 \\
\text { (phi) }\end{array}$ & $\begin{array}{c}\text { Mode } 2 \\
\text { (phi) }\end{array}$ & $\begin{array}{l}D_{10} \\
\text { (phi) }\end{array}$ & $\begin{array}{c}D_{10} \\
(\mathrm{~mm})\end{array}$ & $\begin{array}{l}D_{50} \\
\text { (phi) }\end{array}$ & $\begin{array}{l}D_{50} \\
(\mathrm{~mm})\end{array}$ & $\begin{array}{c}\text { Mean } \\
\text { Size } \\
\text { (phi) }\end{array}$ & $\begin{array}{c}\text { Mean } \\
\text { Size } \\
(\mathrm{mm})\end{array}$ & $\begin{array}{l}\text { Sorting } \\
\text { (phi) }\end{array}$ & Skewness & Kurtosis & LOI \% \\
\hline JL-84 & 73.3 & 24.1 & 2.6 & 24.1 & 0.8 & 1.8 & B & -3.24 & 1.25 & -4.24 & 18.84 & -3.10 & 8.60 & -2.18 & 4.53 & 2.14 & 0.59 & 0.82 & 0.80 \\
\hline $\mathrm{JL}-85$ & 81.2 & 18.5 & 0.3 & 18.5 & & & $\mathrm{~B}$ & -4.24 & -0.24 & -4.62 & 24.62 & -3.85 & 14.43 & -3.00 & 8.01 & 1.74 & 0.66 & 0.93 & 0.50 \\
\hline JL-86 & 79.5 & 20.3 & 0.2 & 20.3 & & & $\mathrm{~B}$ & -5.24 & -4.24 & -5.40 & 42.11 & -5.01 & 32.22 & -3.56 & 11.75 & 2.23 & 0.87 & 0.91 & 0.50 \\
\hline JL-87 & 0.1 & 90.0 & 9.9 & 90.0 & 2.7 & 7.2 & U & 2.74 & & 1.88 & 0.27 & 2.58 & 0.17 & 2.57 & 0.17 & 1.46 & 0.36 & 4.79 & 1.50 \\
\hline JL-88 & 0.0 & 2.2 & 97.8 & 2.2 & 33.8 & 64.0 & U & 7.50 & & 5.56 & 0.02 & 9.24 & 0.00 & 9.34 & 0.00 & 2.90 & 0.01 & 0.81 & 7.60 \\
\hline JL-89 & 5.4 & 92.6 & 2.0 & 92.6 & & & U & 1.75 & & 0.09 & 0.94 & 1.52 & 0.35 & 1.46 & 0.36 & 1.05 & -0.22 & 1.48 & 0.80 \\
\hline JL-90 & 89.1 & 11.0 & 0.0 & 11.0 & & & U & -2.24 & & -3.24 & 9.47 & -2.34 & 5.06 & -2.29 & 4.88 & 1.00 & 0.28 & 1.37 & \\
\hline JL-91 & 70.8 & 27.4 & 1.8 & 27.4 & 0.5 & 1.3 & $\mathrm{U}$ & -5.24 & & -5.39 & 41.87 & -4.96 & 31.17 & -3.18 & 9.04 & 2.62 & 0.87 & 0.59 & 0.90 \\
\hline JL-92 & 87.1 & 12.5 & 0.4 & 12.5 & & & $U$ & -4.24 & & -4.79 & 27.74 & -4.11 & 17.28 & -3.66 & 12.67 & 1.53 & 0.64 & 1.82 & 0.70 \\
\hline JL-93 & 1.2 & 91.2 & 7.6 & 91.2 & 2.0 & 5.6 & U & 2.74 & & 2.01 & 0.25 & 2.59 & 0.17 & 2.57 & 0.17 & 1.27 & 0.33 & 4.28 & 1.20 \\
\hline JL-94 & 8.7 & 83.0 & 8.3 & 83.0 & 2.0 & 6.3 & B & 2.74 & -3.74 & -0.27 & 1.21 & 2.67 & 0.16 & 2.49 & 0.18 & 2.34 & -0.16 & 6.66 & 1.20 \\
\hline JL-95 & 8.1 & 74.6 & 17.3 & 74.6 & 5.0 & 12.3 & B & 2.74 & -3.24 & -0.21 & 1.16 & 2.73 & 0.15 & 3.09 & 0.12 & 3.16 & 0.24 & 5.34 & 2.30 \\
\hline JL-96 & 0.0 & 0.6 & 99.4 & 0.6 & 32.6 & 66.8 & U & 7.50 & & 6.19 & 0.01 & 9.51 & 0.00 & 9.62 & 0.00 & 2.67 & 0.04 & 0.75 & 8.93 \\
\hline JL-97 & 0.0 & 4.2 & 95.8 & 4.2 & 37.2 & 58.6 & U & 8.48 & & 4.74 & 0.04 & 8.81 & 0.00 & 8.87 & 0.00 & 3.14 & 0.01 & 0.78 & 8.60 \\
\hline JL-98 & 89.4 & 9.9 & 0.7 & 9.9 & & & U & -5.24 & & -5.38 & 41.56 & -4.87 & 29.32 & -4.34 & 20.28 & 1.63 & 0.74 & 2.73 & 1.00 \\
\hline JL-99 & 78.0 & 17.9 & 4.1 & 17.9 & 1.3 & 2.8 & U & -4.73 & & -5.32 & 40.03 & -4.72 & 26.37 & -3.01 & 8.03 & 2.80 & 0.83 & 1.22 & 2.80 \\
\hline JL-100 & 79.9 & 19.7 & 0.4 & 19.7 & & & $B$ & -5.24 & 0.25 & -5.28 & 38.94 & -3.86 & 14.54 & -3.12 & 8.66 & 2.16 & 0.49 & 0.90 & 0.50 \\
\hline JL-101 & 14.4 & 80.7 & 4.9 & 80.7 & & & U & 1.25 & & -1.58 & 2.99 & 0.76 & 0.59 & 0.58 & 0.67 & 1.59 & -0.10 & 1.34 & 0.70 \\
\hline JL-102 & 0.0 & 92.0 & 8.0 & 92.0 & 2.4 & 5.6 & U & 2.74 & & 2.14 & 0.23 & 2.80 & 0.14 & 2.83 & 0.14 & 1.28 & 0.42 & 4.06 & 1.10 \\
\hline JL-103 & 0.0 & 86.0 & 14.0 & 86.0 & 5.0 & 9.0 & $\mathrm{u}$ & 3.24 & & 2.46 & 0.18 & 3.13 & 0.11 & 3.20 & 0.11 & 1.61 & 0.47 & 4.32 & 1.80 \\
\hline JL-104 & 16.4 & 69.5 & 14.1 & 69.5 & 5.6 & 8.5 & B & 3.24 & -4.24 & -4.10 & 17.17 & 2.95 & 0.13 & 1.78 & 0.29 & 3.54 & -0.32 & 5.12 & 1.80 \\
\hline
\end{tabular}




\section{Jeffreys Ledge Survey (2002-2005) (5): Grain Size Distribution}

\begin{tabular}{|c|c|c|c|c|c|c|c|c|c|c|c|c|c|c|c|c|c|c|c|c|c|c|c|c|c|c|c|c|c|c|c|c|}
\hline Sample ID & $\begin{array}{c}\text { Class } \\
\% \\
\text { phi } \\
-5.5\end{array}$ & $\begin{array}{c}\text { Class } \\
\% \\
\text { phi } \\
-5.0\end{array}$ & $\begin{array}{c}\text { Class } \\
\% \\
\text { phi } \\
-4.5 \\
\end{array}$ & $\begin{array}{c}\text { Class } \\
\% \\
\text { phi } \\
-4.0 \\
\end{array}$ & $\begin{array}{c}\text { Class } \\
\% \\
\text { phi } \\
-3.5 \\
\end{array}$ & $\begin{array}{c}\text { Class } \\
\% \\
\text { phi } \\
-3.0 \\
\end{array}$ & $\begin{array}{c}\text { Class } \\
\% \\
\text { phi } \\
-2.5 \\
\end{array}$ & $\begin{array}{c}\text { Class } \\
\% \\
\text { phi } \\
-2.0\end{array}$ & $\begin{array}{c}\text { Class } \\
\% \\
\text { phi } \\
-1.5\end{array}$ & $\begin{array}{c}\text { Class } \\
\% \\
\text { phi } \\
-1.0\end{array}$ & $\begin{array}{c}\text { Class } \\
\% \\
\text { phi } \\
-0.5\end{array}$ & $\begin{array}{c}\text { Class } \\
\% \\
\text { phi } \\
0.0 \\
\end{array}$ & $\begin{array}{c}\text { Class } \\
\% \\
\text { phi } \\
0.5 \\
\end{array}$ & $\begin{array}{c}\text { Class } \\
\% \\
\text { phi } \\
1.0 \\
\end{array}$ & $\begin{array}{c}\text { Class } \\
\% \\
\text { phi } \\
1.5\end{array}$ & $\begin{array}{c}\text { Class } \\
\% \\
\text { phi } \\
2.0\end{array}$ & $\begin{array}{c}\text { Class } \\
\% \\
\text { phi } \\
2.5 \\
\end{array}$ & $\begin{array}{c}\text { Class } \\
\% \\
\text { phi } \\
3.0\end{array}$ & $\begin{array}{c}\text { Class } \\
\% \\
\text { phi } \\
3.5\end{array}$ & $\begin{array}{c}\text { Class } \\
\% \\
\text { phi } \\
4.0\end{array}$ & $\begin{array}{c}\text { Class } \\
\% \\
\text { phi } \\
>4.0\end{array}$ & $\begin{array}{c}\text { Class } \\
\% \\
\text { phi } \\
5.0\end{array}$ & $\begin{array}{c}\text { Class } \\
\% \\
\text { phi } \\
6.0\end{array}$ & $\begin{array}{c}\text { Class } \\
\% \\
\text { phi } \\
7.0\end{array}$ & $\begin{array}{c}\text { Class } \\
\% \\
\text { phi } \\
8.0\end{array}$ & $\begin{array}{c}\text { Class } \\
\% \\
\text { phi } \\
9.0\end{array}$ & $\begin{array}{c}\text { Class } \\
\% \\
\text { phi } \\
10.0\end{array}$ & $\begin{array}{c}\text { Class } \\
\% \\
\text { phi } \\
>10.0\end{array}$ & $\begin{array}{c}\text { Class } \\
\% \\
\text { phi } \\
11.0\end{array}$ & $\begin{array}{c}\text { Class } \\
\% \\
\text { phi } \\
12.0\end{array}$ & $\begin{array}{c}\text { Class } \\
\% \\
\text { phi } \\
13.0\end{array}$ & $\begin{array}{c}\text { Class } \\
\% \\
\text { phi } \\
14.0\end{array}$ \\
\hline JL-84 & 0.00 & 0.00 & 0.00 & 19.49 & 15.18 & 19.49 & 8.58 & 5.02 & 3.20 & 2.37 & 2.41 & 3.22 & 3.69 & 4.33 & 4.66 & 3.85 & 1.22 & 0.44 & 0.20 & 0.14 & & 0.11 & 0.17 & 0.15 & 0.29 & 0.44 & 0.44 & 0.91 & & & & \\
\hline L-85 & 0.00 & 0.00 & 13.84 & 28.77 & 25.53 & 1.69 & 4.09 & 0.97 & 2.46 & 3.84 & 4.92 & 5.34 & 3.66 & 2.67 & 1.46 & 0.38 & 0.08 & 0.03 & 0.01 & 0.01 & 0.26 & & & & & & & & & & & \\
\hline LL-86 & 0.00 & 53.45 & 7.38 & 12.45 & 0.00 & 0.49 & 0.94 & 1.00 & 1.49 & 2.29 & 3.11 & 4.09 & 4.40 & 3.98 & 3.02 & 1.45 & 0.24 & 0.04 & 0.01 & 0.01 & & 0.02 & 0.01 & 0.01 & 0.02 & 0.03 & 0.02 & 0.08 & & & & \\
\hline JL-87 & 0.00 & 0.00 & 0.00 & 0.00 & 0.00 & 0.00 & 0.00 & 0.00 & 0.00 & 0.13 & 0.33 & 0.36 & 0.79 & 1.07 & 1.60 & 7.50 & 30.30 & 41.26 & 5.91 & 0.86 & & 0.58 & 0.38 & 0.67 & 1.05 & 1.32 & 1.51 & & 1.09 & 1.09 & $\begin{array}{l}9 \\
9\end{array}$ & $\begin{array}{ll}9 & 1.09 \\
\end{array}$ \\
\hline JL-88 & 0.00 & 0.00 & 0.00 & 0.00 & 0.00 & 0.00 & 0.00 & 0.00 & 0.00 & 0.00 & 0.00 & 0.00 & 0.00 & 0.00 & 0.00 & 0.00 & 0.00 & 0.00 & 0.00 & 2.11 & & 4.55 & 6.00 & 11.45 & 511.86 & 510.90 & 11.59 & & 10.38 & 810.38 & 810.38 & 810.38 \\
\hline L-89 & 0.00 & 0.00 & 0.00 & 0.00 & 0.00 & 0.00 & 2.23 & 0.00 & 1.97 & 1.25 & 1.28 & 2.23 & 5.52 & 12.81 & 21.53 & 25.16 & 16.32 & 6.24 & 1.06 & 0.41 & 2.01 & & & & & & & & & & & \\
\hline L-90 & 0.00 & 0.00 & 0.00 & 0.00 & 0.00 & 20.11 & 22.55 & 24.44 & 16.14 & 5.81 & 2.77 & 1.83 & 1.50 & 1.25 & 1.24 & 1.50 & 0.70 & 0.13 & 0.03 & 0.01 & & & & & & & & & & & & \\
\hline L-91 & 0.00 & 49.48 & 16.54 & 0.00 & 0.00 & 0.00 & 0.48 & 1.42 & 1.54 & 1.38 & 2.01 & 3.29 & 4.87 & 5.75 & 5.61 & 4.05 & 1.36 & 0.33 & 0.05 & 0.05 & & 0.07 & 0.08 & 0.13 & 0.19 & 0.26 & 0.29 & 0.79 & & & & \\
\hline JL-92 & 0.00 & 0.00 & 26.87 & 30.02 & 12.79 & 8.20 & 4.92 & 2.02 & 1.14 & 1.17 & 1.59 & 1.89 & 2.00 & 1.98 & 2.21 & 1.79 & 0.82 & 0.15 & 0.02 & 0.02 & & 0.03 & 0.02 & 0.03 & $\begin{array}{l}3 \quad 0.02 \\
\end{array}$ & 0.09 & 0.04 & 0.20 & & & & \\
\hline JL-93 & 0.00 & 0.00 & 0.00 & 0.00 & 0.00 & 1.10 & 0.00 & 0.00 & 0.00 & 0.06 & 0.30 & 0.32 & 0.35 & 0.45 & 0.77 & 6.32 & 31.06 & 43.06 & 7.55 & 1.04 & & 0.55 & 0.37 & 0.44 & 0.72 & 1.07 & 1.37 & & 0.78 & 0.78 & $\begin{array}{l}3 \quad 0.78 \\
\end{array}$ & $\begin{array}{l}3 \\
\end{array}$ \\
\hline JL-94 & 0.00 & 0.00 & 0.00 & 0.00 & 7.75 & 0.00 & 0.00 & 0.28 & 0.10 & 0.53 & 0.85 & 1.14 & 1.49 & 1.76 & 1.80 & 3.30 & 14.82 & 43.70 & 12.39 & 1.76 & & 0.51 & 0.38 & 0.48 & 0.62 & 1.37 & 1.30 & & 0.92 & 0.92 & 0.92 & $\begin{array}{l}2 \\
2\end{array}$ \\
\hline JL-95 & 0.00 & 0.00 & 0.00 & 0.00 & 0.00 & 7.51 & 0.00 & 0.00 & 0.42 & 0.19 & 1.00 & 1.54 & 1.75 & 1.91 & 2.33 & 3.69 & 12.85 & 34.33 & 12.47 & 2.73 & & 1.20 & 0.41 & 1.52 & 1.89 & 2.28 & 2.48 & & 1.87 & 1.87 & 1.87 & $7 \quad 1.87$ \\
\hline JL-96 & 0.00 & 0.00 & 0.00 & 0.00 & 0.00 & 0.00 & 0.00 & 0.00 & 0.00 & 0.00 & 0.00 & 0.00 & 0.00 & 0.00 & 0.00 & 0.00 & 0.00 & 0.00 & 0.00 & 0.65 & & 0.31 & 7.00 & 10.73 & 314.58 & 811.16 & 510.57 & & 11.25 & 511.25 & 511.25 & 511.25 \\
\hline JL-97 & 0.00 & 0.00 & 0.00 & 0.00 & 0.00 & 0.00 & 0.00 & 0.00 & 0.00 & 0.00 & 0.00 & 0.00 & 0.01 & 0.01 & 0.01 & 0.05 & 0.07 & 0.35 & 0.95 & 2.67 & & 7.97 & 8.41 & 10.41 & 110.46 & 510.27 & 710.81 & & 9.39 & 9.39 & 9.39 & $\begin{array}{l}9 \\
9\end{array}$ \\
\hline JL-98 & 0.00 & 44.78 & 24.81 & 9.21 & 0.00 & 4.06 & 3.85 & 1.49 & 0.83 & 0.35 & 0.68 & 0.91 & 1.40 & 1.71 & 1.80 & 1.75 & 1.02 & 0.45 & 0.09 & 0.05 & & 0.05 & 0.05 & 0.07 & 0.07 & 0.10 & 0.16 & 0.28 & & & & \\
\hline JL-99 & 0.00 & 30.43 & 36.23 & 0.00 & 1.66 & 3.92 & 2.07 & 1.53 & 1.22 & 0.89 & 0.87 & 1.29 & 1.97 & 2.28 & 2.44 & 2.96 & 3.72 & 1.82 & 0.37 & 0.22 & & 0.22 & 0.27 & 0.34 & 0.46 & 0.79 & 0.42 & 1.63 & & & & \\
\hline JL-100 & 0.00 & 24.59 & 11.56 & 8.81 & 18.78 & 5.06 & 3.86 & 3.04 & 2.07 & 2.17 & 2.80 & 3.91 & 4.73 & 4.72 & 2.55 & 0.72 & 0.16 & 0.05 & 0.02 & 0.01 & & 0.02 & 0.02 & 0.02 & 0.03 & 0.05 & 0.05 & 0.22 & & & & \\
\hline $\mathrm{JL}-101$ & 0.00 & 0.00 & 0.00 & 0.00 & 0.00 & 0.00 & 2.86 & 4.64 & 3.06 & 3.79 & 6.38 & 9.08 & 12.84 & 414.13 & 15.75 & 16.03 & 5.10 & 1.09 & 0.19 & 0.12 & & 0.22 & 0.32 & 0.10 & 0.26 & 0.68 & 0.56 & & 0.70 & 0.70 & 0.70 & 0.70 \\
\hline JL-102 & 0.00 & 0.00 & 0.00 & 0.00 & 0.00 & 0.00 & 0.00 & 0.00 & 0.00 & 0.00 & 0.22 & 0.25 & 0.30 & 0.34 & 0.49 & 3.46 & 17.30 & 45.01 & 20.37 & 4.28 & & 0.86 & 0.48 & 0.40 & 0.64 & 0.90 & 1.14 & & 0.89 & 0.89 & 0.89 & 0.89 \\
\hline $\mathrm{JL}-103$ & 0.00 & 0.00 & 0.00 & 0.00 & 0.00 & 0.00 & 0.00 & 0.00 & 0.00 & 0.00 & 0.01 & 0.08 & 0.12 & 0.13 & 0.25 & 1.35 & 8.36 & 31.18 & 32.45 & 12.04 & & 2.07 & 0.71 & 0.84 & 1.37 & 1.37 & 1.80 & & 1.47 & 1.47 & 1.47 & 1.47 \\
\hline JL-104 & 0.00 & 0.00 & 0.00 & 12.65 & 0.00 & 1.01 & 1.06 & 0.69 & 0.59 & 0.41 & 0.62 & 0.59 & 0.70 & 0.73 & 0.89 & 1.63 & 5.92 & 25.12 & 24.27 & 9.03 & & 2.69 & 0.81 & 0.81 & 1.25 & 2.20 & 1.04 & & 1.33 & 1.33 & 1.33 & 1.33 \\
\hline
\end{tabular}


Jeffreys Ledge Survey (2002-2005) (6): Identification, Location, and Description

\begin{tabular}{|c|c|c|c|c|c|c|c|c|}
\hline Sample ID & $\begin{array}{c}\text { Original } \\
\text { Sample ID }\end{array}$ & $\begin{array}{c}\text { Latitude } \\
\text { WGS84 }\end{array}$ & $\begin{array}{c}\text { Longitude } \\
\text { WGS84 }\end{array}$ & $\begin{array}{l}\text { Reliability } \\
\text { Ranking for } \\
\text { Positioning }\end{array}$ & $\begin{array}{c}\text { Water } \\
\text { Depth } \\
\text { (m) }\end{array}$ & $\begin{array}{c}\text { Sample } \\
\text { Collected }\end{array}$ & $\begin{array}{c}\text { Sampler } \\
\text { Type }\end{array}$ & $\begin{array}{c}\text { Total Wt } \\
\text { (gm) }\end{array}$ \\
\hline JL-105 & JL162b & 42.833030 & -70.113800 & 1 & 131 & $11 / 17 / 2004$ & $\mathrm{BC}$ & 24.80 \\
\hline JL-106 & JL162c & 42.833920 & -70.113880 & 1 & 133 & $7 / 26 / 2005$ & $\mathrm{SH}$ & 23.50 \\
\hline JL-107 & JL163 & 42.820810 & -70.396390 & 1 & 135 & $9 / 13 / 2002$ & $\mathrm{BC}$ & 9.60 \\
\hline JL-108 & JL164 & 42.820800 & -70.380070 & 1 & 134 & $9 / 13 / 2002$ & $\mathrm{BC}$ & 6.80 \\
\hline JL-109 & JL165 & 42.820750 & -70.363450 & 1 & 124 & $9 / 13 / 2002$ & $\mathrm{BC}$ & 10.30 \\
\hline JL-110 & JL174 & 42.820430 & -70.213750 & 1 & 56 & $9 / 23 / 2004$ & $\mathrm{SH}$ & 273.40 \\
\hline JL-111 & JL178 & 42.820870 & -70.147060 & 1 & 112 & $10 / 8 / 2002$ & $B C$ & 69.90 \\
\hline JL-112 & JL181 & 42.808310 & -70.396540 & 1 & 112 & $9 / 13 / 2002$ & $\mathrm{BC}$ & 12.50 \\
\hline JL-113 & JL182a & 42.808340 & -70.379820 & 1 & 119 & $9 / 13 / 2002$ & $\mathrm{BC}$ & 9.40 \\
\hline JL-114 & JL182b & 42.808200 & -70.380380 & 1 & 120 & $11 / 17 / 2004$ & $\mathrm{BC}$ & 11.30 \\
\hline JL-115 & JL183a & 42.808270 & -70.363370 & 1 & 124 & $9 / 13 / 2002$ & $\mathrm{BC}$ & 10.00 \\
\hline JL-116 & JL183b & 42.808320 & -70.363950 & 1 & 128 & $11 / 17 / 2004$ & $\mathrm{BC}$ & 9.90 \\
\hline JL-117 & JL184 & 42.808370 & -70.346870 & 1 & 113 & $9 / 13 / 2002$ & $\mathrm{BC}$ & 11.60 \\
\hline JL-118 & JL186b & 42.807780 & -70.313470 & 1 & 54 & $9 / 21 / 2004$ & $\mathrm{SH}$ & 392.60 \\
\hline JL-119 & JL191 & 42.808080 & -70.229900 & 1 & 56 & $9 / 23 / 2004$ & $\mathrm{SH}$ & 176.20 \\
\hline $\mathrm{JL}-120$ & JL192 & 42.807980 & -70.213750 & 1 & 85 & $9 / 23 / 2004$ & $\mathrm{SH}$ & 99.00 \\
\hline JL-121 & JL200b & 42.796020 & -70.380130 & 1 & 89 & $8 / 12 / 2002$ & $\mathrm{BC}$ & 24.70 \\
\hline JL-122 & JL201 & 42.796240 & -70.363640 & 1 & 106 & $8 / 12 / 2002$ & $\mathrm{BC}$ & 16.00 \\
\hline JL-123 & JL202 & 42.796240 & -70.346780 & 1 & 110 & $8 / 12 / 2002$ & $\mathrm{BC}$ & 10.80 \\
\hline JL-124 & JL203b & 42.795970 & -70.330040 & 1 & 91 & $8 / 12 / 2002$ & $\mathrm{BC}$ & 60.90 \\
\hline
\end{tabular}


Jeffreys Ledge Survey (2002-2005) (6): Sediment Classifications

\begin{tabular}{|c|c|c|c|c|c|c|c|c|c|}
\hline Sample ID & $\begin{array}{l}\text { CMECS Substrate } \\
\text { Component } \\
\text { Group (Specific) }\end{array}$ & $\begin{array}{l}\text { CMECS Substrate } \\
\text { Component } \\
\text { Subgroup (Specific) }\end{array}$ & $\begin{array}{l}\text { Textural Group } \\
\text { from \%GSM } \\
\text { (Gradistat) }\end{array}$ & $\begin{array}{l}\text { Textural Group } \\
\text { from } \% S Z C \\
\text { (Gradistat) } \\
\end{array}$ & $\begin{array}{l}\text { Sediment Name from } \\
\text { \%GSM } \\
\text { and Mode (Gradistat) }\end{array}$ & $\begin{array}{l}\text { Sediment Name from } \\
\text { \%GSM and Mode } \\
\text { (Wentworth Scale) }\end{array}$ & $\begin{array}{l}\text { Sediment } \\
\text { Classification from } \\
\text { Mean Phi (Gradistat) }\end{array}$ & $\begin{array}{l}\text { Classification } \\
\text { from Mean Phi } \\
\text { (Wentworth) }\end{array}$ & $\begin{array}{l}\text { Sorting } \\
\text { (Gradistat) }\end{array}$ \\
\hline $\mathrm{JL}-105$ & Slightly Pebbly & $\begin{array}{l}\text { Slightly Pebbly Silty- } \\
\text { Clayey Very Fine Sand }\end{array}$ & $\begin{array}{l}\text { Slightly Gravelly } \\
\text { Muddy Sand }\end{array}$ & N/A & $\begin{array}{l}\text { Slightly Medium Gravelly } \\
\text { Muddy Very Fine Sand }\end{array}$ & $\begin{array}{l}\text { Slightly Pebbly Muddy } \\
\text { Very Fine Sand }\end{array}$ & Very Coarse Silt & Coarse Silt & $\begin{array}{l}\text { Very Poorly } \\
\text { Sorted }\end{array}$ \\
\hline $\mathrm{JL}-106$ & Slightly Granuley & $\begin{array}{l}\text { Slightly Granul ey Silty- } \\
\text { Clayey Very Fine Sand }\end{array}$ & $\begin{array}{l}\text { Slightly Gravelly } \\
\text { Muddy Sand }\end{array}$ & Muddy Sand & $\begin{array}{l}\text { Slightly Very Fine Gravelly } \\
\text { Muddy Very Fine Sand }\end{array}$ & $\begin{array}{l}\text { Slightly Granular } \\
\text { Muddy Very Fine Sand }\end{array}$ & Very Fine Sand & Very Fine Sand & Poorly Sorted \\
\hline $\mathrm{JL}-107$ & Mud & Silt-Clay & Mud & Mud & Mud & Mud & Clay & Clay & $\begin{array}{l}\text { Very Poorly } \\
\text { Sorted }\end{array}$ \\
\hline $\mathrm{JL}-108$ & Mud & Silt-Clay & Mud & Mud & Mud & Mud & Clay & Clay & $\begin{array}{l}\text { Very Poorly } \\
\text { Sorted }\end{array}$ \\
\hline $\mathrm{JL}-109$ & Mud & Silt-Clay & Mud & Mud & Mud & Mud & Very Fine Silt & Clay & $\begin{array}{l}\text { Very Poorly } \\
\text { Sorted }\end{array}$ \\
\hline $\mathrm{JL}-110$ & Pebble Mixes & Sandy Pebble Gravel & Sandy Gravel & $\mathrm{N} / \mathrm{A}$ & Sandy Coarse Gravel & Sandy Pebble Gravel & Fine Gravel & Pebble Gravel & $\begin{array}{l}\text { Very Poorly } \\
\text { Sorted }\end{array}$ \\
\hline JL-111 & Pebbly & $\begin{array}{l}\text { Pebbly Clayey Fine } \\
\text { Sand }\end{array}$ & $\begin{array}{l}\text { Gravelly Muddy } \\
\text { Sand }\end{array}$ & $\mathrm{N} / \mathrm{A}$ & $\begin{array}{l}\text { Medium Gravelly Muddy } \\
\text { Fine Sand }\end{array}$ & $\begin{array}{l}\text { Pebbly Muddy Fine } \\
\text { Sand }\end{array}$ & Fine Sand & Fine Sand & $\begin{array}{l}\text { Very Poorly } \\
\text { Sorted }\end{array}$ \\
\hline $\mathrm{JL}-112$ & Sandy Mud & Sandy Silt-Clay & Sandy Mud & Sandy Mud & Very Fine Sandy Mud & Very Fine Sandy Mud & Medium Silt & Fine Silt & $\begin{array}{l}\text { Very Poorly } \\
\text { Sorted }\end{array}$ \\
\hline $\mathrm{JL}-113$ & Mud & Silt-Clay & Mud & Mud & Mud & Mud & Very Fine Silt & Clay & $\begin{array}{l}\text { Very Poorly } \\
\text { Sorted }\end{array}$ \\
\hline $\mathrm{JL}-114$ & Mud & Silt-Clay & Mud & Mud & Mud & Mud & Very Fine Silt & Clay & $\begin{array}{l}\text { Very Poorly } \\
\text { Sorted }\end{array}$ \\
\hline $\mathrm{JL}-115$ & Mud & Silt-Clay & Mud & Mud & Mud & Mud & Clay & Clay & $\begin{array}{l}\text { Very Poorly } \\
\text { Sorted }\end{array}$ \\
\hline $\mathrm{JL}-116$ & Mud & Silt-Clay & Mud & Mud & Mud & Mud & Clay & Clay & $\begin{array}{l}\text { Very Poorly } \\
\text { Sorted }\end{array}$ \\
\hline $\mathrm{JL}-117$ & Sandy Mud & Sandy Silt-Clay & Sandy Mud & Sandy Mud & Very Fine Sandy Mud & Very Fine Sandy Mud & Very Fine Silt & Clay & $\begin{array}{l}\text { Very Poorly } \\
\text { Sorted }\end{array}$ \\
\hline $\mathrm{JL}-118$ & Gravel & Pebble Gravel & Gravel & $\mathrm{N} / \mathrm{A}$ & Very Coarse Gravel & Pebble Gravel & Very Coarse Gravel & Pebble Gravel & $\begin{array}{l}\text { Moderately } \\
\text { Sorted }\end{array}$ \\
\hline $\mathrm{JL}-119$ & Pebbly & Pebbly Coarse Gravel & Gravelly Sand & $\mathrm{N} / \mathrm{A}$ & $\begin{array}{l}\text { Coarse Gravelly Coarse } \\
\text { Sand }\end{array}$ & Pebbly Coarse Sand & Very Coarse Sand & $\begin{array}{l}\text { Very Coarse } \\
\text { Sand }\end{array}$ & $\begin{array}{l}\text { Very Poorly } \\
\text { Sorted }\end{array}$ \\
\hline $\mathrm{JL}-120$ & Slightly Granuley & $\begin{array}{l}\text { Slightly Granuley } \\
\text { Coarse Sand }\end{array}$ & $\begin{array}{l}\text { Slighty Gravelly } \\
\text { Sand }\end{array}$ & $\mathrm{N} / \mathrm{A}$ & $\begin{array}{l}\text { Slightly Very Fine Gravelly } \\
\text { Coarse Sand }\end{array}$ & $\begin{array}{l}\text { Slightly Granular } \\
\text { Coarse Sand }\end{array}$ & Coarse Sand & Coarse Sand & Poorly Sorted \\
\hline $\mathrm{JL}-121$ & Slightly Granuley & $\begin{array}{l}\text { Slightly Granul ey Silty- } \\
\text { Clayey Fine Sand }\end{array}$ & $\begin{array}{l}\text { Slightly Gravelly } \\
\text { Muddy Sand }\end{array}$ & $\mathrm{N} / \mathrm{A}$ & $\begin{array}{l}\text { Slightly Very Fine Gravelly } \\
\text { Muddy Fine Sand }\end{array}$ & $\begin{array}{l}\text { Slightly Granular } \\
\text { Muddy Fine Sand }\end{array}$ & Very Fine Sand & Very Fine Sand & $\begin{array}{l}\text { Very Poorly } \\
\text { Sorted }\end{array}$ \\
\hline $\mathrm{JL}-122$ & Mud & Silt-Clay & Mud & Mud & Mud & Mud & Very Fine Silt & Clay & $\begin{array}{l}\text { Very Poorly } \\
\text { Sorted }\end{array}$ \\
\hline $\mathrm{JL}-123$ & Mud & Silt-Clay & Mud & Mud & Mud & Mud & Very Fine Silt & Clay & $\begin{array}{l}\text { Very Poorly } \\
\text { Sorted }\end{array}$ \\
\hline $\mathrm{JL}-124$ & Pebbly & Pebbly Medium Sand & Gravelly Sand & N/A & $\begin{array}{l}\text { Medium Gravelly Medium } \\
\text { Sand }\end{array}$ & Pebbly Medium Sand & Medium Sand & Medium Sand & Poorly Sorted \\
\hline
\end{tabular}




\section{Jeffreys Ledge Survey (2002-2005) (6): Grain Size Statistics}

\begin{tabular}{|c|c|c|c|c|c|c|c|c|c|c|c|c|c|c|c|c|c|c|c|}
\hline Sample ID & $\begin{array}{c}\text { Gravel } \\
\%\end{array}$ & $\begin{array}{c}\text { Sand } \\
\%\end{array}$ & $\begin{array}{c}\text { Mud } \\
\%\end{array}$ & $\begin{array}{c}\text { Sand } \\
\%\end{array}$ & $\begin{array}{c}\text { Silt } \\
\%\end{array}$ & $\begin{array}{c}\text { Clay } \\
\% \\
\end{array}$ & Modes & $\begin{array}{c}\text { Mode } 1 \\
\text { (phi) }\end{array}$ & $\begin{array}{c}\text { Mode } 2 \\
\text { (phi) }\end{array}$ & $\begin{array}{c}D_{10} \\
\text { (phi) }\end{array}$ & $\begin{array}{c}\mathrm{D}_{10} \\
(\mathrm{~mm})\end{array}$ & $\begin{array}{c}D_{50} \\
\text { (phi) }\end{array}$ & $\begin{array}{c}D_{50} \\
(\mathrm{~mm})\end{array}$ & $\begin{array}{c}\text { Mean } \\
\text { Size } \\
\text { (phi) }\end{array}$ & $\begin{array}{l}\text { Mean } \\
\text { Size } \\
(\mathrm{mm})\end{array}$ & $\begin{array}{c}\text { Sorting } \\
\text { (phi) }\end{array}$ & Skewness & Kurtosis & LOI \% \\
\hline JL-106 & 0.1 & 84.6 & 15.3 & 84.6 & 6.2 & 9.1 & $\mathrm{U}$ & 2.74 & & 2.53 & 0.17 & 3.14 & 0.11 & 3.24 & 0.11 & 1.58 & 0.52 & 4.00 & 2.00 \\
\hline JL-107 & 0.0 & 0.0 & 100.0 & 0.0 & 34.4 & 65.6 & U & 9.48 & & 5.86 & 0.02 & 9.58 & 0.00 & 9.54 & 0.00 & 2.79 & -0.04 & 0.78 & 8.00 \\
\hline JL-108 & 0.0 & 1.0 & 99.0 & 1.0 & 35.7 & 63.3 & $\mathrm{U}$ & 7.50 & & 5.80 & 0.02 & 9.20 & 0.00 & 9.36 & 0.00 & 2.81 & 0.05 & 0.79 & 13.20 \\
\hline JL-109 & 0.0 & 6.1 & 93.9 & 6.1 & 34.6 & 59.3 & $\mathrm{U}$ & 8.48 & & 4.53 & 0.04 & 8.73 & 0.00 & 8.78 & 0.00 & 3.21 & 0.00 & 0.81 & 10.00 \\
\hline JL-110 & 63.5 & 35.6 & 0.8 & 35.6 & & & $\mathrm{~B}$ & -5.24 & 0.25 & -5.23 & 37.45 & -2.77 & 6.83 & -2.44 & 5.44 & 2.41 & 0.20 & 0.58 & 0.50 \\
\hline JL-111 & 9.2 & 74.4 & 16.3 & 74.4 & 4.7 & 11.6 & $\mathrm{~B}$ & 2.74 & -3.74 & -0.61 & 1.53 & 2.82 & 0.14 & 2.63 & 0.16 & 3.10 & -0.01 & 4.52 & 1.90 \\
\hline JL-112 & 0.0 & 43.7 & 56.3 & 43.7 & 23.0 & 33.3 & $\mathrm{~B}$ & 3.24 & 7.50 & 2.80 & 0.14 & 4.86 & 0.03 & 6.31 & 0.01 & 3.60 & 0.54 & 0.74 & 4.10 \\
\hline JL-113 & 0.0 & 4.3 & 95.8 & 4.2 & 37.2 & 58.6 & $\mathrm{U}$ & 8.48 & & 4.97 & 0.03 & 8.73 & 0.00 & 8.81 & 0.00 & 3.15 & 0.03 & 0.78 & 6.80 \\
\hline JL-114 & 0.0 & 4.3 & 95.8 & 4.2 & 37.0 & 58.8 & $\mathrm{U}$ & 8.48 & & 4.67 & 0.04 & 8.78 & 0.00 & 8.85 & 0.00 & 3.16 & 0.02 & 0.79 & 7.10 \\
\hline JL-115 & 0.0 & 1.5 & 98.5 & 1.5 & 35.4 & 63.1 & $\mathrm{U}$ & 8.48 & & 5.50 & 0.02 & 9.08 & 0.00 & 9.24 & 0.00 & 2.90 & 0.05 & 0.80 & 7.80 \\
\hline JL-116 & 0.0 & 1.9 & 98.1 & 1.9 & 34.1 & 64.0 & U & 7.50 & & 5.54 & 0.02 & 9.29 & 0.00 & 9.34 & 0.00 & 2.90 & 0.00 & 0.78 & 8.00 \\
\hline JL-117 & 0.0 & 13.1 & 86.9 & 13.1 & 36.6 & 50.3 & $\mathrm{U}$ & 3.73 & & 3.74 & 0.07 & 8.05 & 0.00 & 8.12 & 0.00 & 3.48 & 0.04 & 0.72 & 6.30 \\
\hline JL-118 & 96.8 & 3.1 & 0.1 & 3.1 & & & $\mathrm{U}$ & -5.74 & & -5.87 & 58.53 & -5.39 & 41.96 & -5.17 & 36.03 & 0.88 & 0.56 & 1.34 & 0.80 \\
\hline JL-119 & 25.2 & 74.7 & 0.1 & 74.7 & & & $\mathrm{~B}$ & 0.25 & -4.73 & -4.62 & 24.64 & 0.03 & 0.98 & -0.89 & 1.85 & 2.08 & -0.58 & 1.47 & 0.40 \\
\hline JL-120 & 2.6 & 94.9 & 2.5 & 94.9 & 0.6 & 1.9 & $\mathrm{U}$ & 0.75 & & -0.38 & 1.30 & 0.84 & 0.56 & 0.87 & 0.55 & 1.02 & 0.06 & 1.01 & 0.60 \\
\hline JL-121 & 2.9 & 74.0 & 23.1 & 74.0 & 7.9 & 15.2 & $\mathrm{U}$ & 2.74 & & -0.14 & 1.10 & 2.12 & 0.23 & 3.38 & 0.10 & 3.77 & 0.53 & 1.88 & 2.50 \\
\hline JL-124 & 6.3 & 88.7 & 5.0 & 88.7 & 1.8 & 3.2 & B & 1.75 & -3.24 & -0.29 & 1.22 & 1.49 & 0.36 & 1.39 & 0.38 & 1.40 & -0.11 & 1.59 & 0.91 \\
\hline
\end{tabular}




\section{Jeffreys Ledge Survey (2002-2005) (6): Grain Size Distribution}

\begin{tabular}{|c|c|c|c|c|c|c|c|c|c|c|c|c|c|c|c|c|c|c|c|c|c|c|c|c|c|c|c|c|c|c|c|c|}
\hline Sample ID & $\begin{array}{c}\text { Class } \\
\% \\
\text { phi } \\
-5.5\end{array}$ & $\begin{array}{c}\text { Class } \\
\% \\
\text { phi } \\
-5.0\end{array}$ & $\begin{array}{c}\text { Class } \\
\% \\
\text { phi } \\
-4.5\end{array}$ & $\begin{array}{c}\text { Class } \\
\% \\
\text { phi } \\
-4.0\end{array}$ & $\begin{array}{c}\text { Class } \\
\% \\
\text { phi } \\
-3.5\end{array}$ & $\begin{array}{c}\text { Class } \\
\% \\
\text { phi } \\
-3.0\end{array}$ & $\begin{array}{c}\text { Class } \\
\% \\
\text { phi } \\
-2.5\end{array}$ & $\begin{array}{c}\text { Class } \\
\% \\
\text { phi } \\
-2.0\end{array}$ & $\begin{array}{c}\text { Class } \\
\% \\
\text { phi } \\
-1.5\end{array}$ & $\begin{array}{c}\text { Class } \\
\% \\
\text { phi } \\
-1.0\end{array}$ & $\begin{array}{c}\text { Class } \\
\% \\
\text { phi } \\
-0.5\end{array}$ & $\begin{array}{c}\text { Class } \\
\% \\
\text { phi } \\
0.0\end{array}$ & $\begin{array}{c}\text { Class } \\
\% \\
\text { phi } \\
0.5\end{array}$ & $\begin{array}{c}\text { Class } \\
\% \\
\text { phi } \\
1.0\end{array}$ & $\begin{array}{c}\text { Class } \\
\% \\
\text { phi } \\
1.5\end{array}$ & $\begin{array}{c}\text { Class } \\
\% \\
\text { phi } \\
2.0\end{array}$ & $\begin{array}{c}\text { Class } \\
\% \\
\text { phi } \\
2.5\end{array}$ & $\begin{array}{c}\text { Class } \\
\% \\
\text { phi } \\
3.0\end{array}$ & $\begin{array}{c}\text { Class } \\
\% \\
\text { phi } \\
3.5\end{array}$ & $\begin{array}{c}\text { Class } \\
\% \\
\text { phi } \\
4.0\end{array}$ & $\begin{array}{c}\text { Class } \\
\% \\
\text { phi } \\
>4.0\end{array}$ & $\begin{array}{c}\text { Class } \\
\% \\
\text { phi } \\
5.0\end{array}$ & $\begin{array}{c}\text { Class } \\
\% \\
\text { phi } \\
6.0\end{array}$ & $\begin{array}{c}\text { Class } \\
\% \\
\text { phi } \\
7.0\end{array}$ & $\begin{array}{c}\text { Class } \\
\% \\
\text { phi } \\
8.0\end{array}$ & $\begin{array}{c}\text { Class } \\
\% \\
\text { phi } \\
9.0\end{array}$ & $\begin{array}{c}\text { Class } \\
\% \\
\text { phi } \\
10.0\end{array}$ & $\begin{array}{c}\text { Class } \\
\% \\
\text { phi } \\
>10.0\end{array}$ & $\begin{array}{c}\text { Class } \\
\% \\
\text { phi } \\
11.0\end{array}$ & $\begin{array}{c}\text { Class } \\
\% \\
\text { phi } \\
12.0\end{array}$ & $\begin{array}{c}\text { Class } \\
\% \\
\text { phi } \\
13.0\end{array}$ & $\begin{array}{c}\text { Class } \\
\% \\
\text { phi } \\
14.0\end{array}$ \\
\hline JL-105 & 0.00 & 0.00 & 0.00 & 0.00 & 0.00 & 4.24 & 0.00 & 0.00 & 0.00 & 0.00 & 0.04 & 0.19 & 0.86 & 0.33 & 0.41 & 1.19 & 5.06 & 24.03 & 325.10 & 13.67 & & 4.87 & 1.33 & 1.57 & 2.28 & 2.88 & 2.70 & & 2.31 & 2.31 & 2.31 & $\begin{array}{l}1 \quad 2.31 \\
\end{array}$ \\
\hline L-106 & 0.00 & 0.00 & 0.00 & 0.00 & 0.00 & 0.00 & 0.00 & 0.00 & 0.00 & 0.06 & 0.05 & 0.08 & 0.14 & 0.20 & 0.32 & 0.94 & 4.61 & 34.80 & 30.81 & 12.65 & & 3.45 & 0.94 & 0.62 & 1.21 & 1.66 & 1.70 & & 1.44 & 1.44 & 1.44 & $\begin{array}{l}4 \\
4.44\end{array}$ \\
\hline L-107 & 0.00 & 0.00 & 0.00 & 0.00 & 0.00 & 0.00 & 0.00 & 0.00 & 0.00 & 0.00 & 0.00 & 0.00 & 0.00 & 0.00 & 0.00 & 0.00 & 0.00 & 0.00 & 0.00 & 0.00 & & 3.95 & 7.07 & 10.55 & 12.79 & 5.72 & 16.53 & & 10.85 & 10.85 & 10.85 & 510.85 \\
\hline L-108 & 0.00 & 0.00 & 0.00 & 0.00 & 0.00 & 0.00 & 0.00 & 0.00 & 0.00 & 0.00 & 0.00 & 0.00 & 0.00 & 0.00 & 0.01 & 0.08 & 0.19 & 0.23 & 0.19 & 0.28 & & 4.13 & 6.19 & 11.65 & 13.79 & 9 10.69 & 11.43 & & 10.29 & 10.29 & 10.29 & 910.25 \\
\hline JL-109 & 0.00 & 0.00 & 0.00 & 0.00 & 0.00 & 0.00 & 0.00 & 0.00 & 0.00 & 0.00 & 0.00 & 0.02 & 0.78 & 0.01 & 0.02 & 0.05 & 0.18 & 0.80 & 1.36 & 2.80 & & 7.53 & 7.72 & 9.71 & 9.66 & 12.43 & 10.15 & & 9.19 & 9.19 & 9.19 & $\begin{array}{l}9 \\
9.19 \\
\end{array}$ \\
\hline JL-110 & 0.00 & 19.46 & 11.38 & 7.98 & 4.62 & 4.19 & 5.31 & 3.72 & 3.83 & 3.03 & 4.38 & 6.85 & 8.96 & 8.25 & 4.90 & 1.84 & 0.35 & 0.08 & 0.02 & 0.01 & & 0.03 & 0.02 & 0.06 & 0.08 & 0.11 & 0.15 & 0.39 & & & & \\
\hline L-111 & 0.00 & 0.00 & 0.00 & 0.00 & 6.35 & 0.00 & 1.18 & 1.72 & 0.00 & 0.00 & 1.00 & 1.42 & 2.27 & 2.72 & 3.12 & 4.51 & 9.77 & 24.22 & 19.96 & 5.44 & & 1.64 & 0.60 & 0.92 & 1.53 & 1.99 & 1.94 & & 1.93 & 1.93 & 1.93 & 1.93 \\
\hline L-112 & 0.00 & 0.00 & 0.00 & 0.00 & 0.00 & 0.00 & 0.00 & 0.00 & 0.00 & 0.00 & 0.23 & 0.15 & 0.38 & 0.44 & 0.77 & 1.40 & 2.38 & 6.77 & 15.88 & 315.26 & & 7.48 & 4.60 & 4.80 & 6.20 & 5.88 & 6.24 & & 5.29 & 5.29 & 5.29 & $\begin{array}{l}9 \\
9\end{array}$ \\
\hline $\mathrm{JL}-113$ & 0.00 & 0.00 & 0.00 & 0.00 & 0.00 & 0.00 & 0.00 & 0.00 & 0.00 & 0.00 & 0.00 & 0.02 & 0.02 & 0.03 & 0.03 & 0.05 & 0.17 & 0.35 & 0.82 & 2.72 & & 8.39 & 8.77 & 9.67 & 10.37 & 711.65 & 9.62 & & 9.33 & 9.33 & 9.33 & $\begin{array}{l}3 \quad 9.33 \\
\end{array}$ \\
\hline $\mathrm{JL}-114$ & 0.00 & 0.00 & 0.00 & 0.00 & 0.00 & 0.00 & 0.00 & 0.00 & 0.00 & 0.00 & 0.00 & 0.00 & 0.02 & 0.04 & 0.02 & 0.02 & 0.07 & 0.49 & 0.81 & 2.72 & & 8.76 & 7.47 & 9.38 & 11.34 & 411.03 & 9.74 & & 9.53 & 9.53 & 9.53 & $\begin{array}{l}3 \quad 9.53 \\
\end{array}$ \\
\hline JL-115 & 0.00 & 0.00 & 0.00 & 0.00 & 0.00 & 0.00 & 0.00 & 0.00 & 0.00 & 0.00 & 0.00 & 0.00 & 0.00 & 0.00 & 0.00 & 0.00 & 0.00 & 0.00 & 0.00 & 1.47 & & 4.79 & 7.68 & 10.87 & 12.07 & 711.97 & 10.62 & & 10.14 & 10.14 & 10.14 & 410.1 \\
\hline JL-116 & 0.00 & 0.00 & 0.00 & 0.00 & 0.00 & 0.00 & 0.00 & 0.00 & 0.00 & 0.00 & 0.00 & 0.00 & 0.00 & 0.00 & 0.00 & 0.00 & 0.00 & 0.00 & 0.00 & 1.83 & & 3.79 & 8.23 & 10.45 & 11.71 & 10.45 & 11.11 & & 10.61 & 10.61 & 10.61 & 110.6 \\
\hline JL-117 & 0.00 & 0.00 & 0.00 & 0.00 & 0.00 & 0.00 & 0.00 & 0.00 & 0.00 & 0.00 & 0.06 & 0.04 & 0.02 & 0.01 & 0.02 & 0.07 & 0.35 & 2.30 & 3.94 & 6.17 & & 11.17 & 77.66 & 8.57 & 9.26 & 8.18 & 9.87 & & 8.08 & 8.08 & 8.08 & 8.08 \\
\hline JL-118 & 45.69 & 22.07 & 12.10 & 11.81 & 0.00 & 2.15 & 1.36 & 0.84 & 0.51 & 0.27 & 0.28 & 0.23 & 0.31 & 0.38 & 0.47 & 0.67 & 0.56 & 0.16 & 0.02 & 0.01 & & 0.01 & 0.01 & 0.01 & 0.01 & 0.02 & 0.02 & 0.04 & & & & \\
\hline JL-119 & 0.00 & 0.00 & 13.87 & 0.00 & 2.98 & 0.00 & 1.15 & 1.21 & 2.23 & 3.77 & 9.25 & 14.50 & 18.44 & 18.72 & 10.93 & 2.62 & 0.17 & 0.02 & 0.00 & 0.00 & 0.14 & & & & & & & & & & & \\
\hline $\mathrm{JL}-120$ & 0.00 & 0.00 & 0.00 & 0.00 & 0.00 & 0.00 & 0.29 & 0.07 & 0.70 & 1.55 & 4.86 & 11.48 & 17.71 & 19.77 & 17.24 & 12.87 & 7.51 & 3.05 & 0.28 & 0.09 & & 0.17 & 0.09 & 0.15 & 0.24 & 0.51 & 0.40 & 0.98 & & & & \\
\hline JL-121 & 0.00 & 0.00 & 0.00 & 0.00 & 0.00 & 0.00 & 0.00 & 0.00 & 1.26 & 1.65 & 3.49 & 5.11 & 7.34 & 8.92 & 9.44 & 10.43 & 9.61 & 11.06 & 6.08 & 2.45 & & 1.80 & 1.50 & 2.02 & 2.55 & 2.73 & 2.81 & & 2.43 & 2.43 & 2.43 & 2.43 \\
\hline $\mathrm{JL}-122$ & 0.00 & 0.00 & 0.00 & 0.00 & 0.00 & 0.00 & 0.00 & 0.00 & 0.00 & 0.00 & 0.00 & 0.02 & 0.03 & 0.03 & 0.05 & 0.09 & 0.11 & 0.54 & 1.79 & 6.45 & & 10.59 & 8.19 & 9.03 & 9.84 & 9.59 & 8.16 & & 8.87 & 8.87 & 8.87 & 7.87 \\
\hline $\mathrm{JL}-123$ & 0.00 & 0.00 & 0.00 & 0.00 & 0.00 & 0.00 & 0.00 & 0.00 & 0.00 & 0.00 & 0.09 & 0.11 & 0.03 & 0.02 & 0.03 & 0.07 & 0.27 & 0.90 & 2.51 & 4.45 & & 7.71 & 16.98 & 2.85 & 10.19 & 910.10 & 9.27 & & 8.61 & 8.61 & 8.61 & 18.61 \\
\hline JL-124 & 0.00 & 0.00 & 0.00 & 0.00 & 0.00 & 3.55 & 0.00 & 0.31 & 1.26 & 1.14 & 2.17 & 3.84 & 7.47 & 13.44 & 16.97 & 20.48 & 14.72 & 7.57 & 1.55 & 0.49 & & 0.44 & 0.29 & 0.43 & 0.65 & 0.63 & 0.63 & 1.9 & & & & \\
\hline
\end{tabular}


Section 4: Cutter Thesis Survey (2002) 
Cutter Thesis Survey (2002): Identification, Location, and Description

\begin{tabular}{|c|c|c|c|c|c|c|c|}
\hline Sample ID & $\begin{array}{c}\text { Original } \\
\text { Sample ID }\end{array}$ & $\begin{array}{c}\text { Latitude } \\
\text { WGS84 }\end{array}$ & $\begin{array}{c}\text { Longitude } \\
\text { WGS84 }\end{array}$ & $\begin{array}{l}\text { Reliability } \\
\text { Ranking for } \\
\text { Positioning }\end{array}$ & $\begin{array}{c}\text { Water } \\
\text { Depth } \\
(\mathrm{m}) \\
\end{array}$ & $\begin{array}{c}\text { Sample } \\
\text { Collected }\end{array}$ & $\begin{array}{c}\text { Sampler } \\
\text { Type }\end{array}$ \\
\hline $01 \_2$ & 200209_01_2 & 43.072333 & -70.699850 & Unknown & & $9 / 4 / 2002$ & Shipek \\
\hline $01 \_4$ & 200209_01_4 & 43.066250 & -70.706217 & Unknown & & $9 / 4 / 2002$ & Shipek \\
\hline $01 \_5$ & 200209_01_5 & 43.070050 & -70.706433 & Unknown & & $9 / 4 / 2002$ & Shipek \\
\hline $01 \_7$ & 200209_01_7 & 43.069450 & -70.701583 & Unknown & & $9 / 5 / 2002$ & Shipek \\
\hline $02 \_1$ & 200209_02_1 & 43.065817 & -70.706500 & Unknown & & $9 / 4 / 2002$ & Boxcorer \\
\hline $02 \_10$ & 200209_02_10 & 43.066267 & -70.703200 & Unknown & & $9 / 5 / 2002$ & Shipek \\
\hline $02 \_2$ & 200209_02_2 & 43.065167 & -70.706033 & Unknown & & $9 / 4 / 2002$ & Shipek \\
\hline $02 \_7$ & 200209_02_7 & 43.071850 & -70.702750 & Unknown & & $9 / 5 / 2002$ & Shipek \\
\hline $02 \_7$ & 200209_02_7rl & & & Unknown & & & \\
\hline 03_1 & 200209_03_1 & 43.076200 & -70.704250 & Unknown & & $9 / 4 / 2002$ & Shipek \\
\hline 03_10 & 200209_03_10 & 43.068833 & -70.701650 & Unknown & & $9 / 5 / 2002$ & Shipek \\
\hline $03 \_2$ & 200209_03_2 & 43.063600 & -70.700583 & Unknown & & $9 / 4 / 2002$ & Shipek \\
\hline $04.1 \_2$ & 200209_04.1_2 & 43.069517 & -70.705467 & Unknown & & $9 / 4 / 2002$ & Shipek \\
\hline $04.1 \_3$ & 200209_04.1_3 & 43.065200 & -70.707350 & Unknown & & $9 / 4 / 2002$ & Shipek \\
\hline $04.1 \_4$ & 200209_04.1_4 & 43.070417 & -70.701767 & Unknown & & $9 / 5 / 2002$ & Shipek \\
\hline $04.1 \_5$ & 200209_04.1_5 & 43.066267 & -70.703233 & Unknown & & $9 / 5 / 2002$ & Shipek \\
\hline $04 \_1$ & 200209_04_1 & 43.066033 & -70.704150 & Unknown & & $9 / 4 / 2002$ & Boxcorer \\
\hline $04 \_2$ & 200209_04_2 & 43.069283 & -70.705233 & Unknown & & $9 / 4 / 2002$ & Shipek \\
\hline $04 \_3$ & 200209_04_3 & 43.066150 & -70.704250 & Unknown & & $9 / 5 / 2002$ & Shipek \\
\hline $04 \_5$ & 200209_04_5 & 43.069267 & -70.704067 & Unknown & & $9 / 4 / 2002$ & Shipek \\
\hline $05 \_3$ & 200209_05_3 & 43.064450 & -70.700233 & Unknown & & $9 / 5 / 2002$ & Shipek \\
\hline $05 \_8$ & 200209_05_8 & 43.067133 & -70.702233 & Unknown & & $9 / 5 / 2002$ & Shipek \\
\hline $05 \_9$ & 200209_05_9 & 43.064017 & -70.699783 & Unknown & & $9 / 5 / 2002$ & Shipek \\
\hline $06 \_1$ & 200209_06_1 & 43.069667 & -70.707200 & Unknown & & $9 / 4 / 2002$ & Shipek \\
\hline $06 \_2$ & 200209_06_2 & 43.070983 & -70.705367 & Unknown & & $9 / 4 / 2002$ & Shipek \\
\hline $06 \_3$ & 200209_06_3 & 43.065900 & -70.700683 & Unknown & & $9 / 4 / 2002$ & Shipek \\
\hline $06 \_4$ & 200209_06_4 & 43.070383 & -70.703633 & Unknown & & $9 / 4 / 2002$ & Shipek \\
\hline $06 \_5$ & 200209_06_5 & 43.071883 & -70.702517 & Unknown & & $9 / 4 / 2002$ & Shipek \\
\hline $06 \_8$ & 200209_06_8 & 43.069133 & -70.703283 & Unknown & & $9 / 4 / 2002$ & Shipek \\
\hline 07_11 & 200209_07_11 & 43.066533 & -70.707500 & Unknown & & $9 / 5 / 2002$ & Diver \\
\hline $07 \_3$ & 200209_07_3 & 43.065050 & -70.703850 & Unknown & & $9 / 4 / 2002$ & Shipek \\
\hline $07 \_5$ & 200209_07_5 & 43.068300 & -70.700200 & Unknown & & $9 / 4 / 2002$ & Shipek \\
\hline 07_9 & 200209_07_9 & 43.065150 & -70.700267 & Unknown & & $9 / 5 / 2002$ & Shipek \\
\hline
\end{tabular}


Section 5: OOA Survey (1997-2005) 
OOA Survey (1997-2005) (1): Identification, Location, and Description

\begin{tabular}{|c|c|c|c|c|c|c|c|}
\hline Sample ID & $\begin{array}{c}\text { Original } \\
\text { Sample ID }\end{array}$ & $\begin{array}{l}\text { Latitude } \\
\text { WGS84 }\end{array}$ & $\begin{array}{l}\text { Longitude } \\
\text { WGS84 }\end{array}$ & $\begin{array}{l}\text { Reliability } \\
\text { Ranking for } \\
\text { Positioning }\end{array}$ & $\begin{array}{l}\text { Water } \\
\text { Depth } \\
\text { (m) }\end{array}$ & $\begin{array}{l}\text { Sample } \\
\text { Collected }\end{array}$ & $\begin{array}{c}\text { Sampler } \\
\text { Type }\end{array}$ \\
\hline OOA_1 & A1097G01 & 42.947000 & -70.633333 & 1 & 50 & $10 / 21 / 1997$ & $B C$ \\
\hline OOA_2 & A1097G02 & 42.947000 & -70.632833 & 1 & 50 & $10 / 21 / 1997$ & $\mathrm{BC}$ \\
\hline OOA_4 & A1097G04 & 42.949167 & -70.619167 & 1 & 53 & $10 / 21 / 1997$ & $B C$ \\
\hline OOA_6 & A1097G06 & 42.944167 & -70.631833 & 1 & 56 & $10 / 21 / 1997$ & $B C$ \\
\hline OOA_7 & A1097G07 & 42.943167 & -70.633500 & 1 & 41 & 10/21/1997 & $B C$ \\
\hline OOA_8 & A1197G01 & 42.949000 & -70.619000 & 1 & 55 & 11/26/1997 & $B C$ \\
\hline OOA_10 & A1197G03 & 42.948167 & -70.618167 & 1 & 57 & $11 / 26 / 1997$ & $B C$ \\
\hline OOA_13 & A1197G06 & 42.943333 & -70.620333 & 1 & 58 & $11 / 26 / 1997$ & $B C$ \\
\hline OOA_14 & A1197G07 & 42.947000 & -70.620167 & 1 & 53 & $11 / 26 / 1997$ & $B C$ \\
\hline OOA_15 & A1197G08 & 42.941000 & -70.623833 & 1 & 56 & $11 / 26 / 1997$ & $B C$ \\
\hline OOA_17 & A1297G01 & 42.940833 & -70.619167 & 1 & & $12 / 18 / 1997$ & $\mathrm{BC}$ \\
\hline OOA_18 & A1297G02 & 42.947000 & -70.633333 & 1 & & $12 / 18 / 1997$ & $B C$ \\
\hline OOA_19 & A1297G03 & 42.943333 & -70.620500 & 1 & & $12 / 18 / 1997$ & $B C$ \\
\hline OOA_21 & A1297G05 & 42.927333 & -70.611333 & 1 & & $12 / 18 / 1997$ & $B C$ \\
\hline OOA_22 & A1297G06 & 42.941167 & -70.623500 & 1 & & $12 / 18 / 1997$ & $B C$ \\
\hline OOA_23 & A0398G01 & 42.947333 & -70.620500 & 1 & 55 & $3 / 23 / 1998$ & $B C$ \\
\hline OOA_24 & A0398G02 & 42.948000 & -70.618333 & 1 & 56 & $3 / 23 / 1998$ & $B C$ \\
\hline OOA_25 & A0398G03 & 42.946833 & -70.615667 & 1 & 57 & $3 / 23 / 1998$ & $B C$ \\
\hline OOA_26 & A0398G04 & 42.946667 & -70.614000 & 1 & 58 & $3 / 23 / 1998$ & $B C$ \\
\hline OOA_27 & A0398G05 & 42.946167 & -70.611833 & 1 & 60 & $3 / 23 / 1998$ & $B C$ \\
\hline OOA_28 & A0498G01 & 42.946500 & -70.619000 & 1 & & 4/18/1998 & $B C$ \\
\hline OOA_29 & $\mathrm{A} 0498 \mathrm{G} 02$ & 42.945833 & -70.615833 & 1 & & 4/18/1998 & $\mathrm{BC}$ \\
\hline OOA_30 & A0498G03 & 42.945667 & -70.614500 & 1 & & $4 / 18 / 1998$ & $B C$ \\
\hline OOA_31 & A0498G04 & 42.944833 & -70.613167 & 1 & & 4/18/1998 & $B C$ \\
\hline OOA_32 & A0498G05 & 42.944333 & -70.611500 & 1 & & 4/18/1998 & $\mathrm{BC}$ \\
\hline OOA_33 & A0598G01 & 42.939833 & -70.624833 & 1 & & $5 / 15 / 1998$ & $B C$ \\
\hline OOA_35 & A0698G02 & 42.952767 & -70.623717 & 1 & 49 & $6 / 26 / 1998$ & $B C$ \\
\hline OOA_36 & A0698G03 & 42.951750 & -70.623233 & 1 & 50 & $6 / 26 / 1998$ & $\mathrm{BC}$ \\
\hline OOA_37 & A0698G04 & 42.951383 & -70.623983 & 1 & 50 & $6 / 26 / 1998$ & $\mathrm{BC}$ \\
\hline OOA_39 & A0698G06 & 42.950733 & -70.622400 & 1 & 52 & $6 / 26 / 1998$ & $\mathrm{BC}$ \\
\hline OOA_41 & A0698G08 & 42.951233 & -70.623167 & 1 & 50 & $6 / 26 / 1998$ & $B C$ \\
\hline OOA_43 & A0798G02 & 42.952933 & -70.623883 & 1 & 49 & $7 / 22 / 1998$ & $\mathrm{BC}$ \\
\hline OOA_45 & A0798G04 & 42.940600 & -70.629867 & 1 & 56 & $7 / 22 / 1998$ & $B C$ \\
\hline OOA_47 & A0798G06 & 42.940583 & -70.619667 & 1 & 59 & $7 / 22 / 1998$ & $B C$ \\
\hline OOA_48 & A0798G07 & 42.920350 & -70.617333 & 1 & 67 & $7 / 22 / 1998$ & $\mathrm{BC}$ \\
\hline OOA_52 & A0798G11 & 42.950500 & -70.633000 & 1 & 53 & $7 / 22 / 1998$ & $B C$ \\
\hline
\end{tabular}


OOA Survey (1997-2005) (1): Sediment Classifications and Grain Size Statistics

\begin{tabular}{|c|c|c|c|c|c|c|c|c|c|c|c|c|c|c|c|c|}
\hline Sample ID & $\begin{array}{l}\text { Textural Group from \%GSM } \\
\text { (Gradistat) }\end{array}$ & $\begin{array}{l}\text { Textural Group } \\
\text { from \%SZC } \\
\text { (Gradistat) }\end{array}$ & $\begin{array}{l}\text { Sediment Classification } \\
\text { from Mean Phi } \\
\text { (Wentworth) }\end{array}$ & Sorting (Gradistat) & $\begin{array}{c}\text { Gravel } \\
\% \\
\end{array}$ & $\begin{array}{c}\text { Sand } \\
\%\end{array}$ & $\begin{array}{c}\text { Mud } \\
\%\end{array}$ & $\begin{array}{c}\text { Sand } \\
\%\end{array}$ & $\begin{array}{l}\text { Silt } \\
\% \\
\end{array}$ & $\begin{array}{c}\text { Clay } \\
\%\end{array}$ & $\begin{array}{c}\text { Mean } \\
\text { Size } \\
\text { (phi) }\end{array}$ & $\begin{array}{c}\text { Mean } \\
\text { Size } \\
(\mathrm{mm}) \\
\end{array}$ & $\begin{array}{c}\text { Sorting } \\
\text { (phi) }\end{array}$ & Skewness & Kurtosis & LOI \% \\
\hline OOA_1 & Muddy Sandy Gravel & & & & 55.0 & 37.0 & 8.0 & & & & & & & & & \\
\hline OOA_2 & Muddy Sand & Muddy Sand & Coarse Silt & Very Poorly Sorted & 0.0 & 65.0 & 35.0 & 65.0 & 17.0 & 18.0 & 4.57 & 0.04 & 3.33 & 0.65 & 1.34 & 2.20 \\
\hline OOA_4 & Muddy Sand & Muddy Sand & Very Fine Sand & Very Poorly Sorted & 0.0 & 79.0 & 21.0 & 79.0 & 11.0 & 10.0 & 3.53 & 0.09 & 2.42 & 0.31 & 5.33 & 1.60 \\
\hline OOA_6 & Muddy Sand & Muddy Sand & Very Fine Sand & Poorly Sorted & 0.0 & 81.0 & 19.0 & 81.0 & 12.0 & 7.0 & 3.67 & 0.08 & 1.41 & 0.70 & 4.78 & 1.20 \\
\hline OOA_7 & Muddy Sand & Muddy Sand & Very Fine Sand & Poorly Sorted & 0.0 & 84.0 & 16.0 & 84.0 & 8.0 & 8.0 & 3.13 & 0.11 & 1.64 & 0.48 & 4.80 & 1.30 \\
\hline OOA_8 & Muddy Sand & Muddy Sand & Very Fine Sand & Poorly Sorted & 0.0 & 88.0 & 12.0 & 88.0 & 7.0 & 5.0 & 3.33 & 0.10 & 1.07 & 0.39 & 3.83 & 1.20 \\
\hline OOA_10 & Muddy Sand & Muddy Sand & Coarse Silt & Very Poorly Sorted & 0.0 & 69.0 & 31.0 & 69.0 & 17.0 & 14.0 & 4.13 & 0.06 & 2.99 & 0.49 & 2.05 & 2.40 \\
\hline OOA_13 & Muddy Sand & Muddy Sand & Very Fine Sand & Very Poorly Sorted & 0.0 & 74.0 & 26.0 & 74.0 & 13.0 & 13.0 & 3.77 & 0.07 & 2.98 & 0.62 & 2.46 & 2.10 \\
\hline OOA_14 & Muddy Sand & Muddy Sand & Medium Silt & Very Poorly Sorted & 0.0 & 63.0 & 37.0 & 63.0 & 21.0 & 16.0 & 5.10 & 0.03 & 2.85 & 0.74 & 1.90 & 1.60 \\
\hline OOA_15 & Muddy Sand & Muddy Sand & Coarse Silt & Poorly Sorted & 0.0 & 78.0 & 22.0 & 78.0 & 13.0 & 9.0 & 4.03 & 0.06 & 1.85 & 0.75 & 8.81 & 1.40 \\
\hline OOA_17 & Muddy Sandy Gravel & & & & 46.0 & 44.0 & 10.0 & & & & & & & & & 1.40 \\
\hline OOA_18 & Muddy Sand & Muddy Sand & Very Fine Sand & Poorly Sorted & 0.0 & 80.0 & 20.0 & 80.0 & 10.0 & 10.0 & 3.67 & 0.08 & 1.75 & 0.62 & 5.04 & 1.70 \\
\hline OOA_19 & Muddy Sand & Muddy Sand & Very Fine Sand & Very Poorly Sorted & 0.0 & 78.0 & 22.0 & 78.0 & 12.0 & 10.0 & 3.63 & 0.08 & 2.03 & 0.45 & 3.61 & 1.30 \\
\hline OOA_21 & Muddy Sand & Muddy Sand & Coarse Silt & Poorly Sorted & 0.0 & 71.0 & 29.0 & 71.0 & 18.0 & 11.0 & 4.13 & 0.06 & 1.98 & 0.65 & 3.61 & 1.90 \\
\hline OOA_22 & Muddy Sand & Silty Sand & Very Fine Sand & Poorly Sorted & 0.0 & 80.0 & 20.0 & 80.0 & 16.0 & 4.0 & 3.73 & 0.08 & 1.16 & 0.56 & 4.26 & 1.40 \\
\hline OOA_23 & Muddy Sand & Muddy Sand & Very Fine Sand & Poorly Sorted & 5.0 & 80.0 & 15.0 & 84.0 & 10.0 & 6.0 & 3.34 & 0.10 & 1.70 & -0.07 & 4.41 & 1.90 \\
\hline OOA_24 & Muddy Sand & Muddy Sand & Coarse Silt & Very Poorly Sorted & 7.0 & 66.0 & 27.0 & 73.0 & 15.0 & 12.0 & 4.03 & 0.06 & 2.90 & 0.31 & 2.63 & 4.60 \\
\hline OOA_25 & Gravelly Muddy Sand & Silty Sand & Very Fine Sand & Very Poorly Sorted & 7.0 & 68.0 & 25.0 & 75.0 & 23.0 & 2.0 & 3.53 & 0.09 & 2.78 & 0.24 & 1.93 & 2.60 \\
\hline OOA_26 & Gravelly Muddy Sand & & Fine Sand & Very Poorly Sorted & 17.0 & 62.0 & 21.0 & & & & 2.13 & 0.23 & 3.48 & -0.12 & 1.88 & 3.70 \\
\hline OOA_27 & Muddy Sand & Muddy Sand & Very Fine Sand & Very Poorly Sorted & 0.0 & 76.0 & 24.0 & 76.0 & 13.0 & 11.0 & 3.60 & 0.08 & 2.44 & 0.47 & 2.84 & 2.20 \\
\hline OOA_28 & Gravelly Muddy Sand & & Fine Sand & Extremely Poorly Sorted & 25.0 & 39.0 & 36.0 & & & & 2.73 & 0.15 & 4.28 & -0.06 & 0.64 & 2.80 \\
\hline OOA_29 & Gravelly Muddy Sand & & Very Fine Sand & Very Poorly Sorted & 15.0 & 52.0 & 33.0 & & & & 3.40 & 0.09 & 3.88 & 0.12 & 1.25 & 1.90 \\
\hline OOA_30 & Gravelly Muddy Sand & & Very Fine Sand & Very Poorly Sorted & 14.0 & 58.0 & 28.0 & & & & 3.27 & 0.10 & 3.63 & 0.06 & 1.42 & 2.00 \\
\hline OOA_31 & Gravelly Muddy Sand & & Very Fine Sand & Very Poorly Sorted & 9.0 & 68.0 & 23.0 & & & & 3.33 & 0.10 & 2.66 & 0.03 & 3.02 & 1.40 \\
\hline OOA_32 & Muddy Sand & Silty Sand & Coarse Silt & Very Poorly Sorted & 0.0 & 73.0 & 27.0 & 73.0 & 19.0 & 8.0 & 4.37 & 0.05 & 2.18 & 0.53 & 2.32 & 1.90 \\
\hline OOA_33 & Gravelly Muddy Sand & Silty Sand & Very Fine Sand & Very Poorly Sorted & 5.0 & 72.0 & 23.0 & 77.0 & 19.0 & 4.0 & 3.50 & 0.09 & 2.52 & 0.13 & 1.79 & 1.60 \\
\hline OOA_35 & Muddy Sand & Silty Sand & Very Fine Sand & Very Poorly Sorted & 0.0 & 78.0 & 22.0 & 78.0 & 19.0 & 3.0 & 3.54 & 0.09 & 2.05 & 0.62 & 1.94 & 2.30 \\
\hline OOA_36 & Muddy Sand & Silty Sand & Very Fine Sand & Very Poorly Sorted & 5.0 & 74.0 & 21.0 & 79.0 & 17.0 & 4.0 & 3.79 & 0.07 & 2.53 & 0.41 & 2.88 & 2.30 \\
\hline OOA_37 & Muddy Sand & Silty Sand & Fine Sand & Poorly Sorted & 4.0 & 80.0 & 16.0 & 84.0 & 12.0 & 4.0 & 2.98 & 0.13 & 1.75 & 0.32 & 2.99 & 1.70 \\
\hline OOA_39 & Muddy Sand & Silty Sand & Very Fine Sand & Poorly Sorted & 0.0 & 81.0 & 19.0 & 81.0 & 14.0 & 5.0 & 3.37 & 0.10 & 1.80 & 0.56 & 2.47 & 1.90 \\
\hline OOA_41 & Muddy Sand & Silty Sand & Very Fine Sand & Poorly Sorted & 0.0 & 78.0 & 22.0 & 78.0 & 18.0 & 4.0 & 3.77 & 0.07 & 1.93 & 0.63 & 2.28 & 2.00 \\
\hline OOA_43 & Sand & Sand & Fine Sand & Poorly Sorted & 1.0 & 89.0 & 10.0 & 90.0 & 9.0 & 1.0 & 2.50 & 0.18 & 1.17 & 0.26 & 2.77 & 1.10 \\
\hline OOA_45 & Muddy Sand & Silty Sand & Very Fine Sand & Poorly Sorted & 0.0 & 84.0 & 16.0 & 84.0 & 11.0 & 5.0 & 3.47 & 0.09 & 1.05 & 0.45 & 4.34 & 1.30 \\
\hline OOA_47 & Muddy Sand & Silty Sand & Fine Sand & Poorly Sorted & 3.0 & 82.0 & 15.0 & 85.0 & 14.0 & 1.0 & 2.97 & 0.13 & 1.72 & -0.18 & 2.53 & 1.20 \\
\hline OOA_48 & Sandy Mud & Sandy Silt & Coarse Silt & Very Poorly Sorted & 0.0 & 42.0 & 58.0 & 42.0 & 52.0 & 6.0 & 4.70 & 0.04 & 2.19 & 0.02 & 0.99 & 4.80 \\
\hline OOA_52 & Sandy Mud & Sandy Silt & Coarse Silt & Very Poorly Sorted & 3.0 & 47.0 & 50.0 & 50.0 & 46.0 & 4.0 & 4.47 & 0.05 & 2.03 & 0.13 & 0.77 & 3.90 \\
\hline
\end{tabular}


OOA Survey (1997-2005) (2): Identification, Location, and Description

\begin{tabular}{|c|c|c|c|c|c|c|c|}
\hline Sample ID & $\begin{array}{l}\text { Original } \\
\text { Sample ID }\end{array}$ & $\begin{array}{l}\text { Latitude } \\
\text { WGS84 }\end{array}$ & $\begin{array}{l}\text { Longitude } \\
\text { WGS84 }\end{array}$ & $\begin{array}{l}\text { Reliability } \\
\text { Ranking for } \\
\text { Positioning }\end{array}$ & $\begin{array}{c}\text { Water } \\
\text { Depth } \\
\text { (m) }\end{array}$ & $\begin{array}{l}\text { Sample } \\
\text { Collected }\end{array}$ & $\begin{array}{c}\text { Sampler } \\
\text { Type }\end{array}$ \\
\hline OOA_53 & A0798G12 & 42.959667 & -70.647833 & 1 & 45 & $7 / 22 / 1998$ & $B C$ \\
\hline OOA_54 & A0798G13 & 42.940000 & -70.649500 & 1 & 54 & $7 / 22 / 1998$ & $\mathrm{BC}$ \\
\hline OOA_57 & A0798G16 & 43.016667 & -70.628833 & 1 & 32 & $7 / 22 / 1998$ & $B C$ \\
\hline OOA_58 & A0798G17 & 43.053333 & -70.670000 & 1 & 22 & $7 / 22 / 1998$ & $B C$ \\
\hline OOA_59 & A0798G18 & 43.053667 & -70.666833 & 1 & 18 & $7 / 22 / 1998$ & $B C$ \\
\hline OOA_61 & A0798G20 & 43.066667 & -70.701000 & 1 & 21 & $7 / 22 / 1998$ & $B C$ \\
\hline OOA_62 & A0898G01 & 42.960500 & -70.649500 & 1 & 41 & $8 / 25 / 1998$ & $B C$ \\
\hline OOA_64 & A0898G03 & 42.950833 & -70.651333 & 1 & 46 & $8 / 25 / 1998$ & $B C$ \\
\hline OOA_66 & A0898G05 & 42.940000 & -70.650500 & 1 & 51 & $8 / 25 / 1998$ & $B C$ \\
\hline OOA_70 & A0898G09 & 42.920500 & -70.650500 & 1 & 58 & $8 / 25 / 1998$ & $B C$ \\
\hline OOA_74 & A0898G13 & 42.930667 & -70.635833 & 1 & 57 & $8 / 25 / 1998$ & $\mathrm{BC}$ \\
\hline OOA_76 & A0898G15 & 42.940667 & -70.635000 & 1 & 53 & 8/25/1998 & $B C$ \\
\hline OOA_77 & A0898G16 & 42.951333 & -70.635333 & 1 & 51 & $8 / 25 / 1998$ & $B C$ \\
\hline OOA_78 & A0898G17 & 42.960333 & -70.620500 & 1 & 37 & $8 / 25 / 1998$ & $B C$ \\
\hline OOA_80 & A0898G19 & 42.950500 & -70.620333 & 1 & 52 & $8 / 25 / 1998$ & $B C$ \\
\hline OOA_81 & A0898G20 & 42.939833 & -70.620500 & 1 & 58 & $8 / 25 / 1998$ & $B C$ \\
\hline OOA_84 & A0898G23 & 42.920667 & -70.621000 & 1 & 66 & $8 / 25 / 1998$ & $B C$ \\
\hline OOA_85 & A0998G01 & 43.076667 & -70.700333 & 1 & 17 & 9/15/1998 & $B C$ \\
\hline OOA_86 & A0998G02 & 42.960833 & -70.559667 & 1 & 74 & 9/15/1998 & $B C$ \\
\hline OOA_90 & A0998G06 & 42.925667 & -70.642167 & 1 & 52 & 9/15/1998 & $B C$ \\
\hline OOA_93 & A0998G09 & 42.942333 & -70.642167 & 1 & 51 & 9/15/1998 & $B C$ \\
\hline OOA_94 & A0998G10 & 42.950167 & -70.642333 & 1 & & $9 / 15 / 1998$ & $\mathrm{BC}$ \\
\hline OOA_95 & A1098G01 & 42.918333 & -70.627333 & 1 & 64 & $10 / 23 / 1998$ & $B C$ \\
\hline OOA_96 & A1098G02 & 42.920667 & -70.650500 & 1 & 57 & $10 / 23 / 1998$ & $\mathrm{BC}$ \\
\hline OOA_97 & $\mathrm{A} 1098 \mathrm{G} 03$ & 42.940667 & -70.638333 & 1 & 50 & $10 / 23 / 1998$ & $\mathrm{BC}$ \\
\hline OOA_98 & A1098G04 & 42.943333 & -70.630500 & 1 & 52 & $10 / 23 / 1998$ & $B C$ \\
\hline OOA_100 & A1098G06 & 42.950333 & -70.599667 & 1 & 62 & $10 / 23 / 1998$ & $B C$ \\
\hline OOA_101 & A0399G01 & 42.938667 & -70.648333 & 1 & 51 & $3 / 25 / 1999$ & $\mathrm{BC}$ \\
\hline OOA_102 & A0399G02 & 42.940000 & -70.620167 & 1 & 58 & $3 / 25 / 1999$ & $\mathrm{BC}$ \\
\hline OOA_103 & A0399G03 & 42.941667 & -70.629833 & 1 & 54 & $3 / 25 / 1999$ & $\mathrm{BC}$ \\
\hline OOA_104 & A0399G04 & 42.941333 & -70.633167 & 1 & 52 & $3 / 25 / 1999$ & $B C$ \\
\hline OOA_105 & A0399G05 & 42.945833 & -70.633000 & 1 & 51 & $3 / 25 / 1999$ & $\mathrm{BC}$ \\
\hline OOA_109 & G3 & 42.941667 & -70.629667 & 1 & 54 & $3 / 25 / 1999$ & $B C$ \\
\hline OOA_110 & G5 & 42.946000 & -70.633333 & 1 & 50 & $3 / 26 / 1999$ & $B C$ \\
\hline OOA_111 & G7 & 42.943667 & -70.631500 & 1 & 52 & $3 / 27 / 1999$ & $\mathrm{BC}$ \\
\hline OOA_113 & A0499G02 & 42.941000 & -70.633333 & 1 & 55 & 4/15/1999 & $B C$ \\
\hline
\end{tabular}


OOA Survey (1997-2005) (2): Sediment Classifications and Grain Size Statistics

\begin{tabular}{|c|c|c|c|c|c|c|c|c|c|c|c|c|c|c|c|c|}
\hline Sample ID & $\begin{array}{l}\text { Textural Group from \%GSM } \\
\text { (Gradistat) }\end{array}$ & $\begin{array}{l}\text { Textural Group } \\
\text { from \%SZC } \\
\text { (Gradistat) }\end{array}$ & $\begin{array}{l}\text { Sediment Classification } \\
\text { from Mean Phi } \\
\text { (Wentworth) }\end{array}$ & Sorting (Gradistat) & $\begin{array}{c}\text { Gravel } \\
\%\end{array}$ & $\begin{array}{c}\text { Sand } \\
\%\end{array}$ & $\begin{array}{c}\text { Mud } \\
\%\end{array}$ & $\begin{array}{c}\text { Sand } \\
\%\end{array}$ & $\begin{array}{c}\text { Silt } \\
\%\end{array}$ & $\begin{array}{c}\text { Clay } \\
\%\end{array}$ & $\begin{array}{l}\text { Mean } \\
\text { Size } \\
\text { (phi) }\end{array}$ & $\begin{array}{l}\text { Mean } \\
\text { Size } \\
(\mathrm{mm})\end{array}$ & $\begin{array}{c}\text { Sorting } \\
\text { (phi) }\end{array}$ & Skewness & Kurtosis & LOI \% \\
\hline OOA_53 & Muddy Sand & Silty Sand & Very Fine Sand & Poorly Sorted & 4.0 & 76.0 & 20.0 & 80.0 & 18.0 & 2.0 & 3.73 & 0.08 & 1.89 & 0.29 & 3.05 & 2.20 \\
\hline OOA_54 & Muddy Sand & Silty Sand & Very Fine Sand & Poorly Sorted & 0.0 & 78.0 & 22.0 & 79.0 & 19.0 & 2.0 & 3.80 & 0.07 & 1.28 & 0.53 & 3.10 & 1.50 \\
\hline OOA_57 & Gravel & & Pebble Gravel & Moderately Well Sorted & 98.0 & 1.0 & 1.0 & & & & -4.03 & 16.37 & 0.56 & 0.52 & 1.30 & \\
\hline OOA_58 & Gravel & & & & & & & & & & & & & & & \\
\hline OOA_59 & Gravel & & & & & & & & & & & & & & & \\
\hline OOA_61 & Sandy Gravel & & Pebble Gravel & Very Poorly Sorted & 77.0 & 22.0 & 1.0 & & & & -2.33 & 5.04 & 2.46 & 0.78 & 0.87 & \\
\hline OOA_62 & Sand & Sand & Fine Sand & Moderately Well Sorted & 3.0 & 93.0 & 4.0 & 96.0 & 2.0 & 2.0 & 2.93 & 0.13 & 0.64 & -0.29 & 1.52 & 0.70 \\
\hline OOA_64 & Muddy Sand & Muddy Sand & Very Fine Sand & Poorly Sorted & 1.0 & 81.0 & 18.0 & 82.0 & 9.0 & 9.0 & 3.53 & 0.09 & 1.60 & 0.63 & 5.53 & 1.50 \\
\hline OOA_66 & Muddy Sand & Muddy Sand & Coarse Silt & Very Poorly Sorted & 1.0 & 67.0 & 32.0 & 68.0 & 17.0 & 15.0 & 4.60 & 0.04 & 2.69 & 0.69 & 2.48 & 2.20 \\
\hline OOA_70 & Gravelly Muddy Sand & & Very Fine Sand & Very Poorly Sorted & 13.0 & 60.0 & 27.0 & & & & 3.73 & 0.08 & 3.98 & 0.13 & 2.74 & 2.10 \\
\hline OOA_74 & Muddy Sand & Muddy Sand & Very Fine Sand & Poorly Sorted & 2.0 & 83.0 & 15.0 & 85.0 & 8.0 & 7.0 & 3.27 & 0.10 & 1.61 & 0.25 & 4.98 & 1.30 \\
\hline OOA_76 & Muddy Sand & Muddy Sand & Very Fine Sand & Poorly Sorted & 0.0 & 82.0 & 18.0 & 82.0 & 11.0 & 7.0 & 3.60 & 0.08 & 1.41 & 0.65 & 4.99 & 1.30 \\
\hline OOA_77 & Muddy Sand & Muddy Sand & Coarse Silt & Very Poorly Sorted & 0.0 & 76.0 & 24.0 & 76.0 & 11.0 & 13.0 & 4.00 & 0.06 & 2.52 & 0.73 & 3.06 & 2.40 \\
\hline OOA_78 & Sandy Gravel & & Granule Gravel & Poorly Sorted & 52.0 & 47.0 & 1.0 & & & & -1.40 & 2.64 & 1.73 & -0.18 & 1.07 & 0.90 \\
\hline OOA_80 & Muddy Sand & Muddy Sand & Very Fine Sand & Poorly Sorted & 0.0 & 86.0 & 14.0 & 86.0 & 6.0 & 8.0 & 3.80 & 0.07 & 1.66 & 0.46 & 3.87 & 1.30 \\
\hline OOA_81 & Muddy Sand & Muddy Sand & Very Fine Sand & Poorly Sorted & 0.0 & 82.0 & 18.0 & 82.0 & 10.0 & 8.0 & 3.57 & 0.08 & 1.63 & 0.48 & 8.81 & 1.40 \\
\hline OOA_84 & Gravelly Muddy Sand & & Very Fine Sand & Very Poorly Sorted & 14.0 & 56.0 & 30.0 & & & & 3.13 & 0.11 & 3.86 & 0.06 & 1.99 & 2.90 \\
\hline OOA_85 & Gravelly Sand & & Coarse Sand & Poorly Sorted & 10.0 & 88.0 & 2.0 & & & & 0.17 & 0.89 & 1.12 & 0.21 & 1.29 & 1.10 \\
\hline OOA_86 & Sandy Mud & Sandy Mud & Fine Silt & Very Poorly Sorted & 0.0 & 32.0 & 68.0 & 32.0 & 40.0 & 28.0 & 6.30 & 0.01 & 3.18 & 0.61 & 0.87 & 4.00 \\
\hline OOA_90 & Gravel & & Pebble Gravel & Poorly Sorted & 87.0 & 9.0 & 3.0 & & & & -3.10 & 8.59 & 1.44 & 0.89 & 7.38 & \\
\hline OOA_93 & Muddy Sand & Muddy Sand & Very Fine Sand & Poorly Sorted & 1.0 & 80.0 & 19.0 & 81.0 & 10.0 & 9.0 & 3.50 & 0.09 & 1.79 & 0.39 & 4.61 & 1.70 \\
\hline OOA_94 & Muddy Sand & Muddy Sand & Very Fine Sand & Poorly Sorted & 0.0 & 81.0 & 19.0 & 81.0 & 9.0 & 10.0 & 3.57 & 0.08 & 1.83 & 0.65 & 5.04 & 1.80 \\
\hline OOA_95 & Muddy Sand & Muddy Sand & Coarse Silt & Very Poorly Sorted & 1.0 & 64.0 & 35.0 & 65.0 & 22.0 & 13.0 & 4.50 & 0.04 & 2.38 & 0.64 & 3.09 & 2.10 \\
\hline OOA_96 & Muddy Sand & Muddy Sand & Coarse Silt & Very Poorly Sorted & 0.0 & 71.0 & 29.0 & 71.0 & 16.0 & 13.0 & 4.50 & 0.04 & 2.41 & 0.76 & 3.46 & 2.10 \\
\hline OOA_97 & Muddy Sand & Muddy Sand & Coarse Silt & Very Poorly Sorted & 0.0 & 75.0 & 25.0 & 75.0 & 13.0 & 12.0 & 4.17 & 0.06 & 2.05 & 0.76 & 4.56 & 2.00 \\
\hline OOA_98 & Muddy Sand & Muddy Sand & Very Fine Sand & Poorly Sorted & 1.0 & 80.0 & 19.0 & 81.0 & 11.0 & 8.0 & 3.63 & 0.08 & 1.52 & 0.66 & 6.48 & 1.50 \\
\hline OOA_100 & Muddy Sand & Muddy Sand & Very Fine Sand & Very Poorly Sorted & 0.0 & 77.0 & 23.0 & 77.0 & 12.0 & 11.0 & 3.83 & 0.07 & 2.21 & 0.67 & 3.89 & 2.00 \\
\hline OOA_101 & Muddy Sand & Muddy Sand & Very Fine Sand & Poorly Sorted & 0.0 & 77.0 & 23.0 & 77.0 & 13.0 & 10.0 & 3.87 & 0.07 & 1.65 & 0.75 & 4.74 & 1.87 \\
\hline OOA_102 & Muddy Sand & Muddy Sand & Very Fine Sand & Very Poorly Sorted & 4.0 & 75.0 & 21.0 & 79.0 & 11.0 & 10.0 & 3.43 & 0.09 & 2.67 & 0.16 & 3.37 & 1.67 \\
\hline OOA_103 & Muddy Sand & Muddy Sand & Very Fine Sand & Poorly Sorted & 0.0 & 81.0 & 19.0 & 81.0 & 12.0 & 7.0 & 3.70 & 0.08 & 1.40 & 0.49 & 4.85 & 1.47 \\
\hline OOA_104 & Muddy Sand & Muddy Sand & Very Fine Sand & Poorly Sorted & 3.0 & 77.0 & 20.0 & 80.0 & 12.0 & 8.0 & 3.63 & 0.08 & 1.50 & 0.49 & 4.63 & 1.55 \\
\hline OOA_105 & Muddy Sand & Muddy Sand & Very Fine Sand & Poorly Sorted & 2.0 & 85.0 & 13.0 & 87.0 & 7.0 & 6.0 & 3.37 & 0.10 & 1.39 & 0.32 & 4.27 & 1.34 \\
\hline OOA_109 & Muddy Sand & Muddy Sand & Very Fine Sand & Poorly Sorted & 0.0 & 82.0 & 18.0 & 82.0 & 12.0 & 6.0 & 3.67 & 0.08 & 1.20 & 0.46 & 4.30 & 1.00 \\
\hline OOA_110 & Muddy Sand & Muddy Sand & Very Fine Sand & Poorly Sorted & 0.0 & 83.0 & 17.0 & 83.0 & 10.0 & 7.0 & 3.50 & 0.09 & 1.37 & 0.35 & 4.67 & 1.40 \\
\hline OOA_111 & Muddy Sand & Muddy Sand & Very Fine Sand & Poorly Sorted & 0.0 & 86.0 & 14.0 & 86.0 & 8.0 & 6.0 & 3.53 & 0.09 & 1.26 & 0.42 & 4.64 & 1.40 \\
\hline OOA_113 & Muddy Sand & Muddy Sand & Very Fine Sand & Poorly Sorted & 0.0 & 85.0 & 15.0 & 85.0 & 8.0 & 7.0 & 3.53 & 0.09 & 1.34 & 0.40 & 5.48 & 1.50 \\
\hline
\end{tabular}


OOA Survey (1997-2005) (3): Identification, Location, and Description

\begin{tabular}{|c|c|c|c|c|c|c|c|}
\hline Sample ID & $\begin{array}{c}\text { Original } \\
\text { Sample ID }\end{array}$ & $\begin{array}{l}\text { Latitude } \\
\text { WGS84 }\end{array}$ & $\begin{array}{l}\text { Longitude } \\
\text { WGS84 }\end{array}$ & $\begin{array}{l}\text { Reliability } \\
\text { Ranking for } \\
\text { Positioning }\end{array}$ & $\begin{array}{c}\text { Water } \\
\text { Depth } \\
\text { (m) }\end{array}$ & $\begin{array}{l}\text { Sample } \\
\text { Collected }\end{array}$ & $\begin{array}{c}\text { Sampler } \\
\text { Type }\end{array}$ \\
\hline OOA_115 & A0499G04 & 42.943000 & -70.634167 & 1 & 53 & $4 / 15 / 1999$ & $B C$ \\
\hline OOA_116 & A0499G05 & 42.945667 & -70.632500 & 1 & 53 & $4 / 15 / 1999$ & $\mathrm{BC}$ \\
\hline OOA_117 & A0499G06 & 42.945833 & -70.629500 & 1 & 53 & 4/15/1999 & $B C$ \\
\hline OOA_118 & A0499G07 & 42.942833 & -70.627500 & 1 & 56 & 4/15/1999 & $B C$ \\
\hline OOA_119 & A0499G08 & 42.941167 & -70.629833 & 1 & 56 & 4/15/1999 & $B C$ \\
\hline OOA_120 & A0599G01 & 42.932333 & -70.631833 & 1 & 64 & 5/21/1999 & $B C$ \\
\hline OOA_121 & A0599G02 & 42.923000 & -70.651833 & 1 & 65 & $5 / 21 / 1999$ & $B C$ \\
\hline OOA_122 & A0599G03 & 42.927167 & -70.612833 & 1 & 71 & $5 / 21 / 1999$ & $B C$ \\
\hline OOA_123 & A0599G04 & 42.934833 & -70.602333 & 1 & 74 & $5 / 21 / 1999$ & $B C$ \\
\hline OOA_124 & A0599G05 & 42.938333 & -70.602000 & 1 & 73 & $5 / 21 / 1999$ & $B C$ \\
\hline OOA_127 & A0599G08 & 42.946000 & -70.632167 & 1 & 53 & $5 / 26 / 1999$ & $\mathrm{BC}$ \\
\hline OOA_132 & A0599G13 & 42.945833 & -70.629333 & 1 & 53 & $5 / 26 / 1999$ & $B C$ \\
\hline OOA_133 & A0599G14 & 42.943667 & -70.632000 & 1 & 53 & $5 / 26 / 1999$ & $B C$ \\
\hline OOA_134 & A0599G15 & 42.943667 & -70.629167 & 1 & 55 & $5 / 26 / 1999$ & $B C$ \\
\hline OOA_135 & A0599G16 & 42.943333 & -70.631833 & 1 & 53 & $5 / 26 / 1999$ & $B C$ \\
\hline OOA_136 & A0599G17 & 42.943667 & -70.629167 & 1 & 53 & $5 / 26 / 1999$ & $B C$ \\
\hline OOA_137 & A0599G18 & 42.941500 & -70.632000 & 1 & 53 & $5 / 26 / 1999$ & $B C$ \\
\hline OOA_138 & A0599G19 & 42.941500 & -70.628833 & 1 & & $5 / 26 / 1999$ & $B C$ \\
\hline OOA_139 & A0699G01 & 42.941167 & -70.631000 & 1 & 53 & 6/7/1999 & $B C$ \\
\hline OOA_142 & A0699G04 & 42.942500 & -70.631000 & 1 & 52 & $6 / 7 / 1999$ & $B C$ \\
\hline OOA_143 & A0699G05 & 42.943333 & -70.630667 & 1 & 52 & 6/7/1999 & $B C$ \\
\hline OOA_144 & A0699G06 & 42.942500 & -70.628667 & 1 & 53 & $6 / 7 / 1999$ & $\mathrm{BC}$ \\
\hline OOA_146 & A0699G08 & 42.943333 & -70.632833 & 1 & 51 & $6 / 7 / 1999$ & $B C$ \\
\hline OOA_148 & A0799G03 & 42.945333 & -70.629167 & 1 & 53 & 7/12/1999 & $B C$ \\
\hline OOA_149 & A0799G04 & 42.944333 & -70.630167 & 1 & 54 & 7/12/1999 & $\mathrm{BC}$ \\
\hline OOA_150 & A0799G05 & 42.944500 & -70.631500 & 1 & 53 & 7/12/1999 & $B C$ \\
\hline OOA_152 & A0799C01 & 42.943500 & -70.630667 & 1 & 54 & 7/12/1999 & $B C$ \\
\hline OOA_153 & A0799C02 & 42.943333 & -70.631000 & 1 & 54 & 7/12/1999 & $\mathrm{BC}$ \\
\hline OOA_154 & A0799C03 & 42.941667 & -70.630833 & 1 & 55 & 7/12/1999 & $\mathrm{BC}$ \\
\hline OOA_155 & A0799C04 & 42.940833 & -70.630333 & 1 & 55 & 7/12/1999 & $\mathrm{BC}$ \\
\hline OOA_156 & A0799C05 & 42.943333 & -70.634167 & 1 & 52 & 7/12/1999 & $B C$ \\
\hline OOA_157 & A0799C06 & 42.942833 & -70.626167 & 1 & 56 & 7/12/1999 & $\mathrm{BC}$ \\
\hline OOA_158 & A0899G01 & 42.945000 & -70.630667 & 1 & 51 & 8/3/1999 & $B C$ \\
\hline OOA_159 & A0899G02 & 42.943833 & -70.631000 & 1 & 51 & 8/3/1999 & $B C$ \\
\hline OOA_160 & A0899C01 & 42.945167 & -70.630833 & 1 & 51 & 8/3/1999 & $\mathrm{BC}$ \\
\hline OOA_161 & A0899C02 & 42.944000 & -70.631000 & 1 & 51 & 8/3/1999 & $B C$ \\
\hline
\end{tabular}


OOA Survey (1997-2005) (3): Sediment Classifications and Grain Size Statistics

\begin{tabular}{|c|c|c|c|c|c|c|c|c|c|c|c|c|c|c|c|c|}
\hline Sample ID & $\begin{array}{l}\text { Textural Group from \%GSM } \\
\text { (Gradistat) }\end{array}$ & $\begin{array}{l}\text { Textural Group } \\
\text { from \%SZC } \\
\text { (Gradistat) }\end{array}$ & $\begin{array}{l}\text { Sediment Classification } \\
\text { from Mean Phi } \\
\text { (Wentworth) }\end{array}$ & Sorting (Gradistat) & $\begin{array}{c}\text { Gravel } \\
\%\end{array}$ & $\begin{array}{c}\text { Sand } \\
\%\end{array}$ & $\begin{array}{c}\text { Mud } \\
\%\end{array}$ & $\begin{array}{c}\text { Sand } \\
\%\end{array}$ & $\begin{array}{c}\text { Silt } \\
\%\end{array}$ & $\begin{array}{c}\text { Clay } \\
\%\end{array}$ & $\begin{array}{c}\text { Mean } \\
\text { Size } \\
\text { (phi) }\end{array}$ & $\begin{array}{c}\text { Mean } \\
\text { Size } \\
(\mathrm{mm}) \\
\end{array}$ & $\begin{array}{l}\text { Sorting } \\
\text { (phi) }\end{array}$ & Skewness & Kurtosis & LOI \% \\
\hline OOA_115 & Muddy Sand & Muddy Sand & Very Fine Sand & Poorly Sorted & 1.0 & 83.0 & 16.0 & 84.0 & 9.0 & 7.0 & 3.57 & 0.08 & 1.29 & 0.48 & 4.32 & 1.60 \\
\hline OOA_116 & Gravelly Muddy Sand & & Very Fine Sand & Very Poorly Sorted & 7.0 & 81.0 & 12.0 & & & & 3.10 & 0.12 & 2.36 & -0.12 & 5.81 & 1.60 \\
\hline OOA_117 & Muddy Sand & Muddy Sand & Very Fine Sand & Poorly Sorted & 1.0 & 86.0 & 13.0 & 87.0 & 8.0 & 5.0 & 3.23 & 0.11 & 1.25 & 0.26 & 4.17 & 1.70 \\
\hline OOA_118 & Muddy Sand & Muddy Sand & Very Fine Sand & Poorly Sorted & 1.0 & 82.0 & 17.0 & 83.0 & 10.0 & 7.0 & 3.52 & 0.09 & 1.34 & 0.44 & 4.41 & 1.60 \\
\hline OOA_119 & Muddy Sand & Muddy Sand & Very Fine Sand & Poorly Sorted & 0.0 & 80.0 & 20.0 & 80.0 & 12.0 & 8.0 & 3.70 & 0.08 & 1.42 & 0.67 & 4.41 & 1.50 \\
\hline OOA_120 & Muddy Sand & Muddy Sand & Coarse Silt & Very Poorly Sorted & 3.0 & 61.0 & 36.0 & 64.0 & 19.0 & 17.0 & 4.77 & 0.04 & 3.07 & 0.62 & 1.84 & 2.61 \\
\hline OOA_121 & Muddy Sand & Muddy Sand & Very Fine Sand & Very Poorly Sorted & 1.0 & 75.0 & 24.0 & 76.0 & 12.0 & 12.0 & 3.98 & 0.06 & 2.59 & 0.58 & 3.05 & 1.88 \\
\hline OOA_122 & Muddy Sand & Muddy Sand & Coarse Silt & Very Poorly Sorted & 1.0 & 66.0 & 33.0 & 67.0 & 20.0 & 13.0 & 4.57 & 0.04 & 2.58 & 0.58 & 2.66 & 1.87 \\
\hline OOA_123 & Sandy Mud & Sandy Mud & Medium Silt & Very Poorly Sorted & 3.0 & 39.0 & 58.0 & 42.0 & 32.0 & 26.0 & 5.75 & 0.02 & 3.78 & 0.40 & 0.98 & 3.35 \\
\hline OOA_124 & Sandy Mud & Sandy Mud & Fine Silt & Very Poorly Sorted & 2.0 & 38.0 & 60.0 & 40.0 & 30.0 & 30.0 & 6.00 & 0.02 & 3.79 & 0.32 & 0.89 & 5.29 \\
\hline OOA_127 & Muddy Sand & Muddy Sand & Very Fine Sand & Poorly Sorted & 1.0 & 84.0 & 15.0 & 85.0 & 8.0 & 7.0 & 3.38 & 0.10 & 1.45 & 0.38 & 4.15 & 1.42 \\
\hline OOA_132 & Muddy Sand & Muddy Sand & Very Fine Sand & Poorly Sorted & 1.0 & 81.0 & 18.0 & 82.0 & 9.0 & 9.0 & 3.33 & 0.10 & 1.98 & 0.37 & 3.55 & 1.61 \\
\hline OOA_133 & Muddy Sand & Muddy Sand & Very Fine Sand & Poorly Sorted & 0.0 & 81.0 & 19.0 & 81.0 & 11.0 & 8.0 & 3.63 & 0.08 & 1.46 & 0.66 & 4.39 & 1.53 \\
\hline OOA_134 & Muddy Sand & Muddy Sand & Very Fine Sand & Poorly Sorted & 0.0 & 81.0 & 19.0 & 81.0 & 11.0 & 8.0 & 3.67 & 0.08 & 1.43 & 0.58 & 4.99 & 1.40 \\
\hline OOA_135 & Muddy Sand & Muddy Sand & Very Fine Sand & Poorly Sorted & 0.0 & 81.0 & 19.0 & 81.0 & 11.0 & 8.0 & 3.65 & 0.08 & 1.53 & 0.65 & 4.85 & 1.41 \\
\hline OOA_136 & Muddy Sand & Muddy Sand & Very Fine Sand & Poorly Sorted & 0.0 & 82.0 & 18.0 & 82.0 & 10.0 & 8.0 & 3.67 & 0.08 & 1.34 & 0.67 & 4.47 & 1.37 \\
\hline OOA_137 & Muddy Sand & Muddy Sand & Very Fine Sand & Poorly Sorted & 1.0 & 79.0 & 20.0 & 80.0 & 12.0 & 8.0 & 3.77 & 0.07 & 1.52 & 0.58 & 4.39 & 1.52 \\
\hline OOA_138 & Muddy Sand & Muddy Sand & Very Fine Sand & Poorly Sorted & 0.0 & 80.0 & 20.0 & 80.0 & 12.0 & 8.0 & 3.70 & 0.08 & 1.43 & 0.69 & 4.54 & 1.52 \\
\hline OOA_139 & Muddy Sand & Muddy Sand & Very Fine Sand & Poorly Sorted & 0.0 & 84.0 & 16.0 & 84.0 & 10.0 & 6.0 & 3.53 & 0.09 & 1.22 & 0.43 & 4.47 & 1.22 \\
\hline OOA_142 & Muddy Sand & Muddy Sand & Very Fine Sand & Poorly Sorted & 3.0 & 81.0 & 16.0 & 84.0 & 10.0 & 6.0 & 3.68 & 0.08 & 1.48 & 0.58 & 3.28 & 1.39 \\
\hline OOA_143 & Muddy Sand & Muddy Sand & Very Fine Sand & Poorly Sorted & 1.0 & 80.0 & 19.0 & 81.0 & 11.0 & 8.0 & 3.75 & 0.07 & 1.58 & 0.62 & 2.78 & 1.79 \\
\hline OOA_144 & Muddy Sand & Muddy Sand & Very Fine Sand & Poorly Sorted & 0.0 & 81.0 & 19.0 & 81.0 & 12.0 & 7.0 & 3.67 & 0.08 & 1.36 & 0.59 & 4.29 & 1.36 \\
\hline OOA_146 & Muddy Sand & Muddy Sand & Very Fine Sand & Poorly Sorted & 0.0 & 80.0 & 20.0 & 80.0 & 12.0 & 8.0 & 3.72 & 0.08 & 1.43 & 0.57 & 5.86 & 1.47 \\
\hline OOA_148 & Muddy Sand & Muddy Sand & Very Fine Sand & & 0.0 & 84.0 & 16.0 & 84.0 & 9.0 & 7.0 & 3.47 & 0.09 & & 0.46 & 4.41 & 1.50 \\
\hline OOA_149 & Muddy Sand & Muddy Sand & Very Fine Sand & & 0.0 & 86.0 & 14.0 & 86.0 & 8.0 & 6.0 & 3.47 & 0.09 & & 0.43 & 4.68 & 1.50 \\
\hline OOA_150 & Muddy Sand & Muddy Sand & Very Fine Sand & & 3.0 & 81.0 & 16.0 & 84.0 & 10.0 & 6.0 & 3.43 & 0.09 & & 0.37 & 5.02 & 1.80 \\
\hline OOA_152 & Muddy Sand & Muddy Sand & Very Fine Sand & & 0.0 & 84.0 & 16.0 & 84.0 & 9.0 & 7.0 & 3.70 & 0.08 & & 0.70 & 2.89 & 1.50 \\
\hline OOA_153 & Muddy Sand & Muddy Sand & Very Fine Sand & & 0.0 & 83.0 & 17.0 & 83.0 & 10.0 & 7.0 & 3.62 & 0.08 & & 0.54 & 4.48 & 1.70 \\
\hline OOA_154 & Muddy Sand & Muddy Sand & Very Fine Sand & & 0.0 & 81.0 & 19.0 & 81.0 & 12.0 & 7.0 & 3.67 & 0.08 & & 0.57 & 4.22 & 1.60 \\
\hline OOA_155 & Muddy Sand & Muddy Sand & Very Fine Sand & & 0.0 & 82.0 & 18.0 & 82.0 & 11.0 & 7.0 & 3.75 & 0.07 & & 0.60 & 2.59 & 1.40 \\
\hline OOA_156 & Muddy Sand & Muddy Sand & Very Fine Sand & & 0.0 & 84.0 & 16.0 & 84.0 & 10.0 & 6.0 & 3.53 & 0.09 & & 0.52 & 4.51 & 1.30 \\
\hline OOA_157 & Muddy Sand & Muddy Sand & Very Fine Sand & & 0.0 & 81.0 & 12.0 & 81.0 & 12.0 & 7.0 & 3.68 & 0.08 & & 0.66 & 4.51 & 1.70 \\
\hline OOA_158 & Muddy Sand & Muddy Sand & Very Fine Sand & & 0.0 & 78.0 & 22.0 & 78.0 & 12.0 & 10.0 & 3.93 & 0.07 & & 0.68 & 4.46 & 1.90 \\
\hline OOA_159 & Muddy Sand & Muddy Sand & Very Fine Sand & & 2.0 & 80.0 & 18.0 & 82.0 & 10.0 & 8.0 & 3.62 & 0.08 & & 0.43 & 4.57 & 1.60 \\
\hline OOA_160 & Muddy Sand & Muddy Sand & Very Fine Sand & & 0.0 & 80.0 & 20.0 & 80.0 & 11.0 & 9.0 & 3.73 & 0.08 & & 0.67 & 4.29 & 1.90 \\
\hline OOA_161 & Muddy Sand & Muddy Sand & Very Fine Sand & & 0.0 & 83.0 & 17.0 & 83.0 & 10.0 & 7.0 & 3.63 & 0.08 & & 0.60 & 4.63 & 1.70 \\
\hline
\end{tabular}


OOA Survey (1997-2005) (4): Identification, Location, and Description

\begin{tabular}{|c|c|c|c|c|c|c|c|}
\hline Sample ID & $\begin{array}{c}\text { Original } \\
\text { Sample ID }\end{array}$ & $\begin{array}{c}\text { Latitude } \\
\text { WGS84 }\end{array}$ & $\begin{array}{c}\text { Longitude } \\
\text { WGS84 }\end{array}$ & $\begin{array}{l}\text { Reliability } \\
\text { Ranking for } \\
\text { Positioning }\end{array}$ & $\begin{array}{c}\text { Water } \\
\text { Depth } \\
\text { (m) }\end{array}$ & $\begin{array}{c}\text { Sample } \\
\text { Collected }\end{array}$ & $\begin{array}{c}\text { Sampler } \\
\text { Type }\end{array}$ \\
\hline OOA_162 & A0899C03 & 42.942500 & -70.631167 & 1 & 51 & 8/3/1999 & $\mathrm{BC}$ \\
\hline OOA_163 & A0899C04 & 42.941667 & -70.630833 & 1 & 52 & $8 / 3 / 1999$ & $B C$ \\
\hline OOA_164 & A0899C05 & 42.945500 & -70.632833 & 1 & 50 & $8 / 3 / 1999$ & $B C$ \\
\hline OOA_165 & A0899C06 & 42.942833 & -70.626833 & 1 & 53 & $8 / 3 / 1999$ & $\mathrm{BC}$ \\
\hline OOA_168 & A0500C01 & 42.947167 & -70.633000 & 1 & 50 & $5 / 6 / 2000$ & $B C$ \\
\hline OOA_169 & $\mathrm{A} 0500 \mathrm{CO} 2$ & 42.945000 & -70.633333 & 1 & 51 & $5 / 6 / 2000$ & $B C$ \\
\hline OOA_170 & $\mathrm{A} 0500 \mathrm{CO} 3$ & 42.943833 & -70.630333 & 1 & 53 & $5 / 6 / 2000$ & $B C$ \\
\hline OOA_171 & $\mathrm{A} 0500 \mathrm{CO} 4$ & 42.943500 & -70.627333 & 1 & 54 & $5 / 6 / 2000$ & $B C$ \\
\hline OOA_172 & A0500C05 & 42.944333 & -70.625500 & 1 & 54 & $5 / 6 / 2000$ & $B C$ \\
\hline OOA_173 & A0500C06 & 42.941167 & -70.630333 & 1 & 54 & $5 / 6 / 2000$ & $B C$ \\
\hline OOA_174 & A0500C07 & 42.946333 & -70.628333 & 1 & 53 & $5 / 6 / 2000$ & $B C$ \\
\hline OOA_175 & A0600C01 & 42.946667 & -70.628500 & 1 & 52 & $6 / 26 / 2000$ & $B C$ \\
\hline OOA_176 & A0600C02 & 42.944667 & -70.640667 & 1 & 50 & $6 / 26 / 2000$ & $B C$ \\
\hline OOA_177 & A0600C03 & 42.943833 & -70.633833 & 1 & 51 & $6 / 26 / 2000$ & $B C$ \\
\hline OOA_178 & A0600C04 & 42.943833 & -70.630667 & 1 & 52 & $6 / 26 / 2000$ & $B C$ \\
\hline OOA_179 & A0600C05 & 42.944000 & -70.627333 & 1 & 53 & $6 / 26 / 2000$ & $B C$ \\
\hline OOA_180 & A0600C06 & 42.944000 & -70.621000 & 1 & 56 & $6 / 26 / 2000$ & $B C$ \\
\hline OOA_181 & A0600C07 & 42.941667 & -70.630167 & 1 & 53 & $6 / 26 / 2000$ & $B C$ \\
\hline OOA_182 & A0600C08 & 42.937000 & -70.630167 & 1 & 52 & $6 / 26 / 2000$ & $B C$ \\
\hline OOA_183 & A0801CO1 & 42.947933 & -70.628100 & 1 & 50.4 & $8 / 14 / 2001$ & $B C$ \\
\hline OOA_184 & $\mathrm{A} 0801 \mathrm{CO} 2$ & 42.944650 & -70.640717 & 1 & 49.3 & $8 / 14 / 2001$ & $B C$ \\
\hline OOA_185 & A0801CO3 & 42.944133 & -70.633983 & 1 & 50.3 & $8 / 14 / 2001$ & $B C$ \\
\hline OOA_186 & $\mathrm{A} 0801 \mathrm{CO} 4$ & 42.943967 & -70.630667 & 1 & 51.7 & $8 / 14 / 2001$ & $B C$ \\
\hline OOA_187 & A0801CO5 & 42.944267 & -70.627183 & 1 & 52 & $8 / 14 / 2001$ & $B C$ \\
\hline OOA_188 & A0801CO6 & 42.943717 & -70.621900 & 1 & 54.3 & $8 / 14 / 2001$ & $B C$ \\
\hline OOA_189 & A0801CO7 & 42.941317 & -70.631417 & 1 & 52.1 & $8 / 14 / 2001$ & $B C$ \\
\hline OOA_190 & A0801CO8 & 42.938283 & -70.630567 & 1 & 52 & $8 / 14 / 2001$ & $B C$ \\
\hline OOA_191 & $\mathrm{A} 0702 \mathrm{CO} 1$ & 42.947167 & -70.628733 & 1 & 53 & $7 / 19 / 2002$ & $B C$ \\
\hline OOA_192 & $\mathrm{A} 0702 \mathrm{CO} 2$ & 42.943617 & -70.641400 & 1 & 51 & $7 / 19 / 2002$ & $B C$ \\
\hline OOA_193 & $\mathrm{A} 0702 \mathrm{CO} 3$ & 42.942783 & -70.633967 & 1 & 52 & $7 / 19 / 2002$ & $B C$ \\
\hline OOA_194 & $\mathrm{A} 0702 \mathrm{CO} 4$ & 42.943700 & -70.631100 & 1 & 53 & $7 / 19 / 2002$ & $B C$ \\
\hline OOA_195 & $\mathrm{A} 0702 \mathrm{CO} 5$ & 42.942867 & -70.627983 & 1 & 54 & $7 / 19 / 2002$ & $B C$ \\
\hline OOA_196 & $\mathrm{A} 0702 \mathrm{CO} 6$ & 42.943233 & -70.621250 & 1 & 57 & $7 / 19 / 2002$ & $B C$ \\
\hline OOA_197 & $\mathrm{A} 0702 \mathrm{CO}$ & 42.941017 & -70.631183 & 1 & 54 & $7 / 19 / 2002$ & $B C$ \\
\hline OOA_198 & $\mathrm{A} 0702 \mathrm{CO} 8$ & 42.937417 & -70.630467 & 1 & 49 & $7 / 19 / 2002$ & $B C$ \\
\hline
\end{tabular}


OOA Survey (1997-2005) (4): Sediment Classifications and Grain Size Statistics

\begin{tabular}{|c|c|c|c|c|c|c|c|c|c|c|c|c|c|c|c|c|}
\hline Sample ID & $\begin{array}{l}\text { Textural Group from \%GSM } \\
\text { (Gradistat) }\end{array}$ & $\begin{array}{l}\text { Textural Group } \\
\text { from \%SZC } \\
\text { (Gradistat) }\end{array}$ & $\begin{array}{l}\text { Sediment Classification } \\
\text { from Mean Phi } \\
\text { (Wentworth) }\end{array}$ & Sorting (Gradistat) & $\begin{array}{c}\text { Gravel } \\
\%\end{array}$ & $\begin{array}{c}\text { Sand } \\
\%\end{array}$ & $\begin{array}{c}\text { Mud } \\
\%\end{array}$ & $\begin{array}{c}\text { Sand } \\
\%\end{array}$ & $\begin{array}{c}\text { Silt } \\
\%\end{array}$ & $\begin{array}{c}\text { Clay } \\
\%\end{array}$ & $\begin{array}{c}\text { Mean } \\
\text { Size } \\
\text { (phi) }\end{array}$ & $\begin{array}{c}\text { Mean } \\
\text { Size } \\
(\mathrm{mm}) \\
\end{array}$ & $\begin{array}{c}\text { Sorting } \\
\text { (phi) }\end{array}$ & Skewness & Kurtosis & LOI \% \\
\hline OOA_162 & Muddy Sand & Muddy Sand & Very Fine Sand & & 0.0 & 84.0 & 16.0 & 84.0 & 11.0 & 5.0 & 3.52 & 0.09 & & 0.35 & 3.31 & 1.50 \\
\hline OOA_163 & Muddy Sand & Muddy Sand & Very Fine Sand & & 0.0 & 88.0 & 12.0 & 88.0 & 5.0 & 7.0 & 3.53 & 0.09 & & 0.46 & 4.92 & 1.60 \\
\hline OOA_164 & Muddy Sand & Muddy Sand & Very Fine Sand & & 0.0 & 87.0 & 13.0 & 87.0 & 7.0 & 6.0 & 3.30 & 0.10 & & 0.32 & 3.61 & 1.60 \\
\hline OOA_165 & Muddy Sand & Muddy Sand & Very Fine Sand & & 0.0 & 84.0 & 16.0 & 84.0 & 11.0 & 5.0 & 3.47 & 0.09 & & 0.45 & 3.85 & 1.60 \\
\hline OOA_168 & Muddy Sand & Muddy Sand & Very Fine Sand & Poorly Sorted & 0.0 & 84.0 & 16.0 & 84.0 & 8.0 & 8.0 & 3.40 & 0.09 & 1.49 & 0.49 & 4.51 & 1.47 \\
\hline OOA_169 & Muddy Sand & Muddy Sand & Very Fine Sand & Poorly Sorted & 0.0 & 84.0 & 16.0 & 84.0 & 8.0 & 8.0 & 3.45 & 0.09 & 1.39 & 0.66 & 7.19 & 1.38 \\
\hline OOA_170 & Muddy Sand & Muddy Sand & Very Fine Sand & Poorly Sorted & 2.0 & 81.0 & 17.0 & 83.0 & 10.0 & 7.0 & 3.48 & 0.09 & 1.37 & 0.46 & 4.13 & 1.37 \\
\hline OOA_171 & Muddy Sand & Muddy Sand & Very Fine Sand & Poorly Sorted & 0.0 & 77.0 & 23.0 & 77.0 & 13.0 & 10.0 & 3.90 & 0.07 & 1.77 & 0.69 & 4.92 & 1.73 \\
\hline OOA_172 & Muddy Sand & Muddy Sand & Very Fine Sand & Poorly Sorted & 0.0 & 84.0 & 16.0 & 84.0 & 9.0 & 7.0 & 3.34 & 0.10 & 1.51 & 0.34 & 4.96 & 1.35 \\
\hline OOA_173 & Muddy Sand & Muddy Sand & Very Fine Sand & Poorly Sorted & 0.0 & 77.0 & 23.0 & 77.0 & 13.0 & 10.0 & 3.83 & 0.07 & 1.58 & 0.72 & 4.64 & 1.75 \\
\hline OOA_174 & Muddy Sand & Muddy Sand & Very Fine Sand & Poorly Sorted & 0.0 & 82.0 & 18.0 & 82.0 & 9.0 & 9.0 & 3.42 & 0.09 & 1.56 & 0.55 & 5.04 & 1.53 \\
\hline OOA_175 & Muddy Sand & Muddy Sand & Very Fine Sand & Poorly Sorted & 1.0 & 82.0 & 17.0 & 83.0 & 9.0 & 8.0 & 3.41 & 0.09 & 1.60 & 0.54 & 5.16 & 1.44 \\
\hline OOA_176 & Muddy Sand & Muddy Sand & Very Fine Sand & Very Poorly Sorted & 2.0 & 78.0 & 20.0 & 80.0 & 10.0 & 10.0 & 3.43 & 0.09 & 2.20 & 0.38 & 3.92 & 1.82 \\
\hline OOA_177 & Muddy Sand & Muddy Sand & Very Fine Sand & Poorly Sorted & 0.0 & 78.0 & 22.0 & 78.0 & 13.0 & 9.0 & 3.78 & 0.07 & 1.63 & 0.71 & 4.69 & 1.68 \\
\hline OOA_178 & Muddy Sand & Muddy Sand & Very Fine Sand & Poorly Sorted & 1.0 & 80.0 & 19.0 & 81.0 & 11.0 & 8.0 & 3.62 & 0.08 & 1.52 & 0.64 & 5.17 & 1.48 \\
\hline OOA_179 & Muddy Sand & Muddy Sand & Very Fine Sand & Poorly Sorted & 0.0 & 81.0 & 19.0 & 81.0 & 10.0 & 9.0 & 3.56 & 0.08 & 1.54 & 0.56 & 5.04 & 1.24 \\
\hline OOA_180 & Muddy Sand & Muddy Sand & Coarse Silt & Very Poorly Sorted & 0.0 & 70.0 & 30.0 & 70.0 & 15.0 & 15.0 & 4.13 & 0.06 & 3.05 & 0.55 & 1.76 & 2.26 \\
\hline OOA_181 & Muddy Sand & Muddy Sand & Very Fine Sand & Poorly Sorted & 0.0 & 80.0 & 20.0 & 80.0 & 12.0 & 8.0 & 3.68 & 0.08 & 1.46 & 0.68 & 4.73 & 1.54 \\
\hline OOA_182 & Gravelly Muddy Sand & & Very Fine Sand & Very Poorly Sorted & 8.0 & 71.0 & 21.0 & & & & 3.50 & 0.09 & 2.58 & 0.05 & 5.59 & 2.35 \\
\hline OOA_183 & Muddy Sand & Muddy Sand & Very Fine Sand & Poorly Sorted & 0.0 & 85.0 & 15.0 & 85.0 & 8.0 & 7.0 & 3.25 & 0.11 & 1.50 & 0.41 & 4.21 & 1.50 \\
\hline OOA_184 & Muddy Sand & & Very Fine Sand & Very Poorly Sorted & 4.0 & 76.0 & 20.0 & & & & 3.43 & 0.09 & 2.36 & 0.31 & 3.96 & 2.00 \\
\hline OOA_185 & Muddy Sand & Muddy Sand & Very Fine Sand & Poorly Sorted & 0.0 & 83.0 & 17.0 & 83.0 & 10.0 & 7.0 & 3.52 & 0.09 & 1.34 & 0.57 & 4.24 & 1.40 \\
\hline OOA_186 & Muddy Sand & Muddy Sand & Very Fine Sand & Poorly Sorted & 0.0 & 79.0 & 21.0 & 79.0 & 12.0 & 9.0 & 3.73 & 0.08 & 1.67 & 0.67 & 4.12 & 2.10 \\
\hline OOA_187 & Muddy Sand & & Very Fine Sand & Poorly Sorted & 5.0 & 78.0 & 17.0 & & & & 3.38 & 0.10 & 1.80 & 0.31 & 5.22 & 1.60 \\
\hline OOA_188 & Muddy Sand & Muddy Sand & Very Fine Sand & Very Poorly Sorted & 0.0 & 77.0 & 23.0 & 77.0 & 13.0 & 10.0 & 3.55 & 0.09 & 2.10 & 0.47 & 3.30 & 2.00 \\
\hline OOA_189 & Muddy Sand & Muddy Sand & Very Fine Sand & Poorly Sorted & 0.0 & 81.0 & 19.0 & 81.0 & 12.0 & 7.0 & 3.65 & 0.08 & 1.42 & 0.61 & 4.22 & 1.50 \\
\hline OOA_190 & Muddy Sand & Muddy Sand & Very Fine Sand & Poorly Sorted & 0.0 & 81.0 & 19.0 & 81.0 & 11.0 & 8.0 & 3.65 & 0.08 & 1.48 & 0.63 & 4.45 & 1.60 \\
\hline OOA_191 & Muddy Sand & Muddy Sand & Very Fine Sand & Poorly Sorted & 0.0 & 82.0 & 18.0 & 82.0 & 9.0 & 9.0 & 3.47 & 0.09 & 1.63 & 0.64 & 4.74 & 1.80 \\
\hline OOA_192 & Muddy Sand & Muddy Sand & Very Fine Sand & Poorly Sorted & 0.0 & 80.0 & 20.0 & 80.0 & 11.0 & 9.0 & 3.63 & 0.08 & 1.75 & 0.53 & 4.30 & 1.70 \\
\hline OOA_193 & Muddy Sand & Muddy Sand & Very Fine Sand & Poorly Sorted & 0.0 & 80.0 & 20.0 & 80.0 & 12.0 & 8.0 & 3.72 & 0.08 & 1.46 & 0.65 & 5.05 & 1.50 \\
\hline OOA_194 & Muddy Sand & Muddy Sand & Very Fine Sand & Poorly Sorted & 0.0 & 83.0 & 17.0 & 83.0 & 10.0 & 7.0 & 3.54 & 0.09 & 1.39 & 0.56 & 4.23 & 1.40 \\
\hline OOA_195 & Muddy Sand & Muddy Sand & Very Fine Sand & Poorly Sorted & 1.0 & 80.0 & 19.0 & 81.0 & 11.0 & 8.0 & 3.63 & 0.08 & 1.56 & 0.48 & 4.68 & 1.50 \\
\hline OOA_196 & Muddy Sand & Muddy Sand & Very Fine Sand & Very Poorly Sorted & 0.0 & 80.0 & 20.0 & 80.0 & 11.0 & 9.0 & 3.50 & 0.09 & 2.04 & 0.41 & 3.89 & 1.80 \\
\hline OOA_197 & Muddy Sand & Muddy Sand & Very Fine Sand & Poorly Sorted & 0.0 & 80.0 & 20.0 & 80.0 & 12.0 & 8.0 & 3.70 & 0.08 & 1.53 & 0.57 & 4.57 & 1.50 \\
\hline OOA_198 & Muddy Sand & Muddy Sand & Very Fine Sand & Poorly Sorted & 0.0 & 78.0 & 22.0 & 78.0 & 13.0 & 9.0 & 3.87 & 0.07 & 1.60 & 0.66 & 5.40 & 1.80 \\
\hline
\end{tabular}


OOA Survey (1997-2005) (5): Identification, Location, and Description

\begin{tabular}{|c|c|c|c|c|c|c|c|}
\hline Sample ID & $\begin{array}{c}\text { Original } \\
\text { Sample ID }\end{array}$ & $\begin{array}{l}\text { Latitude } \\
\text { WGS84 }\end{array}$ & $\begin{array}{c}\text { Longitude } \\
\text { WGS84 }\end{array}$ & $\begin{array}{l}\text { Reliability } \\
\text { Ranking for } \\
\text { Positioning }\end{array}$ & $\begin{array}{l}\text { Water } \\
\text { Depth } \\
\text { (m) }\end{array}$ & $\begin{array}{l}\text { Sample } \\
\text { Collected }\end{array}$ & $\begin{array}{c}\text { Sampler } \\
\text { Type }\end{array}$ \\
\hline OOA_199 & 1 & 42.947250 & -70.636367 & 1 & 50 & $7 / 25 / 2002$ & $B C$ \\
\hline OOA_201 & 2 & 42.944983 & -70.634717 & 1 & 51 & $7 / 25 / 2002$ & $\mathrm{BC}$ \\
\hline OOA_203 & 3 & 42.944300 & -70.635517 & 1 & 51 & $7 / 25 / 2002$ & $\mathrm{BC}$ \\
\hline OOA_204 & 4 & 42.945300 & -70.632933 & 1 & 52 & $7 / 25 / 2002$ & $B C$ \\
\hline OOA_206 & 5 & 42.945250 & -70.625233 & 1 & 54 & $7 / 25 / 2002$ & $B C$ \\
\hline OOA_208 & 6 & 42.948400 & -70.624317 & 1 & 53 & $7 / 25 / 2002$ & $B C$ \\
\hline OOA_209 & 7 & 42.944550 & -70.624067 & 1 & 55 & $7 / 25 / 2002$ & $\mathrm{BC}$ \\
\hline OOA_210 & 8 & 42.942617 & -70.637000 & 1 & 52 & $7 / 25 / 2002$ & $B C$ \\
\hline OOA_211 & 9 & 42.942117 & -70.634767 & 1 & 53 & $7 / 25 / 2002$ & $B C$ \\
\hline OOA_212 & 10 & 42.941933 & -70.628617 & 1 & 54 & $7 / 25 / 2002$ & $B C$ \\
\hline OOA_213 & 11 & 42.942017 & -70.629167 & 1 & 55 & $7 / 25 / 2002$ & $B C$ \\
\hline OOA_214 & 12 & 42.941100 & -70.628200 & 1 & 56 & $7 / 25 / 2002$ & $B C$ \\
\hline OOA_215 & 13 & 42.939667 & -70.632033 & 1 & 55 & $7 / 25 / 2002$ & $B C$ \\
\hline OOA_216 & A0803CO1 & 42.947400 & -70.629067 & 1 & 52 & $8 / 11 / 2003$ & $\mathrm{BC}$ \\
\hline OOA_217 & $\mathrm{A} 0803 \mathrm{CO} 2$ & 42.944333 & -70.640750 & 1 & 52 & $8 / 11 / 2003$ & $\mathrm{BC}$ \\
\hline OOA_218 & $\mathrm{A} 0803 \mathrm{CO} 3$ & 42.944850 & -70.635517 & 1 & 51 & $8 / 11 / 2003$ & $\mathrm{BC}$ \\
\hline OOA_219 & $\mathrm{A} 0803 \mathrm{CO} 4$ & 42.944817 & -70.630683 & 1 & 54 & $8 / 11 / 2003$ & $B C$ \\
\hline OOA_220 & A0803CO5 & 42.943717 & -70.628383 & 1 & 55 & $8 / 11 / 2003$ & $\mathrm{BC}$ \\
\hline OOA_221 & A0803CO6 & 42.944633 & -70.621700 & 1 & 57 & $8 / 11 / 2003$ & $\mathrm{BC}$ \\
\hline OOA_222 & A0803CO7 & 42.941200 & -70.630933 & 1 & 55 & $8 / 11 / 2003$ & $\mathrm{BC}$ \\
\hline OOA_223 & A0803CO8 & 42.938550 & -70.630950 & 1 & 55 & $8 / 11 / 2003$ & $\mathrm{BC}$ \\
\hline OOA_224 & A0204C01 & 42.947717 & -70.628367 & 1 & 53 & $2 / 3 / 2004$ & $B C$ \\
\hline OOA_225 & A0204C02 & 42.944767 & -70.640767 & 1 & 51 & $2 / 3 / 2004$ & $B C$ \\
\hline OOA_226 & A0204C03 & 42.936317 & -70.635217 & 1 & 52 & $2 / 3 / 2004$ & $B C$ \\
\hline OOA_227 & $\mathrm{A} 0204 \mathrm{CO} 04$ & 42.944700 & -70.630367 & 1 & 54 & $2 / 3 / 2004$ & $\mathrm{BC}$ \\
\hline OOA_228 & A0204C05 & 42.943767 & -70.628233 & 1 & 55 & $2 / 3 / 2004$ & $B C$ \\
\hline OOA_229 & A0204C06 & 42.944867 & -70.621417 & 1 & 58 & $2 / 3 / 2004$ & $B C$ \\
\hline OOA_230 & $\mathrm{A} 0204 \mathrm{C07}$ & 42.941267 & -70.631317 & 1 & 55 & $2 / 3 / 2004$ & $B C$ \\
\hline OOA_231 & A0204C08 & 42.938667 & -70.630567 & 1 & 55 & $2 / 3 / 2004$ & $\mathrm{BC}$ \\
\hline OOA_232 & A0804C01 & 42.947633 & -70.629383 & 1 & 49 & $8 / 4 / 2004$ & $\mathrm{BC}$ \\
\hline
\end{tabular}


OOA Survey (1997-2005) (5): Sediment Classifications and Grain Size Statistics

\begin{tabular}{|c|c|c|c|c|c|c|c|c|c|c|c|c|c|c|c|c|}
\hline Sample ID & $\begin{array}{l}\text { Textural Group from \%GSM } \\
\text { (Gradistat) }\end{array}$ & $\begin{array}{l}\text { Textural Group } \\
\text { from \%SZC } \\
\text { (Gradistat) } \\
\end{array}$ & $\begin{array}{l}\text { Sediment Classification } \\
\text { from Mean Phi } \\
\text { (Wentworth) }\end{array}$ & Sorting (Gradistat) & $\begin{array}{c}\text { Gravel } \\
\% \\
\end{array}$ & $\begin{array}{c}\text { Sand } \\
\%\end{array}$ & $\begin{array}{c}\text { Mud } \\
\% \\
\end{array}$ & $\begin{array}{c}\text { Sand } \\
\%\end{array}$ & $\begin{array}{l}\text { Silt } \\
\% \\
\end{array}$ & $\begin{array}{c}\text { Clay } \\
\%\end{array}$ & $\begin{array}{c}\text { Mean } \\
\text { Size } \\
\text { (phi) }\end{array}$ & $\begin{array}{c}\text { Mean } \\
\text { Size } \\
(\mathrm{mm}) \\
\end{array}$ & $\begin{array}{c}\text { Sorting } \\
\text { (phi) }\end{array}$ & Skewness & Kurtosis & LOI \% \\
\hline OOA_199 & Muddy Sand & Muddy Sand & Very Fine Sand & Poorly Sorted & 0.0 & 82.0 & 18.0 & 82.0 & 10.0 & 8.0 & 3.60 & 0.08 & 1.53 & 0.60 & 4.57 & 1.60 \\
\hline OOA_201 & Muddy Sand & Muddy Sand & Very Fine Sand & Poorly Sorted & 1.0 & 82.0 & 17.0 & 82.0 & 9.0 & 8.0 & 3.60 & 0.08 & 1.58 & 0.57 & 5.53 & 1.60 \\
\hline OOA_203 & Muddy Sand & Muddy Sand & Very Fine Sand & Poorly Sorted & 0.0 & 83.0 & 17.0 & 83.0 & 9.0 & 8.0 & 3.53 & 0.09 & 1.45 & 0.56 & 5.19 & 1.70 \\
\hline OOA_204 & Muddy Sand & Muddy Sand & Very Fine Sand & Poorly Sorted & 0.0 & 84.0 & 16.0 & 84.0 & 9.0 & 7.0 & 3.37 & 0.10 & 1.38 & 0.43 & 3.64 & 1.40 \\
\hline OOA_206 & Muddy Sand & Muddy Sand & Very Fine Sand & Poorly Sorted & 0.0 & 82.0 & 18.0 & 82.0 & 10.0 & 8.0 & 3.40 & 0.09 & 1.60 & 0.45 & 4.74 & 1.60 \\
\hline OOA_208 & Muddy Sand & Muddy Sand & Very Fine Sand & Poorly Sorted & 0.0 & 83.0 & 17.0 & 83.0 & 9.0 & 8.0 & 3.33 & 0.10 & 1.76 & 0.54 & 3.87 & 1.50 \\
\hline OOA_209 & Muddy Sand & Muddy Sand & Very Fine Sand & Poorly Sorted & 0.0 & 82.0 & 18.0 & 82.0 & 10.0 & 8.0 & 3.37 & 0.10 & 1.72 & 0.38 & 3.83 & 1.80 \\
\hline OOA_210 & Muddy Sand & Muddy Sand & Very Fine Sand & Poorly Sorted & 0.0 & 80.0 & 20.0 & 80.0 & 11.0 & 9.0 & 3.67 & 0.08 & 1.45 & 0.57 & 4.67 & 1.60 \\
\hline OOA_211 & Muddy Sand & Muddy Sand & Very Fine Sand & Poorly Sorted & 0.0 & 79.0 & 21.0 & 79.0 & 13.0 & 8.0 & 3.80 & 0.07 & 1.50 & 0.61 & 5.19 & 1.70 \\
\hline OOA_212 & Muddy Sand & Muddy Sand & Very Fine Sand & Poorly Sorted & 0.0 & 82.0 & 18.0 & 82.0 & 12.0 & 6.0 & 3.63 & 0.08 & 1.19 & 0.59 & 4.23 & 1.50 \\
\hline OOA_213 & Muddy Sand & Muddy Sand & Very Fine Sand & Poorly Sorted & 0.0 & 77.0 & 23.0 & 77.0 & 14.0 & 9.0 & 3.87 & 0.07 & 1.58 & 0.65 & 4.57 & 1.80 \\
\hline OOA_214 & Muddy Sand & Muddy Sand & Very Fine Sand & Poorly Sorted & 0.0 & 78.0 & 22.0 & 78.0 & 13.0 & 9.0 & 3.83 & 0.07 & 1.56 & 0.63 & 5.33 & 1.60 \\
\hline OOA_215 & Muddy Sand & Muddy Sand & Very Fine Sand & Poorly Sorted & 0.0 & 79.0 & 21.0 & 79.0 & 13.0 & 8.0 & 3.77 & 0.07 & 1.46 & 0.57 & 5.12 & 1.50 \\
\hline OOA_216 & Muddy Sand & Muddy Sand & Very Fine Sand & Poorly Sorted & 0.0 & 81.0 & 19.0 & 81.0 & 10.0 & 9.0 & 3.55 & 0.09 & 1.78 & 0.56 & 3.87 & 1.87 \\
\hline OOA_217 & Muddy Sand & Muddy Sand & Very Fine Sand & Poorly Sorted & 0.0 & 84.0 & 16.0 & 84.0 & 9.0 & 7.0 & 3.27 & 0.10 & 1.61 & 0.25 & 3.71 & 1.71 \\
\hline OOA_218 & Muddy Sand & Muddy Sand & Very Fine Sand & Poorly Sorted & 0.0 & 80.0 & 20.0 & 80.0 & 11.0 & 9.0 & 3.68 & 0.08 & 1.59 & 0.67 & 4.34 & 1.80 \\
\hline OOA_219 & Muddy Sand & Muddy Sand & Very Fine Sand & Poorly Sorted & 0.0 & 79.0 & 21.0 & 79.0 & 12.0 & 9.0 & 3.67 & 0.08 & 1.70 & 0.61 & 4.23 & 2.08 \\
\hline OOA_220 & Muddy Sand & Muddy Sand & Coarse Silt & Very Poorly Sorted & 0.0 & 74.0 & 26.0 & 74.0 & 14.0 & 12.0 & 4.32 & 0.05 & 2.16 & 0.81 & 3.61 & 2.42 \\
\hline OOA_221 & Muddy Sand & Muddy Sand & Very Fine Sand & Very Poorly Sorted & 0.0 & 76.0 & 24.0 & 76.0 & 13.0 & 11.0 & 3.72 & 0.08 & 2.39 & 0.51 & 3.06 & 2.20 \\
\hline OOA_222 & Muddy Sand & Muddy Sand & Very Fine Sand & Poorly Sorted & 0.0 & 76.0 & 24.0 & 76.0 & 14.0 & 10.0 & 3.87 & 0.07 & 1.68 & 0.67 & 5.17 & 2.15 \\
\hline OOA_223 & Muddy Sand & Muddy Sand & Very Fine Sand & Poorly Sorted & 0.0 & 82.0 & 18.0 & 82.0 & 11.0 & 8.0 & 3.65 & 0.08 & 1.46 & 0.63 & 4.39 & 2.06 \\
\hline OOA_224 & Muddy Sand & Muddy Sand & Very Fine Sand & Poorly Sorted & 0.0 & 86.0 & 14.0 & 86.0 & 8.0 & 6.0 & 3.27 & 0.10 & 1.41 & 0.43 & 4.27 & 1.10 \\
\hline OOA_225 & Muddy Sand & Muddy Sand & Very Fine Sand & Poorly Sorted & 3.0 & 81.0 & 16.0 & 84.0 & 9.0 & 7.0 & 3.10 & 0.12 & 1.98 & 0.13 & 3.58 & 1.50 \\
\hline OOA_226 & Muddy Sand & Muddy Sand & Very Fine Sand & Poorly Sorted & 1.0 & 80.0 & 19.0 & 81.0 & 10.0 & 9.0 & 3.67 & 0.08 & 1.60 & 0.61 & 4.63 & 1.50 \\
\hline OOA_227 & Muddy Sand & Muddy Sand & Very Fine Sand & Poorly Sorted & 0.0 & 82.0 & 18.0 & 82.0 & 10.0 & 8.0 & 3.60 & 0.08 & 1.55 & 0.64 & 5.05 & 1.30 \\
\hline OOA_228 & Muddy Sand & Muddy Sand & Very Fine Sand & Poorly Sorted & 0.0 & 83.0 & 17.0 & 83.0 & 10.0 & 7.0 & 3.53 & 0.09 & 1.38 & 0.54 & 4.85 & 1.30 \\
\hline OOA_229 & Muddy Sand & Muddy Sand & Coarse Silt & Very Poorly Sorted & 0.0 & 70.0 & 30.0 & 70.0 & 16.0 & 14.0 & 4.43 & 0.05 & 2.79 & 0.64 & 2.35 & 2.30 \\
\hline OOA_230 & Muddy Sand & Muddy Sand & Very Fine Sand & Poorly Sorted & 0.0 & 84.0 & 16.0 & 84.0 & 10.0 & 6.0 & 3.53 & 0.09 & 1.16 & 0.41 & 4.23 & 1.20 \\
\hline OOA_231 & Muddy Sand & Muddy Sand & Very Fine Sand & Poorly Sorted & 0.0 & 83.0 & 17.0 & 83.0 & 10.0 & 7.0 & 3.57 & 0.08 & 1.39 & 0.46 & 4.92 & 1.30 \\
\hline OOA_232 & Muddy Sand & Muddy Sand & Very Fine Sand & Poorly Sorted & 0.0 & 80.1 & 19.9 & 80.1 & 11.3 & 8.6 & 3.60 & 0.08 & 1.68 & 0.53 & 4.43 & 1.80 \\
\hline
\end{tabular}


OOA Survey (1997-2005) (6): Identification, Location, and Description

\begin{tabular}{|c|c|c|c|c|c|c|c|}
\hline Sample ID & $\begin{array}{c}\text { Original } \\
\text { Sample ID }\end{array}$ & $\begin{array}{c}\text { Latitude } \\
\text { WGS84 }\end{array}$ & $\begin{array}{c}\text { Longitude } \\
\text { WGS84 }\end{array}$ & $\begin{array}{l}\text { Reliability } \\
\text { Ranking for } \\
\text { Positioning }\end{array}$ & $\begin{array}{c}\text { Water } \\
\text { Depth } \\
\text { (m) }\end{array}$ & $\begin{array}{c}\text { Sample } \\
\text { Collected }\end{array}$ & $\begin{array}{c}\text { Sampler } \\
\text { Type }\end{array}$ \\
\hline OOA_233 & $\mathrm{A} 0804 \mathrm{CO} 2$ & 42.944267 & -70.640617 & 1 & 49 & $8 / 4 / 2004$ & $\mathrm{BC}$ \\
\hline OOA_236 & A0804C03 & 42.944700 & -70.635083 & 1 & 49 & $8 / 4 / 2004$ & $\mathrm{BC}$ \\
\hline OOA_237 & A0804C04 & 42.944950 & -70.630617 & 1 & 51 & $8 / 4 / 2004$ & $B C$ \\
\hline OOA_238 & A0804C05 & 42.944100 & -70.629150 & 1 & 52 & $8 / 4 / 2004$ & $\mathrm{BC}$ \\
\hline OOA_239 & A0804C06 & 42.944550 & -70.622150 & 1 & 54 & $8 / 4 / 2004$ & $B C$ \\
\hline OOA_240 & A0804C07 & 42.941350 & -70.630950 & 1 & 52 & $8 / 4 / 2004$ & $\mathrm{BC}$ \\
\hline OOA_241 & A0804C08 & 42.938817 & -70.631267 & 1 & 53 & $8 / 4 / 2004$ & $B C$ \\
\hline OOA_242 & $\mathrm{A} 0705 \mathrm{C01}$ & 42.941483 & -70.631283 & 1 & & $7 / 6 / 2005$ & $\mathrm{BC}$ \\
\hline OOA_243 & $\mathrm{A} 0705 \mathrm{CO} 2$ & 42.941817 & -70.630883 & 1 & & $7 / 6 / 2005$ & $\mathrm{BC}$ \\
\hline OOA_244 & $\mathrm{A} 0705 \mathrm{CO}$ & 42.943883 & -70.634167 & 1 & & $7 / 6 / 2005$ & $\mathrm{BC}$ \\
\hline OOA_245 & $\mathrm{A} 0705 \mathrm{CO} 4$ & 42.943883 & -70.634200 & 1 & & $7 / 6 / 2005$ & $B C$ \\
\hline OOA_246 & A0605C05 & 42.943950 & -70.634333 & 1 & 50.8 & $6 / 5 / 2005$ & $\mathrm{BC}$ \\
\hline OOA_247 & A0605C06 & 42.944033 & -70.631617 & 1 & 51.5 & $6 / 5 / 2005$ & $\mathrm{BC}$ \\
\hline OOA_248 & A0605C07 & 42.941250 & -70.631100 & 1 & 53 & $6 / 5 / 2005$ & $B C$ \\
\hline OOA_249 & $\mathrm{A} 0605 \mathrm{C08}$ & 42.941300 & -70.634067 & 1 & 52 & $6 / 5 / 2005$ & $B C$ \\
\hline OOA_251 & A0605C10 & 42.945067 & -70.630417 & 1 & 52 & $6 / 5 / 2005$ & $\mathrm{BC}$ \\
\hline OOA_252 & A0605C11 & 42.940417 & -70.630567 & 1 & 54 & $6 / 5 / 2005$ & $\mathrm{BC}$ \\
\hline OOA_253 & $\mathrm{A} 0605 \mathrm{C} 12$ & 42.940483 & -70.635683 & 1 & 53 & $6 / 5 / 2005$ & $B C$ \\
\hline OOA_254 & A0605C13 & 42.947483 & -70.638317 & 1 & 50 & $6 / 5 / 2005$ & $B C$ \\
\hline OOA_255 & $\mathrm{A} 0605 \mathrm{C} 14$ & 42.947450 & -70.629100 & 1 & 51.5 & $6 / 5 / 2005$ & $\mathrm{BC}$ \\
\hline OOA_256 & A0605C15 & 42.943950 & -70.628417 & 1 & 54 & $6 / 5 / 2005$ & $\mathrm{BC}$ \\
\hline OOA_257 & $\mathrm{A} 0605 \mathrm{C} 16$ & 42.938300 & -70.628467 & 1 & 57 & $6 / 5 / 2005$ & $B C$ \\
\hline OOA_258 & $\mathrm{A} 0605 \mathrm{C} 17$ & 42.938533 & -70.631067 & 1 & 55 & $6 / 5 / 2005$ & $\mathrm{BC}$ \\
\hline OOA_259 & A0605C18 & 42.938767 & -70.638467 & 1 & 55 & $6 / 5 / 2005$ & $B C$ \\
\hline OOA_260 & $\mathrm{A} 0605 \mathrm{C} 19$ & 42.944317 & -70.641667 & 1 & 54 & $6 / 5 / 2005$ & $\mathrm{BC}$ \\
\hline OOA_261 & $\mathrm{A} 0605 \mathrm{C} 20$ & 42.944883 & -70.621817 & 1 & 50 & $6 / 5 / 2005$ & $B C$ \\
\hline
\end{tabular}


OOA Survey (1997-2005) (6): Sediment Classifications and Grain Size Statistics

\begin{tabular}{|c|c|c|c|c|c|c|c|c|c|c|c|c|c|c|c|c|}
\hline Sample ID & $\begin{array}{l}\text { Textural Group from \%GSM } \\
\text { (Gradistat) }\end{array}$ & $\begin{array}{l}\text { Textural Group } \\
\text { from \%SZC } \\
\text { (Gradistat) } \\
\end{array}$ & $\begin{array}{l}\text { Sediment Classification } \\
\text { from Mean Phi } \\
\text { (Wentworth) }\end{array}$ & Sorting (Gradistat) & $\begin{array}{c}\text { Gravel } \\
\%\end{array}$ & $\begin{array}{c}\text { Sand } \\
\%\end{array}$ & $\begin{array}{c}\text { Mud } \\
\%\end{array}$ & $\begin{array}{c}\text { Sand } \\
\%\end{array}$ & $\begin{array}{c}\text { Silt } \\
\%\end{array}$ & $\begin{array}{c}\text { Clay } \\
\%\end{array}$ & $\begin{array}{c}\text { Mean } \\
\text { Size } \\
\text { (phi) }\end{array}$ & $\begin{array}{c}\text { Mean } \\
\text { Size } \\
(\mathrm{mm}) \\
\end{array}$ & $\begin{array}{c}\text { Sorting } \\
\text { (phi) }\end{array}$ & Skewness & Kurtosis & LOI \% \\
\hline OOA_233 & Muddy Sand & Muddy Sand & Very Fine Sand & Poorly Sorted & 2.1 & 82.3 & 15.6 & 84.4 & 9.1 & 6.5 & 3.27 & 0.10 & 1.58 & 0.21 & 4.07 & 1.50 \\
\hline OOA_236 & Muddy Sand & Muddy Sand & Very Fine Sand & Poorly Sorted & 1.2 & 81.5 & 17.3 & 82.7 & 10.4 & 6.9 & 3.53 & 0.09 & 1.38 & 0.53 & 4.48 & 1.70 \\
\hline OOA_237 & Muddy Sand & Muddy Sand & Very Fine Sand & Poorly Sorted & 0.3 & 83.7 & 16.0 & 84.0 & 9.9 & 6.1 & 3.43 & 0.09 & 1.32 & 0.39 & 4.04 & 1.40 \\
\hline OOA_238 & Sandy Mud & Sandy Mud & Very Fine Silt & Very Poorly Sorted & 0.8 & 18.0 & 81.2 & 18.8 & 31.4 & 49.8 & 7.90 & 0.00 & 3.62 & -0.02 & 0.72 & 8.90 \\
\hline OOA_239 & Muddy Sand & Muddy Sand & Very Fine Sand & Very Poorly Sorted & 0.3 & 80.1 & 19.6 & 80.4 & 11.2 & 9.0 & 3.30 & 0.10 & 2.04 & 0.36 & 2.84 & 1.70 \\
\hline OOA_240 & Muddy Sand & Muddy Sand & Very Fine Sand & Poorly Sorted & 0.0 & 79.7 & 20.3 & 79.7 & 13.4 & 6.9 & 3.77 & 0.07 & 1.39 & 0.56 & 4.78 & 1.50 \\
\hline OOA_241 & Muddy Sand & Muddy Sand & Coarse Silt & Very Poorly Sorted & 0.0 & 70.7 & 29.3 & 70.7 & 16.5 & 12.8 & 4.50 & 0.04 & 2.20 & 0.74 & 3.17 & 2.50 \\
\hline OOA_242 & Muddy Sand & Muddy Sand & Very Fine Sand & Poorly Sorted & 0.0 & 76.0 & 24.0 & 76.0 & 15.0 & 9.0 & 3.85 & 0.07 & 1.71 & 0.64 & 4.42 & 2.52 \\
\hline OOA_243 & Muddy Sand & Muddy Sand & Very Fine Sand & Poorly Sorted & 0.0 & 81.0 & 19.0 & 81.0 & 13.0 & 9.0 & 3.65 & 0.08 & 1.35 & 0.56 & 3.94 & 1.66 \\
\hline OOA_244 & Slightly Gravelly Muddy Sand & Muddy Sand & Very Fine Sand & Poorly Sorted & 1.0 & 85.0 & 14.0 & 86.0 & 6.0 & 9.0 & 3.45 & 0.09 & 1.45 & 0.49 & 4.96 & 1.86 \\
\hline OOA_245 & Slightly Gravelly Muddy Sand & Muddy Sand & Very Fine Sand & Poorly Sorted & 0.0 & 83.0 & 17.0 & 83.0 & 11.0 & 6.0 & 3.54 & 0.09 & 1.29 & 0.54 & 3.90 & 1.83 \\
\hline OOA_246 & Muddy Sand & Muddy Sand & Very Fine Sand & Poorly Sorted & 0.0 & 79.0 & 21.0 & 79.0 & 13.0 & 8.0 & 3.69 & 0.08 & 1.50 & 0.64 & 4.07 & 1.34 \\
\hline OOA_247 & Muddy Sand & Muddy Sand & Coarse Silt & Poorly Sorted & 0.0 & 73.0 & 27.0 & 73.0 & 18.0 & 9.0 & 4.05 & 0.06 & 1.97 & 0.73 & 3.34 & 2.00 \\
\hline OOA_248 & Slightly Gravelly Muddy Sand & Muddy Sand & Very Fine Sand & Poorly Sorted & 4.0 & 78.0 & 18.0 & 82.0 & 11.0 & 7.0 & 3.58 & 0.08 & 1.55 & 0.45 & 4.53 & 1.55 \\
\hline OOA_249 & Muddy Sand & Muddy Sand & Very Fine Sand & Poorly Sorted & 0.0 & 75.0 & 25.0 & 75.0 & 15.0 & 10.0 & 3.88 & 0.07 & 1.69 & 0.64 & 4.30 & 1.78 \\
\hline OOA_251 & Gravelly Muddy Sand & & Very Fine Sand & Very Poorly Sorted & 11.0 & 74.0 & 14.0 & & & & 3.18 & 0.11 & 2.36 & -0.11 & 6.09 & 1.58 \\
\hline OOA_252 & Muddy Sand & Muddy Sand & Coarse Silt & Poorly Sorted & 0.0 & 72.0 & 28.0 & 72.0 & 17.0 & 11.0 & 4.19 & 0.05 & 2.00 & 0.71 & 3.18 & 2.41 \\
\hline OOA_253 & Muddy Sand & Muddy Sand & Very Fine Sand & Poorly Sorted & 0.0 & 79.0 & 21.0 & 79.0 & 13.0 & 8.0 & 3.72 & 0.08 & 1.50 & 0.57 & 4.11 & 1.46 \\
\hline OOA_254 & Slightly Gravelly Muddy Sand & Muddy Sand & Very Fine Sand & Poorly Sorted & 2.0 & 82.0 & 16.0 & 84.0 & 9.0 & 7.0 & 3.33 & 0.10 & 1.54 & 0.37 & 4.76 & 1.57 \\
\hline OOA_255 & Muddy Sand & Muddy Sand & Very Fine Sand & Poorly Sorted & 0.0 & 83.0 & 17.0 & 83.0 & 10.0 & 7.0 & 3.38 & 0.10 & 1.57 & 0.49 & 3.96 & 1.59 \\
\hline OOA_256 & Slightly Gravelly Muddy Sand & Muddy Sand & Very Fine Sand & Poorly Sorted & 1.0 & 76.0 & 23.0 & 77.0 & 14.0 & 9.0 & 3.73 & 0.08 & 1.81 & 0.62 & 4.19 & 2.05 \\
\hline OOA_257 & Muddy Sand & Muddy Sand & Coarse Silt & Very Poorly Sorted & 0.0 & 73.0 & 27.0 & 73.0 & 15.0 & 12.0 & 4.83 & 0.04 & 2.72 & 0.85 & 1.87 & 2.17 \\
\hline OOA_258 & Muddy Sand & Muddy Sand & Very Fine Sand & Poorly Sorted & 0.0 & 76.0 & 24.0 & 76.0 & 15.0 & 10.0 & 3.81 & 0.07 & 1.63 & 0.64 & 4.16 & 1.56 \\
\hline OOA_259 & Muddy Sand & Muddy Sand & Very Fine Sand & Poorly Sorted & 0.0 & 74.0 & 26.0 & 74.0 & 17.0 & 9.0 & 3.88 & 0.07 & 1.67 & 0.62 & 3.86 & 1.51 \\
\hline OOA_260 & Slightly Gravelly Muddy Sand & Muddy Sand & Very Fine Sand & Poorly Sorted & 1.0 & 83.0 & 16.0 & 84.0 & 9.0 & 7.0 & 3.22 & 0.11 & 1.74 & 0.27 & 3.81 & 1.50 \\
\hline OOA_261 & Muddy Sand & Muddy Sand & Very Fine Sand & Very Poorly Sorted & 0.0 & 76.0 & 24.0 & 76.0 & 14.0 & 10.0 & 3.52 & 0.09 & 2.15 & 0.44 & 3.22 & 1.80 \\
\hline
\end{tabular}


Section 6: MMS Year 2 Survey (1985), Year 4 Survey (1988), Year 8 Survey (1992), and Year 10 Survey (1994) 
MMS Year 2 Survey (1985): Identification, Location, and Description

\begin{tabular}{|c|c|c|c|c|c|c|c|}
\hline Sample ID & $\begin{array}{c}\text { Original } \\
\text { Sample ID }\end{array}$ & $\begin{array}{c}\text { Latitude } \\
\text { WGS84 }\end{array}$ & $\begin{array}{c}\text { Longitude } \\
\text { WGS84 }\end{array}$ & $\begin{array}{l}\text { Reliability } \\
\text { Ranking for } \\
\text { Positioning }\end{array}$ & $\begin{array}{c}\text { Water } \\
\text { Depth } \\
\text { (m) }\end{array}$ & $\begin{array}{c}\text { Sample } \\
\text { Collected }\end{array}$ & $\begin{array}{c}\text { Sampler } \\
\text { Type }\end{array}$ \\
\hline MMS_Y2_1 & 1 & 42.994833 & -70.632333 & 3 & 19 & $8 / 12 / 1985$ or $8 / 14 / 1985$ & Shipek \\
\hline MMS_Y2_2 & 2 & 42.990500 & -70.627667 & 3 & 23 & $8 / 12 / 1985$ or $8 / 14 / 1985$ & Shipek \\
\hline MMS_Y2_3 & 3 & 42.987000 & -70.665500 & 3 & 24 & $8 / 12 / 1985$ or $8 / 14 / 1985$ & Shipek \\
\hline MMS_Y2_4 & 4 & 42.990167 & -70.637667 & 3 & 15 & $8 / 12 / 1985$ or $8 / 14 / 1985$ & Shipek \\
\hline MMS_Y2_5 & 5 & 42.988000 & -70.638833 & 3 & 20 & $8 / 12 / 1985$ or $8 / 14 / 1985$ & Shipek \\
\hline MMS_Y2_6 & 6 & 42.992167 & -70.642500 & 3 & 20 & $8 / 12 / 1985$ or $8 / 14 / 1985$ & Shipek \\
\hline MMS_Y2_7 & 7 & 42.987833 & -70.646000 & 3 & 20 & $8 / 12 / 1985$ or $8 / 14 / 1985$ & Shipek \\
\hline MMS_Y2_8 & 8 & 42.998667 & -70.649000 & 3 & 23 & $8 / 12 / 1985$ or $8 / 14 / 1985$ & Shipek \\
\hline MMS_Y2_9 & 9 & 42.984333 & -70.648833 & 3 & 24 & $8 / 12 / 1985$ or $8 / 14 / 1985$ & Shipek \\
\hline MMS_Y2_10 & 10 & 42.989167 & -70.652500 & 3 & 24 & $8 / 12 / 1985$ or $8 / 14 / 1985$ & Shipek \\
\hline MMS_Y2_11 & 11 & 42.993667 & -70.656333 & 3 & 26 & $8 / 12 / 1985$ or $8 / 14 / 1985$ & Shipek \\
\hline MMS_Y2_12 & 12 & 42.989000 & -70.659333 & 3 & 28 & $8 / 12 / 1985$ or $8 / 14 / 1985$ & Shipek \\
\hline MMS_Y2_13 & 13 & 42.985167 & -70.655167 & 3 & 23 & $8 / 12 / 1985$ or $8 / 14 / 1985$ & Shipek \\
\hline MMS_Y2_14 & 14 & 42.981833 & -70.659500 & 3 & 26 & $8 / 12 / 1985$ or $8 / 14 / 1985$ & Shipek \\
\hline MMS_Y2_15 & 15 & 42.986000 & -70.662833 & 3 & 30 & $8 / 12 / 1985$ or $8 / 14 / 1985$ & Shipek \\
\hline MMS_Y2_16 & 16 & 42.981833 & -70.666500 & 3 & 27 & $8 / 12 / 1985$ or $8 / 14 / 1985$ & Shipek \\
\hline MMS_Y2_17 & 18 & 42.990000 & -70.666000 & 3 & 24 & $8 / 12 / 1985$ or $8 / 14 / 1985$ & Shipek \\
\hline MMS_Y2_18 & 20 & 42.996667 & -70.659167 & 3 & 26 & $8 / 12 / 1985$ or $8 / 14 / 1985$ & Shipek \\
\hline MMS_Y2_19 & 22 & 42.996000 & -70.645167 & 3 & 27 & $8 / 12 / 1985$ or $8 / 14 / 1985$ & Shipek \\
\hline MMS_Y2_20 & 25 & 42.987500 & -70.625333 & 3 & 9 & $8 / 12 / 1985$ or $8 / 14 / 1985$ & Shipek \\
\hline MMS_Y2_21 & 26 & 42.983833 & -70.628833 & 3 & & $8 / 12 / 1985$ or $8 / 14 / 1985$ & Shipek \\
\hline MMS_Y2_22 & 28 & 42.985000 & -70.642667 & 3 & & $8 / 12 / 1985$ or $8 / 14 / 1985$ & Shipek \\
\hline MMS_Y2_23 & 30 & 42.981667 & -70.653167 & 3 & & $8 / 12 / 1985$ or $8 / 14 / 1985$ & Shipek \\
\hline MMS_Y2_24 & 32 & 42.929667 & -70.773000 & 3 & 25 & $8 / 12 / 1985$ or $8 / 14 / 1985$ & Shipek \\
\hline MMS_Y2_25 & 34 & 42.929167 & -70.765833 & 3 & & $8 / 12 / 1985$ or $8 / 14 / 1985$ & Shipek \\
\hline MMS_Y2_26 & 36 & 42.932333 & -70.755667 & 3 & 25 & $8 / 12 / 1985$ or $8 / 14 / 1985$ & Shipek \\
\hline MMS_Y2_27 & 38 & 42.939833 & -70.749500 & 3 & 25 & $8 / 12 / 1985$ or $8 / 14 / 1985$ & Shipek \\
\hline MMS_Y2_28 & 40 & 42.947667 & -70.750333 & 3 & 25 & $8 / 12 / 1985$ or $8 / 14 / 1985$ & Shipek \\
\hline MMS_Y2_29 & $42 \mathrm{~A}$ & 42.979000 & -70.719500 & 3 & 24 & $8 / 12 / 1985$ or $8 / 14 / 1985$ & Shipek \\
\hline MMS_Y2_30 & $42 B$ & 42.979167 & -70.719667 & 3 & 24 & $8 / 12 / 1985$ or $8 / 14 / 1985$ & Shipek \\
\hline MMS_Y2_31 & 44 & 42.988000 & -70.701333 & 3 & 24 & $8 / 12 / 1985$ or $8 / 14 / 1985$ & Shipek \\
\hline MMS_Y2_32 & $46 \mathrm{~A}$ & 42.984167 & -70.692667 & 3 & 25 & $8 / 12 / 1985$ or $8 / 14 / 1985$ & Shipek \\
\hline MMS_Y2_33 & $46 B$ & 42.980333 & -70.689333 & 3 & 27 & $8 / 12 / 1985$ or $8 / 14 / 1985$ & Shipek \\
\hline MMS_Y2_34 & 48 & 43.037000 & -70.662500 & 3 & 24 & $8 / 12 / 1985$ or $8 / 14 / 1985$ & Shipek \\
\hline MMS_Y2_35 & 50 & 43.039333 & -70.677333 & 3 & 23 & $8 / 12 / 1985$ or $8 / 14 / 1985$ & Shipek \\
\hline
\end{tabular}


MMS Year 2 Survey (1985): Sediment Classifications and Grain Size Statistics

\begin{tabular}{|c|c|c|c|c|c|c|c|c|c|c|c|c|c|c|c|}
\hline Sample ID & $\begin{array}{l}\text { Textural Group from \%GSM } \\
\text { (Gradistat) }\end{array}$ & $\begin{array}{l}\text { Textural Group } \\
\text { from \%SZC } \\
\text { (Gradistat) }\end{array}$ & $\begin{array}{l}\text { Sediment Classification } \\
\text { from Mean Phi } \\
\text { (Wentworth) }\end{array}$ & Sorting (Gradistat) & $\begin{array}{c}\text { Gravel } \\
\%\end{array}$ & $\begin{array}{c}\text { Sand } \\
\%\end{array}$ & $\begin{array}{c}\text { Mud } \\
\%\end{array}$ & $\begin{array}{c}\text { Sand } \\
\%\end{array}$ & $\begin{array}{c}\text { Silt } \\
\%\end{array}$ & $\begin{array}{c}\text { Clay } \\
\%\end{array}$ & $\begin{array}{c}\text { Mean } \\
\text { Size } \\
\text { (phi) }\end{array}$ & $\begin{array}{l}\text { Mean } \\
\text { Size } \\
(\mathrm{mm})\end{array}$ & $\begin{array}{c}\text { Sorting } \\
\text { (phi) }\end{array}$ & Skewness & Kurtosis LOI \% \\
\hline MMS_Y2_1 & Muddy Sandy Gravel & & Coarse Sand & Extremely Poorly Sorted & 31.0 & 54.0 & 15.0 & & & & 0.10 & 0.93 & 4.15 & -0.02 & 1.25 \\
\hline MMS_Y2_2 & Sand & Sand & Medium Sand & Moderately Sorted & 2.0 & 96.0 & 2.0 & 98.0 & & & 1.43 & 0.37 & 0.77 & -0.45 & 1.51 \\
\hline MMS_Y2_3 & Sand & Sand & Medium Sand & Moderately Well Sorted & 2.0 & 97.0 & 2.0 & 99.0 & & & 1.07 & 0.48 & 0.56 & 0.06 & 1.26 \\
\hline MMS_Y2_4 & Sand & Sand & Medium Sand & Well Sorted & 1.0 & 98.0 & 1.0 & 99.0 & & & 1.05 & 0.48 & 0.48 & -0.03 & 1.55 \\
\hline MMS_Y2_5 & Sand & Sand & Coarse Sand & Moderately Well Sorted & 1.0 & 98.0 & 1.0 & 99.0 & & & 0.97 & 0.51 & 0.63 & 0.07 & 1.70 \\
\hline MMS_Y2_6 & Gravelly Sand & & Coarse Sand & Poorly Sorted & 12.0 & 87.0 & 1.0 & & & & 0.58 & 0.67 & 1.12 & -0.40 & 1.80 \\
\hline MMS_Y2_7 & Gravelly Sand & & Very Coarse Sand & Poorly Sorted & 22.0 & 78.0 & 1.0 & & & & -0.12 & 1.09 & 1.51 & -0.56 & 1.46 \\
\hline MMS_Y2_8 & Sandy Gravel & & Very Coarse Sand & Very Poorly Sorted & 44.0 & 55.0 & 1.0 & & & & -0.60 & 1.52 & 2.07 & -0.38 & 0.55 \\
\hline MMS_Y2_9 & Sand & Sand & Medium Sand & Moderately Well Sorted & 2.0 & 96.0 & 2.0 & 98.0 & & & 1.50 & 0.35 & 0.63 & -0.21 & 1.38 \\
\hline MMS_Y2_10 & Gravelly Sand & & Coarse Sand & Poorly Sorted & 16.0 & 81.0 & 3.0 & & & & 0.92 & 0.53 & 1.70 & -0.53 & 1.55 \\
\hline MMS_Y2_11 & Gravelly Sand & & Coarse Sand & Very Poorly Sorted & 28.0 & 69.0 & 3.0 & & & & 0.25 & 0.84 & 2.10 & -0.26 & 0.76 \\
\hline MMS_Y2_12 & Sand & & Fine Sand & Poorly Sorted & 5.0 & 88.0 & 7.0 & & & & 2.15 & 0.23 & 1.26 & 0.19 & 2.84 \\
\hline MMS_Y2_13 & Sand & & Medium Sand & Moderately Sorted & 3.0 & 95.0 & 2.0 & & & & 1.12 & 0.46 & 0.85 & -0.11 & 1.10 \\
\hline MMS_Y2_14 & Sand & & Medium Sand & Moderately Sorted & 0.0 & 98.0 & 2.0 & 98.0 & & & 1.58 & 0.33 & 0.73 & -0.21 & 1.12 \\
\hline MMS_Y2_15 & Muddy Sand & & Fine Sand & Poorly Sorted & 3.0 & 82.0 & 15.0 & & & & 2.93 & 0.13 & 1.52 & 0.35 & 5.51 \\
\hline MMS_Y2_16 & Sand & & Fine Sand & Moderately Well Sorted & 1.0 & 95.0 & 4.0 & & & & 2.33 & 0.20 & 0.68 & -0.02 & 1.21 \\
\hline MMS_Y2_17 & Sand & & Medium Sand & Moderately Sorted & 1.0 & 97.0 & 2.0 & & & & 1.83 & 0.28 & 0.77 & -0.21 & 1.33 \\
\hline MMS_Y2_18 & Sandy Gravel & & Very Coarse Sand & Very Poorly Sorted & 50.0 & 49.0 & 1.0 & & & & -0.78 & 1.72 & 2.25 & 0.13 & 0.58 \\
\hline MMS_Y2_19 & Gravelly Sand & & Very Coarse Sand & Very Poorly Sorted & 27.0 & 70.0 & 3.0 & & & & -0.22 & 1.16 & 2.24 & -0.42 & 0.81 \\
\hline MMS_Y2_20 & Gravel & & & & & & & & & & & 1.00 & & & \\
\hline MMS_Y2_21 & Sandy Gravel & & Very Coarse Sand & Very Poorly Sorted & 39.0 & 60.0 & 1.0 & & & & -0.07 & 1.05 & 2.49 & -0.27 & 0.62 \\
\hline MMS_Y2_22 & Sand & & Medium Sand & Moderately Well Sorted & 3.0 & 95.0 & 2.0 & & & & 1.22 & 0.43 & 0.55 & -0.17 & 1.14 \\
\hline MMS_Y2_23 & Gravelly Sand & & Medium Sand & Poorly Sorted & 14.0 & 86.0 & 0.0 & & & & 1.23 & 0.43 & 1.33 & -0.64 & 2.42 \\
\hline MMS_Y2_24 & Sand & Sand & Fine Sand & Moderately Sorted & 0.0 & 97.0 & 3.0 & 97.0 & & & 2.10 & 0.23 & 0.80 & 0.25 & 1.13 \\
\hline MMS_Y2_25 & Sand & Sand & Fine Sand & Moderately Well Sorted & 1.0 & 98.0 & 1.0 & 98.0 & & & 2.20 & 0.22 & 0.65 & -0.04 & 1.11 \\
\hline MMS_Y2_26 & Sand & Sand & Fine Sand & Moderately Sorted & 1.0 & 96.0 & 4.0 & 96.0 & & & 2.67 & 0.16 & 0.70 & 0.07 & 1.18 \\
\hline MMS_Y2_27 & Sandy Gravel & & Granule Gravel & Poorly Sorted & 69.0 & 31.0 & 0.0 & & & & -1.75 & 3.36 & 1.69 & 0.29 & 0.85 \\
\hline MMS_Y2_28 & Muddy Sand & & Fine Sand & Poorly Sorted & 1.0 & 88.0 & 11.0 & & & & 2.90 & 0.13 & 1.07 & 0.13 & 1.84 \\
\hline MMS_Y2_29 & Gravelly Sand & & Coarse Sand & Poorly Sorted & 22.0 & 75.0 & 3.0 & & & & 0.43 & 0.74 & 1.86 & -0.35 & 1.00 \\
\hline MMS_Y2_30 & Gravelly Sand & & Medium Sand & Poorly Sorted & 8.0 & 91.0 & 1.0 & & & & 1.55 & 0.34 & 1.45 & -0.37 & 1.82 \\
\hline MMS_Y2_31 & Sandy Gravel & & Very Coarse Sand & Very Poorly Sorted & 43.0 & 56.0 & 1.0 & & & & -0.28 & 1.21 & 2.37 & -0.41 & 0.50 \\
\hline MMS_Y2_32 & Gravel & & & & & & & & & & & 1.00 & & & \\
\hline MMS_Y2_33 & Gravelly Sand & & Coarse Sand & Very Poorly Sorted & 26.0 & 72.0 & 2.0 & & & & 0.70 & 0.62 & 2.01 & -0.46 & 0.69 \\
\hline MMS_Y2_34 & Gravel & & Pebble Gravel & Poorly Sorted & 83.0 & 17.0 & 0.0 & & & & -2.70 & 6.50 & 1.82 & 0.72 & 1.60 \\
\hline MMS_Y2_35 & Gravelly Sand & & Coarse Sand & Very Poorly Sorted & 22.0 & 77.0 & 1.0 & & & & 0.30 & 0.81 & 2.54 & -0.72 & 1.86 \\
\hline
\end{tabular}


MMS Year 4 Survey; Core Tops (1988): Identification, Location, and Description

\begin{tabular}{|lccccccc|}
\hline & $\begin{array}{c}\text { Original } \\
\text { Sample ID }\end{array}$ & $\begin{array}{c}\text { Latitude } \\
\text { WGS84 }\end{array}$ & $\begin{array}{c}\text { Longitude } \\
\text { WGS84 }\end{array}$ & $\begin{array}{c}\text { Reliability } \\
\text { Ranking for } \\
\text { Positioning }\end{array}$ & $\begin{array}{c}\text { Water } \\
\text { Depth } \\
(\mathbf{m})\end{array}$ & $\begin{array}{c}\text { Sample } \\
\text { Collected }\end{array}$ & $\begin{array}{c}\text { Sampler } \\
\text { Type }\end{array}$ \\
\hline MMS_Y4_1 & A1 & 42.995000 & -70.643333 & 3 & 21 & $10 / 1 / 1988$ & Vibracore \\
\hline MMS_Y4_2 & A2 & 42.986667 & -70.646667 & 3 & 22 & $10 / 1 / 1988$ & Vibracore \\
\hline MMS_Y4_3 & A3 & 42.983333 & -70.660000 & 3 & 25 & $10 / 1 / 1988$ & Vibracore \\
\hline MMS_Y4_4 & A6(2) & 42.931667 & -70.763333 & 3 & 23 & $10 / 1 / 1988$ & Vibracore \\
\hline MMS_Y4_5 & A7(1) & 42.948333 & -70.755000 & 3 & 24 & $10 / 1 / 1988$ & Vibracore \\
\hline
\end{tabular}

MMS Year 8 Survey (1992): Identification, Location, and Description

\begin{tabular}{|c|c|c|c|c|c|c|c|}
\hline Sample ID & $\begin{array}{c}\text { Original } \\
\text { Sample ID }\end{array}$ & $\begin{array}{c}\text { Latitude } \\
\text { WGS84 }\end{array}$ & $\begin{array}{c}\text { Longitude } \\
\text { WGS84 }\end{array}$ & $\begin{array}{l}\text { Reliability } \\
\text { Ranking for } \\
\text { Positioning }\end{array}$ & $\begin{array}{l}\text { Water } \\
\text { Depth } \\
\text { (m) }\end{array}$ & $\begin{array}{l}\text { Sample } \\
\text { Collected }\end{array}$ & $\begin{array}{c}\text { Sampler } \\
\text { Type }\end{array}$ \\
\hline MMS_Y8_1 & NHS-1 & 42.836833 & -70.812833 & 3 & & 1992 & Shipek \\
\hline MMS_Y8_2 & NHS-2 & 42.837000 & -70.801667 & 3 & & 1992 & Shipek \\
\hline MMS_Y8_3 & NHS-3 & 42.836333 & -70.789000 & 3 & & 1992 & Shipek \\
\hline MMS_Y8_4 & NHS-4 & 42.835667 & -70.778167 & 3 & & 1992 & Shipek \\
\hline MMS_Y8_5 & NHS-5 & 42.833833 & -70.766667 & 3 & & 1992 & Shipek \\
\hline MMS_Y8_6 & NHS-6 & 42.850167 & -70.767667 & 3 & & 1992 & Shipek \\
\hline MMS_Y8_7 & NHS-7 & 42.850500 & -70.784500 & 3 & & 1992 & Shipek \\
\hline MMS_Y8_8 & NHS-8 & 42.851167 & -70.799833 & 3 & & $\sim 1992$ & Shipek \\
\hline MMS_Y8_9 & NHS-9 & 42.851333 & -70.811833 & 3 & & $\sim 1992$ & Shipek \\
\hline MMS_Y8_10 & NHS-10 & 42.884500 & -70.810167 & 3 & & $\sim 1992$ & Shipek \\
\hline MMS_Y8_11 & NHS-11 & 42.884500 & -70.794833 & 3 & & $\sim 1992$ & Shipek \\
\hline MMS_Y8_12 & NHS-12 & 42.883833 & -70.781500 & 3 & & 1992 & Shipek \\
\hline MMS_Y8_15 & NHS-15 & 42.915333 & -70.749667 & 3 & & 1992 & Shipek \\
\hline MMS_Y8_18 & NHS-18 & 42.949333 & -70.778333 & 3 & & 1992 & Shipek \\
\hline MMS_Y8_19 & NHS-19 & 42.951500 & -70.760667 & 3 & & 1992 & Shipek \\
\hline MMS_Y8_20 & NHS-20 & 42.952000 & -70.744333 & 3 & & 1992 & Shipek \\
\hline MMS_Y8_21 & NHS-21 & 42.951167 & -70.727333 & 3 & & 1992 & Shipek \\
\hline MMS_Y8_22 & NHS-22 & 42.950167 & -70.708333 & 3 & & 1992 & Shipek \\
\hline
\end{tabular}


MMS Year 4 Survey; Core Tops (1988): Sediment Classifications and Grain Size Statistics

\begin{tabular}{|c|c|c|c|c|c|c|c|c|c|c|c|c|c|c|c|c|}
\hline Sample ID & $\begin{array}{l}\text { Textural Group from \%GSM } \\
\text { (Gradistat) }\end{array}$ & $\begin{array}{l}\text { Textural Group } \\
\text { from \%SZC } \\
\text { (Gradistat) }\end{array}$ & $\begin{array}{l}\text { Sediment Classification } \\
\text { from Mean Phi } \\
\text { (Wentworth) }\end{array}$ & Sorting (Gradistat) & $\begin{array}{c}\text { Gravel } \\
\%\end{array}$ & $\begin{array}{c}\text { Sand } \\
\%\end{array}$ & $\begin{array}{c}\text { Mud } \\
\%\end{array}$ & $\begin{array}{c}\text { Sand } \\
\%\end{array}$ & $\begin{array}{c}\text { Silt } \\
\%\end{array}$ & $\begin{array}{c}\text { Clay } \\
\%\end{array}$ & $\begin{array}{c}\text { Mean } \\
\text { Size } \\
\text { (phi) }\end{array}$ & $\begin{array}{c}\text { Mean } \\
\text { Size } \\
(\mathrm{mm})\end{array}$ & $\begin{array}{c}\text { Sorting } \\
\text { (phi) }\end{array}$ & Skewness & Kurtosis & LOI \% \\
\hline MMS_Y4_1 & Sand & & & & 2.0 & 97.0 & 1.0 & & & & & & & & & \\
\hline MMS_Y4_2 & Sand & & & & 1.0 & 98.0 & 1.0 & & & & & & & & & \\
\hline MMS_Y4_3 & Sand & & & & 0.0 & 99.0 & 1.0 & & & & & & & & & \\
\hline MMS_Y4_4 & Sand & & & & 3.0 & 95.0 & 2.0 & & & & & & & & & \\
\hline MMS_Y4_5 & Sand & & & & 3.0 & 92.0 & 5.0 & & & & & & & & & \\
\hline
\end{tabular}

MMS Year 8 Survey (1992): Sediment Classifications and Grain Size Statistics

\begin{tabular}{|c|c|c|c|c|c|c|c|c|c|c|c|c|c|c|c|c|}
\hline Sample ID & $\begin{array}{l}\text { Textural Group from \%GSM } \\
\text { (Gradistat) }\end{array}$ & $\begin{array}{l}\text { Textural Group } \\
\text { from \%SZC } \\
\text { (Gradistat) }\end{array}$ & $\begin{array}{l}\text { Sediment Classification } \\
\text { from Mean Phi } \\
\text { (Wentworth) }\end{array}$ & Sorting (Gradistat) & $\begin{array}{c}\text { Gravel } \\
\%\end{array}$ & $\begin{array}{l}\text { Sand } \\
\%\end{array}$ & $\begin{array}{c}\text { Mud } \\
\%\end{array}$ & $\begin{array}{c}\text { Sand } \\
\%\end{array}$ & $\begin{array}{c}\text { Silt } \\
\%\end{array}$ & $\begin{array}{c}\text { Clay } \\
\%\end{array}$ & $\begin{array}{c}\text { Mean } \\
\text { Size } \\
\text { (phi) }\end{array}$ & $\begin{array}{c}\text { Mean } \\
\text { Size } \\
(\mathrm{mm})\end{array}$ & $\begin{array}{l}\text { Sorting } \\
\text { (phi) }\end{array}$ & Skewness & Kurtosis & LOI \% \\
\hline MMS_Y8_1 & Sand & Sand & Medium Sand & Moderately Well Sorted & 0.0 & 99.0 & 1.0 & 99.0 & & & 1.62 & 0.33 & 0.62 & -0.32 & 0.86 & \\
\hline MMS_Y8_2 & Sand & Sand & Fine Sand & Well Sorted & 0.0 & 98.0 & 2.0 & 98.0 & & & 2.67 & 0.16 & 0.37 & -0.03 & 1.07 & \\
\hline MMS_Y8_3 & Gravelly Sand & & Coarse Sand & Moderately Sorted & 5.0 & 93.0 & 2.0 & & & & 0.62 & 0.65 & 0.86 & -0.02 & 1.23 & \\
\hline MMS_Y8_4 & Gravelly Sand & & Coarse Sand & Poorly Sorted & 13.0 & 86.0 & 1.0 & & & & 0.42 & 0.75 & 1.07 & -0.42 & 1.31 & \\
\hline MMS_Y8_5 & Gravelly Sand & & Coarse Sand & Moderately Sorted & 7.0 & 92.0 & 1.0 & & & & 0.57 & 0.67 & 0.96 & -0.14 & 1.01 & \\
\hline MMS_Y8_6 & Slightly Gravelly Sand & Sand & Coarse Sand & Moderately Sorted & 4.0 & 96.0 & 0.0 & 100.0 & 0.0 & 0.0 & 0.65 & 0.64 & 0.73 & -0.13 & 1.18 & \\
\hline MMS_Y8_7 & Gravelly Sand & & Coarse Sand & Poorly Sorted & 17.0 & 83.0 & 0.0 & & & & 0.73 & 0.60 & 1.00 & -0.03 & 1.10 & \\
\hline MMS_Y8_8 & Sand & Sand & Fine Sand & Moderately Well Sorted & 0.0 & 95.0 & 5.0 & 95.0 & & & 2.95 & 0.13 & 0.53 & 0.16 & 1.20 & \\
\hline MMS_Y8_9 & Sand & Sand & Fine Sand & Moderately Sorted & 0.0 & 98.0 & 2.0 & 98.0 & & & 2.20 & 0.22 & 0.72 & -0.11 & 1.43 & \\
\hline MMS_Y8_10 & Slightly Gravelly Sand & Sand & Fine Sand & Moderately Well Sorted & 1.0 & 97.0 & 2.0 & 97.0 & & & 2.52 & 0.17 & 0.67 & -0.28 & 1.38 & \\
\hline MMS_Y8_11 & Sand & Sand & Very Fine Sand & Moderately Well Sorted & 0.0 & 93.0 & 7.0 & 93.0 & 4.0 & 3.0 & 3.15 & 0.11 & 0.51 & 0.25 & 1.45 & \\
\hline MMS_Y8_12 & Sandy Gravel & & Granule Gravel & Very Poorly Sorted & 59.0 & 40.0 & 1.0 & & & & -1.37 & 2.58 & 2.21 & 0.30 & 0.55 & \\
\hline MMS_Y8_15 & Gravelly Muddy Sand & & Medium Sand & Very Poorly Sorted & 10.0 & 78.0 & 12.0 & & & & 1.53 & 0.35 & 2.83 & 0.19 & 3.01 & \\
\hline MMS_Y8_18 & Sand & Sand & Fine Sand & Well Sorted & 0.0 & 98.0 & 2.0 & 98.0 & & & 2.85 & 0.14 & 0.47 & 0.12 & 1.27 & \\
\hline MMS_Y8_19 & Sandy Gravel & & Granule Gravel & Very Poorly Sorted & 59.0 & 40.0 & 1.0 & & & & -1.60 & 3.03 & 2.02 & 0.19 & 0.65 & \\
\hline MMS_Y8_20 & Gravel & & Pebble Gravel & Very Poorly Sorted & 80.0 & 16.0 & 4.0 & & & & -2.95 & 7.73 & 2.43 & 0.94 & 1.08 & \\
\hline MMS_Y8_21 & Gravel & & Pebble Gravel & Very Poorly Sorted & 80.0 & 17.0 & 3.0 & & & & -2.43 & 5.39 & 2.08 & 0.59 & 1.56 & \\
\hline MMS_Y8_22 & Slightly Gravelly Sand & & Fine Sand & Poorly Sorted & 4.0 & 88.0 & 8.0 & 92.0 & 4.0 & 4.0 & 2.93 & 0.13 & 1.30 & 0.12 & 4.85 & \\
\hline
\end{tabular}


MMS Year 10 Survey (1994): Identification, Location, and Description

\begin{tabular}{|c|c|c|c|c|c|c|c|}
\hline Sample ID & $\begin{array}{c}\text { Original } \\
\text { Sample ID }\end{array}$ & $\begin{array}{l}\text { Latitude } \\
\text { WGS84 }\end{array}$ & $\begin{array}{c}\text { Longitude } \\
\text { WGS84 }\end{array}$ & $\begin{array}{l}\text { Reliability } \\
\text { Ranking for } \\
\text { Positioning }\end{array}$ & $\begin{array}{l}\text { Water } \\
\text { Depth } \\
\text { (m) }\end{array}$ & $\begin{array}{c}\text { Sample } \\
\text { Collected }\end{array}$ & $\begin{array}{c}\text { Sampler } \\
\text { Type }\end{array}$ \\
\hline MMS_Y10_1 & NHS-101 & 42.930667 & -70.711000 & 3 & 39 & $8 / 11 / 1994$ & Shipek \\
\hline MMS_Y10_3 & NHS-103 & 42.946000 & -70.724833 & 3 & 34 & $8 / 11 / 1994$ & Shipek \\
\hline MMS_Y10_5 & NHS-105 & 42.961333 & -70.737833 & 3 & 24 & $8 / 11 / 1994$ & Shipek \\
\hline MMS_Y10_6 & NHS-106 & 42.969167 & -70.744500 & 3 & 16 & $8 / 11 / 1994$ & Shipek \\
\hline MMS_Y10_7 & NHS-107 & 42.961833 & -70.751167 & 3 & 20 & $8 / 11 / 1994$ & Shipek \\
\hline MMS_Y10_8 & NHS-108A & 42.954167 & -70.744167 & 3 & 27 & $8 / 11 / 1994$ & Shipek \\
\hline MMS_Y10_9 & NHS-108B & 42.954667 & -70.744000 & 3 & 27 & $8 / 11 / 1994$ & Shipek \\
\hline MMS_Y10_10 & NHS-109 & 42.946333 & -70.737500 & 3 & 33 & $8 / 11 / 1994$ & Shipek \\
\hline MMS_Y10_13 & NHS-111 & 42.930667 & -70.724500 & 3 & 37 & $8 / 11 / 1994$ & Shipek \\
\hline MMS_Y10_14 & NHS-112 & 42.923167 & -70.717833 & 3 & 41 & $8 / 11 / 1994$ & Shipek \\
\hline MMS_Y10_18 & NHS-115 & 42.932000 & -70.737833 & 3 & 36 & $8 / 11 / 1994$ & Shipek \\
\hline MMS_Y10_21 & NHS-116C & 42.940000 & -70.744667 & 3 & 30 & $8 / 11 / 1994$ & Shipek \\
\hline MMS_Y10_22 & NHS-117 & 42.946833 & -70.751000 & 3 & 27 & $8 / 11 / 1994$ & Shipek \\
\hline MMS_Y10_28 & NHS-120B & 42.939667 & -70.757833 & 3 & 27 & $8 / 11 / 1994$ & Shipek \\
\hline MMS_Y10_29 & NHS-121 & 42.932333 & -70.751500 & 3 & 29 & $8 / 11 / 1994$ & Shipek \\
\hline MMS_Y10_33 & NHS-123B & 42.916667 & -70.751167 & 3 & 32 & $8 / 11 / 1994$ & Shipek \\
\hline MMS_Y10_35 & NHS-124B & 42.925167 & -70.757833 & 3 & 28 & $8 / 11 / 1994$ & Shipek \\
\hline MMS_Y10_36 & NHS-125 & 42.932667 & -70.764500 & 3 & 25 & $8 / 11 / 1994$ & Shipek \\
\hline MMS_Y10_38 & NHS-126B & 42.944500 & -70.774000 & 3 & 16 & $8 / 11 / 1994$ & Shipek \\
\hline MMS_Y10_39 & NHS-127 & 42.949000 & -70.777333 & 3 & 11 & $8 / 11 / 1994$ & Shipek \\
\hline MMS_Y10_40 & NHS-128 & 42.935167 & -70.779333 & 3 & 15 & $8 / 11 / 1994$ & Shipek \\
\hline MMS_Y10_41 & NHS-129 & 42.925667 & -70.770167 & 3 & 22 & $8 / 11 / 1994$ & Shipek \\
\hline MMS_Y10_44 & NHS-131 & 42.918833 & -70.777167 & 3 & 21 & $8 / 11 / 1994$ & Shipek \\
\hline MMS_Y10_45 & NHS-132A & 42.929000 & -70.787333 & 3 & 10 & $8 / 11 / 1994$ & Shipek \\
\hline MMS_Y10_46 & NHS-132B & 42.929667 & -70.786500 & 3 & 9 & $8 / 11 / 1994$ & Shipek \\
\hline MMS_Y10_47 & NHS-133A & 42.911500 & -70.784500 & 3 & 17 & $8 / 11 / 1994$ & Shipek \\
\hline MMS_Y10_48 & NHS-133B & 42.911500 & -70.784500 & 3 & 17 & $8 / 11 / 1994$ & Shipek \\
\hline MMS_Y10_49 & NHS-134 & 42.911833 & -70.797667 & 3 & 7 & $8 / 11 / 1994$ & Shipek \\
\hline MMS_Y10_50 & NHS-135 & 42.900500 & -70.800500 & 3 & 9 & $8 / 11 / 1994$ & Shipek \\
\hline MMS_Y10_51 & NHS-136A & 42.904667 & -70.790333 & 3 & 12 & $8 / 11 / 1994$ & Shipek \\
\hline MMS_Y10_52 & NHS-136B & 42.905167 & -70.789833 & 3 & 12 & 8/11/1994 & Shipek \\
\hline
\end{tabular}


MMS Year 10 Survey (1994): Sediment Classifications and Grain Size Statistics

\begin{tabular}{|c|c|c|c|c|c|c|c|c|c|c|c|c|c|c|c|c|}
\hline Sample ID & $\begin{array}{l}\text { Textural Group from \%GSM } \\
\text { (Gradistat) }\end{array}$ & $\begin{array}{l}\text { Textural Group } \\
\text { from \%SZC } \\
\text { (Gradistat) }\end{array}$ & $\begin{array}{l}\text { Sediment Classification } \\
\text { from Mean Phi } \\
\text { (Wentworth) }\end{array}$ & Sorting (Gradistat) & $\begin{array}{c}\text { Gravel } \\
\%\end{array}$ & $\begin{array}{c}\text { Sand } \\
\%\end{array}$ & $\begin{array}{c}\text { Mud } \\
\%\end{array}$ & $\begin{array}{c}\text { Sand } \\
\%\end{array}$ & $\begin{array}{c}\text { Silt } \\
\%\end{array}$ & $\begin{array}{c}\text { Clay } \\
\%\end{array}$ & $\begin{array}{c}\text { Mean } \\
\text { Size } \\
\text { (phi) }\end{array}$ & $\begin{array}{c}\text { Mean } \\
\text { Size } \\
(\mathrm{mm}) \\
\end{array}$ & $\begin{array}{c}\text { Sorting } \\
\text { (phi) }\end{array}$ & Skewness & Kurtosis & LOI \% \\
\hline MMS_Y10_1 & Muddy Sand & Muddy Sand & Very Fine Sand & Poorly Sorted & 0.0 & 78.0 & 22.0 & 78.0 & 11.0 & 11.0 & 3.93 & 0.07 & 1.75 & 0.78 & 3.01 & 1.30 \\
\hline MMS_Y10_3 & Sand & & Very Fine Sand & Moderately Well Sorted & 0.0 & 95.0 & 5.0 & & & & 3.20 & 0.11 & 0.62 & -1.63 & 3.55 & 0.30 \\
\hline MMS_Y10_5 & Sandy Gravel & & Very Coarse Sand & Very Poorly Sorted & 65.0 & 32.0 & 3.0 & & & & -0.80 & 1.74 & 2.43 & 0.54 & 0.86 & 1.20 \\
\hline MMS_Y10_6 & Sandy Gravel & & Granule Gravel & Poorly Sorted & 72.0 & 27.0 & 1.0 & & & & -1.87 & 3.66 & 1.13 & 0.00 & 0.80 & 0.30 \\
\hline MMS_Y10_7 & Sandy Gravel & & Very Coarse Sand & Poorly Sorted & 52.0 & 45.0 & 3.0 & & & & -0.87 & 1.83 & 1.99 & 0.29 & 1.25 & 0.90 \\
\hline MMS_Y10_8 & Sandy Gravel & & Granule Gravel & Very Poorly Sorted & 60.0 & 38.0 & 2.0 & & & & -1.43 & 2.69 & 2.33 & 0.42 & 0.71 & 0.50 \\
\hline MMS_Y10_9 & Muddy Sand & Silty Sand & Very Fine Sand & Poorly Sorted & 0.0 & 76.0 & 24.0 & 76.0 & 21.0 & 3.0 & 3.60 & 0.08 & 1.84 & 0.51 & 1.69 & 2.40 \\
\hline MMS_Y10_10 & Muddy Sandy Gravel & & Granule Gravel & Very Poorly Sorted & 70.0 & 20.0 & 10.0 & & & & -1.37 & 2.58 & 3.43 & 0.72 & 0.79 & 1.30 \\
\hline MMS_Y10_13 & Muddy Sandy Gravel & & Granule Gravel & Very Poorly Sorted & 72.0 & 23.0 & 5.0 & & & & -1.23 & 2.35 & 2.55 & 0.69 & 1.15 & 1.00 \\
\hline MMS_Y10_14 & Muddy Sandy Gravel & & Pebble Gravel & Very Poorly Sorted & 71.0 & 25.0 & 4.0 & & & & -2.53 & 5.78 & 2.54 & 0.41 & 0.97 & 1.50 \\
\hline MMS_Y10_18 & Muddy Sandy Gravel & & Medium Sand & Very Poorly Sorted & 34.0 & 45.0 & 21.0 & & & & 1.50 & 0.35 & 3.88 & 0.12 & 0.97 & 2.00 \\
\hline MMS_Y10_21 & Sand & Sand & Fine Sand & Moderately Sorted & 4.0 & 92.0 & 4.0 & 96.0 & 3.0 & 1.0 & 2.80 & 0.14 & 0.95 & -0.27 & 2.52 & 0.80 \\
\hline MMS_Y10_22 & Sand & Sand & Fine Sand & Moderately Sorted & 0.0 & 95.0 & 5.0 & 95.0 & 4.0 & 1.0 & 2.43 & 0.19 & 0.77 & 0.11 & 1.07 & 0.90 \\
\hline MMS_Y10_28 & Gravelly Sand & & Medium Sand & Poorly Sorted & 13.0 & 86.0 & 1.0 & & & & 1.50 & 0.35 & 1.48 & -0.47 & 2.42 & 0.50 \\
\hline MMS_Y10_29 & Sand & Sand & Fine Sand & Moderately Sorted & 4.0 & 90.0 & 6.0 & 94.0 & 5.0 & 1.0 & 2.50 & 0.18 & 0.94 & -0.11 & 1.78 & 1.00 \\
\hline MMS_Y10_33 & Gravelly Muddy Sand & & Medium Sand & Very Poorly Sorted & 26.0 & 64.0 & 10.0 & & & & 1.00 & 0.50 & 3.17 & -0.31 & 1.16 & 1.30 \\
\hline MMS_Y10_35 & Sand & Sand & Fine Sand & Moderately Well Sorted & 0.0 & 98.0 & 2.0 & 98.0 & 1.0 & 1.0 & 2.33 & 0.20 & 0.58 & 0.10 & 1.17 & 0.50 \\
\hline MMS_Y10_36 & Gravelly Sand & & Very Coarse Sand & Poorly Sorted & 27.0 & 72.0 & 1.0 & & & & -0.47 & 1.39 & 1.12 & 0.21 & 1.29 & 0.40 \\
\hline MMS_Y10_38 & Sand & Sand & Fine Sand & Moderately Sorted & 0.0 & 96.0 & 4.0 & 96.0 & 3.0 & 1.0 & 2.90 & 0.13 & 0.70 & -0.26 & 1.14 & 0.60 \\
\hline MMS_Y10_39 & Sand & Sand & Very Fine Sand & Well Sorted & 0.0 & 98.0 & 2.0 & 98.0 & 2.0 & 0.0 & 3.00 & 0.13 & 0.44 & 0.00 & 1.09 & 0.50 \\
\hline MMS_Y10_40 & Sand & Sand & Fine Sand & Moderately Well Sorted & 0.0 & 98.0 & 2.0 & 98.0 & 2.0 & 0.0 & 2.87 & 0.14 & 0.61 & -0.18 & 1.50 & 0.50 \\
\hline MMS_Y10_41 & Sand & Sand & Fine Sand & Moderately Well Sorted & 0.0 & 98.0 & 2.0 & 98.0 & 1.0 & 1.0 & 2.57 & 0.17 & 0.58 & -0.10 & 1.17 & 0.50 \\
\hline MMS_Y10_44 & Sand & Sand & Fine Sand & Moderately Well Sorted & 0.0 & 97.0 & 3.0 & 97.0 & 2.0 & 1.0 & 2.63 & 0.16 & 0.66 & 0.08 & 1.00 & 0.60 \\
\hline MMS_Y10_45 & Sand & Sand & Very Fine Sand & Well Sorted & 0.0 & 95.0 & 5.0 & & & & 3.07 & 0.12 & 0.39 & -0.14 & 1.15 & 0.40 \\
\hline MMS_Y10_46 & Sand & Sand & Fine Sand & Well Sorted & 0.0 & 98.0 & 2.0 & 98.0 & 1.0 & 1.0 & 2.90 & 0.13 & 0.36 & 0.00 & 1.43 & 0.60 \\
\hline MMS_Y10_47 & Sand & Sand & Very Fine Sand & Moderately Well Sorted & 0.0 & 95.0 & 5.0 & & & & 3.17 & 0.11 & 0.50 & -0.11 & 1.48 & 0.50 \\
\hline MMS_Y10_48 & Sandy Gravel & & Granule Gravel & Poorly Sorted & 63.0 & 35.0 & 2.0 & & & & -1.57 & 2.97 & 1.87 & 0.28 & 1.14 & 0.60 \\
\hline MMS_Y10_49 & Sand & Sand & Fine Sand & Moderately Well Sorted & 0.0 & 99.0 & 1.0 & 99.0 & 0.5 & 0.5 & 2.93 & 0.13 & 0.64 & -0.33 & 1.78 & 0.40 \\
\hline MMS_Y10_50 & Sandy Gravel & & Granule Gravel & Very Poorly Sorted & 48.0 & 52.0 & 0.0 & & & & -1.43 & 2.69 & 2.03 & 0.44 & 0.54 & 0.60 \\
\hline MMS_Y10_51 & Sand & Sand & Very Fine Sand & Well Sorted & 0.0 & 98.0 & 2.0 & & & & 3.17 & 0.11 & 0.37 & -0.11 & 1.33 & 0.50 \\
\hline MMS_Y10_52 & Sand & Sand & Very Fine Sand & Well Sorted & 0.0 & 98.0 & 2.0 & 98.0 & 1.0 & 1.0 & 3.13 & 0.11 & 0.37 & 0.11 & 1.07 & 0.60 \\
\hline
\end{tabular}


Section 7: Flight Survey (1972) 
Flight Survey (1972) (1): Identification, Location, and Description

\begin{tabular}{|c|c|c|c|c|c|c|c|}
\hline Sample ID & $\begin{array}{c}\text { Original } \\
\text { Sample ID }\end{array}$ & $\begin{array}{c}\text { Latitude } \\
\text { WGS84 }\end{array}$ & $\begin{array}{c}\text { Longitude } \\
\text { WGS84 }\end{array}$ & $\begin{array}{l}\text { Reliability } \\
\text { Ranking for } \\
\text { Positioning }\end{array}$ & $\begin{array}{c}\text { Water } \\
\text { Depth } \\
\text { (m) } \\
\end{array}$ & $\begin{array}{c}\text { Sample } \\
\text { Collected }\end{array}$ & $\begin{array}{c}\text { Sampler } \\
\text { Type }\end{array}$ \\
\hline WFS_1 & 101 & 42.975000 & -70.275000 & 4 & & $5 / 18 / 1971$ & Gravity Corer \\
\hline WFS_2 & 102 & 42.983333 & -70.216667 & 4 & & $5 / 18 / 1971$ & Gravity Corer \\
\hline WFS_3 & 103 & 43.000000 & -70.158333 & 4 & & $5 / 18 / 1971$ & Gravity Corer \\
\hline WFS_4 & 104 & 43.008333 & -70.133333 & 4 & & $5 / 18 / 1971$ & Gravity Corer \\
\hline WFS_5 & 105 & 43.063333 & -70.480000 & 4 & & $5 / 19 / 1971$ & Gravity Corer \\
\hline WFS_6 & 106 & 43.050000 & -70.433333 & 4 & & $5 / 19 / 1971$ & Gravity Corer \\
\hline WFS_7 & 107 & 43.041667 & -70.405000 & 4 & & $5 / 19 / 1971$ & Gravity Corer \\
\hline WFS_8 & 108 & 43.008333 & -70.383333 & 4 & & $5 / 19 / 1971$ & Gravity Corer \\
\hline WFS_9 & 109 & 42.980000 & -70.353333 & 4 & & $5 / 19 / 1971$ & Gravity Corer \\
\hline WFS_10 & 202 & 42.998333 & -70.503333 & 4 & & $6 / 18 / 1971$ & Gravity Corer \\
\hline WFS_11 & 203 & 42.983333 & -70.458333 & 4 & & $6 / 18 / 1971$ & Gravity Corer \\
\hline WFS_12 & 204 & 42.971667 & -70.416667 & 4 & & $6 / 18 / 1971$ & Gravity Corer \\
\hline WFS_13 & 206 & 42.950000 & -70.320000 & 4 & & $6 / 18 / 1971$ & Gravity Corer \\
\hline WFS_14 & 207 & 42.953333 & -70.371667 & 4 & & $6 / 18 / 1971$ & Gravity Corer \\
\hline WFS_15 & 208 & 42.958333 & -70.416667 & 4 & & $6 / 18 / 1971$ & Gravity Corer \\
\hline WFS_16 & 210 & 42.966667 & -70.505000 & 4 & & $6 / 18 / 1971$ & Gravity Corer \\
\hline WFS_17 & 211 & 43.021667 & -70.275000 & 4 & & $6 / 19 / 1971$ & Gravity Corer \\
\hline WFS_18 & 212 & 43.043333 & -70.316667 & 4 & & $6 / 19 / 1971$ & Gravity Corer \\
\hline WFS_19 & 213 & 43.063333 & -70.358333 & 4 & & $6 / 19 / 1971$ & Gravity Corer \\
\hline WFS_20 & 214 & 43.080000 & -70.395000 & 4 & & $6 / 19 / 1971$ & Gravity Corer \\
\hline WFS_21 & 215 & 43.096667 & -70.421667 & 4 & & $6 / 19 / 1971$ & Gravity Corer \\
\hline WFS_22 & 216 & 43.086667 & -70.458333 & 4 & & $6 / 19 / 1971$ & Gravity Corer \\
\hline WFS_23 & 301 & 42.938333 & -70.275000 & 4 & & $7 / 1 / 1971$ & Gravity Corer \\
\hline WFS_24 & 302 & 42.925000 & -70.230000 & 4 & & $7 / 1 / 1971$ & Gravity Corer \\
\hline WFS_25 & 304 & 42.905000 & -70.150000 & 4 & & $7 / 1 / 1971$ & Gravity Corer \\
\hline WFS_26 & 305 & 42.893333 & -70.103333 & 4 & & $7 / 2 / 1971$ & Gravity Corer \\
\hline WFS_27 & 306 & 42.888333 & -70.088333 & 4 & & 7/2/1971 & Gravity Corer \\
\hline WFS_28 & 307 & 42.886667 & -70.075000 & 4 & & $7 / 2 / 1971$ & Gravity Corer \\
\hline WFS_29 & 401 & 43.111667 & -70.388333 & 4 & & $7 / 15 / 1971$ & Gravity Corer \\
\hline WFS_30 & 402 & 43.096667 & -70.351667 & 4 & & $7 / 15 / 1971$ & Gravity Corer \\
\hline
\end{tabular}


Flight Survey (1972) (1): Sediment Classifications and Grain Size Statistics

\begin{tabular}{|c|c|c|c|c|c|c|c|c|c|c|c|c|c|c|c|}
\hline Sample ID & $\begin{array}{l}\text { Textural Group from \%GSM } \\
\text { (Gradistat) }\end{array}$ & $\begin{array}{l}\text { Textural Group } \\
\text { from \%SZC } \\
\text { (Gradistat) } \\
\end{array}$ & $\begin{array}{l}\text { Sediment Classification } \\
\text { from Mean Phi } \\
\text { (Wentworth) }\end{array}$ & Sorting (Gradistat) & $\begin{array}{c}\text { Gravel } \\
\%\end{array}$ & $\begin{array}{c}\text { Sand } \\
\%\end{array}$ & $\begin{array}{c}\text { Mud } \\
\%\end{array}$ & $\begin{array}{c}\text { Sand } \\
\%\end{array}$ & $\begin{array}{c}\text { Silt } \\
\%\end{array}$ & $\begin{array}{c}\text { Clay } \\
\%\end{array}$ & $\begin{array}{c}\text { Mean } \\
\text { Size } \\
\text { (phi) } \\
\end{array}$ & $\begin{array}{c}\text { Mean } \\
\text { Size } \\
(\mathrm{mm}) \\
\end{array}$ & $\begin{array}{c}\text { Sorting } \\
\text { (phi) }\end{array}$ & Skewness & Kurtosis LOI \% \\
\hline WFS_1 & Mud & Mud & Clay & Very Poorly Sorted & 0.0 & 1.0 & 99.0 & 1.0 & 43.0 & 56.0 & 8.65 & 0.00 & 2.06 & & \\
\hline WFS_2 & Mud & Mud & Clay & Very Poorly Sorted & 0.0 & 2.0 & 98.0 & 2.0 & 41.0 & 57.0 & 8.55 & 0.00 & 2.04 & & \\
\hline WFS_3 & Mud & Mud & Clay & Very Poorly Sorted & 0.0 & 2.0 & 98.0 & 2.0 & 41.0 & 57.0 & 9.15 & 0.00 & 2.13 & & \\
\hline WFS_4 & Mud & Mud & Clay & Very Poorly Sorted & 0.0 & 2.0 & 98.0 & 2.0 & 44.0 & 54.0 & 8.38 & 0.00 & 2.15 & & \\
\hline WFS_5 & Sandy Mud & Sandy Silt & Medium Silt & Poorly Sorted & 0.0 & 25.0 & 75.0 & 25.0 & 62.0 & 13.0 & 5.47 & 0.02 & 1.88 & & \\
\hline WFS_6 & Sandy Mud & Sandy Silt & Medium Silt & Very Poorly Sorted & 0.0 & 25.0 & 75.0 & 25.0 & 60.0 & 15.0 & 5.49 & 0.02 & 2.03 & & \\
\hline WFS_7 & Mud & Mud & Very Fine Silt & Very Poorly Sorted & 0.0 & 9.0 & 91.0 & 9.0 & 54.0 & 37.0 & 7.10 & 0.01 & 2.59 & & \\
\hline WFS_8 & Mud & Mud & Very Fine Silt & Very Poorly Sorted & 0.0 & 1.0 & 99.0 & 1.0 & 64.0 & 35.0 & 7.38 & 0.01 & 2.04 & & \\
\hline WFS_9 & Mud & Mud & Very Fine Silt & Very Poorly Sorted & 0.0 & 1.0 & 99.0 & 1.0 & 51.0 & 48.0 & 7.90 & 0.00 & 2.07 & & \\
\hline WFS_10 & Mud & Mud & Very Fine Silt & Very Poorly Sorted & 0.0 & 6.0 & 94.0 & 6.0 & 60.0 & 34.0 & 7.03 & 0.01 & 2.60 & & \\
\hline WFS_11 & Mud & Mud & Very Fine Silt & Very Poorly Sorted & 0.0 & 2.0 & 98.0 & 2.0 & 67.0 & 31.0 & 7.10 & 0.01 & 2.27 & & \\
\hline WFS_12 & Mud & Mud & Very Fine Sand & Moderately Sorted & 0.0 & 2.0 & 98.0 & 2.0 & 51.0 & 47.0 & 3.75 & 0.07 & 0.89 & & \\
\hline WFS_13 & Mud & Mud & Very Fine Silt & Very Poorly Sorted & 0.0 & 2.0 & 98.0 & 2.0 & 52.0 & 46.0 & 7.88 & 0.00 & 2.05 & & \\
\hline WFS_14 & Gravelly Muddy Sand & & Medium Silt & Very Poorly Sorted & 19.0 & 55.0 & 26.0 & & & & 5.24 & 0.03 & 2.30 & & \\
\hline WFS_15 & Sandy Mud & Sandy Mud & Fine Silt & Very Poorly Sorted & 3.0 & 22.0 & 75.0 & 25.0 & 47.0 & 28.0 & 6.42 & 0.01 & 2.51 & & \\
\hline WFS_16 & Sandy Mud & Sandy Silt & Medium Silt & Very Poorly Sorted & 0.0 & 13.0 & 87.0 & 13.0 & 70.0 & 17.0 & 5.84 & 0.02 & 2.07 & & \\
\hline WFS_17 & Mud & Mud & Very Fine Silt & Very Poorly Sorted & 0.0 & 1.0 & 99.0 & 1.0 & 47.0 & 52.0 & 7.52 & 0.01 & 2.96 & & \\
\hline WFS_18 & Mud & Mud & Very Fine Silt & Very Poorly Sorted & 0.0 & 5.0 & 95.0 & 5.0 & 50.0 & 45.0 & 7.86 & 0.00 & 2.37 & & \\
\hline WFS_19 & Sandy Mud & Sandy Mud & Very Fine Silt & Very Poorly Sorted & 0.0 & 20.0 & 80.0 & 20.0 & 44.0 & 36.0 & 7.25 & 0.01 & 2.69 & & \\
\hline WFS_20 & Gravelly Mud & & Fine Silt & Very Poorly Sorted & 20.0 & 23.0 & 57.0 & & & & 6.17 & 0.01 & 2.72 & & \\
\hline WFS_21 & Muddy Sand & Muddy Sand & Coarse Silt & Poorly Sorted & 1.0 & 61.0 & 38.0 & 62.0 & 25.0 & 13.0 & 4.80 & 0.04 & 1.88 & & \\
\hline WFS_22 & Sandy Mud & Sandy Silt & Medium Silt & Very Poorly Sorted & 0.0 & 21.0 & 79.0 & 21.0 & 62.0 & 17.0 & 5.64 & 0.02 & 2.15 & & \\
\hline WFS_23 & Sandy Mud & Sandy Mud & Very Fine Silt & Very Poorly Sorted & 3.0 & 20.0 & 77.0 & 23.0 & 34.0 & 43.0 & 7.25 & 0.01 & 2.73 & & \\
\hline WFS_24 & Gravelly Mud & Sandy Mud & Very Fine Silt & Very Poorly Sorted & 9.0 & 17.0 & 74.0 & 24.0 & 30.0 & 46.0 & 7.40 & 0.01 & 2.81 & & \\
\hline WFS_25 & Mud & Mud & Very Fine Silt & Very Poorly Sorted & 0.0 & 1.0 & 99.0 & 1.0 & 58.0 & 41.0 & 7.76 & 0.00 & 2.20 & & \\
\hline WFS_26 & Sandy Gravel & & Fine Silt & Very Poorly Sorted & 45.0 & 54.0 & 1.0 & & & & 6.22 & 0.01 & 2.34 & & \\
\hline WFS_27 & Sandy Mud & Sandy Mud & Medium Silt & Very Poorly Sorted & 2.0 & 27.0 & 71.0 & 28.0 & 47.0 & 25.0 & 5.94 & 0.02 & 2.40 & & \\
\hline WFS_28 & Sandy Mud & Sandy Mud & Medium Silt & Very Poorly Sorted & 1.0 & 44.0 & 55.0 & 45.0 & 36.0 & 19.0 & 5.54 & 0.02 & 2.28 & & \\
\hline WFS_29 & Sandy Mud & Sandy Mud & Fine Silt & Very Poorly Sorted & 0.0 & 12.0 & 88.0 & 11.0 & 60.0 & 29.0 & 6.60 & 0.01 & 2.45 & & \\
\hline WFS_30 & Gravelly Mud & & Fine Silt & Very Poorly Sorted & 25.0 & 24.0 & 51.0 & & & & 6.00 & 0.02 & 2.71 & & \\
\hline
\end{tabular}


Flight Survey (1972) (2): Identification, Location, and Description

\begin{tabular}{|c|c|c|c|c|c|c|c|}
\hline Sample ID & $\begin{array}{c}\text { Original } \\
\text { Sample ID }\end{array}$ & $\begin{array}{l}\text { Latitude } \\
\text { WGS84 }\end{array}$ & $\begin{array}{l}\text { Longitude } \\
\text { WGS84 }\end{array}$ & $\begin{array}{l}\text { Reliability } \\
\text { Ranking for } \\
\text { Positioning }\end{array}$ & $\begin{array}{l}\text { Water } \\
\text { Depth } \\
\text { (m) }\end{array}$ & $\begin{array}{l}\text { Sample } \\
\text { Collected }\end{array}$ & $\begin{array}{c}\text { Sampler } \\
\text { Type }\end{array}$ \\
\hline WFS_31 & 403 & 43.080000 & -70.316667 & 4 & & $7 / 15 / 1971$ & Gravity Corer \\
\hline WFS_32 & 404 & 43.063333 & -70.283333 & 4 & & $7 / 15 / 1971$ & Gravity Corer \\
\hline WFS_33 & 405 & 43.041667 & -70.236667 & 4 & & $7 / 15 / 1971$ & Gravity Corer \\
\hline WFS_34 & 406 & 43.068333 & -70.200000 & 4 & & $7 / 15 / 1971$ & Gravity Corer \\
\hline WFS_35 & 407 & 43.088333 & -70.241667 & 4 & & $7 / 15 / 1971$ & Gravity Corer \\
\hline WFS_36 & 408 & 43.103333 & -70.276667 & 4 & & $7 / 15 / 1971$ & Gravity Corer \\
\hline WFS_37 & 409 & 43.120000 & -70.311667 & 4 & & $7 / 16 / 1971$ & Gravity Corer \\
\hline WFS_38 & 410 & 43.136667 & -70.341667 & 4 & & $7 / 16 / 1971$ & Gravity Corer \\
\hline WFS_39 & 411 & 43.156667 & -70.308333 & 4 & & $7 / 16 / 1971$ & Gravity Corer \\
\hline WFS_40 & 412 & 43.140000 & -70.266667 & 4 & & $7 / 16 / 1971$ & Gravity Corer \\
\hline WFS_41 & 413 & 43.123333 & -70.233333 & 4 & & $7 / 16 / 1971$ & Gravity Corer \\
\hline WFS_42 & 415 & 43.106667 & -70.196667 & 4 & & $7 / 16 / 1971$ & Gravity Corer \\
\hline WFS_43 & 416 & 43.090000 & -70.163333 & 4 & & $7 / 16 / 1971$ & Gravity Corer \\
\hline WFS_44 & 501 & 43.005000 & -70.245000 & 4 & & $7 / 29 / 1971$ & Gravity Corer \\
\hline WFS_45 & 502 & 43.025000 & -70.200000 & 4 & & $7 / 29 / 1971$ & Gravity Corer \\
\hline WFS_46 & 503 & 43.050000 & -70.158333 & 4 & & $7 / 29 / 1971$ & Gravity Corer \\
\hline WFS_47 & 504 & 43.068333 & -70.120000 & 4 & & $7 / 29 / 1971$ & Gravity Corer \\
\hline WFS_48 & 506 & 43.103333 & -70.120000 & 4 & & $7 / 29 / 1971$ & Gravity Corer \\
\hline WFS_49 & 507 & 43.123333 & -70.158333 & 4 & & $7 / 29 / 1971$ & Gravity Corer \\
\hline WFS_50 & 508 & 43.160000 & -70.225000 & 4 & & $7 / 29 / 1971$ & Gravity Corer \\
\hline WFS_51 & 509 & 43.183333 & -70.263333 & 4 & & $7 / 30 / 1971$ & Gravity Corer \\
\hline WFS_52 & 510 & 42.948333 & -70.471667 & 4 & & $7 / 30 / 1971$ & Gravity Corer \\
\hline WFS_53 & 513 & 42.895000 & -70.355000 & 4 & & $7 / 30 / 1971$ & Gravity Corer \\
\hline WFS_54 & 514 & 42.875000 & -70.316667 & 4 & & $7 / 30 / 1971$ & Gravity Corer \\
\hline WFS_55 & 601 & 42.953333 & -70.215000 & 4 & & $8 / 12 / 1971$ & Gravity Corer \\
\hline WFS_56 & 603 & 42.970000 & -70.138333 & 4 & & $8 / 12 / 1971$ & Gravity Corer \\
\hline WFS_57 & 604 & 42.978333 & -70.105000 & 4 & & $8 / 12 / 1971$ & Gravity Corer \\
\hline WFS_58 & 605 & 42.941667 & -70.105000 & 4 & & $8 / 12 / 1971$ & Gravity Corer \\
\hline WFS_59 & 607 & 42.925000 & -70.343333 & 4 & & $8 / 13 / 1971$ & Gravity Corer \\
\hline WFS_60 & 608 & 42.906667 & -70.305000 & 4 & & $8 / 13 / 1971$ & Gravity Corer \\
\hline WFS_61 & 609 & 42.888333 & -70.266667 & 4 & & $8 / 13 / 1971$ & Gravity Corer \\
\hline
\end{tabular}


Flight Survey (1972) (2): Sediment Classifications and Grain Size Statistics

\begin{tabular}{|c|c|c|c|c|c|c|c|c|c|c|c|c|c|c|c|c|}
\hline Sample ID & $\begin{array}{l}\text { Textural Group from \%GSM } \\
\text { (Gradistat) }\end{array}$ & $\begin{array}{l}\text { Textural Group } \\
\text { from \%SZC } \\
\text { (Gradistat) } \\
\end{array}$ & $\begin{array}{l}\text { Sediment Classification } \\
\text { from Mean Phi } \\
\text { (Wentworth) }\end{array}$ & Sorting (Gradistat) & $\begin{array}{c}\text { Gravel } \\
\%\end{array}$ & $\begin{array}{c}\text { Sand } \\
\%\end{array}$ & $\begin{array}{c}\text { Mud } \\
\% \\
\end{array}$ & $\begin{array}{c}\text { Sand } \\
\%\end{array}$ & $\begin{array}{c}\text { Silt } \\
\% \\
\end{array}$ & $\begin{array}{c}\text { Clay } \\
\%\end{array}$ & $\begin{array}{c}\text { Mean } \\
\text { Size } \\
\text { (phi) }\end{array}$ & $\begin{array}{c}\text { Mean } \\
\text { Size } \\
(\mathrm{mm})\end{array}$ & $\begin{array}{c}\text { Sorting } \\
\text { (phi) }\end{array}$ & Skewness & Kurtosis & LOI \% \\
\hline WFS_31 & Mud & Mud & Clay & Very Poorly Sorted & 0.0 & 0.0 & 100.0 & 0.0 & 45.0 & 55.0 & 8.51 & 0.00 & 2.18 & & & \\
\hline WFS_32 & Mud & Mud & Clay & Very Poorly Sorted & 0.0 & 1.0 & 99.0 & 1.0 & 46.0 & 53.0 & 8.40 & 0.00 & 2.05 & & & \\
\hline WFS_33 & Mud & Mud & Clay & Poorly Sorted & 0.0 & 1.0 & 99.0 & 1.0 & 41.0 & 58.0 & 8.70 & 0.00 & 1.97 & & & \\
\hline WFS_34 & Mud & Mud & Clay & Poorly Sorted & 0.0 & 0.0 & 100.0 & 0.0 & 42.0 & 58.0 & 8.74 & 0.00 & 1.90 & & & \\
\hline WFS_35 & Sandy Mud & Sandy Mud & Clay & Very Poorly Sorted & 1.0 & 10.0 & 89.0 & 11.0 & 34.0 & 55.0 & 8.09 & 0.00 & 2.64 & & & \\
\hline WFS_36 & Mud & Mud & Clay & Very Poorly Sorted & 0.0 & 1.0 & 99.0 & 1.0 & 46.0 & 53.0 & 8.30 & 0.00 & 2.36 & & & \\
\hline WFS_37 & Gravelly Mud & & Very Fine Silt & Very Poorly Sorted & 12.0 & 19.0 & 69.0 & & & & 7.21 & 0.01 & 2.91 & & & \\
\hline WFS_38 & Mud & Mud & Fine Silt & Very Poorly Sorted & 0.0 & 1.0 & 99.0 & 1.0 & 48.0 & 51.0 & 6.51 & 0.01 & 2.32 & & & \\
\hline WFS_39 & Mud & Mud & Fine Silt & Very Poorly Sorted & 0.0 & 8.0 & 92.0 & 8.0 & 70.0 & 22.0 & 6.32 & 0.01 & 2.76 & & & \\
\hline WFS_40 & Muddy Gravel & & Fine Silt & Very Poorly Sorted & 35.0 & 11.0 & 54.0 & & & & 6.27 & 0.01 & 2.76 & & & \\
\hline WFS_41 & Gravelly Mud & & Clay & Very Poorly Sorted & 12.0 & 28.0 & 60.0 & & & & 8.11 & 0.00 & 2.41 & & & \\
\hline WFS_42 & Mud & Mud & Clay & Very Poorly Sorted & 0.0 & 8.0 & 92.0 & 8.0 & 37.0 & 55.0 & 8.30 & 0.00 & 2.35 & & & \\
\hline WFS_43 & Mud & Mud & Clay & Poorly Sorted & 0.0 & 1.0 & 99.0 & 1.0 & 40.0 & 59.0 & 8.63 & 0.00 & 1.91 & & & \\
\hline WFS_44 & Mud & Clay & Clay & Very Poorly Sorted & 0.0 & 1.0 & 99.0 & 1.0 & 28.0 & 71.0 & 9.03 & 0.00 & 2.83 & & & \\
\hline WFS_45 & Mud & Clay & Clay & Poorly Sorted & 0.0 & 0.0 & 100.0 & 0.0 & 37.0 & 63.0 & 8.88 & 0.00 & 1.87 & & & \\
\hline WFS_46 & Mud & Clay & Clay & Poorly Sorted & 0.0 & 0.0 & 100.0 & 0.0 & 37.0 & 63.0 & 8.90 & 0.00 & 1.87 & & & \\
\hline WFS_47 & Mud & Clay & Clay & Very Poorly Sorted & 0.0 & 1.0 & 99.0 & 1.0 & 37.0 & 62.0 & 8.74 & 0.00 & 2.07 & & & \\
\hline WFS_48 & Sandy Mud & Sandy Clay & Very Fine Silt & Very Poorly Sorted & 0.0 & 16.0 & 84.0 & 16.0 & 31.0 & 53.0 & 7.51 & 0.01 & 2.82 & & & \\
\hline WFS_49 & Gravelly Mud & Sandy Clay & Clay & Very Poorly Sorted & 5.0 & 9.0 & 86.0 & 14.0 & 29.0 & 57.0 & 8.13 & 0.00 & 2.80 & & & \\
\hline WFS_50 & Mud & Mud & Very Fine Silt & Very Poorly Sorted & 0.0 & 2.0 & 98.0 & 2.0 & 49.0 & 49.0 & 7.86 & 0.00 & 2.46 & & & \\
\hline WFS_51 & Mud & Mud & Clay & Very Poorly Sorted & 0.0 & 0.0 & 100.0 & 0.0 & 53.0 & 47.0 & 8.00 & 0.00 & 2.25 & & & \\
\hline WFS_52 & Mud & Mud & Very Fine Silt & Very Poorly Sorted & 0.0 & 2.0 & 98.0 & 2.0 & 67.0 & 31.0 & 7.11 & 0.01 & 2.31 & & & \\
\hline WFS_53 & Muddy Sandy Gravel & & Very Fine Sand & Poorly Sorted & 50.0 & 35.0 & 15.0 & & & & 3.78 & 0.07 & 1.06 & & & \\
\hline WFS_54 & Gravelly Muddy Sand & & Medium Silt & Very Poorly Sorted & 6.0 & 66.0 & 28.0 & & & & 5.03 & 0.03 & 2.11 & & & \\
\hline WFS_55 & Mud & Mud & Clay & Very Poorly Sorted & 0.0 & 1.0 & 99.0 & 1.0 & 39.0 & 60.0 & 8.77 & 0.00 & 2.03 & & & \\
\hline WFS_56 & Mud & Mud & Clay & Very Poorly Sorted & 0.0 & 9.0 & 91.0 & 9.0 & 36.0 & 55.0 & 8.10 & 0.00 & 2.68 & & & \\
\hline WFS_57 & Muddy Sandy Gravel & & Coarse Silt & Poorly Sorted & 32.0 & 48.0 & 20.0 & & & & 4.68 & 0.04 & 1.91 & & & \\
\hline WFS_58 & Gravelly Mud & & Fine Silt & Very Poorly Sorted & 8.0 & 43.0 & 49.0 & & & & 6.00 & 0.02 & 2.78 & & & \\
\hline WFS_59 & Mud & Mud & Clay & Poorly Sorted & 0.0 & 0.0 & 100.0 & 0.0 & 41.0 & 59.0 & 8.68 & 0.00 & 1.99 & & & \\
\hline WFS_60 & Mud & Mud & Clay & Very Poorly Sorted & 0.0 & 0.0 & 100.0 & 0.0 & 41.0 & 59.0 & 8.52 & 0.00 & 2.24 & & & \\
\hline WFS_61 & Sandy Mud & Sandy Mud & Very Fine Silt & Very Poorly Sorted & 0.0 & 26.0 & 74.0 & 26.0 & 30.0 & 44.0 & 7.32 & 0.01 & 2.82 & & & \\
\hline
\end{tabular}


Section 8: Mills Survey (1971) 
Mills Survey (1971) (1): Identification, Location, and Description

\begin{tabular}{|c|c|c|c|c|c|c|c|}
\hline Sample ID & $\begin{array}{c}\text { Original } \\
\text { Sample ID }\end{array}$ & $\begin{array}{c}\text { Latitude } \\
\text { WGS84 }\end{array}$ & $\begin{array}{c}\text { Longitude } \\
\text { WGS84 }\end{array}$ & $\begin{array}{l}\text { Reliability } \\
\text { Ranking for } \\
\text { Positioning }\end{array}$ & $\begin{array}{c}\text { Water } \\
\text { Depth } \\
(\mathrm{m})\end{array}$ & $\begin{array}{l}\text { Sample } \\
\text { Collected }\end{array}$ & $\begin{array}{c}\text { Sampler } \\
\text { Type }\end{array}$ \\
\hline MS_1 & 1 & 43.046667 & -70.698333 & 4 & 12 & $6 / 5 / 1972-11 / 30 / 1972$ & Shipek \\
\hline MS_2 & 2 & 43.046667 & -70.690000 & 4 & 14 & $6 / 5 / 1972-11 / 30 / 1972$ & Shipek \\
\hline MS_3 & 3 & 43.038333 & -70.668333 & 4 & 24 & $6 / 5 / 1972-11 / 30 / 1972$ & Shipek \\
\hline MS_4 & 4 & 43.031667 & -70.646667 & 4 & 27 & $6 / 5 / 1972-11 / 30 / 1972$ & Shipek \\
\hline MS_5 & 5 & 43.026667 & -70.630000 & 4 & 33 & $6 / 5 / 1972-11 / 30 / 1972$ & Shipek \\
\hline MS_6 & 6 & 43.021667 & -70.605000 & 4 & 37 & $6 / 5 / 1972-11 / 30 / 1972$ & Shipek \\
\hline MS_7 & 8 & 42.993333 & -70.626667 & 4 & 17 & $6 / 5 / 1972-11 / 30 / 1972$ & Shipek \\
\hline MS_8 & 9 & 43.008333 & -70.645000 & 4 & 24 & $6 / 5 / 1972-11 / 30 / 1972$ & Shipek \\
\hline MS_9 & 10 & 43.018333 & -70.653333 & 4 & 23 & $6 / 5 / 1972-11 / 30 / 1972$ & Shipek \\
\hline MS_10 & 11 & 43.030000 & -70.676667 & 4 & 17 & $6 / 5 / 1972-11 / 30 / 1972$ & Shipek \\
\hline MS_11 & 12 & 43.041667 & -70.691667 & 4 & 14 & $6 / 5 / 1972-11 / 30 / 1972$ & Shipek \\
\hline MS_12 & 13 & 43.040000 & -70.696667 & 4 & 14 & $6 / 5 / 1972-11 / 30 / 1972$ & Shipek \\
\hline MS_13 & 14 & 43.025000 & -70.688333 & 4 & 19 & $6 / 5 / 1972-11 / 30 / 1972$ & Shipek \\
\hline MS_14 & 15 & 43.008333 & -70.680000 & 4 & 24 & $6 / 5 / 1972-11 / 30 / 1972$ & Shipek \\
\hline MS_15 & 16 & 42.993333 & -70.671667 & 4 & 26 & $6 / 5 / 1972-11 / 30 / 1972$ & Shipek \\
\hline MS_16 & 17 & 42.978333 & -70.665000 & 4 & 23 & $6 / 5 / 1972-11 / 30 / 1972$ & Shipek \\
\hline MS_17 & 18 & 42.971667 & -70.703333 & 4 & 26 & $6 / 5 / 1972-11 / 30 / 1972$ & Shipek \\
\hline MS_18 & 19 & 42.988333 & -70.705000 & 4 & 20 & $6 / 5 / 1972-11 / 30 / 1972$ & Shipek \\
\hline MS_19 & 20 & 43.005000 & -70.701667 & 4 & 22 & $6 / 5 / 1972-11 / 30 / 1972$ & Shipek \\
\hline MS_20 & 21 & 43.025000 & -70.700000 & 4 & 15 & $6 / 5 / 1972-11 / 30 / 1972$ & Shipek \\
\hline MS_21 & 22 & 43.038333 & -70.700000 & 4 & 15 & $6 / 5 / 1972-11 / 30 / 1972$ & Shipek \\
\hline MS_22 & 25 & 43.038333 & -70.508333 & 4 & 26 & $6 / 5 / 1972-11 / 30 / 1972$ & Shipek \\
\hline MS_23 & 26 & 43.040000 & -70.703333 & 4 & 13 & $6 / 5 / 1972-11 / 30 / 1972$ & Shipek \\
\hline MS_24 & 27 & 43.023333 & -70.711667 & 4 & 16 & $6 / 5 / 1972-11 / 30 / 1972$ & Shipek \\
\hline MS_25 & 28 & 43.008333 & -70.720000 & 4 & 18 & $6 / 5 / 1972-11 / 30 / 1972$ & Shipek \\
\hline MS_26 & 29 & 42.998333 & -70.715000 & 4 & 21 & $6 / 5 / 1972-11 / 30 / 1972$ & Shipek \\
\hline MS_27 & 30 & 42.991667 & -70.726667 & 4 & 19 & $6 / 5 / 1972-11 / 30 / 1972$ & Shipek \\
\hline MS_28 & 31 & 43.045000 & -70.641667 & 4 & 27 & $6 / 5 / 1972-11 / 30 / 1972$ & Shipek \\
\hline MS_29 & 32 & 43.046667 & -70.665000 & 4 & 22 & $6 / 5 / 1972-11 / 30 / 1972$ & Shipek \\
\hline MS_30 & 33 & 43.046667 & -70.688333 & 4 & 9 & $6 / 5 / 1972-11 / 30 / 1972$ & Shipek \\
\hline MS_31 & 34 & 42.976667 & -70.735000 & 4 & 10 & $6 / 5 / 1972-11 / 30 / 1972$ & Shipek \\
\hline MS_32 & 35 & 42.965000 & -70.740000 & 4 & 20 & $6 / 5 / 1972-11 / 30 / 1972$ & Shipek \\
\hline
\end{tabular}


Mills Survey (1971) (1): Sediment Classifications and Grain Size Statistics

\begin{tabular}{|c|c|c|c|c|c|c|c|c|c|c|c|c|c|c|c|c|}
\hline Sample ID & $\begin{array}{l}\text { Textural Group from \%GSM } \\
\text { (Gradistat) }\end{array}$ & $\begin{array}{l}\text { Textural Group } \\
\text { from \%SZC } \\
\text { (Gradistat) }\end{array}$ & $\begin{array}{l}\text { Sediment Classification } \\
\text { from Mean Phi } \\
\text { (Wentworth) }\end{array}$ & Sorting (Gradistat) & $\begin{array}{c}\text { Gravel } \\
\%\end{array}$ & $\begin{array}{l}\text { Sand } \\
\%\end{array}$ & $\begin{array}{c}\text { Mud } \\
\%\end{array}$ & $\begin{array}{c}\text { Sand } \\
\%\end{array}$ & $\begin{array}{c}\text { Silt } \\
\%\end{array}$ & $\begin{array}{c}\text { Clay } \\
\%\end{array}$ & $\begin{array}{c}\text { Mean } \\
\text { Size } \\
\text { (phi) }\end{array}$ & $\begin{array}{c}\text { Mean } \\
\text { Size } \\
(\mathrm{mm})\end{array}$ & $\begin{array}{l}\text { Sorting } \\
\text { (phi) }\end{array}$ & Skewness & Kurtosis & LOI \% \\
\hline MS_1 & Gravelly Sand & & Medium Sand & Poorly Sorted & 7.0 & 92.0 & 1.0 & & & & 1.20 & 0.44 & 1.21 & -0.13 & & \\
\hline MS_2 & Gravelly Sand & & Medium Sand & Poorly Sorted & 17.0 & 82.0 & 1.0 & & & & 1.10 & 0.47 & 1.82 & -0.38 & & \\
\hline MS_3 & Sandy Gravel & & Very Coarse Sand & Very Poorly Sorted & 42.0 & 57.0 & 1.0 & & & & -0.50 & 1.41 & 2.36 & -0.34 & & \\
\hline MS_4 & Sandy Gravel & & Granule Gravel & Very Poorly Sorted & 63.0 & 35.0 & 2.0 & & & & -1.50 & 2.83 & 2.32 & 0.43 & & \\
\hline MS_5 & Sandy Gravel & & Granule Gravel & Poorly Sorted & 70.0 & 29.0 & 1.0 & & & & -1.50 & 2.83 & 1.50 & 0.46 & & \\
\hline MS_6 & Sandy Gravel & & Granule Gravel & Very Poorly Sorted & 59.0 & 39.0 & 2.0 & & & & -1.50 & 2.83 & 2.61 & 0.35 & & \\
\hline MS_7 & Gravelly Sand & & Fine Sand & Poorly Sorted & 7.0 & 90.0 & 3.0 & & & & 2.10 & 0.23 & 1.31 & -0.34 & & \\
\hline MS_8 & Gravelly Muddy Sand & & Coarse Sand & Very Poorly Sorted & 28.0 & 64.0 & 8.0 & & & & 0.30 & 0.81 & 2.57 & -0.01 & & \\
\hline MS_9 & Sandy Gravel & & Very Coarse Sand & Poorly Sorted & 42.0 & 57.0 & 1.0 & & & & -0.50 & 1.41 & 1.90 & -0.03 & & \\
\hline MS_10 & Gravelly Sand & & Coarse Sand & Poorly Sorted & 11.0 & 88.0 & 1.0 & & & & 0.20 & 0.87 & 1.08 & -0.10 & & \\
\hline MS_11 & Sand & Sand & Very Fine Sand & Very Well Sorted & 0.0 & 99.0 & 1.0 & 99.0 & 0.0 & 1.0 & 3.00 & 0.13 & 0.34 & -0.19 & & \\
\hline MS_12 & Sand & Sand & Very Fine Sand & Well Sorted & 0.0 & 99.0 & 1.0 & 99.0 & 1.0 & 0.0 & 3.00 & 0.13 & 0.36 & -0.01 & & \\
\hline MS_13 & Sandy Gravel & & Granule Gravel & Very Poorly Sorted & 68.0 & 31.0 & 1.0 & & & & -1.90 & 3.73 & 2.27 & 0.35 & & \\
\hline MS_14 & Sandy Gravel & & Granule Gravel & Very Poorly Sorted & 66.0 & 31.0 & 2.0 & & & & -1.80 & 3.48 & 2.48 & 0.50 & & \\
\hline MS_15 & Gravelly Sand & & Coarse Sand & Very Poorly Sorted & 23.0 & 75.0 & 2.0 & & & & 0.60 & 0.66 & 2.09 & -0.52 & & \\
\hline MS_16 & Gravelly Sand & & Medium Sand & Poorly Sorted & 15.0 & 83.0 & 2.0 & & & & 1.40 & 0.38 & 1.62 & -0.66 & & \\
\hline MS_17 & Sandy Gravel & & Very Coarse Sand & Very Poorly Sorted & 53.0 & 45.0 & 2.0 & & & & -0.70 & 1.62 & 2.54 & 0.34 & & \\
\hline MS_18 & Gravelly Sand & & Medium Sand & Poorly Sorted & 5.0 & 91.0 & 4.0 & & & & 1.10 & 0.47 & 1.20 & -0.31 & & \\
\hline MS_19 & Sandy Gravel & & Very Coarse Sand & Very Poorly Sorted & 46.0 & 52.0 & 2.0 & & & & -0.20 & 1.15 & 2.39 & 0.00 & & \\
\hline MS_20 & Sand & Sand & Very Fine Sand & Very Well Sorted & 0.0 & 99.0 & 1.0 & 99.0 & 1.0 & 0.0 & 3.10 & 0.12 & 0.29 & -0.50 & & \\
\hline MS_21 & Sand & Sand & Fine Sand & Moderately Well Sorted & 0.0 & 98.0 & 2.0 & 98.0 & 1.0 & 1.0 & 2.70 & 0.15 & 0.50 & 0.09 & & \\
\hline MS_22 & Muddy Sandy Gravel & & Coarse Sand & Extremely Poorly Sorted & 51.0 & 29.0 & 20.0 & & & & 0.70 & 0.62 & 4.07 & 0.68 & & \\
\hline MS_23 & Muddy Sand & & Fine Sand & Poorly Sorted & 2.0 & 83.0 & 15.0 & 83.0 & 12.0 & 3.0 & 2.90 & 0.13 & 1.30 & 0.10 & & \\
\hline MS_24 & Muddy Sandy Gravel & & Very Coarse Sand & Very Poorly Sorted & 63.0 & 29.0 & 8.0 & & & & -1.00 & 2.00 & 2.95 & 0.62 & & \\
\hline MS_25 & Gravelly Muddy Sand & & Medium Sand & Very Poorly Sorted & 22.0 & 69.0 & 9.0 & & & & 1.20 & 0.44 & 2.59 & -0.23 & & \\
\hline MS_26 & Sand & & Very Fine Sand & Moderately Well Sorted & 2.0 & 91.0 & 7.0 & 91.0 & 6.0 & 2.0 & 3.10 & 0.12 & 0.68 & 0.10 & & \\
\hline MS_27 & Gravel & & Granule Gravel & Poorly Sorted & 80.0 & 17.0 & 3.0 & & & & -2.00 & 4.00 & 1.37 & 0.26 & & \\
\hline MS_28 & Sandy Gravel & & Granule Gravel & Very Poorly Sorted & 67.0 & 29.0 & 4.0 & & & & -1.90 & 3.73 & 2.60 & 0.73 & & \\
\hline MS_29 & Muddy Sandy Gravel & & Granule Gravel & Very Poorly Sorted & 75.0 & 20.0 & 5.0 & & & & -1.70 & 3.25 & 2.24 & 0.52 & & \\
\hline MS_30 & Sand & Sand & Fine Sand & Moderately Well Sorted & 0.0 & 97.0 & 3.0 & 97.0 & 3.0 & 0.0 & 2.50 & 0.18 & 0.58 & 0.17 & & \\
\hline MS_31 & Sand & & Fine Sand & Moderately Well Sorted & 1.0 & 97.0 & 2.0 & 97.0 & 1.0 & 1.0 & 2.50 & 0.18 & 0.59 & 0.04 & & \\
\hline MS_32 & Sandy Gravel & & Granule Gravel & Very Poorly Sorted & 65.0 & 31.0 & 4.0 & & & & -1.60 & 3.03 & 2.33 & 0.48 & & \\
\hline
\end{tabular}


Mills Survey (1971) (2): Identification, Location, and Description

\begin{tabular}{|c|c|c|c|c|c|c|c|}
\hline Sample ID & $\begin{array}{c}\text { Original } \\
\text { Sample ID }\end{array}$ & $\begin{array}{c}\text { Latitude } \\
\text { WGS84 }\end{array}$ & $\begin{array}{l}\text { Longitude } \\
\text { WGS84 }\end{array}$ & $\begin{array}{l}\text { Reliability } \\
\text { Ranking for } \\
\text { Positioning }\end{array}$ & $\begin{array}{c}\text { Water } \\
\text { Depth } \\
\text { (m) }\end{array}$ & $\begin{array}{l}\text { Sample } \\
\text { Collected }\end{array}$ & $\begin{array}{c}\text { Sampler } \\
\text { Type }\end{array}$ \\
\hline MS_33 & 36 & 42.948333 & -70.748333 & 4 & 24 & $6 / 5 / 1972-11 / 30 / 1972$ & Shipek \\
\hline MS_34 & 37 & 42.946667 & -70.705000 & 4 & 38 & $6 / 5 / 1972-11 / 30 / 1972$ & Shipek \\
\hline MS_35 & 38 & 42.955000 & -70.705000 & 4 & 34 & $6 / 5 / 1972-11 / 30 / 1972$ & Shipek \\
\hline MS_36 & 39 & 42.961667 & -70.651667 & 4 & 38 & $6 / 5 / 1972-11 / 30 / 1972$ & Shipek \\
\hline MS_37 & 40 & 42.956667 & -70.651667 & 4 & 37 & $6 / 5 / 1972-11 / 30 / 1972$ & Shipek \\
\hline MS_38 & 41 & 42.946667 & -70.645000 & 4 & 44 & $6 / 5 / 1972-11 / 30 / 1972$ & Shipek \\
\hline MS_39 & 42 & 42.968333 & -70.595000 & 4 & 56 & $6 / 5 / 1972-11 / 30 / 1972$ & Shipek \\
\hline MS_40 & 43 & 42.965000 & -70.580000 & 4 & 59 & $6 / 5 / 1972-11 / 30 / 1972$ & Shipek \\
\hline MS_41 & 44 & 42.945000 & -70.565000 & 4 & 78 & $6 / 5 / 1972-11 / 30 / 1972$ & Shipek \\
\hline MS_42 & 46 & 42.990000 & -70.558333 & 4 & 66 & $6 / 5 / 1972-11 / 30 / 1972$ & Shipek \\
\hline MS_43 & 47 & 42.975000 & -70.543333 & 4 & 72 & $6 / 5 / 1972-11 / 30 / 1972$ & Shipek \\
\hline MS_44 & 48 & 42.961667 & -70.528333 & 4 & 75 & $6 / 5 / 1972-11 / 30 / 1972$ & Shipek \\
\hline MS_45 & 49 & 42.960000 & -70.516667 & 4 & 76 & $6 / 5 / 1972-11 / 30 / 1972$ & Shipek \\
\hline MS_46 & 50 & 42.943333 & -70.508333 & 4 & 84 & $6 / 5 / 1972-11 / 30 / 1972$ & Shipek \\
\hline MS_47 & 51 & 43.040000 & -70.531667 & 4 & 62 & $6 / 5 / 1972-11 / 30 / 1972$ & Shipek \\
\hline MS_48 & 52 & 43.040000 & -70.553333 & 4 & 61 & $6 / 5 / 1972-11 / 30 / 1972$ & Shipek \\
\hline MS_49 & 53 & 43.041667 & -70.575000 & 4 & 40 & $6 / 5 / 1972-11 / 30 / 1972$ & Shipek \\
\hline MS_50 & 56 & 43.048333 & -70.623333 & 4 & 32 & $6 / 5 / 1972-11 / 30 / 1972$ & Shipek \\
\hline MS_51 & 58 & 43.048333 & -70.686667 & 4 & 20 & $6 / 5 / 1972-11 / 30 / 1972$ & Shipek \\
\hline MS_52 & 60 & 43.053333 & -70.651667 & 4 & 21 & $6 / 5 / 1972-11 / 30 / 1972$ & Shipek \\
\hline MS_53 & 61 & 43.056667 & -70.633333 & 4 & 14 & $6 / 5 / 1972-11 / 30 / 1972$ & Shipek \\
\hline MS_54 & 62 & 43.058333 & -70.620000 & 4 & 24 & $6 / 5 / 1972-11 / 30 / 1972$ & Shipek \\
\hline MS_55 & 63 & 43.058333 & -70.601667 & 4 & 34 & $6 / 5 / 1972-11 / 30 / 1972$ & Shipek \\
\hline MS_56 & 64 & 43.061667 & -70.578333 & 4 & 40 & $6 / 5 / 1972-11 / 30 / 1972$ & Shipek \\
\hline MS_57 & 65 & 43.068333 & -70.551667 & 4 & 53 & $6 / 5 / 1972-11 / 30 / 1972$ & Shipek \\
\hline MS_58 & 66 & 43.070000 & -70.528333 & 4 & 53 & $6 / 5 / 1972-11 / 30 / 1972$ & Shipek \\
\hline MS_59 & 70 & 43.105000 & -70.611667 & 4 & 15 & $6 / 5 / 1972-11 / 30 / 1972$ & Shipek \\
\hline MS_60 & 72 & 43.106667 & -70.565000 & 4 & 31 & $6 / 5 / 1972-11 / 30 / 1972$ & Shipek \\
\hline MS_61 & 75 & 43.090000 & -70.498333 & 4 & 62 & $6 / 5 / 1972-11 / 30 / 1972$ & Shipek \\
\hline MS_62 & 76 & 43.090000 & -70.475000 & 4 & 62 & $6 / 5 / 1972-11 / 30 / 1972$ & Shipek \\
\hline MS_63 & 77 & 43.090000 & -70.451667 & 4 & 75 & $6 / 5 / 1972-11 / 30 / 1972$ & Shipek \\
\hline MS_64 & 78 & 43.090000 & -70.440000 & 4 & 75 & 6/5/1972 - 11/30/1972 & Shipek \\
\hline
\end{tabular}


Mills Survey (1971) (2): Sediment Classifications and Grain Size Statistics

\begin{tabular}{|c|c|c|c|c|c|c|c|c|c|c|c|c|c|c|c|}
\hline Sample ID & $\begin{array}{l}\text { Textural Group from \%GSM } \\
\text { (Gradistat) }\end{array}$ & $\begin{array}{l}\text { Textural Group } \\
\text { from \%SZC } \\
\text { (Gradistat) } \\
\end{array}$ & $\begin{array}{l}\text { Sediment Classification } \\
\text { from Mean Phi } \\
\text { (Wentworth) }\end{array}$ & Sorting (Gradistat) & $\begin{array}{c}\text { Gravel } \\
\% \\
\end{array}$ & $\begin{array}{c}\text { Sand } \\
\%\end{array}$ & $\begin{array}{c}\text { Mud } \\
\%\end{array}$ & $\begin{array}{c}\text { Sand } \\
\%\end{array}$ & $\begin{array}{c}\text { Silt } \\
\% \\
\end{array}$ & $\begin{array}{c}\text { Clay } \\
\%\end{array}$ & $\begin{array}{c}\text { Mean } \\
\text { Size } \\
\text { (phi) }\end{array}$ & $\begin{array}{c}\text { Mean } \\
\text { Size } \\
(\mathrm{mm}) \\
\end{array}$ & $\begin{array}{c}\text { Sorting } \\
\text { (phi) }\end{array}$ & Skewness & Kurtosis LOI \% \\
\hline MS_33 & Sand & Sand & Fine Sand & Poorly Sorted & 0.0 & 90.0 & 9.0 & 90.0 & 8.0 & 1.0 & 2.80 & 0.14 & 1.05 & 0.23 & \\
\hline MS_34 & Sand & Sand & Fine Sand & Moderately Sorted & 0.0 & 93.0 & 6.0 & 93.0 & 4.0 & 2.0 & 2.70 & 0.15 & 0.93 & -0.15 & \\
\hline MS_35 & Sandy Gravel & & Coarse Sand & Poorly Sorted & 47.0 & 52.0 & 1.0 & & & & 0.90 & 0.54 & 1.08 & 0.01 & \\
\hline MS_36 & Sand & Sand & Very Fine Sand & Well Sorted & 0.0 & 92.0 & 8.0 & 92.0 & 5.0 & 3.0 & 3.20 & 0.11 & 0.47 & 0.14 & \\
\hline MS_37 & Muddy Sand & Silty Sand & Very Fine Sand & Moderately Sorted & 0.0 & 89.0 & 11.0 & 89.0 & 10.0 & 1.0 & 3.30 & 0.10 & 0.75 & 0.30 & \\
\hline MS_38 & Muddy Sand & & Very Fine Sand & Poorly Sorted & 3.0 & 80.0 & 17.0 & 80.0 & 11.0 & 6.0 & 3.30 & 0.10 & 1.41 & 0.39 & \\
\hline MS_39 & Muddy Sandy Gravel & & Very Coarse Sand & Extremely Poorly Sorted & 57.0 & 27.0 & 16.0 & & & & -0.80 & 1.74 & 4.20 & 0.58 & \\
\hline MS_40 & Gravelly Muddy Sand & & Medium Sand & Very Poorly Sorted & 26.0 & 57.0 & 17.0 & & & & 1.30 & 0.41 & 3.50 & 0.15 & \\
\hline MS_41 & Gravelly Mud & & Medium Silt & Very Poorly Sorted & 11.0 & 23.0 & 66.0 & & & & 5.50 & 0.02 & 3.76 & 0.05 & \\
\hline MS_42 & Muddy Sand & & Coarse Silt & Very Poorly Sorted & 1.0 & 58.0 & 41.0 & 58.0 & 26.0 & 15.0 & 4.80 & 0.04 & 2.83 & 0.57 & \\
\hline MS_43 & Sandy Mud & Sandy Mud & Fine Silt & Very Poorly Sorted & 0.0 & 18.0 & 82.0 & 18.0 & 52.0 & 30.0 & 6.60 & 0.01 & 2.61 & 0.15 & \\
\hline MS_44 & Sandy Gravel & & Pebble Gravel & Very Poorly Sorted & 78.0 & 19.0 & 3.0 & & & & -2.60 & 6.06 & 2.36 & 0.78 & \\
\hline MS_45 & Muddy Sandy Gravel & & Granule Gravel & Extremely Poorly Sorted & 65.0 & 20.0 & 15.0 & & & & -1.40 & 2.64 & 4.18 & 0.91 & \\
\hline MS_46 & Sandy Mud & Sandy Silt & Fine Silt & Very Poorly Sorted & 0.0 & 14.0 & 86.0 & 14.0 & 66.0 & 20.0 & 6.20 & 0.01 & 2.17 & -0.01 & \\
\hline MS_47 & Muddy Sand & Muddy Sand & Very Fine Sand & Poorly Sorted & 0.0 & 77.0 & 23.0 & 77.0 & 14.0 & 9.0 & 3.90 & 0.07 & 1.80 & 0.70 & \\
\hline MS_48 & Sandy Mud & Sandy Mud & Medium Silt & Very Poorly Sorted & 0.0 & 47.0 & 53.0 & 47.0 & 28.0 & 25.0 & 5.60 & 0.02 & 3.42 & 0.58 & \\
\hline MS_49 & Sandy Gravel & & Granule Gravel & Poorly Sorted & 67.0 & 30.0 & 3.0 & & & & -1.80 & 3.48 & 1.72 & 0.34 & \\
\hline MS_50 & Gravelly Sand & & Coarse Sand & Poorly Sorted & 13.0 & 83.0 & 4.0 & & & & 0.80 & 0.57 & 1.69 & 0.12 & \\
\hline MS_51 & Sand & Sand & Fine Sand & Moderately Well Sorted & 0.0 & 97.0 & 3.0 & 97.0 & 2.0 & 1.0 & 2.30 & 0.20 & 0.65 & 0.11 & \\
\hline MS_52 & Gravel & & Pebble Gravel & Poorly Sorted & 87.0 & 13.0 & 0.0 & & & & -2.70 & 6.50 & 1.34 & 0.42 & \\
\hline MS_53 & Gravel & & Pebble Gravel & Poorly Sorted & 83.0 & 14.0 & 3.0 & & & & -2.50 & 5.66 & 1.63 & 0.72 & \\
\hline MS_54 & Gravel & & Pebble Gravel & Moderately Sorted & 96.0 & 3.0 & 1.0 & & & & -3.00 & 8.00 & 0.84 & 0.53 & \\
\hline MS_55 & Muddy Sandy Gravel & & Granule Gravel & Very Poorly Sorted & 63.0 & 30.0 & 7.0 & & & & -1.90 & 3.73 & 2.52 & 0.59 & \\
\hline MS_56 & Muddy Sandy Gravel & & Very Coarse Sand & Very Poorly Sorted & 51.0 & 41.0 & 8.0 & & & & -0.70 & 1.62 & 3.10 & 0.31 & \\
\hline MS_57 & Muddy Sand & & Very Fine Sand & Poorly Sorted & 3.0 & 72.0 & 25.0 & 72.0 & 23.0 & 2.0 & 3.50 & 0.09 & 1.58 & 0.47 & \\
\hline MS_58 & Muddy Sandy Gravel & & Very Coarse Sand & Very Poorly Sorted & 45.0 & 45.0 & 10.0 & & & & -0.40 & 1.32 & 3.11 & 0.23 & \\
\hline MS_59 & Muddy Sandy Gravel & & Pebble Gravel & Very Poorly Sorted & 79.0 & 17.0 & 4.0 & & & & -2.30 & 4.92 & 2.53 & 0.82 & \\
\hline MS_60 & Sandy Gravel & & Medium Sand & Very Poorly Sorted & 61.0 & 35.0 & 4.0 & & & & 1.00 & 0.50 & 2.51 & 0.47 & \\
\hline MS_61 & Muddy Sand & Silty Sand & Very Fine Sand & Moderately Well Sorted & 0.0 & 87.0 & 13.0 & 87.0 & 10.0 & 3.0 & 3.30 & 0.10 & 0.61 & 0.33 & \\
\hline MS_62 & Muddy Sandy Gravel & & Very Coarse Sand & Very Poorly Sorted & 66.0 & 20.0 & 14.0 & & & & -0.90 & 1.87 & 3.67 & 0.69 & \\
\hline MS_63 & Sandy Mud & Sandy Silt & Coarse Silt & Very Poorly Sorted & 0.0 & 44.0 & 56.0 & 44.0 & 42.0 & 14.0 & 4.90 & 0.03 & 2.19 & 0.67 & \\
\hline MS_64 & Muddy Sand & Silty Sand & Coarse Silt & Poorly Sorted & 0.0 & 63.0 & 37.0 & 63.0 & 28.0 & 9.0 & 4.00 & 0.06 & 1.52 & 0.70 & \\
\hline
\end{tabular}


Mills Survey (1971) (3): Identification, Location, and Description

\begin{tabular}{|c|c|c|c|c|c|c|c|}
\hline Sample ID & $\begin{array}{c}\text { Original } \\
\text { Sample ID }\end{array}$ & $\begin{array}{c}\text { Latitude } \\
\text { WGS84 }\end{array}$ & $\begin{array}{c}\text { Longitude } \\
\text { WGS84 }\end{array}$ & $\begin{array}{l}\text { Reliability } \\
\text { Ranking for } \\
\text { Positioning }\end{array}$ & $\begin{array}{c}\text { Water } \\
\text { Depth } \\
\text { (m) }\end{array}$ & $\begin{array}{c}\text { Sample } \\
\text { Collected }\end{array}$ & $\begin{array}{c}\text { Sampler } \\
\text { Type }\end{array}$ \\
\hline MS_65 & 79 & 43.090000 & -70.416667 & 4 & 81 & $6 / 5 / 1972-11 / 30 / 1972$ & Shipek \\
\hline MS_66 & 80 & 43.090000 & -70.388333 & 4 & 90 & $6 / 5 / 1972-11 / 30 / 1972$ & Shipek \\
\hline MS_67 & 81 & 43.091667 & -70.366667 & 4 & 93 & $6 / 5 / 1972-11 / 30 / 1972$ & Shipek \\
\hline MS_68 & Sta $2-1$ & 43.075000 & -70.396667 & 4 & 62 & $6 / 5 / 1972-11 / 30 / 1972$ & Shipek \\
\hline MS_69 & Sta $2-2$ & 42.998333 & -70.503333 & 4 & 85 & $6 / 5 / 1972-11 / 30 / 1972$ & Shipek \\
\hline MS_70 & Sta $2-3$ & 42.983333 & -70.458333 & 4 & 95 & $6 / 5 / 1972-11 / 30 / 1972$ & Shipek \\
\hline MS_71 & Sta $2-8$ & 42.958333 & -70.416667 & 4 & 107 & $6 / 5 / 1972-11 / 30 / 1972$ & Shipek \\
\hline MS_72 & Sta $2-9$ & 42.963333 & -70.461667 & 4 & 97 & $6 / 5 / 1972-11 / 30 / 1972$ & Shipek \\
\hline MS_73 & Sta $2-10$ & 42.966667 & -70.505000 & 4 & 87 & $6 / 5 / 1972-11 / 30 / 1972$ & Shipek \\
\hline MS_74 & Sta 4-1 & 43.111667 & -70.388333 & 4 & 70 & $6 / 5 / 1972-11 / 30 / 1972$ & Shipek \\
\hline MS_75 & Sta $4-2$ & 43.096667 & -70.351667 & 4 & 117 & $6 / 5 / 1972-11 / 30 / 1972$ & Shipek \\
\hline MS_76 & Sta 7-6 & 43.036667 & -70.385000 & 4 & 109 & $6 / 5 / 1972-11 / 30 / 1972$ & Shipek \\
\hline MS_77 & Sta $7-7$ & 43.055000 & -70.421667 & 4 & 101 & $6 / 5 / 1972-11 / 30 / 1972$ & Shipek \\
\hline MS_78 & Sta 7-8 & 43.051667 & -70.336667 & 4 & 87 & $6 / 5 / 1972-11 / 30 / 1972$ & Shipek \\
\hline MS_80 & Sta 3 & 43.005000 & -70.473333 & 4 & 91 & $6 / 5 / 1972-11 / 30 / 1972$ & Shipek \\
\hline MS_81 & Sta 4 & 43.020000 & -70.461667 & 4 & 91 & $6 / 5 / 1972-11 / 30 / 1972$ & Shipek \\
\hline MS_82 & Sta 5 & 43.015000 & -70.445000 & 4 & 94 & $6 / 5 / 1972-11 / 30 / 1972$ & Shipek \\
\hline MS_83 & Sta 6 & 43.006667 & -70.421667 & 4 & 99 & $6 / 5 / 1972-11 / 30 / 1972$ & Shipek \\
\hline MS_84 & Sta 8 & 43.030000 & -70.435000 & 4 & 91 & $6 / 5 / 1972-11 / 30 / 1972$ & Shipek \\
\hline MS_85 & Sta 9 & 43.034167 & -70.453333 & 4 & 91 & $6 / 5 / 1972-11 / 30 / 1972$ & Shipek \\
\hline
\end{tabular}


Mills Survey (1971) (3): Sediment Classifications and Grain Size Statistics

\begin{tabular}{|c|c|c|c|c|c|c|c|c|c|c|c|c|c|c|c|c|}
\hline Sample ID & $\begin{array}{l}\text { Textural Group from \%GSM } \\
\text { (Gradistat) }\end{array}$ & $\begin{array}{l}\text { Textural Group } \\
\text { from \%SZC } \\
\text { (Gradistat) } \\
\end{array}$ & $\begin{array}{l}\text { Sediment Classification } \\
\text { from Mean Phi } \\
\text { (Wentworth) } \\
\end{array}$ & Sorting (Gradistat) & $\begin{array}{c}\text { Gravel } \\
\%\end{array}$ & $\begin{array}{c}\text { Sand } \\
\%\end{array}$ & $\begin{array}{c}\text { Mud } \\
\%\end{array}$ & $\begin{array}{c}\text { Sand } \\
\%\end{array}$ & $\begin{array}{c}\text { Silt } \\
\% \\
\end{array}$ & $\begin{array}{c}\text { Clay } \\
\% \\
\end{array}$ & $\begin{array}{c}\text { Mean } \\
\text { Size } \\
\text { (phi) } \\
\end{array}$ & $\begin{array}{c}\text { Mean } \\
\text { Size } \\
(\mathrm{mm}) \\
\end{array}$ & $\begin{array}{c}\text { Sorting } \\
\text { (phi) }\end{array}$ & Skewness & Kurtosis & LOI \% \\
\hline MS_65 & Muddy Sand & & Coarse Silt & Very Poorly Sorted & 4.0 & 58.0 & 38.0 & 58.0 & 21.0 & 17.0 & 4.60 & 0.04 & 3.12 & 0.59 & & \\
\hline MS_66 & Sandy Mud & Sandy Mud & Fine Silt & Very Poorly Sorted & 0.0 & 20.0 & 80.0 & 20.0 & 52.0 & 28.0 & 6.20 & 0.01 & 2.70 & 0.43 & & \\
\hline MS_67 & Muddy Gravel & & Fine Silt & Extremely Poorly Sorted & 41.0 & 7.0 & 52.0 & & & & 6.70 & 0.01 & 5.15 & 0.74 & & \\
\hline MS_68 & Muddy Sand & & Fine Sand & Very Poorly Sorted & 5.0 & 78.0 & 17.0 & 78.0 & 9.0 & 8.0 & 2.70 & 0.15 & 2.48 & 0.43 & & \\
\hline MS_69 & Mud & Mud & Very Fine Silt & Very Poorly Sorted & 0.0 & 7.0 & 93.0 & 7.0 & 54.0 & 39.0 & 7.10 & 0.01 & 2.94 & 0.32 & & \\
\hline MS_70 & Mud & Mud & Very Fine Silt & Very Poorly Sorted & 0.0 & 4.0 & 96.0 & 4.0 & 57.0 & 39.0 & 7.30 & 0.01 & 3.04 & 0.48 & & \\
\hline MS_71 & Sandy Mud & & Fine Silt & Very Poorly Sorted & 2.0 & 17.0 & 81.0 & 17.0 & 50.0 & 31.0 & 6.60 & 0.01 & 3.29 & 0.27 & & \\
\hline MS_72 & Muddy Sand & & Fine Sand & Poorly Sorted & 3.0 & 84.0 & 13.0 & 84.0 & 7.0 & 6.0 & 2.50 & 0.18 & 1.93 & 0.32 & & \\
\hline MS_73 & Sandy Mud & & Medium Silt & Very Poorly Sorted & 1.0 & 39.0 & 60.0 & 39.0 & 39.0 & 21.0 & 5.40 & 0.02 & 3.13 & 0.43 & & \\
\hline MS_74 & Sandy Mud & & Fine Silt & Very Poorly Sorted & 1.0 & 15.0 & 84.0 & 15.0 & 53.0 & 31.0 & 6.70 & 0.01 & 2.74 & 0.46 & & \\
\hline MS_75 & Sandy Mud & Sandy Mud & Very Fine Silt & Very Poorly Sorted & 0.0 & 12.0 & 88.0 & 12.0 & 46.0 & 42.0 & 7.50 & 0.01 & 3.33 & 0.18 & & \\
\hline MS_76 & Muddy Gravel & & Medium Silt & Extremely Poorly Sorted & 32.0 & 18.0 & 50.0 & & & & 5.00 & 0.03 & 4.13 & 0.28 & & \\
\hline MS_77 & Sandy Mud & & Medium Silt & Very Poorly Sorted & 3.0 & 40.0 & 57.0 & 40.0 & 37.0 & 20.0 & 5.30 & 0.03 & 3.16 & 0.41 & & \\
\hline MS_78 & Muddy Sand & & Medium Silt & Very Poorly Sorted & 2.0 & 52.0 & 46.0 & 52.0 & 26.0 & 20.0 & 5.10 & 0.03 & 2.97 & 0.58 & & \\
\hline MS_80 & Mud & Mud & Fine Silt & Very Poorly Sorted & 0.0 & 10.0 & 90.0 & 10.0 & 60.0 & 30.0 & 6.80 & 0.01 & 2.79 & 0.31 & & \\
\hline MS_81 & Mud & Mud & Very Fine Silt & Very Poorly Sorted & 0.0 & 5.0 & 95.0 & 5.0 & 56.0 & 39.0 & 7.30 & 0.01 & 2.77 & 0.26 & & \\
\hline MS_82 & Mud & Mud & Very Fine Silt & Very Poorly Sorted & 0.0 & 3.0 & 97.0 & 3.0 & 52.0 & 45.0 & 7.60 & 0.01 & 2.66 & 0.26 & & \\
\hline MS_83 & Sandy Mud & Sandy Mud & Fine Silt & Very Poorly Sorted & 0.0 & 17.0 & 83.0 & 17.0 & 48.0 & 35.0 & 6.40 & 0.01 & 2.84 & 0.33 & & \\
\hline MS_84 & Sandy Mud & Sandy Mud & Fine Silt & Very Poorly Sorted & 0.0 & 16.0 & 84.0 & 16.0 & 56.0 & 28.0 & 6.20 & 0.01 & 2.78 & 0.63 & & \\
\hline MS_85 & Gravelly Mud & & Coarse Silt & Extremely Poorly Sorted & 18.0 & 17.0 & 65.0 & & & & 4.80 & 0.04 & 5.03 & -0.12 & & \\
\hline
\end{tabular}




\section{Appendix B: New Hampshire Continental Shelf 2016-2017 Field Campaign Station Locations}

The stations sampled during the New Hampshire Continental Shelf 2016-2017 Field Campaign were targeted at locations where there was high-resolution bathymetry available and also where ground truth was most needed. The bathymetry includes MBES surveys conducted by the UNH CCOM/JHC Hydrographic Field Course (Ocean Engineering 972) (all GSH or SH), NOS surveys (NSBE), and lidar surveys by the USGS (Shoals) (see Ward et al., 2021h). Here, the stations are grouped by survey or location and year the bathymetry was run. The Station IDs indicate the type of survey (e.g., SH, GSH, Shoals) and/or location (NSBE), the year of the bathymetry survey (i.e., 06, 07, etc.), and the station number (e.g., T03, T04, etc. or S01, S02, etc.). The " $Y$ " in the "Video Recorded" and "Sample Collected" columns indicates video or bottom sediment samples were taken at that station. The "Y" below the "Analyzed (Reported)" column indicates that grain size analysis was run on the sample and is available in the database. Not all samples were analyzed due to size or redundancy at a station.

The sediment grain size data and seafloor photographs from the New Hampshire Continental Shelf 20162017 Field Campaign are also available for viewing at the UNH CCOM/JHC web site: (https://maps.ccom.unh.edu/portal/apps/webappviewer/index.html?id=28df035fe82c423cb3517295d9 bbc24c).

The sediment grain size data for the New Hampshire Continental Shelf 2016-2017 Field Campaign is shown in Appendix C. The database New Hampshire Continental Shelf Geophysical Database: 2016-2017 Field Campaign - Stations and Sediment Data is also available as a Microsoft Excel file at the UNH Scholars Repository (https://dx.doi.org/10.34051/d/2021.2).

All stations with video had seafloor photographs extracted. The seafloor and sample photographs from each station where sediment samples were collected and analyzed are not included in this report due to the size of the databases, but are available at the UNH Scholars Repository: New Hampshire Continental Shelf Geophysical Database: 2016-2017 Field Campaign - Seafloor and Sample Photographs and Sediment Data (https://dx.doi.org/10.34051/d/2021.1) and New Hampshire Continental Shelf Geophysical Database: 2016-2017 Field Campaign - Seafloor Photographs (https://dx.doi.org/10.34051/d/2021.5). 
Appendix B: (continued)

\begin{tabular}{|c|c|c|c|c|c|c|c|}
\hline Station ID & $\begin{array}{l}\text { Latitude } \\
\text { WGS84 }\end{array}$ & $\begin{array}{c}\text { Longitude } \\
\text { WGS84 }\end{array}$ & $\begin{array}{c}\text { Water } \\
\text { Depth (m) }\end{array}$ & $\begin{array}{l}\text { Sample } \\
\text { Collected }\end{array}$ & $\begin{array}{c}\text { Video } \\
\text { Recorded }\end{array}$ & $\begin{array}{l}\text { Sample } \\
\text { Collected }\end{array}$ & $\begin{array}{c}\text { Analyzed } \\
\text { (Reported) }\end{array}$ \\
\hline GSH06_T03 & 43.066875 & -70.670733 & 11.0 & $8 / 1 / 2017$ & $\mathrm{Y}$ & $\mathrm{Y}$ & $\mathrm{Y}$ \\
\hline GSH06_T04 & 43.071793 & -70.660163 & 15.2 & $8 / 1 / 2017$ & $Y$ & $Y$ & $\mathrm{Y}$ \\
\hline GSH06_T06 & 43.078768 & -70.656497 & 10.0 & $8 / 1 / 2017$ & $\mathrm{Y}$ & & \\
\hline GSH06_T07 & 43.088600 & -70.653268 & 8.5 & $8 / 1 / 2017$ & $\mathrm{Y}$ & $Y$ & $\mathrm{Y}$ \\
\hline Shoals07_S01 & 42.872299 & -70.805992 & 9.4 & $10 / 13 / 2016$ & $\mathrm{Y}$ & & \\
\hline Shoals07_S02 & 42.868036 & -70.810027 & 8.5 & $10 / 13 / 2016$ & $Y$ & $\mathrm{Y}$ & $Y$ \\
\hline Shoals07_S03 & 42.868160 & -70.803958 & 9.1 & $10 / 13 / 2016$ & $Y$ & & \\
\hline Shoals07_S04 & 42.864090 & -70.806587 & 14.6 & $10 / 13 / 2016$ & $\mathrm{Y}$ & & \\
\hline Shoals07_S05 & 42.861377 & -70.806588 & 9.1 & $10 / 13 / 2016$ & $Y$ & & \\
\hline Shoals07_S06 & 42.858927 & -70.806217 & 15.8 & $10 / 13 / 2016$ & $Y$ & & \\
\hline Shoals07_S07 & 42.855850 & -70.807233 & 13.4 & $10 / 13 / 2016$ & $Y$ & $Y$ & $Y$ \\
\hline Shoals07_S08 & 42.855847 & -70.803396 & 16.8 & $10 / 13 / 2016$ & $\mathrm{Y}$ & $\mathrm{Y}$ & $Y$ \\
\hline Shoals07_S09 & 42.854926 & -70.805300 & 12.0 & $10 / 6 / 2016$ & $\mathrm{Y}$ & $\mathrm{Y}$ & $\mathrm{Y}$ \\
\hline Shoals07_S09-2 & 42.854691 & -70.805010 & 11.9 & $10 / 13 / 2016$ & $\mathrm{Y}$ & & \\
\hline Shoals07_S11 & 42.849072 & -70.805784 & 13.4 & $10 / 6 / 2016$ & $Y$ & $\mathrm{Y}$ & $Y$ \\
\hline Shoals07_S11-2 & 42.849118 & -70.805506 & 12.2 & $10 / 13 / 2016$ & $Y$ & & \\
\hline Shoals07_S12 & 42.846389 & -70.805687 & 11.3 & $10 / 6 / 2016$ & $Y$ & & \\
\hline Shoals07_S13 & 42.843429 & -70.801355 & 15.2 & $10 / 6 / 2016$ & $Y$ & $\mathrm{Y}$ & $\mathrm{Y}$ \\
\hline Shoals07_S13-2 & 42.843243 & -70.801207 & 14.0 & $10 / 13 / 2016$ & $\mathrm{Y}$ & & \\
\hline Shoals07_S14 & 42.829714 & -70.805199 & 8.8 & $10 / 6 / 2016$ & $Y$ & $\mathrm{Y}$ & $\mathrm{Y}$ \\
\hline Shoals07_S14-3 & 42.829303 & -70.805617 & 7.6 & $10 / 13 / 2016$ & $\mathrm{Y}$ & & \\
\hline GSH12_T01 & 43.061225 & -70.665870 & 20.1 & $8 / 1 / 2017$ & $\mathrm{Y}$ & & \\
\hline GSH12_T02 & 43.065934 & -70.661847 & 16.8 & $8 / 1 / 2017$ & $\mathrm{Y}$ & & \\
\hline GSH12_T03 & 43.062239 & -70.659768 & 14.9 & $8 / 1 / 2017$ & $Y$ & & \\
\hline GSH12_T05 & 43.054176 & -70.655580 & 18.9 & $8 / 1 / 2017$ & $\mathrm{Y}$ & & \\
\hline GSH12_T06 & 43.050548 & -70.655044 & 29.9 & $8 / 1 / 2017$ & $Y$ & $\mathrm{Y}$ & $Y$ \\
\hline GSH12_T08 & 43.065093 & -70.650458 & 12.8 & $8 / 1 / 2017$ & $\mathrm{Y}$ & & \\
\hline GSH12_T09 & 43.060070 & -70.648875 & 20.7 & $7 / 19 / 2017$ & $Y$ & & \\
\hline GSH12_T10 & 43.053340 & -70.643997 & 26.8 & $7 / 19 / 2017$ & $Y$ & $Y$ & $\mathrm{Y}$ \\
\hline GSH12_T11 & 43.047794 & -70.642528 & 29.0 & $7 / 19 / 2017$ & $Y$ & & \\
\hline GSH12_T12 & 43.044385 & -70.635119 & 32.0 & $7 / 19 / 2017$ & $\mathrm{Y}$ & $Y$ & $Y$ \\
\hline
\end{tabular}


Appendix B: Continued

\begin{tabular}{|c|c|c|c|c|c|c|c|}
\hline Station ID & $\begin{array}{l}\text { Latitude } \\
\text { WGS84 }\end{array}$ & $\begin{array}{c}\text { Longitude } \\
\text { WGS84 }\end{array}$ & $\begin{array}{c}\text { Water } \\
\text { Depth (m) }\end{array}$ & $\begin{array}{c}\text { Sample } \\
\text { Collected }\end{array}$ & $\begin{array}{c}\text { Video } \\
\text { Recorded }\end{array}$ & $\begin{array}{c}\text { Sample } \\
\text { Collected }\end{array}$ & $\begin{array}{c}\text { Analyzed } \\
\text { (Reported) }\end{array}$ \\
\hline GSH12_T13 & 43.074722 & -70.651201 & 10.7 & $7 / 19 / 2017$ & $Y$ & & \\
\hline GSH12_T14 & 43.068737 & -70.643519 & 16.8 & $8 / 1 / 2017$ & $Y$ & & \\
\hline GSH12_T15 & 43.063468 & -70.640147 & 14.6 & $8 / 1 / 2017$ & $\mathrm{Y}$ & & \\
\hline GSH12_T16 & 43.050815 & -70.631221 & 20.4 & $7 / 19 / 2017$ & $Y$ & & \\
\hline GSH12_T17 & 43.062383 & -70.631459 & 26.5 & 7/19/2017 & $Y$ & $Y$ & $Y$ \\
\hline GSH12_T18 & 43.078743 & -70.645144 & 15.2 & $8 / 1 / 2017$ & $Y$ & $Y$ & $Y$ \\
\hline GSH12_T19 & 43.082730 & -70.646555 & 10.1 & $8 / 1 / 2017$ & $Y$ & & \\
\hline GSH12_T20 & 43.082758 & -70.650398 & 14.3 & $8 / 1 / 2017$ & $Y$ & $Y$ & $Y$ \\
\hline GSH12_T21 & 43.088377 & -70.645729 & 18.3 & $8 / 1 / 2017$ & $Y$ & $Y$ & $Y$ \\
\hline GSH12_T22 & 43.091586 & -70.650512 & 9.4 & $7 / 19 / 2017$ & $Y$ & $Y$ & $Y$ \\
\hline GSH12_T23 & 43.095915 & -70.645111 & 12.2 & 7/19/2017 & $Y$ & $Y$ & $Y$ \\
\hline GSH12_T24 & 43.089942 & -70.655696 & 7.3 & $8 / 1 / 2017$ & $\mathrm{Y}$ & $\mathrm{Y}$ & $Y$ \\
\hline GSH12_T50 & 43.055388 & -70.628376 & 30.5 & $7 / 19 / 2017$ & $Y$ & & \\
\hline GSH12_T51 & 43.055253 & -70.623333 & 33.2 & $7 / 19 / 2017$ & $Y$ & $Y$ & $Y$ \\
\hline GSH12_T52 & 43.056485 & -70.632533 & 18.3 & $7 / 19 / 2017$ & $Y$ & & \\
\hline SH12-01-A & 43.048528 & -70.649317 & 27.1 & $6 / 28 / 2012$ & $\mathrm{Y}$ & $\mathrm{Y}$ & \\
\hline $\mathrm{SH} 12-02$ & 43.053003 & -70.644819 & 23.5 & $6 / 28 / 2012$ & $Y$ & $Y$ & $Y$ \\
\hline SH12-03-A & 43.052022 & -70.641728 & 17.1 & $6 / 28 / 2012$ & $\mathrm{Y}$ & & \\
\hline SH12-03-B & 43.051731 & -70.641808 & 17.1 & $6 / 28 / 2012$ & $Y$ & & \\
\hline $\mathrm{SH} 12-03-\mathrm{C}$ & 43.052122 & -70.641561 & N/A & $6 / 28 / 2012$ & $Y$ & & \\
\hline $\mathrm{SH} 12-04$ & 43.048156 & -70.639286 & 24.4 & $6 / 28 / 2012$ & $Y$ & $Y$ & $Y$ \\
\hline SH12-05 & 43.043894 & -70.634703 & 32.6 & $6 / 28 / 2012$ & $\mathrm{Y}$ & $\mathrm{Y}$ & $Y$ \\
\hline SH12-06-A & 43.050539 & -70.632264 & 21.6 & $6 / 28 / 2012$ & $Y$ & & \\
\hline SH12-06-B & 43.050553 & -70.632428 & N/A & $6 / 28 / 2012$ & $Y$ & & \\
\hline SH12-07 & 43.050233 & -70.629839 & N/A & $6 / 28 / 2012$ & $Y$ & & \\
\hline SH12-08 & 43.052706 & -70.624306 & 33.2 & $6 / 28 / 2012$ & $Y$ & $Y$ & \\
\hline SH12-09 & 43.055544 & -70.623825 & 33.5 & $6 / 28 / 2012$ & $Y$ & $Y$ & $\mathrm{Y}$ \\
\hline $\mathrm{SH} 12-10$ & 43.061981 & -70.649350 & N/A & $6 / 28 / 2012$ & $Y$ & $Y$ & \\
\hline SH12-11 & 43.057017 & -70.650367 & N/A & $6 / 28 / 2012$ & $Y$ & $Y$ & $Y$ \\
\hline SH12-12 & 43.094919 & -70.639372 & N/A & $6 / 28 / 2012$ & $Y$ & $Y$ & $Y$ \\
\hline SH12-13 & 43.097294 & -70.648036 & 10.1 & $6 / 28 / 2012$ & $Y$ & $Y$ & $Y$ \\
\hline SH12-14 & 43.096169 & -70.646881 & 11.3 & $6 / 28 / 2012$ & $Y$ & $Y$ & $Y$ \\
\hline SH12-15-A & 43.093869 & -70.648525 & 8.2 & $6 / 28 / 2012$ & $Y$ & & \\
\hline SH12-15-B & 43.093020 & -70.647627 & 13.7 & $6 / 28 / 2012$ & $Y$ & $Y$ & $Y$ \\
\hline SH12-16 & 43.091936 & -70.651581 & 8.8 & $6 / 28 / 2012$ & $Y$ & $Y$ & $Y$ \\
\hline SH12-17 & 43.063233 & -70.663283 & 22.6 & $6 / 28 / 2012$ & $Y$ & $Y$ & $Y$ \\
\hline
\end{tabular}


Appendix B: Continued

\begin{tabular}{|c|c|c|c|c|c|c|c|}
\hline Station ID & $\begin{array}{l}\text { Latitude } \\
\text { WGS84 }\end{array}$ & $\begin{array}{c}\text { Longitude } \\
\text { WGS84 }\end{array}$ & $\begin{array}{c}\text { Water } \\
\text { Depth (m) }\end{array}$ & $\begin{array}{c}\text { Sample } \\
\text { Collected }\end{array}$ & $\begin{array}{c}\text { Video } \\
\text { Recorded }\end{array}$ & $\begin{array}{l}\text { Sample } \\
\text { Collected }\end{array}$ & $\begin{array}{c}\text { Analyzed } \\
\text { (Reported) }\end{array}$ \\
\hline GSH13-T10 & 43.026381 & -70.714468 & 11.0 & $7 / 17 / 2017$ & $Y$ & & \\
\hline GSH13-T11 & 43.024318 & -70.713641 & 13.4 & $7 / 17 / 2017$ & $Y$ & $\mathrm{Y}$ & $Y$ \\
\hline GSH13-T13 & 43.022075 & -70.719898 & 11.0 & $7 / 17 / 2017$ & $Y$ & $Y$ & $\mathrm{Y}$ \\
\hline GSH13-T14 & 43.020354 & -70.722841 & 9.4 & $7 / 17 / 2017$ & $Y$ & $Y$ & $Y$ \\
\hline GSH13-T15 & 43.017187 & -70.719325 & 15.5 & $7 / 17 / 2017$ & $\mathrm{Y}$ & $\mathrm{Y}$ & $\mathrm{Y}$ \\
\hline GSH13-T16 & 43.014625 & -70.722427 & 13.4 & 7/17/2017 & Y & & \\
\hline GSH13-T17 & 43.014386 & -70.726680 & 8.5 & $7 / 17 / 2017$ & $Y$ & $\mathrm{Y}$ & $Y$ \\
\hline GSH14_T12 & 42.992188 & -70.726966 & 17.1 & $7 / 18 / 2017$ & $Y$ & & \\
\hline GSH14_T14 & 42.983649 & -70.738158 & 15.8 & $7 / 18 / 2017$ & Y & $\mathrm{Y}$ & Y \\
\hline GSH14_T15 & 42.982267 & -70.736127 & 18.9 & $7 / 18 / 2017$ & $Y$ & $Y$ & $Y$ \\
\hline GSH14_T16 & 42.978441 & -70.736979 & 19.8 & $7 / 18 / 2017$ & $\mathrm{Y}$ & $\mathrm{Y}$ & $Y$ \\
\hline GSH14_T17 & 42.977165 & -70.740457 & 19.5 & $11 / 1 / 2016$ & $Y$ & $\mathrm{Y}$ & Y \\
\hline GSH14_T18 & 42.974451 & -70.743121 & 17.4 & $11 / 1 / 2016$ & Y & & \\
\hline GSH14_T19 & 42.972294 & -70.745278 & 16.2 & $11 / 1 / 2016$ & Y & & \\
\hline GSH14_T20 & 42.974777 & -70.749365 & 17.4 & $11 / 1 / 2016$ & $Y$ & $Y$ & $Y$ \\
\hline GSH14_T21 & 42.974018 & -70.750086 & 15.8 & $7 / 18 / 2017$ & $\mathrm{Y}$ & & \\
\hline GSH14_T23 & 42.978113 & -70.746629 & 15.8 & $11 / 1 / 2016$ & $Y$ & & \\
\hline GSH14_T24 & 42.979850 & -70.745290 & 14.9 & $7 / 18 / 2017$ & $\mathrm{Y}$ & $\mathrm{Y}$ & $Y$ \\
\hline GSH14_T25 & 42.981999 & -70.745452 & 15.2 & $11 / 1 / 2016$ & $Y$ & & \\
\hline GSH14_T27 & 42.986960 & -70.743435 & 12.2 & $7 / 18 / 2017$ & $Y$ & & \\
\hline GSH14_T35 & 42.979467 & -70.742292 & 16.5 & $7 / 18 / 2017$ & $\mathrm{Y}$ & $\mathrm{Y}$ & $Y$ \\
\hline GSH14_T36 & 42.979925 & -70.752487 & 8.2 & $7 / 18 / 2017$ & $\mathrm{Y}$ & $\mathrm{Y}$ & Y \\
\hline SH14_BS-01A & 42.972965 & -70.743245 & 15.8 & $6 / 30 / 2014$ & $Y$ & & \\
\hline SH14_BS-02 & 42.979475 & -70.741855 & 18.3 & $6 / 30 / 2014$ & $\mathrm{Y}$ & $Y$ & $Y$ \\
\hline SH14_BS-Extra & 42.983121 & -70.752974 & 8.1 & $6 / 30 / 2014$ & $Y$ & $Y$ & \\
\hline SH14_BS-03A & 42.983972 & -70.736481 & 20.3 & $6 / 30 / 2014$ & $\mathrm{Y}$ & $\mathrm{Y}$ & $\mathrm{Y}$ \\
\hline SH14_BS-03B & 42.983936 & -70.736288 & 20.9 & $6 / 30 / 2014$ & $\mathrm{Y}$ & $\mathrm{Y}$ & $\mathrm{Y}$ \\
\hline SH14_BS-04A & 42.990993 & -70.731050 & 16.9 & $6 / 30 / 2014$ & Y & $\mathrm{Y}$ & \\
\hline SH14_BS-04B & 42.991207 & -70.731278 & 16.8 & $6 / 30 / 2014$ & Y & & \\
\hline SH14_BS-05 & 42.996231 & -70.740064 & 10.6 & $6 / 30 / 2014$ & $\mathrm{Y}$ & $Y$ & $Y$ \\
\hline SH14_BS-06 & 42.995856 & -70.728489 & 17.7 & $7 / 1 / 2014$ & $Y$ & $Y$ & $\mathrm{Y}$ \\
\hline SH14_BS-07 & 43.000470 & -70.727247 & 16.5 & $7 / 1 / 2014$ & $Y$ & $\mathrm{Y}$ & $Y$ \\
\hline SH14_BS-08 & 43.000737 & -70.722898 & 19.8 & $7 / 1 / 2014$ & $Y$ & $Y$ & $Y$ \\
\hline SH14_BS-09 & 43.006318 & -70.720523 & 8.8 & $7 / 1 / 2014$ & $Y$ & $Y$ & $Y$ \\
\hline SH14_BS-10 & 43.008803 & -70.714451 & 20.7 & $7 / 1 / 2014$ & $Y$ & $Y$ & $Y$ \\
\hline SH14_BS-11 & 42.996592 & -70.727623 & 17.4 & $7 / 1 / 2014$ & $Y$ & $Y$ & \\
\hline GSH15_T01 & 42.930871 & -70.790805 & 7.6 & $11 / 1 / 2016$ & $Y$ & $Y$ & $Y$ \\
\hline
\end{tabular}


Appendix B: Continued

\begin{tabular}{|c|c|c|c|c|c|c|c|}
\hline Station ID & $\begin{array}{c}\text { Latitude } \\
\text { WGS84 }\end{array}$ & $\begin{array}{c}\text { Longitude } \\
\text { WGS84 }\end{array}$ & $\begin{array}{c}\text { Water } \\
\text { Depth (m) }\end{array}$ & $\begin{array}{l}\text { Sample } \\
\text { Collected }\end{array}$ & $\begin{array}{c}\text { Video } \\
\text { Recorded }\end{array}$ & $\begin{array}{l}\text { Sample } \\
\text { Collected }\end{array}$ & $\begin{array}{c}\text { Analyzed } \\
\text { (Reported) }\end{array}$ \\
\hline GSH15_T02 & 42.926577 & -70.790042 & 8.8 & $11 / 1 / 2016$ & $Y$ & $\mathrm{Y}$ & $Y$ \\
\hline GSH15_T04 & 42.943020 & -70.777881 & 14.3 & $11 / 1 / 2016$ & $Y$ & & \\
\hline GSH15_T05 & 42.947430 & -70.775635 & 13.1 & $11 / 1 / 2016$ & $Y$ & $\mathrm{Y}$ & $\mathrm{Y}$ \\
\hline GSH15_T06 & 42.941297 & -70.771655 & 15.2 & $11 / 1 / 2016$ & $\mathrm{Y}$ & & \\
\hline GSH15_T07 & 42.935006 & -70.767757 & 21.9 & $10 / 6 / 2016$ & $\mathrm{Y}$ & $\mathrm{Y}$ & $Y$ \\
\hline GSH15_T08 & 42.932906 & -70.773033 & 20.1 & $11 / 1 / 2016$ & $Y$ & $Y$ & $Y$ \\
\hline GSH15_T09 & 42.927505 & -70.777355 & 18.3 & $11 / 1 / 2016$ & $Y$ & & \\
\hline GSH15_T10 & 42.922464 & -70.780157 & 15.8 & $10 / 6 / 2016$ & $Y$ & $\mathrm{Y}$ & $Y$ \\
\hline GSH15_T12 & 42.924009 & -70.786317 & 12.2 & $11 / 1 / 2016$ & $Y$ & & \\
\hline GSH15_T14 & 42.944763 & -70.761670 & 23.5 & $11 / 1 / 2016$ & $\mathrm{Y}$ & $Y$ & $Y$ \\
\hline GSH15_T15 & 42.941103 & -70.762389 & 82.0 & $11 / 1 / 2016$ & $Y$ & $Y$ & $Y$ \\
\hline GSH15_T17 & 42.951865 & -70.764080 & 14.3 & $11 / 1 / 2016$ & Y & & \\
\hline GSH15_T18 & 42.957298 & -70.767873 & 9.8 & $11 / 1 / 2016$ & $\mathrm{Y}$ & & \\
\hline GSH15_T19 & 42.955194 & -70.761460 & 12.8 & $11 / 1 / 2016$ & Y & & \\
\hline GSH16-T01 & 43.045297 & -70.697871 & 16.8 & $7 / 10 / 2017$ & $Y$ & $\mathrm{Y}$ & $Y$ \\
\hline GSH16-T02 & 43.042899 & -70.697748 & 17.1 & $7 / 10 / 2017$ & $Y$ & $Y$ & $Y$ \\
\hline GSH16-T03 & 43.041998 & -70.694267 & 16.2 & $7 / 10 / 2017$ & $Y$ & Y & $\mathrm{Y}$ \\
\hline GSH16-T04 & 43.039079 & -70.686425 & 21.3 & $7 / 10 / 2017$ & $\mathrm{Y}$ & $\mathrm{Y}$ & $\mathrm{Y}$ \\
\hline GSH16-T05 & 43.035313 & -70.683228 & 25.9 & $7 / 10 / 2017$ & $Y$ & $Y$ & $Y$ \\
\hline GSH16-T06 & 43.031908 & -70.682273 & 27.7 & $7 / 10 / 2017$ & $Y$ & $Y$ & $\mathrm{Y}$ \\
\hline GSH16-T07 & 43.026767 & -70.677968 & 25.9 & $7 / 11 / 2017$ & $Y$ & $Y$ & $Y$ \\
\hline GSH16-T08 & 43.022048 & -70.682323 & 23.5 & $7 / 11 / 2017$ & $Y$ & & \\
\hline GSH16-T09 & 43.018776 & -70.687632 & 18.3 & $7 / 11 / 2017$ & $Y$ & & \\
\hline GSH16-T10 & 43.021826 & -70.702667 & 8.8 & $7 / 11 / 2017$ & $Y$ & & \\
\hline GSH16-T11 & 43.024464 & -70.704939 & 18.9 & $7 / 11 / 2017$ & $\mathrm{Y}$ & $Y$ & $Y$ \\
\hline GSH16-T12 & 43.025693 & -70.710856 & 15.8 & $7 / 11 / 2017$ & $Y$ & $Y$ & $Y$ \\
\hline GSH16-T13 & 43.030201 & -70.708662 & 16.5 & $7 / 11 / 2017$ & $Y$ & & \\
\hline GSH16-T14 & 43.030153 & -70.702878 & 17.7 & $7 / 11 / 2017$ & Y & $Y$ & $Y$ \\
\hline GSH16-T15 & 43.031050 & -70.698484 & 17.7 & $7 / 11 / 2017$ & $Y$ & $Y$ & $Y$ \\
\hline GSH16-T16 & 43.033065 & -70.698736 & 16.8 & $7 / 11 / 2017$ & $Y$ & & \\
\hline GSH16-T17 & 43.033956 & -70.693845 & 18.3 & $7 / 11 / 2017$ & $Y$ & $\mathrm{Y}$ & $\mathrm{Y}$ \\
\hline GSH16-T18 & 43.036400 & -70.695581 & 16.8 & $7 / 11 / 2017$ & $Y$ & $Y$ & $Y$ \\
\hline GSH16-TSO6 & 42.855997 & -70.794752 & 18.0 & $10 / 13 / 2016$ & $Y$ & $Y$ & $Y$ \\
\hline GSH16-TS09 & 42.857124 & -70.799412 & 16.5 & $10 / 13 / 2016$ & $Y$ & $Y$ & $Y$ \\
\hline GSH16-TS10 & 42.853463 & -70.799742 & 17.4 & $10 / 13 / 2016$ & $Y$ & $Y$ & $Y$ \\
\hline SH18-T01 & 43.110880 & 70.621355 & 25.0 & $7 / 9 / 2018$ & $Y$ & $Y$ & $Y$ \\
\hline SH18-T02 & 43.111675 & 70.624758 & 18.2 & $7 / 9 / 2018$ & $Y$ & $Y$ & \\
\hline
\end{tabular}


Appendix B: Continued

\begin{tabular}{|c|c|c|c|c|c|c|c|}
\hline Station ID & $\begin{array}{l}\text { Latitude } \\
\text { WGS84 }\end{array}$ & $\begin{array}{c}\text { Longitude } \\
\text { WGS84 }\end{array}$ & $\begin{array}{c}\text { Water } \\
\text { Depth (m) }\end{array}$ & $\begin{array}{c}\text { Sample } \\
\text { Collected }\end{array}$ & $\begin{array}{c}\text { Video } \\
\text { Recorded }\end{array}$ & $\begin{array}{l}\text { Sample } \\
\text { Collected }\end{array}$ & $\begin{array}{c}\text { Analyzed } \\
\text { (Reported) }\end{array}$ \\
\hline SH18-T03 & 43.112021 & 70.617811 & 22.5 & $7 / 9 / 2018$ & $\mathrm{Y}$ & $\mathrm{Y}$ & \\
\hline SH18-T04 & 43.115655 & 70.625815 & 13.6 & $7 / 9 / 2018$ & $Y$ & $\mathrm{Y}$ & \\
\hline SH18-T05 & 43.097957 & 70.635421 & 14.7 & $7 / 9 / 2018$ & $Y$ & $Y$ & \\
\hline SH18-T06 & 43.098228 & 70.630833 & 14.7 & $7 / 9 / 2018$ & $Y$ & $Y$ & \\
\hline SH18-T07 & 43.100331 & 70.629278 & 21.3 & $7 / 9 / 2018$ & $\mathrm{Y}$ & $\mathrm{Y}$ & $Y$ \\
\hline SH18-T08 & 43.098002 & 70.625083 & 22.3 & $7 / 9 / 2018$ & $Y$ & & \\
\hline SH18-T09 & 43.095290 & 70.627049 & 20.0 & $7 / 9 / 2018$ & $\mathrm{Y}$ & $\mathrm{Y}$ & $\mathrm{Y}$ \\
\hline SH18-T10 & 43.085579 & 70.636676 & 27.1 & $7 / 9 / 2018$ & $\mathrm{Y}$ & $\mathrm{Y}$ & $Y$ \\
\hline SH18-T11 & 43.078903 & 70.640364 & 13.9 & $7 / 9 / 2018$ & $Y$ & $Y$ & \\
\hline SH18-T12 & 43.075575 & 70.638538 & 10.3 & $7 / 9 / 2018$ & $Y$ & & \\
\hline NSBE-T01 & 42.968322 & -70.660788 & 43.6 & $8 / 8 / 2017$ & $\mathrm{Y}$ & $Y$ & $Y$ \\
\hline NSBE-T02 & 42.971764 & -70.653901 & 40.5 & $8 / 8 / 2017$ & $Y$ & $Y$ & $Y$ \\
\hline NSBE-T03 & 42.976383 & -70.643870 & 35.7 & $8 / 7 / 2017$ & $Y$ & $Y$ & $Y$ \\
\hline NSBE-T04 & 42.990159 & -70.638573 & 20.7 & $8 / 7 / 2017$ & $Y$ & $Y$ & $Y$ \\
\hline NSBE-T05 & 42.986699 & -70.645589 & 24.4 & $8 / 7 / 2017$ & $Y$ & $Y$ & $Y$ \\
\hline NSBE-T06 & 42.981125 & -70.655257 & 26.8 & $8 / 7 / 2017$ & $\mathrm{Y}$ & & \\
\hline NSBE-T06a & 42.980573 & -70.655550 & 27.1 & $8 / 7 / 2017$ & $Y$ & $Y$ & $Y$ \\
\hline NSBE-T07 & 42.977264 & -70.669788 & 24.7 & $8 / 7 / 2017$ & $\mathrm{Y}$ & & \\
\hline NSBE-T08 & 42.980381 & -70.672999 & 21.0 & $8 / 7 / 2017$ & $Y$ & & \\
\hline NSBE-T09a & 42.981201 & -70.677443 & 21.9 & $8 / 7 / 2017$ & $Y$ & & \\
\hline NSBE-T10 & 42.976370 & -70.678328 & 37.5 & $8 / 7 / 2017$ & $\mathrm{Y}$ & $\mathrm{Y}$ & $Y$ \\
\hline NSBE-T11 & 42.981649 & -70.686066 & 30.8 & $8 / 8 / 2017$ & $Y$ & & \\
\hline NSBE-T12 & 42.984786 & -70.685358 & 32.3 & $8 / 8 / 2017$ & $Y$ & $Y$ & $Y$ \\
\hline NSBE-T13 & 42.987658 & -70.660873 & 30.5 & $8 / 8 / 2017$ & $Y$ & $Y$ & $Y$ \\
\hline NSBE-T14 & 42.990282 & -70.668139 & 26.8 & $8 / 8 / 2017$ & $Y$ & $Y$ & $Y$ \\
\hline NSBE-T15 & 42.991793 & -70.674793 & 29.9 & $8 / 8 / 2017$ & $\mathrm{Y}$ & $Y$ & $Y$ \\
\hline NSBE-T16 & 42.996550 & -70.670892 & 33.5 & $8 / 8 / 2017$ & $\mathrm{Y}$ & & \\
\hline NSBE-T17 & 42.991445 & -70.689473 & 19.5 & $8 / 16 / 2017$ & $Y$ & & \\
\hline NSBE-T18 & 42.990468 & -70.695505 & 17.4 & $8 / 16 / 2017$ & $Y$ & & \\
\hline NSBE-T19 & 42.989167 & -70.699695 & 21.3 & $8 / 8 / 2017$ & $Y$ & & \\
\hline NSBE-T20 & 42.987371 & -70.694519 & 25.3 & $8 / 8 / 2017$ & $\mathrm{Y}$ & $Y$ & $\mathrm{Y}$ \\
\hline NSBE-T21a & 42.991405 & -70.702695 & 27.4 & $8 / 8 / 2017$ & $\mathrm{Y}$ & $Y$ & $Y$ \\
\hline NSBE-T22 & 43.001453 & -70.657417 & 19.2 & $8 / 15 / 2017$ & $Y$ & & \\
\hline NSBE-T23 & 43.005541 & -70.668076 & 17.1 & $8 / 15 / 2017$ & $\mathrm{Y}$ & & \\
\hline NSBE-T24 & 43.011734 & -70.676721 & 18.9 & $8 / 16 / 2017$ & $Y$ & & \\
\hline NSBE-T25 & 43.009708 & -70.664556 & 27.7 & $8 / 15 / 2017$ & $Y$ & & \\
\hline NSBE-T26 & 43.003448 & -70.672623 & 26.8 & $8 / 15 / 2017$ & $Y$ & & \\
\hline NSBE-T27 & 43.015685 & -70.676396 & 27.4 & $8 / 16 / 2017$ & $\mathrm{Y}$ & & \\
\hline
\end{tabular}


Appendix B: Continued

\begin{tabular}{|c|c|c|c|c|c|c|c|}
\hline Station ID & $\begin{array}{l}\text { Latitude } \\
\text { WGS84 }\end{array}$ & $\begin{array}{c}\text { Longitude } \\
\text { WGS84 }\end{array}$ & $\begin{array}{c}\text { Water } \\
\text { Depth (m) }\end{array}$ & $\begin{array}{c}\text { Sample } \\
\text { Collected }\end{array}$ & $\begin{array}{c}\text { Video } \\
\text { Recorded }\end{array}$ & $\begin{array}{l}\text { Sample } \\
\text { Collected }\end{array}$ & $\begin{array}{c}\text { Analyzed } \\
\text { (Reported) }\end{array}$ \\
\hline NSBE-T28 & 42.978978 & -70.674840 & 19.8 & $8 / 15 / 2017$ & $Y$ & & \\
\hline NSBE-T29 & 42.979621 & -70.679554 & 26.5 & $8 / 15 / 2017$ & $Y$ & $Y$ & $Y$ \\
\hline NSBE-T30 & 42.985811 & -70.671644 & 26.2 & $8 / 15 / 2017$ & $Y$ & $Y$ & $Y$ \\
\hline NSBE-T31 & 42.987403 & -70.669282 & 33.5 & $8 / 15 / 2017$ & $\mathrm{Y}$ & & \\
\hline NSBE-T32 & 42.985106 & -70.663309 & 34.7 & $8 / 15 / 2017$ & $Y$ & $Y$ & $\mathrm{Y}$ \\
\hline NSBE-T33 & 42.984001 & -70.673906 & 20.1 & $8 / 15 / 2017$ & $Y$ & & \\
\hline NSBE-T34 & 42.982058 & -70.702832 & 18.9 & $8 / 16 / 2017$ & $Y$ & & \\
\hline NSBE-T35 & 42.990936 & -70.712882 & 13.7 & $8 / 16 / 2017$ & $Y$ & & \\
\hline NSBE-T36 & 42.995784 & -70.710278 & 25.6 & $8 / 16 / 2017$ & $Y$ & $Y$ & $Y$ \\
\hline NSBE-T37 & 42.997882 & -70.688657 & 14.3 & $8 / 16 / 2017$ & $Y$ & & \\
\hline NSBE-T38 & 43.000245 & -70.701154 & 14.6 & $8 / 16 / 2017$ & $Y$ & & \\
\hline NSBE-T39 & 43.005076 & -70.694332 & 23.5 & $8 / 16 / 2017$ & $Y$ & $Y$ & $Y$ \\
\hline NSBE-T40 & 42.973863 & -70.698420 & 32.9 & $8 / 16 / 2017$ & $Y$ & $Y$ & $\mathrm{Y}$ \\
\hline NSBE-T41 & 42.983621 & -70.710607 & 16.8 & $8 / 16 / 2017$ & $\mathrm{Y}$ & & \\
\hline NSBE-T42 & 42.971760 & -70.670194 & 37.2 & $8 / 16 / 2017$ & $Y$ & $Y$ & $Y$ \\
\hline NSBE-T43 & 43.007699 & -70.664900 & 27.4 & $8 / 15 / 2017$ & $\mathrm{Y}$ & & \\
\hline NSBE-T45 & 43.026969 & -70.648673 & 33.5 & $8 / 16 / 2017$ & $Y$ & $Y$ & $Y$ \\
\hline NSBE-T47 & 43.006194 & -70.679682 & 26.8 & $8 / 16 / 2017$ & $Y$ & & \\
\hline NSBE-T52 & 43.022767 & -70.666995 & 21.6 & $8 / 16 / 2017$ & $Y$ & & \\
\hline NSBE-T59 & 42.998502 & -70.648205 & 27.4 & $8 / 15 / 2017$ & $Y$ & & \\
\hline NSBE-T60 & 42.995995 & -70.652881 & 21.9 & $8 / 15 / 2017$ & $Y$ & & \\
\hline NSBE-T61 & 42.992317 & -70.659907 & 30.2 & $8 / 16 / 2017$ & $Y$ & $Y$ & $Y$ \\
\hline NSBE-T62 & 42.985456 & -70.653036 & 23.8 & $8 / 15 / 2017$ & $Y$ & $Y$ & $Y$ \\
\hline NSBE-T64 & 42.978964 & -70.663495 & 25.6 & $8 / 15 / 2017$ & $Y$ & $Y$ & $Y$ \\
\hline
\end{tabular}




\section{Appendix C: New Hampshire Continental Shelf 2016-2017 Field Campaign: Grain Size Data}

Sediment grain size data from the major field campaign conducted in 2016-2017 on the NH continental shelf are presented here, along with grain size data from the UNH Ocean Engineering 972 Hydrographic Field Course classes in 2012, 2014, and 2018. In total, thirteen one-day cruises provided 150 samples for grain size analysis.

This appendix provides complete descriptions for each sample including identification, station and sample characteristics, sediment classifications, grain size statistics, and grain size distribution. Samples were analyzed with standard sieve and pipette analyses after Folk (1980). The sediment grain size classifications include: CMECS (Coastal and Marine Ecological Classification Standard; FGDC, 2012); Gradistat (Blott and Pye, 2001); and Wentworth (Wentworth, 1922; described in Folk, 1954, 1980). Statistics are based on the phi scale and include the graphic mean, sorting, skewness, and kurtosis (Folk, 1980).

The grain size data is grouped by survey or location and year the bathymetry was run. The Station IDs indicate the type of survey (e.g., SH, GSH, Shoals) and/or location (NSBE), the year of the bathymetry survey (i.e., 06, 07, etc.), and the station number (e.g., T03, T04, etc. or S01, S02, etc.). If needed, the cruises are divided into separate sections [e.g., NSBE (1), NSBE (2), etc.]. Data for each cruise is spread across four pages, with each sample identified in the first column by the Sample ID. Each sample has a "Reliability Ranking for Positioning" which gives an estimate of the uncertainty of the location of the sample classified from 1 to 4 . Explanation of uncertainty and the numbering system is given in "Positioning Uncertainty of Station Locations" and in Table 3.

The sediment grain size data and seafloor photographs from the New Hampshire Continental Shelf 20162017 Field Campaign are also available for viewing at the UNH CCOM/JHC web site: (https://maps.ccom.unh.edu/portal/apps/webappviewer/index.html?id=28df035fe82c423cb3517295d9 bbc24c).

The sediment grain size database New Hampshire Continental Shelf Geophysical Database: 2016-2017 Field Campaign - Stations and Sediment Data is also available as a Microsoft Excel file at the UNH Scholars Repository (https://dx.doi.org/10.34051/d/2021.2).

"Single sample summaries" presenting the grain size data along with photographs of the seafloor and the sediment samples are also available through the UNH Scholars Repository: New Hampshire Continental Shelf Geophysical Database: 2016-2017 Field Campaign - Seafloor and Sample Photographs and Sediment Data (https://dx.doi.org/10.34051/d/2021.1) . 
Section 1: GSH12 
GSH12: Identification, Location, and Description

\begin{tabular}{|c|c|c|c|c|c|c|c|c|}
\hline Sample ID & Global Sample ID & Station ID & $\begin{array}{l}\text { Latitude } \\
\text { WGS84 }\end{array}$ & $\begin{array}{c}\text { Longitude } \\
\text { WGS84 }\end{array}$ & $\begin{array}{l}\text { Reliability } \\
\text { Ranking for } \\
\text { Positioning }\end{array}$ & $\begin{array}{c}\text { Water } \\
\text { Depth }(\mathrm{m})\end{array}$ & $\begin{array}{l}\text { Sample } \\
\text { Collected }\end{array}$ & $\begin{array}{c}\text { Total Wt } \\
\text { (gm) }\end{array}$ \\
\hline GSH12-T06-S1 & GSH12-T06-S1_8/7/2017 & GSH12-T06 & 43.050548 & -70.655044 & 1 & 29.9 & $8 / 7 / 2017$ & 1071.1 \\
\hline GSH12-T06-S2 & GSH12-T06-S2_8/7/2017 & GSH12-T06 & 43.050665 & -70.654846 & 1 & 29.3 & $8 / 7 / 2017$ & 861.1 \\
\hline GSH12-T10-S1 & GSH12-T10-S1_7/19/2017 & GSH12-T10 & 43.053340 & -70.643999 & 1 & 26.8 & $7 / 19 / 2017$ & 301.2 \\
\hline GSH12-T10-S2 & GSH12-T10-S2_7/19/2017 & GSH12-T10 & 43.053312 & -70.643906 & 1 & 26.5 & $7 / 19 / 2017$ & 381.8 \\
\hline GSH12-T12-S1 & GSH12-T12-S1_7/19/2017 & GSH12-T12 & 43.044385 & -70.635119 & 1 & 32.0 & $7 / 19 / 2017$ & 259.0 \\
\hline GSH12-T12-S2 & GSH12-T12-S2_7/19/2017 & GSH12-T12 & 43.044436 & -70.635434 & 1 & 32.0 & $7 / 19 / 2017$ & 361.0 \\
\hline GSH12-T17-S1 & GSH12-T17-S1_7/19/2017 & GSH12-T17 & 43.062383 & -70.631459 & 1 & 26.5 & $7 / 19 / 2017$ & 307.1 \\
\hline GSH12-T17-S2 & GSH12-T17-S2_7/19/2017 & GSH12-T17 & 43.062393 & -70.631335 & 1 & 26.8 & $7 / 19 / 2017$ & 249.5 \\
\hline GSH12-T18-S1 & GSH12-T18-S1_8/1/2017 & GSH12-T18 & 43.078743 & -70.645144 & 1 & 15.2 & $8 / 1 / 2017$ & 336.7 \\
\hline GSH12-T18-S2 & GSH12-T18-S2_8/1/2017 & GSH12-T18 & 43.078727 & -70.645136 & 1 & 15.2 & $8 / 1 / 2017$ & 598.9 \\
\hline GSH12-T20-S1 & GSH12-T20-S1_8/1/2017 & GSH12-T20 & 43.082758 & -70.650398 & 2 & 14.3 & $8 / 1 / 2017$ & 305.2 \\
\hline GSH12-T2O-S2 & GSH12-T20-S2_8/1/2017 & GSH12-T20 & 43.082805 & -70.650454 & 1 & 13.7 & $8 / 1 / 2017$ & 263.3 \\
\hline GSH12-T21-S1 & GSH12-T21-S1_8/1/2017 & GSH12-T21 & 43.088377 & -70.645729 & 1 & 18.3 & $8 / 1 / 2017$ & 458.9 \\
\hline GSH12-T21-S2 & GSH12-T21-S2_8/1/2017 & GSH12-T21 & 43.088509 & -70.645753 & 1 & 18.3 & $8 / 1 / 2017$ & 1068.9 \\
\hline GSH12-T22-S1 & GSH12-T22-S1_7/19/2017 & GSH12-T22 & 43.091586 & -70.650512 & 1 & 9.4 & $7 / 19 / 2017$ & 95.7 \\
\hline GSH12-T22-S2 & GSH12-T22-S2_7/19/2017 & GSH12-T22 & 43.091839 & -70.651043 & 1 & 9.1 & $7 / 19 / 2017$ & 89.3 \\
\hline GSH12-T23-S1 & GSH12-T23-S1_7/19/2017 & GSH12-T23 & 43.095915 & -70.645111 & 1 & 12.2 & $7 / 19 / 2017$ & 105.9 \\
\hline GSH12-T23-S2 & GSH12-T23-S2_7/19/2017 & GSH12-T23 & 43.096115 & -70.645381 & 1 & 12.2 & $7 / 19 / 2017$ & 89.4 \\
\hline GSH12-T24-S1 & GSH12-T24-S1_8/1/2017 & GSH12-T24 & 43.089942 & -70.655696 & 1 & 7.3 & $8 / 1 / 2017$ & 87.0 \\
\hline GSH12-T24-S2 & GSH12-T24-S2_8/1/2017 & GSH12-T24 & 43.089975 & -70.655647 & 1 & 7.3 & $8 / 1 / 2017$ & 111.2 \\
\hline GSH12-T51-S1 & GSH12-T51-S1_7/19/2017 & GSH12-T51 & 43.055253 & -70.623333 & 1 & 33.2 & $7 / 19 / 2017$ & 549.6 \\
\hline GSH12-T51-S2 & GSH12-T51-S2_7/19/2017 & GSH12-T51 & 43.055192 & -70.623168 & 1 & 33.5 & $7 / 19 / 2017$ & 411.4 \\
\hline
\end{tabular}




\section{GSH12: Sediment Classifications}

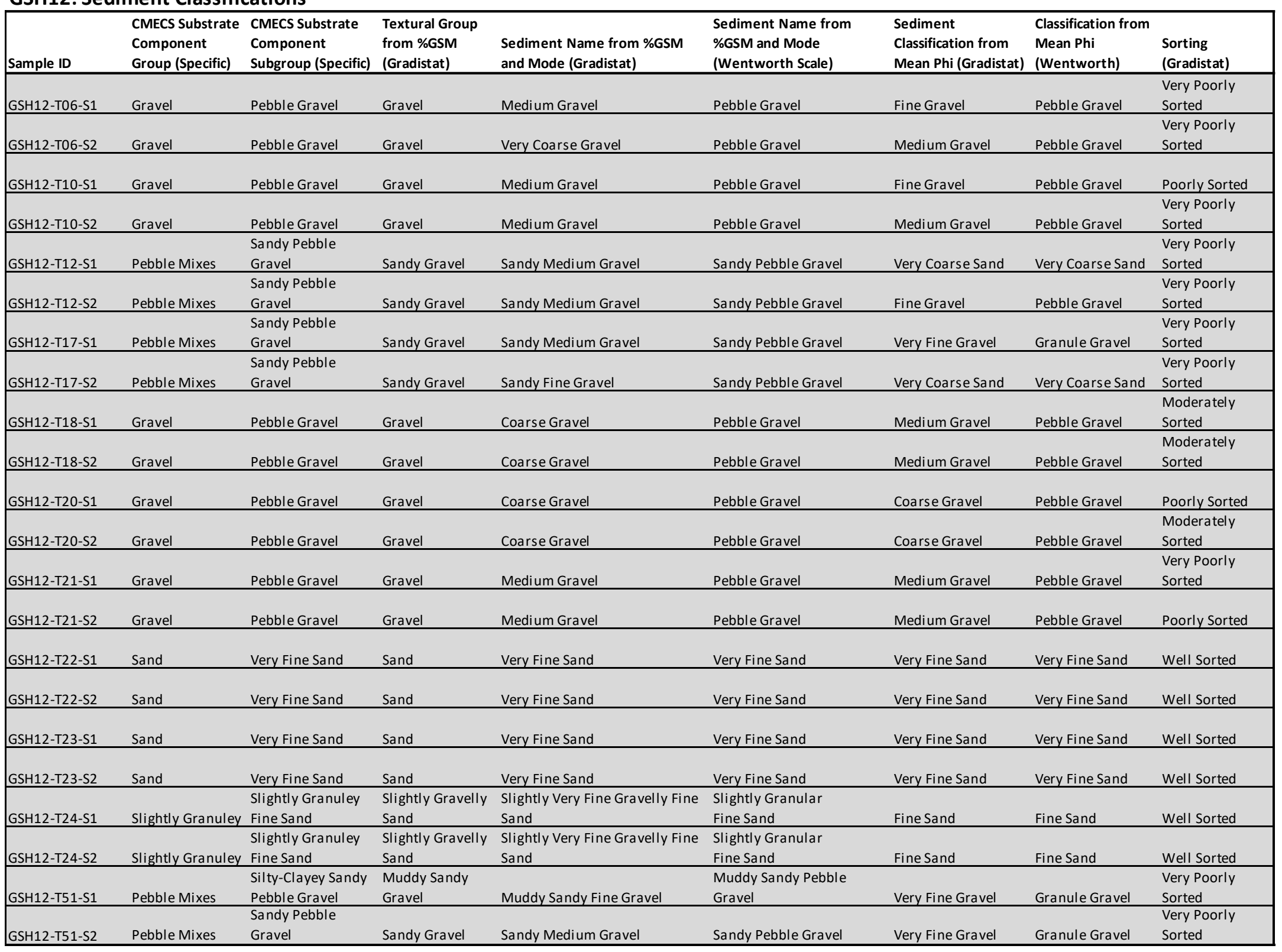


GSH12: Grain Size Statistics

\begin{tabular}{|c|c|c|c|c|c|c|c|c|c|c|c|c|c|c|c|c|c|c|c|c|}
\hline Sample ID & $\begin{array}{c}\text { Gravel } \\
\%\end{array}$ & $\begin{array}{c}\text { Pebble } \\
\%\end{array}$ & $\begin{array}{c}\text { Granule } \\
\%\end{array}$ & Sand \% & Mud \% & Silt \% & Clay $\%$ & Modes & $\begin{array}{c}\text { Mode } 1 \\
\text { (phi) }\end{array}$ & $\begin{array}{c}\text { Mode } 2 \\
\text { (phi) }\end{array}$ & $\begin{array}{c}\mathrm{D}_{10} \\
\text { (phi) }\end{array}$ & $\begin{array}{c}D_{10} \\
(\mathrm{~mm})\end{array}$ & $\begin{array}{l}D_{50} \\
\text { (phi) }\end{array}$ & $\begin{array}{c}D_{50} \\
(\mathrm{~mm})\end{array}$ & $\begin{array}{c}\text { Mean } \\
\text { Size } \\
\text { (phi) }\end{array}$ & $\begin{array}{c}\text { Mean } \\
\text { Size } \\
(\mathrm{mm})\end{array}$ & $\begin{array}{c}\text { Sorting } \\
\text { (phi) }\end{array}$ & Skewness & Kurtosis & $\begin{array}{r}\text { LOI \% } \\
450^{\circ} \mathrm{C}\end{array}$ \\
\hline GSH12-T06-S1 & 80.11 & 77.08 & 3.03 & 17.85 & 2.04 & 1.27 & 0.77 & $\mathrm{~B}$ & -4.73 & 2.24 & -5.09 & 34.13 & -3.72 & 13.15 & -2.69 & 6.47 & 2.55 & 0.58 & 1.41 & N/A \\
\hline GSH12-T06-S2 & 84.81 & 83.37 & 1.44 & 13.59 & 1.60 & $\mathrm{~N} / \mathrm{A}$ & $\mathrm{N} / \mathrm{A}$ & B & -5.38 & 2.24 & -5.74 & 53.52 & -4.06 & 16.71 & -3.74 & 13.34 & 2.26 & 0.40 & 1.63 & 0.86 \\
\hline GSH12-T10-S1 & 84.31 & 83.75 & 0.56 & 15.09 & 0.60 & $\mathrm{~N} / \mathrm{A}$ & $\mathrm{N} / \mathrm{A}$ & U & -3.24 & $\mathrm{~N} / \mathrm{A}$ & -3.24 & 9.47 & -3.06 & 8.32 & -2.80 & 6.97 & 1.31 & 0.54 & 2.58 & 0.73 \\
\hline GSH12-T10-S2 & 83.17 & 77.71 & 5.45 & 16.33 & 0.50 & $\mathrm{~N} / \mathrm{A}$ & N/A & $\mathrm{B}$ & -5.24 & -3.24 & -5.29 & 39.23 & -3.40 & 10.54 & -3.14 & 8.80 & 2.11 & 0.30 & 1.04 & 0.46 \\
\hline GSH12-T12-S1 & 45.04 & 39.65 & 5.39 & 51.67 & 3.29 & 1.65 & 1.64 & $\mathrm{~B}$ & -3.24 & 1.75 & -3.62 & 12.26 & -0.20 & 1.15 & -0.42 & 1.34 & 2.48 & -0.08 & 0.57 & 0.64 \\
\hline GSH12-T12-S2 & 74.74 & 70.16 & 4.59 & 24.00 & 1.26 & $\mathrm{~N} / \mathrm{A}$ & $\mathrm{N} / \mathrm{A}$ & $\mathrm{B}$ & -3.74 & 2.24 & -4.33 & 20.11 & -3.18 & 9.09 & -2.05 & 4.14 & 2.38 & 0.63 & 1.01 & 0.57 \\
\hline GSH12-T17-S1 & 60.62 & 51.29 & 9.32 & 38.31 & 1.07 & N/A & $\mathrm{N} / \mathrm{A}$ & $\mathrm{B}$ & -3.74 & 0.75 & -4.10 & 17.14 & -2.13 & 4.36 & -1.67 & 3.18 & 2.19 & 0.30 & 0.66 & 0.55 \\
\hline GSH12-T17-S2 & 34.50 & 24.18 & 10.32 & 62.74 & 2.76 & 1.43 & 1.33 & B & 0.75 & -3.24 & -3.27 & 9.67 & -0.01 & 1.01 & -0.37 & 1.29 & 2.14 & -0.22 & 0.87 & 0.82 \\
\hline GSH12-T18-S1 & 99.04 & 98.48 & 0.55 & 0.58 & 0.38 & N/A & $\mathrm{N} / \mathrm{A}$ & $\mathrm{B}$ & -4.73 & -2.74 & -4.80 & 27.80 & -3.42 & 10.70 & -3.59 & 12.06 & 0.90 & -0.19 & 0.64 & N/A \\
\hline GSH12-T18-S2 & 97.99 & 94.83 & 3.16 & 1.69 & 0.32 & $\mathrm{~N} / \mathrm{A}$ & $\mathrm{N} / \mathrm{A}$ & U & -4.73 & $\mathrm{~N} / \mathrm{A}$ & -4.78 & 27.46 & -3.95 & 15.48 & -3.87 & 14.65 & 0.86 & 0.26 & 1.03 & N/A \\
\hline GSH12-T2O-S1 & 90.81 & 87.47 & 3.34 & 8.37 & 0.82 & $\mathrm{~N} / \mathrm{A}$ & $\mathrm{N} / \mathrm{A}$ & U & -5.24 & $\mathrm{~N} / \mathrm{A}$ & -5.28 & 38.72 & -4.29 & 19.62 & -4.01 & 16.11 & 1.68 & 0.51 & 1.70 & N/A \\
\hline GSH12-T2O-S2 & 96.32 & 95.20 & 1.12 & 3.10 & 0.58 & N/A & $\mathrm{N} / \mathrm{A}$ & U & -4.73 & $\mathrm{~N} / \mathrm{A}$ & -5.30 & 39.26 & -4.76 & 27.13 & -4.69 & 25.75 & 0.76 & 0.41 & 2.91 & N/A \\
\hline GSH12-T21-S1 & 85.58 & 82.24 & 3.34 & 12.95 & 1.47 & N/A & $\mathrm{N} / \mathrm{A}$ & B & -3.74 & -5.24 & -5.17 & 36.08 & -3.60 & 12.11 & -3.38 & 10.44 & 2.10 & 0.38 & 2.22 & 0.67 \\
\hline GSH12-T21-S2 & 85.73 & 83.83 & 1.90 & 12.97 & 1.30 & 0.97 & 0.33 & $\mathrm{~B}$ & -3.74 & -4.73 & -4.87 & 29.32 & -3.67 & 12.70 & -3.44 & 10.82 & 1.90 & 0.45 & 2.26 & N/A \\
\hline GSH12-T22-S1 & 0.01 & 0.00 & 0.01 & 98.40 & 1.59 & $\mathrm{~N} / \mathrm{A}$ & $\mathrm{N} / \mathrm{A}$ & U & 3.24 & $\mathrm{~N} / \mathrm{A}$ & 2.58 & 0.17 & 3.12 & 0.12 & 3.08 & 0.12 & 0.40 & -0.03 & 0.98 & 0.49 \\
\hline GSH12-T22-S2 & 0.01 & 0.00 & 0.01 & 96.91 & 3.08 & $\mathrm{~N} / \mathrm{A}$ & $\mathrm{N} / \mathrm{A}$ & U & 3.24 & $\mathrm{~N} / \mathrm{A}$ & 2.58 & 0.17 & 3.12 & 0.11 & 3.09 & 0.12 & 0.41 & -0.01 & 1.02 & 0.75 \\
\hline GSH12-T23-S1 & 0.00 & 0.00 & 0.00 & 97.15 & 2.85 & $\mathrm{~N} / \mathrm{A}$ & $\mathrm{N} / \mathrm{A}$ & U & 3.24 & $\mathrm{~N} / \mathrm{A}$ & 2.61 & 0.16 & 3.18 & 0.11 & 3.16 & 0.11 & 0.43 & -0.01 & 1.08 & 0.61 \\
\hline GSH12-T23-S2 & 0.00 & 0.00 & 0.00 & 94.18 & 5.82 & 4.67 & 1.15 & U & 3.24 & $\mathrm{~N} / \mathrm{A}$ & 2.64 & 0.16 & 3.24 & 0.11 & 3.25 & 0.11 & 0.49 & 0.09 & 1.34 & 0.72 \\
\hline GSH12-T24-S1 & 0.15 & 0.00 & 0.15 & 99.10 & 0.75 & N/A & N/A & 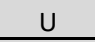 & 2.74 & $\mathrm{~N} / \mathrm{A}$ & 2.48 & 0.18 & 2.82 & 0.14 & 2.86 & 0.14 & 0.37 & 0.08 & 1.11 & 0.45 \\
\hline GSH12-T24-S2 & 0.04 & 0.00 & 0.04 & 99.08 & 0.88 & N/A & $\mathrm{N} / \mathrm{A}$ & U & 2.74 & $\mathrm{~N} / \mathrm{A}$ & 2.50 & 0.18 & 2.86 & 0.14 & 2.90 & 0.13 & 0.35 & 0.13 & 0.91 & 0.40 \\
\hline GSH12-T51-S1 & 69.54 & 52.43 & 17.11 & 27.18 & 3.28 & 1.85 & 1.43 & $u$ & -3.24 & $\mathrm{~N} / \mathrm{A}$ & -3.49 & 11.27 & -2.11 & 4.32 & -1.51 & 2.86 & 2.05 & 0.45 & 1.05 & 0.67 \\
\hline GSH12-T51-S2 & 57.88 & 43.08 & 14.80 & 38.59 & 3.53 & 2.02 & 1.51 & U & -2.24 & N/A & -3.75 & 13.42 & -1.57 & 2.97 & -1.10 & 2.15 & 2.32 & 0.28 & 0.79 & 0.37 \\
\hline
\end{tabular}




\section{GSH12: Grain Size Distribution}

\begin{tabular}{|c|c|c|c|c|c|c|c|c|c|c|c|c|c|c|c|c|c|c|c|c|c|c|c|c|c|c|c|c|}
\hline Sample ID & $\begin{array}{c}\text { Class } \\
\% \\
\text { phi } \\
-5.5\end{array}$ & $\begin{array}{c}\text { Class } \\
\% \\
\text { phi } \\
-5.0\end{array}$ & $\begin{array}{c}\text { Class } \\
\% \\
\text { phi } \\
-4.5\end{array}$ & $\begin{array}{c}\text { Class } \\
\% \\
\text { phi } \\
-4.0\end{array}$ & $\begin{array}{c}\text { Class } \\
\% \\
\text { phi } \\
-3.5\end{array}$ & $\begin{array}{c}\text { Class } \\
\% \\
\text { phi } \\
-3.0\end{array}$ & $\begin{array}{c}\text { Class } \\
\% \\
\text { phi } \\
-2.5\end{array}$ & $\begin{array}{c}\text { Class } \\
\% \\
\text { phi } \\
-2.0\end{array}$ & $\begin{array}{c}\text { Class } \\
\% \\
\text { phi } \\
-1.5\end{array}$ & $\begin{array}{c}\text { Class } \\
\% \\
\text { phi } \\
-1.0\end{array}$ & $\begin{array}{c}\text { Class } \\
\% \\
\text { phi } \\
-0.5\end{array}$ & $\begin{array}{c}\text { Class } \\
\% \\
\text { phi } \\
0.0\end{array}$ & $\begin{array}{c}\text { Class } \\
\% \\
\text { phi } \\
0.5\end{array}$ & $\begin{array}{c}\text { Class } \\
\% \\
\text { phi } \\
1.0\end{array}$ & $\begin{array}{c}\text { Class } \\
\% \\
\text { phi } \\
1.5\end{array}$ & $\begin{array}{c}\text { Class } \\
\% \\
\text { phi } \\
2.0\end{array}$ & $\begin{array}{c}\text { Class } \\
\% \\
\text { phi } \\
2.5\end{array}$ & $\begin{array}{c}\text { Class } \\
\% \\
\text { phi } \\
3.0\end{array}$ & $\begin{array}{c}\text { Class } \\
\% \\
\text { phi } \\
3.5\end{array}$ & $\begin{array}{c}\text { Class } \\
\% \\
\text { phi } \\
4.0\end{array}$ & $\begin{array}{c}\text { Class } \\
\% \\
\text { phi } \\
>4.0\end{array}$ & $\begin{array}{c}\text { Class } \\
\% \\
\text { phi } \\
5.0\end{array}$ & $\begin{array}{c}\text { Class } \\
\% \\
\text { phi } \\
6.0\end{array}$ & $\begin{array}{c}\text { Class } \\
\% \\
\text { phi } \\
7.0\end{array}$ & $\begin{array}{c}\text { Class } \\
\% \\
\text { phi } \\
8.0\end{array}$ & $\begin{array}{c}\text { Class } \\
\% \\
\text { phi } \\
9.0\end{array}$ & $\begin{array}{c}\text { Class } \\
\% \\
\text { phi } \\
10.0\end{array}$ & $\begin{array}{c}\text { Class } \\
\% \\
\text { phi } \\
>10.0\end{array}$ \\
\hline GSH12- & 0.00 & 12.91 & 21.45 & 4.27 & 20.70 & 10.25 & 5.09 & 2.41 & 1.73 & 1.31 & 1.29 & 1.09 & 1.29 & 2.24 & 2.65 & 2.87 & 3.11 & 2.26 & 0.66 & 0.39 & $\mathrm{~N} / \mathrm{A}$ & 0.60 & 0.31 & 0.19 & 0.17 & 0.16 & 0.14 & 0.47 \\
\hline GSH12-T06-S2 & 20.63 & 7.13 & 10.70 & 13.26 & 13.91 & 12.22 & 4.38 & 1.15 & 0.72 & 0.72 & 0.77 & 0.67 & 0.79 & 1.28 & 1.91 & 2.68 & 2.71 & 1.92 & 0.58 & 0.27 & 1.60 & N/A & N/A & N/A & N/A & $\mathrm{N} / \mathrm{A}$ & N/A & $\mathrm{N} / \mathrm{A}$ \\
\hline GSH12-T10-S1 & 0.00 & 0.00 & 0.00 & 2.75 & 18.89 & 32.10 & 25.29 & 4.72 & 0.44 & 0.13 & 1.64 & 2.36 & 2.38 & 2.17 & 2.04 & 2.24 & 1.50 & 0.53 & 0.11 & 0.11 & 0.60 & $\mathrm{~N} / \mathrm{A}$ & N/A & N/A & N/A & $\mathrm{N} / \mathrm{A}$ & $\mathrm{N} / \mathrm{A}$ & N/A \\
\hline GSH12- & 0.00 & 25.95 & 5.99 & 2.55 & 12.14 & 18.63 & 8.21 & 4.23 & 2.94 & 2.51 & 2.69 & 2.59 & 2.56 & 2.32 & 2.10 & 2.36 & 1.20 & 0.39 & 0.06 & 0.05 & 0.50 & N/A & N/A & $\mathrm{N} / \mathrm{A}$ & $\mathrm{N} / \mathrm{A}$ & $\mathrm{N} / \mathrm{A}$ & $\mathrm{N} / \mathrm{A}$ & N/A \\
\hline GSH12-T12-S1 & 0.00 & 0.00 & 0.00 & 0.00 & 13.41 & 16.07 & 7.05 & 3.17 & 3.09 & 2.31 & 2.94 & 3.29 & 4.16 & 5.85 & 6.90 & 9.50 & 8.90 & 7.97 & 1.75 & 0.49 & N/A & 0.60 & 0.30 & 0.32 & 0.30 & 0.29 & 0.73 & 0.63 \\
\hline GSH12-T12-S2 & 0.00 & 0.00 & 6.08 & 12.25 & 20.17 & 18.49 & 9.11 & 4.05 & 2.36 & 2.22 & 2.26 & 1.67 & 1.74 & 2.76 & 3.31 & 4.16 & 3.92 & 3.31 & 0.66 & 0.21 & 1.26 & $\mathrm{~N} / \mathrm{A}$ & N/A & $\mathrm{N} / \mathrm{A}$ & N/A & $\mathrm{N} / \mathrm{A}$ & $\mathrm{N} / \mathrm{A}$ & N/A \\
\hline GSH12- & 0.00 & 0.00 & 0.00 & 12.56 & 16.29 & 12.71 & 4.74 & 5.00 & 4.89 & 4.44 & 5.38 & 5.17 & 5.84 & 6.87 & 5.49 & 5.14 & 3.11 & 0.92 & 0.23 & 0.16 & 1.07 & N/A & N/A & $\mathrm{N} / \mathrm{A}$ & N/A & N/A & N/A & N/A \\
\hline GSH12 & 0.00 & 0.00 & 0.00 & 5.47 & 1.59 & 6.73 & 5.89 & 4.50 & 4.92 & 5.41 & 7.46 & 8.28 & 9.71 & 11.20 & 9.07 & 8.59 & 5.61 & 2.05 & 0.46 & 0.31 & $\mathrm{~N} / \mathrm{A}$ & 0.43 & 0.35 & 0.34 & 0.31 & 0.32 & 0.36 & 0.64 \\
\hline GSH1 & 0.00 & 0.00 & 27.35 & 12.92 & 6.82 & 21.61 & 23.76 & 6.03 & 0.49 & 0.06 & 0.01 & 0.02 & 0.02 & 0.02 & 0.02 & 0.03 & 0.03 & 0.07 & 0.14 & 0.22 & 0.38 & N/A & N/A & $\mathrm{N} / \mathrm{A}$ & N/A & N/A & $\mathrm{N} / \mathrm{A}$ & N/A \\
\hline GSH12-T18-S2 & 0.00 & 0.00 & 24.78 & 23.14 & 22.26 & 14.02 & 7.87 & 2.76 & 1.62 & 1.54 & 0.85 & 0.22 & 0.12 & 0.08 & 0.06 & 0.06 & 0.07 & 0.06 & 0.07 & 0.10 & 0.32 & N/A & N/A & N/A & N/A & N/A & N/A & N/A \\
\hline GSH1 & 00 & 23.77 & 20.99 & 13.31 & 14.32 & 6.63 & 6.25 & 2.20 & 2.09 & 1.24 & 0.84 & 0.60 & 0.67 & 0.90 & 1.16 & 1.30 & 1.08 & 0.77 & 0.69 & 0.35 & 0.82 & N/A & N/A & $\mathrm{N} / \mathrm{A}$ & N/A & $\mathrm{N} / \mathrm{A}$ & $\mathrm{N} / \mathrm{A}$ & N/A \\
\hline $\mathrm{GSH} 12$ & 0.00 & 26.09 & 54.63 & 4.36 & 3.60 & 3.86 & 1.83 & 0.83 & 0.62 & 0.50 & 0.51 & 0.35 & 0.28 & 0.28 & 0.28 & 0.31 & 0.28 & 0.33 & 0.28 & 0.20 & 0.58 & N/A & N/A & $\mathrm{N} / \mathrm{A}$ & N/A & N/A & N/A & N/A \\
\hline GSH12-T21-S1 & 0.00 & 16.15 & 7.66 & 5.39 & 26.61 & 17.60 & 5.64 & 3.21 & 2.12 & 1.22 & 0.78 & 0.38 & 0.37 & 0.55 & 0.98 & 2.17 & 2.47 & 2.06 & 2.07 & 1.11 & 1.47 & $\mathrm{~N} / \mathrm{A}$ & N/A & $\mathrm{N} / \mathrm{A}$ & N/A & $\mathrm{N} / \mathrm{A}$ & N/A & N/A \\
\hline GSH1 & 00 & 5.98 & 19.09 & 9.71 & 23.52 & 18.47 & 4.72 & 2.34 & 1.13 & 0.78 & 0.63 & 0.39 & 0.35 & 0.64 & 1.11 & 2.18 & 2.40 & 1.96 & 2.17 & 1.12 & $\mathrm{~N} / \mathrm{A}$ & 0.63 & 0.16 & 0.10 & 0.08 & 0.13 & 0.09 & 0.11 \\
\hline GSH12-T22-S1 & 0.00 & 0.00 & 0.00 & 0.00 & 0.00 & 0.00 & 0.00 & 0.00 & 0.01 & 0.00 & 0.01 & 0.02 & 0.02 & 0.03 & 0.08 & 0.29 & 2.32 & 35.50 & 47.95 & 12.20 & 1.59 & N/A & N/A & $\mathrm{N} / \mathrm{A}$ & N/A & N/A & $\mathrm{N} / \mathrm{A}$ & N/A \\
\hline GSH1 & 0.00 & 0.00 & 0.00 & 0.00 & 0.00 & 0.00 & 0.00 & 0.00 & 0.00 & 0.01 & 0.01 & 0.00 & 0.01 & 0.03 & 0.09 & 0.30 & 2.28 & 34.94 & 47.48 & 11.77 & 3.08 & N/A & N/A & N/A & N/A & N/A & N/A & N/A \\
\hline GSH12-T23-S1 & 0.00 & 0.00 & 0.00 & 0.00 & 0.00 & 0.00 & 0.00 & 0.00 & 0.00 & 0.00 & 0.02 & 0.05 & 0.05 & 0.06 & 0.09 & 0.40 & 2.02 & 28.30 & 49.21 & 16.94 & 2.85 & N/A & N/A & $\mathrm{N} / \mathrm{A}$ & $\mathrm{N} / \mathrm{A}$ & N/A & $\mathrm{N} / \mathrm{A}$ & N/A \\
\hline GSH12-T23-S2 & 0.00 & 0.00 & 0.00 & 0.00 & 0.00 & 0.00 & 0.00 & 0.00 & 0.00 & 0.00 & 0.06 & 0.04 & 0.07 & 0.12 & 0.19 & 0.42 & 1.79 & 22.99 & 48.63 & 19.86 & N/A & 4.30 & 0.18 & 0.08 & 0.10 & 0.13 & 0.21 & 0.82 \\
\hline GSH12-T24-S1 & 0.00 & 0.00 & 0.00 & 0.00 & 0.00 & 0.00 & 0.00 & 0.00 & 0.11 & 0.04 & 0.03 & 0.02 & 0.04 & 0.12 & 0.25 & 1.73 & 7.17 & 61.30 & 25.63 & 2.80 & 0.75 & N/A & N/A & $\mathrm{N} / \mathrm{A}$ & N/A & N/A & $\mathrm{N} / \mathrm{A}$ & N/A \\
\hline GSH12-T24-S2 & 0.00 & 0.00 & 0.00 & 0.00 & 0.00 & 0.00 & 0.00 & 0.00 & 0.00 & 0.04 & 0.00 & 0.02 & 0.03 & 0.09 & 0.19 & 0.44 & 5.76 & 59.76 & 29.56 & 3.23 & 0.88 & N/A & N/A & $\mathrm{N} / \mathrm{A}$ & N/A & N/A & $\mathrm{N} / \mathrm{A}$ & N/A \\
\hline GSH12-T51-S1 & 0.00 & 0.00 & 0.00 & 2.80 & 7.33 & 17.43 & 14.29 & 10.59 & 9.97 & 7.14 & 5.62 & 3.43 & 2.98 & 3.09 & 2.25 & 2.91 & 4.13 & 1.98 & 0.47 & 0.32 & $\mathrm{~N} / \mathrm{A}$ & 0.75 & 0.49 & 0.32 & 0.28 & 0.28 & 0.70 & 0.45 \\
\hline SH12-T51-S2 & 0.00 & 0.00 & 0.00 & 6.63 & 6.83 & 13.24 & 8.31 & 8.07 & 8.33 & 6.47 & 6.53 & 5.06 & 4.81 & 4.86 & 3.50 & 4.20 & 6.13 & 2.64 & 0.53 & 0.34 & N/A & 0.80 & 0.63 & 0.33 & 0.27 & 0.28 & 0.61 & 0.61 \\
\hline
\end{tabular}


Section 2: SH12, GSHO6, and GSH13 
SH12, GSH06, and GSH13: Identification, Location, and Description

\begin{tabular}{|c|c|c|c|c|c|c|c|c|}
\hline Sample ID & Global Sample ID & Station ID & $\begin{array}{c}\text { Latitude } \\
\text { WGS84 }\end{array}$ & $\begin{array}{c}\text { Longitude } \\
\text { WGS84 }\end{array}$ & $\begin{array}{l}\text { Reliability } \\
\text { Ranking for } \\
\text { Positioning }\end{array}$ & $\begin{array}{c}\text { Water } \\
\text { Depth }(\mathrm{m})\end{array}$ & $\begin{array}{c}\text { Sample } \\
\text { Collected }\end{array}$ & $\begin{array}{c}\text { Total Wt } \\
\text { (gm) }\end{array}$ \\
\hline $\mathrm{SH} 12-02$ & SH12-02_6/28/2012 & $\mathrm{SH} 12-02$ & 43.053003 & -70.644819 & 1 & 23.5 & $6 / 28 / 2012$ & 693.5 \\
\hline $\mathrm{SH} 12-04$ & SH12-04_6/28/2012 & $\mathrm{SH} 12-04$ & 43.048156 & -70.639286 & 1 & 24.4 & $6 / 28 / 2012$ & 696.9 \\
\hline $\mathrm{SH} 12-05$ & SH12-05_6/28/2012 & SH12-05 & 43.043894 & -70.634703 & 1 & 32.6 & $6 / 28 / 2012$ & 221.2 \\
\hline SH12-09 & SH12-09_6/28/2012 & SH12-09 & 43.055544 & -70.623825 & 1 & 33.5 & $6 / 28 / 2012$ & 324.9 \\
\hline SH12-11 & SH12-11_6/28/2012 & SH12-11 & 43.057017 & -70.650367 & 1 & N/A & $6 / 28 / 2012$ & 71.7 \\
\hline SH12-12 & SH12-12_6/28/2012 & SH12-12 & 43.094919 & -70.639372 & 1 & $\mathrm{~N} / \mathrm{A}$ & $6 / 28 / 2012$ & 280.4 \\
\hline $\mathrm{SH} 12-13$ & SH12-13_6/28/2012 & $\mathrm{SH} 12-13$ & 43.097294 & -70.648036 & 1 & 10.1 & $6 / 28 / 2012$ & 84.2 \\
\hline $\mathrm{SH} 12-14$ & SH12-14_6/28/2012 & SH12-14 & 43.096169 & -70.646881 & 1 & 11.3 & $6 / 28 / 2012$ & 99.8 \\
\hline SH12-15-B & SH12-15-B_6/28/2012 & SH12-15-B & 43.093020 & -70.647627 & 1 & 13.7 & $6 / 28 / 2012$ & 317.6 \\
\hline SH12-16 & SH12-16_6/28/2012 & SH12-16 & 43.091936 & -70.651581 & 1 & 8.8 & $6 / 28 / 2012$ & 132.6 \\
\hline SH12-17 & SH12-17_6/28/2012 & SH12-17 & 43.063233 & -70.663283 & 1 & 22.6 & $6 / 28 / 2012$ & 201.9 \\
\hline GSH06-T03-S1 & GSH06-T03-S1_8/1/2017 & GSH06-T03 & 43.066875 & -70.670733 & 1 & 11.0 & $8 / 1 / 2017$ & 79.8 \\
\hline GSH06-T04-S1 & GSH06-T04-S1_8/1/2017 & GSH06-T04 & 43.071793 & -70.660163 & 1 & 15.2 & $8 / 1 / 2017$ & 495.7 \\
\hline GSH06-T04-S2 & GSH06-T04-S2_8/1/2017 & GSH06-T04 & 43.071747 & -70.660160 & 1 & 15.2 & $8 / 1 / 2017$ & 334.8 \\
\hline GSH06-T07-S1 & GSH06-T07-S1_8/1/2017 & GSH06-T07 & 43.088600 & -70.653268 & 1 & 8.5 & $8 / 1 / 2017$ & 135.4 \\
\hline GSH06-T07-S2 & GSH06-T07-S2_8/1/2017 & GSH06-T07 & 43.088606 & -70.653326 & 1 & 8.5 & $8 / 1 / 2017$ & 98.3 \\
\hline GSH13-T11-S1 & GSH13-T11-S1_7/17/2017 & GSH13-T11 & 43.024318 & -70.713641 & 1 & 13.4 & $7 / 17 / 2017$ & 123.7 \\
\hline GSH13-T13-S1 & GSH13-T13-S1_7/17/2017 & GSH13-T13 & 43.022075 & -70.719898 & 1 & 11.0 & $7 / 17 / 2017$ & 144.4 \\
\hline GSH13-T14-S1 & GSH13-T14-S1_7/17/2017 & GSH13-T14 & 43.020354 & -70.722841 & 1 & 9.4 & $7 / 17 / 2017$ & 123.9 \\
\hline GSH13-T15-S1 & GSH13-T15-S1_7/17/2017 & GSH13-T15 & 43.017187 & -70.719325 & 1 & 15.5 & $7 / 17 / 2017$ & 281.5 \\
\hline GSH13-T15-S2 & GSH13-T15-S2_7/17/2017 & GSH13-T15 & 43.017226 & -70.719428 & 1 & 15.5 & $7 / 17 / 2017$ & 421.3 \\
\hline GSH13-T17-S1 & GSH13-T17-S1_7/17/2017 & GSH13-T17 & 43.014386 & -70.726680 & 1 & 8.5 & $7 / 17 / 2017$ & 89.5 \\
\hline
\end{tabular}


SH12, GSH06, and GSH13: Sediment Classifications

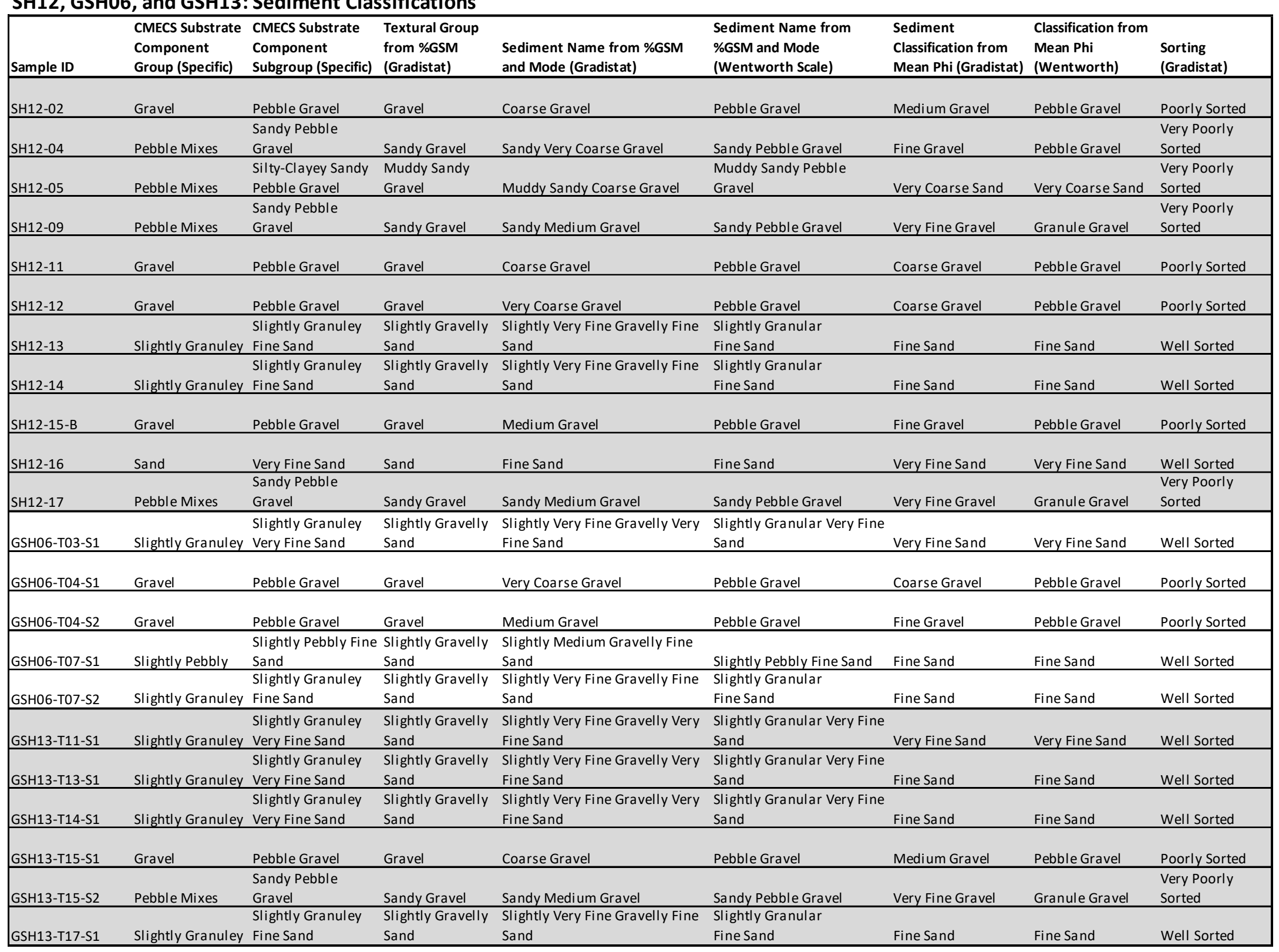


SH12, GSH06, and GSH13: Grain Size Statistics

\begin{tabular}{|c|c|c|c|c|c|c|c|c|c|c|c|c|c|c|c|c|c|c|c|c|}
\hline Sample ID & $\begin{array}{c}\text { Gravel } \\
\%\end{array}$ & $\begin{array}{c}\text { Pebble } \\
\%\end{array}$ & $\begin{array}{c}\text { Granule } \\
\%\end{array}$ & Sand \% & Mud \% & Silt \% & Clay \% & Modes & $\begin{array}{c}\text { Mode } 1 \\
\text { (phi) }\end{array}$ & $\begin{array}{c}\text { Mode } 2 \\
\text { (phi) }\end{array}$ & $\begin{array}{c}\mathrm{D}_{10} \\
\text { (phi) }\end{array}$ & $\begin{array}{c}\mathrm{D}_{10} \\
(\mathrm{~mm})\end{array}$ & $\begin{array}{l}D_{50} \\
\text { (phi) }\end{array}$ & $\begin{array}{c}D_{50} \\
(\mathrm{~mm})\end{array}$ & $\begin{array}{c}\text { Mean } \\
\text { Size } \\
\text { (phi) }\end{array}$ & $\begin{array}{c}\text { Mean } \\
\text { Size } \\
(\mathrm{mm})\end{array}$ & $\begin{array}{l}\text { Sorting } \\
\text { (phi) }\end{array}$ & Skewness & Kurtosis & $\begin{array}{r}\mathrm{LOI} \% \\
450^{\circ} \mathrm{C}\end{array}$ \\
\hline SH12-02 & 86.52 & 82.33 & 4.19 & 12.98 & 0.50 & N/A & $\mathrm{N} / \mathrm{A}$ & $\mathrm{B}$ & -5.24 & -3.24 & -5.25 & 38.08 & -3.92 & 15.16 & -3.55 & 11.69 & 1.80 & 0.42 & 1.23 & 0.82 \\
\hline SH12-04 & 64.43 & 58.59 & 5.84 & 33.87 & 1.70 & $\mathrm{~N} / \mathrm{A}$ & $\mathrm{N} / \mathrm{A}$ & $\mathrm{P}$ & -5.24 & -3.24 & -5.24 & 37.82 & -2.98 & 7.87 & -2.24 & 4.71 & 2.79 & 0.36 & 0.62 & 0.70 \\
\hline SH12-05 & 38.14 & 29.75 & 8.39 & 55.38 & 6.48 & 3.48 & 3.00 & B & 2.24 & -4.73 & -4.55 & 23.49 & 0.77 & 0.59 & -0.02 & 1.01 & 3.04 & -0.26 & 0.82 & 0.98 \\
\hline SH12-09 & 60.69 & 52.11 & 8.58 & 36.18 & 3.13 & N/A & $\mathrm{N} / \mathrm{A}$ & $\mathrm{B}$ & -4.73 & 2.24 & -4.73 & 26.48 & -2.17 & 4.50 & -1.56 & 2.95 & 2.86 & 0.30 & 0.64 & 0.80 \\
\hline SH12-11 & 93.07 & 91.03 & 2.04 & 6.82 & 0.11 & N/A & $\mathrm{N} / \mathrm{A}$ & N/A & $\mathrm{N} / \mathrm{A}$ & $\mathrm{N} / \mathrm{A}$ & -4.83 & 28.48 & -4.10 & 17.12 & -4.01 & 16.14 & 1.05 & 0.40 & 1.79 & $\mathrm{~N} / \mathrm{A}$ \\
\hline SH12-12 & 91.86 & 89.53 & 2.33 & 7.76 & 0.38 & N/A & $\mathrm{N} / \mathrm{A}$ & $\mathrm{N} / \mathrm{A}$ & $\mathrm{N} / \mathrm{A}$ & $\mathrm{N} / \mathrm{A}$ & -5.41 & 42.43 & -5.07 & 33.59 & -4.47 & 22.15 & 1.57 & 0.82 & 1.94 & $\mathrm{~N} / \mathrm{A}$ \\
\hline SH12-13 & 0.01 & 0.00 & 0.01 & 98.99 & 1.00 & N/A & $\mathrm{N} / \mathrm{A}$ & U & 2.74 & $\mathrm{~N} / \mathrm{A}$ & 2.51 & 0.18 & 2.95 & 0.13 & 2.96 & 0.13 & 0.40 & 0.05 & 0.95 & 0.48 \\
\hline SH12-14 & 0.07 & 0.00 & 0.07 & 98.33 & 1.60 & N/A & $\mathrm{N} / \mathrm{A}$ & U & 3.24 & $\mathrm{~N} / \mathrm{A}$ & 2.50 & 0.18 & 2.98 & 0.13 & 2.98 & 0.13 & 0.43 & -0.01 & 1.04 & 0.52 \\
\hline SH12-15-B & 85.82 & 79.66 & 6.17 & 13.45 & 0.73 & N/A & $\mathrm{N} / \mathrm{A}$ & U & -4.24 & $\mathrm{~N} / \mathrm{A}$ & -4.39 & 20.91 & -3.23 & 9.36 & -2.97 & 7.83 & 1.85 & 0.46 & 1.84 & 2.16 \\
\hline SH12-16 & 0.00 & 0.00 & 0.00 & 98.62 & 1.38 & N/A & $\mathrm{N} / \mathrm{A}$ & u & 2.74 & $\mathrm{~N} / \mathrm{A}$ & 2.55 & 0.17 & 3.00 & 0.12 & 3.00 & 0.12 & 0.38 & 0.10 & 0.90 & 0.47 \\
\hline SH12-17 & 63.46 & 52.96 & 10.50 & 35.32 & 1.22 & N/A & $\mathrm{N} / \mathrm{A}$ & B & -3.74 & 1.75 & -3.96 & 15.53 & -2.22 & 4.67 & -1.54 & 2.91 & 2.27 & 0.39 & 0.61 & 0.63 \\
\hline GSH06-T03-S1 & 0.06 & 0.00 & 0.06 & 96.26 & 3.68 & $\mathrm{~N} / \mathrm{A}$ & $\mathrm{N} / \mathrm{A}$ & U & 3.24 & $\mathrm{~N} / \mathrm{A}$ & 2.74 & 0.15 & 3.28 & 0.10 & 3.32 & 0.10 & 0.41 & 0.06 & 1.20 & 0.53 \\
\hline GSH06-T04-S1 & 90.52 & 88.56 & 1.97 & 9.08 & 0.40 & $\mathrm{~N} / \mathrm{A}$ & $\mathrm{N} / \mathrm{A}$ & $\mathrm{N} / \mathrm{A}$ & $\mathrm{N} / \mathrm{A}$ & $\mathrm{N} / \mathrm{A}$ & -5.88 & 58.93 & -5.50 & 45.10 & -4.74 & 26.78 & 1.75 & 0.82 & 1.21 & $\mathrm{~N} / \mathrm{A}$ \\
\hline GSH06-T04-S2 & 80.35 & 67.19 & 13.17 & 18.94 & 0.71 & $\mathrm{~N} / \mathrm{A}$ & N/A & $\mathrm{N} / \mathrm{A}$ & $\mathrm{N} / \mathrm{A}$ & $\mathrm{N} / \mathrm{A}$ & -4.66 & 25.25 & -3.04 & 8.23 & -2.70 & 6.48 & 1.99 & 0.38 & 1.18 & 0.49 \\
\hline GSH06-T07-S1 & 0.69 & 0.69 & 0.00 & 98.83 & 0.48 & $\mathrm{~N} / \mathrm{A}$ & $\mathrm{N} / \mathrm{A}$ & U & 2.74 & $\mathrm{~N} / \mathrm{A}$ & 2.46 & 0.18 & 2.82 & 0.14 & 2.87 & 0.14 & 0.38 & 0.06 & 1.10 & 0.44 \\
\hline GSH06-T07-S2 & 0.49 & 0.19 & 0.30 & 98.89 & 0.62 & $\mathrm{~N} / \mathrm{A}$ & $\mathrm{N} / \mathrm{A}$ & U & 2.74 & $\mathrm{~N} / \mathrm{A}$ & 2.48 & 0.18 & 2.86 & 0.14 & 2.90 & 0.13 & 0.39 & 0.04 & 0.99 & 0.34 \\
\hline GSH13-T11-S1 & 0.01 & 0.00 & 0.01 & 97.64 & 2.35 & N/A & N/A & U & 3.24 & N/A & 2.53 & 0.17 & 3.06 & 0.12 & 3.03 & 0.12 & 0.41 & -0.07 & 1.00 & 0.68 \\
\hline GSH13-T13-S1 & 0.03 & 0.00 & 0.03 & 97.21 & 2.76 & N/A & $\mathrm{N} / \mathrm{A}$ & U & 3.24 & $\mathrm{~N} / \mathrm{A}$ & 2.32 & 0.20 & 3.03 & 0.12 & 2.99 & 0.13 & 0.50 & -0.09 & 1.11 & 0.59 \\
\hline GSH13-T14-S1 & 0.07 & 0.00 & 0.07 & 98.03 & 1.90 & $\mathrm{~N} / \mathrm{A}$ & $\mathrm{N} / \mathrm{A}$ & U & 3.24 & $\mathrm{~N} / \mathrm{A}$ & 2.32 & 0.20 & 3.04 & 0.12 & 2.99 & 0.13 & 0.49 & -0.14 & 1.10 & 0.59 \\
\hline GSH13-T15-S1 & 91.95 & 90.06 & 1.90 & 7.38 & 0.67 & N/A & N/A & U & -4.24 & $\mathrm{~N} / \mathrm{A}$ & -4.72 & 26.32 & -3.99 & 15.93 & -3.78 & 13.75 & 1.35 & 0.54 & 2.07 & $\mathrm{~N} / \mathrm{A}$ \\
\hline GSH13-T15-S2 & 65.46 & 57.71 & 7.74 & 33.10 & 1.44 & N/A & $\mathrm{N} / \mathrm{A}$ & $\mathrm{B}$ & -4.73 & 2.74 & -4.64 & 24.93 & -2.72 & 6.58 & -1.54 & 2.91 & 2.95 & 0.50 & 0.66 & 0.41 \\
\hline GSH13-T17-S1 & 0.04 & 0.00 & 0.04 & 98.63 & 1.33 & N/A & N/A & U & 2.74 & N/A & 2.48 & 0.18 & 2.94 & 0.13 & 2.95 & 0.13 & 0.43 & 0.01 & 1.03 & 0.43 \\
\hline
\end{tabular}


SH12, GSH06, and GSH13: Grain Size Distribution

\begin{tabular}{|c|c|c|c|c|c|c|c|c|c|c|c|c|c|c|c|c|c|c|c|c|c|c|c|c|c|c|c|c|}
\hline & $\begin{array}{c}\text { Class } \\
\% \\
\text { phi } \\
-5.5\end{array}$ & $\begin{array}{c}\text { Class } \\
\% \\
\text { phi } \\
-5.0\end{array}$ & $\begin{array}{c}\text { Class } \\
\% \\
\text { phi } \\
-4.5\end{array}$ & $\begin{array}{c}\text { Class } \\
\% \\
\text { phi } \\
-4.0\end{array}$ & $\begin{array}{c}\text { Class } \\
\% \\
\text { phi } \\
-3.5\end{array}$ & $\begin{array}{c}\text { Class } \\
\% \\
\text { phi } \\
-3.0\end{array}$ & $\begin{array}{c}\text { Class } \\
\% \\
\text { phi } \\
-2.5\end{array}$ & $\begin{array}{c}\text { Class } \\
\% \\
\text { phi } \\
-2.0\end{array}$ & $\begin{array}{c}\text { Class } \\
\% \\
\text { phi } \\
-1.5\end{array}$ & $\begin{array}{c}\text { Class } \\
\% \\
\text { phi } \\
-1.0\end{array}$ & $\begin{array}{c}\text { Class } \\
\% \\
\text { phi } \\
-0.5\end{array}$ & $\begin{array}{c}\text { Class } \\
\% \\
\text { phi } \\
0.0\end{array}$ & $\begin{array}{c}\text { Class } \\
\% \\
\text { phi } \\
0.5\end{array}$ & $\begin{array}{c}\text { Class } \\
\% \\
\text { phi } \\
1.0\end{array}$ & $\begin{array}{c}\text { Class } \\
\% \\
\text { phi } \\
1.5\end{array}$ & $\begin{array}{c}\text { Class } \\
\% \\
\text { phi } \\
2.0\end{array}$ & $\begin{array}{c}\text { Class } \\
\% \\
\text { phi } \\
2.5\end{array}$ & $\begin{array}{c}\text { Class } \\
\% \\
\text { phi } \\
3.0\end{array}$ & $\begin{array}{c}\text { Class } \\
\% \\
\text { phi } \\
3.5\end{array}$ & $\begin{array}{c}\text { Class } \\
\% \\
\text { phi } \\
4.0\end{array}$ & $\begin{array}{c}\text { Class } \\
\% \\
\text { phi } \\
>4.0\end{array}$ & $\begin{array}{c}\text { Class } \\
\% \\
\text { phi } \\
5.0\end{array}$ & $\begin{array}{c}\text { Class } \\
\% \\
\text { phi } \\
6.0\end{array}$ & $\begin{array}{c}\text { Class } \\
\% \\
\text { phi } \\
7.0\end{array}$ & $\begin{array}{c}\text { Class } \\
\% \\
\text { phi } \\
8.0\end{array}$ & $\begin{array}{c}\text { Class } \\
\% \\
\text { phi } \\
9.0\end{array}$ & $\begin{array}{c}\text { Class } \\
\% \\
\text { phi } \\
10.0\end{array}$ & $\begin{array}{c}\text { Class } \\
\% \\
\text { phi } \\
>10.0\end{array}$ \\
\hline 112 & 00 & 21.37 & 14.14 & 13.01 & 9.73 & 14.44 & 6.46 & 3.18 & 2.22 & 1.97 & 2.44 & 2.54 & 2.51 & 2.07 & 1.28 & 1.07 & 0.63 & 0.29 & 0.08 & 0.07 & 0.50 & N/A & $\mathrm{N} / \mathrm{A}$ & N/A & $\mathrm{N} / \mathrm{A}$ & N/A & $\mathrm{V} / \mathrm{A}$ & N/A \\
\hline $\mathrm{H} 12-04$ & 00 & 20.55 & 4.47 & 8.31 & 6.35 & 10.10 & 4.66 & 4.15 & 3.30 & 2.54 & 2.58 & 2.62 & 3.60 & 5.99 & 6.53 & 6.09 & 4.00 & 1.87 & 0.38 & 0.20 & 1.70 & $\mathrm{~N} / \mathrm{A}$ & $\mathrm{N} / \mathrm{A}$ & $\mathrm{N} / \mathrm{A}$ & $\mathrm{N} / \mathrm{A}$ & $\mathrm{N} / \mathrm{A}$ & $\mathrm{N} / \mathrm{A}$ & N/A \\
\hline $112-05$ & 00 & 0.00 & 11.61 & 0.00 & 5.13 & 5.46 & 3.12 & 4.44 & 4.48 & 3.91 & 4.00 & 3.12 & 2.88 & 3.41 & 5.00 & 10.18 & 11.99 & 10.90 & 2.83 & 1.07 & N/A & 1.63 & 0.74 & 0.58 & 0.52 & 0.55 & 0.50 & 1.94 \\
\hline $\mathrm{H} 12-09$ & 00 & 0.00 & 19.66 & 0.00 & 13.30 & 7.84 & 5.33 & 5.98 & 4.58 & 4.00 & 2.93 & 1.65 & 1.93 & 4.45 & 5.64 & 5.84 & 6.88 & 4.66 & 1.53 & 0.67 & 3.13 & N/A & $\mathrm{N} / \mathrm{A}$ & $\mathrm{N} / \mathrm{A}$ & $\mathrm{N} / \mathrm{A}$ & $\mathrm{N} / \mathrm{A}$ & $\mathrm{N} / \mathrm{A}$ & N/A \\
\hline $\mathrm{H} 12-11$ & 0.00 & 0.00 & 33.89 & 20.17 & 27.65 & 3.82 & 3.80 & 1.69 & 0.96 & 1.08 & 2.02 & 1.87 & 1.31 & 0.63 & 0.38 & 0.19 & 0.11 & 0.20 & 0.07 & 0.04 & 0.11 & $\mathrm{~N} / \mathrm{A}$ & $\mathrm{N} / \mathrm{A}$ & $\mathrm{N} / \mathrm{A}$ & N/A & N/A & $\mathrm{N} / \mathrm{A}$ & $\mathrm{N} / \mathrm{A}$ \\
\hline$-112-12$ & 00 & 60.96 & 0.00 & 12.85 & 7.32 & 2.75 & 3.27 & 2.38 & 1.15 & 1.18 & 0.92 & 0.59 & 0.56 & 1.03 & 1.49 & 1.55 & 0.74 & 0.42 & 0.30 & 0.16 & 0.38 & $\mathrm{~N} / \mathrm{A}$ & $\mathrm{N} / \mathrm{A}$ & $\mathrm{N} / \mathrm{A}$ & $\mathrm{N} / \mathrm{A}$ & $\mathrm{N} / \mathrm{A}$ & $\mathrm{N} / \mathrm{A}$ & $\mathrm{N} / \mathrm{A}$ \\
\hline $112-13$ & 00 & 0.00 & 0.00 & 0.00 & 0.00 & 0.00 & 0.00 & 00 & 0.00 & 0.01 & 0.01 & 0.01 & 0.01 & 0.06 & 16 & 0.83 & 5.97 & 47.69 & 38.15 & 6.10 & 1.00 & $\mathrm{~N} / \mathrm{A}$ & $\mathrm{N} / \mathrm{A}$ & $\mathrm{N} / \mathrm{A}$ & $\mathrm{N} / \mathrm{A}$ & $\mathrm{N} / \mathrm{A}$ & N/A & $\mathrm{N} / \mathrm{A}$ \\
\hline $\mathrm{H12}$ & 0 & 0.00 & 0.00 & .00 & 0.00 & 0.00 & 0.00 & 00 & 0.02 & 0.06 & 0.02 & 0.01 & 0.08 & 0.31 & .73 & 1.60 & 5.08 & 44.06 & 39.89 & 6.54 & 1.60 & $\mathrm{~N} / \mathrm{A}$ & $\mathrm{N} / \mathrm{A}$ & N/A & $\mathrm{N} / \mathrm{A}$ & $\mathrm{N} / \mathrm{A}$ & $\mathrm{N} / \mathrm{A}$ & N/A \\
\hline & 00 & 0.00 & 6.35 & 17.81 & 16.54 & 17.38 & 13.33 & 8.24 & 24 & 1.93 & 0.96 & 0.51 & 0.45 & 0.60 & 75 & 0.85 & 0.96 & 3.74 & 3.72 & 0.90 & 0.73 & N/A & $\mathrm{N} / \mathrm{A}$ & $\mathrm{N} / \mathrm{A}$ & $\mathrm{N} / \mathrm{A}$ & $\mathrm{N} / \mathrm{A}$ & $\mathrm{N} / \mathrm{A}$ & $\mathrm{N} / \mathrm{A}$ \\
\hline & 0 & 00 & 00 & .00 & 0.00 & 0.00 & 0.00 & 00 & .00 & 0.00 & 0.00 & 0.01 & 0.02 & 0.01 & .06 & .25 & 3.21 & 46.24 & 41.13 & 7.70 & 1.38 & $\mathrm{~N} / \mathrm{A}$ & $\mathrm{N} / \mathrm{A}$ & $\mathrm{N} / \mathrm{A}$ & $\mathrm{N} / \mathrm{A}$ & $\mathrm{N} / \mathrm{A}$ & N/A & $\mathrm{N} / \mathrm{A}$ \\
\hline$-112-17$ & 00 & 0.00 & 0.00 & 8.15 & 21.88 & 11.15 & 5.36 & 6.41 & 5.64 & 4.86 & 4.18 & 3.07 & 3.06 & 4.54 & 82 & 9.06 & 2.80 & 0.78 & 0.60 & 0.42 & 1.22 & N/A & $\mathrm{N} / \mathrm{A}$ & N/A & N/A & $\mathrm{N} / \mathrm{A}$ & $\mathrm{N} / \mathrm{A}$ & N/A \\
\hline GSH & 00 & 0.00 & 0.00 & 0.00 & 0.00 & 0.00 & 0.00 & 00 & .03 & 0.04 & 0.01 & 0.01 & 0.02 & 0.08 & 22 & .39 & 0.96 & 16.09 & 54.43 & 24.05 & 3.68 & N/A & $\mathrm{N} / \mathrm{A}$ & N/A & $\mathrm{N} / \mathrm{A}$ & $\mathrm{N} / \mathrm{A}$ & N/A & $\mathrm{N} / \mathrm{A}$ \\
\hline & .31 & 0.00 & 15.16 & 0.00 & 8.86 & 9.05 & 3.52 & & 1.18 & 0.79 & 0.78 & 0.95 & & & & & 0.38 & 0.38 & 0.46 & 0.19 & 0.40 & $\mathrm{~N} / \mathrm{A}$ & $\mathrm{N} / \mathrm{A}$ & $\mathrm{N} / \mathrm{A}$ & $\mathrm{N} / \mathrm{A}$ & $\mathrm{N} / \mathrm{A}$ & $\mathrm{N} / \mathrm{A}$ & $\mathrm{N} / \mathrm{A}$ \\
\hline $\mathrm{GSHO}$ & 00 & 0.00 & 15.42 & 3.46 & 16.37 & 16.12 & 9.37 & 6.45 & 6.93 & 6.24 & 5.24 & 2.93 & 1.81 & 1.62 & 1.41 & 1.42 & 1.15 & 1.25 & 1.41 & 0.70 & 0.71 & $\mathrm{~N} / \mathrm{A}$ & $\mathrm{N} / \mathrm{A}$ & $\mathrm{N} / \mathrm{A}$ & $\mathrm{N} / \mathrm{A}$ & $\mathrm{N} / \mathrm{A}$ & N/A & N/A \\
\hline & & 0.00 & 0.00 & 0.00 & 0.00 & 0.69 & 0.00 & & 0.00 & 0.00 & 0.01 & 0.07 & 0.09 & & & & 7.37 & 59.67 & 26.99 & 2.61 & 0.48 & $\mathrm{~N} / \mathrm{A}$ & $\mathrm{N} / \mathrm{A}$ & $\mathrm{N} / \mathrm{A}$ & $\mathrm{N} / \mathrm{A}$ & $\mathrm{N} / \mathrm{A}$ & $\mathrm{N} / \mathrm{A}$ & $\mathrm{N} / \mathrm{A}$ \\
\hline GSHO6- & DO & 0.00 & 0.00 & 0.00 & 0.00 & 0.00 & 0.00 & 19 & 26 & 0.05 & 0.03 & 0.06 & 0.10 & 0.28 & 0.55 & 1.09 & 7.31 & 54.51 & 30.80 & 4.17 & 0.62 & $\mathrm{~N} / \mathrm{A}$ & $\mathrm{N} / \mathrm{A}$ & N/A & $\mathrm{N} / \mathrm{A}$ & $\mathrm{N} / \mathrm{A}$ & N/A & N/A \\
\hline . & & 0.00 & 0.00 & 0.00 & 0.00 & 0.00 & 0.00 & 00 & 0.00 & 0.01 & 0.08 & 0.14 & 0.16 & 0.24 & 0.42 & 0.90 & 3.88 & 37.82 & 47.81 & 6.19 & 2.35 & $\mathrm{~N} / \mathrm{A}$ & $\mathrm{N} / \mathrm{A}$ & $\mathrm{N} / \mathrm{A}$ & $\mathrm{N} / \mathrm{A}$ & $\mathrm{N} / \mathrm{A}$ & N/A & $\mathrm{N} / \mathrm{A}$ \\
\hline GSH13 & & 0.00 & 0.00 & 0.00 & 0.00 & 0.00 & 0.00 & 0.00 & 0.00 & 0.03 & 0.01 & 0.04 & 0.07 & 0.23 & 0.69 & 2.85 & 9.05 & 34.92 & 40.26 & 9.09 & 2.76 & N/A & N/A & N/A & N/A & N/A & $\mathrm{N} / \mathrm{A}$ & N/A \\
\hline $5 \mathrm{H} 13-114-3$ & & 0.00 & 0.00 & 0.00 & 0.00 & 0.00 & 0.00 & 0.00 & 0.06 & 0.01 & 0.03 & 0.08 & 0.13 & 0.42 & 0.72 & 2.67 & 8.86 & 33.80 & 42.50 & 8.82 & 1.90 & $\mathrm{~N} / \mathrm{A}$ & $\mathrm{N} / \mathrm{A}$ & $\mathrm{N} / \mathrm{A}$ & $\mathrm{N} / \mathrm{A}$ & $\mathrm{N} / \mathrm{A}$ & N/A & N/A \\
\hline & & 0.00 & 18.96 & 30.84 & 16.69 & 14.88 & 6.33 & 2.35 & 0.96 & 0.93 & 0.73 & 0.70 & 0.68 & 0.76 & 0.69 & 0.82 & 0.63 & 1.02 & 0.97 & 0.36 & 0.67 & $\mathrm{~N} / \mathrm{A}$ & $\mathrm{N} / \mathrm{A}$ & N/A & $\mathrm{N} / \mathrm{A}$ & $\mathrm{N} / \mathrm{A}$ & N/A & N/A \\
\hline GSH13-T15-S2 & 0.00 & 0.00 & 14.59 & 7.81 & 13.53 & 10.86 & 5.83 & 5.09 & 4.06 & 3.69 & 3.16 & 1.99 & 1.88 & 2.05 & 2.08 & 3.58 & 3.58 & 7.32 & 5.95 & 1.52 & 1.44 & N/A & $\mathrm{N} / \mathrm{A}$ & $\mathrm{N} / \mathrm{A}$ & $\mathrm{N} / \mathrm{A}$ & $\mathrm{N} / \mathrm{A}$ & N/A & N/A \\
\hline SH13-T17-S1 & 0.00 & 0.00 & 0.00 & 0.00 & 0.00 & 0.00 & 0.00 & 0.00 & 0.00 & 0.04 & 0.04 & 0.09 & 0.20 & 0.46 & 0.44 & 1.25 & 7.26 & 45.84 & 37.04 & 6.00 & 1.34 & $\mathrm{~N} / \mathrm{A}$ & N/A & N/A & $\mathrm{N} / \mathrm{A}$ & N/A & N/A & $\mathrm{N} / \mathrm{l}$ \\
\hline
\end{tabular}


Section 3: GSH14 and SH14 


\section{GSH14 and SH14: Identification, Location, and Description}

\begin{tabular}{|c|c|c|c|c|c|c|c|c|}
\hline Sample ID & Global Sample ID & Station ID & $\begin{array}{c}\text { Latitude } \\
\text { WGS84 }\end{array}$ & $\begin{array}{c}\text { Longitude } \\
\text { WGS84 }\end{array}$ & $\begin{array}{l}\text { Reliability } \\
\text { Ranking for } \\
\text { Positioning }\end{array}$ & $\begin{array}{c}\text { Water } \\
\text { Depth }(m)\end{array}$ & $\begin{array}{l}\text { Sample } \\
\text { Collected }\end{array}$ & $\begin{array}{c}\text { Total Wt } \\
\text { (gm) }\end{array}$ \\
\hline GSH14-T14-S1 & GSH14-T14-S1 7/18/2017 & GSH14-T14 & 42.983649 & -70.738158 & 1 & 15.8 & $7 / 18 / 2017$ & 581.6 \\
\hline GSH14-T14-S2 & GSH14-T14-S2_7/18/2017 & GSH14-T14 & 42.983600 & -70.738090 & 1 & 15.8 & $7 / 18 / 2017$ & 357.8 \\
\hline GSH14-T15-S1 & GSH14-T15-S1_7/18/2017 & GSH14-T15 & 42.982267 & -70.736127 & 1 & 18.9 & $7 / 18 / 2017$ & 157.9 \\
\hline GSH14-T16-S1 & GSH14-T16-S1_7/18/2017 & GSH14-T16 & 42.978441 & -70.736979 & 1 & 19.8 & $7 / 18 / 2017$ & 166.0 \\
\hline GSH14-T17-S1 & GSH14-T17-S1_11/1/2016 & GSH14-T17 & 42.977165 & -70.740457 & 1 & 19.5 & $11 / 1 / 2016$ & 45.5 \\
\hline GSH14-T17-S2 & GSH14-T17-S2_11/1/2016 & GSH14-T17 & 42.977288 & -70.740452 & 1 & 19.2 & $11 / 1 / 2016$ & 255.1 \\
\hline GSH14-T20-S2 & GSH14-T20-S2_11/1/2016 & GSH14-T20 & 42.974750 & -70.748936 & 1 & 18.0 & $11 / 1 / 2016$ & 176.0 \\
\hline GSH14-T24-S1 & GSH14-T24-S1_7/18/2017 & GSH14-T24 & 42.979850 & -70.745290 & 1 & 14.9 & $7 / 18 / 2017$ & 93.9 \\
\hline GSH14-T35-S1 & GSH14-T35-S1_7/18/2017 & GSH14-T35 & 42.979467 & -70.742292 & 1 & 16.5 & $7 / 18 / 2017$ & 114.7 \\
\hline GSH14-T36-S1 & GSH14-T36-S1_7/18/2017 & GSH14-T36 & 42.979925 & -70.752487 & 1 & 8.2 & $7 / 18 / 2017$ & 137.8 \\
\hline SH14-BS-02 & SH14_BS-02_6/30/2014 & SH14-BS-02 & 42.979475 & -70.741855 & 1 & 18.3 & $6 / 30 / 2014$ & 36.7 \\
\hline SH14-BS-03A & SH14_BS-03A_6/30/2014 & SH14-BS-03A & 42.983972 & -70.736481 & 1 & 20.3 & $6 / 30 / 2014$ & 484.1 \\
\hline SH14-BS-03B & SH14_BS-03B_6/30/2014 & SH14-BS-03B & 42.983936 & -70.736288 & 1 & 20.9 & $6 / 30 / 2014$ & 385.9 \\
\hline SH14-BS-05 & SH14_BS-05_6/30/2014 & SH14-BS-05 & 42.996231 & -70.740064 & 1 & 10.6 & $6 / 30 / 2014$ & 100.2 \\
\hline SH14-BS-06 & SH14_BS-06_7/1/2014 & SH14-BS-06 & 42.995856 & -70.728489 & 1 & 17.7 & $7 / 1 / 2014$ & 149.1 \\
\hline SH14-BS-07 & SH14_BS-07_7/1/2014 & SH14-BS-07 & 43.000470 & -70.727247 & 1 & 16.5 & $7 / 1 / 2014$ & 130.5 \\
\hline SH14-BS-08 & SH14_BS-08_7/1/2014 & SH14-BS-08 & 43.000737 & -70.722898 & 1 & 19.8 & $7 / 1 / 2014$ & 174.5 \\
\hline SH14-BS-09 & SH14_BS-09_7/1/2014 & SH14-BS-09 & 43.006318 & -70.720523 & 1 & 8.8 & $7 / 1 / 2014$ & 390.5 \\
\hline SH14-BS-10 & SH14_BS-10_7/1/2014 & SH14-BS-10 & 43.008803 & -70.714451 & 1 & 20.7 & $7 / 1 / 2014$ & 112.0 \\
\hline
\end{tabular}


GSH14 and SH14: Sediment Classifications

\begin{tabular}{|c|c|c|c|c|c|c|c|c|}
\hline Sample ID & $\begin{array}{l}\text { CMECS Substrate } \\
\text { Component } \\
\text { Group (Specific) }\end{array}$ & $\begin{array}{l}\text { CMECS Substrate } \\
\text { Component } \\
\text { Subgroup (Specific) }\end{array}$ & $\begin{array}{l}\text { Textural Group } \\
\text { from \%GSM } \\
\text { (Gradistat) }\end{array}$ & $\begin{array}{l}\text { Sediment Name from \%GSM } \\
\text { and Mode (Gradistat) }\end{array}$ & $\begin{array}{l}\text { Sediment Name from } \\
\text { \%GSM and Mode } \\
\text { (Wentworth Scale) }\end{array}$ & $\begin{array}{l}\text { Sediment } \\
\text { Classification from } \\
\text { Mean Phi (Gradistat) }\end{array}$ & $\begin{array}{l}\text { Classification from } \\
\text { Mean Phi } \\
\text { (Wentworth) }\end{array}$ & $\begin{array}{l}\text { Sorting } \\
\text { (Gradistat) }\end{array}$ \\
\hline GSH14-T14-S1 & Pebble Mixes & $\begin{array}{l}\text { Sandy Pebble } \\
\text { Gravel }\end{array}$ & Sandy Gravel & Sandy Medium Gravel & Sandy Pebble Gravel & Very Fine Gravel & Granule Gravel & $\begin{array}{l}\text { Very Poorly } \\
\text { Sorted }\end{array}$ \\
\hline GSH14-T14-S2 & Pebble Mixes & $\begin{array}{l}\text { Sandy Pebble } \\
\text { Gravel }\end{array}$ & Sandy Gravel & Sandy Medium Gravel & Sandy Pebble Gravel & Fine Gravel & Pebble Gravel & Poorly Sorted \\
\hline GSH14-T15-S1 & Pebbly & Pebbly Sand & Gravelly Sand & Fine Gravelly Very Fine Sand & Pebbly Very Fine Sand & Fine Sand & Fine Sand & Poorly Sorted \\
\hline GSH14-T16-S1 & Slightly Pebbly & $\begin{array}{l}\text { Slightly Pebbly Fine } \\
\text { Sand }\end{array}$ & $\begin{array}{l}\text { Slightly Gravelly } \\
\text { Sand }\end{array}$ & $\begin{array}{l}\text { Slightly Medium Gravelly Fine } \\
\text { Sand }\end{array}$ & Slightly Pebbly Fine Sand & Fine Sand & Fine Sand & $\begin{array}{l}\text { Moderately } \\
\text { Sorted }\end{array}$ \\
\hline GSH14-T17-S1 & Slightly Pebbly & $\begin{array}{l}\text { Slightly Pebbly } \\
\text { Muddy Fine Sand }\end{array}$ & $\begin{array}{l}\text { Slightly Gravelly } \\
\text { Muddy Sand }\end{array}$ & $\begin{array}{l}\text { Slightly Fine Gravelly Muddy } \\
\text { Fine Sand }\end{array}$ & $\begin{array}{l}\text { Slightly Pebbly Muddy Fine } \\
\text { Sand }\end{array}$ & Fine Sand & Fine Sand & Poorly Sorted \\
\hline GSH14-T17-S2 & Pebbly & Pebbly Fine Sand & Gravelly Sand & Medium Gravelly Fine Sand & Pebbly Fine Sand & Fine Sand & Fine Sand & Poorly Sorted \\
\hline GSH14-T2O-S2 & Slightly Granuley & $\begin{array}{l}\text { Slightly Granuley } \\
\text { Silty Very Fine Sand }\end{array}$ & $\begin{array}{l}\text { Slightly Gravelly } \\
\text { Muddy Sand }\end{array}$ & $\begin{array}{l}\text { Slightly Very Fine Gravelly Very } \\
\text { Coarse Silty Very Fine Sand }\end{array}$ & $\begin{array}{l}\text { Slightly Granular Very } \\
\text { Coarse Silty Very Fine Sand }\end{array}$ & Very Fine Sand & Very Fine Sand & Poorly Sorted \\
\hline GSH14-T24-S1 & Slightly Granuley & $\begin{array}{l}\text { Slightly Granuley } \\
\text { Very Fine Sand }\end{array}$ & $\begin{array}{l}\text { Slightly Gravelly } \\
\text { Sand }\end{array}$ & $\begin{array}{l}\text { Slightly Very Fine Gravelly Very } \\
\text { Fine Sand }\end{array}$ & $\begin{array}{l}\text { Slightly Granular Very Fine } \\
\text { Sand }\end{array}$ & Fine Sand & Fine Sand & $\begin{array}{l}\text { Moderately } \\
\text { Sorted }\end{array}$ \\
\hline GSH14-T35-S1 & Slightly Granuley & $\begin{array}{l}\text { Slightly Granuley } \\
\text { Silty Fine Sand }\end{array}$ & $\begin{array}{l}\text { Slightly Gravelly } \\
\text { Muddy Sand }\end{array}$ & $\begin{array}{l}\text { Slightly Very Fine Gravelly Very } \\
\text { Coarse Silty Very Fine Sand }\end{array}$ & $\begin{array}{l}\text { Slightly Granular Very } \\
\text { Coarse Silty Fine Sand }\end{array}$ & Very Fine Sand & Very Fine Sand & Poorly Sorted \\
\hline GSH14-T36-S1 & Slightly Pebbly & $\begin{array}{l}\text { Slightly Pebbly Very } \\
\text { Fine Sand }\end{array}$ & $\begin{array}{l}\text { Slightly Gravelly } \\
\text { Sand }\end{array}$ & $\begin{array}{l}\text { Slightly Fine Gravelly Very Fine } \\
\text { Sand }\end{array}$ & $\begin{array}{l}\text { Slightly Pebbly Very Fine } \\
\text { Sand }\end{array}$ & Very Fine Sand & Very Fine Sand & $\begin{array}{l}\text { Moderately Well } \\
\text { Sorted }\end{array}$ \\
\hline SH14-BS-02 & Slightly Granuley & $\begin{array}{l}\text { Slightly Granuley } \\
\text { Silty Very Fine Sand }\end{array}$ & $\begin{array}{l}\text { Slightly Gravelly } \\
\text { Muddy Sand }\end{array}$ & $\begin{array}{l}\text { Slightly Very Fine Gravelly Very } \\
\text { Coarse Silty Very Fine Sand }\end{array}$ & $\begin{array}{l}\text { Slightly Granular Very } \\
\text { Coarse Silty Very Fine Sand }\end{array}$ & Very Fine Sand & Very Fine Sand & Poorly Sorted \\
\hline SH14-BS-03A & Gravel & Pebble Gravel & Gravel & Medium Gravel & Pebble Gravel & Medium Gravel & Pebble Gravel & Poorly Sorted \\
\hline SH14-BS-03B & Pebble Mixes & $\begin{array}{l}\text { Sandy Pebble } \\
\text { Gravel }\end{array}$ & Sandy Gravel & Sandy Medium Gravel & Sandy Pebble Gravel & Very Fine Gravel & Granule Gravel & $\begin{array}{l}\text { Very Poorly } \\
\text { Sorted }\end{array}$ \\
\hline SH14-BS-05 & Sand & Very Fine Sand & Sand & Well Sorted Very Fine Sand & Very Fine Sand & Very Fine Sand & Very Fine Sand & Well Sorted \\
\hline SH14-BS-06 & Slightly Pebbly & $\begin{array}{l}\text { Slightly Pebbly Very } \\
\text { Fine Sand }\end{array}$ & $\begin{array}{l}\text { Slightly Gravelly } \\
\text { Sand }\end{array}$ & $\begin{array}{l}\text { Slightly Medium Gravelly Very } \\
\text { Fine Sand }\end{array}$ & $\begin{array}{l}\text { Slightly Pebbly Very Fine } \\
\text { Sand }\end{array}$ & Very Fine Sand & Very Fine Sand & $\begin{array}{l}\text { Moderately Well } \\
\text { Sorted }\end{array}$ \\
\hline SH14-BS-07 & Pebbly & $\begin{array}{l}\text { Pebbly Very Fine } \\
\text { Sand }\end{array}$ & Gravelly Sand & Medium Gravelly Very Fine Sand & Pebbly Very Fine Sand & Fine Sand & Fine Sand & Poorly Sorted \\
\hline SH14-BS-08 & Pebbly & Pebbly Fine Sand & Gravelly Sand & Medium Gravelly Fine Sand & Pebbly Fine Sand & Coarse Sand & Coarse Sand & $\begin{array}{l}\text { Very Poorly } \\
\text { Sorted }\end{array}$ \\
\hline SH14-BS-09 & Pebble Mixes & $\begin{array}{l}\text { Sandy Pebble } \\
\text { Gravel }\end{array}$ & Sandy Gravel & Sandy Medium Gravel & Sandy Pebble Gravel & Very Coarse Sand & Very Coarse Sand & $\begin{array}{l}\text { Very Poorly } \\
\text { Sorted }\end{array}$ \\
\hline SH14-BS-10 & Pebbly & $\begin{array}{l}\text { Pebbly Very Fine } \\
\text { Sand }\end{array}$ & Gravelly Sand & Medium Gravelly Very Fine Sand & Pebbly Very Fine Sand & Medium Sand & Medium Sand & $\begin{array}{l}\text { Very Poorly } \\
\text { Sorted }\end{array}$ \\
\hline
\end{tabular}


GSH14 and SH14: Grain Size Statistics

\begin{tabular}{|c|c|c|c|c|c|c|c|c|c|c|c|c|c|c|c|c|c|c|c|c|}
\hline Sample ID & $\begin{array}{c}\text { Gravel } \\
\%\end{array}$ & $\begin{array}{c}\text { Pebble } \\
\%\end{array}$ & $\begin{array}{c}\text { Granule } \\
\%\end{array}$ & Sand \% & Mud \% & Silt \% & Clay \% & Modes & $\begin{array}{c}\text { Mode } 1 \\
\text { (phi) }\end{array}$ & $\begin{array}{c}\text { Mode } 2 \\
\text { (phi) }\end{array}$ & $\begin{array}{l}\mathrm{D}_{10} \\
\text { (phi) }\end{array}$ & $\begin{array}{c}D_{10} \\
(\mathrm{~mm})\end{array}$ & $\begin{array}{c}D_{50} \\
\text { (phi) }\end{array}$ & $\begin{array}{c}D_{50} \\
(\mathrm{~mm})\end{array}$ & $\begin{array}{c}\text { Mean } \\
\text { Size } \\
\text { (phi) }\end{array}$ & $\begin{array}{c}\text { Mean } \\
\text { Size } \\
(\mathrm{mm})\end{array}$ & $\begin{array}{l}\text { Sorting } \\
\text { (phi) }\end{array}$ & Skewness & Kurtosis & $\begin{array}{l}\text { LOI \% } \\
450^{\circ} \mathrm{C}\end{array}$ \\
\hline GSH14-T14-S1 & 60.12 & 53.62 & 6.50 & 38.50 & 1.38 & $\mathrm{~N} / \mathrm{A}$ & $\mathrm{N} / \mathrm{A}$ & $\mathrm{T}$ & -3.74 & -0.24 & -4.20 & 18.39 & -2.40 & 5.26 & -1.83 & 3.57 & 2.36 & 0.41 & 0.82 & 0.59 \\
\hline GSH14-T14-S2 & 68.21 & 57.99 & 10.22 & 31.18 & 0.61 & $\mathrm{~N} / \mathrm{A}$ & $\mathrm{N} / \mathrm{A}$ & $\mathrm{B}$ & -3.74 & -0.24 & -4.69 & 25.78 & -2.90 & 7.44 & -2.54 & 5.80 & 1.88 & 0.26 & 0.68 & 0.32 \\
\hline GSH14-T15-S1 & 6.84 & 5.22 & 1.62 & 88.39 & 4.77 & 3.57 & 1.20 & U & 3.24 & $\mathrm{~N} / \mathrm{A}$ & 0.61 & 0.66 & 2.79 & 0.14 & 2.59 & 0.17 & 1.37 & -0.47 & 2.04 & 0.65 \\
\hline GSH14-T16-S1 & 2.60 & 2.49 & 0.10 & 95.91 & 1.49 & $\mathrm{~N} / \mathrm{A}$ & $\mathrm{N} / \mathrm{A}$ & U & 2.74 & $\mathrm{~N} / \mathrm{A}$ & 1.35 & 0.39 & 2.52 & 0.17 & 2.46 & 0.18 & 0.80 & -0.20 & 1.01 & 0.40 \\
\hline GSH14-T17-S1 & 1.86 & 1.73 & 0.14 & 84.90 & 13.24 & $\mathrm{~N} / \mathrm{A}$ & $\mathrm{N} / \mathrm{A}$ & $\mathrm{U}$ & 2.24 & $\mathrm{~N} / \mathrm{A}$ & 1.36 & 0.39 & 2.55 & 0.17 & 2.63 & 0.16 & 1.91 & 0.37 & 2.70 & 1.78 \\
\hline GSH14-T17-S2 & 6.84 & 6.23 & 0.61 & 88.60 & 4.56 & 3.36 & 1.20 & $u$ & 3.24 & $\mathrm{~N} / \mathrm{A}$ & 1.11 & 0.46 & 2.61 & 0.16 & 2.54 & 0.17 & 1.43 & -0.36 & 2.17 & 0.50 \\
\hline GSH14-T20-S2 & 0.03 & 0.00 & 0.03 & 58.08 & 41.89 & 37.62 & 4.27 & $\mathrm{U}$ & 3.24 & $\mathrm{~N} / \mathrm{A}$ & 2.91 & 0.13 & 3.81 & 0.07 & 3.94 & 0.07 & 1.14 & 0.33 & 1.41 & 1.44 \\
\hline GSH14-T24-S1 & 1.83 & 0.88 & 0.95 & 92.94 & 5.23 & 4.24 & 0.99 & U & 3.24 & $\mathrm{~N} / \mathrm{A}$ & 1.52 & 0.35 & 2.84 & 0.14 & 2.69 & 0.15 & 0.89 & -0.24 & 1.08 & 0.62 \\
\hline GSH14-T35-S1 & 0.39 & 0.09 & 0.30 & 78.26 & 21.35 & 19.56 & 1.79 & U & 3.24 & $\mathrm{~N} / \mathrm{A}$ & 2.09 & 0.24 & 3.31 & 0.10 & 3.39 & 0.10 & 1.04 & 0.11 & 1.50 & 0.88 \\
\hline GSH14-T36-S1 & 0.48 & 0.48 & 0.00 & 96.22 & 3.30 & $\mathrm{~N} / \mathrm{A}$ & $\mathrm{N} / \mathrm{A}$ & U & 3.24 & $\mathrm{~N} / \mathrm{A}$ & 2.50 & 0.18 & 3.14 & 0.11 & 3.10 & 0.12 & 0.53 & -0.17 & 1.30 & 0.49 \\
\hline SH14-BS-02 & 0.05 & 0.00 & 0.05 & 73.00 & 26.95 & 22.70 & 4.25 & U & 3.24 & $\mathrm{~N} / \mathrm{A}$ & 2.03 & 0.24 & 3.34 & 0.10 & 3.43 & 0.09 & 1.37 & 0.24 & 1.53 & $\mathrm{~N} / \mathrm{A}$ \\
\hline SH14-BS-03A & 88.03 & 81.23 & 6.79 & 11.00 & 0.97 & $\mathrm{~N} / \mathrm{A}$ & $\mathrm{N} / \mathrm{A}$ & U & -4.24 & $\mathrm{~N} / \mathrm{A}$ & -4.36 & 20.49 & -3.37 & 10.33 & -3.09 & 8.49 & 1.61 & 0.49 & 1.69 & $\mathrm{~N} / \mathrm{A}$ \\
\hline SH14-BS-03B & 70.08 & 57.19 & 12.89 & 28.48 & 1.44 & $\mathrm{~N} / \mathrm{A}$ & $\mathrm{N} / \mathrm{A}$ & U & -3.74 & $\mathrm{~N} / \mathrm{A}$ & -3.75 & 13.47 & -2.41 & 5.32 & -1.79 & 3.45 & 2.08 & 0.50 & 0.97 & 0.37 \\
\hline SH14-BS-05 & 0.00 & 0.00 & 0.00 & 93.47 & 6.53 & 5.97 & 0.56 & U & 3.24 & $\mathrm{~N} / \mathrm{A}$ & 2.72 & 0.15 & 3.25 & 0.10 & 3.28 & 0.10 & 0.47 & 0.15 & 1.64 & 0.64 \\
\hline SH14-BS-06 & 2.62 & 1.90 & 0.72 & 92.60 & 4.78 & 3.13 & 1.65 & U & 3.24 & $\mathrm{~N} / \mathrm{A}$ & 2.26 & 0.21 & 3.09 & 0.12 & 3.03 & 0.12 & 0.67 & -0.31 & 1.90 & 0.71 \\
\hline SH14-BS-07 & 6.24 & 5.52 & 0.72 & 88.76 & 5.00 & 3.52 & 1.48 & U & 3.24 & $\mathrm{~N} / \mathrm{A}$ & 1.78 & 0.29 & 3.02 & 0.12 & 2.90 & 0.13 & 1.31 & -0.49 & 3.24 & 0.73 \\
\hline SH14-BS-08 & 29.57 & 25.72 & 3.85 & 67.08 & 3.35 & $\mathrm{~N} / \mathrm{A}$ & $\mathrm{N} / \mathrm{A}$ & $\mathrm{B}$ & 3.24 & -3.74 & -3.64 & 12.44 & 2.11 & 0.23 & 0.72 & 0.61 & 2.79 & -0.61 & 0.62 & 0.75 \\
\hline SH14-BS-09 & 43.47 & 37.10 & 6.36 & 54.55 & 1.98 & $\mathrm{~N} / \mathrm{A}$ & $\mathrm{N} / \mathrm{A}$ & $\mathrm{B}$ & -3.74 & 1.75 & -3.84 & 14.30 & 0.08 & 0.95 & -0.25 & 1.19 & 2.71 & -0.01 & 0.58 & 0.61 \\
\hline SH14-BS-10 & 17.36 & 15.51 & 1.84 & 78.24 & 4.40 & 2.99 & 1.41 & B & 3.24 & -3.24 & -3.02 & 8.09 & 2.92 & 0.13 & 1.52 & 0.35 & 2.42 & -0.76 & 1.82 & 0.70 \\
\hline
\end{tabular}


GSH14 and SH14: Grain Size Distribution

\begin{tabular}{|c|c|c|c|c|c|c|c|c|c|c|c|c|c|c|c|c|c|c|c|c|c|c|c|c|c|c|c|c|}
\hline & $\begin{array}{c}\text { Class } \\
\% \\
\text { phi } \\
-5.5\end{array}$ & $\begin{array}{c}\text { Class } \\
\% \\
\text { phi } \\
-5.0\end{array}$ & $\begin{array}{c}\text { Class } \\
\% \\
\text { phi } \\
-4.5\end{array}$ & $\begin{array}{c}\text { Class } \\
\% \\
\text { phi } \\
-4.0\end{array}$ & $\begin{array}{c}\text { Class } \\
\% \\
\text { phi } \\
-3.5\end{array}$ & $\begin{array}{c}\text { Class } \\
\% \\
\text { phi } \\
-3.0\end{array}$ & $\begin{array}{c}\text { Class } \\
\% \\
\text { phi } \\
-2.5\end{array}$ & $\begin{array}{c}\text { Class } \\
\% \\
\text { phi } \\
-20\end{array}$ & $\begin{array}{c}\text { Class } \\
\% \\
\text { phi } \\
-1.5\end{array}$ & $\begin{array}{c}\text { Class } \\
\% \\
\text { phi } \\
-10\end{array}$ & $\begin{array}{c}\text { Class } \\
\% \\
\text { phi } \\
-0.5\end{array}$ & $\begin{array}{c}\text { Class } \\
\% \\
\text { phi }\end{array}$ & $\begin{array}{c}\text { Class } \\
\% \\
\text { phi } \\
0.5\end{array}$ & $\begin{array}{c}\text { Class } \\
\% \\
\text { phi }\end{array}$ & $\begin{array}{c}\text { Class } \\
\% \\
\text { phi }\end{array}$ & $\begin{array}{c}\text { Class } \\
\% \\
\text { phi }\end{array}$ & $\begin{array}{c}\text { Class } \\
\% \\
\text { phi }\end{array}$ & $\begin{array}{c}\text { Class } \\
\% \\
\text { phi }\end{array}$ & $\begin{array}{c}\text { Class } \\
\% \\
\text { phi }\end{array}$ & $\begin{array}{c}\text { Class } \\
\% \\
\text { phi }\end{array}$ & $\begin{array}{c}\text { Class } \\
\% \\
\text { phi }\end{array}$ & $\begin{array}{c}\text { Class } \\
\% \\
\text { phi }\end{array}$ & $\begin{array}{c}\text { Class } \\
\% \\
\text { phi }\end{array}$ & $\begin{array}{c}\text { Class } \\
\% \\
\text { phi }\end{array}$ & $\begin{array}{c}\text { Class } \\
\% \\
\text { phi }\end{array}$ & $\begin{array}{c}\text { Class } \\
\% \\
\text { phi }\end{array}$ & $\begin{array}{c}\text { Class } \\
\% \\
\text { phi }\end{array}$ & $\begin{array}{c}\text { Class } \\
\% \\
\text { phi }\end{array}$ \\
\hline Sample ID & -5.5 & -5.0 & -4.5 & -4.0 & -3.5 & -3.0 & -2.5 & -2.0 & -1.5 & -1.0 & -0.5 & 0.0 & 0.5 & 1.0 & 1.5 & 2.0 & 2.5 & 3.0 & 3.5 & 4.0 & $>4.0$ & 5.0 & 6.0 & 7.0 & 8.0 & 9.0 & 10.0 & $>10.0$ \\
\hline GSH14-T14-S1 & 0.00 & 0.00 & 2.84 & 12.24 & 15.61 & 13.36 & 5.12 & 4.44 & 3.43 & 3.07 & 4.73 & 8.10 & 7.95 & 4.24 & 2.24 & 1.34 & 1.06 & 2.90 & 4.78 & 1.16 & 1.38 & $\mathrm{~N} / \mathrm{A}$ & N/A & $\mathrm{N} / \mathrm{A}$ & $\mathrm{N} / \mathrm{A}$ & N/A & N/A & $\mathrm{N} / \mathrm{A}$ \\
\hline GSH14-T14-S2 & 0.00 & 0.00 & 17.02 & 0.00 & 22.02 & 10.08 & 4.26 & 4.60 & 4.43 & 5.79 & 9.06 & 11.44 & 6.98 & 1.47 & 0.32 & 0.17 & 0.13 & 0.38 & 0.85 & 0.38 & 0.61 & N/A & $\mathrm{N} / \mathrm{A}$ & N/A & $\mathrm{N} / \mathrm{A}$ & $\mathrm{N} / \mathrm{A}$ & N/A & $\mathrm{N} / \mathrm{A}$ \\
\hline GSH14-T15-S1 & 0.00 & 0.00 & 0.00 & 0.00 & 0.00 & 1.04 & 2.85 & 1.32 & 0.84 & 0.78 & 1.01 & 0.88 & 0.97 & 1.35 & 3.30 & 9.64 & 11.67 & 23.93 & 28.60 & 7.04 & $\mathrm{~N} / \mathrm{A}$ & 2.85 & 0.39 & 0.18 & 0.14 & 0.14 & 0.18 & 0.87 \\
\hline GSH14-T16-S1 & 0.00 & 0.00 & 0.00 & 0.00 & 1.34 & 0.92 & 0.23 & 0.00 & 0.09 & 0.01 & 0.08 & 0.24 & 0.71 & 2.24 & 5.84 & 14.85 & 21.24 & 26.20 & 21.51 & 3.00 & 1.49 & N/A & $\mathrm{N} / \mathrm{A}$ & $\mathrm{N} / \mathrm{A}$ & $\mathrm{N} / \mathrm{A}$ & $\mathrm{N} / \mathrm{A}$ & N/A & $\mathrm{N} / \mathrm{A}$ \\
\hline GSH14-T17-S1 & 0.00 & 0.00 & 0.00 & 0.00 & 0.00 & 0.00 & 1.67 & 0.05 & 0.00 & 0.14 & 0.21 & 0.45 & 0.83 & 2.44 & 5.82 & 15.61 & 20.85 & 12.60 & 20.91 & 5.18 & N/A & 2.20 & 1.78 & 1.30 & 1.09 & 0.77 & 0.83 & 5.08 \\
\hline GSH14-T17-S2 & 0.00 & 0.00 & 0.00 & 0.00 & 3.46 & 0.96 & 0.75 & 1.06 & 0.23 & 0.38 & 0.30 & 0.34 & 0.51 & 1.21 & 3.59 & 13.01 & 19.39 & 18.89 & 24.66 & 6.69 & $\mathrm{~N} / \mathrm{A}$ & 2.70 & 0.41 & 0.12 & 0.13 & 0.11 & 0.21 & 0.89 \\
\hline GSH14-T2O-S2 & 0.00 & 0.00 & 0.00 & 0.00 & 0.00 & 0.00 & 0.00 & 0.00 & 0.02 & 0.01 & 0.09 & 0.07 & 0.05 & 0.11 & 0.25 & 0.97 & 2.44 & 7.24 & 23.67 & 23.17 & $\mathrm{~N} / \mathrm{A}$ & 28.87 & 6.86 & 1.11 & 0.77 & 0.73 & 1.02 & 2.52 \\
\hline GSH14-T24-S1 & 0.00 & 0.00 & 0.00 & 0.00 & 0.00 & 0.00 & 0.00 & 0.88 & 0.75 & 0.20 & 0.41 & 0.56 & 0.77 & 1.54 & 4.21 & 11.65 & 14.81 & 20.60 & 28.86 & 9.53 & $\mathrm{~N} / \mathrm{A}$ & 3.69 & 0.30 & 0.14 & 0.11 & 0.15 & 0.24 & 0.59 \\
\hline GSH14-T35-S1 & 0.00 & 0.00 & 0.00 & 0.00 & 0.00 & 0.00 & 0.00 & 0.09 & 0.20 & 0.10 & 0.28 & 0.27 & 0.36 & 0.63 & 1.69 & 5.11 & 7.11 & 12.75 & 32.33 & 17.74 & N/A & 14.67 & 4.21 & 0.41 & 0.27 & 0.33 & 0.50 & 0.96 \\
\hline GSH14-T36-S1 & 0.00 & 0.00 & 0.00 & 0.00 & 0.00 & 0.00 & 0.48 & 0.00 & 0.00 & 0.00 & 0.03 & 0.08 & 0.24 & 0.59 & 1.21 & 2.56 & 3.68 & 27.25 & 45.66 & 14.93 & 3.30 & N/A & $\mathrm{N} / \mathrm{A}$ & $\mathrm{N} / \mathrm{A}$ & $\mathrm{N} / \mathrm{A}$ & N/A & $\mathrm{N} / \mathrm{A}$ & $\mathrm{N} / \mathrm{A}$ \\
\hline SH14-BS-02 & 0.00 & 0.00 & 0.00 & 0.00 & 0.00 & 0.00 & 0.00 & 0.00 & 0.00 & 0.05 & 0.10 & 0.17 & 0.18 & 0.43 & 1.53 & 6.89 & 10.16 & 12.46 & 25.40 & 15.69 & N/A & 17.22 & 3.79 & 1.05 & 0.64 & 0.65 & 0.59 & 3.01 \\
\hline SH14-BS-03A & 0.00 & 0.00 & 3.96 & 22.83 & 18.63 & 19.15 & 10.43 & 6.23 & 4.16 & 2.64 & 1.99 & 1.33 & 1.13 & 0.97 & 0.85 & 0.84 & 0.83 & 1.13 & 1.42 & 0.52 & 0.97 & N/A & $\mathrm{N} / \mathrm{A}$ & N/A & $\mathrm{N} / \mathrm{A}$ & $\mathrm{N} / \mathrm{A}$ & $\mathrm{N} / \mathrm{A}$ & $\mathrm{N} / \mathrm{A}$ \\
\hline SH14-BS-03B & 0.00 & 0.00 & 0.00 & 0.00 & 20.71 & 17.34 & 10.66 & 8.48 & 6.82 & 6.07 & 5.39 & 3.95 & 3.54 & 3.43 & 2.98 & 2.46 & 1.82 & 2.01 & 2.16 & 0.74 & 1.44 & N/A & $\mathrm{N} / \mathrm{A}$ & N/A & $\mathrm{N} / \mathrm{A}$ & N/A & $\mathrm{N} / \mathrm{A}$ & $\mathrm{N} / \mathrm{A}$ \\
\hline SH14-BS-05 & 0.00 & 0.00 & 0.00 & 0.00 & 0.00 & 0.00 & 0.00 & 0.00 & 0.00 & 0.00 & 0.03 & 0.01 & 0.02 & 0.02 & 0.07 & 0.28 & 0.97 & 18.78 & 55.60 & 17.68 & $\mathrm{~N} / \mathrm{A}$ & 5.46 & 0.32 & 0.10 & 0.09 & 0.03 & 0.10 & 0.43 \\
\hline SH14-BS-06 & 0.00 & 0.00 & 0.00 & 0.00 & 0.00 & 1.24 & 0.00 & 0.67 & 0.18 & 0.53 & 0.43 & 0.46 & 0.66 & 0.80 & 0.96 & 1.72 & 4.24 & 29.44 & 45.88 & 8.01 & $\mathrm{~N} / \mathrm{A}$ & 2.05 & 0.51 & 0.29 & 0.29 & 0.22 & 0.24 & 1.19 \\
\hline SH14-BS-07 & 0.00 & 0.00 & 0.00 & 0.00 & 0.00 & 2.93 & 2.34 & 0.24 & 0.53 & 0.19 & 0.08 & 0.13 & 0.16 & 0.29 & 0.81 & 4.11 & 10.36 & 26.69 & 34.88 & 11.25 & $\mathrm{~N} / \mathrm{A}$ & 2.56 & 0.54 & 0.25 & 0.18 & 0.18 & 0.25 & 1.05 \\
\hline SH14-BS-08 & 0.00 & 0.00 & 0.00 & 4.45 & 7.87 & 6.24 & 3.98 & 3.18 & 1.95 & 1.90 & 1.71 & 1.21 & 1.09 & 1.62 & 3.20 & 8.56 & 12.54 & 15.43 & 17.23 & 4.48 & 3.35 & N/A & $\mathrm{N} / \mathrm{A}$ & $\mathrm{N} / \mathrm{A}$ & $\mathrm{N} / \mathrm{A}$ & $\mathrm{N} / \mathrm{A}$ & N/A & $\mathrm{N} / \mathrm{A}$ \\
\hline SH14-BS-09 & 0.00 & 0.00 & 0.00 & 6.48 & 11.20 & 10.31 & 5.60 & 3.51 & 3.23 & 3.14 & 3.10 & 2.95 & 3.16 & 3.92 & 5.59 & 9.71 & 8.15 & 8.28 & 7.86 & 1.82 & 1.98 & $\mathrm{~N} / \mathrm{A}$ & $\mathrm{N} / \mathrm{A}$ & N/A & $\mathrm{N} / \mathrm{A}$ & $\mathrm{N} / \mathrm{A}$ & $\mathrm{N} / \mathrm{A}$ & $\mathrm{N} / \mathrm{A}$ \\
\hline SH14-BS-10 & 0.00 & 0.00 & 0.00 & 0.00 & 4.17 & 6.03 & 3.52 & 1.79 & 1.19 & 0.66 & 0.89 & 0.76 & 0.79 & 1.10 & 2.42 & 5.47 & 6.20 & 17.65 & 34.79 & 8.15 & N/A & 2.19 & 0.43 & 0.21 & 0.16 & 0.21 & 0.22 & 0.98 \\
\hline
\end{tabular}


Section 4: GSH15 
GSH15: Identification, Location, and Description

\begin{tabular}{|c|c|c|c|c|c|c|c|c|}
\hline Sample ID & Global Sample ID & Station ID & $\begin{array}{c}\text { Latitude } \\
\text { WGS84 }\end{array}$ & $\begin{array}{c}\text { Longitude } \\
\text { WGS84 }\end{array}$ & $\begin{array}{l}\text { Reliability } \\
\text { Ranking for } \\
\text { Positioning }\end{array}$ & $\begin{array}{c}\text { Water } \\
\text { Depth }(\mathrm{m})\end{array}$ & $\begin{array}{c}\text { Sample } \\
\text { Collected }\end{array}$ & $\begin{array}{c}\text { Total Wt } \\
\text { (gm) }\end{array}$ \\
\hline GSH15-T01-S1 & GSH15-T01-S1_11/1/2016 & GSH15-T01 & 42.930871 & -70.790805 & 1 & 7.6 & $11 / 1 / 2016$ & 210.6 \\
\hline GSH15-T01-S2 & GSH15-T01-S2_11/1/2016 & GSH15-T01 & 42.930622 & -70.791069 & 1 & 7.6 & $11 / 1 / 2016$ & 21.5 \\
\hline GSH15-T02-S1 & GSH15-T02-S1_11/1/2016 & GSH15-T02 & 42.926577 & -70.790042 & 1 & 8.8 & $11 / 1 / 2016$ & 79.5 \\
\hline GSH15-T02-S2 & GSH15-T02-S2_11/1/2016 & GSH15-T02 & 42.926825 & -70.790057 & 1 & 8.8 & $11 / 1 / 2016$ & 62.5 \\
\hline GSH15-T05-S1 & GSH15-T05-S1_11/1/2016 & GSH15-T05 & 42.947430 & -70.775635 & 1 & 13.1 & $11 / 1 / 2016$ & 119.0 \\
\hline GSH15-T05-S2 & GSH15-T05-S2_11/1/2016 & GSH15-T05 & 42.947053 & -70.775637 & 1 & 13.7 & $11 / 1 / 2016$ & 59.3 \\
\hline GSH15-T07-S1 & GSH15-T07-S1_10/6/2016 & GSH15-T07 & 42.935006 & -70.767757 & 1 & 21.9 & $10 / 6 / 2016$ & 270.6 \\
\hline GSH15-T07-S2 & GSH15-T07-S2_10/6/2016 & GSH15-T07 & 42.935032 & -70.768204 & 1 & 21.9 & $10 / 6 / 2016$ & 294.3 \\
\hline GSH15-T08-S1 & GSH15-T08-S1_11/1/2016 & GSH15-T08 & 42.932906 & -70.773033 & 1 & 20.1 & $11 / 1 / 2016$ & 207.0 \\
\hline GSH15-T08-S2 & GSH15-T08-S2_11/1/2016 & GSH15-T08 & 42.932895 & -70.772589 & 1 & 20.1 & $11 / 1 / 2016$ & 156.5 \\
\hline GSH15-T10-S1 & GSH15-T10-S1_10/6/2016 & GSH15-T10 & 42.922464 & -70.780157 & 1 & 15.8 & $10 / 6 / 2016$ & 157.9 \\
\hline GSH15-T10-S2 & GSH15-T10-S2_10/6/2016 & GSH15-T10 & 42.922273 & -70.779691 & 1 & 15.8 & $10 / 6 / 2016$ & 191.9 \\
\hline GSH15-T14-S2 & GSH15-T14-S2_11/1/2016 & GSH15-T14 & 42.944854 & -70.761584 & 1 & 23.8 & $11 / 1 / 2016$ & 102.6 \\
\hline GSH15-T15-S1 & GSH15-T15-S1_11/1/2016 & GSH15-T15 & 42.941103 & -70.762389 & 1 & 25.0 & $11 / 1 / 2016$ & 335.3 \\
\hline GSH15-T15-S2 & GSH15-T15-S2_11/1/2016 & GSH15-T15 & 42.941348 & -70.762455 & 1 & 25.0 & $11 / 1 / 2016$ & 193.5 \\
\hline
\end{tabular}


GSH15: Sediment Classifications

\begin{tabular}{|c|c|c|c|c|c|c|c|c|}
\hline Sample ID & $\begin{array}{l}\text { CMECS Substrate } \\
\text { Component } \\
\text { Group (Specific) }\end{array}$ & $\begin{array}{l}\text { CMECS Substrate } \\
\text { Component } \\
\text { Subgroup (Specific) }\end{array}$ & $\begin{array}{l}\text { Textural Group } \\
\text { from \%GSM } \\
\text { (Gradistat) }\end{array}$ & $\begin{array}{l}\text { Sediment Name from \%GSM } \\
\text { and Mode (Gradistat) }\end{array}$ & $\begin{array}{l}\text { Sediment Name from } \\
\text { \%GSM and Mode } \\
\text { (Wentworth Scale) }\end{array}$ & $\begin{array}{l}\text { Sediment } \\
\text { Classification from } \\
\text { Mean Phi (Gradistat) }\end{array}$ & $\begin{array}{l}\text { Classification from } \\
\text { Mean Phi } \\
\text { (Wentworth) }\end{array}$ & $\begin{array}{l}\text { Sorting } \\
\text { (Gradistat) }\end{array}$ \\
\hline GSH15-T01-S1 & Slightly Granuley & $\begin{array}{l}\text { Slightly Granuley } \\
\text { Very Fine Sand }\end{array}$ & $\begin{array}{l}\text { Slightly Gravelly } \\
\text { Sand }\end{array}$ & $\begin{array}{l}\text { Slightly Very Fine Gravelly Very } \\
\text { Fine Sand }\end{array}$ & $\begin{array}{l}\text { Slightly Granular Very Fine } \\
\text { Sand }\end{array}$ & Very Fine Sand & Very Fine Sand & Well Sorted \\
\hline GSH15-T01-S2 & Slightly Pebbly & $\begin{array}{l}\text { Slightly Pebbly Very } \\
\text { Fine Sand }\end{array}$ & $\begin{array}{l}\text { Slightly Gravelly } \\
\text { Sand }\end{array}$ & $\begin{array}{l}\text { Slightly Fine Gravelly Very Fine } \\
\text { Sand }\end{array}$ & $\begin{array}{l}\text { Slightly Pebbly Very Fine } \\
\text { Sand }\end{array}$ & Very Fine Sand & Very Fine Sand & Well Sorted \\
\hline GSH15-T02-S1 & Sand & Fine Sand & Sand & Fine Sand & Fine Sand & Fine Sand & Fine Sand & Well Sorted \\
\hline GSH15-T02-S2 & Sand & Very Fine Sand & Sand & Very Fine Sand & Very Fine Sand & Very Fine Sand & Very Fine Sand & Well Sorted \\
\hline GSH15-T05-S1 & Sand & Very Fine Sand & Sand & Very Fine Sand & Very Fine Sand & Very Fine Sand & Very Fine Sand & $\begin{array}{l}\text { Moderately Well } \\
\text { Sorted }\end{array}$ \\
\hline GSH15-T05-S2 & Sand & Very Fine Sand & Sand & Very Fine Sand & Very Fine Sand & Very Fine Sand & Very Fine Sand & $\begin{array}{l}\text { Moderately Well } \\
\text { Sorted }\end{array}$ \\
\hline GSH15-T07-S1 & Granuley & $\begin{array}{l}\text { Granuley Very } \\
\text { Coarse Sand }\end{array}$ & Gravelly Sand & $\begin{array}{l}\text { Very Fine Gravelly Very Coarse } \\
\text { Sand }\end{array}$ & $\begin{array}{l}\text { Granular Very Coarse } \\
\text { Sand }\end{array}$ & Very Coarse Sand & Very Coarse Sand & $\begin{array}{l}\text { Moderately } \\
\text { Sorted }\end{array}$ \\
\hline GSH15-T07-S2 & Slightly Granuley & $\begin{array}{l}\text { Slightly Granuley } \\
\text { Coarse Sand }\end{array}$ & $\begin{array}{l}\text { Slightly Gravelly } \\
\text { Sand }\end{array}$ & $\begin{array}{l}\text { Slightly Very Fine Gravelly } \\
\text { Coarse Sand }\end{array}$ & $\begin{array}{l}\text { Slightly Granular Coarse } \\
\text { Sand }\end{array}$ & Coarse Sand & Coarse Sand & $\begin{array}{l}\text { Moderately Well } \\
\text { Sorted }\end{array}$ \\
\hline GSH15-T08-S1 & Slightly Granuley & $\begin{array}{l}\text { Slightly Granuley } \\
\text { Fine Sand }\end{array}$ & $\begin{array}{l}\text { Slightly Gravelly } \\
\text { Sand }\end{array}$ & $\begin{array}{l}\text { Slightly Very Fine Gravelly Fine } \\
\text { Sand }\end{array}$ & $\begin{array}{l}\text { Slightly Granular } \\
\text { Fine Sand }\end{array}$ & Fine Sand & Fine Sand & $\begin{array}{l}\text { Moderately } \\
\text { Sorted }\end{array}$ \\
\hline GSH15-T08-S2 & Slightly Granuley & $\begin{array}{l}\text { Slightly Granuley } \\
\text { Fine Sand }\end{array}$ & $\begin{array}{l}\text { Slightly Gravelly } \\
\text { Sand }\end{array}$ & $\begin{array}{l}\text { Slightly Very Fine Gravelly Fine } \\
\text { Sand }\end{array}$ & $\begin{array}{l}\text { Slightly Granular } \\
\text { Fine Sand }\end{array}$ & Fine Sand & Fine Sand & $\begin{array}{l}\text { Moderately } \\
\text { Sorted }\end{array}$ \\
\hline GSH15-T10-S1 & Slightly Pebbly & $\begin{array}{l}\text { Slightly Pebbly Fine } \\
\text { Sand }\end{array}$ & $\begin{array}{l}\text { Slightly Gravelly } \\
\text { Sand }\end{array}$ & Slightly Fine Gravelly Fine Sand & Slightly Pebbly Fine Sand & Fine Sand & Fine Sand & $\begin{array}{l}\text { Moderately Well } \\
\text { Sorted }\end{array}$ \\
\hline GSH15-T10-S2 & Slightly Granuley & $\begin{array}{l}\text { Slightly Granuley } \\
\text { Fine Sand }\end{array}$ & $\begin{array}{l}\text { Slightly Gravelly } \\
\text { Sand }\end{array}$ & $\begin{array}{l}\text { Slightly Very Fine Gravelly Fine } \\
\text { Sand }\end{array}$ & $\begin{array}{l}\text { Slightly Granular } \\
\text { Fine Sand }\end{array}$ & Fine Sand & Fine Sand & $\begin{array}{l}\text { Moderately Well } \\
\text { Sorted }\end{array}$ \\
\hline GSH15-T14-S2 & Pebbly & $\begin{array}{l}\text { Pebbly Silty Very } \\
\text { Fine Sand }\end{array}$ & $\begin{array}{l}\text { Gravelly Muddy } \\
\text { Sand }\end{array}$ & $\begin{array}{l}\text { Coarse Gravelly Very Coarse } \\
\text { Silty Very Fine Sand }\end{array}$ & $\begin{array}{l}\text { Pebbly Very Coarse Silty } \\
\text { Very Fine Sand }\end{array}$ & Fine Sand & Fine Sand & Poorly Sorted \\
\hline GSH15-T15-S1 & Granule Mixes & $\begin{array}{l}\text { Sandy Granule } \\
\text { Gravel }\end{array}$ & Sandy Gravel & Sandy Very Fine Gravel & Sandy Granule & Very Coarse Sand & Very Coarse Sand & Poorly Sorted \\
\hline GSH15-T15-S2 & Pebble Mixes & $\begin{array}{l}\text { Sandy Pebble } \\
\text { Gravel }\end{array}$ & Sandy Gravel & Sandy Fine Gravel & Sandy Pebble Gravel & Very Coarse Sand & Very Coarse Sand & $\begin{array}{l}\text { Very Poorly } \\
\text { Sorted }\end{array}$ \\
\hline
\end{tabular}


GSH15: Grain Size Statistics

\begin{tabular}{|c|c|c|c|c|c|c|c|c|c|c|c|c|c|c|c|c|c|c|c|c|}
\hline Sample ID & $\begin{array}{c}\text { Gravel } \\
\%\end{array}$ & $\begin{array}{c}\text { Pebble } \\
\%\end{array}$ & $\begin{array}{c}\text { Granule } \\
\%\end{array}$ & Sand \% & Mud \% & Silt \% & Clay \% & Modes & $\begin{array}{c}\text { Mode } 1 \\
\text { (phi) }\end{array}$ & $\begin{array}{c}\text { Mode } 2 \\
\text { (phi) }\end{array}$ & $\begin{array}{l}D_{10} \\
\text { (phi) }\end{array}$ & $\begin{array}{c}D_{10} \\
(\mathrm{~mm})\end{array}$ & $\begin{array}{l}\mathrm{D}_{50} \\
\text { (phi) }\end{array}$ & $\begin{array}{c}D_{50} \\
(\mathrm{~mm})\end{array}$ & $\begin{array}{c}\text { Mean } \\
\text { Size } \\
\text { (phi) }\end{array}$ & $\begin{array}{l}\text { Mean } \\
\text { Size } \\
(\mathrm{mm})\end{array}$ & $\begin{array}{l}\text { Sorting } \\
\text { (phi) }\end{array}$ & Skewness & Kurtosis & $\begin{array}{r}\text { LOI \% } \\
450^{\circ} \mathrm{C}\end{array}$ \\
\hline GSH15-T01-S1 & 0.08 & 0.00 & 0.08 & 97.66 & 2.26 & $\mathrm{~N} / \mathrm{A}$ & $\mathrm{N} / \mathrm{A}$ & $\mathrm{U}$ & 3.24 & $\mathrm{~N} / \mathrm{A}$ & 2.60 & 0.17 & 3.18 & 0.11 & 3.12 & 0.11 & 0.39 & -0.11 & 1.20 & 0.50 \\
\hline GSH15-T01-S2 & 1.45 & 0.74 & 0.71 & 96.19 & 2.36 & N/A & $\mathrm{N} / \mathrm{A}$ & $U$ & 3.24 & N/A & 2.52 & 0.17 & 3.19 & 0.11 & 3.12 & 0.12 & 0.47 & -0.26 & 1.64 & 0.54 \\
\hline GSH15-T02-S1 & 0.00 & 0.00 & 0.00 & 98.70 & 1.30 & $\mathrm{~N} / \mathrm{A}$ & $\mathrm{N} / \mathrm{A}$ & U & 3.24 & N/A & 2.38 & 0.19 & 2.95 & 0.13 & 2.95 & 0.13 & 0.44 & -0.01 & 1.00 & 0.40 \\
\hline GSH15-T02-S2 & 0.00 & 0.00 & 0.00 & 97.98 & 2.02 & $\mathrm{~N} / \mathrm{A}$ & $\mathrm{N} / \mathrm{A}$ & U & 3.24 & $\mathrm{~N} / \mathrm{A}$ & 2.38 & 0.19 & 3.07 & 0.12 & 3.01 & 0.12 & 0.47 & -0.16 & 1.09 & 0.40 \\
\hline GSH15-T05-S1 & 0.00 & 0.00 & 0.00 & 93.25 & 6.75 & 5.94 & 0.81 & U & 3.24 & $\mathrm{~N} / \mathrm{A}$ & 2.51 & 0.18 & 3.12 & 0.12 & 3.12 & 0.12 & 0.57 & 0.09 & 1.29 & 0.60 \\
\hline GSH15-T05-S2 & 0.01 & 0.00 & 0.01 & 94.02 & 5.97 & 4.80 & 1.17 & U & 3.24 & $\mathrm{~N} / \mathrm{A}$ & 2.45 & 0.18 & 3.11 & 0.12 & 3.12 & 0.12 & 0.59 & 0.04 & 1.19 & 0.54 \\
\hline GSH15-T07-S1 & 8.31 & 2.56 & 5.75 & 90.82 & 0.87 & $\mathrm{~N} / \mathrm{A}$ & $\mathrm{N} / \mathrm{A}$ & U & -0.24 & $\mathrm{~N} / \mathrm{A}$ & -0.95 & 1.94 & -0.17 & 1.12 & -0.13 & 1.10 & 0.82 & 0.16 & 1.54 & 0.50 \\
\hline GSH15-T07-S2 & 1.18 & 0.31 & 0.87 & 97.92 & 0.90 & N/A & $\mathrm{N} / \mathrm{A}$ & $U$ & 0.25 & $\mathrm{~N} / \mathrm{A}$ & -0.47 & 1.38 & 0.11 & 0.92 & 0.17 & 0.89 & 0.64 & 0.20 & 1.35 & 0.40 \\
\hline GSH15-T08-S1 & 0.03 & 0.00 & 0.03 & 97.86 & 2.11 & N/A & $\mathrm{N} / \mathrm{A}$ & $U$ & 2.74 & $\mathrm{~N} / \mathrm{A}$ & 1.55 & 0.34 & 2.54 & 0.17 & 2.52 & 0.17 & 0.77 & -0.05 & 1.04 & 0.40 \\
\hline GSH15-T08-S2 & 0.10 & 0.04 & 0.06 & 96.85 & 3.05 & N/A & $\mathrm{N} / \mathrm{A}$ & $\mathrm{U}$ & 2.74 & $\mathrm{~N} / \mathrm{A}$ & 1.55 & 0.34 & 2.56 & 0.17 & 2.55 & 0.17 & 0.80 & -0.03 & 1.06 & 0.40 \\
\hline GSH15-T10-S1 & 0.16 & 0.12 & 0.04 & 96.97 & 2.87 & N/A & $\mathrm{N} / \mathrm{A}$ & $U$ & 3.24 & $\mathrm{~N} / \mathrm{A}$ & 1.85 & 0.28 & 2.85 & 0.14 & 2.79 & 0.14 & 0.69 & -0.11 & 1.00 & 0.40 \\
\hline GSH15-T10-S2 & 0.02 & 0.00 & 0.02 & 97.77 & 2.21 & N/A & N/A & $U$ & 3.24 & $\mathrm{~N} / \mathrm{A}$ & 1.92 & 0.26 & 2.84 & 0.14 & 2.79 & 0.14 & 0.65 & -0.12 & 1.03 & 0.40 \\
\hline GSH15-T14-S2 & 5.05 & 2.91 & 2.14 & 83.10 & 11.85 & 10.10 & 1.75 & $U$ & 3.24 & $\mathrm{~N} / \mathrm{A}$ & 0.97 & 0.51 & 3.03 & 0.12 & 2.83 & 0.14 & 1.45 & -0.33 & 1.66 & 0.90 \\
\hline GSH15-T15-S1 & 38.59 & 16.82 & 21.77 & 61.14 & 0.27 & N/A & $\mathrm{N} / \mathrm{A}$ & B & -0.74 & $\mathrm{~N} / \mathrm{A}$ & -2.59 & 6.01 & -0.76 & 1.69 & -0.80 & 1.74 & 1.33 & 0.00 & 1.36 & 0.60 \\
\hline GSH15-T15-S2 & 45.96 & 32.69 & 13.27 & 50.63 & 3.41 & N/A & N/A & B & -2.74 & 1.25 & -3.22 & 9.31 & -0.68 & 1.60 & -0.57 & 1.48 & 2.26 & 0.13 & 0.80 & 0.57 \\
\hline
\end{tabular}




\section{GSH15: Grain Size Distribution}

\begin{tabular}{|c|c|c|c|c|c|c|c|c|c|c|c|c|c|c|c|c|c|c|c|c|c|c|c|c|c|c|c|c|}
\hline & $\begin{array}{c}\text { Class } \\
\% \\
\text { phi }\end{array}$ & $\begin{array}{c}\text { Class } \\
\% \\
\text { phi }\end{array}$ & $\begin{array}{c}\text { Class } \\
\% \\
\text { phi }\end{array}$ & $\begin{array}{c}\text { Class } \\
\% \\
\text { phi }\end{array}$ & $\begin{array}{c}\text { Class } \\
\% \\
\text { phi }\end{array}$ & $\begin{array}{c}\text { Class } \\
\% \\
\text { phi }\end{array}$ & $\begin{array}{c}\text { Class } \\
\% \\
\text { phi }\end{array}$ & $\begin{array}{c}\text { Class } \\
\% \\
\text { phi }\end{array}$ & $\begin{array}{c}\text { Class } \\
\% \\
\text { phi }\end{array}$ & $\begin{array}{c}\text { Class } \\
\% \\
\text { phi }\end{array}$ & $\begin{array}{c}\text { Class } \\
\% \\
\text { phi }\end{array}$ & $\begin{array}{c}\text { Class } \\
\% \\
\text { phi }\end{array}$ & $\begin{array}{c}\text { Class } \\
\% \\
\text { phi }\end{array}$ & $\begin{array}{c}\text { Class } \\
\% \\
\text { phi }\end{array}$ & $\begin{array}{c}\text { Class } \\
\% \\
\text { phi }\end{array}$ & $\begin{array}{c}\text { Class } \\
\% \\
\text { phi }\end{array}$ & $\begin{array}{c}\text { Class } \\
\% \\
\text { phi }\end{array}$ & $\begin{array}{c}\text { Class } \\
\% \\
\text { phi }\end{array}$ & $\begin{array}{c}\text { Class } \\
\% \\
\text { phi }\end{array}$ & $\begin{array}{c}\text { Class } \\
\% \\
\text { phi }\end{array}$ & $\begin{array}{c}\text { Class } \\
\% \\
\text { phi }\end{array}$ & $\begin{array}{c}\text { Class } \\
\% \\
\text { phi }\end{array}$ & $\begin{array}{c}\text { Class } \\
\% \\
\text { phi }\end{array}$ & $\begin{array}{c}\text { Class } \\
\% \\
\text { phi }\end{array}$ & $\begin{array}{c}\text { Class } \\
\% \\
\text { phi }\end{array}$ & $\begin{array}{c}\text { Class } \\
\% \\
\text { phi }\end{array}$ & $\begin{array}{c}\text { Class } \\
\% \\
\text { phi }\end{array}$ & $\begin{array}{c}\text { Class } \\
\% \\
\text { phi } \\
>10.0\end{array}$ \\
\hline GSH15 & .00 & 0.00 & 0.00 & 0.00 & 0.00 & 0.00 & 0.00 & 0.00 & 0.04 & 0.04 & 0.09 & 0.06 & 0.11 & 0.15 & 0.23 & 0.71 & 2.68 & 4.97 & 56.13 & 12.52 & 2.26 & N/A & N/A & N/A & N/A & N/A & N/A & N/A \\
\hline GSH15-T01-S2 & 0.00 & 0.00 & 0.00 & 0.00 & 0.00 & 0.00 & 0.00 & 0.74 & 0.62 & 0.09 & 0.09 & 0.28 & 0.34 & 0.46 & 0.50 & 1.15 & 4.04 & 18.71 & 57.92 & 12.69 & 2.37 & N/A & N/A & $\mathrm{N} / \mathrm{A}$ & N/A & N/A & N/A & N/A \\
\hline GSH15-T02-S1 & .00 & 0.00 & 0.00 & 0.00 & 0.00 & 0.00 & 0.00 & 0.00 & 0.00 & 0.00 & 0.00 & 0.02 & 0.04 & 0.12 & 0.33 & 0.80 & 10.78 & 42.16 & 38.08 & 6.38 & 1.30 & $\mathrm{~N} / \mathrm{A}$ & $\mathrm{N} / \mathrm{A}$ & N/A & N/A & $\mathrm{N} / \mathrm{A}$ & $\mathrm{N} / \mathrm{A}$ & N/A \\
\hline GSH15-T02-S2 & 0.00 & 0.00 & 0.00 & 0.00 & 0.00 & 0.00 & 0.00 & 0.00 & 0.00 & 0.00 & 0.09 & 0.05 & 0.25 & 0.24 & 0.29 & 1.35 & 9.60 & 31.05 & 46.29 & 8.77 & 2.02 & N/A & N/A & N/A & N/A & N/A & $\mathrm{N} / \mathrm{A}$ & N/A \\
\hline GSH15-T05-S1 & 0.00 & 0.00 & 0.00 & 0.00 & 0.00 & 0.00 & 0.00 & 0.00 & 0.00 & 0.00 & 0.01 & 0.02 & 0.03 & 0.13 & 0.30 & 1.55 & 5.57 & 32.69 & 39.85 & 13.10 & $\mathrm{~N} / \mathrm{A}$ & 5.24 & 0.41 & 0.16 & 0.12 & 0.03 & 0.14 & 0.64 \\
\hline GSH15-T05-S2 & .00 & 0.00 & 0.00 & 0.00 & 0.00 & 0.00 & 0.00 & 0.00 & 0.00 & 0.01 & 0.02 & 0.03 & 0.08 & 0.07 & 0.30 & 2.11 & 7.70 & 30.93 & 36.69 & 16.08 & $\mathrm{~N} / \mathrm{A}$ & 4.44 & 0.32 & 0.02 & 0.03 & 0.03 & 0.14 & 1.00 \\
\hline GSH15-T07-S1 & 0.00 & 0.00 & 0.00 & 0.00 & 0.51 & 1.06 & 0.36 & 0.62 & 1.45 & 4.30 & 18.96 & 34.62 & 21.33 & 6.58 & 3.21 & 2.99 & 1.66 & 0.96 & 0.35 & 0.17 & 0.87 & N/A & $\mathrm{N} / \mathrm{A}$ & $\mathrm{N} / \mathrm{A}$ & N/A & $\mathrm{N} / \mathrm{A}$ & $\mathrm{N} / \mathrm{A}$ & N/A \\
\hline GSH15-T07-S2 & .00 & 0.00 & 0.00 & 0.00 & 0.00 & 0.00 & 0.20 & 0.11 & 0.17 & 0.70 & 7.60 & 33.08 & 35.29 & 12.75 & 4.52 & 2.70 & 1.08 & 0.57 & 0.22 & 0.10 & 0.90 & N/A & N/A & N/A & N/A & N/A & N/A & N/A \\
\hline GSH15-T08-S1 & 0.00 & 0.00 & 0.00 & 0.00 & 0.00 & 0.00 & 0.00 & 0.00 & 0.03 & 0.00 & 0.03 & 0.23 & 0.93 & 2.07 & 4.89 & 15.70 & 22.88 & 26.77 & 18.36 & 5.98 & 2.11 & N/A & N/A & N/A & N/A & N/A & N/A & N/A \\
\hline GSH15-T08-S2 & 0.00 & 0.00 & 0.00 & 0.00 & 0.00 & 0.00 & 0.00 & 0.04 & 0.00 & 0.06 & 0.06 & 0.24 & 0.97 & 2.20 & 4.81 & 14.68 & 22.67 & 26.19 & 17.57 & 7.46 & 3.05 & N/A & N/A & $\mathrm{N} / \mathrm{A}$ & N/A & N/A & $\mathrm{N} / \mathrm{A}$ & N/A \\
\hline GSH15-T10-S1 & 0.00 & 0.00 & 0.00 & 0.00 & 0.00 & 0.00 & 0.12 & 0.00 & 0.02 & 0.02 & 0.04 & 0.07 & 0.14 & 0.74 & 2.49 & 9.13 & 17.30 & 28.10 & 27.96 & 10.99 & 2.87 & N/A & N/A & N/A & N/A & N/A & N/A & N/A \\
\hline GSH15-T10-S2 & 0.00 & 0.00 & 0.00 & 0.00 & 0.00 & 0.00 & 0.00 & 0.00 & 0.01 & 0.02 & 0.01 & 0.03 & 0.11 & 0.50 & 1.87 & 8.78 & 17.24 & 30.63 & 29.84 & 8.75 & 2.21 & $\mathrm{~N} / \mathrm{A}$ & $\mathrm{N} / \mathrm{A}$ & $\mathrm{N} / \mathrm{A}$ & N/A & $\mathrm{N} / \mathrm{A}$ & $\mathrm{N} / \mathrm{A}$ & N/A \\
\hline GSH15-T14-S2 & 0.00 & 0.00 & 0.00 & 2.15 & 0.00 & 0.00 & 0.76 & 0.00 & 1.08 & 1.06 & 1.07 & 1.11 & 1.26 & 1.61 & 3.69 & 9.68 & 12.24 & 12.81 & 25.47 & 14.16 & N/A & 8.18 & 1.32 & 0.33 & 0.28 & 0.11 & 0.18 & 1.47 \\
\hline GSH15-T15-S1 & 0.00 & 0.00 & 0.00 & 0.00 & 2.17 & 2.57 & 6.57 & 5.51 & 8.11 & 13.65 & 23.94 & 14.78 & 7.91 & 5.22 & 3.54 & 2.51 & 0.97 & 0.48 & 0.93 & 0.86 & 0.27 & $\mathrm{~N} / \mathrm{A}$ & $\mathrm{N} / \mathrm{A}$ & $\mathrm{N} / \mathrm{A}$ & N/A & N/A & $\mathrm{N} / \mathrm{A}$ & N/A \\
\hline GSH15-T15-S2 & 0.00 & 0.00 & 0.00 & 0.00 & 5.78 & 7.70 & 11.09 & 8.12 & 7.02 & 6.25 & 6.54 & 6.46 & 6.51 & 6.73 & 7.02 & 6.61 & 3.06 & 2.78 & 3.07 & 1.86 & 3.41 & N/A & N/A & N/A & N/A & N/A & N/A & N/A \\
\hline
\end{tabular}


Section 5: GSH16 


\section{GSH16: Identification, Location, and Description}

\begin{tabular}{|c|c|c|c|c|c|c|c|c|}
\hline Sample ID & Global Sample ID & Station ID & $\begin{array}{c}\text { Latitude } \\
\text { WGS84 }\end{array}$ & $\begin{array}{c}\text { Longitude } \\
\text { WGS84 }\end{array}$ & $\begin{array}{l}\text { Reliability } \\
\text { Ranking for } \\
\text { Positioning }\end{array}$ & $\begin{array}{c}\text { Water } \\
\text { Depth }(m)\end{array}$ & $\begin{array}{c}\text { Sample } \\
\text { Collected }\end{array}$ & $\begin{array}{c}\text { Total Wt } \\
\text { (gm) }\end{array}$ \\
\hline GSH16-T01-S1 & GSH16-T01-S1-7/10/2017 & GSH16-T01 & 43.045297 & -70.697871 & 1 & 16.8 & $7 / 10 / 2017$ & 103.6 \\
\hline GSH16-T02-S1 & GSH16-T02-S1-7/10/2017 & GSH16-T02 & 43.042899 & -70.697748 & 1 & 17.1 & $7 / 10 / 2017$ & 109.5 \\
\hline GSH16-T03-S1 & GSH16-T03-S1-7/10/2017 & GSH16-T03 & 43.041998 & -70.694267 & 1 & 16.2 & $7 / 10 / 2017$ & 102.7 \\
\hline GSH16-T04-S1 & GSH16-T04-S1-7/10/2017 & GSH16-T04 & 43.039079 & -70.686425 & 1 & 21.3 & $7 / 10 / 2017$ & 176.0 \\
\hline GSH16-T05-S1 & GSH16-T05-S1-7/10/2017 & GSH16-T05 & 43.035313 & -70.683228 & 1 & 25.9 & $7 / 10 / 2017$ & 195.0 \\
\hline GSH16-T06-S1 & GSH16-T06-S1-7/10/2017 & GSH16-T06 & 43.031908 & -70.682273 & 1 & 27.7 & $7 / 10 / 2017$ & 319.3 \\
\hline GSH16-T07-S1 & GSH16-T07-S1-7/11/2017 & GSH16-T07 & 43.026767 & -70.677968 & 1 & 25.9 & $7 / 11 / 2017$ & 282.9 \\
\hline GSH16-T11-S1 & GSH16-T11-S1-7/11/2017 & GSH16-T11 & 43.024464 & -70.704939 & 1 & 18.9 & $7 / 11 / 2017$ & 84.9 \\
\hline GSH16-T12-S1 & GSH16-T12-S1-7/11/2017 & GSH16-T12 & 43.025693 & -70.710856 & 1 & 15.8 & $7 / 11 / 2017$ & 101.9 \\
\hline GSH16-T14-S1 & GSH16-T14-S1-7/11/2017 & GSH16-T14 & 43.030153 & -70.702878 & 1 & 17.7 & $7 / 11 / 2017$ & 122.5 \\
\hline GSH16-T15-S1 & GSH16-T15-S1-7/11/2017 & GSH16-T15 & 43.031050 & -70.698484 & 1 & 17.7 & $7 / 11 / 2017$ & 124.1 \\
\hline GSH16-T17-S2 & GSH16-T17-S2-7/11/2017 & GSH16-T17 & 43.033956 & -70.693845 & 1 & 18.3 & $7 / 11 / 2017$ & 103.3 \\
\hline GSH16-T18-S1 & GSH16-T18-S1-7/11/2017 & GSH16-T18 & 43.036400 & -70.695581 & 1 & 16.8 & $7 / 11 / 2017$ & 120.8 \\
\hline GSH16-TS06-S1 & GSH16-TS06-S1-10/13/2016 & GSH16-TS06 & 42.855997 & -70.794752 & 1 & 18.0 & $10 / 13 / 2016$ & 55.1 \\
\hline GSH16-TS06-S2 & GSH16-TS06-S2-10/13/2016 & GSH16-TS06 & 42.855877 & -70.794708 & 1 & 18.0 & $10 / 13 / 2016$ & 32.2 \\
\hline GSH16-TS09-S1 & GSH16-TS09-S1-10/13/2016 & GSH16-TS09 & 42.857124 & -70.799412 & 1 & 16.5 & $10 / 13 / 2016$ & 67.8 \\
\hline GSH16-TS09-S2 & GSH16-TS09-S2-10/13/2016 & GSH16-TS09 & 42.857120 & -70.799449 & 1 & 16.5 & $10 / 13 / 2016$ & 168.4 \\
\hline GSH16-TS10-S1 & GSH16-TS10-S1-10/13/2016 & GSH16-TS10 & 42.853463 & -70.799742 & 1 & 17.4 & $10 / 13 / 2016$ & 209.3 \\
\hline GSH16-TS10-S2 & GSH16-TS10-S2-10/13/2016 & GSH16-TS10 & 42.853333 & -70.799738 & 1 & 17.4 & $10 / 13 / 2016$ & 63.9 \\
\hline
\end{tabular}


GSH16: Sediment Classifications

\begin{tabular}{|c|c|c|c|c|c|c|c|c|}
\hline Sample ID & $\begin{array}{l}\text { CMECS Substrate } \\
\text { Component } \\
\text { Group (Specific) }\end{array}$ & $\begin{array}{l}\text { Component } \\
\text { Subgroup } \\
\text { (Specific) } \\
\end{array}$ & $\begin{array}{l}\text { Textural Group } \\
\text { from \%GSM } \\
\text { (Gradistat) }\end{array}$ & $\begin{array}{l}\text { Sediment Name from \%GSM } \\
\text { and Mode (Gradistat) }\end{array}$ & $\begin{array}{l}\text { Sediment Name from } \\
\text { \%GSM and Mode } \\
\text { (Wentworth Scale) }\end{array}$ & $\begin{array}{l}\text { Sediment } \\
\text { Classification from } \\
\text { Mean Phi (Gradistat) }\end{array}$ & $\begin{array}{l}\text { Classification from } \\
\text { Mean Phi } \\
\text { (Wentworth) }\end{array}$ & $\begin{array}{l}\text { Sorting } \\
\text { (Gradistat) }\end{array}$ \\
\hline GSH16-T01-S1 & Sand & Fine Sand & Sand & Fine Sand & Fine Sand & Fine Sand & Fine Sand & Well Sorted \\
\hline GSH16-T02-S1 & Sand & Fine Sand & Sand & Fine Sand & Fine Sand & Fine Sand & Fine Sand & Well Sorted \\
\hline GSH16-T03-S1 & Sand & Fine Sand & Sand & Fine Sand & Fine Sand & Fine Sand & Fine Sand & $\begin{array}{l}\text { Moderately Well } \\
\text { Sorted }\end{array}$ \\
\hline GSH16-T04-S1 & Pebbly & Pebbly Fine Sand & Gravelly Sand & Medium Gravelly Fine Sand & Pebbly Fine Sand & Medium Sand & Medium Sand & $\begin{array}{l}\text { Very Poorly } \\
\text { Sorted }\end{array}$ \\
\hline GSH16-T05-S1 & Pebbly & Pebbly Fine Sand & Gravelly Sand & Medium Gravelly Fine Sand & Pebbly Fine Sand & Fine Sand & Fine Sand & Poorly Sorted \\
\hline GSH16-T06-S1 & Pebble Mixes & $\begin{array}{l}\text { Sandy Pebble } \\
\text { Gravel }\end{array}$ & Sandy Gravel & Sandy Coarse Gravel & Sandy Pebble Gravel & Very Fine Gravel & Granule Gravel & $\begin{array}{l}\text { Very Poorly } \\
\text { Sorted }\end{array}$ \\
\hline GSH16-T07-S1 & Pebble Mixes & $\begin{array}{l}\text { Sandy Pebble } \\
\text { Gravel }\end{array}$ & Sandy Gravel & Sandy Medium Gravel & Sandy Pebble Gravel & Very Coarse Sand & Very Coarse Sand & $\begin{array}{l}\text { Very Poorly } \\
\text { Sorted }\end{array}$ \\
\hline GSH16-T11-S1 & Slightly Pebbly & $\begin{array}{l}\text { Slightly Pebbly } \\
\text { Fine Sand }\end{array}$ & $\begin{array}{l}\text { Slightly Gravelly } \\
\text { Sand }\end{array}$ & Slightly Fine Gravelly Fine Sand & Slightly Pebbly Fine Sand & Fine Sand & Fine Sand & Well Sorted \\
\hline GSH16-T12-S1 & Sand & Fine Sand & Sand & Fine Sand & Fine Sand & Fine Sand & Fine Sand & Very Well Sorted \\
\hline GSH16-T14-S1 & Slightly Granuley & $\begin{array}{l}\text { Slightly Granuley } \\
\text { Fine Sand }\end{array}$ & $\begin{array}{l}\text { Slightly Gravelly } \\
\text { Sand }\end{array}$ & $\begin{array}{l}\text { Slightly Very Fine Gravelly Fine } \\
\text { Sand }\end{array}$ & $\begin{array}{l}\text { Slightly Granular } \\
\text { Fine Sand }\end{array}$ & Fine Sand & Fine Sand & Well Sorted \\
\hline GSH16-T15-S1 & Slightly Pebbly & $\begin{array}{l}\text { Slightly Pebbly } \\
\text { Fine Sand }\end{array}$ & $\begin{array}{l}\text { Slightly Gravelly } \\
\text { Sand }\end{array}$ & Slightly Fine Gravelly Fine Sand & Slightly Pebbly Fine Sand & Fine Sand & Fine Sand & Well Sorted \\
\hline GSH16-T17-S2 & Slightly Granuley & $\begin{array}{l}\text { Slightly Granuley } \\
\text { Very Fine Sand } \\
\end{array}$ & $\begin{array}{l}\text { Slightly Gravelly } \\
\text { Sand }\end{array}$ & $\begin{array}{l}\text { Slightly Very Fine Gravelly Very } \\
\text { Fine Sand }\end{array}$ & $\begin{array}{l}\text { Slightly Granular Very Fine } \\
\text { Sand }\end{array}$ & Very Fine Sand & Very Fine Sand & Well Sorted \\
\hline GSH16-T18-S1 & Sand & Fine Sand & Sand & Fine Sand & Fine Sand & Fine Sand & Fine Sand & Well Sorted \\
\hline GSH16-TS06-S1 & Sand & Fine Sand & Sand & Fine Sand & Fine Sand & Fine Sand & Fine Sand & $\begin{array}{l}\text { Moderately Well } \\
\text { Sorted }\end{array}$ \\
\hline GSH16-TS06-S2 & Slightly Granuley & $\begin{array}{l}\text { Slightly Granuley } \\
\text { Very Fine Sand }\end{array}$ & $\begin{array}{l}\text { Slightly Gravelly } \\
\text { Sand }\end{array}$ & $\begin{array}{l}\text { Slightly Very Fine Gravelly Very } \\
\text { Fine Sand }\end{array}$ & $\begin{array}{l}\text { Slightly Granular Very Fine } \\
\text { Sand }\end{array}$ & Fine Sand & Fine Sand & $\begin{array}{l}\text { Moderately } \\
\text { Sorted }\end{array}$ \\
\hline GSH16-TS09-S1 & Slightly Granuley & $\begin{array}{l}\text { Slightly Granuley } \\
\text { Fine Sand }\end{array}$ & $\begin{array}{l}\text { Slightly Gravelly } \\
\text { Sand }\end{array}$ & $\begin{array}{l}\text { Slightly Very Fine Gravelly Fine } \\
\text { Sand }\end{array}$ & $\begin{array}{l}\text { Slightly Granular } \\
\text { Fine Sand }\end{array}$ & Fine Sand & Fine Sand & $\begin{array}{l}\text { Moderately } \\
\text { Sorted }\end{array}$ \\
\hline GSH16-TS09-S2 & Slightly Pebbley & $\begin{array}{l}\text { Slightly Pebbly } \\
\text { Fine Sand }\end{array}$ & $\begin{array}{l}\text { Slightly Gravelly } \\
\text { Sand }\end{array}$ & Slightly Fine Gravelly Fine Sand & Slightly Pebbly Fine Sand & Fine Sand & Fine Sand & $\begin{array}{l}\text { Moderately Well } \\
\text { Sorted }\end{array}$ \\
\hline GSH16-TS10-S1 & Slightly Granuley & $\begin{array}{l}\text { Slightly Granuley } \\
\text { Fine Sand }\end{array}$ & $\begin{array}{l}\text { Slightly Gravelly } \\
\text { Sand }\end{array}$ & $\begin{array}{l}\text { Slightly Very Fine Gravelly Fine } \\
\text { Sand }\end{array}$ & $\begin{array}{l}\text { Slightly Granular } \\
\text { Fine Sand }\end{array}$ & Fine Sand & Fine Sand & $\begin{array}{l}\text { Moderately } \\
\text { Sorted }\end{array}$ \\
\hline GSH16-TS10-S2 & Slightly Pebbly & $\begin{array}{l}\text { Slightly Pebbly } \\
\text { Fine Sand }\end{array}$ & $\begin{array}{l}\text { Slightly Gravelly } \\
\text { Sand }\end{array}$ & $\begin{array}{l}\text { Slightly Medium Gravelly Fine } \\
\text { Sand }\end{array}$ & Slightly Pebbly Fine Sand & Fine Sand & Fine Sand & $\begin{array}{l}\text { Moderately } \\
\text { Sorted }\end{array}$ \\
\hline
\end{tabular}


GSH16: Grain Size Statistics

\begin{tabular}{|c|c|c|c|c|c|c|c|c|c|c|c|c|c|c|c|c|c|c|c|c|}
\hline Sample ID & $\begin{array}{c}\text { Gravel } \\
\%\end{array}$ & $\begin{array}{c}\text { Pebble } \\
\%\end{array}$ & $\begin{array}{c}\text { Granule } \\
\%\end{array}$ & Sand \% & Mud \% & Silt \% & Clay \% & Modes & $\begin{array}{c}\text { Mode } 1 \\
\text { (phi) }\end{array}$ & $\begin{array}{c}\text { Mode } 2 \\
\text { (phi) }\end{array}$ & $\begin{array}{c}D_{10} \\
\text { (phi) }\end{array}$ & $\begin{array}{c}\mathrm{D}_{10} \\
(\mathrm{~mm})\end{array}$ & $\begin{array}{c}D_{50} \\
\text { (phi) }\end{array}$ & $\begin{array}{c}D_{50} \\
(\mathrm{~mm}) \\
\end{array}$ & $\begin{array}{c}\text { Mean } \\
\text { Size } \\
\text { (phi) }\end{array}$ & $\begin{array}{c}\text { Mean } \\
\text { Size } \\
(\mathrm{mm})\end{array}$ & $\begin{array}{c}\text { Sorting } \\
\text { (phi) }\end{array}$ & Skewness & Kurtosis & $\begin{array}{r}\mathrm{LO} \% \mathrm{~K} \\
450^{\circ} \mathrm{C} \\
\end{array}$ \\
\hline GSH16-T01-S1 & 0.00 & 0.00 & 0.00 & 98.78 & 1.22 & N/A & $\mathrm{N} / \mathrm{A}$ & $\mathrm{U}$ & 2.74 & $\mathrm{~N} / \mathrm{A}$ & 2.35 & 0.20 & 2.80 & 0.14 & 2.84 & 0.14 & 0.38 & 0.07 & 1.22 & 0.60 \\
\hline GSH16-T02-S1 & 0.00 & 0.00 & 0.00 & 99.19 & 0.81 & N/A & $\mathrm{N} / \mathrm{A}$ & $\mathrm{U}$ & 2.74 & $\mathrm{~N} / \mathrm{A}$ & 2.27 & 0.21 & 2.77 & 0.15 & 2.80 & 0.14 & 0.37 & 0.05 & 1.33 & 0.52 \\
\hline GSH16-T03-S1 & 0.00 & 0.00 & 0.00 & 98.72 & 1.28 & $\mathrm{~N} / \mathrm{A}$ & $\mathrm{N} / \mathrm{A}$ & U & 2.74 & $\mathrm{~N} / \mathrm{A}$ & 1.95 & 0.26 & 2.89 & 0.14 & 2.86 & 0.14 & 0.53 & -0.24 & 1.22 & 0.67 \\
\hline GSH16-T04-S1 & 15.66 & 15.11 & 0.55 & 82.87 & 1.47 & $\mathrm{~N} / \mathrm{A}$ & $\mathrm{N} / \mathrm{A}$ & B & 2.74 & -3.74 & -3.51 & 11.42 & 2.46 & 0.18 & 1.65 & 0.32 & 2.07 & -0.67 & 2.00 & 0.69 \\
\hline GSH16-T05-S1 & 7.70 & 5.88 & 1.81 & 90.82 & 1.48 & $\mathrm{~N} / \mathrm{A}$ & $\mathrm{N} / \mathrm{A}$ & U & 2.74 & $\mathrm{~N} / \mathrm{A}$ & -0.02 & 1.02 & 2.31 & 0.20 & 2.08 & 0.24 & 1.44 & -0.47 & 1.78 & 0.55 \\
\hline GSH16-T06-S1 & 51.74 & 48.31 & 3.43 & 45.87 & 2.39 & $\mathrm{~N} / \mathrm{A}$ & $\mathrm{N} / \mathrm{A}$ & B & -4.73 & 1.25 & -4.63 & 24.74 & -1.51 & 2.85 & -1.18 & 2.26 & 2.89 & 0.17 & 0.61 & 0.80 \\
\hline GSH16-T07-S1 & 52.53 & 43.01 & 9.52 & 43.15 & 4.32 & 2.76 & 1.56 & $\mathrm{~B}$ & -3.74 & 1.75 & -3.80 & 13.88 & -1.27 & 2.41 & -0.94 & 1.91 & 2.58 & 0.24 & 0.68 & 0.74 \\
\hline GSH16-T11-S1 & 0.44 & 0.31 & 0.14 & 96.91 & 2.65 & $\mathrm{~N} / \mathrm{A}$ & $\mathrm{N} / \mathrm{A}$ & U & 3.24 & $\mathrm{~N} / \mathrm{A}$ & 2.49 & 0.18 & 2.98 & 0.13 & 2.97 & 0.13 & 0.45 & -0.07 & 1.15 & 0.40 \\
\hline GSH16-T12-S1 & 0.00 & 0.00 & 0.00 & 98.38 & 1.62 & $\mathrm{~N} / \mathrm{A}$ & $\mathrm{N} / \mathrm{A}$ & U & 2.74 & $\mathrm{~N} / \mathrm{A}$ & 2.52 & 0.17 & 2.86 & 0.14 & 2.91 & 0.13 & 0.33 & 0.19 & 0.81 & 0.63 \\
\hline GSH16-T14-S1 & 0.04 & 0.00 & 0.04 & 98.31 & 1.65 & $\mathrm{~N} / \mathrm{A}$ & $\mathrm{N} / \mathrm{A}$ & U & 2.74 & $\mathrm{~N} / \mathrm{A}$ & 2.49 & 0.18 & 2.85 & 0.14 & 2.89 & 0.13 & 0.38 & 0.06 & 1.01 & 0.58 \\
\hline GSH16-T15-S1 & 0.23 & 0.16 & 0.06 & 97.64 & 2.13 & $\mathrm{~N} / \mathrm{A}$ & $\mathrm{N} / \mathrm{A}$ & U & 2.74 & $\mathrm{~N} / \mathrm{A}$ & 2.53 & 0.17 & 2.93 & 0.13 & 2.96 & 0.13 & 0.36 & 0.13 & 0.81 & 0.70 \\
\hline GSH16-T17-S2 & 0.04 & 0.00 & 0.04 & 97.46 & 2.50 & $\mathrm{~N} / \mathrm{A}$ & $\mathrm{N} / \mathrm{A}$ & U & 3.24 & $\mathrm{~N} / \mathrm{A}$ & 2.54 & 0.17 & 3.04 & 0.12 & 3.02 & 0.12 & 0.40 & 0.00 & 0.95 & 0.60 \\
\hline GSH16-T18-S1 & 0.00 & 0.00 & 0.00 & 98.77 & 1.23 & $\mathrm{~N} / \mathrm{A}$ & $\mathrm{N} / \mathrm{A}$ & U & 2.74 & $\mathrm{~N} / \mathrm{A}$ & 2.51 & 0.18 & 2.94 & 0.13 & 2.95 & 0.13 & 0.36 & -0.01 & 0.84 & 0.57 \\
\hline GSH16-TS06-S1 & 0.00 & 0.00 & 0.00 & 96.13 & 3.87 & $\mathrm{~N} / \mathrm{A}$ & $\mathrm{N} / \mathrm{A}$ & U & 2.74 & $\mathrm{~N} / \mathrm{A}$ & 2.03 & 0.24 & 2.92 & 0.13 & 2.88 & 0.14 & 0.65 & -0.11 & 1.18 & 0.61 \\
\hline GSH16-TS06-S2 & 0.73 & 0.22 & 0.51 & 96.43 & 2.84 & $\mathrm{~N} / \mathrm{A}$ & $\mathrm{N} / \mathrm{A}$ & U & 3.24 & $\mathrm{~N} / \mathrm{A}$ & 1.74 & 0.30 & 2.96 & 0.13 & 2.81 & 0.14 & 0.73 & -0.31 & 1.08 & 0.50 \\
\hline GSH16-TS09-S1 & 0.24 & 0.00 & 0.24 & 95.90 & 3.86 & $\mathrm{~N} / \mathrm{A}$ & $\mathrm{N} / \mathrm{A}$ & U & 3.24 & $\mathrm{~N} / \mathrm{A}$ & 1.79 & 0.29 & 2.86 & 0.14 & 2.79 & 0.14 & 0.73 & -0.16 & 1.10 & 0.48 \\
\hline GSH16-TS09-S2 & 0.09 & 0.08 & 0.01 & 96.25 & 3.66 & $\mathrm{~N} / \mathrm{A}$ & $\mathrm{N} / \mathrm{A}$ & U & 2.74 & $\mathrm{~N} / \mathrm{A}$ & 2.03 & 0.24 & 2.85 & 0.14 & 2.82 & 0.14 & 0.64 & -0.07 & 1.14 & 0.44 \\
\hline GSH16-TS10-S1 & 0.02 & 0.00 & 0.02 & 96.70 & 3.28 & $\mathrm{~N} / \mathrm{A}$ & $\mathrm{N} / \mathrm{A}$ & U & 2.74 & $\mathrm{~N} / \mathrm{A}$ & 1.76 & 0.30 & 2.80 & 0.14 & 2.76 & 0.15 & 0.72 & -0.09 & 1.06 & 0.46 \\
\hline GSH16-TS10-S2 & 0.94 & 0.71 & 0.23 & 95.62 & 3.44 & $\mathrm{~N} / \mathrm{A}$ & $\mathrm{N} / \mathrm{A}$ & $\mathrm{U}$ & 2.74 & $\mathrm{~N} / \mathrm{A}$ & 1.47 & 0.36 & 2.63 & 0.16 & 2.58 & 0.17 & 0.87 & -0.12 & 1.13 & 0.46 \\
\hline
\end{tabular}


GSH16: Grain Size Distribution

\begin{tabular}{|c|c|c|c|c|c|c|c|c|c|c|c|c|c|c|c|c|c|c|c|c|c|c|c|c|c|c|c|c|}
\hline & $\begin{array}{c}\text { Class } \\
\% \\
\text { phi }\end{array}$ & $\begin{array}{c}\text { Class } \\
\% \\
\text { phi }\end{array}$ & $\begin{array}{c}\text { Class } \\
\% \\
\text { phi }\end{array}$ & $\begin{array}{c}\text { Class } \\
\% \\
\text { phi }\end{array}$ & $\begin{array}{c}\text { Class } \\
\% \\
\text { phi }\end{array}$ & $\begin{array}{c}\text { Class } \\
\% \\
\text { phi }\end{array}$ & $\begin{array}{c}\text { Class } \\
\% \\
\text { phi }\end{array}$ & $\begin{array}{c}\text { Class } \\
\% \\
\text { phi }\end{array}$ & $\begin{array}{c}\text { Class } \\
\% \\
\text { phi }\end{array}$ & $\begin{array}{c}\text { Class } \\
\% \\
\text { phi }\end{array}$ & $\begin{array}{c}\text { Class } \\
\% \\
\text { phi }\end{array}$ & $\begin{array}{c}\text { Class } \\
\% \\
\text { phi }\end{array}$ & $\begin{array}{c}\text { Class } \\
\% \\
\text { phi }\end{array}$ & $\begin{array}{c}\text { Class } \\
\% \\
\text { phi }\end{array}$ & $\begin{array}{c}\text { Class } \\
\% \\
\text { phi }\end{array}$ & $\begin{array}{c}\text { Class } \\
\% \\
\text { phi }\end{array}$ & $\begin{array}{c}\text { Class } \\
\% \\
\text { phi }\end{array}$ & $\begin{array}{c}\text { Class } \\
\% \\
\text { phi }\end{array}$ & $\begin{array}{c}\text { Class } \\
\% \\
\text { phi }\end{array}$ & $\begin{array}{c}\text { Class } \\
\% \\
\text { phi }\end{array}$ & $\begin{array}{c}\text { Class } \\
\% \\
\text { phi }\end{array}$ & $\begin{array}{c}\text { Class } \\
\% \\
\text { phi }\end{array}$ & $\begin{array}{c}\text { Class } \\
\% \\
\text { phi } \\
6.0\end{array}$ & $\begin{array}{c}\text { Class } \\
\% \\
\text { phi } \\
70\end{array}$ & $\begin{array}{c}\text { Class } \\
\% \\
\text { phi } \\
8.0\end{array}$ & $\begin{array}{c}\text { Class } \\
\% \\
\text { phi } \\
9.0\end{array}$ & $\begin{array}{c}\text { Class } \\
\% \\
\text { phi } \\
100\end{array}$ & $\begin{array}{c}\text { Class } \\
\% \\
\text { phi }\end{array}$ \\
\hline Sample ID & -5.5 & -5.0 & -4.5 & -4.0 & -3.5 & -3.0 & -2.5 & -2.0 & -1.5 & -1.0 & -0.5 & 0.0 & 0.5 & 1.0 & 1.5 & 2.0 & 2.5 & 3.0 & 3.5 & 4.0 & $>4.0$ & 5.0 & 6.0 & 7.0 & 8.0 & 9.0 & 10.0 & $>10.0$ \\
\hline GSH16-T01-S1 & 0.00 & 0.00 & 0.00 & 0.00 & 0.00 & 0.00 & 0.00 & 0.00 & 0.00 & 0.00 & 0.02 & 0.02 & 0.04 & 0.06 & 0.24 & 2.14 & 10.23 & 61.10 & 22.86 & 2.06 & 1.22 & $\mathrm{~N} / \mathrm{A}$ & $\mathrm{N} / \mathrm{A}$ & $\mathrm{N} / \mathrm{A}$ & N/A & $\mathrm{N} / \mathrm{A}$ & $\mathrm{N} / \mathrm{A}$ & N/A \\
\hline GSH16-T02-S1 & 0.00 & 0.00 & 0.00 & 0.00 & 0.00 & 0.00 & 0.00 & 0.00 & 0.00 & 0.00 & 0.01 & 0.03 & 0.06 & 0.20 & 0.50 & 2.85 & 11.19 & 63.26 & 19.70 & 1.40 & 0.81 & N/A & $\mathrm{N} / \mathrm{A}$ & N/A & N/A & N/A & $\mathrm{N} / \mathrm{A}$ & N/A \\
\hline GSH16-T03-S1 & 0.00 & 0.00 & 0.00 & 0.00 & 0.00 & 0.00 & 0.00 & 0.00 & 0.00 & 0.00 & 0.05 & 0.10 & 0.18 & 0.74 & 3.16 & 6.42 & 7.17 & 41.11 & 36.38 & 3.40 & 1.28 & N/A & $\mathrm{N} / \mathrm{A}$ & N/A & N/A & $\mathrm{N} / \mathrm{A}$ & $\mathrm{N} / \mathrm{A}$ & $\mathrm{N} / \mathrm{A}$ \\
\hline GSH16-T04-S1 & 0.00 & 0.00 & 0.00 & 0.00 & 10.57 & 3.88 & 0.00 & 0.66 & 0.28 & 0.27 & 0.61 & 0.61 & 1.01 & 2.22 & 4.66 & 10.73 & 15.09 & 25.05 & 19.93 & 2.95 & 1.47 & $\mathrm{~N} / \mathrm{A}$ & $\mathrm{N} / \mathrm{A}$ & $\mathrm{N} / \mathrm{A}$ & $\mathrm{N} / \mathrm{A}$ & $\mathrm{N} / \mathrm{A}$ & $\mathrm{N} / \mathrm{A}$ & N/A \\
\hline GSH16-T05-S1 & 0.00 & 0.00 & 0.00 & 0.00 & 1.36 & 1.70 & 1.86 & 0.97 & 1.04 & 0.77 & 0.91 & 1.47 & 2.66 & 4.52 & 7.38 & 14.26 & 16.99 & 26.07 & 14.38 & 2.18 & 1.48 & N/A & $\mathrm{N} / \mathrm{A}$ & N/A & N/A & N/A & $\mathrm{N} / \mathrm{A}$ & N/A \\
\hline GSH16-T06-S1 & 0.00 & 0.00 & 14.13 & 6.40 & 10.26 & 9.76 & 4.39 & 3.37 & 1.77 & 1.66 & 1.66 & 1.67 & 3.60 & 6.55 & 7.78 & 7.04 & 5.74 & 6.93 & 3.76 & 1.13 & 2.39 & $\mathrm{~N} / \mathrm{A}$ & $\mathrm{N} / \mathrm{A}$ & N/A & N/A & N/A & $\mathrm{N} / \mathrm{A}$ & N/A \\
\hline GSH16-T07-S1 & 0.00 & 0.00 & 0.00 & 2.43 & 19.03 & 6.91 & 8.36 & 6.28 & 4.97 & 4.55 & 4.79 & 4.37 & 5.05 & 5.20 & 4.59 & 6.34 & 5.55 & 4.14 & 2.13 & 1.00 & $\mathrm{~N} / \mathrm{A}$ & 0.92 & 0.77 & 0.87 & 0.19 & 0.48 & 0.31 & 0.77 \\
\hline GSH16-T11-S1 & 0.00 & 0.00 & 0.00 & 0.00 & 0.00 & 0.00 & 0.00 & 0.31 & 0.05 & 0.09 & 0.26 & 0.28 & 0.43 & 0.60 & 0.85 & 1.70 & 4.00 & 43.11 & 41.19 & 4.49 & 2.65 & N/A & $\mathrm{N} / \mathrm{A}$ & $\mathrm{N} / \mathrm{A}$ & N/A & $\mathrm{N} / \mathrm{A}$ & $\mathrm{N} / \mathrm{A}$ & N/A \\
\hline GSH16-T12-S1 & 0.00 & 0.00 & 0.00 & 0.00 & 0.00 & 0.00 & 0.00 & 0.00 & 0.00 & 0.00 & 0.03 & 0.02 & 0.03 & 0.05 & 0.03 & 0.58 & 4.29 & 60.58 & 30.01 & 2.76 & 1.63 & N/A & $\mathrm{N} / \mathrm{A}$ & N/A & N/A & $\mathrm{N} / \mathrm{A}$ & N/A & N/A \\
\hline GSH16-T14-S1 & 0.00 & 0.00 & 0.00 & 0.00 & 0.00 & 0.00 & 0.00 & 0.00 & 0.00 & 0.04 & 0.07 & 0.11 & 0.18 & 0.30 & 0.55 & 1.35 & 6.23 & 57.34 & 29.46 & 2.71 & 1.65 & $\mathrm{~N} / \mathrm{A}$ & $\mathrm{N} / \mathrm{A}$ & $\mathrm{N} / \mathrm{A}$ & $\mathrm{N} / \mathrm{A}$ & $\mathrm{N} / \mathrm{A}$ & $\mathrm{N} / \mathrm{A}$ & $\mathrm{N} / \mathrm{A}$ \\
\hline GSH16-T15-S1 & 0.00 & 0.00 & 0.00 & 0.00 & 0.00 & 0.00 & 0.00 & 0.16 & 0.00 & 0.06 & 0.00 & 0.01 & 0.05 & 0.08 & 0.17 & 0.61 & 3.67 & 51.77 & 37.75 & 3.53 & 2.13 & N/A & $\mathrm{N} / \mathrm{A}$ & $\mathrm{N} / \mathrm{A}$ & $\mathrm{N} / \mathrm{A}$ & $\mathrm{N} / \mathrm{A}$ & $\mathrm{N} / \mathrm{A}$ & N/A \\
\hline GSH16-T17-S2 & 0.00 & 0.00 & 0.00 & 0.00 & 0.00 & 0.00 & 0.00 & 0.00 & 0.00 & 0.04 & 0.03 & 0.03 & 0.04 & 0.07 & 0.20 & 1.02 & 3.79 & 40.51 & 45.68 & 6.08 & 2.50 & N/A & $\mathrm{N} / \mathrm{A}$ & N/A & N/A & $\mathrm{N} / \mathrm{A}$ & N/A & N/A \\
\hline GSH16-T18-S1 & 0.00 & 0.00 & 0.00 & 0.00 & 0.00 & 0.00 & 0.00 & 0.00 & 0.00 & 0.00 & 0.01 & 0.02 & 0.03 & 0.05 & 0.17 & 1.35 & 4.89 & 49.07 & 39.62 & 3.56 & 1.23 & N/A & $\mathrm{N} / \mathrm{A}$ & N/A & N/A & N/A & N/A & $\mathrm{N} / \mathrm{A}$ \\
\hline GSH16-TS06-S1 & 0.00 & 0.00 & 0.00 & 0.00 & 0.00 & 0.00 & 0.00 & 0.00 & 0.00 & 0.00 & 0.31 & 0.29 & 0.43 & 0.61 & 1.72 & 5.82 & 13.05 & 32.96 & 29.64 & 11.30 & 3.87 & $\mathrm{~N} / \mathrm{A}$ & $\mathrm{N} / \mathrm{A}$ & N/A & $\mathrm{N} / \mathrm{A}$ & $\mathrm{N} / \mathrm{A}$ & $\mathrm{N} / \mathrm{A}$ & N/A \\
\hline GSH16-TS06-S2 & 0.00 & 0.00 & 0.00 & 0.00 & 0.00 & 0.00 & 0.00 & 0.22 & 0.18 & 0.32 & 0.30 & 0.47 & 0.81 & 0.99 & 2.81 & 7.99 & 15.86 & 21.76 & 36.45 & 8.99 & 2.84 & N/A & N/A & N/A & N/A & $\mathrm{N} / \mathrm{A}$ & N/A & N/A \\
\hline GSH16-TS09-S1 & 0.00 & 0.00 & 0.00 & 0.00 & 0.00 & 0.00 & 0.00 & 0.00 & 0.15 & 0.10 & 0.18 & 0.29 & 0.65 & 1.55 & 2.94 & 7.15 & 17.63 & 26.62 & 29.56 & 9.31 & 3.86 & N/A & $\mathrm{N} / \mathrm{A}$ & N/A & N/A & N/A & N/A & N/A \\
\hline GSH16-TS09-S2 & 0.00 & 0.00 & 0.00 & 0.00 & 0.00 & 0.00 & 0.00 & 0.08 & 0.00 & 0.01 & 0.07 & 0.15 & 0.33 & 0.62 & 1.68 & 5.87 & 16.90 & 33.72 & 28.13 & 8.78 & 3.66 & $\mathrm{~N} / \mathrm{A}$ & $\mathrm{N} / \mathrm{A}$ & N/A & $\mathrm{N} / \mathrm{A}$ & $\mathrm{N} / \mathrm{A}$ & $\mathrm{N} / \mathrm{A}$ & $\mathrm{N} / \mathrm{A}$ \\
\hline GSH16-TS10-S1 & 0.00 & 0.00 & 0.00 & 0.00 & 0.00 & 0.00 & 0.00 & 0.00 & 0.01 & 0.01 & 0.02 & 0.13 & 0.66 & 1.65 & 2.88 & 8.90 & 16.88 & 30.68 & 24.31 & 10.59 & 3.28 & $\mathrm{~N} / \mathrm{A}$ & $\mathrm{N} / \mathrm{A}$ & $\mathrm{N} / \mathrm{A}$ & N/A & N/A & $\mathrm{N} / \mathrm{A}$ & N/A \\
\hline GSH16-TS10-S2 & 0.00 & 0.00 & 0.00 & 0.00 & 0.00 & 0.48 & 0.00 & 0.23 & 0.15 & 0.07 & 0.14 & 0.21 & 1.27 & 3.02 & 4.67 & 12.27 & 19.38 & 27.41 & 18.03 & 9.23 & 3.44 & N/A & $\mathrm{N} / \mathrm{A}$ & N/A & N/A & N/A & $\mathrm{N} / \mathrm{A}$ & N/A \\
\hline
\end{tabular}


Section 6: SH18 and ShoalsO7 
SH18 and Shoals07: Identification, Location, and Description

\begin{tabular}{|c|c|c|c|c|c|c|c|c|}
\hline Sample ID & Global Sample ID & Station ID & $\begin{array}{c}\text { Latitude } \\
\text { WGS84 }\end{array}$ & $\begin{array}{c}\text { Longitude } \\
\text { WGS84 }\end{array}$ & $\begin{array}{l}\text { Reliability } \\
\text { Ranking for } \\
\text { Positioning }\end{array}$ & $\begin{array}{c}\text { Water } \\
\text { Depth }(\mathrm{m})\end{array}$ & $\begin{array}{c}\text { Sample } \\
\text { Collected }\end{array}$ & $\begin{array}{c}\text { Total Wt } \\
(\mathrm{gm})\end{array}$ \\
\hline SH18-T01 & SH18-T01-7/10/2018 & SH18-T01 & 43.110750 & -70.620768 & 1 & 25.0 & $7 / 10 / 2018$ & 141.0 \\
\hline SH18-T07 & SH18-T07-7/10/2018 & SH18-T07 & 43.100621 & -70.629160 & 1 & 21.3 & $7 / 10 / 2018$ & 549.9 \\
\hline SH18-T09 & SH18-T09-7/10/2018 & SH18-T09 & 43.095376 & -70.626840 & 1 & 20.0 & $7 / 10 / 2018$ & 424.1 \\
\hline SH18-T10 & SH18-T10-7/10/2018 & SH18-T10 & 43.085480 & -70.636550 & 1 & 27.1 & $7 / 10 / 2018$ & 93.4 \\
\hline Shoals07-S02-S1 & Shoals07-S02-S1_10/13/2016 & Shoals07-S02 & 42.868036 & -70.810027 & 1 & 8.5 & $10 / 13 / 2016$ & 232.7 \\
\hline Shoals07-S02-S2 & Shoals07-S02-S2_10/13/2016 & Shoals07-S02 & 42.868029 & -70.809822 & 1 & 8.5 & $10 / 13 / 2016$ & 119.5 \\
\hline Shoals07-S07-S1 & Shoals07-S07-S1_10/13/2016 & Shoals07-S07 & 42.855850 & -70.807233 & 1 & 13.4 & $10 / 13 / 2016$ & 401.3 \\
\hline Shoals07-S07-S2 & Shoals07-S07-S2_10/13/2016 & Shoals07-S07 & 42.855950 & -70.807259 & 1 & 13.4 & $10 / 13 / 2016$ & 201.4 \\
\hline Shoals07-S08-S1 & Shoals07-S08-S1_10/13/2016 & Shoals07-S08 & 42.855847 & -70.803396 & 1 & 16.8 & $10 / 13 / 2016$ & 367.5 \\
\hline Shoals07-S08-S2 & Shoals07-S08-S2_10/13/2016 & Shoals07-S08 & 42.855587 & -70.802909 & 1 & 16.8 & $10 / 13 / 2016$ & 209.8 \\
\hline Shoals07-S09-S1 & Shoals07-S09-S1_10/6/2016 & Shoals07-S09 & 42.854926 & -70.805300 & 1 & & $10 / 6 / 2016$ & 306.0 \\
\hline Shoals07-S09-S2 & Shoals07-S09-S2_10/6/2016 & Shoals07-S09 & 42.854867 & -70.804997 & 1 & & $10 / 6 / 2016$ & 136.9 \\
\hline Shoals07-S11-S1 & Shoals07-S11-S1_10/6/2016 & Shoals07-S11 & 42.849072 & -70.805784 & 1 & 13.4 & $10 / 6 / 2016$ & 358.6 \\
\hline Shoals07-S11-S2 & Shoals07-S11-S2_10/6/2016 & Shoals07-S11 & 42.849135 & -70.805405 & 1 & 13.4 & $10 / 6 / 2016$ & 145.7 \\
\hline Shoals07-S13-S1 & Shoals 07-S13-S1_10/6/2016 & Shoals07-S13 & 42.843429 & -70.801355 & 1 & 15.2 & $10 / 6 / 2016$ & 175.1 \\
\hline Shoals07-S13-S2 & Shoals07-S13-S2_10/6/2016 & Shoals07-S13 & 42.843461 & -70.801725 & 1 & 15.2 & $10 / 6 / 2016$ & 189.6 \\
\hline Shoals07-S14-S1 & Shoals07-S14-S1_10/6/2016 & Shoals07-S14 & 42.829714 & -70.805199 & 1 & 8.8 & $10 / 6 / 2016$ & 321.6 \\
\hline Shoals07-S14-S2 & Shoals07-S14-S2_10/6/2016 & Shoals07-S14 & 42.829566 & -70.805736 & 1 & 8.8 & $10 / 6 / 2016$ & 145.9 \\
\hline
\end{tabular}


SH18 and Shoals07: Sediment Classifications

\begin{tabular}{|c|c|c|c|c|c|c|c|c|}
\hline Sample ID & $\begin{array}{l}\text { CMECS Substrate } \\
\text { Component } \\
\text { Group (Specific) }\end{array}$ & $\begin{array}{l}\text { Component } \\
\text { Subgroup } \\
\text { (Specific) } \\
\end{array}$ & $\begin{array}{l}\text { Textural Group } \\
\text { from \%GSM } \\
\text { (Gradistat) }\end{array}$ & $\begin{array}{l}\text { Sediment Name from \%GSM } \\
\text { and Mode (Gradistat) }\end{array}$ & $\begin{array}{l}\text { Sediment Name from } \\
\text { \%GSM and Mode } \\
\text { (Wentworth Scale) }\end{array}$ & $\begin{array}{l}\text { Sediment } \\
\text { Classification from } \\
\text { Mean Phi (Gradistat) }\end{array}$ & $\begin{array}{l}\text { Classification from } \\
\text { Mean Phi } \\
\text { (Wentworth) }\end{array}$ & $\begin{array}{l}\text { Sorting } \\
\text { (Gradistat) }\end{array}$ \\
\hline SH18-T01 & Slightly Pebbly & $\begin{array}{l}\text { Slightly Pebbly } \\
\text { Fine Sand }\end{array}$ & $\begin{array}{l}\text { Slightly Gravelly } \\
\text { Sand }\end{array}$ & Slightly Fine Gravelly Fine Sand & Slightly Pebbly Fine Sand & Fine Sand & Fine Sand & $\begin{array}{l}\text { Moderately Well } \\
\text { Sorted }\end{array}$ \\
\hline SH18-T07 & Gravel & Pebble Gravel & Gravel & Medium Gravel & Pebble Gravel & Fine Gravel & Pebble Gravel & $\begin{array}{l}\text { Very Poorly } \\
\text { Sorted }\end{array}$ \\
\hline SH18-T09 & Gravel & Pebble Gravel & Gravel & Very Coarse Gravel & Pebble Gravel & Very Coarse Gravel & Pebble Gravel & Poorly Sorted \\
\hline SH18-T10 & Slightly Pebbly & $\begin{array}{l}\text { Slightly Pebbly } \\
\text { Fine Sand }\end{array}$ & $\begin{array}{l}\text { Slightly Gravelly } \\
\text { Sand }\end{array}$ & Slightly Fine Gravelly Fine Sand & Slightly Pebbly Fine Sand & Fine Sand & Fine Sand & $\begin{array}{l}\text { Moderately Well } \\
\text { Sorted }\end{array}$ \\
\hline Shoals07-S02-S1 & Slightly Granuley & $\begin{array}{l}\text { Slightly Granuley } \\
\text { Very Fine Sand }\end{array}$ & $\begin{array}{l}\text { Slightly Gravelly } \\
\text { Sand }\end{array}$ & $\begin{array}{l}\text { Slightly Very Fine Gravelly Very } \\
\text { Fine Sand }\end{array}$ & $\begin{array}{l}\text { Slightly Granular Very Fine } \\
\text { Sand }\end{array}$ & Very Fine Sand & Very Fine Sand & Well Sorted \\
\hline Shoals07-S02-S2 & Slightly Granuley & $\begin{array}{l}\text { Slightly Granuley } \\
\text { Very Fine Sand }\end{array}$ & $\begin{array}{l}\text { Slightly Gravelly } \\
\text { Sand }\end{array}$ & $\begin{array}{l}\text { Slightly Very Fine Gravelly Very } \\
\text { Fine Sand }\end{array}$ & $\begin{array}{l}\text { Slightly Granular Very Fine } \\
\text { Sand }\end{array}$ & Very Fine Sand & Very Fine Sand & Well Sorted \\
\hline Shoals07-S07-s1 & Granuley & $\begin{array}{l}\text { Granuley Coarse } \\
\text { Sand }\end{array}$ & Gravelly Sand & Very Fine Gravelly Coarse Sand & Granular Coarse Sand & Coarse Sand & Coarse Sand & Poorly Sorted \\
\hline Shoals07-S07-S2 & Granuley & $\begin{array}{l}\text { Granuley Coarse } \\
\text { Sand }\end{array}$ & Gravelly Sand & Very Fine Gravelly Coarse Sand & Granular Coarse Sand & Coarse Sand & Coarse Sand & Poorly Sorted \\
\hline Shoals07-S08-S1 & Slightly Granuley & $\begin{array}{l}\text { Slightly Granuley } \\
\text { Coarse Sand }\end{array}$ & $\begin{array}{l}\text { Slightly Gravelly } \\
\text { Sand }\end{array}$ & $\begin{array}{l}\text { Slightly Very Fine Gravelly } \\
\text { Coarse Sand }\end{array}$ & $\begin{array}{l}\text { Slightly Granular Coarse } \\
\text { Sand }\end{array}$ & Coarse Sand & Coarse Sand & $\begin{array}{l}\text { Moderately Well } \\
\text { Sorted }\end{array}$ \\
\hline Shoals07-S08-S2 & Granuley & $\begin{array}{l}\text { Granuley Coarse } \\
\text { Sand }\end{array}$ & Gravelly Sand & Very Fine Gravelly Coarse Sand & Granular Coarse Sand & Very Coarse Sand & Very Coarse Sand & Poorly Sorted \\
\hline Shoals07-S09-S1 & Sand & Very Fine Sand & Sand & Very Fine Sand & Very Fine Sand & Fine Sand & Fine Sand & $\begin{array}{l}\text { Moderately } \\
\text { Sorted } \\
\end{array}$ \\
\hline Shoals07-S09-S2 & Slightly Granuley & $\begin{array}{l}\text { Slightly Granuley } \\
\text { Fine Sand }\end{array}$ & $\begin{array}{l}\text { Slightly Gravelly } \\
\text { Sand }\end{array}$ & $\begin{array}{l}\text { Slightly Very Fine Gravelly Fine } \\
\text { Sand }\end{array}$ & $\begin{array}{l}\text { Slightly Granular } \\
\text { Fine Sand }\end{array}$ & Fine Sand & Fine Sand & $\begin{array}{l}\text { Moderately } \\
\text { Sorted }\end{array}$ \\
\hline Shoals07-S11-S1 & Slightly Granuley & $\begin{array}{l}\text { Slightly Granuley } \\
\text { Coarse Sand } \\
\end{array}$ & $\begin{array}{l}\text { Slightly Gravelly } \\
\text { Sand }\end{array}$ & $\begin{array}{l}\text { Slightly Very Fine Gravelly } \\
\text { Coarse Sand }\end{array}$ & $\begin{array}{l}\text { Slightly Granular Coarse } \\
\text { Sand }\end{array}$ & Coarse Sand & Coarse Sand & $\begin{array}{l}\text { Moderately } \\
\text { Sorted }\end{array}$ \\
\hline Shoals07-S11-S2 & Slightly Granuley & $\begin{array}{l}\text { Slightly Granuley } \\
\text { Coarse Sand }\end{array}$ & $\begin{array}{l}\text { Slightly Gravelly } \\
\text { Sand }\end{array}$ & $\begin{array}{l}\text { Slightly Very Fine Gravelly } \\
\text { Coarse Sand }\end{array}$ & $\begin{array}{l}\text { Slightly Granular Coarse } \\
\text { Sand }\end{array}$ & Coarse Sand & Coarse Sand & $\begin{array}{l}\text { Moderately } \\
\text { Sorted }\end{array}$ \\
\hline Shoals07-S13-S1 & Slightly Granuley & $\begin{array}{l}\text { Slightly Granuley } \\
\text { Coarse Sand }\end{array}$ & $\begin{array}{l}\text { Slightly Gravelly } \\
\text { Sand }\end{array}$ & $\begin{array}{l}\text { Slightly Very Fine Gravelly } \\
\text { Coarse Sand }\end{array}$ & $\begin{array}{l}\text { Slightly Granular Coarse } \\
\text { Sand }\end{array}$ & Coarse Sand & Coarse Sand & $\begin{array}{l}\text { Moderately } \\
\text { Sorted }\end{array}$ \\
\hline Shoals07-S13-S2 & Slightly Granuley & $\begin{array}{l}\text { Slightly Granuley } \\
\text { Coarse Sand }\end{array}$ & $\begin{array}{l}\text { Slightly Gravelly } \\
\text { Sand }\end{array}$ & $\begin{array}{l}\text { Slightly Very Fine Gravelly } \\
\text { Coarse Sand }\end{array}$ & $\begin{array}{l}\text { Slightly Granular Coarse } \\
\text { Sand }\end{array}$ & Coarse Sand & Coarse Sand & $\begin{array}{l}\text { Moderately Well } \\
\text { Sorted }\end{array}$ \\
\hline Shoals07-S14-S1 & Slightly Pebbly & $\begin{array}{l}\text { Slightly Pebbly } \\
\text { Fine Sand }\end{array}$ & $\begin{array}{l}\text { Slightly Gravelly } \\
\text { Sand }\end{array}$ & Slightly Fine Gravelly Fine Sand & Slightly Pebbly Fine Sand & Fine Sand & Fine Sand & $\begin{array}{l}\text { Moderately Well } \\
\text { Sorted }\end{array}$ \\
\hline Shoals07-S14-S2 & Sand & Fine Sand & Sand & Fine Sand & Fine Sand & Fine Sand & Fine Sand & $\begin{array}{l}\text { Moderately Well } \\
\text { Sorted }\end{array}$ \\
\hline
\end{tabular}


SH18 and Shoals07: Grain Size Statistics

\begin{tabular}{|c|c|c|c|c|c|c|c|c|c|c|c|c|c|c|c|c|c|c|c|c|}
\hline Sample ID & $\begin{array}{c}\text { Gravel } \\
\%\end{array}$ & $\begin{array}{c}\text { Pebble } \\
\%\end{array}$ & $\begin{array}{c}\text { Granule } \\
\%\end{array}$ & Sand \% & Mud \% & Silt \% & Clay \% & Modes & $\begin{array}{c}\text { Mode } 1 \\
\text { (phi) }\end{array}$ & $\begin{array}{c}\text { Mode } 2 \\
\text { (phi) }\end{array}$ & $\begin{array}{l}D_{10} \\
\text { (phi) }\end{array}$ & $\begin{array}{c}D_{10} \\
(\mathrm{~mm})\end{array}$ & $\begin{array}{c}\mathrm{D}_{50} \\
\text { (phi) }\end{array}$ & $\begin{array}{c}D_{50} \\
(\mathrm{~mm})\end{array}$ & $\begin{array}{c}\text { Mean } \\
\text { Size } \\
\text { (phi) }\end{array}$ & $\begin{array}{c}\text { Mean } \\
\text { Size } \\
(\mathrm{mm})\end{array}$ & $\begin{array}{l}\text { Sorting } \\
\text { (phi) }\end{array}$ & Skewness & Kurtosis & $\begin{array}{l}\mathrm{LOI} \% \\
450^{\circ} \mathrm{C}\end{array}$ \\
\hline SH18-T01 & 1.39 & 1.37 & 0.02 & 96.16 & 2.45 & $\mathrm{~N} / \mathrm{A}$ & $\mathrm{N} / \mathrm{A}$ & $\mathrm{U}$ & 2.74 & $\mathrm{~N} / \mathrm{A}$ & 1.88 & 0.27 & 2.74 & 0.15 & 2.73 & 0.15 & 0.66 & -0.03 & 1.04 & 0.77 \\
\hline SH18-T07 & 81.40 & 70.84 & 10.56 & 17.53 & 1.07 & $\mathrm{~N} / \mathrm{A}$ & $\mathrm{N} / \mathrm{A}$ & $\mathrm{B}$ & -5.24 & -3.24 & -5.19 & 36.58 & -3.13 & 8.76 & -2.92 & 7.55 & 2.17 & 0.26 & 0.95 & 0.83 \\
\hline SH18-T09 & 92.42 & 91.83 & 0.58 & 7.05 & 0.53 & $\mathrm{~N} / \mathrm{A}$ & $\mathrm{N} / \mathrm{A}$ & $\mathrm{U}$ & -5.74 & N/A & -5.87 & 58.44 & -5.39 & 41.79 & -5.23 & 37.61 & 1.40 & 0.60 & 3.22 & N/A \\
\hline SH18-T10 & 1.07 & 0.89 & 0.18 & 97.07 & 1.86 & N/A & N/A & U & 2.24 & $\mathrm{~N} / \mathrm{A}$ & 1.72 & 0.30 & 2.41 & 0.19 & 2.45 & 0.18 & 0.55 & 0.09 & 1.06 & 0.75 \\
\hline Shoals07-S02-S1 & 0.07 & 0.00 & 0.07 & 98.49 & 1.44 & $\mathrm{~N} / \mathrm{A}$ & $\mathrm{N} / \mathrm{A}$ & $\mathrm{U}$ & 3.24 & $\mathrm{~N} / \mathrm{A}$ & 2.49 & 0.18 & 3.09 & 0.12 & 3.03 & 0.12 & 0.45 & -0.16 & 1.09 & 0.40 \\
\hline Shoals07-S02-S2 & 0.10 & 0.00 & 0.10 & 98.43 & 1.47 & $\mathrm{~N} / \mathrm{A}$ & $\mathrm{N} / \mathrm{A}$ & $\mathrm{U}$ & 3.24 & $\mathrm{~N} / \mathrm{A}$ & 2.48 & 0.18 & 3.07 & 0.12 & 3.02 & 0.12 & 0.45 & -0.14 & 1.08 & $\mathrm{~N} / \mathrm{A}$ \\
\hline Shoals07-S07-S1 & 9.29 & 3.68 & 5.60 & 90.29 & 0.42 & $\mathrm{~N} / \mathrm{A}$ & $\mathrm{N} / \mathrm{A}$ & $\mathrm{U}$ & 0.75 & $\mathrm{~N} / \mathrm{A}$ & -0.96 & 1.95 & 0.39 & 0.76 & 0.35 & 0.78 & 1.06 & -0.07 & 1.19 & 0.30 \\
\hline Shoals07-S07-S2 & 14.53 & 3.83 & 10.71 & 85.01 & 0.46 & $\mathrm{~N} / \mathrm{A}$ & $\mathrm{N} / \mathrm{A}$ & $U$ & 0.25 & $\mathrm{~N} / \mathrm{A}$ & -1.29 & 2.44 & 0.14 & 0.91 & 0.06 & 0.96 & 1.02 & -0.11 & 1.06 & N/A \\
\hline Shoals07-S08-S1 & 1.25 & 0.03 & 1.22 & 98.21 & 0.54 & $\mathrm{~N} / \mathrm{A}$ & $\mathrm{N} / \mathrm{A}$ & $U$ & 0.25 & $\mathrm{~N} / \mathrm{A}$ & -0.35 & 1.28 & 0.45 & 0.73 & 0.42 & 0.75 & 0.60 & -0.02 & 1.12 & 0.30 \\
\hline Shoals07-S08-S2 & 19.06 & 8.65 & 10.41 & 80.52 & 0.42 & $\mathrm{~N} / \mathrm{A}$ & $\mathrm{N} / \mathrm{A}$ & $\mathrm{B}$ & 0.25 & -4.24 & -1.84 & 3.57 & 0.11 & 0.93 & -0.09 & 1.07 & 1.23 & -0.35 & 1.42 & $\mathrm{~N} / \mathrm{A}$ \\
\hline Shoals07-S09-S1 & 0.01 & 0.00 & 0.01 & 98.18 & 1.81 & N/A & $\mathrm{N} / \mathrm{A}$ & U & 3.24 & $\mathrm{~N} / \mathrm{A}$ & 1.61 & 0.33 & 2.90 & 0.13 & 2.78 & 0.15 & 0.76 & -0.31 & 1.37 & 0.30 \\
\hline Shoals07-S09-S2 & 0.05 & 0.00 & 0.05 & 98.90 & 1.05 & N/A & $\mathrm{N} / \mathrm{A}$ & $\mathrm{U}$ & 3.24 & N/A & 1.51 & 0.35 & 2.87 & 0.14 & 2.76 & 0.15 & 0.79 & -0.34 & 1.39 & $\mathrm{~N} / \mathrm{A}$ \\
\hline Shoals07-S11-S1 & 4.98 & 1.09 & 3.89 & 94.66 & 0.36 & $\mathrm{~N} / \mathrm{A}$ & $\mathrm{N} / \mathrm{A}$ & $U$ & 0.25 & $\mathrm{~N} / \mathrm{A}$ & -0.71 & 1.63 & 0.28 & 0.82 & 0.28 & 0.82 & 0.77 & 0.02 & 1.13 & 0.30 \\
\hline Shoals07-S11-S2 & 0.14 & 0.00 & 0.14 & 99.45 & 0.41 & N/A & $\mathrm{N} / \mathrm{A}$ & U & 0.75 & N/A & 0.08 & 0.95 & 0.78 & 0.58 & 0.84 & 0.56 & 0.77 & 0.26 & 1.40 & N/A \\
\hline Shoals07-S13-S1 & 2.06 & 0.28 & 1.78 & 97.53 & 0.41 & $\mathrm{~N} / \mathrm{A}$ & $\mathrm{N} / \mathrm{A}$ & U & 0.75 & $\mathrm{~N} / \mathrm{A}$ & -0.48 & 1.40 & 0.56 & 0.68 & 0.54 & 0.69 & 0.84 & -0.01 & 0.93 & 0.30 \\
\hline Shoals07-S13-S2 & 0.22 & 0.03 & 0.19 & 99.41 & 0.37 & $\mathrm{~N} / \mathrm{A}$ & $\mathrm{N} / \mathrm{A}$ & $\mathrm{U}$ & 0.75 & $\mathrm{~N} / \mathrm{A}$ & -0.15 & 1.11 & 0.73 & 0.60 & 0.72 & 0.61 & 0.66 & -0.01 & 0.97 & N/A \\
\hline Shoals07-S14-S1 & 0.18 & 0.14 & 0.04 & 99.25 & 0.57 & $\mathrm{~N} / \mathrm{A}$ & $\mathrm{N} / \mathrm{A}$ & U & 2.74 & $\mathrm{~N} / \mathrm{A}$ & 1.57 & 0.34 & 2.54 & 0.17 & 2.47 & 0.18 & 0.64 & -0.19 & 1.15 & 0.30 \\
\hline Shoals07-S14-S2 & 0.00 & 0.00 & 0.00 & 99.53 & 0.47 & $\mathrm{~N} / \mathrm{A}$ & N/A & U & 2.24 & N/A & 1.63 & 0.32 & 2.45 & 0.18 & 2.43 & 0.19 & 0.58 & -0.08 & 1.10 & N/A \\
\hline
\end{tabular}


SH18 and Shoals07: Grain Size Distribution

\begin{tabular}{|c|c|c|c|c|c|c|c|c|c|c|c|c|c|c|c|c|c|c|c|c|c|c|c|c|c|c|c|c|}
\hline & $\begin{array}{c}\text { Class } \\
\% \\
\text { phi } \\
-5.5\end{array}$ & $\begin{array}{c}\text { Class } \\
\% \\
\text { phi } \\
-5.0\end{array}$ & $\begin{array}{c}\text { Class } \\
\% \\
\text { phi } \\
-4.5\end{array}$ & $\begin{array}{c}\text { Class } \\
\% \\
\text { phi } \\
-4.0\end{array}$ & $\begin{array}{c}\text { Class } \\
\% \\
\text { phi } \\
-3.5\end{array}$ & $\begin{array}{c}\text { Class } \\
\% \\
\text { phi } \\
-3.0\end{array}$ & $\begin{array}{c}\text { Class } \\
\% \\
\text { phi } \\
-2.5\end{array}$ & $\begin{array}{c}\text { Class } \\
\% \\
\text { phi } \\
-2.0\end{array}$ & $\begin{array}{c}\text { Class } \\
\% \\
\text { phi } \\
-1.5\end{array}$ & $\begin{array}{c}\text { Class } \\
\% \\
\text { phi } \\
-1.0\end{array}$ & $\begin{array}{c}\text { Class } \\
\% \\
\text { phi } \\
-0.5\end{array}$ & $\begin{array}{c}\text { Class } \\
\% \\
\text { phi } \\
0.0\end{array}$ & $\begin{array}{c}\text { Class } \\
\% \\
\text { phi } \\
0.5\end{array}$ & $\begin{array}{c}\text { Class } \\
\% \\
\text { phi } \\
1.0\end{array}$ & $\begin{array}{c}\text { Class } \\
\% \\
\text { phi } \\
1.5\end{array}$ & $\begin{array}{c}\text { Class } \\
\% \\
\text { phi } \\
2.0\end{array}$ & $\begin{array}{c}\text { Class } \\
\% \\
\text { phi } \\
2.5\end{array}$ & $\begin{array}{c}\text { Class } \\
\% \\
\text { phi } \\
3.0\end{array}$ & $\begin{array}{c}\text { Class } \\
\% \\
\text { phi } \\
3.5\end{array}$ & $\begin{array}{c}\text { Class } \\
\% \\
\text { phi } \\
4.0\end{array}$ & $\begin{array}{c}\text { Class } \\
\% \\
\text { phi } \\
>4.0\end{array}$ & $\begin{array}{c}\text { Class } \\
\% \\
\text { phi } \\
5.0\end{array}$ & $\begin{array}{c}\text { Class } \\
\% \\
\text { phi } \\
6.0\end{array}$ & $\begin{array}{c}\text { Class } \\
\% \\
\text { phi } \\
7.0\end{array}$ & $\begin{array}{c}\text { Class } \\
\% \\
\text { phi } \\
8.0\end{array}$ & $\begin{array}{c}\text { Class } \\
\% \\
\text { phi } \\
9.0\end{array}$ & $\begin{array}{c}\text { Class } \\
\% \\
\text { phi } \\
10.0\end{array}$ & $\begin{array}{c}\text { Class } \\
\% \\
\text { phi } \\
>10.0\end{array}$ \\
\hline SH18-T01 & 0.00 & 0.00 & 0.00 & 0.00 & 0.00 & 0.00 & 1.03 & 0.34 & 0.00 & 0.02 & 0.11 & 0.26 & 0.31 & 0.44 & 1.35 & 8.02 & 21.51 & 32.94 & 23.42 & 7.80 & 2.45 & N/A & N/A & N/A & N/A & N/A & N/A & N/A \\
\hline H18-T07 & 0.00 & 17.24 & 14.90 & 0.00 & 9.97 & 10.80 & 10.28 & 7.64 & 6.46 & 4.10 & 3.37 & 2.16 & 2.15 & 2.27 & 2.45 & 2.62 & 1.20 & 0.60 & 0.45 & 0.26 & 1.07 & N/A & N/A & N/A & N/A & N/A & N/A & N/A \\
\hline H18-T09 & 44.92 & 24.53 & 15.29 & 0.00 & 2.24 & 3.43 & 1.08 & 0.34 & 0.30 & 0.28 & 0.40 & 0.49 & 0.65 & 0.70 & 0.84 & 1.83 & 1.29 & 0.59 & 0.18 & 0.09 & 0.53 & N/A & N/A & N/A & N/A & N/A & N/A & N/A \\
\hline H18-T10 & 0.00 & 0.00 & 0.00 & 0.00 & 0.00 & 0.00 & 0.72 & 0.16 & 0.00 & 0.18 & 0.08 & 0.10 & 0.15 & 0.35 & 1.99 & 14.12 & 36.77 & 29.77 & 11.19 & 2.55 & 1.86 & N/A & N/A & N/A & N/A & N/A & N/A & N/A \\
\hline hoals07-S02-S1 & 0.00 & 0.00 & 0.00 & 0.00 & 0.00 & 0.00 & 0.00 & 0.00 & 0.04 & 0.04 & 0.03 & 0.08 & 0.18 & 0.33 & 0.37 & 1.16 & 6.59 & 32.42 & 48.51 & 8.80 & 1.44 & N/A & N/A & N/A & N/A & N/A & N/A & N/A \\
\hline Shoals07-S02-S2 & 0.00 & 0.00 & 0.00 & 0.00 & 0.00 & 0.00 & 0.00 & 0.00 & 0.00 & 0.10 & 0.06 & 0.06 & 0.18 & 0.30 & 0.30 & 1.11 & 7.60 & 33.65 & 46.21 & 8.98 & 1.47 & N/A & N/A & N/A & N/A & N/A & N/A & N/A \\
\hline Shoal & 0.00 & 0.00 & 0.00 & 0.00 & 0.00 & 1.54 & 0.54 & 1.60 & 1.68 & 3.92 & 10.14 & 14.95 & 19.60 & 21.69 & 13.00 & 5.59 & 2.73 & 1.52 & 0.93 & 0.15 & 0.42 & N/A & N/A & N/A & N/A & N/A & N/A & N/A \\
\hline Shoals07-S07-S2 & 0.00 & 0.00 & 0.00 & 0.00 & 0.00 & 0.36 & 1.06 & 2.41 & 3.10 & 7.61 & 13.70 & 16.28 & 20.12 & 20.00 & 8.97 & 3.31 & 1.42 & 0.66 & 0.48 & 0.08 & 0.46 & N/A & N/A & N/A & N/A & N/A & N/A & N/A \\
\hline Shoa & 0.00 & 0.00 & 0.00 & 0.00 & 0.00 & 0.00 & 0.00 & 0.03 & 0.25 & 0.97 & 4.66 & 14.76 & 32.60 & 32.15 & 9.47 & 2.26 & 0.95 & 0.71 & 0.54 & 0.13 & 0.54 & N/A & N/A & N/A & N/A & N/A & N/A & N/A \\
\hline Shoals07-S08-S2 & 0.00 & 0.00 & 0.00 & 4.64 & 0.00 & 0.55 & 1.20 & 2.26 & 4.20 & 6.21 & 10.31 & 15.21 & 24.89 & 20.30 & 5.53 & 1.67 & 0.71 & 0.72 & 1.01 & 0.17 & 0.42 & N/A & N/A & N/A & N/A & N/A & N/A & N/A \\
\hline Shoals07-S09-S1 & 0.00 & 0.00 & 0.00 & 0.00 & 0.00 & 0.00 & 0.00 & 0.00 & 0.00 & 0.01 & 0.03 & 0.26 & 1.58 & 3.17 & 3.63 & 6.00 & 11.46 & 29.71 & 33.95 & 8.39 & 1.81 & $\mathrm{~N} / \mathrm{A}$ & N/A & N/A & N/A & N/A & N/A & N/A \\
\hline Shoals07-S09-S2 & 0.00 & 0.00 & 0.00 & 0.00 & 0.00 & 0.00 & 0.00 & 0.00 & 0.03 & 0.02 & 0.14 & 0.57 & 2.55 & 3.62 & 2.91 & 5.31 & 12.67 & 29.29 & 33.98 & 7.86 & 1.05 & N/A & N/A & N/A & N/A & N/A & N/A & N/A \\
\hline Shoals07-S11-S1 & 0.00 & 0.00 & 0.00 & 0.00 & 0.00 & 0.50 & 0.00 & 0.59 & 1.05 & 2.85 & 8.79 & 19.27 & 29.47 & 21.84 & 9.22 & 3.32 & 1.31 & 0.96 & 0.40 & 0.08 & 0.36 & N/A & N/A & N/A & N/A & N/A & N/A & N/A \\
\hline Shoals07-S11-S2 & 0.00 & 0.00 & 0.00 & 0.00 & 0.00 & 0.00 & 0.00 & 0.00 & 0.05 & 0.10 & 0.63 & 5.51 & 23.04 & 36.71 & 17.61 & 5.95 & 2.89 & 4.24 & 2.66 & 0.22 & 0.41 & N/A & N/A & N/A & N/A & N/A & N/A & N/A \\
\hline Shoals07-S13-S1 & 0.00 & 0.00 & 0.00 & 0.00 & 0.00 & 0.00 & 0.03 & 0.25 & 0.39 & 1.39 & 7.79 & 16.75 & 20.73 & 22.16 & 18.60 & 8.35 & 1.61 & 1.01 & 0.45 & 0.08 & 0.41 & N/A & N/A & N/A & N/A & N/A & N/A & N/A \\
\hline Shoals07-S13-S2 & 0.00 & 0.00 & 0.00 & 0.00 & 0.00 & 0.00 & 0.00 & 0.03 & 0.03 & 0.16 & 2.27 & 10.95 & 22.34 & 30.64 & 23.33 & 7.97 & 1.12 & 0.46 & 0.29 & 0.05 & 0.37 & N/A & N/A & N/A & N/A & N/A & N/A & N/A \\
\hline Shoals07-S14-S1 & 0.00 & 0.00 & 0.00 & 0.00 & 0.00 & 0.00 & 0.14 & 0.00 & 0.00 & 0.04 & 0.04 & 0.21 & 0.77 & 2.26 & 4.91 & 11.17 & 26.19 & 36.68 & 15.34 & 1.66 & 0.57 & N/A & N/A & N/A & N/A & N/A & N/A & N/A \\
\hline Shoals07-S14-S2 & 0.00 & 0.00 & 0.00 & 0.00 & 0.00 & 0.00 & 0.00 & 0.00 & 0.00 & 0.00 & 0.01 & 0.10 & 0.38 & 1.58 & 4.44 & 12.62 & 32.55 & 33.70 & 13.10 & 1.04 & 0.47 & N/A & N/A & N/A & N/A & N/A & N/A & N/A \\
\hline
\end{tabular}


Section 7: NSBE 
NSBE (1): Identification, Location, and Description

\begin{tabular}{|c|c|c|c|c|c|c|c|c|}
\hline Sample ID & Global Sample ID & Station ID & $\begin{array}{c}\text { Latitude } \\
\text { WGS84 }\end{array}$ & $\begin{array}{c}\text { Longitude } \\
\text { WGS84 }\end{array}$ & $\begin{array}{l}\text { Reliability } \\
\text { Ranking for } \\
\text { Positioning }\end{array}$ & $\begin{array}{c}\text { Water } \\
\text { Depth }(\mathrm{m})\end{array}$ & $\begin{array}{c}\text { Sample } \\
\text { Collected }\end{array}$ & $\begin{array}{c}\text { Total Wt } \\
(\mathrm{gm})\end{array}$ \\
\hline NSBE-T01-S2 & NSBE-T01-S2_8/8/2017 & NSBE-T01 & 42.968322 & -70.660788 & 1 & 43.6 & $8 / 8 / 2017$ & 53.9 \\
\hline NSBE-T02-S1 & NSBE-T02-S1_8/8/2017 & NSBE-T02 & 42.971764 & -70.653901 & 1 & 40.5 & $8 / 8 / 2017$ & 90.0 \\
\hline NSBE-T03-S1 & NSBE-T03-S1_8/7/2017 & NSBE-T03 & 42.976383 & -70.643870 & 1 & 35.7 & $8 / 7 / 2017$ & 103.4 \\
\hline NSBE-T04-S1 & NSBE-T04-S1_8/7/2017 & NSBE-T04 & 42.990159 & -70.638573 & 1 & 20.7 & $8 / 7 / 2017$ & 173.3 \\
\hline NSBE-T04-S2 & NSBE-T04-S2_8/7/2017 & NSBE-T04 & 42.990297 & -70.638753 & 1 & 20.7 & $8 / 7 / 2017$ & 200.7 \\
\hline NSBE-T05-S1 & NSBE-T05-S1_8/7/2017 & NSBE-T05 & 42.986699 & -70.645589 & 1 & 24.4 & $8 / 7 / 2017$ & 169.4 \\
\hline NSBE-T06a-S1 & NSBE-T06a-S1_8/7/2017 & NSBE-T06a & 42.980573 & -70.655550 & 1 & 27.1 & $8 / 7 / 2017$ & 160.5 \\
\hline NSBE-T10-S1 & NSBE-T10-S1_8/7/2017 & NSBE-T10 & 42.976370 & -70.678328 & 1 & 37.5 & $8 / 7 / 2017$ & 52.7 \\
\hline NSBE-T12-S1 & NSBE-T12-S1_8/8/2017 & NSBE-T12 & 42.984786 & -70.685358 & 1 & 32.3 & $8 / 8 / 2017$ & 193.8 \\
\hline NSBE-T13-S1 & NSBE-T13-S1_8/8/2017 & NSBE-T13 & 42.987658 & -70.660873 & 1 & 30.5 & $8 / 8 / 2017$ & 141.8 \\
\hline NSBE-T13-S2 & NSBE-T13-S2_8/8/2017 & NSBE-T13 & 42.987847 & -70.660993 & 1 & 30.5 & $8 / 8 / 2017$ & 157.1 \\
\hline NSBE-T14-S1 & NSBE-T14-S1_8/8/2017 & NSBE-T14 & 42.990282 & -70.668139 & 1 & 26.8 & $8 / 8 / 2017$ & 208.8 \\
\hline NSBE-T15-S2 & NSBE-T15-S2 8/8/2017 & NSBE-T15 & 42.991793 & -70.674793 & 1 & 29.9 & $8 / 8 / 2017$ & 140.4 \\
\hline NSBE-T20-S1 & NSBE-T20-S1_8/8/2017 & NSBE-T20 & 42.987371 & -70.694519 & 1 & 25.3 & $8 / 8 / 2017$ & 358.1 \\
\hline NSBE-T20-S2 & NSBE-T20-S2_8/8/2017 & NSBE-T20 & 42.987267 & -70.694491 & 1 & 25.3 & $8 / 8 / 2017$ & 251.8 \\
\hline NSBE-T21a-S1 & NSBE-T21a-S1_8/8/2017 & NSBE-T21a & 42.991405 & -70.702695 & 1 & 27.4 & $8 / 8 / 2017$ & 300.2 \\
\hline NSBE-T21a-S2 & NSBE-T21a-S2_8/8/2017 & NSBE-T21a & 42.991447 & -70.702537 & 1 & 27.4 & $8 / 8 / 2017$ & 297.6 \\
\hline NSBE-T29-S1 & NSBE-T29-S1_8/15/2017 & NSBE-T29 & 42.979621 & -70.679554 & 1 & 26.5 & $8 / 15 / 2017$ & 318.5 \\
\hline
\end{tabular}


NSBE (1): Sediment Classifications

\begin{tabular}{|c|c|c|c|c|c|c|c|c|}
\hline Sample ID & $\begin{array}{l}\text { CMECS Substrate } \\
\text { Component } \\
\text { Group (Specific) }\end{array}$ & $\begin{array}{l}\text { Component } \\
\text { Subgroup } \\
\text { (Specific) } \\
\end{array}$ & $\begin{array}{l}\text { Textural Group } \\
\text { from \%GSM } \\
\text { (Gradistat) }\end{array}$ & $\begin{array}{l}\text { Sediment Name from \%GSM } \\
\text { and Mode (Gradistat) }\end{array}$ & $\begin{array}{l}\text { Sediment Name from } \\
\text { \%GSM and Mode } \\
\text { (Wentworth Scale) }\end{array}$ & $\begin{array}{l}\text { Sediment } \\
\text { Classification from } \\
\text { Mean Phi (Gradistat) }\end{array}$ & $\begin{array}{l}\text { Classification from } \\
\text { Mean Phi } \\
\text { (Wentworth) }\end{array}$ & $\begin{array}{l}\text { Sorting } \\
\text { (Gradistat) }\end{array}$ \\
\hline NSBE-T01-S2 & Slightly Pebbly & $\begin{array}{l}\text { Slightly Pebbly } \\
\text { Fine Sand }\end{array}$ & $\begin{array}{l}\text { Slightly Gravelly } \\
\text { Sand }\end{array}$ & Slightly Fine Gravelly Fine Sand & Slightly Pebbly Fine Sand & Fine Sand & Fine Sand & Well Sorted \\
\hline NSBE-T02-S1 & Slightly Pebbly & $\begin{array}{l}\text { Slightly Pebbly } \\
\text { Very Fine Sand }\end{array}$ & $\begin{array}{l}\text { Slightly Gravelly } \\
\text { Sand }\end{array}$ & $\begin{array}{l}\text { Slightly Fine Gravelly Very Fine } \\
\text { Sand }\end{array}$ & $\begin{array}{l}\text { Slightly Pebbly Very Fine } \\
\text { Sand }\end{array}$ & Very Fine Sand & Very Fine Sand & $\begin{array}{l}\text { Moderately } \\
\text { Sorted }\end{array}$ \\
\hline NSBE-T03-S1 & Slightly Pebbly & $\begin{array}{l}\text { Slightly Pebbly } \\
\text { Very Fine Sand }\end{array}$ & $\begin{array}{l}\text { Slightly Gravelly } \\
\text { Sand }\end{array}$ & $\begin{array}{l}\text { Slightly Fine Gravelly Very Fine } \\
\text { Sand }\end{array}$ & $\begin{array}{l}\text { Slightly Pebbly Very Fine } \\
\text { Sand }\end{array}$ & Very Fine Sand & Very Fine Sand & Well Sorted \\
\hline NSBE-T04-S1 & Pebbly & $\begin{array}{l}\text { Pebbly Medium } \\
\text { Sand }\end{array}$ & Gravelly Sand & Medium Gravelly Medium Sand & Pebbly Medium Sand & Coarse Sand & Coarse Sand & Poorly Sorted \\
\hline NSBE-T04-S2 & Pebbly & $\begin{array}{l}\text { Pebbly Medium } \\
\text { Sand }\end{array}$ & Gravelly Sand & Coarse Gravelly Medium Sand & Pebbly Medium Sand & Coarse Sand & Coarse Sand & $\begin{array}{l}\text { Moderately } \\
\text { Sorted }\end{array}$ \\
\hline NSBE-T05-S1 & Slightly Granuley & $\begin{array}{l}\text { Slightly Granuley } \\
\text { Medium Sand }\end{array}$ & $\begin{array}{l}\text { Slightly Gravelly } \\
\text { Sand }\end{array}$ & $\begin{array}{l}\text { Slightly Very Fine Gravelly } \\
\text { Medium Sand }\end{array}$ & $\begin{array}{l}\text { Slightly Granular Medium } \\
\text { Sand }\end{array}$ & Medium Sand & Medium Sand & $\begin{array}{l}\text { Moderately Well } \\
\text { Sorted }\end{array}$ \\
\hline NSBE-T06a-S1 & Sand & Medium Sand & Sand & $\begin{array}{l}\text { Moderately Well Sorted } \\
\text { Medium Sand }\end{array}$ & Medium Sand & Medium Sand & Medium Sand & $\begin{array}{l}\text { Moderately Well } \\
\text { Sorted }\end{array}$ \\
\hline NSBE-T10-S1 & Slightly Pebbly & $\begin{array}{l}\text { Slightly Pebbly } \\
\text { Fine Sand }\end{array}$ & $\begin{array}{l}\text { Slightly Gravelly } \\
\text { Sand }\end{array}$ & Slightly Fine Gravelly Fine Sand & Slightly Pebbly Fine Sand & Medium Sand & Medium Sand & Poorly Sorted \\
\hline NSBE-T12-S1 & Slightly Pebbly & $\begin{array}{l}\text { Slightly Pebbly } \\
\text { Fine Sand }\end{array}$ & $\begin{array}{l}\text { Slightly Gravelly } \\
\text { Sand }\end{array}$ & Slightly Fine Gravelly Fine Sand & Slightly Pebbly Fine Sand & Fine Sand & Fine Sand & $\begin{array}{l}\text { Moderately } \\
\text { Sorted }\end{array}$ \\
\hline NSBE-T13-S1 & Slightly Pebbly & $\begin{array}{l}\text { Slightly Pebbly } \\
\text { Fine Sand }\end{array}$ & $\begin{array}{l}\text { Slightly Gravelly } \\
\text { Sand }\end{array}$ & $\begin{array}{l}\text { Slightly Medium Gravelly Fine } \\
\text { Sand }\end{array}$ & Slightly Pebbly Fine Sand & Fine Sand & Fine Sand & $\begin{array}{l}\text { Moderately Well } \\
\text { Sorted }\end{array}$ \\
\hline NSBE-T13-S2 & Slightly Pebbly & $\begin{array}{l}\text { Slightly Pebbly } \\
\text { Fine Sand }\end{array}$ & $\begin{array}{l}\text { Slightly Gravelly } \\
\text { Sand }\end{array}$ & $\begin{array}{l}\text { Slightly Medium Gravelly Fine } \\
\text { Sand }\end{array}$ & Slightly Pebbly Fine Sand & Fine Sand & Fine Sand & $\begin{array}{l}\text { Moderately } \\
\text { Sorted }\end{array}$ \\
\hline NSBE-T14-S1 & Slightly Granuley & $\begin{array}{l}\text { Slightly Granuley } \\
\text { Fine Sand }\end{array}$ & $\begin{array}{l}\text { Slightly Gravelly } \\
\text { Sand }\end{array}$ & $\begin{array}{l}\text { Slightly Very Fine Gravelly Fine } \\
\text { Sand }\end{array}$ & $\begin{array}{l}\text { Slightly Granular } \\
\text { Fine Sand }\end{array}$ & Medium Sand & Medium Sand & Poorly Sorted \\
\hline NSBE-T15-S2 & Slightly Pebbly & $\begin{array}{l}\text { Slightly Pebbly } \\
\text { Fine Sand }\end{array}$ & $\begin{array}{l}\text { Slightly Gravelly } \\
\text { Sand }\end{array}$ & $\begin{array}{l}\text { Slightly Medium Gravelly Fine } \\
\text { Sand }\end{array}$ & Slightly Pebbly Fine Sand & Fine Sand & Fine Sand & $\begin{array}{l}\text { Moderately Well } \\
\text { Sorted }\end{array}$ \\
\hline NSBE-T20-S1 & Pebble Mixes & $\begin{array}{l}\text { Sandy Pebble } \\
\text { Gravel }\end{array}$ & Sandy Gravel & Sandy Medium Gravel & Sandy Pebble Gravel & Very Coarse Sand & Very Coarse Sand & $\begin{array}{l}\text { Very Poorly } \\
\text { Sorted }\end{array}$ \\
\hline NSBE-T2O-S2 & Pebble Mixes & $\begin{array}{l}\text { Sandy Pebble } \\
\text { Gravel }\end{array}$ & Sandy Gravel & Sandy Fine Gravel & Sandy Pebble Gravel & Coarse Sand & Coarse Sand & $\begin{array}{l}\text { Very Poorly } \\
\text { Sorted }\end{array}$ \\
\hline NSBE-T21a-S1 & Pebble Mixes & $\begin{array}{l}\text { Sandy Pebble } \\
\text { Gravel }\end{array}$ & Sandy Gravel & Sandy Medium Gravel & Sandy Pebble Gravel & Coarse Sand & Coarse Sand & $\begin{array}{l}\text { Very Poorly } \\
\text { Sorted }\end{array}$ \\
\hline NSBE-T21a-S2 & Pebble Mixes & $\begin{array}{l}\text { Sandy Pebble } \\
\text { Gravel }\end{array}$ & Sandy Gravel & Sandy Medium Gravel & Sandy Pebble Gravel & Coarse Sand & Coarse Sand & $\begin{array}{l}\text { Very Poorly } \\
\text { Sorted }\end{array}$ \\
\hline NSBE-T29-S1 & Pebble Mixes & $\begin{array}{l}\text { Sandy Pebble } \\
\text { Gravel }\end{array}$ & Sandy Gravel & Sandy Medium Gravel & Sandy Pebble Gravel & Very Coarse Sand & Very Coarse Sand & $\begin{array}{l}\text { Very Poorly } \\
\text { Sorted }\end{array}$ \\
\hline
\end{tabular}


NSBE (1): Grain Size Statistics

\begin{tabular}{|c|c|c|c|c|c|c|c|c|c|c|c|c|c|c|c|c|c|c|c|c|}
\hline Sample ID & $\begin{array}{c}\text { Gravel } \\
\%\end{array}$ & $\begin{array}{c}\text { Pebble } \\
\%\end{array}$ & $\begin{array}{c}\text { Granule } \\
\%\end{array}$ & Sand \% & Mud \% & Silt \% & Clay \% & Modes & $\begin{array}{c}\text { Mode } 1 \\
\text { (phi) }\end{array}$ & $\begin{array}{c}\text { Mode } 2 \\
\text { (phi) }\end{array}$ & $\begin{array}{c}D_{10} \\
\text { (phi) }\end{array}$ & $\begin{array}{c}D_{10} \\
(\mathrm{~mm})\end{array}$ & $\begin{array}{c}D_{50} \\
\text { (phi) }\end{array}$ & $\begin{array}{c}D_{50} \\
(\mathrm{~mm})\end{array}$ & $\begin{array}{c}\text { Mean } \\
\text { Size } \\
\text { (phi) }\end{array}$ & $\begin{array}{c}\text { Mean } \\
\text { Size } \\
(\mathrm{mm})\end{array}$ & $\begin{array}{l}\text { Sorting } \\
\text { (phi) }\end{array}$ & Skewness & Kurtosis & $\begin{array}{l}\text { LOI \% } \\
450^{\circ} \mathrm{C}\end{array}$ \\
\hline NSBE-T01-S2 & 1.21 & 1.09 & 0.12 & 95.00 & 3.79 & N/A & N/A & $U$ & 2.74 & N/A & 2.48 & 0.18 & 2.94 & 0.13 & 2.96 & 0.13 & 0.46 & 0.07 & 1.14 & N/A \\
\hline NSBE-T02-S1 & 3.13 & 2.80 & 0.33 & 89.60 & 7.27 & 4.03 & 3.24 & U & 3.24 & $\mathrm{~N} / \mathrm{A}$ & 2.48 & 0.18 & 3.10 & 0.12 & 3.05 & 0.12 & 0.72 & 0.00 & 2.12 & 0.98 \\
\hline NSBE-T03-S1 & 0.54 & 0.42 & 0.12 & 95.68 & 3.78 & N/A & N/A & $\mathrm{U}$ & 3.24 & N/A & 2.49 & 0.18 & 3.05 & 0.12 & 3.02 & 0.12 & 0.48 & -0.09 & 1.19 & 0.72 \\
\hline NSBE-T04-S1 & 5.62 & 5.11 & 0.52 & 94.28 & 0.10 & N/A & N/A & $U$ & 1.25 & N/A & 0.07 & 0.95 & 1.01 & 0.50 & 0.94 & 0.52 & 1.04 & -0.47 & 3.11 & 0.31 \\
\hline NSBE-T04-S2 & 6.18 & 5.46 & 0.72 & 93.69 & 0.13 & N/A & N/A & $\mathrm{U}$ & 1.25 & N/A & 1.25 & 0.42 & 1.02 & 0.49 & 0.92 & 0.53 & 0.95 & -0.47 & 2.44 & 0.32 \\
\hline NSBE-T05-S1 & 1.22 & 0.41 & 0.80 & 98.54 & 0.24 & N/A & N/A & $U$ & 1.25 & N/A & 0.35 & 0.78 & 1.21 & 0.43 & 1.18 & 0.44 & 0.59 & -0.15 & 0.97 & 0.32 \\
\hline NSBE-T06a-S1 & 0.00 & 0.00 & 0.00 & 99.56 & 0.44 & N/A & N/A & U & 1.75 & N/A & 0.83 & 0.56 & 1.70 & 0.31 & 1.65 & 0.32 & 0.56 & -0.17 & 1.18 & 0.42 \\
\hline NSBE-T10-S1 & 3.75 & 2.82 & 0.93 & 92.40 & 3.85 & N/A & N/A & $U$ & 2.24 & N/A & 0.46 & 0.73 & 2.06 & 0.24 & 1.88 & 0.27 & 1.08 & -0.26 & 1.07 & 0.85 \\
\hline NSBE-T12-S1 & 3.89 & 3.18 & 0.71 & 90.21 & 5.90 & 3.11 & 2.79 & U & 2.74 & N/A & 1.69 & 0.31 & 2.71 & 0.15 & 2.68 & 0.16 & 0.93 & -0.10 & 3.21 & 0.76 \\
\hline NSBE-T13-S1 & 2.02 & 1.26 & 0.76 & 95.91 & 2.07 & N/A & N/A & U & 2.24 & N/A & 1.12 & 0.46 & 2.18 & 0.22 & 2.14 & 0.23 & 0.69 & -0.25 & 1.53 & 0.91 \\
\hline NSBE-T13-S2 & 0.99 & 0.65 & 0.34 & 95.57 & 3.44 & N/A & N/A & U & 2.24 & N/A & 1.10 & 0.47 & 2.21 & 0.22 & 2.17 & 0.22 & 0.76 & -0.17 & 1.57 & 0.97 \\
\hline NSBE-T14-S1 & 4.37 & 1.42 & 2.95 & 94.49 & 1.14 & $\mathrm{~N} / \mathrm{A}$ & N/A & $U$ & 2.24 & $\mathrm{~N} / \mathrm{A}$ & 0.13 & 0.91 & 1.96 & 0.26 & 1.79 & 0.29 & 1.06 & -0.36 & 1.31 & 0.48 \\
\hline NSBE-T15-S2 & 1.60 & 1.55 & 0.05 & 95.70 & 2.70 & N/A & N/A & U & 2.24 & N/A & 1.54 & 0.34 & 2.33 & 0.20 & 2.31 & 0.20 & 0.62 & -0.07 & 1.17 & 0.77 \\
\hline NSBE-T20-S1 & 51.93 & 48.90 & 3.04 & 46.72 & 1.35 & N/A & N/A & B & -3.24 & 2.24 & -3.46 & 11.00 & -1.64 & 3.12 & -0.86 & 1.81 & 2.45 & 0.39 & 0.51 & 0.53 \\
\hline NSBE-T20-S2 & 31.17 & 27.82 & 3.35 & 66.67 & 2.16 & N/A & N/A & B & 2.24 & -3.24 & -3.17 & 9.03 & 1.83 & 0.28 & 0.53 & 0.69 & 2.36 & -0.66 & 0.56 & 0.56 \\
\hline NSBE-T21a-S1 & 40.50 & 38.08 & 2.42 & 57.48 & 2.02 & N/A & N/A & B & 1.75 & -3.74 & -3.70 & 13.03 & 1.33 & 0.40 & 0.05 & 0.97 & 2.49 & -0.59 & 0.52 & 0.54 \\
\hline NSBE-T21a-S2 & 31.90 & 29.63 & 2.27 & 64.83 & 3.27 & N/A & N/A & B & 1.75 & -3.24 & -3.44 & 10.82 & 1.64 & 0.32 & 0.30 & 0.81 & 2.47 & -0.63 & 0.57 & 0.84 \\
\hline NSBE-T29-S1 & 48.74 & 39.38 & 9.37 & 48.53 & 2.73 & N/A & N/A & B & -3.74 & 1.25 & -3.75 & 13.49 & -0.84 & 1.79 & -0.74 & 1.67 & 2.50 & 0.09 & 0.64 & 0.62 \\
\hline
\end{tabular}


NSBE (1): Grain Size Distribution

\begin{tabular}{|c|c|c|c|c|c|c|c|c|c|c|c|c|c|c|c|c|c|c|c|c|c|c|c|c|c|c|c|c|}
\hline & $\begin{array}{c}\text { Class } \\
\% \\
\text { phi }\end{array}$ & $\begin{array}{c}\text { Class } \\
\% \\
\text { phi }\end{array}$ & $\begin{array}{c}\text { Class } \\
\% \\
\text { phi }\end{array}$ & $\begin{array}{c}\text { Class } \\
\% \\
\text { phi }\end{array}$ & $\begin{array}{c}\text { Class } \\
\% \\
\text { phi }\end{array}$ & $\begin{array}{c}\text { Class } \\
\% \\
\text { phi }\end{array}$ & $\begin{array}{c}\text { Class } \\
\% \\
\text { phi }\end{array}$ & $\begin{array}{c}\text { Class } \\
\% \\
\text { phi }\end{array}$ & $\begin{array}{c}\text { Class } \\
\% \\
\text { phi }\end{array}$ & $\begin{array}{c}\text { Class } \\
\% \\
\text { phi }\end{array}$ & $\begin{array}{c}\text { Class } \\
\% \\
\text { phi }\end{array}$ & $\begin{array}{c}\text { Class } \\
\% \\
\text { phi }\end{array}$ & $\begin{array}{c}\text { Class } \\
\% \\
\text { phi }\end{array}$ & $\begin{array}{c}\text { Class } \\
\% \\
\text { phi }\end{array}$ & $\begin{array}{c}\text { Class } \\
\% \\
\text { phi }\end{array}$ & $\begin{array}{c}\text { Class } \\
\% \\
\text { phi }\end{array}$ & $\begin{array}{c}\text { Class } \\
\% \\
\text { phi }\end{array}$ & $\begin{array}{c}\text { Class } \\
\% \\
\text { phi }\end{array}$ & $\begin{array}{c}\text { Class } \\
\% \\
\text { phi } \\
\end{array}$ & $\begin{array}{c}\text { Class } \\
\% \\
\text { phi }\end{array}$ & $\begin{array}{c}\text { Class } \\
\% \\
\text { phi }\end{array}$ & $\begin{array}{c}\text { Class } \\
\% \\
\text { phi }\end{array}$ & $\begin{array}{c}\text { Clas } \\
\% \\
\text { phi }\end{array}$ & $\begin{array}{c}\text { Class } \\
\% \\
\text { phi } \\
7.0\end{array}$ & $\begin{array}{c}\text { Class } \\
\% \\
\text { phi } \\
8.0\end{array}$ & $\begin{array}{c}\text { Class } \\
\% \\
\text { phi } \\
9.0\end{array}$ & $\begin{array}{c}\text { Class } \\
\% \\
\text { phi } \\
10.0\end{array}$ & $\begin{array}{c}\text { Class } \\
\% \\
\text { phi } \\
>10.0\end{array}$ \\
\hline Sample ID & -5.5 & -5.0 & -4.5 & -4.0 & -3.5 & -3.0 & -2.5 & -2.0 & -1.5 & -1.0 & -0.5 & 0.0 & 0.5 & 1.0 & 1.5 & 2.0 & 2.5 & 3.0 & 3.5 & 4.0 & $>4.0$ & 5.0 & 6.0 & 7.0 & 8.0 & 9.0 & 10.0 & $>10.0$ \\
\hline VSBE-T01-S2 & 0.00 & 0.00 & 0.00 & 0.00 & 0.00 & 0.00 & 1.09 & 0.00 & 0.00 & 0.12 & 0.12 & 0.11 & 0.11 & 0.24 & 0.54 & 0.07 & 6.85 & 45.88 & 36.67 & 4.41 & 3.79 & $\mathrm{~N} / \mathrm{A}$ & $\mathrm{N} / \mathrm{A}$ & $\mathrm{N} / \mathrm{A}$ & $\mathrm{N} / \mathrm{A}$ & $\mathrm{N} / \mathrm{A}$ & N/A & $\mathrm{N} / \mathrm{A}$ \\
\hline VSBE-T02-S1 & 0.00 & 0.00 & 0.00 & 0.00 & 0.00 & 0.00 & 2.44 & 0.36 & 0.10 & 0.23 & 0.17 & 0.12 & 0.14 & 0.24 & 0.47 & 1.39 & 4.22 & 30.29 & 44.56 & 8.00 & N/A & 2.25 & 0.68 & 0.57 & 0.53 & 0.42 & 0.68 & 2.14 \\
\hline VSBE-T03-S1 & 0.00 & 0.00 & 0.00 & 0.00 & 0.00 & 0.00 & 0.00 & 0.42 & 0.07 & 0.05 & 0.17 & 0.12 & 0.17 & 0.37 & 0.74 & 1.71 & 5.13 & 36.13 & 43.90 & 7.23 & 3.78 & $\mathrm{~N} / \mathrm{A}$ & $\mathrm{N} / \mathrm{A}$ & N/A & N/A & $\mathrm{N} / \mathrm{A}$ & $\mathrm{N} / \mathrm{A}$ & $\mathrm{N} / \mathrm{A}$ \\
\hline VSBE-T04-S1 & 0.00 & 0.00 & 0.00 & 0.00 & 5.11 & 0.00 & 0.00 & 0.00 & 0.22 & 0.29 & 0.86 & 2.30 & 8.62 & 31.41 & 42.80 & 7.95 & 0.28 & 0.04 & 0.01 & 0.01 & 0.10 & N/A & $\mathrm{N} / \mathrm{A}$ & $\mathrm{N} / \mathrm{A}$ & $\mathrm{N} / \mathrm{A}$ & $\mathrm{N} / \mathrm{A}$ & $\mathrm{N} / \mathrm{A}$ & $\mathrm{N} / \mathrm{A}$ \\
\hline VSBE-T04-S2 & 0.00 & 0.00 & 0.00 & 3.07 & 1.13 & 0.48 & 0.42 & 0.36 & 0.24 & 0.48 & 0.91 & 2.66 & 10.28 & 27.94 & 41.48 & 9.90 & 0.36 & 0.09 & 0.07 & 0.02 & 0.13 & N/A & $\mathrm{N} / \mathrm{A}$ & N/A & N/A & N/A & $\mathrm{N} / \mathrm{A}$ & $\mathrm{N} / \mathrm{A}$ \\
\hline VSBE- & 00 & 0.00 & 0.00 & 0.00 & 0.00 & 0.00 & 0.00 & 0.41 & 0.29 & 0.52 & 0.90 & 2.43 & 7.64 & 23.06 & 34.39 & 27.52 & 2.38 & 0.17 & 0.03 & 0.01 & 0.25 & $\mathrm{~N} / \mathrm{A}$ & $\mathrm{N} / \mathrm{A}$ & $\mathrm{N} / \mathrm{A}$ & N/A & $\mathrm{N} / \mathrm{A}$ & $\mathrm{N} / \mathrm{A}$ & $\mathrm{N} / \mathrm{A}$ \\
\hline NSBE-T06a-S1 & 0.00 & 0.00 & 0.00 & 0.00 & 0.00 & 0.00 & 0.00 & 0.00 & 0.00 & 0.00 & 0.09 & 0.43 & 2.98 & 9.80 & 17.91 & 45.83 & 18.40 & 3.76 & 0.31 & 0.05 & 0.44 & $\mathrm{~N} / \mathrm{A}$ & $\mathrm{N} / \mathrm{A}$ & $\mathrm{N} / \mathrm{A}$ & $\mathrm{N} / \mathrm{A}$ & $\mathrm{N} / \mathrm{A}$ & $\mathrm{N} / \mathrm{A}$ & $\mathrm{N} / \mathrm{A}$ \\
\hline NSBE- & .00 & 0.00 & 0.00 & 0.00 & 0.00 & 0.00 & 2.51 & 0.31 & 0.64 & 0.28 & 0.69 & 1.51 & 4.34 & 10.67 & 12.46 & 13.49 & 23.11 & 20.96 & 4.18 & 0.98 & 3.85 & $\mathrm{~N} / \mathrm{A}$ & $\mathrm{N} / \mathrm{A}$ & N/A & $\mathrm{N} / \mathrm{A}$ & $\mathrm{N} / \mathrm{A}$ & $\mathrm{N} / \mathrm{A}$ & $\mathrm{N} / \mathrm{A}$ \\
\hline NSBE-T12-S1 & 0.00 & 0.00 & 0.00 & 0.00 & 0.00 & 1.56 & 1.07 & 0.55 & 0.34 & 0.37 & 0.35 & 0.45 & 0.55 & 0.98 & 2.07 & 4.46 & 13.86 & 51.15 & 13.34 & 3.01 & N/A & 1.47 & 0.7 & 0.51 & 0.43 & 0.36 & 0.50 & 1.93 \\
\hline NSBE-T13-S1 & 0.00 & 0.00 & 0.00 & 0.00 & 0.00 & 1.09 & 0.17 & 0.00 & 0.19 & 0.57 & 0.53 & 1.05 & 1.82 & 3.12 & 5.92 & 19.46 & 41.52 & 19.76 & 2.42 & 0.31 & 2.07 & $\mathrm{~N} / \mathrm{A}$ & $\mathrm{N} / \mathrm{A}$ & $\mathrm{N} / \mathrm{A}$ & $\mathrm{N} / \mathrm{A}$ & $\mathrm{N} / \mathrm{A}$ & $\mathrm{N} / \mathrm{A}$ & $\mathrm{N} / \mathrm{A}$ \\
\hline NSBE- & 0.00 & 0.00 & 0.00 & 0.00 & 0.00 & 0.57 & 0.00 & 0.08 & 0.10 & 0.24 & 0.91 & 1.62 & 2.04 & 3.28 & 5.94 & 18.22 & 39.07 & 21.07 & 2.94 & 0.48 & 3.44 & $\mathrm{~N} / \mathrm{A}$ & $\mathrm{N} / \mathrm{A}$ & $\mathrm{N} / \mathrm{A}$ & $\mathrm{N} / \mathrm{A}$ & $\mathrm{N} / \mathrm{A}$ & $\mathrm{N} / \mathrm{A}$ & N/A \\
\hline NSBE-T14-S1 & 0.00 & 0.00 & 0.00 & 0.00 & 0.00 & 0.54 & 0.43 & 0.45 & 1.21 & 1.74 & 2.22 & 2.52 & 3.30 & 6.94 & 11.94 & 20.53 & 25.48 & 18.46 & 2.80 & 0.30 & 1.14 & $\mathrm{~N} / \mathrm{A}$ & $\mathrm{N} / \mathrm{A}$ & N/A & N/A & $\mathrm{N} / \mathrm{A}$ & $\mathrm{N} / \mathrm{A}$ & N/A \\
\hline NSBE-T15-S2 & 0.00 & 0.00 & 0.00 & 0.00 & 0.00 & 1.55 & 0.00 & 0.00 & 0.03 & 0.02 & 0.07 & 0.09 & 0.18 & 1.09 & 5.38 & 17.65 & 34.17 & 31.18 & 5.24 & 0.66 & 2.70 & $\mathrm{~N} / \mathrm{A}$ & $\mathrm{N} / \mathrm{A}$ & $\mathrm{N} / \mathrm{A}$ & N/A & $\mathrm{N} / \mathrm{A}$ & $\mathrm{N} / \mathrm{A}$ & $\mathrm{N} / \mathrm{A}$ \\
\hline NSBE-T20-S1 & 0.00 & 0.00 & 0.00 & 2.05 & 6.62 & 24.54 & 10.88 & 4.80 & 1.59 & 1.45 & 1.63 & 1.58 & 1.85 & 2.32 & 2.37 & 6.60 & 18.14 & 10.42 & 1.52 & 0.28 & 1.35 & $\mathrm{~N} / \mathrm{A}$ & $\mathrm{N} / \mathrm{A}$ & $\mathrm{N} / \mathrm{A}$ & $\mathrm{N} / \mathrm{A}$ & $\mathrm{N} / \mathrm{A}$ & $\mathrm{N} / \mathrm{A}$ & $\mathrm{N} / \mathrm{A}$ \\
\hline NSBE-T2O-S2 & 0.00 & 0.00 & 0.00 & 0.00 & 3.50 & 10.14 & 9.58 & 4.60 & 1.82 & 1.53 & 1.51 & 1.77 & 2.38 & 2.85 & 3.52 & 10.39 & 25.20 & 15.97 & 2.66 & 0.42 & 2.16 & $\mathrm{~N} / \mathrm{A}$ & $\mathrm{N} / \mathrm{A}$ & N/A & N/A & $\mathrm{N} / \mathrm{A}$ & N/A & N/A \\
\hline NSBE-T21a-S1 & 0.00 & 0.00 & 0.00 & 0.00 & 17.37 & 14.56 & 4.70 & 1.45 & 1.31 & 1.11 & 1.20 & 0.99 & 1.22 & 2.32 & 5.69 & 20.26 & 16.58 & 7.25 & 1.57 & 0.40 & 2.02 & $\mathrm{~N} / \mathrm{A}$ & $\mathrm{N} / \mathrm{A}$ & N/A & N/A & N/A & $\mathrm{N} / \mathrm{A}$ & N/A \\
\hline NSBE-T21a-S2 & 0.00 & 0.00 & 0.00 & 0.00 & 8.61 & 13.32 & 6.23 & 1.47 & 1.23 & 1.05 & 1.18 & 0.96 & 1.22 & 2.11 & 5.65 & 23.58 & 17.83 & 9.33 & 2.42 & 0.54 & 3.27 & $\mathrm{~N} / \mathrm{A}$ & $\mathrm{N} / \mathrm{A}$ & N/A & $\mathrm{N} / \mathrm{A}$ & $\mathrm{N} / \mathrm{A}$ & $\mathrm{N} / \mathrm{A}$ & $\mathrm{N} / \mathrm{A}$ \\
\hline NSBE-T29-S1 & 0.00 & 0.00 & 0.00 & 2.05 & 16.63 & 5.69 & 8.24 & 6.78 & 4.90 & 4.47 & 4.04 & 3.76 & 4.30 & 6.15 & 7.42 & 6.70 & 6.91 & 6.49 & 2.14 & 0.61 & 2.73 & $\mathrm{~N} / \mathrm{A}$ & $\mathrm{N} / \mathrm{A}$ & N/A & N/A & $\mathrm{N} / \mathrm{A}$ & N/A & N/A \\
\hline
\end{tabular}


NSBE (2): Identification, Location, and Description

\begin{tabular}{|c|c|c|c|c|c|c|c|c|}
\hline Sample ID & Global Sample ID & Station ID & $\begin{array}{c}\text { Latitude } \\
\text { WGS84 }\end{array}$ & $\begin{array}{c}\text { Longitude } \\
\text { WGS84 }\end{array}$ & $\begin{array}{l}\text { Reliability } \\
\text { Ranking for } \\
\text { Positioning }\end{array}$ & $\begin{array}{c}\text { Water } \\
\text { Depth }(\mathrm{m})\end{array}$ & $\begin{array}{c}\text { Sample } \\
\text { Collected }\end{array}$ & $\begin{array}{c}\text { Total Wt } \\
\text { (gm) }\end{array}$ \\
\hline NSBE-T30-S1 & NSBE-T30-S1_8/15/2017 & NSBE-T30 & 42.985811 & -70.671644 & 1 & 26.2 & $8 / 15 / 2017$ & 154.7 \\
\hline NSBE-T32-S1 & NSBE-T32-S1_8/15/2017 & NSBE-T32 & 42.985106 & -70.663309 & 1 & 34.7 & $8 / 15 / 2017$ & 112.7 \\
\hline NSBE-T36-S1 & NSBE-T36-S1_8/16/2017 & NSBE-T36 & 42.995784 & -70.710278 & 1 & 25.6 & $8 / 16 / 2017$ & 205.3 \\
\hline NSBE-T36-S2 & NSBE-T36-S2_8/16/2017 & NSBE-T36 & 42.995662 & -70.710267 & 1 & 25.6 & $8 / 16 / 2017$ & 205.5 \\
\hline NSBE-T39-S1 & NSBE-T39-S1_8/16/2017 & NSBE-T39 & 43.005076 & -70.694332 & 1 & 23.5 & $8 / 16 / 2017$ & 362.2 \\
\hline NSBE-T39-S2 & NSBE-T39-S2_8/16/2017 & NSBE-T39 & 43.004815 & -70.694574 & 1 & 23.8 & $8 / 16 / 2017$ & 312.0 \\
\hline NSBE-T40-S1 & NSBE-T40-S1_8/16/2017 & NSBE-T40 & 42.973863 & -70.698420 & 1 & 32.9 & $8 / 16 / 2017$ & 809.3 \\
\hline NSBE-T40-S2 & NSBE-T40-S2_8/16/2017 & NSBE-T40 & 42.973978 & -70.698061 & 1 & 32.9 & $8 / 16 / 2017$ & 462.5 \\
\hline NSBE-T42-S1 & NSBE-T42-S1_8/16/2017 & NSBE-T42 & 42.971760 & -70.670194 & 1 & 37.2 & $8 / 16 / 2017$ & 130.0 \\
\hline NSBE-T45-S1 & NSBE-T45-S1_8/16/2017 & NSBE-T45 & 43.026969 & -70.648673 & 1 & 33.5 & $8 / 16 / 2017$ & 230.6 \\
\hline NSBE-T45-S2 & NSBE-T45-S2_8/16/2017 & NSBE-T45 & 43.026608 & -70.649007 & 1 & 33.5 & $8 / 16 / 2017$ & 318.4 \\
\hline NSBE-T61-S1 & NSBE-T61-S1_8/16/2017 & NSBE-T61 & 42.992317 & -70.659907 & 1 & 30.2 & $8 / 16 / 2017$ & 213.6 \\
\hline NSBE-T61-S2 & NSBE-T61-S2_8/16/2017 & NSBE-T61 & 42.992124 & -70.660016 & 1 & 30.2 & $8 / 16 / 2017$ & 183.3 \\
\hline NSBE-T62-S1 & NSBE-T62-S1_8/15/2017 & NSBE-T62 & 42.985456 & -70.653036 & 1 & 23.8 & $8 / 15 / 2017$ & 328.7 \\
\hline NSBE-T62-S2 & NSBE-T62-S2_8/15/2017 & NSBE-T62 & 42.985586 & -70.652722 & 1 & 23.8 & $8 / 15 / 2017$ & 195.4 \\
\hline NSBE-T64-S1 & NSBE-T64-S1_8/15/2017 & NSBE-T64 & 42.978964 & -70.663495 & 1 & 25.6 & $8 / 15 / 2017$ & 182.2 \\
\hline NSBE-T64-S2 & NSBE-T64-S2_8/15/2017 & NSBE-T64 & 42.979064 & -70.663322 & 1 & 25.6 & $8 / 15 / 2017$ & 112.7 \\
\hline
\end{tabular}


NSBE (2): Sediment Classifications

\begin{tabular}{|c|c|c|c|c|c|c|c|c|}
\hline Sample ID & $\begin{array}{l}\text { CMECS Substrate } \\
\text { Component } \\
\text { Group (Specific) } \\
\end{array}$ & $\begin{array}{l}\text { CMECS Substrate } \\
\text { Component } \\
\text { Subgroup (Specific) }\end{array}$ & $\begin{array}{l}\text { Textural Group } \\
\text { from \%GSM } \\
\text { (Gradistat) }\end{array}$ & $\begin{array}{l}\text { Sediment Name from \%GSM } \\
\text { and Mode (Gradistat) }\end{array}$ & $\begin{array}{l}\text { Sediment Name from } \\
\text { \%GSM and Mode } \\
\text { (Wentworth Scale) }\end{array}$ & $\begin{array}{l}\text { Sediment } \\
\text { Classification from } \\
\text { Mean Phi (Gradistat) }\end{array}$ & $\begin{array}{l}\text { Classification from } \\
\text { Mean Phi } \\
\text { (Wentworth) }\end{array}$ & $\begin{array}{l}\text { Sorting } \\
\text { (Gradistat) }\end{array}$ \\
\hline NSBE-T30-S1 & Slightly Granuley & $\begin{array}{l}\text { Slightly Granuley } \\
\text { Fine Sand }\end{array}$ & $\begin{array}{l}\text { Slightly Gravelly } \\
\text { Sand }\end{array}$ & $\begin{array}{l}\text { Slightly Very Fine Gravelly Fine } \\
\text { Sand }\end{array}$ & $\begin{array}{l}\text { Slightly Granular } \\
\text { Fine Sand }\end{array}$ & Fine Sand & Fine Sand & $\begin{array}{l}\text { Moderately } \\
\text { Sorted }\end{array}$ \\
\hline NSBE-T32-S1 & Slightly Pebbly & $\begin{array}{l}\text { Slightly Pebbly Fine } \\
\text { Sand }\end{array}$ & $\begin{array}{l}\text { Slightly Gravelly } \\
\text { Sand }\end{array}$ & $\begin{array}{l}\text { Slightly Medium Gravelly Fine } \\
\text { Sand }\end{array}$ & Slightly Pebbly Fine Sand & Fine Sand & Fine Sand & Poorly Sorted \\
\hline NSBE-T36-S1 & Pebbly & $\begin{array}{l}\text { Pebbly Silty-Clayey } \\
\text { Fine Sand }\end{array}$ & $\begin{array}{l}\text { Gravelly Muddy } \\
\text { Sand }\end{array}$ & $\begin{array}{l}\text { Medium Gravelly Very Coarse } \\
\text { Silty Fine Sand }\end{array}$ & $\begin{array}{l}\text { Pebbly Very Coarse Silty } \\
\text { Fine Sand }\end{array}$ & Fine Sand & Fine Sand & $\begin{array}{l}\text { Very Poorly } \\
\text { Sorted }\end{array}$ \\
\hline NSBE-T36-S2 & Pebbly & Pebbly Fine Sand & Gravelly Sand & Medium Gravelly Fine Sand & Pebbly Fine Sand & Medium Sand & Medium Sand & $\begin{array}{l}\text { Very Poorly } \\
\text { Sorted }\end{array}$ \\
\hline NSBE-T39-S1 & Pebble Mixes & $\begin{array}{l}\text { Sandy Pebble } \\
\text { Gravel }\end{array}$ & Sandy Gravel & Sandy Medium Gravel & Sandy Pebble Gravel & Very Coarse Sand & Very Coarse Sand & $\begin{array}{l}\text { Very Poorly } \\
\text { Sorted }\end{array}$ \\
\hline NSBE-T39-S2 & Pebble Mixes & $\begin{array}{l}\text { Sandy Pebble } \\
\text { Gravel }\end{array}$ & Sandy Gravel & Sandy Medium Gravel & Sandy Pebble Gravel & Coarse Sand & Coarse Sand & $\begin{array}{l}\text { Very Poorly } \\
\text { Sorted }\end{array}$ \\
\hline NSBE-T40-S1 & Gravel & Pebble Gravel & Gravel & Very Coarse Gravel & Pebble Gravel & Medium Gravel & Pebble Gravel & $\begin{array}{l}\text { Very Poorly } \\
\text { Sorted }\end{array}$ \\
\hline NSBE-T40-S2 & Pebble Mixes & $\begin{array}{l}\text { Silty-Clayey Sandy } \\
\text { Pebble Gravel }\end{array}$ & $\begin{array}{l}\text { Muddy Sandy } \\
\text { Gravel }\end{array}$ & $\begin{array}{l}\text { Muddy Sandy Very Coarse } \\
\text { Gravel }\end{array}$ & $\begin{array}{l}\text { Muddy Sandy Pebble } \\
\text { Gravel }\end{array}$ & Very Fine Gravel & Granule Gravel & $\begin{array}{l}\text { Very Poorly } \\
\text { Sorted }\end{array}$ \\
\hline NSBE-T42-S1 & Slightly Pebbly & $\begin{array}{l}\text { Slightly Pebbly Fine } \\
\text { Sand }\end{array}$ & $\begin{array}{l}\text { Slightly Gravelly } \\
\text { Sand }\end{array}$ & $\begin{array}{l}\text { Slightly Medium Gravelly Fine } \\
\text { Sand }\end{array}$ & Slightly Pebbly Fine Sand & Fine Sand & Fine Sand & Well Sorted \\
\hline NSBE-T45-S1 & Pebble Mixes & $\begin{array}{l}\text { Sandy Pebble } \\
\text { Gravel }\end{array}$ & Sandy Gravel & Sandy Medium Gravel & Sandy Pebble Gravel & Coarse Sand & Coarse Sand & $\begin{array}{l}\text { Very Poorly } \\
\text { Sorted }\end{array}$ \\
\hline NSBE-T45-S2 & Pebble Mixes & $\begin{array}{l}\text { Sandy Pebble } \\
\text { Gravel }\end{array}$ & Sandy Gravel & Sandy Medium Gravel & Sandy Pebble Gravel & Very Fine Gravel & Granule Gravel & $\begin{array}{l}\text { Very Poorly } \\
\text { Sorted }\end{array}$ \\
\hline NSBE-T61-S1 & Pebble Mixes & $\begin{array}{l}\text { Sandy Pebble } \\
\text { Gravel }\end{array}$ & Sandy Gravel & Sandy Medium Gravel & Sandy Pebble Gravel & Coarse Sand & Coarse Sand & $\begin{array}{l}\text { Very Poorly } \\
\text { Sorted }\end{array}$ \\
\hline NSBE-T61-S2 & Pebbly & Pebbly Fine Sand & Gravelly Sand & Medium Gravelly Fine Sand & Pebbly Fine Sand & Medium Sand & Medium Sand & Poorly Sorted \\
\hline NSBE-T62-S1 & Pebbly & Pebbly Coarse Sand & Gravelly Sand & Medium Gravelly Coarse Sand & Pebbly Coarse Sand & Coarse Sand & Coarse Sand & Poorly Sorted \\
\hline NSBE-T62-S2 & Slightly Pebbly & $\begin{array}{l}\text { Slightly Pebbly } \\
\text { Coarse Sand }\end{array}$ & $\begin{array}{l}\text { Slightly Gravelly } \\
\text { Sand }\end{array}$ & $\begin{array}{l}\text { Slightly Medium Gravelly } \\
\text { Coarse Sand }\end{array}$ & $\begin{array}{l}\text { Slightly Pebbly Coarse } \\
\text { Sand }\end{array}$ & Coarse Sand & Coarse Sand & $\begin{array}{l}\text { Moderately } \\
\text { Sorted }\end{array}$ \\
\hline NSBE-T64-S1 & Slightly Granuley & $\begin{array}{l}\text { Slightly Granuley } \\
\text { Medium Sand }\end{array}$ & $\begin{array}{l}\text { Slightly Gravelly } \\
\text { Sand }\end{array}$ & $\begin{array}{l}\text { Slightly Very Fine Gravelly } \\
\text { Medium Sand }\end{array}$ & $\begin{array}{l}\text { Slightly Granular Medium } \\
\text { Sand }\end{array}$ & Medium Sand & Medium Sand & $\begin{array}{l}\text { Moderately Well } \\
\text { Sorted }\end{array}$ \\
\hline NSBE-T64-S2 & Slightly Granuley & $\begin{array}{l}\text { Slightly Granuley } \\
\text { Medium Sand }\end{array}$ & $\begin{array}{l}\text { Slightly Gravelly } \\
\text { Sand }\end{array}$ & $\begin{array}{l}\text { Slightly Very Fine Gravelly } \\
\text { Medium Sand }\end{array}$ & $\begin{array}{l}\text { Slightly Granular Medium } \\
\text { Sand }\end{array}$ & Medium Sand & Medium Sand & $\begin{array}{l}\text { Moderately Well } \\
\text { Sorted }\end{array}$ \\
\hline
\end{tabular}




\section{NSBE (2): Grain Size Statistics}

\begin{tabular}{|c|c|c|c|c|c|c|c|c|c|c|c|c|c|c|c|c|c|c|c|c|}
\hline Sample ID & $\begin{array}{c}\text { Gravel } \\
\%\end{array}$ & $\begin{array}{c}\text { Pebble } \\
\%\end{array}$ & $\begin{array}{c}\text { Granule } \\
\%\end{array}$ & Sand \% & Mud \% & Silt \% & Clay $\%$ & Modes & $\begin{array}{c}\text { Mode } 1 \\
\text { (phi) }\end{array}$ & $\begin{array}{c}\text { Mode } 2 \\
\text { (phi) }\end{array}$ & $\begin{array}{r}D_{10} \\
\text { (phi) }\end{array}$ & $\begin{array}{c}D_{10} \\
(\mathrm{~mm})\end{array}$ & $\begin{array}{c}D_{50} \\
\text { (phi) }\end{array}$ & $\begin{array}{r}\mathrm{D}_{50} \\
(\mathrm{~mm}) \\
\end{array}$ & $\begin{array}{c}\text { Mean } \\
\text { Size } \\
\text { (phi) }\end{array}$ & $\begin{array}{c}\text { Mean } \\
\text { Size } \\
(\mathrm{mm})\end{array}$ & $\begin{array}{c}\text { Sorting } \\
\text { (phi) }\end{array}$ & Skewness & Kurtosis & $\begin{array}{r}\text { LOI \% } \\
450^{\circ} \mathrm{C} \\
\end{array}$ \\
\hline NSBE-T30-S1 & 1.26 & 0.16 & 1.10 & 96.50 & 2.24 & N/A & $\mathrm{N} / \mathrm{A}$ & U & 2.24 & $\mathrm{~N} / \mathrm{A}$ & 1.25 & 0.42 & 2.28 & 0.21 & 2.25 & 0.21 & 0.78 & -0.22 & 1.43 & 0.49 \\
\hline NSBE-T32-S1 & 1.19 & 0.80 & 0.39 & 90.03 & 8.78 & 4.32 & 4.46 & U & 2.74 & $\mathrm{~N} / \mathrm{A}$ & 2.06 & 0.24 & 2.76 & 0.15 & 2.78 & 0.15 & 1.18 & 0.34 & 3.95 & 0.90 \\
\hline NSBE-T36-S1 & 11.76 & 7.87 & 3.88 & 74.77 & 13.47 & 8.41 & 5.06 & U & 2.74 & $\mathrm{~N} / \mathrm{A}$ & -1.47 & 2.76 & 2.63 & 0.16 & 2.20 & 0.22 & 2.55 & -0.18 & 2.61 & 1.40 \\
\hline NSBE-T36-S2 & 18.38 & 13.08 & 5.30 & 77.93 & 3.69 & N/A & $\mathrm{N} / \mathrm{A}$ & B & 2.74 & -3.24 & -2.56 & 5.89 & 2.01 & 0.25 & 1.20 & 0.44 & 2.18 & -0.53 & 1.11 & 0.79 \\
\hline NSBE-T39-S1 & 50.86 & 49.30 & 1.56 & 47.33 & 1.81 & N/A & $\mathrm{N} / \mathrm{A}$ & B & -3.24 & 2.24 & -3.90 & 14.91 & -1.59 & 3.00 & -0.96 & 1.95 & 2.61 & 0.30 & 0.53 & 0.54 \\
\hline NSBE-T39-S2 & 41.46 & 40.84 & 0.62 & 55.54 & 3.00 & N/A & $\mathrm{N} / \mathrm{A}$ & B & -3.24 & 2.24 & -3.75 & 13.44 & 1.45 & 0.37 & 0.20 & 0.87 & 2.65 & -0.55 & 0.53 & 0.61 \\
\hline NSBE-T40-S1 & 82.27 & 77.93 & 4.34 & 16.10 & 1.63 & N/A & $\mathrm{N} / \mathrm{A}$ & B & -5.74 & -3.74 & -5.81 & 55.99 & -4.76 & 27.08 & -3.66 & 12.68 & 2.57 & 0.68 & 1.12 & 0.75 \\
\hline NSBE-T40-S2 & 55.82 & 50.13 & 5.69 & 39.61 & 4.57 & 2.28 & 2.29 & $\mathrm{P}$ & -5.24 & -3.74 & -5.24 & 37.87 & -2.02 & 4.06 & -1.64 & 3.12 & 3.19 & 0.21 & 0.69 & 0.91 \\
\hline NSBE-T42-S1 & 1.63 & 1.60 & 0.04 & 94.78 & 3.59 & $\mathrm{~N} / \mathrm{A}$ & $\mathrm{N} / \mathrm{A}$ & U & 2.24 & $\mathrm{~N} / \mathrm{A}$ & 1.80 & 0.29 & 2.43 & 0.19 & 2.45 & 0.18 & 0.50 & 0.07 & 1.17 & 0.68 \\
\hline NSBE-T45-S1 & 31.90 & 29.25 & 2.65 & 66.01 & 2.09 & N/A & $\mathrm{N} / \mathrm{A}$ & B & 2.24 & -3.74 & -3.66 & 12.62 & 1.84 & 0.28 & 0.37 & 0.78 & 2.59 & -0.68 & 0.53 & 0.73 \\
\hline NSBE-T45-S2 & 61.00 & 53.05 & 7.95 & 37.78 & 1.22 & N/A & $\mathrm{N} / \mathrm{A}$ & B & -3.24 & 2.24 & -4.06 & 16.66 & -2.30 & 4.93 & -1.36 & 2.56 & 2.54 & 0.46 & 0.64 & 0.54 \\
\hline NSBE-T61-S1 & 30.89 & 26.88 & 4.02 & 66.23 & 2.88 & N/A & $\mathrm{N} / \mathrm{A}$ & B & 2.24 & -3.24 & -3.18 & 9.07 & 1.41 & 0.38 & 0.30 & 0.81 & 2.30 & -0.56 & 0.57 & 0.75 \\
\hline NSBE-T61-S2 & 12.17 & 10.54 & 1.63 & 85.42 & 2.41 & $\mathrm{~N} / \mathrm{A}$ & $\mathrm{N} / \mathrm{A}$ & U & 2.24 & $\mathrm{~N} / \mathrm{A}$ & -2.20 & 4.60 & 2.01 & 0.25 & 1.58 & 0.34 & 1.61 & -0.56 & 1.88 & 0.75 \\
\hline NSBE-T62-S1 & 13.55 & 9.02 & 4.53 & 86.22 & 0.23 & N/A & N/A & U & 0.75 & $\mathrm{~N} / \mathrm{A}$ & -1.77 & 3.41 & 0.70 & 0.62 & 0.53 & 0.69 & 1.28 & -0.38 & 1.68 & 0.57 \\
\hline NSBE-T62-S2 & 3.72 & 2.53 & 1.19 & 95.44 & 0.84 & N/A & N/A & U & 0.75 & $\mathrm{~N} / \mathrm{A}$ & -0.11 & 1.08 & 0.82 & 0.56 & 0.83 & 0.56 & 0.74 & -0.07 & 1.07 & 0.46 \\
\hline NSBE-T64-S1 & 0.05 & 0.00 & 0.05 & 99.37 & 0.58 & N/A & N/A & U & 1.75 & $\mathrm{~N} / \mathrm{A}$ & 0.66 & 0.63 & 1.75 & 0.30 & 1.66 & 0.32 & 0.66 & -0.26 & 1.01 & 0.46 \\
\hline NSBE-T64-S2 & 0.04 & 0.00 & 0.04 & 99.17 & 0.79 & N/A & N/A & U & 1.75 & $N / A$ & 1.00 & 0.50 & 1.90 & 0.27 & 1.87 & 0.27 & 0.61 & -0.14 & 1.29 & 0.45 \\
\hline
\end{tabular}


NSBE (2): Grain Size Distribution

\begin{tabular}{|c|c|c|c|c|c|c|c|c|c|c|c|c|c|c|c|c|c|c|c|c|c|c|c|c|c|c|c|c|}
\hline & $\begin{array}{c}\text { Class } \\
\% \\
\text { phi } \\
-5.5\end{array}$ & $\begin{array}{c}\text { Class } \\
\% \\
\text { phi } \\
-5.0\end{array}$ & $\begin{array}{c}\text { Class } \\
\% \\
\text { phi } \\
-4.5\end{array}$ & $\begin{array}{c}\text { Class } \\
\% \\
\text { phi } \\
-4.0\end{array}$ & $\begin{array}{c}\text { Class } \\
\% \\
\text { phi } \\
-3.5\end{array}$ & $\begin{array}{c}\text { Class } \\
\% \\
\text { phi } \\
-3.0\end{array}$ & $\begin{array}{c}\text { Class } \\
\% \\
\text { phi } \\
-2.5\end{array}$ & $\begin{array}{c}\text { Class } \\
\% \\
\text { phi } \\
-2.0\end{array}$ & $\begin{array}{c}\text { Class } \\
\% \\
\text { phi }\end{array}$ & $\begin{array}{c}\text { Class } \\
\% \\
\text { phi }\end{array}$ & $\begin{array}{c}\text { Class } \\
\% \\
\text { phi }\end{array}$ & $\begin{array}{c}\text { Class } \\
\% \\
\text { phi }\end{array}$ & $\begin{array}{c}\text { Class } \\
\% \\
\text { phi }\end{array}$ & $\begin{array}{c}\text { Class } \\
\% \\
\text { phi } \\
1.0\end{array}$ & $\begin{array}{c}\text { Class } \\
\% \\
\text { phi }\end{array}$ & $\begin{array}{c}\text { Class } \\
\% \\
\text { phi } \\
2.0\end{array}$ & $\begin{array}{c}\text { Class } \\
\% \\
\text { phi }\end{array}$ & $\begin{array}{c}\text { Class } \\
\% \\
\text { phi } \\
\end{array}$ & $\begin{array}{c}\text { Class } \\
\% \\
\text { phi } \\
\end{array}$ & $\begin{array}{c}\text { Class } \\
\% \\
\text { phi }\end{array}$ & $\begin{array}{c}\text { Class } \\
\% \\
\text { phi }\end{array}$ & $\begin{array}{c}\text { Class } \\
\% \\
\text { phi }\end{array}$ & $\begin{array}{c}\text { Class } \\
\% \\
\text { phi }\end{array}$ & $\begin{array}{c}\text { Class } \\
\% \\
\text { phi }\end{array}$ & $\begin{array}{c}\text { Class } \\
\% \\
\text { phi }\end{array}$ & $\begin{array}{c}\text { Class } \\
\% \\
\text { phi }\end{array}$ & $\begin{array}{c}\text { Class } \\
\% \\
\text { phi }\end{array}$ & $\begin{array}{c}\text { Class } \\
\% \\
\text { phi } \\
>10.0\end{array}$ \\
\hline NSBE-T30-S1 & 0.00 & 0.00 & 0.00 & 0.00 & 0.00 & 0.00 & 0.00 & 0.16 & 0.29 & 0.81 & 1.23 & 1.74 & 1.55 & 1.97 & 4.52 & 19.29 & 30.67 & 29.49 & 5.34 & 0.70 & 2.24 & N/A & N/A & N/A & N/A & N/A & N/A & N/A \\
\hline NSBE-T32-S1 & 0.00 & 0.00 & 0.00 & 0.00 & 0.00 & 0.63 & 0.00 & 0.17 & 0.22 & 0.17 & 0.12 & 0.24 & 0.31 & 0.68 & 1.54 & 3.82 & 16.35 & 47.50 & 16.14 & 3.34 & N/A & 1.79 & 1.00 & 0.38 & 1.15 & 0.75 & 0.69 & 3.01 \\
\hline NSBE-T36-S1 & 0.00 & 0.00 & 0.00 & 0.00 & 1.72 & 2.49 & 2.24 & 1.42 & 2.09 & 1.79 & 1.87 & 1.63 & 2.03 & 2.92 & 3.66 & 6.36 & 12.87 & 23.07 & 14.22 & 6.14 & N/A & 3.36 & 2.40 & 1.64 & 1.01 & 0.84 & 2.63 & 1.60 \\
\hline NSBE-T36-S2 & 0.00 & 0.00 & 0.00 & 0.00 & 3.50 & 4.89 & 1.88 & 2.81 & 2.62 & 2.69 & 2.81 & 2.64 & 3.24 & 4.77 & 7.05 & 10.93 & 12.95 & 20.95 & 10.24 & 2.36 & 3.69 & N/A & N/A & N/A & N/A & $\mathrm{N} / \mathrm{A}$ & N/A & N/A \\
\hline NSBE-T39-S1 & 0.00 & 0.00 & 0.00 & 6.69 & 16.69 & 17.31 & 7.21 & 1.40 & 0.87 & 0.69 & 0.90 & 0.88 & 1.13 & 2.35 & 5.35 & 11.17 & 13.08 & 9.72 & 2.20 & 0.56 & 1.81 & $\mathrm{~N} / \mathrm{A}$ & $\mathrm{N} / \mathrm{A}$ & N/A & N/A & $\mathrm{N} / \mathrm{A}$ & $\mathrm{N} / \mathrm{A}$ & N/A \\
\hline NSBE-T39-S2 & 0.00 & 0.00 & 0.00 & 2.56 & 15.19 & 16.58 & 5.61 & 0.91 & 0.29 & 0.33 & 0.57 & 0.56 & 0.82 & 1.81 & 5.23 & 13.14 & 14.97 & 14.02 & 3.68 & 0.75 & 3.00 & N/A & $\mathrm{N} / \mathrm{A}$ & N/A & $\mathrm{N} / \mathrm{A}$ & N/A & $\mathrm{N} / \mathrm{A}$ & N/A \\
\hline NSBE-T40-S1 & 28.45 & 18.31 & 7.28 & 1.04 & 8.99 & 6.87 & 4.01 & 2.98 & 2.48 & 1.87 & 1.89 & 1.47 & 1.51 & 1.91 & 1.79 & 1.96 & 2.09 & 2.54 & 0.74 & 0.22 & 1.63 & N/A & $\mathrm{N} / \mathrm{A}$ & N/A & N/A & $\mathrm{N} / \mathrm{A}$ & N/A & N/A \\
\hline NSBE-T40-S2 & 0.00 & 20.66 & 0.00 & 7.49 & 8.49 & 6.43 & 4.08 & 2.98 & 2.94 & 2.75 & 3.33 & 3.56 & 4.83 & 5.78 & 4.98 & 4.14 & 4.02 & 5.85 & 2.35 & 0.78 & N/A & 0.79 & 0.57 & 0.46 & 0.46 & 0.40 & 0.35 & 1.55 \\
\hline NSBE-T42-S1 & 0.00 & 0.00 & 0.00 & 0.00 & 0.00 & 1.30 & 0.30 & 0.00 & 0.00 & 0.04 & 0.07 & 0.12 & 0.15 & 0.41 & 1.39 & 10.43 & 39.87 & 36.88 & 4.90 & 0.56 & 3.59 & N/A & $\mathrm{N} / \mathrm{A}$ & N/A & N/A & $\mathrm{N} / \mathrm{A}$ & N/A & N/A \\
\hline NSBE-T45-S1 & 0.00 & 0.00 & 0.00 & 0.00 & 15.06 & 9.68 & 2.38 & 2.12 & 1.50 & 1.15 & 1.46 & 1.48 & 1.50 & 1.95 & 3.66 & 11.67 & 23.37 & 17.00 & 3.43 & 0.49 & 2.09 & $\mathrm{~N} / \mathrm{A}$ & $\mathrm{N} / \mathrm{A}$ & N/A & $\mathrm{N} / \mathrm{A}$ & $\mathrm{N} / \mathrm{A}$ & N/A & N/A \\
\hline NSBE-T45-S2 & 0.00 & 0.00 & 0.00 & 11.36 & 14.05 & 14.49 & 8.25 & 4.89 & 4.30 & 3.65 & 4.44 & 3.81 & 3.23 & 2.66 & 2.45 & 5.21 & 8.97 & 5.78 & 1.02 & 0.21 & 1.22 & N/A & $\mathrm{N} / \mathrm{A}$ & N/A & N/A & $\mathrm{N} / \mathrm{A}$ & N/A & N/A \\
\hline NSBE-T61-S1 & 0.00 & 0.00 & 0.00 & 0.00 & 1.63 & 13.34 & 8.76 & 3.13 & 1.96 & 2.06 & 2.64 & 3.04 & 3.46 & 4.58 & 6.42 & 13.83 & 20.87 & 9.65 & 1.41 & 0.32 & 2.88 & $\mathrm{~N} / \mathrm{A}$ & N/A & N/A & $\mathrm{N} / \mathrm{A}$ & $\mathrm{N} / \mathrm{A}$ & N/A & N/A \\
\hline NSBE-T61-S2 & 0.00 & 0.00 & 0.00 & 0.00 & 2.43 & 3.28 & 3.54 & 1.30 & 0.94 & 0.69 & 1.42 & 2.21 & 3.51 & 4.44 & 7.43 & 18.33 & 27.33 & 17.30 & 2.93 & 0.53 & 2.41 & N/A & N/A & N/A & N/A & $\mathrm{N} / \mathrm{A}$ & N/A & N/A \\
\hline NSBE-T62-S1 & 0.00 & 0.00 & 0.00 & 0.00 & 2.56 & 2.69 & 1.95 & 1.83 & 2.18 & 2.35 & 3.33 & 6.64 & 15.94 & 26.33 & 18.04 & 13.46 & 2.19 & 0.24 & 0.03 & 0.01 & 0.23 & N/A & $\mathrm{N} / \mathrm{A}$ & N/A & $\mathrm{N} / \mathrm{A}$ & $\mathrm{N} / \mathrm{A}$ & N/A & N/A \\
\hline NSBE-T62-S2 & 0.00 & 0.00 & 0.00 & 0.00 & 0.00 & 1.37 & 0.40 & 0.77 & 0.35 & 0.83 & 1.78 & 5.82 & 19.24 & 29.82 & 22.08 & 15.12 & 1.26 & 0.19 & 0.09 & 0.03 & 0.84 & N/A & N/A & N/A & N/A & N/A & N/A & N/A \\
\hline NSBE-T64-S1 & 0.00 & 0.00 & 0.00 & 0.00 & 0.00 & 0.00 & 0.00 & 0.00 & 0.00 & 0.05 & 0.19 & 0.95 & 5.08 & 11.26 & 13.92 & 36.19 & 27.24 & 4.32 & 0.19 & 0.03 & 0.58 & N/A & N/A & N/A & N/A & N/A & N/A & N/A \\
\hline NSBE-T64-S2 & 0.00 & 0.00 & 0.00 & 0.00 & 0.00 & 0.00 & 0.00 & 0.00 & 0.00 & 0.04 & 0.16 & 0.71 & 3.04 & 5.99 & 10.13 & 37.10 & 31.65 & 9.74 & 0.60 & 0.05 & 0.79 & N/A & N/A & N/A & N/A & N/A & N/A & N/A \\
\hline
\end{tabular}

